\title{
OBSTACLES AND CHALLENGES AFFECTING THE MOVE TOWARDS UNIVERSAL HEALTHCARE COVERAGE IN NIGERIA
}

\author{
By Jenson Gawain Fofah
}

This thesis is submitted to Swansea University in fulfilment of the requirement for the degree of Doctor of Philosophy (PhD)

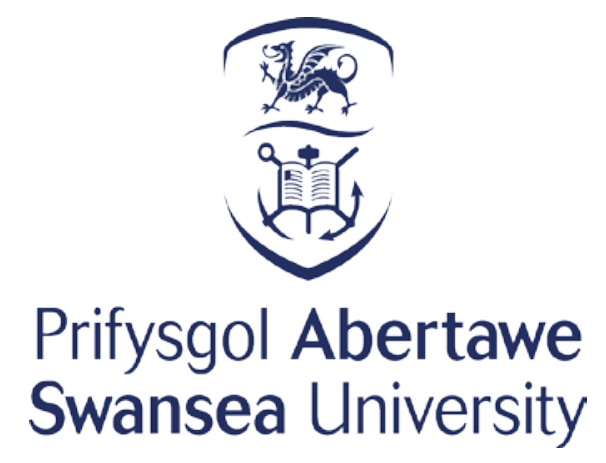




\begin{abstract}
There is increasing pressure for low and middle-income countries to move towards achieving universal healthcare coverage (UHC). According to WHO (2013), UHC guarantees the right to affordable health care for every individual, without financial hardship. The Director-General to the World Health Organization, Margaret Chan (WHO, 2010 p. 1), says UHC "is the single most powerful concept that public health has to offer"."

In recent years, UHC has come onto the policy agenda, but Nigeria has been criticised for its slow progress.

This study investigates why and how the UHC policy is developed by focusing on the roles and interactions of policy actors, their policy setting, and 'how' their actions influence the policy process and outcome of UHC so far in Nigeria. It will provide evidence of Nigeria's policy process that will enhance the understanding of the politics of such health care policy implementation processes, which is fundamental to the success of policy networks of UHC in low- and middle-income settings.

This is an empirical study using a mixed method approach involving quantitative and qualitative research components. The study combines the social network analysis (the quantitative component) with a more general policy process framework (the qualitative component). Data collected between March 2016 and February 2017 involved face-to-face structured interviews, face-to-face semi-structured interviews and documentary analysis to identify members of relevant policy networks and describe the pattern of relationships and influence within the UHC discourse.

The SNA analysis displayed a full structural network taxonomy of the UHC policy process and identified key members of the UHC discourse into four key institutions and organisations- such as the power actors, peripheral actors, gatekeepers or brokers, isolated actors, and policy actors connected to the power actors.

The theoretical policy process framework highlights five key gaps that exist as challenges and obstacles which impedes the implementation process of UHC Nigeria. These challenges and obstacles include, changing political climate, concerns about system capacity and finance, poor coordination between federal and state levels, corruption and problems with the private sector - HMOs. These challenges and obstacles limit the government's ability to provide social and financial risk protection and access to quality health services to vulnerable Nigerian communities.

Drawing on the theoretical framework and intervention, this thesis concludes that the political, policy, financial and organizational constraints of the health system limit UHC Nigeria implementation progress. Addressing the reasons why these issues ensue would be helpful in taking strategic steps towards achieving financial protection and access to basic health services through UHC for many Nigerians.
\end{abstract}


This work has not previously been accepted in substance for any degree and is not being concurrently submitted in candidature for any degree.

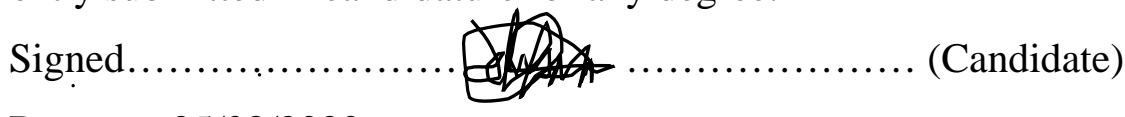

Date......25/08/2020.

This thesis is the result of my own investigations, except where otherwise stated.

Signed.................................. (Candidate)

I hereby give consent for my thesis, if accepted, to be available for photocopying and for interlibrary loans after expiry of a bar on access approved by Swansea University.

Signed. (Candidate)

Date......25/08/2020 
To God be the glory. I dedicate this thesis to Capt. Jenson Edward Fofah, a source of advice and encouragement, who sadly passed away in 2015. Your guidance will be forever missed especially for inspiring and trusting my capability to push on to this level of study. I am indebted to Professor David Hughes, my supervisor, who has been a shining light, mentor, and constant guide throughout the whole process of the $\mathrm{PhD}$ candidature (Thank you sir). Also, I would like to thank my additional research supervisors’ Dr David Rea and Dr Ian Beech for all their kind support during the doctoral process.

My sincere thanks to Mrs Maria Davis for making my stay in Swansea comfortable, stress free and memorable.

Without the support of my family, Mrs Joyce E. Fofah, Mrs Jennifer T. King, Brume Fofah and Xiao Qian, and their intercessory prayers to protect and guide me, I would not have completed this research.

To the Rawles, my adoptive family, I express my love and gratitude for keeping me safe, sheltered and fed during these far from easy years.

Special thanks are due to Dr Garba Safiyanu and special mentions go to my fellow students Aminatul Saadiah binti Abdul Jamil, Soludo Eze, Matthew Swain, Chukwudi Oliver Okolie, Peng Zhao, Leslie Ezeoke, and Chinwe Otugo Onyegegbu- and to friends in RUH Bath Hospital, Birmingham Women's Hospital, Morriston Hospital, and elsewhere in Nigeria, China, Italy, the United States of America and the United Kingdom. 


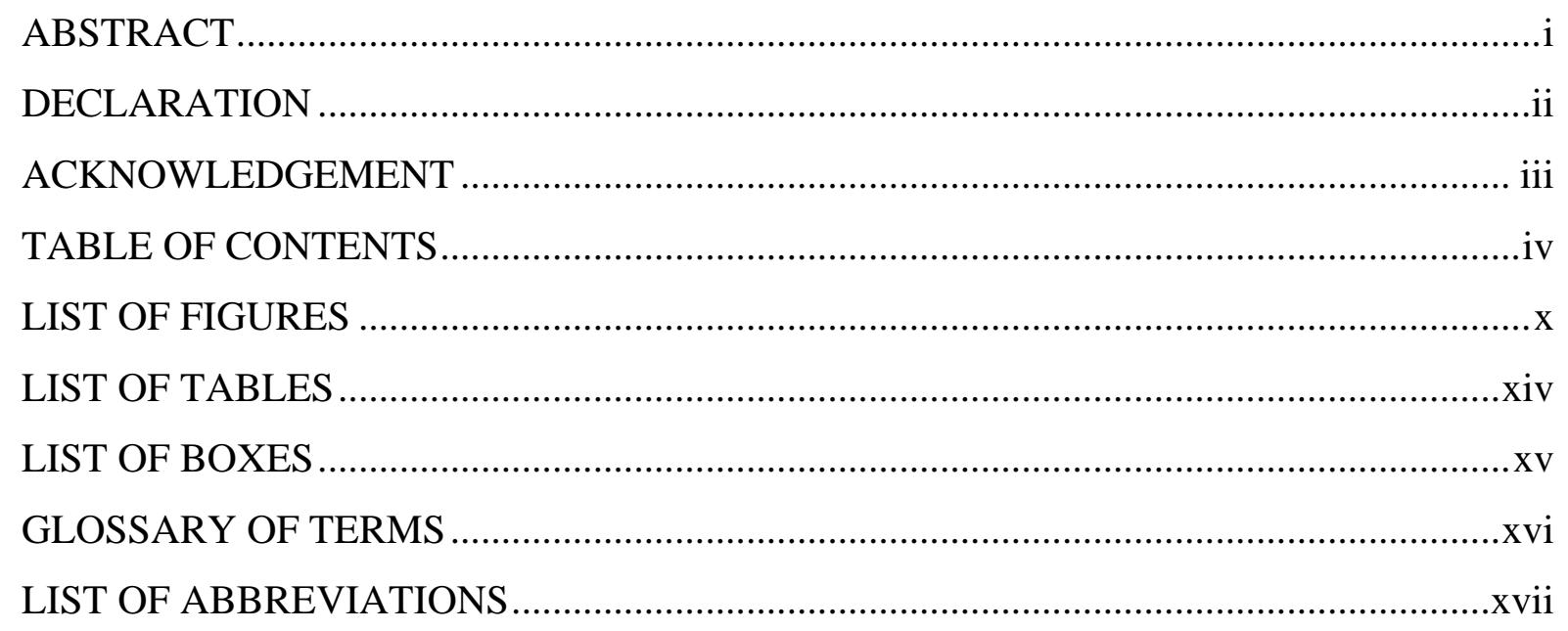

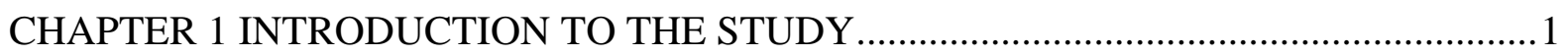

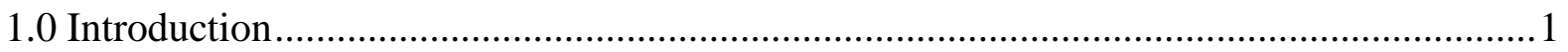

1.1 Why achieving UHC in Nigeria is important ……………......................................

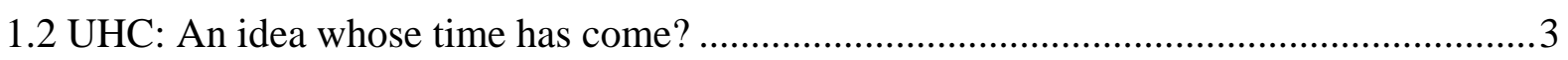

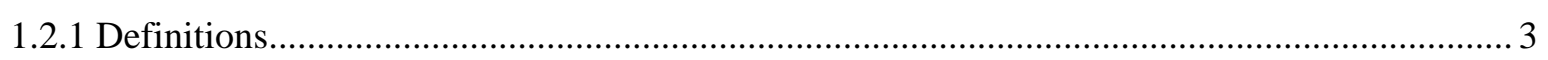

1.2.2 Increasing Global Interest in UHC................................................................................... 4

1.2.3 Dimensions of UHC ................................................................................................. 7

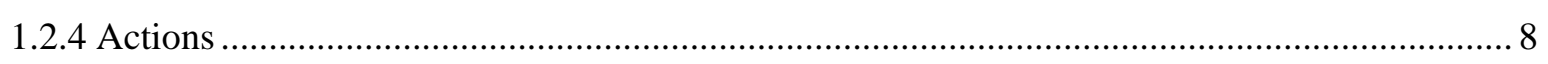

1.3 UHC in the Sustainable Development Goals era..........................................................

1.4 Country profile......................................................................................................14

1.4.1 Geography/demographics .................................................................................... 14

1.4.2 Economic Context.................................................................................................... 15

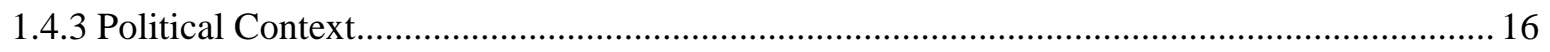

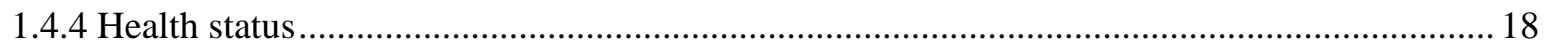

1.5 Overview of the Nigerian Health System ...................................................................20

1.6 Chronological background and Timeline....................................................................22

1.7 The Structure of the Nigerian health system...............................................................25

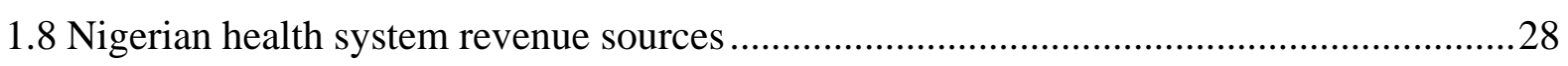

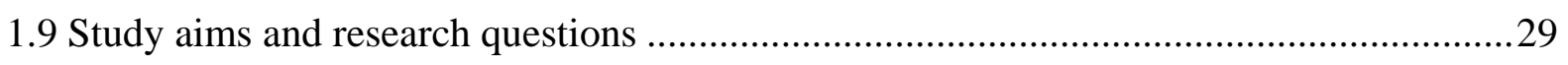

1.10 Addressing the questions through a mixed-methods study............................................29

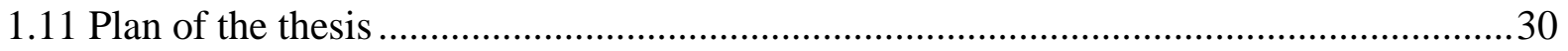




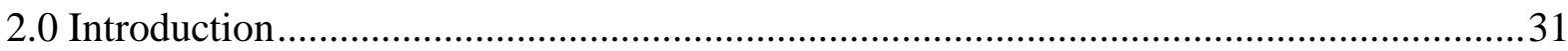

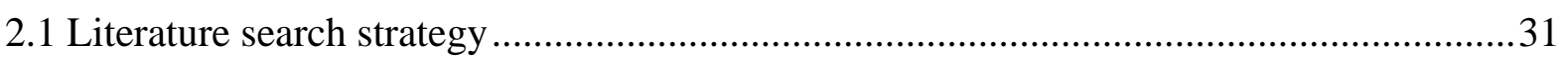

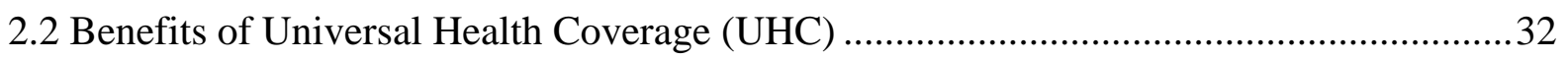

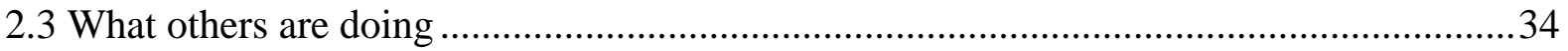

2.4 Systematic comparison of UHC implementation in other countries ...............................41

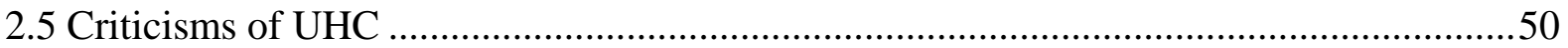

2.6 Universal Health Coverage (UHC) in Nigeria...........................................................57

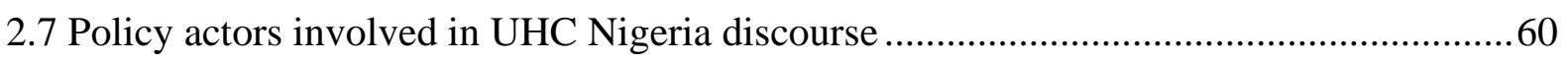

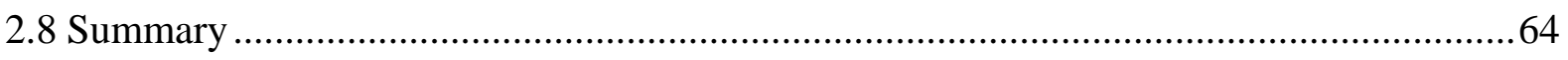

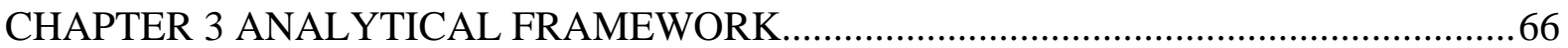

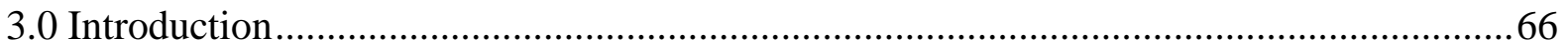

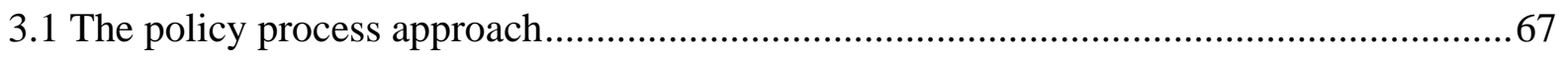

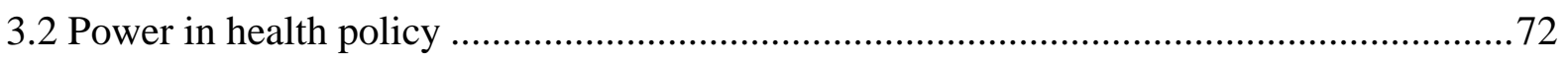

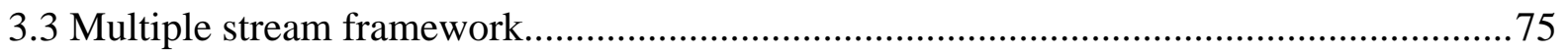

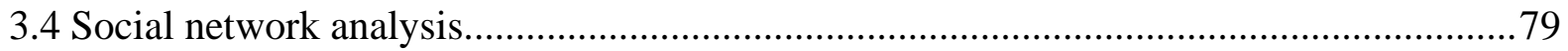

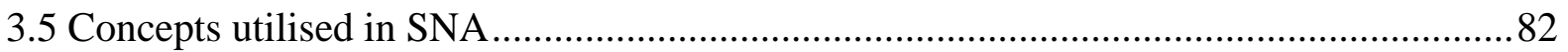

3.6 Social network analysis and policy process in health context ........................................ 87

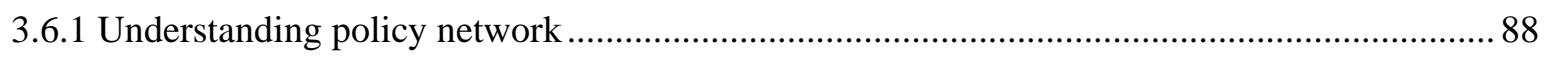

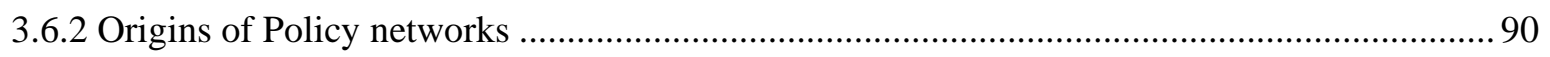

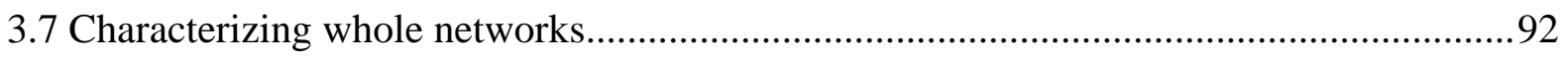

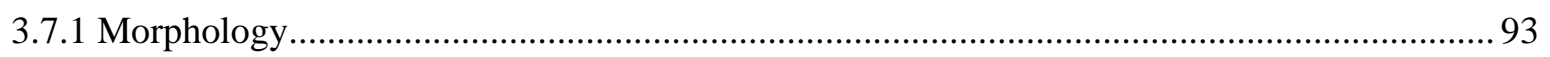

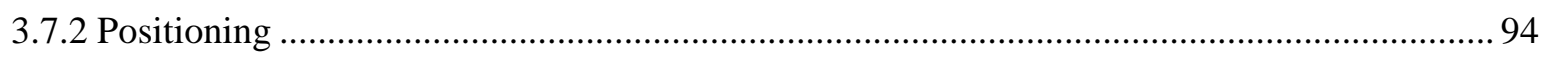

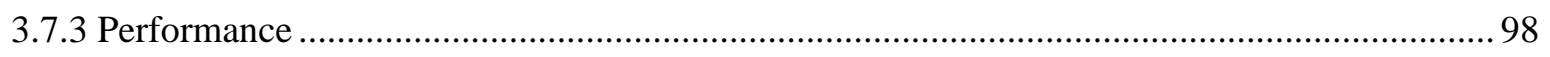

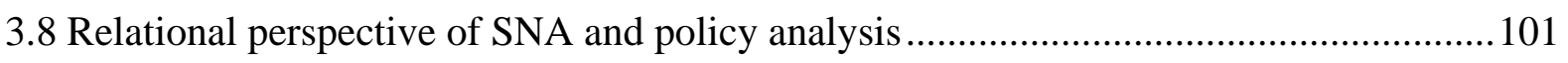

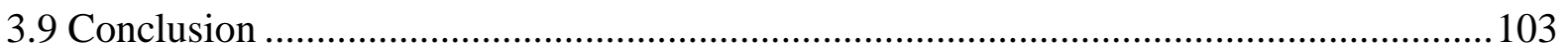

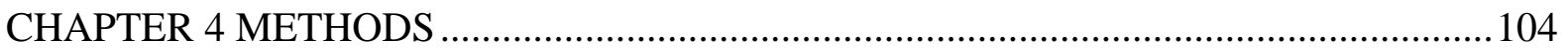

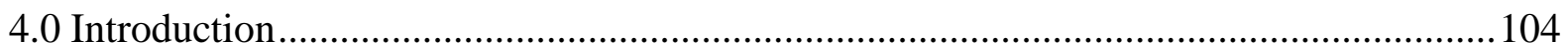

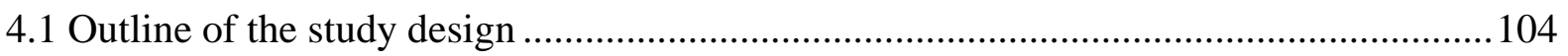

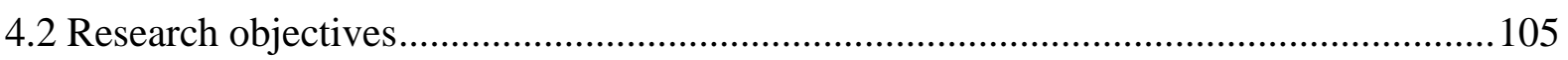

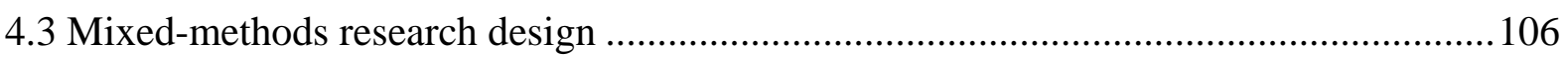

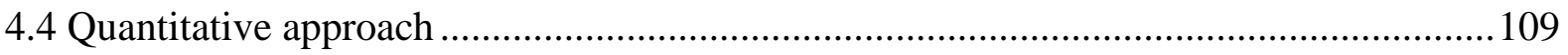




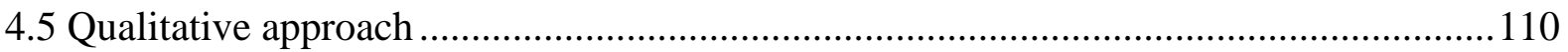

4.6 Justification for combining SNA and qualitative interviews .........................................113

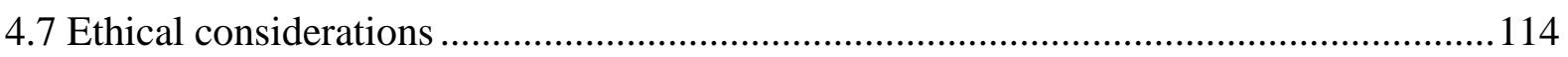

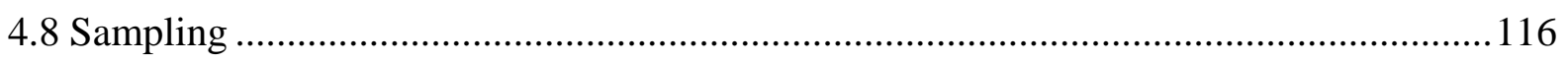

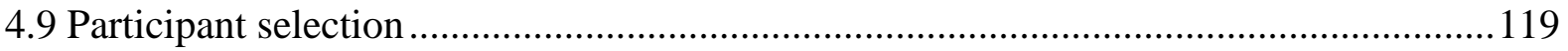

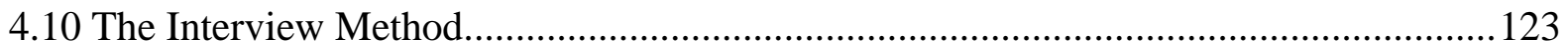

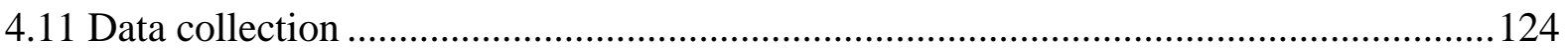

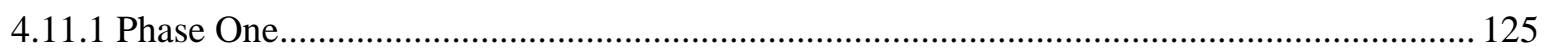

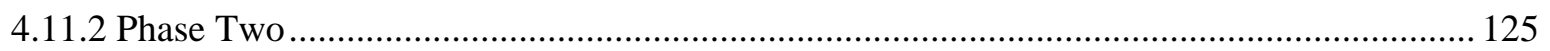

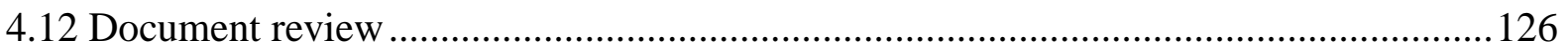

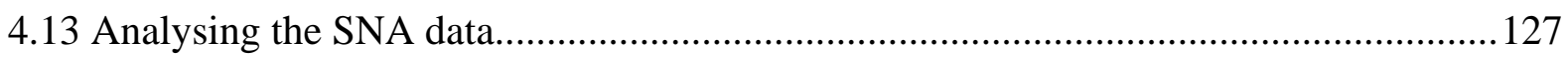

4.14 Analysing the qualitative interviews............................................................................132

4.14.1 Identifying themes in interview transcripts...................................................................... 133

4.15 Data management...............................................................................................135

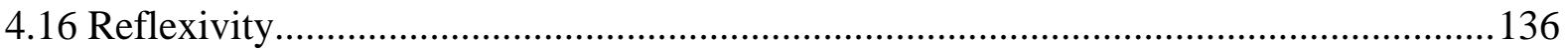

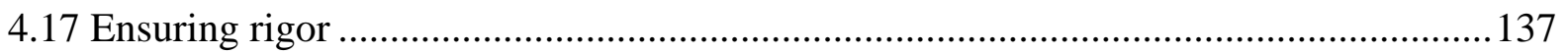

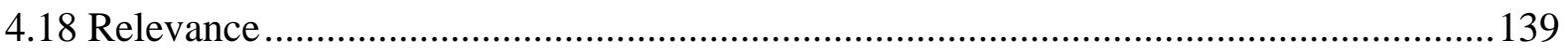

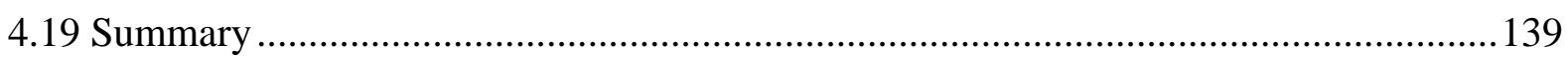

CHAPTER 5 ANALYZING THE UHC POLICY NETWORK …………………………....140

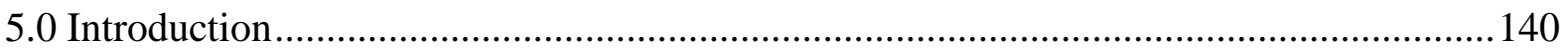

5.1 The network actors and stakeholder groups................................................................141

5.2 Network size, density, fragmentation and transitivity ………………….......................144

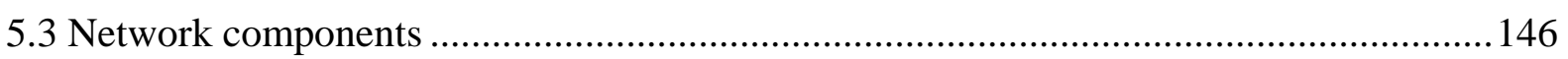

5.4 Network Components and the 'closeness' of links between actors ....................................148

5.5 Investigating core group interactions via measures of centrality.......................................162

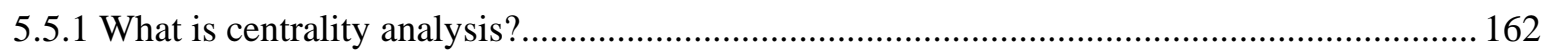

5.5.2 Degree centrality and power actors.................................................................................... 163

5.5.3 Betweenness centrality and the gatekeepers ......................................................................... 164

5.5.4 Closeness centrality and influence through proximity ............................................................ 166

5.5.5 Eigenvector centrality and how policy actors link to power actors ........................................168

5.6 Identifying Multi-relational Policy Actors within the Policy Network ............................169

5.7 Conflict in the Policy Network ......................................................................................171

5.7.1 Peripheral Policy Actors ................................................................................................. 173 


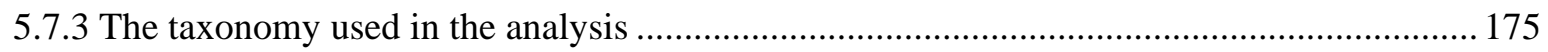

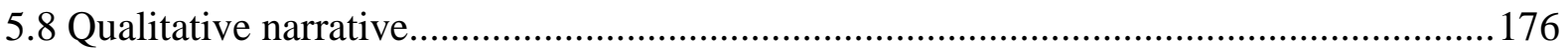

\section{CHAPTER 6 UNDERSTANDING THE UHC NETWORK VIA POLICY PROCESS}

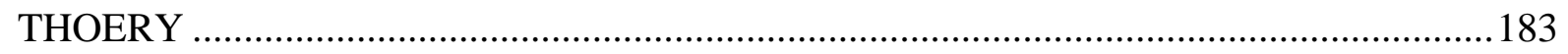

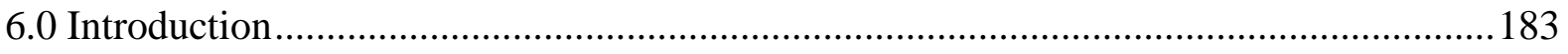

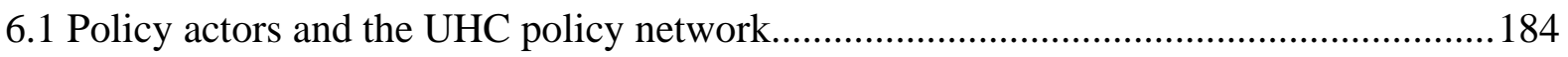

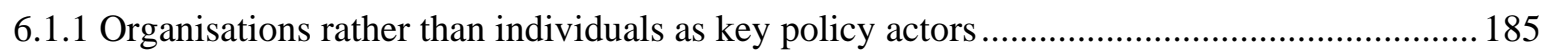

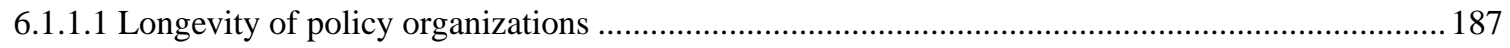

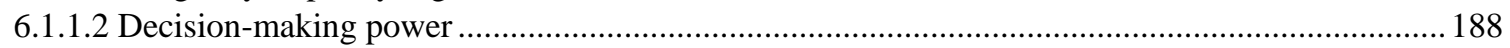

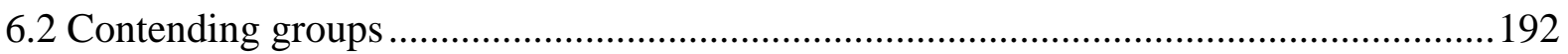

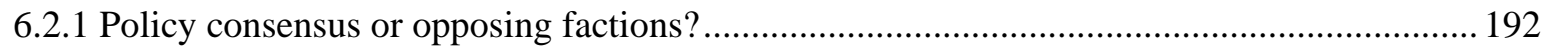

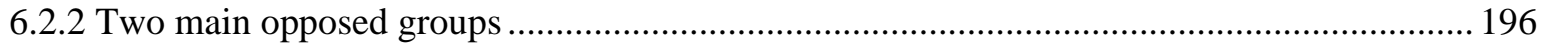

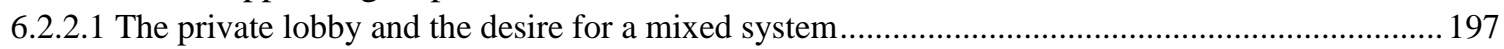

6.2.2.2 The coalition supporting UHC led by the public sector ................................................................... 198

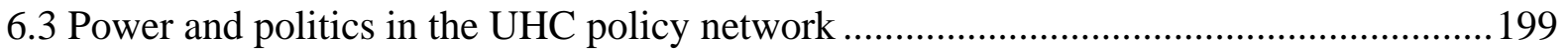

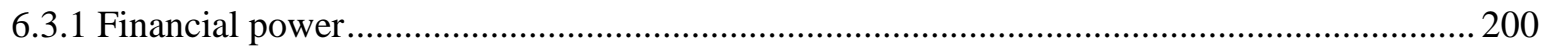

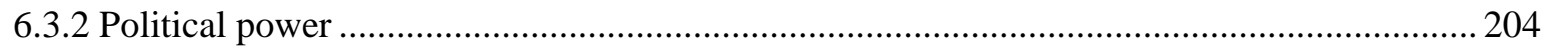

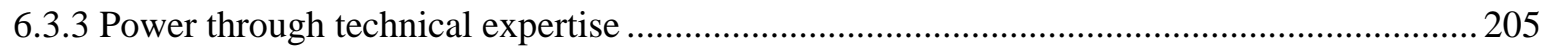

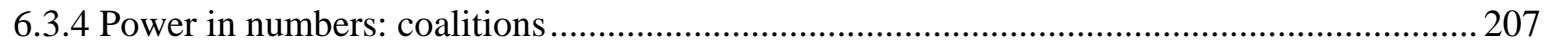

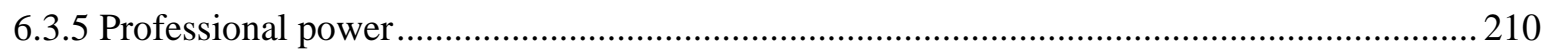

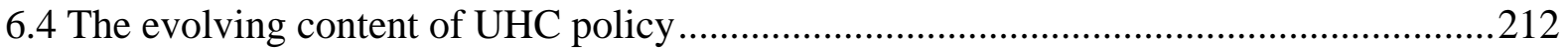

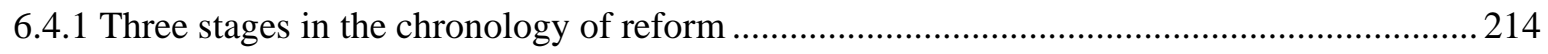

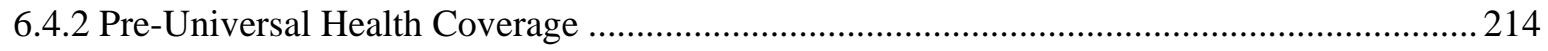

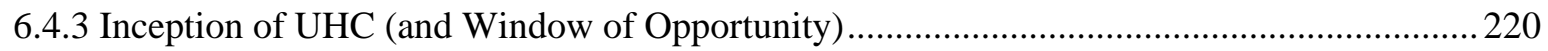

6.4.4 Post-2015 policy developments and implementation issues ..................................................224

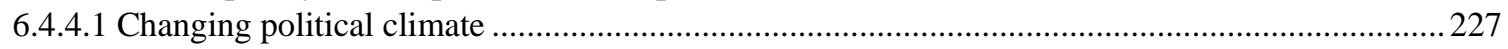

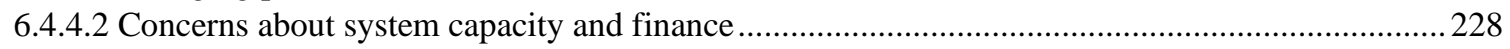

6.4.4.3 Poor coordination between federal and state levels .........................................................................229

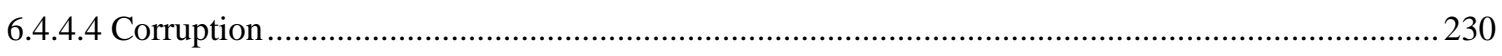

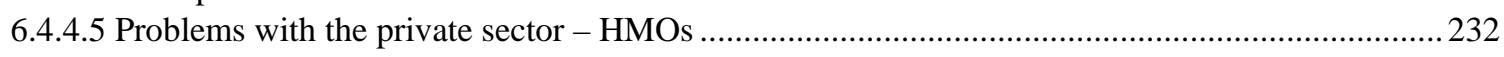

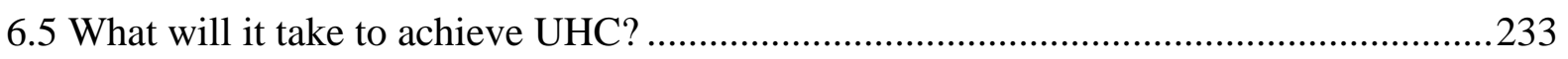

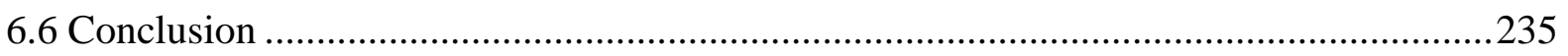

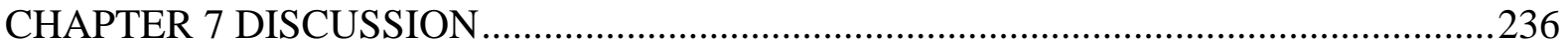

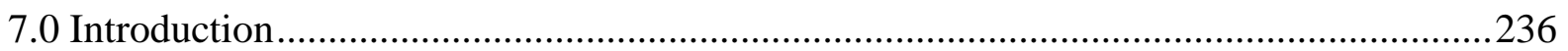

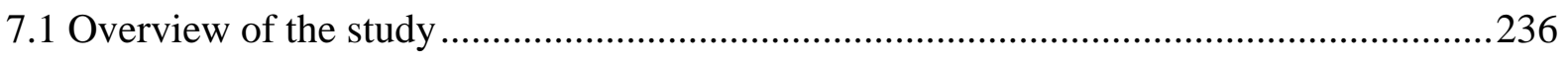


7.2 RQ 1: Who are the influential actors and organizations shaping UHC policy in Nigeria?

7.3 RQ 2: What are the patterns of influence and power relationships within the policy network?

7.4 RQ3. What are the respective roles of domestic and international actors in shaping policy?

7.5 RQ4. Are there disagreements within the policy network over the approach to UHC to be pursued, or the feasibility or desirability of UHC policies?

7.6 RQ5. What are the main gains and setbacks to date, as seen by policy actors?

7.7 RQ6. How far along the path towards UHC do policy actors believe Nigeria has travelled, and what is their assessment of the prospects of achieving adequate coverage?

7.8 RQ7. What general lessons can Nigeria provide regarding the policy process associated with UHC reform? 255

7.9 What the research adds to the wider literature .258

7.10 Limitations of the study 261

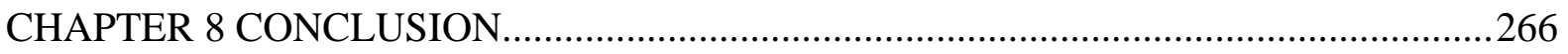

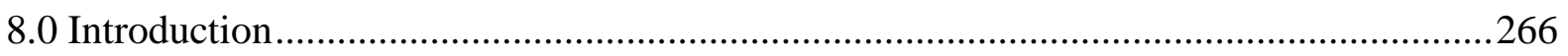

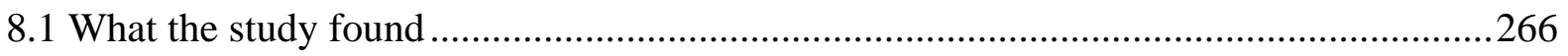

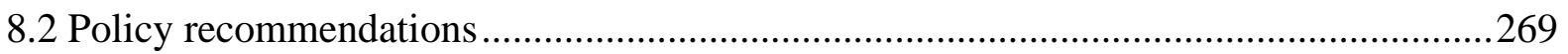

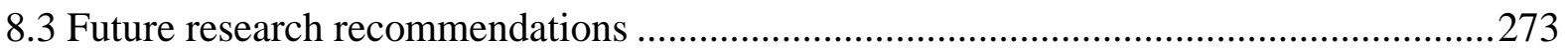

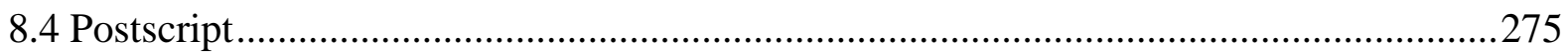

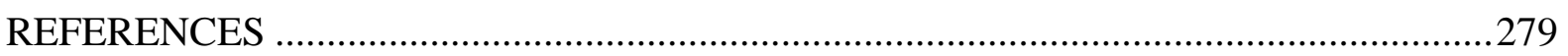

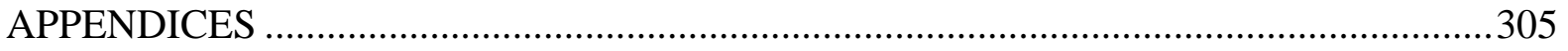

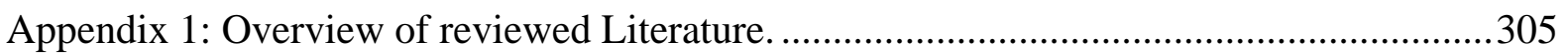

Appendix 2: Ethical approval for the National Health Research Ethics Committee in Nigeria

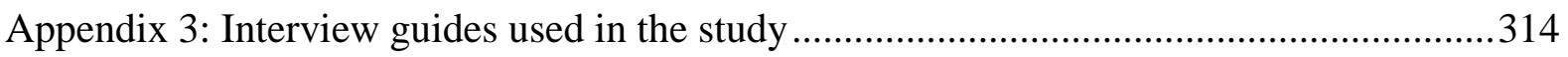

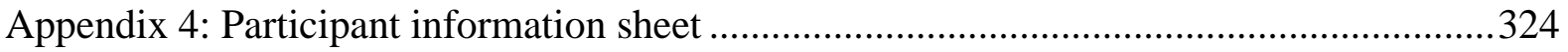

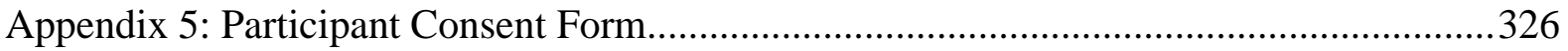

Appendix 6: Examples of SNA measures from the read relationship of the policy network.

Appendix 7: Examples of SNA measures from the discussion relationship of the policy network. 
Appendix 8: Examples of SNA measures from the coalition relationship of the policy network. 342

Appendix 9: Examples of SNA measures from the affiliation relationship of the policy network. 347

Appendix 10: Examples of SNA measures from the conflict relationship of the policy network. .351

Appendix 11: Examples of SNA measures from the advice relationship of the policy network. 357

Appendix 12: Example of a reflexive diary..... 


\section{LIST OF FIGURES}

Figure 1: Current Health Expenditure (CHE) for Selected African Countries.........................2

Figure 2: The three dimensions of UHC.....................................................................

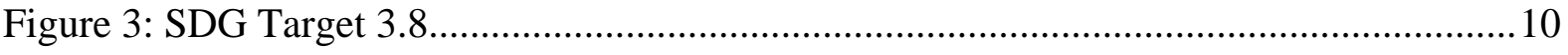

Figure 4: UHC service coverage index scores for selected countries..................................11

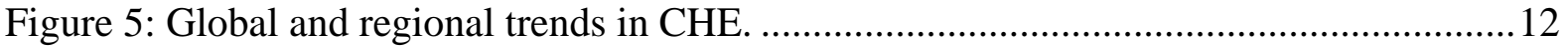

Figure 6: Global and regional impoverishment caused by out-of-pocket expenditures..........13

Figure 7: Shows the political map of Nigeria and neighbouring countries. ...........................14

Figure 8: Key stakeholders in the Nigerian healthcare system............................................22

Figure 9: Nigeria’s pluralistic healthcare system.................................................................25

Figure 10: LMIC countries (GNI per capita of $\$ 1,046$ to $\$ 4,125)$. ........................................35

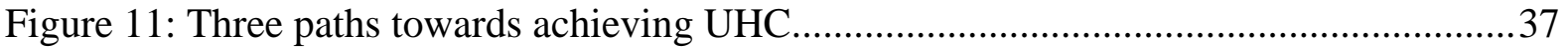

Figure 12: A health system features for selected countries within the global north and south.

Figure 13: A marketplace of health insurance groups in Netherland. …................................52

Figure 14: Percentage of patients seen within four hours for medical consultations. .............53

Figure 15: The performance of lethal diseases and other fields in the National Health Service

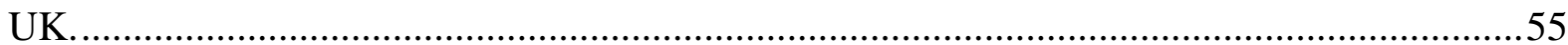

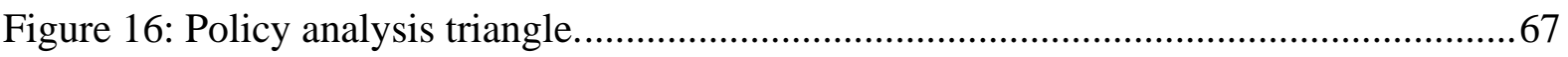

Figure 17: A theoretical framework for understanding how the UHC policy rose to the top of

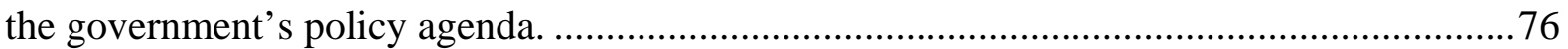

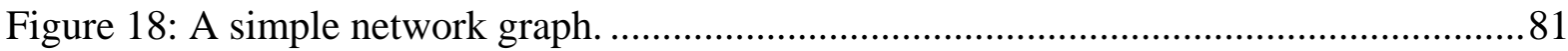

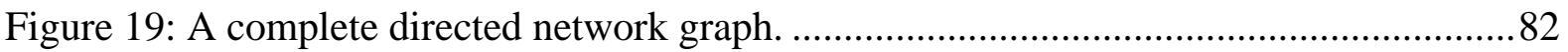

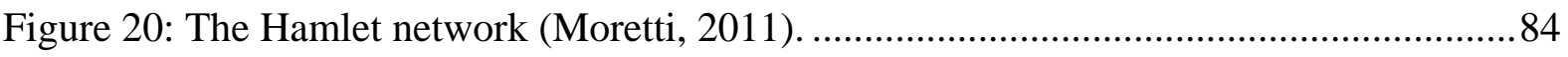

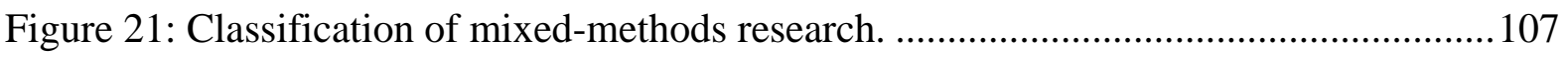

Figure 22: Study with equally weighted, combination of quantitative and qualitative methods.

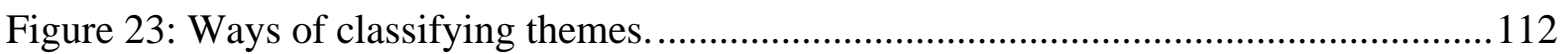

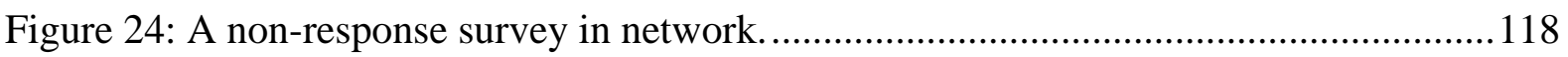

Figure 25: Component analysis of the “advice” relationship of the policy network.............130

Figure 26: Using thematic analysis to map out findings....................................................135

Figure 27: Size of the directed and non-valued policy network. .........................................144

Figure 28: Component analysis of the "read” relationship of the policy network.................147 
Figure 29: Visualisation of the k-core measure of the (bipartite graph) "read" relationship of the policy network showing the presence of eight subgroups.

Figure 30: Visualisation of the k-core measure of the (bipartite graph) “discussion” relationship of the policy network showing the presence of eight subgroups. 151

Figure 31: Visualisation of the k-core measure of the (bipartite graph) "coalition” relationship of the policy network showing the presence of eight subgroups. 152

Figure 32: Visualisation of the k-core measure of the (bipartite graph) “affiliation” relationship of the policy network showing the presence of eight subgroups. 153

Figure 33: Visualisation of the k-core measure of the (bipartite graph) "conflict” relationship of the policy network showing the presence of eight subgroups. .154

Figure 34: Visualisation of the k-core measure of the bipartite graph “advice” relationship of the policy network showing the presence of eight subgroups. 155

Figure 35: A core-periphery network of the bipartite graph "Read" relationship of the policy network showing the presence of core and periphery subgroups. .156

Figure 36: A core-periphery network of the bipartite graph "discussion” relationship of the policy network showing the presence of core and periphery subgroups. 157

Figure 37: A core-periphery network of the bipartite graph “coalition” relationship of the policy network showing the presence of core and periphery subgroups. 158

Figure 38: A core-periphery network of the bipartite graph "affiliation" relationship of the policy network showing the presence of core and periphery subgroups.

Figure 39: A core-periphery network of the bipartite graph “conflict” relationship of the policy network showing the presence of core and periphery subgroups. 160

Figure 40: A core-periphery network of the bipartite graph "advice” relationship of the policy network showing the presence of core and periphery subgroups. 161

Figure 41: Visualisation of the degree centrality measure of the 2-mode (bipartite graph) "read" relationship of the policy network.

Figure 42: Visualisation of the betweenness centrality measure of the 2-mode (bipartite graph) "read" relationship of the policy network.

Figure 43: Visualization of the blocks and cut-points of the 2-mode (bipartite graph) "read" relationship of the policy network. 166

Figure 44: Visualization of closeness centrality of the 2-mode (bipartite graph) "read" relationship of the policy network.

Figure 45: Visualisation of the eigenvector centrality measure of the 2-mode (bipartite graph) "read" relationship of the policy network.

Figure 46: Association between all the networks representing each relationship by a QAP correlation. .170

Figure 47: Visualisation of the core group of the 2-mode (dichotomised graph) "conflict" relationship of the policy network. 
Figure 48: Visualisation of degree centrality measure of the 2-mode (bipartite graph) "conflict” relationship between respondents.

Figure 49: Visualization of cut-points/block measure of the 2-mode (bipartite graph) “conflict” relationship of the policy network. .173

Figure 50: Taxonomy of the policy network analysed in four stages. 175

Figure 51: A timeline of progress towards UHC in the Nigeria health system. 213

Figure 52: Indicators for universal health coverage service index. .278

Figure 53: Visualization of 2-mode (dichotomized graph) “read” relationship of the policy network.

Figure 54: Visualization of (policy organisation-to-policy organisation) “read” relationship of the policy network.

Figure 55: Visualization of (respondent-by-respondent) "read" relationship of the policy network. .330

Figure 56: Visualization of (respondent-to-policy organisation) "read" relationship of the policy network. 331

Figure 57: Visualization of (dichotomized graph) "discussion” relationship of the policy network.

Figure 58: Visualization of the degree centrality measure of (bipartite graph) "discussion” relationship of the policy network. .333

Figure 59: Visualization of the eigenvector centrality measure of the (bipartite graph) “discussion” relationship of the policy network.

Figure 60: Visualization of the betweenness centrality measure of the (bipartite graph) "discussion" relationship of the policy network.

Figure 61: Visualization of the closeness centrality measure of the (bipartite graph) "discussion" relationship of the policy network. 336

Figure 62: Visualization of the blocks and cut-points of the (bipartite graph) "discussion” relationship of the policy network.

Figure 63: Visualisation of the cut-points of the (bipartite graph) "discussion” relationship of the policy network. 338

Figure 64: Visualization of the cut-point of the (bipartite graph) “discussion” relationship of the policy network (a).

Figure 65: Visualisation of the isolate and cut-point of the (bipartite graph) “discussion” ..340

Figure 66: Visualization of the isolates and isolates cut-points of the (bipartite) "discussion” relationship of the policy network.

Figure 67: Visualization of (dichotomized graph) “coalition” relationship of the policy network.

Figure 68: Visualization of the degree centrality measure of the (bipartite graph) "coalition" relationship of the policy network. .343 
Figure 69: Visualization of the eigenvector centrality measure of the (bipartite graph) "coalition" relationship of the policy network

Figure 70: Visualization of the betweenness centrality measure of the (bipartite graph) "coalition" relationship of the policy network.

Figure 71: Visualization of the closeness centrality measure of the (bipartite graph) "coalition” relationship of the policy network.

Figure 72: Visualization of (dichotomized graph) “affiliation” relationship of the policy network.

Figure 73: Visualization of the degree centrality measure of the (bipartite graph) "affiliation” relationship of the policy network.

Figure 74: Visualization of the eigenvector centrality measure of the (bipartite graph) "affiliation” relationship of the policy network.

Figure 75: Visualisation of the betweenness centrality measure of the (bipartite graph) "affiliation" relationship of the policy network.

Figure 76: Visualization of the closeness centrality measure of the (bipartite graph) "affiliation” relationship of the policy network.

Figure 77: Visualization of (dichotomized graph) “conflict” relationship of the policy network .352

Figure 78: Visualization of (respondent-to-policy organisation) “conflict” relationship of the policy network.

Figure 79: Visualization of the eigenvector centrality measure of the (bipartite graph) "conflict” relationship of the policy network.

Figure 80: Visualization of the betweenness centrality measure of the (bipartite graph) "conflict” relationship of the policy network.....

Figure 81: Visualisation of the closeness centrality measure of the (bipartite graph) "conflict” relationship of the policy network. .356

Figure 82: Visualization of (dichotomized graph) “advice” relationship of the policy network. ...357

Figure 83: Visualization of the degree centrality measure of the (bipartite graph) "advice" relationship of the policy network.

Figure 84: Visualization of the eigenvector centrality measure of the (bipartite graph) "advice” relationship of the policy network. 359

Figure 85: Visualisation of the betweenness centrality measure of the (bipartite graph) "advice” relationship of the policy network. 360 


\section{LIST OF TABLES}

Table 1: Timeline of key events in Nigeria’s health system..............................................24

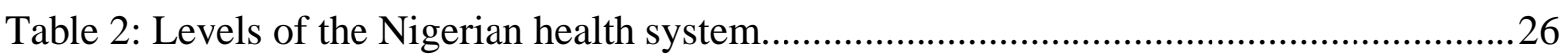

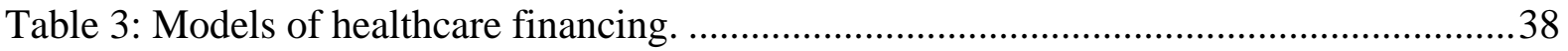

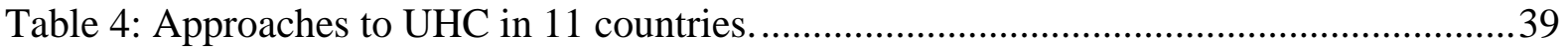

Table 5: Methods for identifying themes from semi-structured qualitive interviews. ..........111

Table 6: Example of coding techniques used in the analysis..............................................133

Table 7: Taxonomy of respondents involved in the second round of structured SNA interviews.

Table 8: Taxonomy of policy organisation involved in the second round of interviews. .....143

Table 9: Measures of cohesion for six relationships of the policy network. 145

Table 10: Relevant periphery nodes of six relationships of the policy network using the closeness centrality and cut-point/block measures. ............................................................174

Table 11: Centrality of key institutions for all relationships of the policy network..............176

Table 12: Centrality of key institutions for the "read" relationship of the policy network...177

Table 13: Centrality of key institutions for the "discussion” relationship of the policy network. 177

Table 14: Centrality of key institutions for the "coalition” relationship of the policy network.

Table 15: Centrality of key institutions for the "affiliation" relationship of the policy network. 177

Table 16: Centrality of key institutions for the "conflict" relationship of the policy network. 178

Table 17: Centrality of key institutions for the "advice" relationship of the policy network. 178

Table 18: The nature of the UHC network. 191 


\section{LIST OF BOXES}

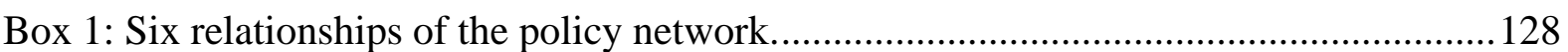




\section{GLOSSARY OF TERMS}

\begin{tabular}{|c|c|}
\hline Betweenness centrality & $\begin{array}{l}\text { 'Betweenness centrality' measure refers to the degree } \\
\text { in which an actor (a node) in the policy network lies } \\
\text { or is connected between two or more actors to gain } \\
\text { considerable influence within the network (Borgatti et } \\
\text { al., 2013) }\end{array}$ \\
\hline Centrality & $\begin{array}{l}\text { 'Centrality' measures the value, prominence or the } \\
\text { influence of an actor (a node) within a given social } \\
\text { network (Bellotti, 2015) }\end{array}$ \\
\hline Closeness centrality & $\begin{array}{l}\text { 'Closeness centrality' measure refers to the inverted } \\
\text { total of the shortest distance or path to every actor } \\
\text { (every node) within the policy network (Borgatti et } \\
\text { al., 2013). }\end{array}$ \\
\hline Degree centrality & $\begin{array}{l}\text { 'Degree centrality' measure refers to the number of } \\
\text { connections or ties an actor develops within the policy } \\
\text { network (Prell, 2012) }\end{array}$ \\
\hline Eigenvector centrality & $\begin{array}{l}\text { 'Eigenvector centrality' measure refers to the } \\
\text { importance of an actor due to its connection or ties } \\
\text { with other influential actors inside the policy network } \\
\text { (Prell, 2012). }\end{array}$ \\
\hline Policy entrepreneur & $\begin{array}{l}\text { 'Policy entrepreneur' is a person that seizes the } \\
\text { opportunity to influence policy outcomes for their } \\
\text { self-interest (Ashford et al., 2006). }\end{array}$ \\
\hline Window of Opportunity & $\begin{array}{l}\text { 'Window of opportunity' in health policy refers to an } \\
\text { opening created when three policy streams (politics, } \\
\text { policy and problem) couple together for a policy } \\
\text { solution (Kingdon, 2010). }\end{array}$ \\
\hline
\end{tabular}


CBHI Community-based health insurance

FMoH Federal ministry of health

GDP Gross domestic product

HMO Health maintenance organization

LMICs Low- and middle-income countries

MDGs Millennium Development Goals

NHIS National health insurance scheme

NGO Non-Governmental Organization

NMA Nigerian medical association

OOPS Out-of-pocket spending

PPP Public-Private Partnership

SDGs Sustainable Development Goals

SHI Social health insurance

$\mathrm{SMoH} \quad$ State ministry of health

SNA Social network analysis

UHC Universal health coverage

UNICEF United Nations Children's Fund

USAIDS United States Agency for International Development

WHO World Health Organization 


\section{CHAPTER 1}

\subsection{Introduction}

This study investigates how the idea of universal healthcare coverage (UHC) came onto the policy agenda of a developing country, Nigeria, the actors who promoted or opposed the emerging UHC policies, and the way those policies developed as they were debated within the policy network. It is a case study of movement towards a policy objective - UHC - that Nigeria has still to achieve but is progressing slowly towards. The research is intended to shed light on how such a policy was advanced through a series of incremental steps and was slowed through disagreements over the means by which UHC could be achieved and nature of the reforms that would be necessary, as well as downright opposition. Special attention is given to the network of actors and organisations that were directly or indirectly involved in the policy debates, and the policy process via which a series of reforms were introduced with the aim of progressively improving healthcare coverage for the Nigerian population. Systematic data on the nature of the UHC policy network is combined with accounts from qualitative interviews with key stakeholders to build a picture of how policy to improve access and coverage evolved, the opposing views concerning how UHC should be taken forward, the respective roles of domestic and international actors, and the obstacles encountered.

\subsection{Why achieving UHC in Nigeria is important}

The author started this research with a strong conviction that healthcare in Africa needed radical improvement, and a feeling that a country with Nigeria's resources was doing much worse than should have been the case.

For example, it was clear from published statistics that Nigeria, although one of the wealthier African nations, was spending less in health in GDP terms than several of its neighbours. Figure 1 shows that the 3.9 per cent of GDP allocated by Nigeria for Current Health Expenditure (CHE) in 2016 is dramatically low in comparison to other African countries. For instance, in 2015 South Africa spent 9.1 per cent of GDP on CHE, while Sierra Leone, Burkina 
Faso, Malawi, Rwanda, and Namibia spent respectively 16.5 per cent, 6.7 per cent, 9.8 per cent, 6.7 per cent and 9.1per cent of GDP on CHE (The World Bank, 2020).

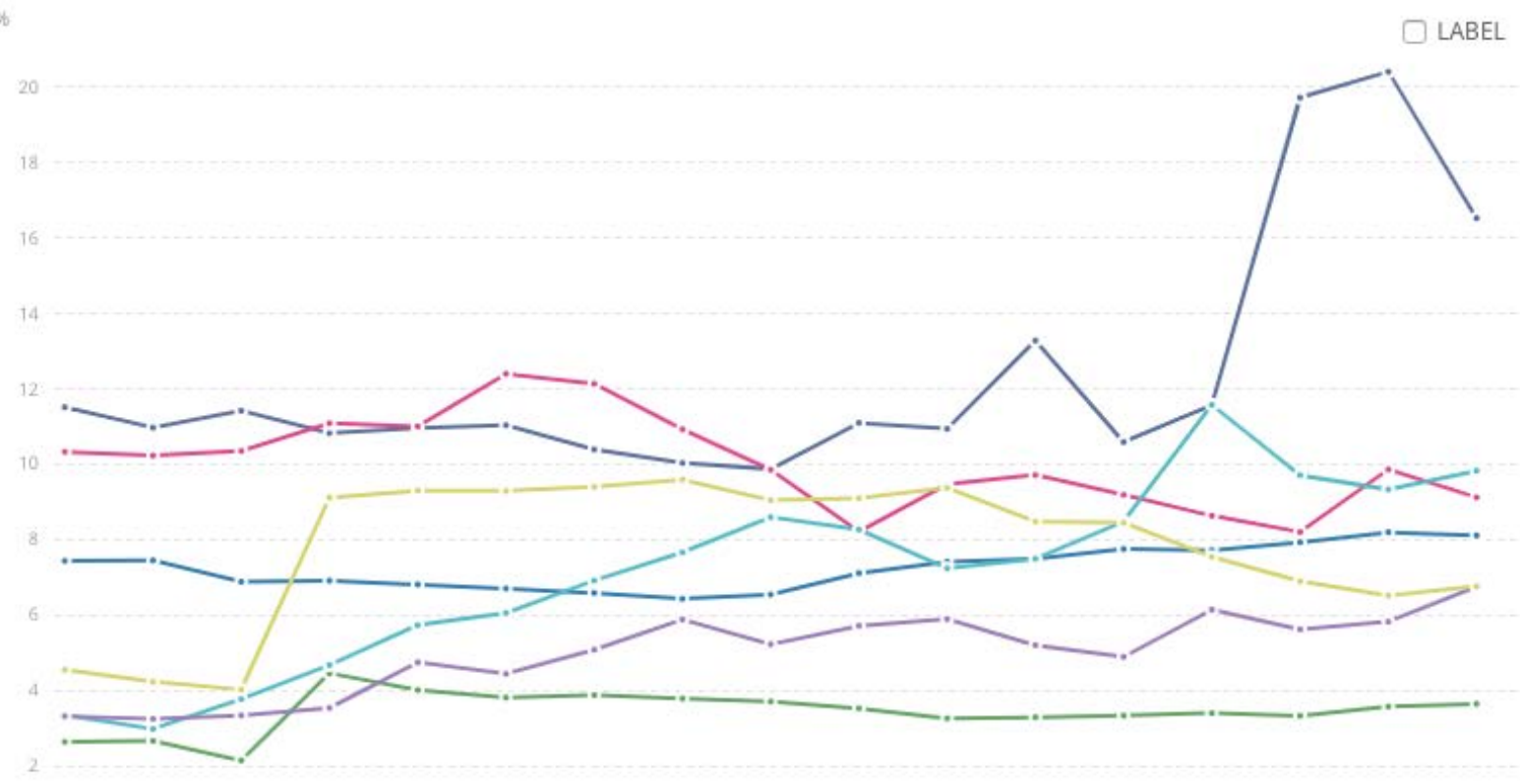

Figure 1: Current Health Expenditure (CHE) for Selected African Countries.

This shows CHE as \% Gross Domestic Product (GDP) for Nigeria X, South Africa X, Sierra Leone X, Burkina Faso X, Rwanda X, Malawi X and Namibia X in 2016. Adopted from The World Bank (2020). Key: crossed colour codes represent the Selected African Countries.

Low levels of health expenditure down through the years had translated into poor outcomes on many important health indices. Even after earlier reforms that aimed to expand coverage, a 2016 study showed that Nigeria scored at or below the African average in a number of key areas (Adewole and Osungbade, 2016). Poor health indices were evident in a low average life expectancy of 54 years, high infant and under 5 mortality rates of 88 and 143 per 1000 live births respectively, and a maternal mortality ratio of 630 per 100,000 live births. There was clearly considerable scope for improvement.

At the same time the author was aware of a global movement whereby many developing countries had either achieved to aspired or to achieve UHC, a movement which had featured in two recent World Health Reports (WHO, 2010, 2013), and had gained the support of leading 
international experts (Savedoff, 2012; Kruk, 2013; Sen, 2015). The student knew that policy makers in Nigeria too were beginning to talk about UHC as path to improving health outcomes but formed the impression of a slow and rather stilted policy process that warranted investigation. He reasoned that a study that examined the nature of the ongoing policies debates and any barriers that were slowing progress towards UHC would provide valuable lessons for policy makers.

\subsection{UHC: An idea whose time has come?}

\subsubsection{Definitions}

UHC is based on the premise that all communities and people should be eligible to use the preventive, curative, promotive, rehabilitative and palliative services of the health sector as and when they need to in order to maintain a good state of health (Atun et al., 2015). The definition of UHC involves three key objectives, which are that (WHO, 2017):

1. Individuals must have equal access to good quality health services irrespective of their social status.

2. The health services provided should significantly improve the health status of the recipient.

3. Health services should be sufficiently affordable to protect the recipient from financial hardship and impoverishment.

An early precursor to UHC was WHO’s 1978 “Health for All”, Alma Ata Declaration, which asserted that health is a fundamental human right. While in practice Alma Ata set a target only for achieving a basic level of primary care for all, the drive towards UHC sets the more ambitious target of delivering comprehensive care for the population. The UNs' current healthrelated Sustainable Development Goals (SDGs) includes a target to: 'Achieve universal health coverage, including financial risk protection, access to quality essential health-care services and access to safe, effective, quality and affordable essential medicines and vaccines for all' (Chapman, 2016; WHO, 2018, goal 3.8).

The UHC policy aims to prevent premature deaths and reduce disease by ensuring access to health services that are good enough to serve people in need (Bloom et al., 2018). According to Hsiao et al (2014), financial expenditure related to health exposes people to impoverishment 
and financial hardship because unintended health-related issues like accidents, disease, infection, illness and sudden death constitute a major burden on total household expenditure. The financial burden obtained from the incurred health expenses can result in financial destitution that affects the future of a family for generations. UHC not only safeguards immediate health problems but prevents financial impoverishment that amplifies ill health over time.

In recent years, there has been an emerging commitment to achieving UHC goals. Universal Health Coverage has been regarded as the third health transition with global impact. The first transition included public health improvements such as sanitation and basic sewage management. The second transition is the epidemiological transition which saw diseases of affluence supplant communicable diseases. UHC is described by the WHO as a crucial development required to improve global public health (Frenk, 2015; Ramke et al., 2017; Hsiao et al., 2014).

\subsubsection{Increasing Global Interest in UHC}

After a period when international bodies such as the World Bank and IMF supported neoliberal policies that translated into a bigger role for private corporations and coverage gaps for those who could not pay, the tide has swung back towards policies that favour UHC. The 2010 World Health Report, Health Financing: The Path to Universal Coverage (WHO, 2010) was particularly influential in building momentum for reform. A May 2011 World Health Assembly resolution requested the Director General to convey to the UN Secretary-General the importance of UHC for discussion by a forthcoming session of the UN General Assembly, and in December 2012 the Assembly endorsed a resolution on Global Health and Foreign Policy urging member countries to accelerate progress toward UHC. Further support came in 2014 when the sixty-seventh World Health Assembly passed a resolution in support of UHC. Other developments include the publication by WHO and the World Bank of the first UHC global monitoring report, Tracking Universal Health Coverage, in 2015, and the Lancet Commission on Investing in Health’s (CIH) Global Health 2035 report (Jamison et al., 2013), which proposes an investment framework for improving global health through UHC. UHC has been included in the United Nations Sustainable Development Goals (see section 1.5). It was the subject of a High-Level Meeting in September 2019 at the UN General Assembly, which 
produced a political declaration whereby the signatories "strongly recommit to achieve universal health coverage by 2030” (United Nations, 2019).

The People's Republic of China, long criticized for health policies that signalled left but turned right, is now improving coverage for its rural population (Lia et al, 2011). Brazil, Rwanda, Chile, Columbia, Mexico, Turkey and South Korea all have ongoing reform programmes. Other low- and middle-income countries, such as India, Ghana, Mali, Kenya, Philippines, Indonesia, Malaysia and Vietnam - as well as Nigeria - cooperate as members of the Joint Learning Network for Universal Coverage and are widening coverage, mainly through the creation of public or voluntary health insurance schemes and closed-end financing mechanisms.

Sharing of UHC experiences can be a valuable tool not just for planning UHC reforms, but for consolidating and maintaining progress made. There is now an extensive network of support organisations that encourage both global North to South knowledge transfer, and - perhaps even more important in the recent past - South to South knowledge transfer. The Joint Learning Network for UHC, with funding from bodies that include the WHO, the World Bank Group and the Rockefeller Foundation, has since 2009 functioned as an umbrella network for organising conferences, workshops and exchange visits, and collecting knowledge resources, such as technical information on effective financing mechanisms (Nachuk, 2013). Entities based in the global North, such as Results 4 Development, Access Help, and the Lancet Commissions have also played a part. In recent years a number of regional networks that allow geographically proximate countries to coordinate actions have also appeared, including the ASEAN+3 UHC network, and the European Union-Luxembourg-WHO Partnership for UHC. In Africa, there is the "Health for All” campaign, supported by the governments of Ethiopia, Kenya and Nigeria, with financial support from the Rockefeller Foundation (Management Sciences for Health, 2020.), and One-by-One’s “Target 2030: UHC for Africa” campaign, associated with Access Challenge (One by One, 2020.). Other pressure groups such as the African Health Economics and Policy Association, Amref Health Africa, and the People's Health Movement have also pushed for progress towards UHC. There are signs that some African countries are looking to Asia for lessons about UHC success, as is illustrated by recent cooperation projects between Kenya and Thailand, and Egypt and Japan (World Economic Forum, 2019). 
Universal Health Coverage (UHC) is considered to be a long-term public health and development objective. Although international human rights law does not provide any absolute guarantee of UHC, a growing series of international declarations, resolutions and agreements are pushing countries towards providing a basic level of healthcare and social protection. While different countries are meeting this challenge in different ways, otherwise dissimilar healthcare systems are increasingly sharing the objective of extending coverage (see: Marten et al., 2014). Usually this will involve new financing mechanisms or service delivery arrangements that extend access to basic healthcare to previously under-served population groups. This is what governments striving for UHC seek to achieve through major health system reform - the provision of quality health care and financial protection for the whole population (O'Connell et al., 2014).

Inequitable access to health services can be observed in many countries, and often originates from political and socioeconomic structures that are highly resistant to change. Progress is only likely if reformists can mobilise countervailing power to equalise access (Kutzin and Susan, 2016). The reform experiences of a growing number of LMICs and UMICs show that the drive to UHC can overcome political opposition and have a positive influence on the social determinants of health (Hsiao et al., 2014). One key step in breaking the negative relationship between social disadvantage and poor health is to make essential health care available at affordable cost (Balabanova et al, 2011; Gupta and Shahabuddin, 2018). The cost of health care should not be a burden on people. UHC systems usually provide free or low-cost benefits in order to address the healthcare needs of the people.

Many debates about UHC wrestle with uncertainty concerning the effectiveness of different policy options for widening coverage. A sound health financing framework is crucial for successful implementation of UHC reforms, and policy makers need to weigh up the benefits and costs of different incentive structures and resource allocation mechanisms. Typically, the financial resources allocated to healthcare are pooled from various sources such as government budgets, payroll taxes/employment-based contributions and prepayment insurance schemes, and the overall balance will be crucial (Schieber et al. 2006; WHO, 2019). For instance, Nigerian policy makers have deliberated the relative attractiveness of tax-funded services versus encouraging cost-sharing from users via health insurance or out-of-pocket spending (Dionne and Mitton, 2018). 


\subsubsection{Dimensions of UHC}

The 2012 World Health Report elaborates further on the concept of UHC by classifying it into three dimensions: 'Who gets health coverage?', 'Which health services are provided?' and 'What proportion of health costs are covered' (see Figure 2).

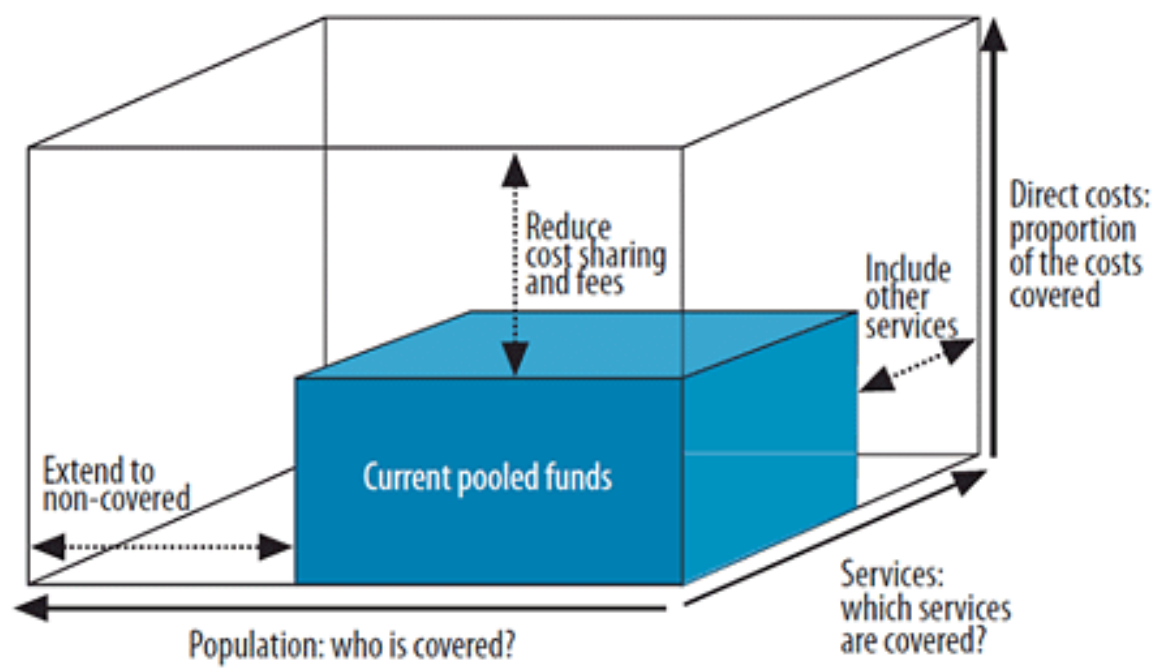

Figure 2: The three dimensions of UHC.

\section{Source: WHO 2008.}

Who gets health coverage: This dimension concerns the coverage of health services provided for the population under UHC? In this dimension, 'universal' is the key word, meaning that the healthcare provided should be available to the whole population. However, the 2010 World Health Report notes that in reality no high-income countries have achieved universal provision without health system trade-offs. For instance, the United Kingdom's National Health Service provides free healthcare only for those “ordinarily resident” in the country, which means that universal access does not apply to ineligible overseas visitors or UK nationals who have been absent from the UK for more than 6 months (Department of Health and Social Care, 2020).

What health services are provided: This concerns the packages of necessary treatments that are provided free or at affordable cost. How this is organised will depend on the financing and service delivery arrangements. Typically, UHC systems are funded from a mix of government tax revenues and payroll tax or contributions-based social health insurance schemes and may be delivered by both public and private sector service providers. These are various mechanisms to limit out-of-pocket payments from service users to providers, including government funding 
of public hospitals and direct reimbursement of healthcare charges to providers by insurance funds. Some UHC systems levy user co-payments or other forms of cost sharing, but these need to be sufficiently affordable that financial hardship is avoided (WHO, 2017).

Providing financial protection: Transitioning to UHC means that a health system avoids creating significant financial barriers to access. According to the 2012 UNGA Resolution (Ooms et al., 2014), UHC requires "that all people have access, without discrimination, to nationally determined sets of the needed promotive, preventive, curative and rehabilitative basic health services". It further recognises that "when managing the transition of the health system to universal coverage, each option will need to be developed within the particular epidemiological, economic, socio-cultural, political and structural context of each country in accordance with the principle of national ownership” (Ooms et al., 2014: 5). WHO (2010) suggests that LMICs that lack the financial capability to introduce the prepayment and pooling schemes necessary to provide cover for poorer citizens, may need to supplement domestic funding with foreign aid support?

In conclusion, UHC is a multi-dimensional concept, which involves making available an adequate range of healthcare services to the whole population, at low or zero cost at the point of need (Savedoff, 2012).

\subsubsection{Actions}

Actions related to UHC are focused on changing and improving the health needs of people and their communities. For example, on March 10th, 2014, the African Presidential Summit on UHC was hosted by the Nigerian government under the theme of 'Universal Health Coverage, The Vehicle for Sustainable Growth and Development' (WHO, 2015). This health forum saw the 16 African countries in attendance declare their commitment to improving their respective health systems by allocating about 15 percent of their national budgets annually to achieve UHC. The forum resulted in a series of actions undertaken by national policymakers to make progress towards UHC.

In Nigeria's case, one of the most significant developments involved earmarking additional funds to support the widening of coverage. There are commendable efforts by the federal government and the Ministry of Health $(\mathrm{MoH})$ in exploring practical, assertive and innovative 
ways to improve domestic health financing despite low expenditure on health and weak health system resource mobilisation caused by the country's fiscal constraints (Aregbeshola, 2018).

UHC is gathering momentum and becoming the main point of attention in many global health policy conversations. A variety of related actions can be observed. Typically, an action plan includes (1) building and maintaining an adequate health platform that provides curative medicines and care, (2) ensuring wide access to healthcare at affordable rates, (3) quick access to necessary medicines and (4) the availability of appropriate human resources for the health platform and system to utilise (Boerma, et al., 2014). Many studies have highlighted that achieving UHC depends on building a capable workforce that goes wider than just front-line care and includes the personnel to staff the necessary governance systems, including management and financing (Barros et al., 2015; Onwujekwe et al., 2019).

\subsection{UHC in the Sustainable Development Goals era}

The progress towards Universal Health Coverage (UHC) has coincided with the replacement of the Millennium Development Goals (MDGs) with an even more ambitious set of Sustainable Development Goals (SDGs), and UHC and the SDGs have to an extent become intertwined. Planning to achieve the SDGs presents policy makers in developing countries with the complex task of managing a broad range of activities including developing infrastructure and setting milestones. UN member states are required to monitor and report progress, including progress on goals related to UHC (Wirtz et al., 2017). The goal that relates directly to UHC is 3.8 (quoted in section 1.3.1), which is seen as central to achieving the objectives set in other domains (Hogan et al., 2018). The UN Statistical Commission has developed two indicators which can be used to monitor progress in achieving goal 3.8: SDG indicator 3.8.1 measures the quality and range of the health services covered across households, while SDG indicator 3.8.2 measures the quantity of household health expenditure as a portion of total household income. Hogan and associates' (2018) study examines progress towards meeting SDG 3.8.1, and associated research by Wagstaff et al (2017) does the same for SDG 3.8.2. An inter-agency, Expert Group report on SDG Indicators explains that 3.8.1 relates to "coverage of essential health services (defined as the average coverage of essential services based on tracer interventions that include reproductive, maternal, new-born, and child health, infectious diseases, non-communicable diseases and service capacity and access, among the general and 
most disadvantaged populations)” (Hogan et al., 2018 p.1) (see Figure 3). SDG indicator 3.8.2 concerns the extent of CHE, and how far out-of-pocket household health expenditure depletes total household budgets, so as to force a trade-off between securing health care or buying other basic essentials such as food and education (WHO, 2017) (see Figure 3). SDG indicators 3.8.1 and 3.8.2 thus respectively describe the comprehensiveness of the healthcare that is available and how much households spend on care. The World Health Organisation (2017) considers that the SDGs are intended to "leave no one behind" and that it is important to monitor implementation. This section describes the outcomes of the recent efforts made by selected countries when it comes to monitoring their journey toward UHC.

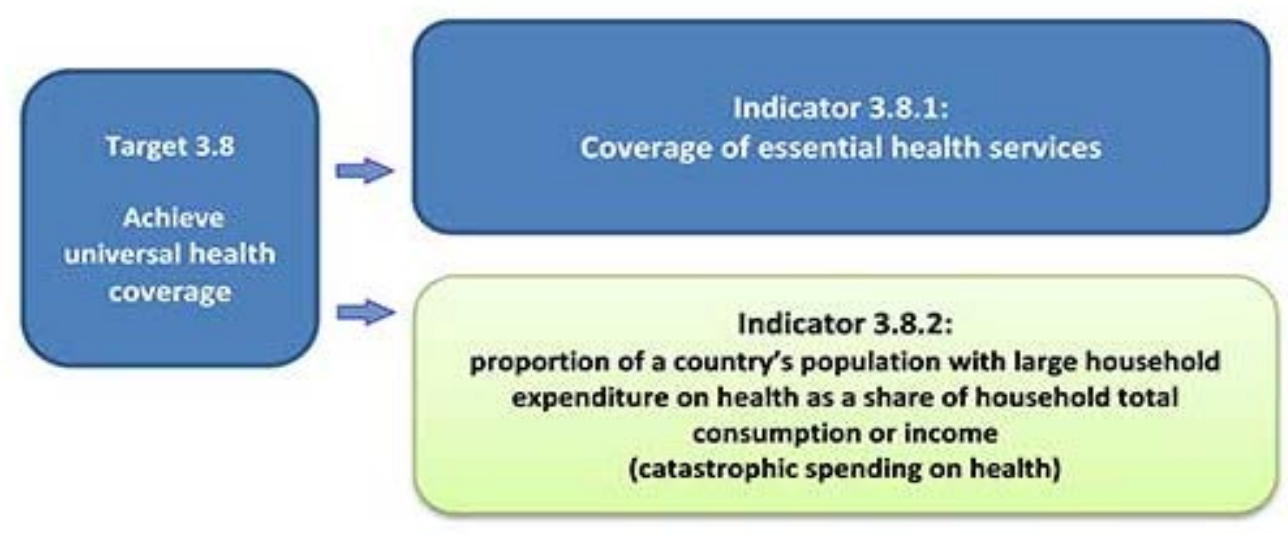

\section{Figure 3: SDG Target 3.8.}

This shows the indicators used to assess progress in achieving SDG Goal 3.8. Source: WHO (2017).

Hogan et al. (2018) developed an index using 16 tracer indicators to measure progress in 183 countries towards achieving target 3.8.1. Across 52 countries, for which data was sufficient to allow measurement, coverage was between $1 \%$ and $66 \%$ lower for the poorest population quintile compared with the average for the national population as a whole. Diagram 3 illustrates the varying levels of service coverage between countries. At the top end are Europe. North America, Australia, New Zealand, and East Asia with index scores of about 77. Against this, Sub-Saharan Africa and Southern Asia have the lowest index of 42 and 53 respectively. Within the former region, Nigeria’s service coverage index is 42 (see Figure 12). 


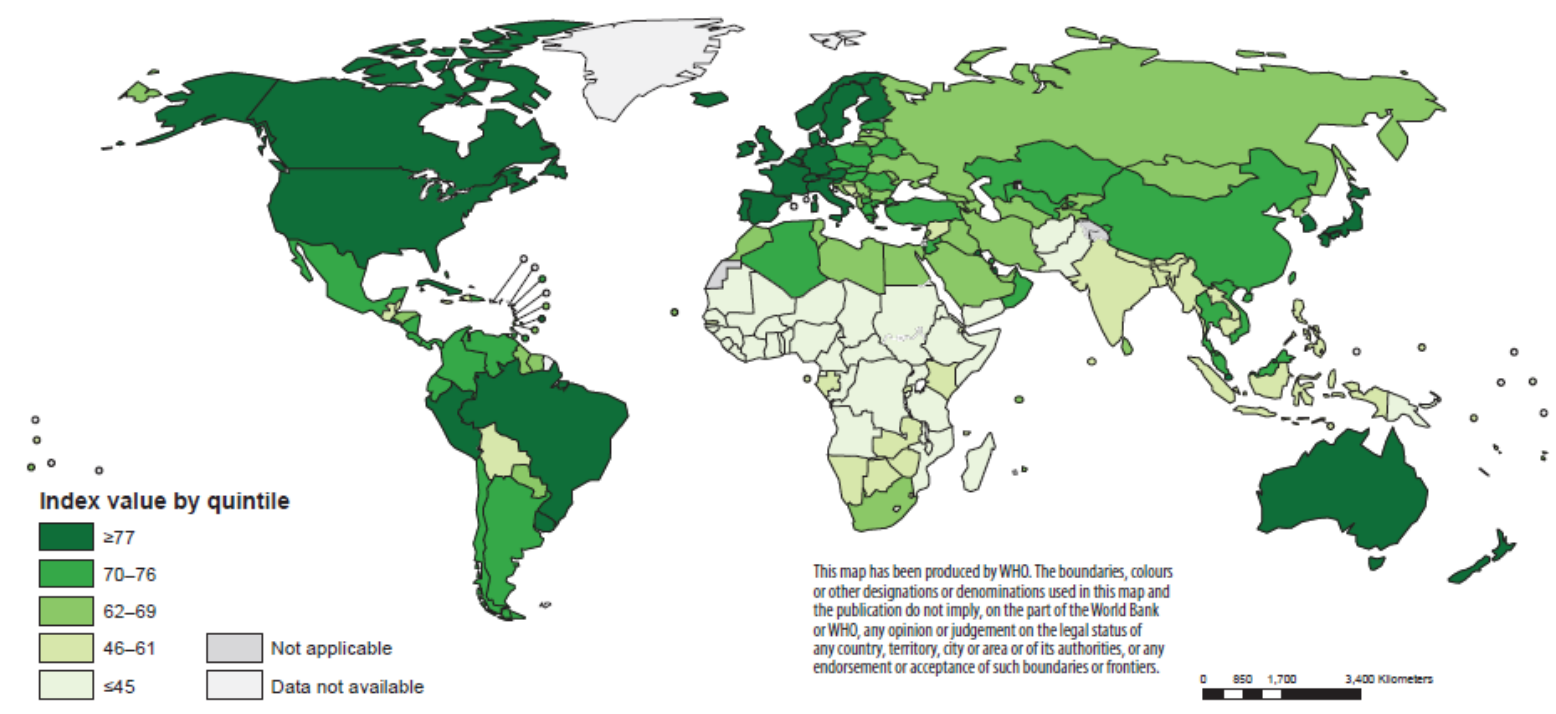

SDG: Sustainable Development Goal; UHC: universal health coverage.

Figure 4: UHC service coverage index scores for selected countries.

Progress towards SDG 3.8.1 based on index developed by the WHO, IBRD and The World Bank (2017).

Regarding Indicator 3.8.2, Wagstaff et al (2017) found that the worldwide prevalence of household catastrophic spending on health at the $10 \%$ threshold was projected to be $9 \cdot 7 \%$ in 2000, 11.4\% in 2005, and $11 \cdot 7 \%$ in 2010. This would mean that in 2010 about 808 million individuals experienced catastrophic health spending that is above 10 per cent of a family's total income, which is approximately 11.7 per cent of the global population (see Figure 5). Recent data show that the Latin American and Caribbean regions have the highest rate at the 10 per cent threshold of about 14.8 per cent of the population, with Asia coming next with about 12.8 per cent affected (see Figure 5). Many African countries are witnessing an unprecedented rise in household debt caused by CHE for health, with an increase in numbers affected of over per cent annually. Africa now follows close behind Asia where numbers of households affected are rising by less than 4 per cent per annum (see Figure 5). The current incidence rate of catastrophic expenditure related to SDG-UHC indicator 3.8.2 at 10 per cent of the total household expenditure shows $27 \%$ of Nigerians suffered CHE at the $10 \%+$ of household income rate. 


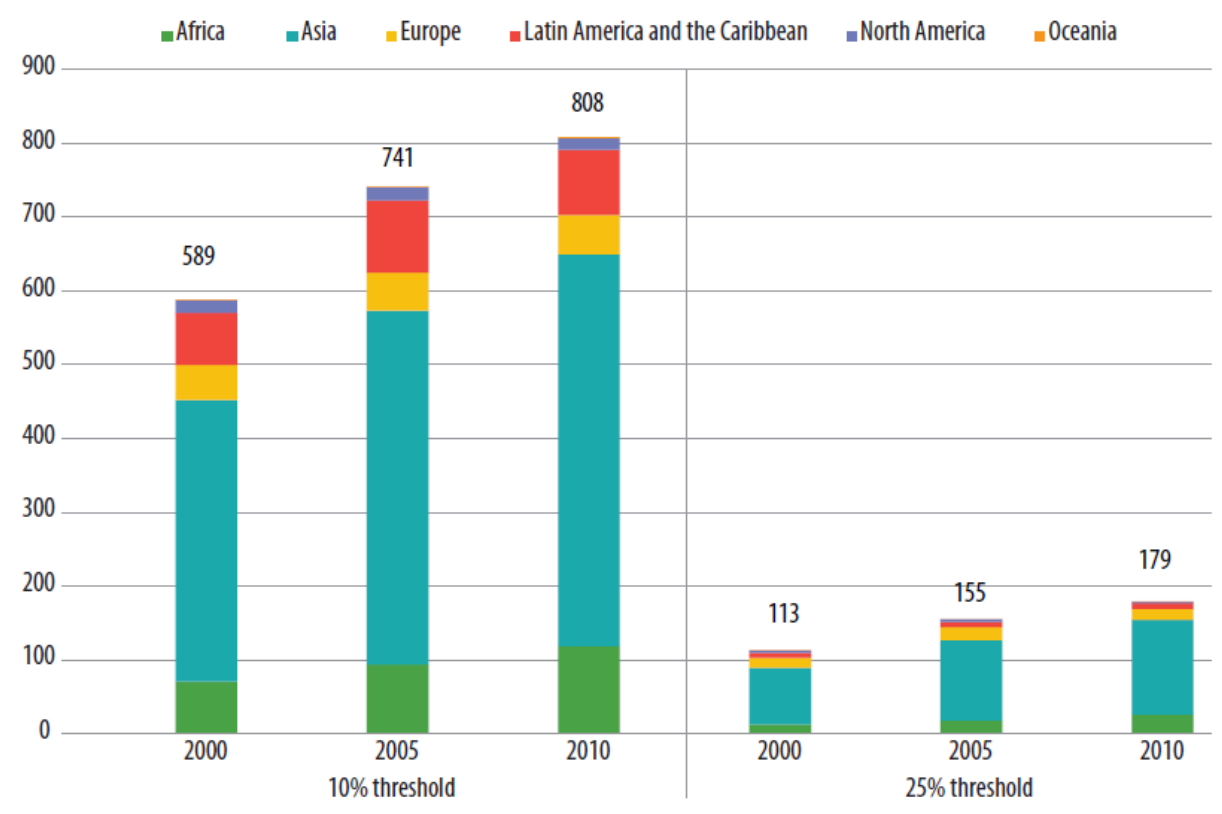

Figure 5: Global and regional trends in CHE.

This figure shows the trends between 2000 and 2010 in catastrophic health expenditure in the various regions, using CHE as in SDG indicator 3.8.2. Source: WHO (2017).

The literature suggests that by 2010 the poverty rates in the African continent were among the highest in the world at an average income (based on 2011 purchasing power parities) of \$1.90a-day. Since 2000, the level of impoverishing health expenditure has decreased in several African countries, at both the 2011 PPP \$1.90 and 2011 PPP \$ 3.10 figures (see Figure 6) (WHO, IBRD, The World Bank, 2017). 


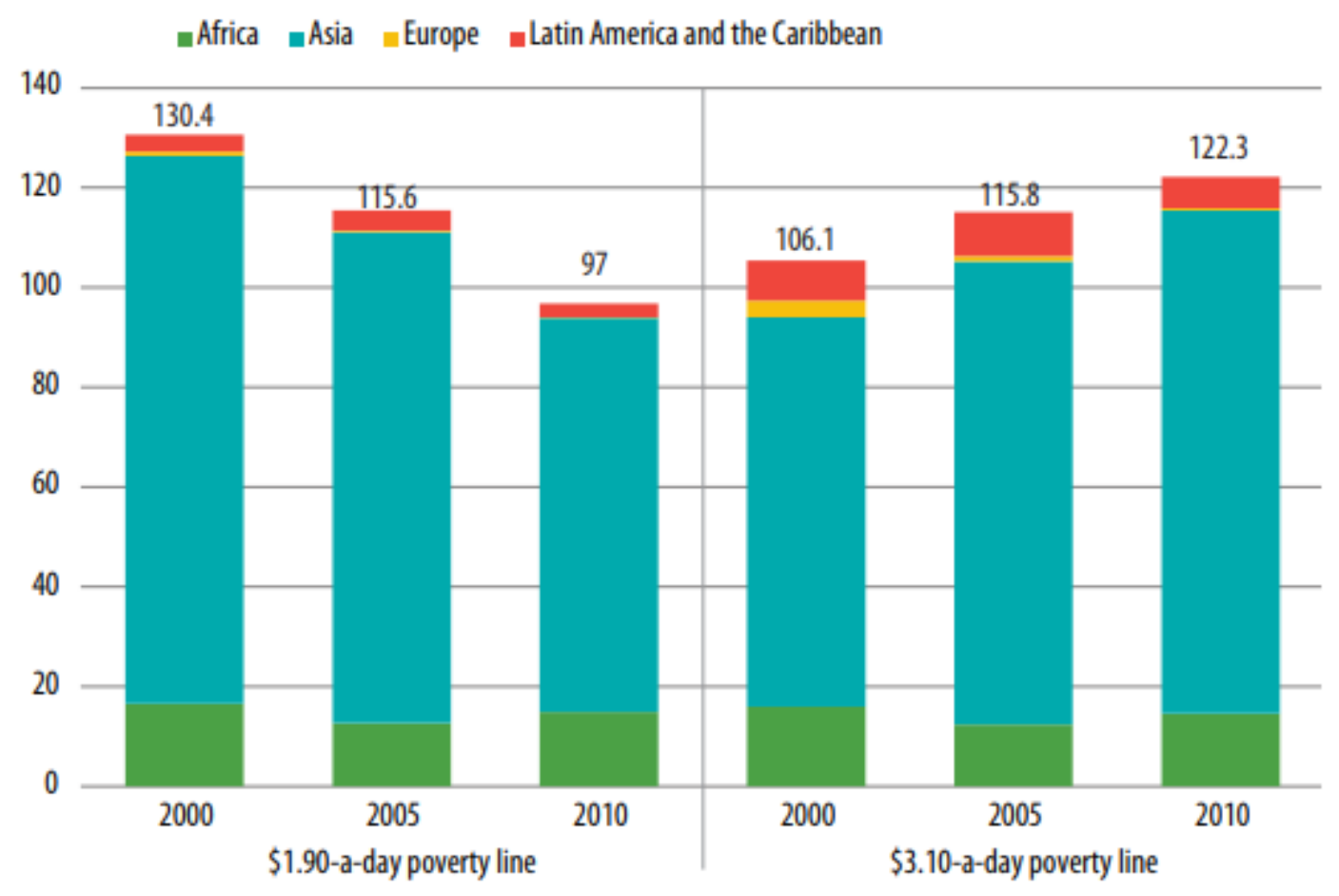

Figure 6: Global and regional impoverishment caused by out-of-pocket expenditures.

This figure shows the proportion of population falling below \$1.90-a-day and \$3.10-a-day poverty lines. Source: WHO, IBRD, The World Bank (2017).

For the above reason, LMICs including Nigeria, Rwanda, Zimbabwe, and Ghana, have sought to avoid budgetary over-reach in their plans to introduce UHC. The governments of these countries have generally had to refrain from big-bang reforms that introduce comprehensive coverage for all in a single step, and instead take the path of incremental progression towards UHC over time. Some may be able to progress faster than others, and countries with greater resources and capacities can utilise their domestic resources to do so (Wirtz et al., 2017; Kieny et al., 2017 and WHO and World Bank Group, 2017).

The chapter now moves on to provide necessary background information on Nigeria and its healthcare system. A brief account of the Nigerian economy. Politics and demographics are given, followed by an overview of the Nigerian healthcare system, including its recent development and current structure. 


\subsection{Country profile}

\subsubsection{Geography/demographics}

The Federal Republic of Nigeria is located in West Africa between Cameroon to the east and the Republic of Benin to the west, with the Atlantic Ocean bordering it to the south and Niger to its north (see Figure 7). The Federal Capital of Nigeria is Abuja; the country contains thirtysix states. Nigeria contains over 400 ethnic groups (with three major ethnic groups, Igbo, Yoruba, and Hausa), offering an extreme diversity of ethnic groupings together with multiple dialects and languages.

The country became independent from the United Kingdom on 1st October 1960 (Deekor et al., 2016). Nigeria measures $1200 \mathrm{~km}$ from east to west, and $1050 \mathrm{~km}$ from north to south. The Nigerian coast features mountains in the east and lowlands in the north; the Niger valley features high plateaus. In the north there is dry grassland containing a diverse range of plants and animals; the south is dominated by tropical forests.

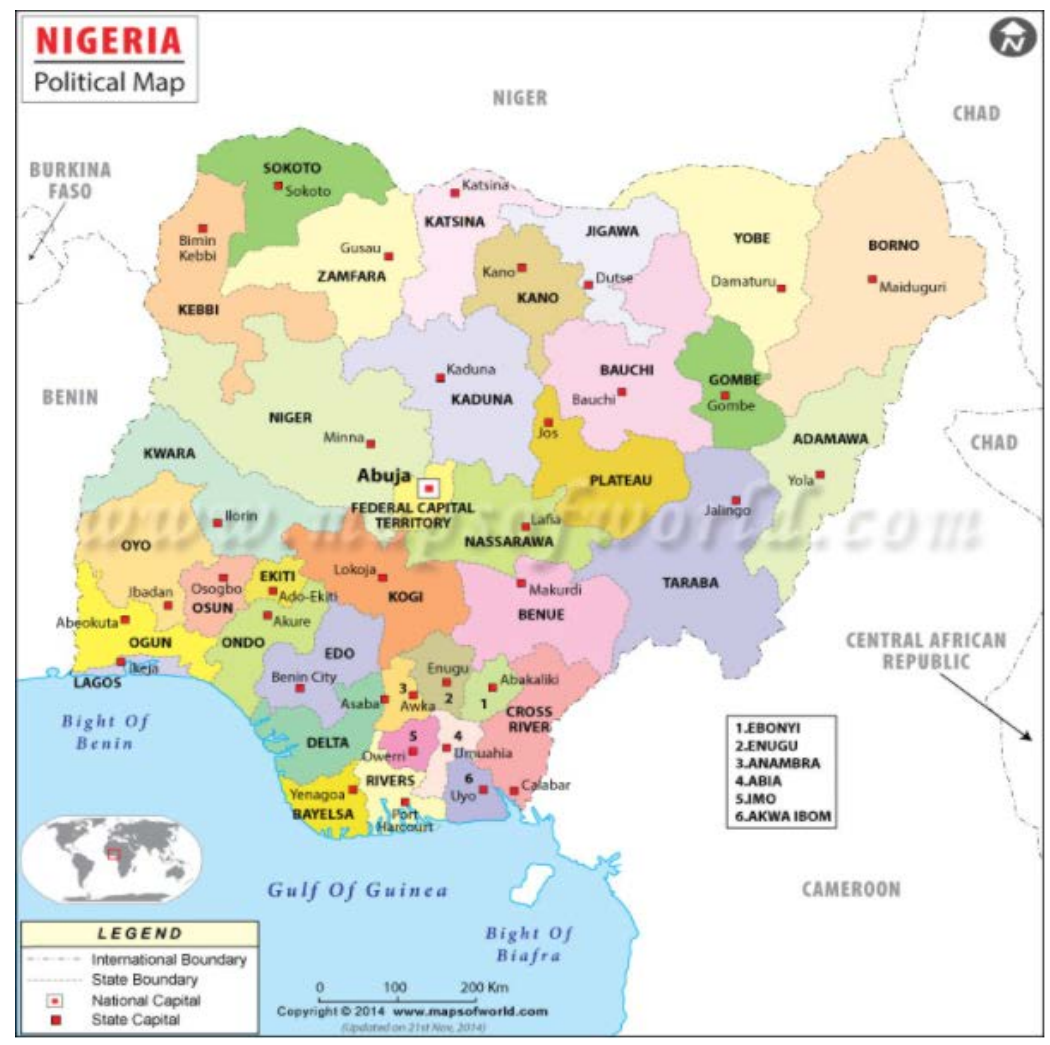

Figure 7: Shows the political map of Nigeria and neighbouring countries.

Source: Maps of World (2020). 
The Nigerian National Bureau of Statistics (2019) estimates that, based on a 2019 United Nations estimate, the Nigerian population is approximately 195,875,237. The largest Nigerian ethnic group is the Hausa-Fulani followed by the Yoruba, Igbo, Urhobos, Nupe, and Tiv. There are many languages spoken in the country, the official language being English. Non-English languages are widely spoken and include Igbo, Hausa, and Yoruba. The country is divided along Religious lines between Christianity and Islam; many northern Nigerians follow Islam, whilst those in middle and south regions are predominantly Christians (Dakar et al., 2016).

\subsubsection{Economic Context}

In 2015, Nigeria had the largest African economy, surpassing South Africa's economy, which had previously held this position for decades. Nigerian Gross Domestic Product (GDP) is in the top 30 of global economies, with total revenue of N7.166tn in 2018 (Bugit, 2018). National income comes primarily from oil production revenues, but also from export of natural resources such as palm oil, coal, rubber, and cocoa. The Nigerian economy is thus vulnerable to fluctuations in oil prices and domestic/global oil production levels (Odekunle et al., 2016). Nigeria is a tropical region with large quantities of agricultural land, with the agricultural sector delivering approximately 20 per cent of GDP in 2013. Although agriculture is still the Nigerian economy's largest sector and employs two-thirds of the workforce, hurdles to production have restricted sector performance.

Nigeria possesses the largest oil reserves in Africa and is the sixth largest oil-producing country globally, with a maximum crude oil production capacity of 2.5 million barrels per day and gas and oil reserves of 192 trillion cubic feet and 37 billion barrels respectively (Gwaambuka, 2018). Crude oil constitutes 90 per cent of exports and approximately 75 per cent of government revenue. According to the World Bank (2018), Nigeria’s GDP grew by 5 per cent from 2010 to 2014 but contracted by 2.7 per cent in 2015 and 1.6 per cent in 2016 because of a decline in global oil prices and the sabotage of many oil installations in the country. Nonetheless, recent research shows that Nigeria has gradually recovered from the 2008 recession with an increase in GDP of 0.8 per cent from 2017 to 2018, primarily driven by increases in oil production. The Central Bank of Nigeria (2018) reported that the Nigerian economy grew by 1.8 per cent in the third quarter of 2018, an increase on the 1.5 per cent expansion in the third quarter of 2017. Quarterly, the economy grew by 9.5 per cent, greater 
than a 2.9 per cent increase in the previous quarter. The Nigerian GDP Annual Growth Rate averaged 3.85\% from 1982-2018, achieving a high of 19.17 per cent in the fourth quarter of 2004 and a record low of -7.81 per cent in the fourth quarter of 1983 . Nonetheless, the government is committed to improving Nigerian lives through the implementation of economic reforms as described in the Economic Recovery and Growth Plan 2017-2020, predicting future growth by 2.8 per cent from 2019 (CBN, 2018). There has been significant socio-economic progress in Nigeria since 2005, when the Human Development Index value in the country increased by 14.1 per cent from 2003 (the base year) projected at 0.443 to 0.534 in 2018 (UNDP, 2018).

Nigerian socio-economic progress could be further improved through overcoming a number of specific developmental challenges and obstacles, including dependency on crude oil production, renovation of dilapidated infrastructure, diversification of the economy, improvements to governance and accountability problems and offering better living standards for the population. Research has suggested that these challenges and obstacles increase inequality in Nigeria, with the gap between the rich and the poor widening. Other research has highlighted Nigeria's inequality as one of the key causes of an increase in the Islamist Boko Haram insurgency in the north of the country (Dunn, 2018). The increasing divide between the North and South has been underscored by a lack of economic development in the north compared to the south. The Nigerian government is aware of these challenges and has committed to providing access to essential services and reducing poverty through the introduction of inclusive development policies (World Bank Group, 2018; Odekunle et al., 2016; CBN, 2018).

\subsubsection{Political Context}

Politically, Nigeria was created in the 19th century, when the British Empire colonised coastal areas around the Niger River. Using the divide and rule method, the British empire divided/controlled the formation of empires, kingdoms, and city-states (including the Oyo and Benin kingdoms in the south, and the Hausa kingdoms and the Borno dynasty in the north), pitting ethnic groups against each other in the western, eastern, and northern regions (Gov. Ng, 2019). In 1960, after half a century of British direct rule, Nigeria achieved independence and created a republic, falling under military rule after the 1966 coup by Maj-Gen. Johnson Aguiyi- 
Ironsi. Under military rule (1966-1999), the country experienced eight military coups (in January and July 1966, 1975, 1976, 1983, 1985, 1990 and 1993). It also underwent the alleged Vatsa coup of December 1985, a civil war (between 6 Jul 1967 - 15 Jan 1970), having eight Military Heads of States and two short term non-military Head of States (1979-1983 and August 1993-November 1993) during the period (Gov. Ng, 2019). In 1999, Nigeria transitioned from a military rule to democracy through the election of former military Head of State Olusegun Mathew Okikiola Aremu Obasanjo, who represented the People’s Democratic Party (PDP). Ever since, Nigeria has adhered to a democratic political system, wherein all individuals have equal rights and freedom of choice to realise their principles and defend and promote their interests via multi party-political platforms whose candidates promise to realise their ideals upon election (Aregbeshola and Adewale, 2011).

Traditionally, the political sphere has been characterised by a two-party system, with various opposition and ruling parties holding a central role (Kim S-Y, 2006). Historically, political parties have reflected the country's Muslim-Christian divide, being headed by former Military leaders and characterised by limited democratic processes with a heavy reliance on a plutocratic ruling class (Adeleye, 2013; Brown, 2013). Muhammadu Buhari (the President since 29 May 2015) is from the All-Progressives Congress Party (APC), which achieved 54 per cent of the votes cast. In this election they defeated the main opposition party, the People's Democratic Party (PDP), securing 196 seats in the House of Representatives, against the PDP's 133 and the minority All Progressives Grand Alliance party’s five. In the Senate, the upper chamber of Nigeria's bicameral legislature, the APC took 60 seats and PDP 48 (The Commonwealth, 2019).

Nigeria's judiciary is independent from its legislature. The Chief Justice presides over the Supreme Court, which is made up of up to 15 Associate Justices. It is the final court of appeal with jurisdiction over disputes between states, or between the federal government and a state, especially relating to funding and resource allocation, and over election disputes. The Federal Court of Appeal has a president and at least 35 justices, at least three of them having Sharia Law expertise and another three having experience in Nigeria's customary law. Beneath these, there is also a Federal High Court, with each of the 36 states also having a high court. States are entitled to have a Sharia court of appeal and a customary court of appeal. Federal court judges are appointed by the president on recommendations from the National Judicial Council and are approved by the Senate. High court judges are appointed by state governors based on 
recommendations from the National Judicial Council, and approved by the state assembly (Commonwealth Governance, 2019).

Critics have labelled Nigeria's democracy as profoundly undemocratic. They highlight several key problems, including the inflation of census figures to make some regions look populous, the ability of stronger political parties to manipulate election results in their favour and the preponderance of corruption and lack of transparency (Abdulmalik et al., 2016). The Nigerian health sector in particular has been widely disrupted by this rise in corruption, which has led to the inability to provide financial resources in order to meet public health sector requirements. A lack of financial governance has also led to improper deployment of state resources (Uzochukwu et al., 2016). Nigeria is affiliated with a diverse set of international organisations, including the United Nations (UN), the African Union (AU), the African Development Bank, the International Labor Organization (ILO), IMF, the International Olympic Committee (IOC), the World Health Organization (WHO) and the World Trade Organization (AU, 2017).

\subsubsection{Health status}

Nigeria's health status is crucially dependent on its socioeconomic context. In a population of over 200 million, 60 per cent of Nigerians live in rural areas (Odekunle et al., 2016). There is high social inequality, particularly in regard to access to healthcare services. About 49 per cent of rural inhabitants in Nigeria have access to adequate social amenities such as roads, food, sanitation, and clean water, with around 51 per cent of Nigerians lacking access to clean water facilities. This compares with other 70 per cent of Nigerians in urban areas who have access to these social amenities (Uzochukwu et al., 2016).

Nigeria's total healthcare expenditure is one of the lowest among sub-Sahara African countries and indeed worldwide. Nigeria spends less than 5 per cent of its GDP on health, which is less than the OECD average of about 8.9 per cent in 2017. Approximately 75 per cent of health expenditure takes the form of household out-of-pocket (OOP) payments, which increases the levels of poverty and impoverishment in the country (FMoH, 2017). The OOP expenditure as percentage of current health expenditure (CHE) in Nigeria has fluctuated and has tended to decrease from the period 2001 - 2015, ending in 2015 at 72.24 per cent, an increase by 0.01 per cent over the previous year (World Bank Group, 2017). The UHC service index of Nigeria is 42 compared to the global UHC service coverage index of 64, or 46 for the continent of 
Africa. Northern Africa having an index of 64 and Sub-Sahara Africa 42 (World Bank Group, 2017).

Nigeria's key health indicators offer further insights into the limitations of the country's health system. Key health indicators such as life expectancy, the burden of communicable diseases, maternal mortality rate, and infant mortality rate in Nigeria are amongst the lowest in subSaharan African countries. This deterioration is the combination of years of poor governance, lack of accountability, economic recession and political instability (WHO, 2017). The World Health Organization (2018) states that Nigeria has the third highest infant mortality rate globally (9 per cent), after Pakistan (10 per cent) and India (24 per cent) and followed by the Democratic Republic of the Congo (4 per cent) and Ethiopia (3 per cent). Nigeria's infant mortality rate is 64.6 per 1000 live births compared to the sub-Saharan African average of 51.5 per 1000 live births and a global average of 39.1 per 1000 live births (World Bank Group, 2018). The Nigerian Minister of Health, Isaac Adewole, confirmed that the country had made significant progress in decreasing the rate from 102.5 per 1000 live births in 2003 to 64.6 per 1000 live births in 2017 (Punchng, 2017). Likewise, the country has a high maternal mortality rate of about 814 per 100,000 live births, compared to an African regional average of 547 per 100,000 live births and a global average of 216 per 100,000 live births. Nigeria is considered one of the most dangerous places to deliver a child and offering the fourth worst national maternal mortality ratio, after Sierra Leone, Central African Republic, and Chad. Total life expectancy in Nigeria is approximately 54 years, below the sub-Saharan African average of 61 years and a global average of 72 years (WHO, 2018).

USAID's (2019) research shows that the number of new HIV infections in Nigeria stands at 130,000 and AIDS-related deaths at around 53,000 deaths. About 1.9 million Nigerians live with HIV, with 55 per cent adults having access to antiretroviral drugs, and 32 per cent of pregnant women with HIV having access to treatments preventing mother-to-child transmission of the disease. Mother-to-child transmission of HIV has infected approximately 35 per cent of children in Nigeria (USAIDS et al., 2019). Thus, the Federal Ministry of Health (FMoH) focuses on implementing policies such as the UHC policy in response to Nigeria's poor key health indicators. Current policies aim to improve health outcomes related to maternal mortality, the disease burden of HIV/AIDS, infant mortality, and life expectancy, areas where Nigeria had failed to achieve MDG targets (FMOH, 2017). 


\subsection{Overview of the Nigerian Health System}

This section reviews Nigeria's healthcare system in regard to its structural framework, development and operational health strategy. In recent years Nigeria's health system has been profoundly affected by wider political and economic events, particularly the recovery from military rule, crude oil price fluctuations and the 2008 global recession which seriously undermined its health goals. For instance, between 2015 and 2018 proposed health expenditure dropped from 5.78 per cent to 3.9 per cent of GDP, significantly lower than other Sub-Sahara African countries like Zambia (6.2 per cent), Tanzania (6.8 per cent), Malawi (7.2 per cent), Kenya (7.5 per cent) and South Africa (7.5 per cent). Significantly, all the above countries (including Nigeria) had committed to spend 15 per cent of GDP on health in the 2015 Abuja Declaration (McIntyre et al., 2018). The World Health Organization (2017) has indicated that Nigeria's health expenditure is directly related to its health system's poor performance, highlighted starkly by the recent outbreaks of Ebola virus, Monkey pox, Yellow fever, Cholera and Lassa fever, together with high maternal and infant deaths. As suggested above, this health crisis has its roots in Nigeria's social and economic context; a key example of this can be seen in the recent outbreak of the Ebola Virus, which created national health insecurity in most of Nigeria. This insecurity prevailed despite significant contributions from international health organizations, which ensured that the country had the capacity to deal with and tackle the deadly virus in a manner comparable to more developed, western countries. Moreover, it is clear that sustainable social and economic growth within Nigeria depends to a significant extent on improved performance from the healthcare sector (Abdulmalik et al., 2016).

The World Health Organization rates Nigeria 187th out of 191 countries in regard to health system performance and suggests that one-third of 700 health facilities are dysfunctional, placing millions of Nigerians in need of health support (Mathew, 2019). The country is also held back by poorly functioning primary health care services which cannot cope with the emergence of non-communicable diseases (such cancer, heart related diseases and diabetes), weak accident and emergency responses and low healthcare coverage under the NHIS scheme. Overall health indicators suggest that Nigeria, compared to other Sub-Saharan countries, performs worse in terms of healthcare delivery, with Nigeria failing to achieve most of the health related MDGs by 2015. For example, in 2017, Ghana allocated 6 per cent of GDP to health, while Nigeria budgeted just 4.7 per cent. Despite Nigeria having the largest economy in Africa, Ghana is ranked 135 out of 191 countries, 52 places ahead of Nigeria in the world 
health ranking. Instead of having one of the most developed health systems in Sub-Sahara Africa, Nigeria healthcare suffers from a lack of effective regulation and governance arrangements (Wollum et al., 2015). Moreover, a lack of healthcare facilities, especially in rural areas, has created many potential healthcare issues. Each level of the government, from Federal to local, faces challenges in meeting healthcare systems requirements due to a lack of services, resources and coordination in the health system, impeding the sector's overall development. There is also profound inequity in the supply of medicines to people suffering from health issues in rural areas (Shuaib et al., 2014).

These issues are compounded by negligence and a lack of responsibility from various levels of government in providing proper healthcare services (Deekor et al., 2016). Despite the government's failure to meet the healthcare requirements of Nigerian people, many international organizations are engaged in providing effective health facilities and services, including the World Health Organization and the United Nations Children's Fund (UNICEF). The institutional structure of healthcare in Nigeria is based on policies produced and regulated by the Federal Ministry of Health. The Nigerian Ministry of Health is responsible for establishing state health institutions to provide the maximum possible healthcare services to the people of Nigeria (Nwadiuko et al., 2016). This institutional structure is divided into three tiers, at Federal, State and local levels of government, but is supported by other key stakeholders such as the National Health Insurance Scheme (NHIS), National Primary Health Care Development Agency (NPHCDA), Health Maintenance Organizations (HMOs), Ward Health Authorities, NGOs and development partners and Donors (such as UN Bodies and External Government Agencies) (see Figure 8). These stakeholders are engaged in healthcare system regulation, including service provision and financing across all of Nigeria (Uzochukwu et al., 2015). 


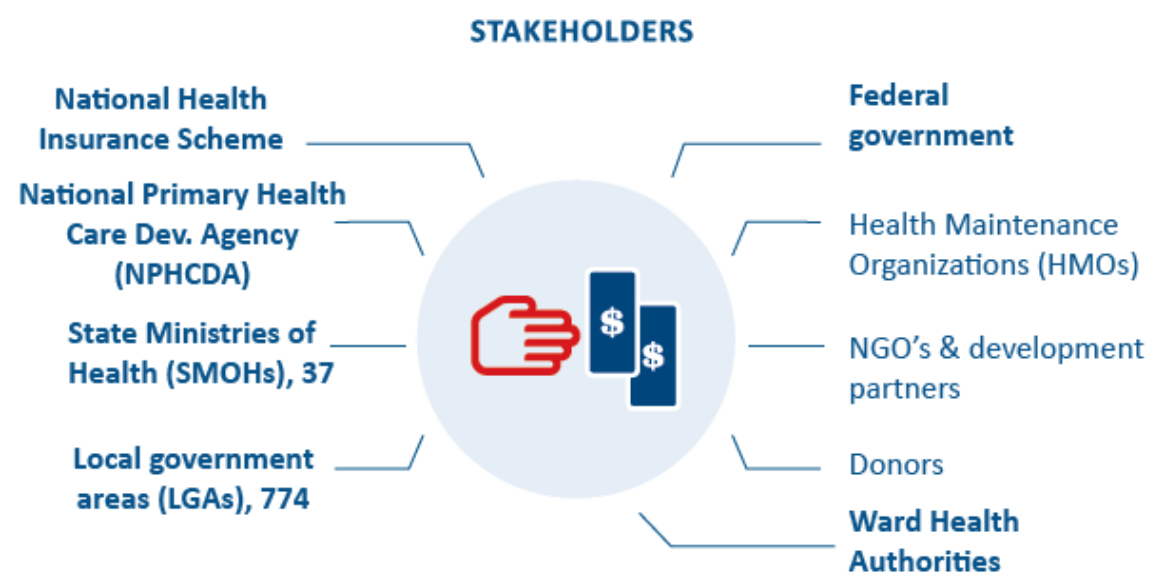

Figure 8: Key stakeholders in the Nigerian healthcare system.

\section{Source: Pharm Access Foundation (2016).}

When there is a lack of proper healthcare a country cannot maintain steady economic progress (Deekor et al., 2016). Research by the National Council on Health also supports the argument that healthy Nigerians will help the economy to thrive. However, in contemporary Nigeria, difficulties in healthcare provision are becoming an obstacle to economic development, with citizens suffering due to a lack of facilities available related to health infrastructure. Leadership in particular has consistently been a crucial factor in developing the Nigerian health sector's ability to achieve the required improvements. The Federal Government, together with key stakeholders are required to address health problems to reduce the national burden related to the inadequate healthcare system and establish healthcare infrastructures which would improve its healthcare system and policy (Uzochukwu et al., 2016).

\subsection{Chronological background and Timeline}

The earliest roots of Nigerian healthcare can be traced back to the early fifteenth century and the Kingdom of Nri. During the 1400s healthcare in Nigeria mainly depended on traditional medicines and treatments, many of which are still observed in parts of the country (Uzochukwu et al., 2016). From 1472 to 1880 many European Missionaries, including the Portuguese and Spanish, began to explore Nigeria and establish colonies (Nwadiuko et al., 2016). Doctors from Europe introduced a western-style healthcare system, primarily for the benefit of European incomers. From 1880-1945 the influence of British missionaries became prominent within Nigeria, culminating in the British Imperial annexation of the country and the establishment of 
British Nigeria (Odekunle et al., 2016). During the era of British colonisation new healthcare practices and healthcare infrastructure were introduced. After World War II healthcare policies initially followed the pre-war pattern, and Nigeria's colonial development plan of 1945 built on the colonial heritage.

After Nigeria's independence in 1960, the newly established FMoH formulated a National Development Plan and National Health Policy to improve the health system. After the 1978 Alma-Ata Declaration much of the focus shifted to primary healthcare-led policies. Following a period of military rule and a transition to democracy in 1999, the FMoH oversaw the launch of two five-year strategic plans, two successive revisions of the National Health Policy and the implementation of NHIS. This last crucial change mostly focused on achieving Millennium Development Goals (MDGs) targets before 2015 and UHC for Nigerians in the healthcare sector (Abdulmalik et al., 2016) (see Table 1 for key chronology events of the Nigerian health system). Due to failures in reaching these MDGs targets, the Nigerian government has refocused its attention on improving financing arrangements so as to increase the prospect of successful implementation of health policies. Despite this focus, less than 5 per cent of the population have health insurance coverage, and these health care issues are significant challenges to the Nigerian government (Uzochukwu et al., 2016). With the world shifting from the MDGs to SDGs, the Nigerian government has increasingly linked the aim of improving access to healthcare to the SDG targets. Much of the ongoing policy debate as the present study started was concerned with agreeing milestones for widening healthcare coverage, in line with SDG3 - “Ensure healthy lives and promote well-being for all at all ages” (WHO, 2017). 


\begin{tabular}{|c|c|c|}
\hline Year/Period & Type of event & Key Nigerian health system events \\
\hline 1880 & Organization & The Church Missionary Society was the first medical institution to open in Nigeria. \\
\hline $1945-1955$ & Policy & $\begin{array}{l}\text { Healthcare became regionalized under the launch of the First Colonial Development plan in Nigeria. The objective of the health policy was to provide } \\
\text { subsidized public health services for many civil servants and their dependents while hospital owned by religious groups provided health care to their } \\
\text { members and those in need. }\end{array}$ \\
\hline 1951 & Organization & The Nigerian Medical Association (NMA) is established. \\
\hline $1952-1954$ & Policy & Regional autonomy of medical services transferred to the goveminent. \\
\hline 1954 & Policy & $\begin{array}{l}\text { The expansion of health services to individuals living in rural areas presented under an overall national policy called the"Policy for Medical and Health } \\
\text { Services" for Nationwide Health Care Services. }\end{array}$ \\
\hline 1956-1962 & Policy & The Second Colonial Development plan implemented to improve the health sy stem in Nigeria. \\
\hline 1960 & Background & Nigerian Independence \\
\hline 1962-1968 & Policy & Implementing the first National Development by an independent govenument for the health sy stem. \\
\hline 1965 & Organization & $\begin{array}{l}\text { Nigeria transitioned from the United Nigeria Insurance Company (UNIC) to a Health Maintenance Organization (HMO) sty le of a healthcare insurance } \\
\text { scheme. }\end{array}$ \\
\hline 1970-1975 & Policy & The Second National Development Plan launched by the FMoH to improve healthcare. \\
\hline 1975-1980 & Policy & The Third National Development Plan launched by the FMoH to improve the health sy stem. \\
\hline 1981-1985 & Policy & The Fourth National Development Phan launched by the FMoHt to improve healthcare. \\
\hline 1987 & Policy & $\begin{array}{l}\text { The FMoH launched the Primary Health Care (PHC) with the following objectives: Improving vaccination programs, improving on the health system } \\
\text { reaction to the communicable disease epidemic, promoting better access to matemal and child care, and investing in family planning programs; and } \\
\text { increasing the human resource capability of tack ling widespread health difficulties. }\end{array}$ \\
\hline 1988 & Policy & Introduction of National Health Policy Plans \\
\hline 1999-2005 & Policy & The Federal Govenument created the National Health Insurance Scheme (NHIS) Act, which became operational in 2005. \\
\hline 2004 & Policy & A revised National Health Policy \\
\hline 2004-2007 & Policy & The FMOH implemented the Health Sector Reforn Program (HSRP) \\
\hline 2006 & Policy & $\begin{array}{l}\text { New National Health Policy is adopted. The plan includes a re-designed National health insurance scheme that protects citizens against high costs of } \\
\text { treatment, and a fair financing of health care providers. }\end{array}$ \\
\hline $2004-2008$ & Policy & The FMoH launched the Five Year Strategic Plan for projected health system improvement in Nigeria. \\
\hline $2009-2012$ & Policy & Implementing the National Human Resource For Health Strategic Plan \\
\hline 2009-2015 & Policy & The FMoH adopted the National Strategic Health Development Plan-One \\
\hline 2014 & Policy & The 2014 Abuja Declaration on Universal Health Coverage \\
\hline 2016 & Policy & A revised National Health Policy \\
\hline $2017-2022$ & Policy & A revised National Strategic Health Development Plan-Two (2017-2022) \\
\hline
\end{tabular}

Table 1: Timeline of key events in Nigeria's health system.

Source: Ajovi Scott-Emuakpor (2010) and BBC (2018). 


\subsection{The Structure of the Nigerian health system}

The Nigerian health system is organized into a three-tier structure of primary, secondary and tertiary tiers, which are accountable to all levels of government (Federal, State, and Local government, as well as at the Community level) for all major health system functions, including stewardship, financing and healthcare delivery. However, each level of government also uses three distinct models of health services provision, these being the public, private and faithbased models (see Figure 9). Pharm Access Foundation (2016) estimates that public health sector healthcare provision remains a concurrent responsibility for about 73 per cent of the three tiers of government, while the private health sector provides about 12 per cent of healthcare delivery and faith-based institutions constitute about 1 per cent of healthcare provision. The remaining 14 per cent of healthcare provision is not specified due to a lack of data surrounding these issues (see Figure 9) (Pharm Access Foundation, 2016).

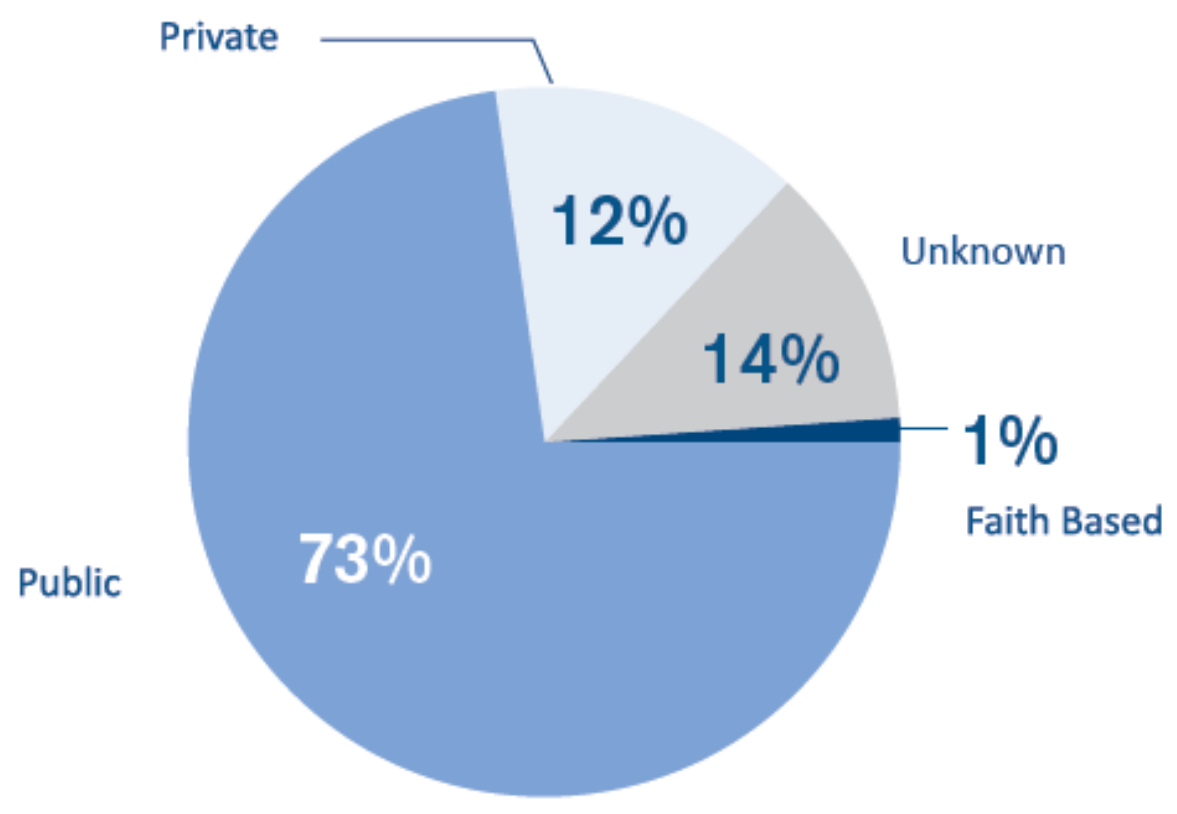

Figure 9: Nigeria’s pluralistic healthcare system.

Source: Pharm Access Foundation (2016).

The Federal level of the health sector is responsible for policy development, regulation, and overall stewardship, providing health service through tertiary care and teaching hospitals (47 in total) and national laboratories. The State level of the health sector is responsible for 
secondary hospitals (3768 in total), which include district hospitals, comprehensive health centres and specialist and general hospitals and for the regulation and technical support for primary healthcare services. Local healthcare, which is the least-well funded and organized level of government, is responsible for primary health care in each ward (29,854 facilities in total), including dispensaries and health posts, health centres and clinics. As a result of poor financing, primary healthcare services in many local areas are a weak base for the healthcare system (Table 2) (MedCOI, 2017 and Pharm Access Foundation, 2016).

\begin{tabular}{|c|c|c|}
\hline Responsibility of: & $\begin{array}{l}\text { Levels of Care and Responsibilities } \\
\text { (ca. } 34,140 \text { healthcare facilities)* }\end{array}$ & Type of healthcare facilities \\
\hline Federal Government & $\begin{array}{l}\text { Tertiary Care } 47 \\
\text { Public: } 85 \% \text {, Private: } 9 \% \text {, Faith- } \\
\text { Based: } 4 \%\end{array}$ & Teaching Hospitals. \\
\hline State Government & $\begin{array}{l}\text { Secondary Care } \quad 3,768 \\
\text { Public: } 32 \% \text {, Private: } 28 \% \text {, Faith- } \\
\text { Based: } 4 \%\end{array}$ & $\begin{array}{c}\text { District Hospitals, Comprehensive } \\
\text { Health Centres and Specialist and } \\
\text { General Hospitals. }\end{array}$ \\
\hline Local Government & $\begin{array}{l}\text { Primary Care } 29,854 \\
\text { Public: } 79 \% \text {, Private: } 10 \% \text {, Faith- } \\
\text { Based: } 1 \%\end{array}$ & $\begin{array}{l}\text { Dispensary and Health Posts (30\%), } \\
\text { Health Centres (44\%) and Clinics } \\
(26 \%) .\end{array}$ \\
\hline
\end{tabular}

Table 2: Levels of the Nigerian health system.

Source: Pharm Access Foundation (2016).

While the organizational charts convey the impression of a well-coordinated system, its practical workings are far from seamless. There is often a duplication and confusion of roles and responsibilities among different government tiers. This results in weaknesses in 
coordinating and tracking performance and benchmarking, especially at community level, where primary healthcare services are often poorly implemented (Pharm Access Foundation, 2015). The system for referrals between these four levels is inconsistent and often ignored. Ailments that are supposed to be managed at the primary level are often managed at the tertiary level. This is because the other levels are very weak, with inadequate infrastructure, personnel and other deficiencies (GOV.UK, 2018). All four government levels, employ a full range of personnel, including doctors, nurses, midwives, community health extension workers and allied medical professionals such as laboratory scientists, pharmacists, dentists and managers. Health workers in the public sector are paid by the government level that employs them.

Generally speaking, the Federal Government is responsible for the funding of tertiary hospitals, the state governments look after secondary hospitals, and the local government authorities take care of the PHC facilities. Nonetheless, there are a few limited exceptions: in some states, health professionals working within PHC facilities might be employed by the Federal Government, while some higher-level health professionals employed by the Federal parastatals or vertical programs may also work at the secondary and primary levels. Nigeria has one of the largest stocks of human resources for health in Africa, but these still do not meet the country's needs. In 2016, an inventory of healthcare personnel indicated a total of 65,759 doctors (38.9 per 100000 population), 249,566 nurses and midwives (148 per 100000 population), 505 dentists (0.001 per 1000 population), 5,483 dental technicians ( $>0.04$ per 1000 population), 19,225 medical laboratory scientists (11.3 per 100000 population), 2,818 physiotherapists (1.7 per 100000 population), 1.286 radiographers (0.76 per 100000 population) 5,986 community health officers (CHOs) (3.5 per 100000 population), 42,938 community health extension workers (CHEWs) (25.3 per 100000 population), 28,458 junior community health extension workers (JCHEWs) (16.8 per 100000 population) and 16,979 pharmacists (10 per 100000 population) (FMoH, 2016).

The Federal Ministry of Health has established a national health workforce registry, although this is not yet fully functional, and the registry's data are not regularly updated. At present, the regulatory bodies maintain health workers' records in their jurisdiction, but the records are often not up to date and are therefore inadequate for planning (FMoH, 2016). There are more doctors per capita in the south than in the north, though no large regional disparities exist in regard to the number of nurses. Most doctors and nurses work in secondary and tertiary level hospitals or in private practices. The United Kingdom and the United States are the top 
destinations for migrant doctors. An Organisation for Economic Co-operation and Development (OECD) report identified Nigeria as the leading African source of foreign-born nurses practising in OECD countries. In spite of this brain drain, there does not appear to be any official action taken either to retain the health professionals or to re-attract those in the diaspora. With Nigeria's estimated 193 million people, medical tourism to external destinations will continue to thrive (Eriki et al., 2015).

\subsection{Nigerian health system revenue sources}

There are various revenue sources for financing a health system that include direct and indirect taxes, OOP payments, donor funding, voluntary payments, co-payments, and mandatory payments that are adjusted into revenue pooling to minimise the risks associated with healthrelated problems (Nkwo et al., 2015). In Nigeria, healthcare financing is focused on several sources, primarily out-of-pocket payments, donor funding, tax revenues and various types of health insurance (Akazili and James et al., 2017). The World Bank Group (2017) states that the National Health Accounts estimate that the bulk of Nigeria's health funding is borne by households' OOP expenditure: these OOP fees are the combination of facility entrance fees, drug costs, material costs and the consultation fees. OOP payments tend to have a regressive impact, since they take a higher proportion of the income of the poor than of the wealthy (Fabricant, Kamara and Mills, 1999). Household expenditure as a proportion of total health expenditure (THE) varied between 72.2 per cent in 2015 and 83.1 per cent in 2003. Nonetheless, Nigeria's OOP expenditure as a share of current health expenditure has fluctuated substantially in recent years, decreasing between 2001 and 2015. A recent WHO report (2017) suggests that this has massive implications for those seeking to access health services without suffering financial hardship. The report shows that, although they may have access to some health services, more than half of the world's 7.3 billion people do not receive all of the essential services they need. Regarding financial protection, over 800 million people spend at least 10 per cent of their household budgets to pay for health care, and about 100 million people are pushed into extreme poverty due to their health expenditure (WHO, 2017). The federal budgetary component of health expenditure has been at best 5 per cent of the national budget, even though African countries committed to an allocation of 15 per cent in the 2014 Presidential Summit on Universal Health Coverage (UHC) in Abuja, Nigeria (MedCOI, 2017). Nigerian health expenditure as a percentage of GDP is lower compared to other Sub-Saharan 
African countries such as Zambia (6.2 per cent), Tanzania (6.8 per cent), Malawi (7.2 per cent), Kenya (7.5 per cent) and South Africa (7.5 per cent) (McIntyre et al., 2018).

\subsection{Study aims and research questions}

With the above considerations in mind, the student has formulated the following general study aims, and a set of related research questions:

Aims

1. To investigate the roles and interactions of policy actors in the UHC policy process and how they impede or advance progress in extending coverage.

2. To investigate the extent of consensus or disagreement between influential actors and interest groups on the direction of UHC policy, and how that has affected progress.

3. To identify the barriers and obstacles which affect Nigeria's process of health policy towards achieving UHC.

Research questions

1. Who are the influential actors and organizations shaping UHC policy in Nigeria?

2. What are the patterns of influence and power relationships within the policy network?

3. What are the respective roles of domestic and international actors in shaping policy?

4. Is there disagreement within the policy network over the approach to UHC to be pursued, or the feasibility or desirability of UHC policies?

5. What are the main gains and setbacks to date, as seen by policy actors?

6. How far along the path towards UHC do policy actors believe Nigeria has travelled, and what is their assessment of the prospects of achieving adequate coverage?

7. What general lessons can Nigeria provide regarding the policy process associated with UHC reform?

\subsection{Addressing the questions through a mixed-methods study}

Answering the above research questions will require an empirical investigation of the social interactions between policy actors and policy organisations involved in the formulation and implementation of UHC-related policies in Nigeria. It will be necessary to investigate patterns of social interaction, influence and conflict within the network of actors and organisations that 
shapes policy. The study methodology will be explained in detail in Chapter 3. For now, it is enough to say that the breadth and complexity of the policy network, as well as the elite character of the actors, made direct observation infeasible, so that the research needed to turn to accounts of action in the network provided by policy actors themselves. This was approached via a mixed-methods study. The two main components of field work were face-to-face structured interviews (involving quantitative analysis) and face-to-face semi-structured interviews (involving qualitative analysis) with key policymakers/stakeholders. This was supplemented by analysis of relevant documents.

\subsection{Plan of the thesis}

Having outlined the scope and aims of the study in the present chapter, we will turn in Chapter 2 to review relevant background literature, and in Chapter 3 to discuss the study methodology, devoting separate sections to the different approaches required for a quantitative social network analysis (SNA), and a qualitative analysis of in-depth semi-structured interviews with the same network actors. This is followed by the two main findings chapters relating to the SNA data and data from the qualitative interviews. The thesis then moves on to a Discussion chapter, intended to bring the findings from the two major study components together, and ends with a Conclusions chapter. 


\section{CHAPTER 2}

\section{LITERATURE REVIEW}

\subsection{Introduction}

This chapter reviews relevant literature on universal health coverage (UHC) and the development of UHC-related policies in Nigeria. This includes a discussion of "normative" literature on the benefits of UHC, a review of what other countries is doing, criticisms of UHC, and consideration of policies so far introduced in Nigeria. The main purpose of conducting this literature review is to provide background for the empirical findings presented in subsequent chapters on the current Nigerian policy discourse on UHC and the steps so far taken to widen coverage.

\subsection{Literature search strategy}

The literature review used two main search strategies, namely electronic and manual searching. The electronic search mechanism gathered relevant information from 1997 to 2020 using several web databases, such as Science Direct, Medline, Google Scholar, the World Bank Group website, the WHO website, the International Labour Organization website, and the Ministry of Health Nigeria website. The keywords used in the search strategy included "universal coverage”, “universal health coverage”, “UHC”, “health insurance”, “dimensions of UHC” AND “Nigeria’s health system”, “Sub-Saharan Africa”, “health” or "health care*” AND “health financing*” or “UHC in Nigeria” AND “impact”. Manual searching involved the use of textbooks, official documents, and articles in order to gather data relevant to the thesis. This included searches of the reference lists of relevant articles for additional sources, and 'forward chaining' by identifying sources that cited a relevant article (Cooper et al, 2018). In this initial search phase, 278 articles were identified, and their abstracts were checked for relevance to the topic and 89 pieces of research were then considered in detail. At the time of writing up the thesis, over 50 additional sources have been reviewed to bring the discussion up to date. 


\subsection{Benefits of Universal Health Coverage (UHC)}

This section provides a brief review of literature that argues that UHC brings multiple benefits. Several authors have commented on the beneficial effect of increasing access to quality health services for individuals who are in need of these services. A recent study (Moreno-Serra and Smith, 2012; Moreno-Serra and Smith, 2015) using data from 153 countries found that increased health coverage improves the lives of the population, and especially the most vulnerable. Better healthcare is found to be accompanied by a reduction of financial hardship and poverty. For instance, countries such as Brazil, Niger, Thailand, and Ghana have seen significant improvements in their population's health and economic situation since they implemented their respective UHC policies.

In Brazil, about three-quarters of the population (roughly 140 million people) have access to health services, boosting the country's health indicators, with infant mortality falling from 46 per 1000 live births in 1990 to about 14.8 per 1000 live births in 2017 (World Health Statistics, 2018). Life expectancy at birth in Brazil rose to 75.5 years old in 2017 from 69 years old in 2007. Sub-Saharan countries such as Niger also saw improvements when governments introduced policies supporting progress towards UHC, which included free health care for pregnant woman and children, and decentralising health programs (WHO, 2018). To take one indicator, Niger has seen a 5.1 per cent decrease in infant mortality from 226 deaths per 1000 live births in 2000 to 81.1 in 2017. Of course, it is essential to keep in mind that developing countries such as this must necessarily extend coverage one step at a time, rather than achieving UHC immediately (WHO, 2018).

Other studies reviewed the financial benefits of introducing UHC for the health sector or the broader economy. In many developing countries the health sector is like any other market system, with a network of buyers and sellers who shape the demand and supply of services reimbursed through out-of-pocket payments. Studies show that about 150 million people suffer from financial hardship, while 100 million people are left impoverished yearly by the need to pay out-of-pocket for treatments (Tahsina et al., 2018; Cotlear et al., 2015 and Zhang et al., 2019). Other research by Krishna (2013) showed that Gujarat, a state in India, saw 88 per cent of households fall below the poverty line due to unaffordable healthcare expenditures. Even some developed countries, such as the United States are facing a situation where high health costs cause personal bankruptcies and impoverishment, something that the Obama reforms sought to counter (Konish, 2019). UHC protects individuals and households from the 
consequences of catastrophic health expenditure and even from episodes of financial hardship and delayed treatment-seeking associated with variable health costs (Wagstaff et al., 2018). For instance, from 2000 to 2006, Thailand saw the prevalence of catastrophic health expenditure (at the 10 per cent threshold) for the poorest quintile of households drop from $4.0 \%$ to $0.9 \%$, after introducing UHC in 2001 (Limwattananon et al., 2011). Meanwhile in Mexico after the introduction of "Seguro Popular", a social health protection program that provided health coverage to about 53 million people, the percentage of Mexicans affected by CHE fell from $3.1 \%$ to $2.0 \%$, while those officially defined as living in poverty fell from $3.3 \%$ to $0.8 \%$ in 2000-2010 (WHO, IBRD, and the World Bank, 2017). In conclusion, UHC allows households to keep funds they would have spent on care, while on average leaving them healthier because of improved access. The extra money in the hands of poorer people leads to increased spending on basic goods and boosts the economy (OECD, 2017).

According to Ramke and associates (2017) the move to UHC reflects, but also amplifies the societal value placed on social solidarity and social protection for the disadvantaged in many countries. Their study showed that the new developments in UHC led to a shift in collective values that made an international consensus on a set of ambitious Sustainable Development Goals (SDGs) possible. According to this line of argument improved social solidarity in the healthcare field has helped move opinion to support advances in social protection in other areas.

Studies by Wirtz et al. (2017) and Tangcharoensathien et al (2015) found that implementing UHC eliminated many health-based barriers and hurdles to education. Children who remain untreated face various health issues and disabilities, which include visual problems and hearing difficulties affecting their education.

Stigler and associates (2016) argue that the emerging consensus on the value of UHC creates a climate in which national governments increasingly regard health as a basic human right of their citizens. These authors suggest that the right to healthcare is a basic need for all people but note that many African countries face difficulties in funding the infrastructure and services necessary to make this equal right to health a reality.

In Nigeria, inequality still exists between the public sector and the private sector. Prices of healthcare services in the private sector are higher than those in the public sector. Insofar as UHC removes the price mechanism as the main means of accessing healthcare it removes a very important dimension of social inequality. Under UHC persons gain access to healthcare 
based on their needs rather than ability to pay (Schmidt et al., 2015). The typical pattern as coverage is extended is that access to care broadens from the wealthy and those employed in the formal sector to progressively include dependants, the unemployed, older people and the chronically sick. As everybody is eligible for treatment, the possibility of demands for extra payments to access care is reduced, so that services are provided directly to the people without being affected by factors such as corruption (Reich et al., 2016; Reeves et al., 2015b).

It has been argued that apart from its positive impacts on health, UHC has potential to improve the economic well-being of individuals and communities and increase social and political stability (Bloom et al, 2004; Lorentzen et al., 2008). It may accelerate economic growth by improving school attendance, educational attainment, and workplace attendance and productivity. Better health improves the economic circumstances of households by allowing regular employment with few absences (Bloom, 2011; Bloom et al, 2018). A variety of healthcare interventions have been shown to have positive effects on educational attainment and earnings, including iodine and iron supplementation, de-worming, and malaria eradication programmes (Bloom et al, 2018). Regarding economic growth, investment in UHC is likely to generate positive outcomes from the economy (Reeves et al., 2015a; Wirtz et al., 2017). On average, a 10-year increase in life expectancy results in a 1\% increase in annual GDP (Bloom et al., 2018). All of the reviewed studies reported that one of the main benefits of the UHC system is that it provides social protection for disadvantaged groups who previously lacked access to care.

\subsection{What others are doing}

While it would be premature to say that international law provides an absolute right to essential health services for all recognised by all countries, there is increasing global pressure to move in this direction, supported by agreements such as the 1966 Covenant on Economic, Social and Cultural Rights, and other international declarations, resolutions at international conferences, and domestic legislation (Office of the UN High Commissioner for Human Rights/ WHO, 2008). The literature search suggested that about 75 out of the 192 countries have passed legislation to provide universal health coverage for their citizens or residents. 58 of the 75 countries have exceeded the threshold for achieving SDG Target 3.8.1 based on $>90 \%$ of the population having access to at least a minimum health package (WHO, 2019). Figure 10 shows 
the progress of countries in the LMIC category, which includes Nigeria. The countries that have not adopted UHC tend to expand their health care coverage slowly, leaving some people vulnerable and without access to health care for extended periods of time. A recent World Bank Group report in 2015 recommends that governments should focus more on "how" they can achieve UHC, while dealing with the "what?” and "why?” questions naturally during different phases of the policy process (Cotlear et al., 2015).

Armenia
Bangladesh
Bhutan
Bolivia
Cabo Verde
Cameroon
Congo, Rep.
Cóte d'lvoire
Djibouti
Egypt, Arab Rep.
El Salvador
Georgia
Ghana
Guatemala
Guyana
Honduras
India

Indonesia
Kenya
Kiribati
Kosovo
Kyrgyz Republic
Lao PDR
Lesotho
Mauritania
Micronesia, Fed. Sts.
Moldova
Morocco
Myanmar
Nicaragua
Nigeria
Pakistan
Papua New Guinea
Philippines

Samoa
São Tomé and Principe
Senegal
Solomon Islands
Sri Lanka
Sudan
Swaziland
Syrian Arab Republic
Tajikistan
Timor-Leste
Ukraine
Uzbekistan
Vanuatu
Vietnam
West Bank and Gaza
Yemen, Rep.
Zambia

Figure 10: LMIC countries (GNI per capita of $\$ 1,046$ to $\$ 4,125$ ).

\section{Source: The World Bank website (2018).}

The literature suggests that governments within the sub-Saharan Africa region (especially in Nigeria) are struggling to deal with the growing healthcare needs of their populations, the rise of health service costs and the challenges associated with the implementation of UHC in their respective health systems (Greer and Maeda, 2015; Ramke et al., 2017). These foregoing studies suggest that a country involved in UHC needs to have a reliable, effective and robust healthcare system in order to tackle the impoverishment and financial hardship that result from out-of-pocket payments.

Expanding health coverage depends on political commitment and sufficient political will to see through what a difficult implementation process is often. Usually, a strong cooperative effort between policymakers, health representative groups (labour unions), political parties and policy organisations is required, Generally the combination of supportive actors, propitious events and resources necessary to make progress will only be intermittently present, at those times when a "window of opportunity" opens (see Evans et al., 2012). This does not mean that success depends on all the desirable prerequisites for UHC coming to the fore at once, as 
sometimes the absence of a desirable condition can be overcome. Countries like the United Kingdom, Thailand, Turkey, Rwanda and Indonesia embarked on UHC reforms in periods when their economies were not strong. Similarly, the heavy burden of reconstruction after World War II did not prevent France and Japan successfully implementing UHC. Most of these countries began their efforts to achieve UHC through the provision of a minimum health package at comparatively low levels of income. They have developed their healthcare systems incrementally to progressively extend coverage over time (WHO, 2010; Ikegami et al., 2014; Maede et al., 2014; Green and Maede, 2015).

Much of the mainstream policy commentary on establishing UHC is framed in terms of the first two of the above approaches. For instance, the World Health Organization/International Bank for Reconstruction and Development/The World Bank (2015) has argued that achieving UHC in developing countries will generally involve a major role for governments operating in a multi-agency environment, not least by putting in place an adequate public financing mechanism or negotiating external financial assistance. This is because political commitment alone is not sufficient to secure movement towards UHC if resources are inadequate. For instance, several African countries that declared their political commitment to UHC in the 2014 Abuja declaration have fallen well short of realising this ambition (World Bank, 2016). How UHC is achieved varies depending on the country (Wagstaff, 2007; McKenna et al., 2017), but the Commonwealth Fund identifies three common pathways (see: Seervai et al., 2017). These pathways are single-payer coverage, regulated private health coverage and mixed publicprivate coverage (see Figure 11). 


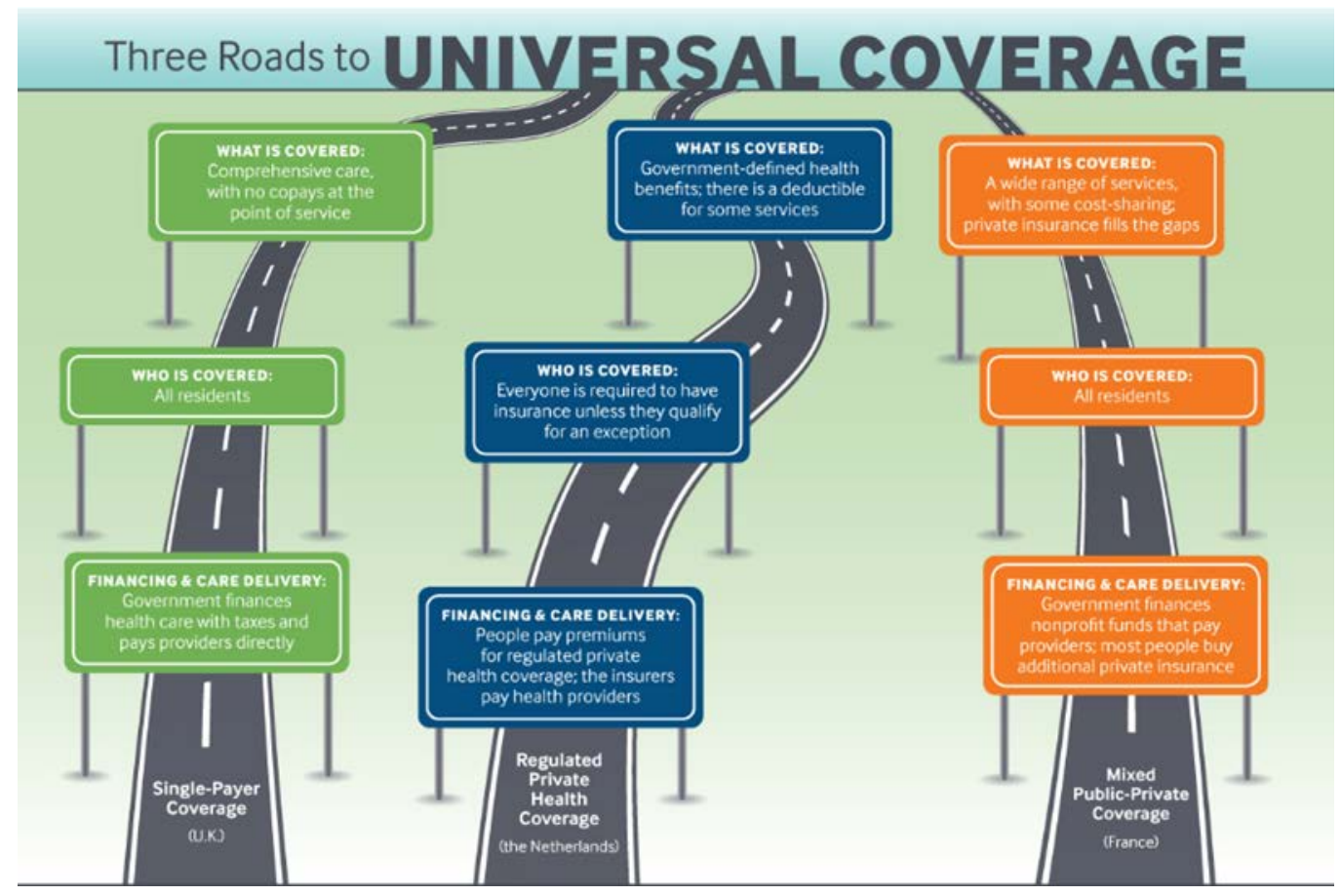

Figure 11: Three paths towards achieving UHC.

\section{Source: Commonwealth Fund (Seervai et al., 2017).}

Single-payer coverage: This is a largely public system in which government healthcare facilities are directly involved in providing care for citizens or residents, with most finance coming from the single source of government revenues, typically tax receipts. Among several countries around the world that use a single-payer system are Canada, the United Kingdom, Taiwan, Sweden and South Korea. Within this general type there are several variations in the details of funding, for example, involving integrated versus purchaser/provider split systems, centralised versus decentralised structures, public hospitals versus a public-private mix, and salaried doctors versus doctors who are independent contractors, even when practicing in public hospitals.

Regulated private health coverage: This pathway toward UHC is regulated by the government, but the organisation and delivery of healthcare to the population is left to the private sector. Such a UHC system can be seen in the Netherlands, where private but not-for-profit insurance funds compete against each other to attract insured members. In this variant of social health insurance funding comes from pooled premium contributions made by businesses and their staff, which are then redistributed using a risk-adjustment formula to prevent insurers from declining joiners with pre-existing conditions. Most providers too are in the independent sector. 
Mixed public-private coverage: This system achieves UHC through a two-tier system that involves both the public and private sectors, with both having a significant role in the financing and delivery of health care. For example, France's health system uses a mixture of payroll taxes and government tax revenues to support not-for-profit insurance funds that provide coverage for the populace. Similar to the regulated private health coverage system, the government stays at arms' length, overseeing negotiations between insurance funds and health providers, but rarely intervening directly.

According to the World Bank (2017), the way universal coverage is implemented is determined by the choice between available financing models, with consequences for the revenue source, the groups covered, the way that risk pooling is achieved, and the type of providers (see Table $3)$.

\begin{tabular}{|c|l|l|l|l|}
\hline $\begin{array}{c}\text { Health care } \\
\text { financing } \\
\text { Model }\end{array}$ & \multicolumn{1}{|c|}{$\begin{array}{c}\text { Revenue } \\
\text { Source } \\
\text { Covered }\end{array}$} & \multicolumn{1}{|c|}{$\begin{array}{c}\text { Groups } \\
\text { Organization }\end{array}$} & $\begin{array}{c}\text { Pare } \\
\text { Provision }\end{array}$ \\
\hline $\begin{array}{c}\text { National Health } \\
\text { Service }\end{array}$ & $\begin{array}{l}\text { General } \\
\text { revenues }\end{array}$ & $\begin{array}{l}\text { Entire } \\
\text { population }\end{array}$ & $\begin{array}{l}\text { Central } \\
\text { government }\end{array}$ & Public providers \\
\hline $\begin{array}{c}\text { Social Health } \\
\text { Insurance }\end{array}$ & Payroll taxes & $\begin{array}{l}\text { Specific } \\
\text { groups }\end{array}$ & $\begin{array}{l}\text { Semi- } \\
\text { autonomous } \\
\text { organizations }\end{array}$ & $\begin{array}{l}\text { Own, public, or } \\
\text { private facilities }\end{array}$ \\
\hline $\begin{array}{c}\text { Community- } \\
\text { based Health } \\
\text { Insurance }\end{array}$ & $\begin{array}{l}\text { Private } \\
\text { voluntary } \\
\text { contributions }\end{array}$ & $\begin{array}{l}\text { Contributing } \\
\text { members }\end{array}$ & Non-profit plans & $\begin{array}{l}\text { NGOs or private } \\
\text { facilities }\end{array}$ \\
\hline $\begin{array}{c}\text { Voluntary Health } \\
\text { Insurance }\end{array}$ & $\begin{array}{l}\text { Private } \\
\text { voluntary } \\
\text { contributions }\end{array}$ & $\begin{array}{l}\text { Contributing } \\
\text { members }\end{array}$ & $\begin{array}{l}\text { For- and non- } \\
\text { profit insurance } \\
\text { organizations }\end{array}$ & $\begin{array}{l}\text { Private and } \\
\text { public facilities }\end{array}$ \\
\hline $\begin{array}{c}\text { Out-of-Pocket } \\
\text { Payments } \\
\text { (including public } \\
\text { user fees) }\end{array}$ & $\begin{array}{l}\text { Individual } \\
\text { payments to } \\
\text { providers }\end{array}$ & & None & $\begin{array}{l}\text { Public and } \\
\text { private facilities } \\
\text { public facilities) }\end{array}$ \\
\hline
\end{tabular}

Table 3: Models of healthcare financing.

Source: World Bank (2018). N.B. Payroll taxes in this classification includes employer and employee contributions in SHI systems that label them in that way.

There are few comparative studies of how well different countries are doing in achieving UHC. A study by the World Bank and the Government of Japan (see Maeda et al., 2014; Reich et al., 2016) compared the common challenges, opportunities and insights observable in eleven 
countries (Bangladesh, Brazil, Ethiopia, France, Ghana, Indonesia, Japan, Peru, Thailand, Turkey, and Vietnam), representing a range of national income levels. The study categorised the countries committed to UHC into four different groups. Category one comprised countries at the stage where UHC is high on the policy agenda. Category two comprised countries that had implemented the UHC policy but are struggling to extend health coverage. Category three comprised countries that have achieved the World Bank/WHO UHC criterion (health coverage $>90$ percent of the population) and were working to maintain that level of coverage. Category four consisted of countries where UHC was fully functional, but policy makers still needed to make adjustments to cope with changing demand for services (see Table 4). The study report concluded that the path towards UHC is a challenging one that needs political support, technical understanding and substantial financial investment in the health system.

\begin{tabular}{|c|c|c|c|c|}
\hline & Group 1 & Group 2 & Group 3 & Group 4 \\
\hline $\begin{array}{l}\text { Status of UHC policies } \\
\text { and progrmmes }\end{array}$ & $\begin{array}{l}\text { Agenda-setting piloting new } \\
\text { programmes and developing new } \\
\text { systems }\end{array}$ & $\begin{array}{l}\text { Initial programmes and systems in place, } \\
\text { implementation in progress; need for further } \\
\text { systems development and capacity building to } \\
\text { address remaining population not yet covered }\end{array}$ & $\begin{array}{l}\text { Strong political leadership and citizen } \\
\text { demand lead to new investments and } \\
\text { UHC policy reforms; systems and } \\
\text { programmes develop to meet new } \\
\text { demands }\end{array}$ & $\begin{array}{l}\text { Mature systems and programmes; } \\
\text { continuous adjuments required to } \\
\text { meet changing demands and rising } \\
\text { costs }\end{array}$ \\
\hline Status of health coverage & $\begin{array}{l}\text { Low population coverage, at the early } \\
\text { stage of UHC }\end{array}$ & $\begin{array}{l}\text { Substantial share of population gain access to } \\
\text { services with financial protection, but } \\
\text { population coverage is not yet universal and } \\
\text { coverage gaps remain in access to services } \\
\text { and financial protection }\end{array}$ & $\begin{array}{l}\text { Universal population coverage achieved } \\
\text { but countries are focusing on } \\
\text { improving financial protection and } \\
\text { quality of services }\end{array}$ & $\begin{array}{l}\text { Universal coverage with } \\
\text { comprehensive access to health } \\
\text { services and effective financial } \\
\text { protection }\end{array}$ \\
\hline Participating countries & Bangladesh and Ehiopia & Ghana, Indonesia, Peru and Vietnam & Brazil, Thailand, and Turkey & France and Japan \\
\hline
\end{tabular}

\section{Table 4: Approaches to UHC in 11 countries.}

Source: Maeda et al., (2014), Reich et al. (2016 p.812).

The factors of accessibility and availability mostly affect health outcomes and health service coverage. The availability of an effective, high-quality workforce, capable of good team working, is also important for successful UHC reform (Hsiao et al., 2014). Ensuring equitable access to key services has a cost and is likely to require significant investment in facilities and professional training (Culyer et al., 2018). The other critical elements for effective health coverage include a proper system of supply and procurement of health technologies and medicines, quality governance, and reliable health information systems. UHC is not only about the health coverage itself, but also the process of managing, funding and delivering the coverage. A UHC system aims to adjust itself to meet the requirements and needs of the communities and people it serves (Maruthappu et al., 2016). Health services, including 
complementary and traditional services and medicines, are managed so as to be responsive to the needs and expectations of the public. System managers must be able to monitor and reorient healthcare over time in order to make sure that it is delivered in the most effective and efficient manner. This builds public support for UHC and increases resilience by ensuring that people will defend a UHC system when opponents seek to dismantle it (Ottersen et al., 2017).

All around the world, UHC systems are on the rise, making affordable care available to ever larger numbers of people (Hsiao et al., 2014). However, the task of ensuring health for all is far from complete, and there are still huge areas where access to healthcare is uneven or unavailable to many. The joint World Health Organization/International Bank for Reconstruction and Development/The World Bank (2015) publication, Tracking universal health coverage: First global monitoring report, states that around four hundred million people in the world do not have access to essential and basic healthcare services, and estimates that around $6 \%$ of people in less developed countries fall into poverty due to spending on healthcare (World Health Organization/International Bank for Reconstruction and Development/The World Bank, 2015).

Several developed countries with established UHC systems face challenges in maintaining them (McKee et al., 2013; Reeves et al., 2015). Economic disturbances and austerity have affected some of the southern European countries, as well as some developing countries that previously provided relatively wide coverage, such as Venezuela (Bello et al., 2017). UHC emerged in the mid-20th century, mainly in high-income countries, albeit in a period of postwar austerity (Gupta and Chowdhury, 2014). A review of this history is beyond the scope of this thesis. Suffice it to say that the national health service and social health insurance system approaches developed in European countries, evolved into two variants of UHC (the Beveridge and Bismarck models) that influenced former colonies and others attracted by Western approaches (Roemer, 1993). On the Asian continent, the “Tiger” economies, including Taiwan and South Korea, used their strong financial resources to implement diverse but effective health care systems under the banner of UHC. Later, as previously described, others such as Malaysia and Thailand also achieved UHC, but these newer systems too are now experiencing challenges of sustainability in times of financial restraint, population changes, and political turbulence (Mueller and Morgan, 2018). 
Even African and Latin American pathfinder countries have been affected by the problems that maturing UHC systems face. In Ghana, a founder member of the Joint Learning Network, the introduction of a UHC program had initially brought a good basic benefits package, an effective payments mechanism, promotive health care and automatic enrolment of patients into the health care programs (Atun et al., 2015). However, economic problems in recent years, together with the unwillingness of the Government to prioritise health over other areas of public expenditure, have resulted in a slowing of planned coverage expansions (Hsiao et al., 2014). This has led to a maldistribution of financial and human resources in health facilities in Ghana's different regions, with a markedly better level of services in the Upper East Region of Ghana compared with less fortunate regions (Amoah and Phillips, 2018).

Argentina provides an even more dramatic Latin American example of the fluctuating fortunes of a UHC system in a middle-income country. Under Peron the nation introduced a progressive social health insurance and tax funded public system that seemed to offer high promise, but economic crisis in the 1990s, and restructuring and decentralisation after a World Bank rescue package, left the system in disarray. By the mid-2000s it was being portrayed in the health policy literature as a prime example of social health insurance gone wrong (Lloyd-Sharrock, 2006; Cavegnero, 2008), and of "an unequal struggle between equity and the market" (Penchaszadeh et al., 2012). The recent history has been one of incremental change that still left significant coverage gaps and failed to address problems of pluralism and fragmentation (Belló and Becerril-Montekio, 2011; Boerma et al., 2014; Atun et al, 2015). The system has been described as one of "nominal” or "aspirational” UHC, meaning that although there is near universal enrolment in programs, many people remain unable to access services (Rubinstein et al, 2018).

\subsection{Systematic comparison of UHC implementation in other countries}

Accountability, transparency and data revision are key to pinpointing areas for improvement when evaluating healthcare systems performance. Comparing other health systems offers policy makers a suggestion to improve their health system relative to their counterparts (Barua and Moir, 2020).

This section examines the factors such as long-term political commitment, expanding financial protection and human resources for health, which facilitated UHC implementation in other 
countries using a systematic comparison approach and discusses which factors the writer thinks are missing in Nigeria. Two definite questions come to mind when evaluating the performance of Nigeria's healthcare: (1) “what are the health outcomes of Nigerians?” and (2) "How is the Nigerian health system performing?”

About 26 health indicators are carefully drawn out from the WHO, IBRD and the World Bank (2015; 2019) and Barua and Moir (2020) reports comparing the performance of several health systems committed to UHC. These 26 health indicators are categorised into three features focusing on (1) the structure of a health system, (2) the financing of a health system and (3) the performance of health systems (note that this feature further splits into indicators such as health outcomes, health and capital resources and incidence of catastrophic expenditure per cent (see Figure 12). Comparing the Nigerian health system to other countries with UHC provides a corresponding value of Nigeria's structure, financing and performance. Furthermore, the countries picked for the comparative analysis were chosen based on the following criteria (WHO, 2015):

- must be a United Nations member state,

- must have UHC commitments,

- and are categorized according to their income level.

Figure 12 attempts to compare the health system performance of 20 countries committed to UHC and explores how their unique experiences can offer insights into the factors missing in Nigeria's UHC pursuit. The missing factors emerging from the comparative approach include the following: long-term political commitment, expanding financial protection and addressing human resources for health. 


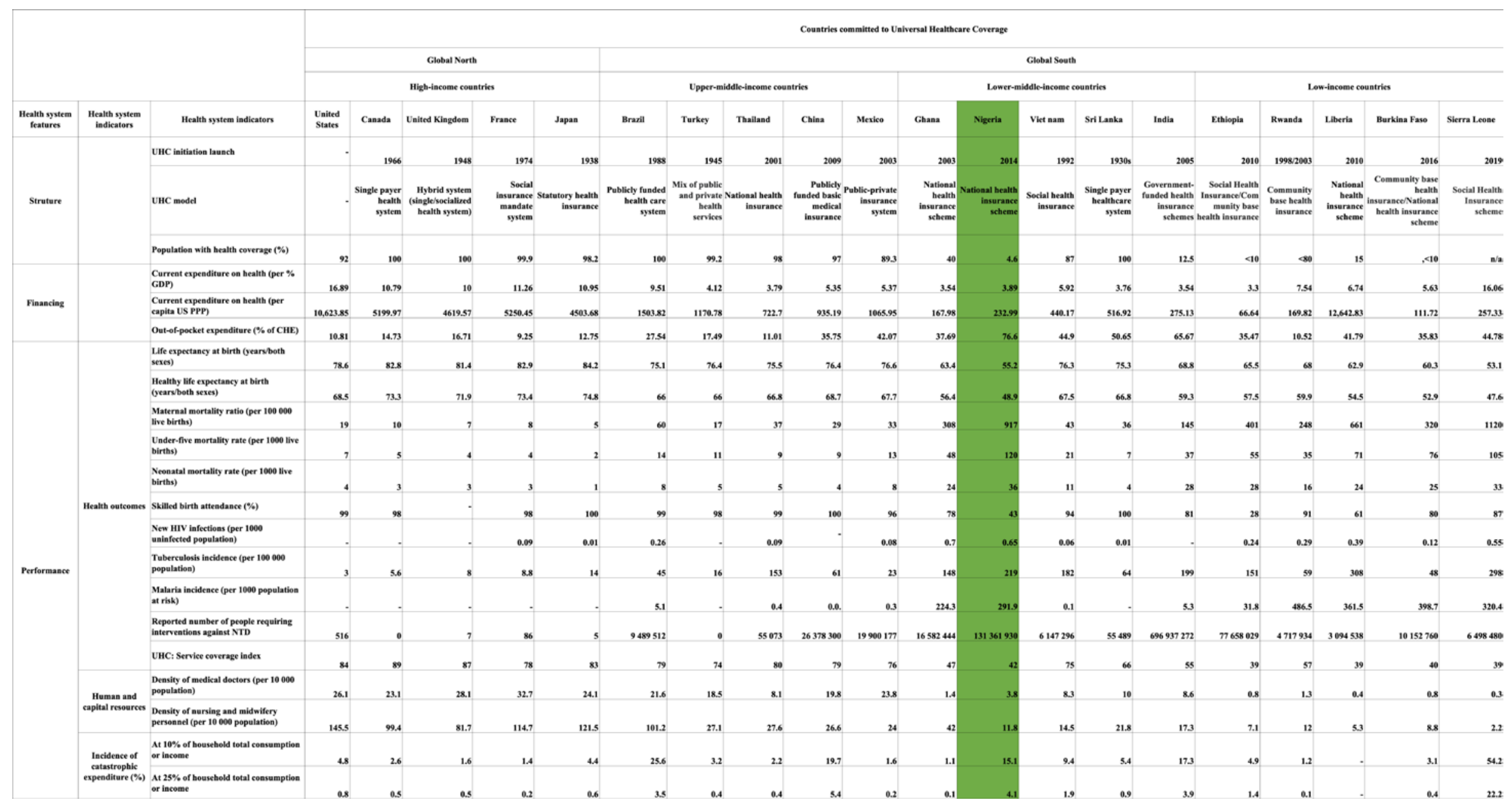

Figure 12: A health system features for selected countries within the global north and south. Source: WHO (2020); The World Bank Group (2020); WHO, IBRD and the World Bank Group (2019) and KPMG (2017). 
Long-term political commitment: strong political leadership and long-term commitment alleviate the possibilities of achieving and sustaining UHC in many health systems. A political leadership capable of resilient mobilisation, adaptable and sustainable social environment can provide the political continuity that accommodates various stakeholders without losing sight of the UHC goals (Maeda et al., 2014).

Thus, the commitment to UHC in 2014 by the Nigerian government is laudable, and further legal strides towards implementing health policies such as the Basic Health Care Provision Fund (BHCPF), the 2014 National Health Act (2014 NHAct) and the 1\% Consolidation Revenue Fund (1\%CRF) that ensure extra increases to public financing are a step in the right direction for the health system.

However, these policies on their own cannot guarantee UHC in Nigeria. According to Aregbeshola (2017), Nigerian policy makers lack the political will to make UHC a reality and, without long-term political commitment, years of meticulously shaped policy solutions have little influence on UHC implementation (Maeda et al., 2014).

Notably, achieving UHC is complex but possible, and requires cooperation between interest groups, policy makers, stakeholders and government officials to ensure its realisation within the stipulated date. The Nigerian President, Muhammadu Buhari, is yet to demonstrate political will, similar to his predecessor (Former President Goodluck Jonathan) towards Universal Health Coverage implementation. Policy makers expect more response from the Buhari administration because the declining health outcomes affect socioeconomic development (Ewelike, 2020).

UHC countries such as the United Kingdom, France, Japan, Brazil, Turkey, Thailand and Mexico have achieved expanded health coverage through long-term political commitment. These countries adopted UHC initiatives in response to several social, political and economic crisis. For instance, Thailand, and Turkey's pledge towards UHC occurred during a severe financial crisis that hit both nations; or Brazil through the democratisation movement; and in France and Japan through the post-World War 2 restoration efforts.

Although these moments of crisis or upheaval mobilised national solidarity needed to embark on such a health system reform, these actions were not enough to drive and sustain the change. McKee et al. (2013) suggest that strong political leadership drove a sustained commitment to 
long-term reforms with the legal basis for UHC advocacy in countries such as the United Kingdom, France, Japan, Brazil, Turkey, Thailand and Mexico.

For instance, it took Japan consistent political commitment to UHC since launching the residence-based National Health Insurance (NHI) under the National Health Insurance Law in 1938 to health reforms in 2004. After World War 2, two-thirds of the population received health coverage until the National Health Insurance Law amendment in 1958, which authorised mandatory access to healthcare through reformed NHI programmes to all municipalities in Japan. Following this, health coverage in Japan expanded from the former sector to the informal sector, self-employed, farmers, vulnerable and unemployed individuals, achieving UHC by 1961 (WHO, 2018). Under a single payment scheme, healthcare prices are set according to the global price revision rate, which helps the Japanese government every two years to control public spending on health. In 2004, health reforms in Japan saw improvements in government-run hospitals with greater autonomy and flexibility to manage hospital affairs (WHO, 2018).

These health systems demonstrate that attaining and sustaining UHC is a complicated process that involves a long-term political commitment that persists despite political change.

Expanding financial protection: Financial protection is a vital objective of SDG-3.8, which ensures individuals get affordable care without becoming impoverished, and over 95\% of uninsured Nigeria fall into poverty due to health-related costs. Consequently, Nigeria has a low life expectancy at birth (55 yrs.) in Figure 12 - almost twenty-nine years lower than Japan's life expectancy at birth.

Using the incidence of catastrophic expenditure (\%) (total household consumption or income exceeding $10 \%$ or $25 \%$ ) and out-of-pocket expenditure (OOP) indictors in Figure 12 to compare the level of out-of-pocket health spending with each country. Most notably, China and Nigeria have the highest percentage of incidence of catastrophic expenditure, with the outof-pocket health spending exceeding $10 \%$ and $25 \%$ of their household expenditure, respectively (WHO, IBRD and the World Bank, 2019). Nigerian households spend $15.1 \%$ on catastrophic OOP health payments, on average at a $10 \%$ threshold of total consumption expenditure, which is higher than Canada, the United Kingdom, France, Japan, Brazil, Turkey, Thailand, Mexico, Ghana, Vietnam, Sri Lanka, India, Ethiopia, Rwanda, Liberia, Burkina Faso and Sudan, but less than China (see Figure 12). 
Aregbeshola (2019) attributes this excessive dependence on OOP expenditures to the failure of the NHIS whose objective is to prevent impoverishment from accessing healthcare. Since its inception in 2005, the NHIS has provided health cover to about $4.6 \%$ of the population leaving the remaining Nigerians spending OOP for care. Furthermore, the author states that many middle-class households experience higher catastrophic health payments than lower-class households because lower-classed households tend to pursue local herbal remedies or resort to self-medication.

To crowd out the cost of OOP expenditure, the World Health Organisation (2015) suggests increasing public spending on health to facilitate health coverage expansion across the population. Although the WHO shows no direct link between increased public spending on health and financial health protection, experiences across several health systems show that increasing spending on health using organised financial mechanisms such as pooled prepayment mechanisms reduce OOP spending for many households (WHO, IBRD and the World Bank, 2019). For instance, many within the health communities encourage pre-payment financial mechanisms such as tax or social insurance as a better approach to reduce catastrophic health expenditure for many households. Countries such as Canada, the UK, France, Japan, Thailand, Mexico, Rwanda, Sri Lanka, and Ghana (with predominantly publicly financed system) show how pre-payment mechanisms reduce catastrophic health expenditure and impoverishment from healthcare consumption (WHO, IBRD and the World Bank, 2019).

As a pre-payment mechanism, the NHIS Nigeria has failed to live up to its expectation of expanding access to affordable health services for uninsured, poor and vulnerable Nigerians. The failure lies within the government's inability to match the 2001 Abuja declaration target of earmarking at least $15 \%$ of the annual budget for healthcare improvement (Aregbeshola and Khan, 2018).

As countries become wealthier, their healthcare spending improves and substantial reliance on public expenditure phases out OPP over time, but there is an anomaly to this trend - the United States health system (WHO, 2003). The United States is the only country within the highincome group that spends (approximately 18\% of GDP) more on healthcare. With 27.2 million Americans without health insurance, higher healthcare costs are the root cause of household debt, impoverishment and poverty (Tikkanen et al., 2020). 
Several lessons demonstrate that achieving UHC is possible in Nigeria's income level and experiences from other countries demonstrate that increasing public spending on health expands financial protection and crowds out catastrophic health expenditure and impoverishment. Without government action towards significant health reforms that increase health budget and reduce household reliance on OPP spending, achieving UHC in Nigeria may be far off.

Furthermore, socioeconomic development supports health coverage expansion, but it does not guarantee equitable coverage. Many progressive Nigerian policy makers would prefer that the Nigerian government avoid enacting policies that evolve into a US-style health system (marketoriented system) in favour of a welfare state-style health system (social-oriented system) seen in Europe (France, the United Kingdom, and Turkey) and Asia (China, Japan, Thailand, Viet Nam, and Sri Lanka). These health systems (that are all heralded as UHC success stories) are predominantly public health financing system capable of redistributing resources to reduce disparities in accessing health care services (Aregbeshola, 2019).

Human resources for health: Figure 12 suggest that the health workforce density in Nigeria is among the lowest in the world at approximately 3.8 per 10,000 population for medical doctors and 11.8 per 10,000 population for nurses. For instance, in 2018, France indicated a workforce density of 32.7 per 10,000 population for medical doctors and 114.7 per 10,000 population for nurses, while the United States, United Kingdom, Canada, Brazil, Thailand, India and Sri Lanka indicated, respectively, 26.1/145.5 per, 28.1/81.7 per, 23.1/99.4 per, 21.6/101.2 per, 8.1/27.6 per, 8.6/17.3 per and 10/21.8 per 10,000 population of doctors/nurses (WHO, 2020) (see Figure 1 for further comparison). The World Health Organisation (2016) found that approximately 1 in 4 doctors, and 1 in 20 nurses employed in Western countries happened to receive training from Africa, which instigates a shortfall of around 1.5 million healthcare personnel within Africa.

Adeloye et al. (2017) attribute Nigeria's underperformance in health outcomes to health workers relocating into high-income countries, and such setback results in a brain-drain on the health system. The brain-drain creates a massive gap in Nigeria's health workforce compounded by the government's incompetence to deploy strategic national policy guidance, transfer or redistribute health workers around the country. 
The workforce for health is central to Nigeria's UHC ambitions, and this requires an efficient and productive distribution of health workers fit for purpose, which equips the health system to meet the growing demand for health (Szabo et al., 2020).

Mature health systems with consistent political and technical commitment maintain and improve universal health coverage through reformist policies on the workforce for health (WHO, 2016).

Every pathway towards UHC attainment differs according to contextual factors specific to the health systems, but efforts towards scaling up the health workforce distribution are similar.

For many health systems, adapting to an analytical framework of the health workforce dimensions of availability, accessibility, acceptability and quality is a well-known transformative process used to regulate the health workforce to meet growing healthcare demands (WHO, 2016).

Allocating health workforce is a technical and political process that requires stringent planning, coordination, education and management efforts on the part of the private and public sectors (Sales et al., 2013). Several authors with success stories show how their health systems align political commitment and thorough technical strategies on workforce resources for UHC achievement. For instance, Badr et al. (2013) present a case study on Sudan accomplishment for strengthening the human workforce for health through information, coordination and accountability mechanisms. Sudan's health system, with political support, used a “country coordination and facilitation” mechanism to mobilise human resource for health experiencing worker shortages, deprived management and fragmented health services to establish a robust health information system and developed a participatory policy making responsive to universal health coverage achievement.

Tangcharoensathien et al. (2013) suggested that Thai healthcare workers contributed to developing UHC in the health system. Pre-UHC achievement, Thailand was struggling with poor health outcomes, low health service coverage and a weak local health infrastructure which were improved through measures that redistributed the accessibility of health workers fit for purpose along national guidelines. Post-UHC achievement, Thailand, with the help of a sound $\mathrm{HRH}$ and governance, succeeded in increasing the capacity of nurses to $210 \%$ and doctors to 186\% from 1991 to 2009 (Campbell et al., 2013), increased maternal and child health services 
and expanded health workforce resulting to a fountaining and attained UHC (Tangcharoensathien et al., 2013).

Campbell et al., (2013) say substantial health workers and expansive health coverage fosters an effective and equitable UHC. In Mexico, health reforms between 1995 and 2003 increased the human resources on health policies to match the population demands. Programa de Calidad (PAC), an expansive health coverage programme initiated in 1996, addressed the inequity of health workers accessibility in underserved areas and adequately improved health outcomes for Mexico’s health system. For instance, the level of well-trained nurses and doctors increased $80 \%$ (for nurses) and 170\% (for doctors) outperforming the population's health demands of $30 \%$. Furthermore, infant mortality and under-five mortality reduced from 32.6 to 14.6 per 1000 live births and from 41 to 17.8 per 1000 live births respectively during this period (Alcalde-Rabanal et al., 2013).

These success stories - from Sudan, Thailand and Mexico - show attempts of health outcomes by improving the availability, accessibility, acceptability and quality of the health workforce (Sales et al., 2013). Despite the contextual factors specific to each health system, Padilha et al. (2013) indicate that political leadership is central to HRH development and it ensures continuity, stability and coordination between different sectors and government officials on human resource development efforts.

Lessons from these health systems suggest that substantial commitment from the Nigerian government towards improving human resources increases the likelihood of UHC achievement is needed. A Global Health Workforce Alliance (Campbell et al., 2013) report offers a 10-point agenda for success in strengthening the availability, accessibility, acceptability and quality of the health workforce in any health system context of universal health coverage. The 10-point agenda is as follows:

- Understanding that health workforce is central to translating universal health coverage into good healthcare outcomes for the health system.

- Providing competent and well-trained workforce required to compensate the population's demand for health services.

- Introducing national policy goals focused on improving healthcare workforce for the health system. 
- Building an effective Information Technology (IT) system that encapsulates evidence base data and strategic intelligence needed to monitor, sustain, survey and manage policy objectives.

- Monitoring and evaluating are key in sustaining the technical capacity to create and apply policies.

- Increasing political will at all levels facilitates and encourages policy continuity that maintains UHC policy on the right path.

- Systematic reforms on several governing institutions creates a stable environment for the healthcare workforce.

- Adopting cost-effective measures for health workers reforms.

- Reassure the international community of the government commitment toward substantial support and assistance for health systems development.

- Increase collaboration with global associates in tackling technical difficulties by reinforcing appropriate global health governance and mechanisms

The key lessons from the comparative analysis are consistent with evidence-based policy and practice. This section exposes missing factors such as addressing the human resources for health, expanding financial protection and long-term political commitment that is likely to facilitate Nigeria's UHC attainment. With these missing factors in mind, the Nigerian government response through good governance, political commitment and strong leadership can meet the population health needs, achieve UHC and provide adequate service delivery, otherwise the prevailing health crisis may continue (Adeloye et al., 2017).

\subsection{Criticisms of UHC}

Regardless of UHC benefits, many criticise the UHC vision, or problems arising from the way it is implemented. Free market liberals warn that UHC advocates should not assume the values underlying the policy are shared by all (Missoni, 2010; World Health Organization/International Bank for Reconstruction and Development/The World Bank, 2015). They point to the ongoing debate surrounding health reforms in the United States (which might be going the opposite way towards UHC under Obama's ACA and President Biden promising more (Biden, 2021)), echoed elsewhere in the world, where some argue that health is a matter of individual responsibility and that dissenting citizens should not be forced to participate in 
solidarity-based health programs that require mandatory contributions or support through taxation (Boudreau, 2017; Amadeo, 2020). Regarding the way UHC is financed, critics contend that developing countries are often compelled to comply with directions from international donor bodies, for example, to channel money towards selective interventions, such as the GOBI-FFF program (advocated by UNICEF) rather than investing in a wider range of primary healthcare services (Verrecchia et al., 2018; Reis, 2016; Brearley, 2013; WHO, 2013). Another issue is the tendency of international agencies to provide consultancy that recommends something resembling a standard package. This is probably less of a problem than in the past, but it is important to understand that UHC is not a 'one size fits all' approach and needs to be tailored to suit particular national contexts (Cheng and Wilson, 2015).

There are, however, undeniable criticisms facing many health systems with UHC, especially towards their policy approach tailored to their national context. Some mature and successful UHC health systems still encounter unintended consequences (such as the overall deterioration in the quality and variety of health services and persistent health inequities) caused by their policy approach (Lim, 2019; Krugman, 2021). A brief outline explores the policy criticisms from three mature health system - the Netherlands, the United Kingdom and France purposefully selected for having achieved UHC through various pathways, such as comprehensive coverage, single-payer coverage, regulated private health coverage and mixed public-private coverage (Montgomery, 2020).

In the Netherlands, the government implemented the 2006 health reforms to transit from an ineffective public and private insurance system to a regulated private health coverage system. The government under the Authority for Consumers and Markets, Health Care Authority and the Health Care Institute act as a regulator for the health insurance scheme responsible for healthcare competition, price, care quality standards and basic health package (Altenburg-van den Broek and Lynch, 2010).

After these reforms, the Dutch health system ensured every individual access to quality and affordable care and, through mandatory health insurance and managed competition, achieved UHC with a UHC service coverage index of 86. Improvements in the Dutch health outcomes saw life expectancy at birth move from 78.2 years in 2000 to 81.6 years in 2015, above the OECD average (OECD/European Observatory on Health Systems and Policies, 2017).

The transition towards a regulated private health coverage system improved the mortality rates, beating the OECD estimates average, and other health outcomes such as avoidable 
hospitalisations and survival rates showed better estimates than the OECD average due to efficient primary and secondary care. Although mortality rates on non-communicable diseases caused by tobacco use, obesity, and alcohol demonstrate a mixed picture, the Dutch government is attentive to the health issue (OECD/European Observatory on Health Systems and Policies, 2017).

\section{Market Share of Dutch Health Insurers}

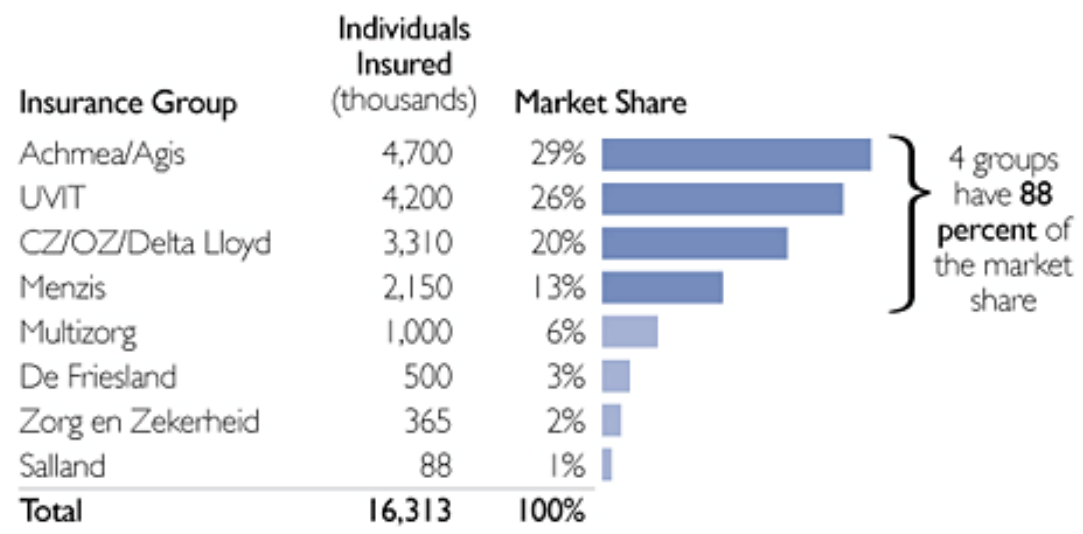

Figure 13: A marketplace of health insurance groups in Netherland. Source: Lynch and Altenburg-van den Broek (2010).

Despite access to quality health services, good health outcomes and stable public funding in the Netherlands, critics argue against the managed competition as a guiding principle of its health reform in creating market value and curbing healthcare costs (Lynch and Altenburg-van den Broek, 2010). They consider the Dutch health system an exceedingly uncompetitive oligopoly market where, since its 2006 health reforms, approximately $88 \%$ of the market share is captured by the four largest health insurance companies (see Figure 13) (Dutch Health Authority, 2010).

Notwithstanding the government's intention to reduce healthcare costs and create value for the consumer through market competition, the health market still emerges as oligopolistic. Even supporters of the Dutch health system consider its health insurance market very uncompetitive.

Since 2008, the oligopolistic market has grown to a point where health insurance companies are able to use their buyer power (monopsony) to negotiate with pharmaceuticals companies. There is now, in many instances, less competition and consumer choice. Hence, rather than creating a new system, many policy makers propose an idea for the government to share its 
supply-side influence with health insurance companies (Altenburg-van den Broek and Lynch, 2010).

In the United Kingdom, the National Health Service (NHS) adopts a single-payer system to achieve universal health coverage, but with a small private sector providing elective surgery, and nursing home care. Unlike its European counterparts, the NHS provides healthcare free at the point of contact. European counterparts such as France, Germany and Italy adopt a social health insurance system of universal health coverage backed by the government with healthcare financing mechanisms similar to the NHS. In contrast to the NHS, where the entire population has free health coverage, countries like Germany, Italy and France have a considerable portion of its citizens without public insurance cover but having subsided private insurance cover (Dayan et al., 2018; Krugman, 2020; Montgomery, 2020).

The writer's analysis shows a mixture of opinion of the NHS performance where its supporters are highly impressed with the system, and its critics allege that the system does not perform as well as sometimes claimed.

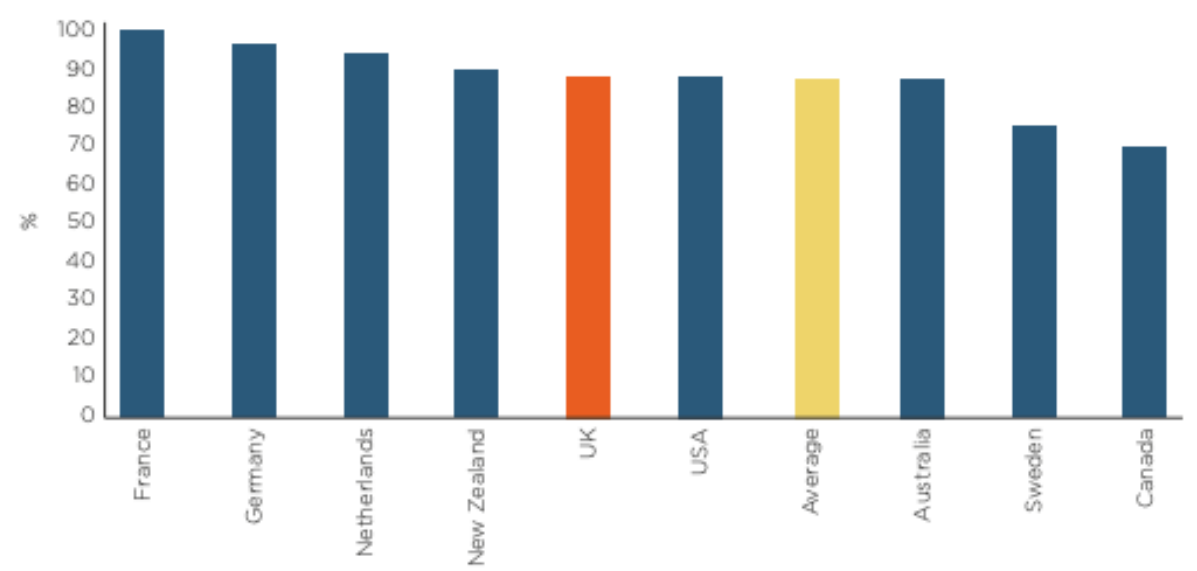

Figure 14: Percentage of patients seen within four hours for medical consultations. Source: Dayan et al./Health Foundation (2018).

Compared to other OECD health systems, the NHS is good at reducing the financial risk of healthcare costs at the point of consumption, making the British less inclined to postpone getting medical treatment due to cost. Consultations in the UK are free, lower than the OECD average (see Figure 14), higher than countries such as Germany (2.6\%), Spain (2.8\%) and Sweden (3.9\%) due to travel costs (Dayan, Ward, Gardner and Kelly, 2018). For instance, 
almost $4.2 \%$ of British people only avoid health consultation because of travel cost and the cost of abstaining from work (The Commonwealth Fund, 2017).

NHS waiting times and patient treatment satisfaction compares well with similar health systems. While data are limited, a Commonwealth Fund report suggests that $88 \%$ of British people have to wait few hours to receive treatment when visiting the Emergency Department (because these visits are not all emergencies, and there is a triage system where patients who visit the Emergency Department without urgent medical need become less of a priority) (see Figure 14) (Molloy, Gardner and Thorlby, 2017). Furthermore, the NHS performs well on waiting times for selected procedures such as cataract surgery and hip and knee replacement. For instance, only the Netherlands (37 days) and Italy (50 days) publish shorter mean average wait times for cataract surgery than the UK (73 days), while hip and knee replacements waiting times for the UK have a mean average of 97 days above the Netherlands (42 days) and below Spain (150 days) (OECD, 2017; 2018).

The NHS is well-organised in providing low administrative costs and cheaper use of generic medicines (Dayan et al., 2018); however, critics still call attention to several problems, such as low efficiency and underproduction or overproduction of care existing within the health system (Xie, 2012).

Low efficiency: this is a problem created when the government's intervention on a particular health issue is exacerbated, thereby running the risk of discouraging motivations. For instance, nurses and doctors within the NHS are public servants employed by the government without profit-seeking motivation. The after-effects create long waiting queues at hospitals and a bottleneck of patients requiring free and non-urgent treatment, making the system operate slow and inefficiently. A proportion of patients then move towards private care for diagnostic services and for elective surgery rather than wait (Xie, 2012). 


\begin{tabular}{lll}
\hline & Relative performance & Relative change over time \\
\hline Breast cancer & Poor & Improving \\
\hline Colorectal cancer & Poor & Improving \\
\hline Lung cancer & Poor & Improving \\
\hline Pancreatic cancer & Poor & Improving \\
\hline Diabetes & Good & Unclear \\
\hline Kidney disease* & Good & Unclear \\
\hline Chronic obstructive pulmonary disease & Poor & Unclear \\
\hline Lower respiratory tract infection* & Poor & Improving \\
\hline Suicide* & Good & Unclear \\
\hline Dementia* & Unclear & Unclear \\
\hline Stroke & Poor & Improving \\
\hline Heart attack & Poor & Unclear \\
\hline Amenable mortality & Poor & Unclear \\
\hline Patient experience & Good & Unclear \\
\hline Birth & Poor & Unclear \\
\hline
\end{tabular}

Figure 15: The performance of lethal diseases and other fields in the National Health Service UK. Source: Dayan et al. (2018)

Low efficacy translates into poor healthcare outcomes, which essentially influences the quality of the NHS. For instance, compared to the United States, the NHS performs poorly on successful medical care that could save lives. Figure 15 shows a summary of how well the NHS performs in each of the 12 most lethal diseases. Most notably, health outcome measures for lethal diseases such as breast, colorectal, lung and pancreatic cancer show relatively low performance (see Figure 15) (Agency for Healthcare Research and Quality, 2016; Institute for Healthcare Improvement, 2018).

Underproduction or overproduction of healthcare: in a single-payer and socialised medicine system such as the NHS, the supply and provision of healthcare services is publicly funded by the government budget centrally into the NHS, leaving the health system at risk of underproduction or overproduction (specifically where untested drugs/treatments are used) for care. There is a divide of information or asymmetric information between central planners and consumer demand for health (this is equally true of other systems, especially private provision in the US), in effect improbable to deliver health services at an optimal level (Xie, 2012).

The Nigerian health system if it is looking to adopt the UK health system approach towards achieving universal health coverage should build an institution that provides doctors and GPs a comprehensive assessment to deal with the low efficiency issue (as is also being done in the UK with Health Improvement and other initiatives (Maguire, 2019). Furthermore, introducing 
a managed competition among health providers could regulate underproduction or overproduction of healthcare, emphasising consumers' right to choose hospitals freely.

In France, the healthcare system was ranked number one by the World Health Organisation in 2000 (Rodwin, 2003). France adopts a mixed public-private system of universal health coverage structured around the Bismarck approach where the entire population has compulsory health coverage through the statutory health insurance system (SHI), which extends to every worker (in 1945), seniors (in 1945), self-employed (in 1966), unemployed (in 2000) and immigrants (in 2016) (Tikkanen et al., 2020).

With health spending over $11 \%$ of GDP above the OECD average (8.8\%), the French health system covers the costs for a comprehensive health service, which includes hospital, physician, prescription drugs and long-term care, and excludes co-insurance, co-payments and consultation charges that exceed government contributions. SHI is financed primarily through employee and employer payroll tax, self-employed income tax, national income tax and earmarked tax, state subsidies and tax levied on tobacco and alcohol (Tikkanen et al., 2020).

Health spending per GDP in France improves health outcomes and patient satisfaction where OOP expenditure represents $2 \%$ of household expenditure (because $95 \%$ of the population own supplemental insurance, which reduces OOP cost on services such as dental, hearing, and vision care) (OECD,2019).

Furthermore, the curative care or medicine displays a high standard in its 30-day mortality rate after heart attacks and strokes of about 20\% and 10\% lower than the OECD average. The French health system is considerably better at preventing hospitalisations for asthma and chronic obstructive pulmonary diseases (at 150 per 100,000 population) less than the OECD average (of 237 per 100,000 population), while avoidable admissions for diabetes and heart failure are above the OECD average. Antibiotics prescription in France is 25\% above the OECD average (OECD, 2019).

Criticism of the French health system exists despite its achievement of universal health coverage under a public-private health insurance system; critics identify prominent health disparities associated with the mixed public-private system model (Rodwin, 2003; Vigneron, 2012). For instance, the geographic distribution of health resources creates disparities in mortality outcomes across France. Under the SHI, where equality is the catchphrase of the entire healthcare system, experiencing health disparities is rather unfortunate. Access to care 
services reduces as the geographical distance extends from urban to remote areas (Vigneron, 2012). For instance, many public health policy solutions attend more to urban areas than in remote areas. In France, the number of doctors in remote areas is 2.7 doctors per 1000 population, compared with a 3.9 per 1000 population in urban areas (OECD, 2019). Some critics suggest that a geographical analysis of the health system can help spread access to medical services to undersupplied areas and people most in need of care.

Another criticism is the perceived problem of uneven distribution of quality health services where some listed hospitals in France are considered dangerous to the public due to low-quality care delivery. The lack of coordination and case management services by these hospitals has increased the awareness of healthcare issues surrounding patients with chronic diseases and older patients. Critics suggest these issues are aggravated by the radical nature of the French health system (Rodwin, 2003; Vigneron, 2012; OECD, 2019), but it ranked first in the world in 2000. It still comes out well in rankings, although ranking depends on what is seen as most important (see Global Burden of Disease Study 2016) (Ryan et al., 2017).

Moving forward, UHC in Nigeria needs to learn from these experiences, acknowledge the problems associated with the various health financing models and provide credible policy solutions that fit its national context before reforming the healthcare system towards UHC 2030.

\subsection{Universal Health Coverage (UHC) in Nigeria}

The push towards UHC in Nigeria is often discussed in the context of its disappointing outcomes on key health indices. Nationally, Nigeria ranks among the worst performers on indices of global health issues and is ranked among the 10 worst countries in the world with respect to disease burdens (Muhammad et al., 2017). Most notably, Nigeria has the highest global burden of malaria, with about 40 per cent of global malaria deaths occurring in the country. 90 per cent of Nigerians are at risk of malaria, which kills more than 300,000 Nigerians annually, and with 11 per cent of maternal deaths linked to the disease. Furthermore, Nigeria is rated as the second worst country with respect to maternal and child deaths globally, second only to India. Some 15 per cent of global maternal deaths occur in Nigeria alone, and the deaths of new-born babies in the country represent a quarter of the total number of deaths of children under five worldwide (Thisday, 2018). Many reform advocates see UHC as the crucial policy needed to improve this situation. They have welcomed the Government's recent commitment 
to ensure that all citizens have access to affordable and high-quality healthcare. Nonetheless, critics note that in 201773 per cent of Nigerians were still paying out of pocket for their healthcare (Aregbesola, 2017).

The path towards UHC in Nigeria has been long and not always smooth. The country's first attempt at building a healthcare system was made in 1962, when the then Federal Minister of Health, Dr. M. A. Majekodunmi, presented a bill to parliament for enactment in Lagos, though the bill was rejected. In 1988, the then Minister of Health commissioned a committee that recommended the template for the present-day National Health Insurance Scheme (NHIS). The law was signed into force in May 1999, but only became operational in 2005 (Medical World, 2015). The original intention had been that the NHIS would expand to provide UHC within ten years (Adewole and Osungbade, 2016), but at the time of the study still covered only a very small percentage of the population (estimated by the FMoH (2016) as around 3\%). This left Nigeria at a critical inflection point in its effort to improve access to healthcare and to move towards UHC. President Jonathan had made a formal declaration at the African Presidential Summit on UHC in 2014 committing the country to achieving UHC, and in October of that same year the National Health Act (NHA) was signed into law, providing a legal framework for healthcare provision to Nigerians and for the organization and management of the health system, and earmarking finance to support progress towards UHC. Yet at the time of the fieldwork the strength of this commitment and the pace of future progress remained uncertain.

In Nigeria the chosen route to UHC was health insurance through the NHIS (Onoka, Hanson, and Hanefeld, 2015; Okpani and Abimbola, 2015; Adewole and Osungbade, 2016). NHIS’s main formal sector social health insurance scheme, which covers all federal government employees, as well as organised private sector enterprises with at least 10 employees, is funded through payroll-based premiums. NHIS in Nigeria functions as a pre-payment plan, where participants pay a fixed regular amount. These funds are pooled, and the finance is channelled to Health Maintenance Organisations (HMOs) to pay for those needing medical attention. As such, it is primarily a risk-sharing arrangement, which can improve resource mobilisation and equity. The NHIS operates as a regulator overseeing the HMOs, and co-ordinates and manages several insurance schemes aimed at particular groups alongside the main Formal Sector Program (NHIS, 2018).

The NHIS has implemented community-based health insurance (CBHIS) programmes for those in the informal sector, although so far with limited uptake (Odeyemi, 2014). These 
schemes are based on a community or occupational group, with a requirement that 50 per cent of the group or a minimum of a thousand members enrol in a new scheme. Members are required to pay monthly or seasonal fees in advance, which is a challenging requirement for poorer people. Additionally, the NHIS oversees targeted schemes for children under five, tertiary-level students, prison inmates, disabled persons, and the police, armed forces, and other uniformed services.

To speed up the attainment of UHC, legislation of the Health Act allowed states to create their own health insurance schemes. Many states have taken advantage of this opportunity, including Lagos, Oyo, Ekiti, Delta, Bayelsa, Cross River, Akwa Ibom, Kwara, Abia, Adamawa, Kano, Anambra, Sokoto, and Enugu states (Awosusi et al., 2015). Nonetheless, most states have been reluctant to join because of the anticipated increase in wage bills. Some states like Lagos, Ogun and Kwara are also implementing CBHIS. The NHIS schemes are relatively unusual in that they give private sector health maintenance organisations (HMOs) a substantial role by licensing them to facilitate the provision of healthcare benefits to scheme members, something that is said to have discouraged some states from getting more closely involved (Onoka, Hanson, and Hanefeld, 2015). The financial contribution coming from the public insurance schemes is relatively low because of the low levels of enrolment, even by African standards. A recent four-nation study showed that health insurance uptake in Nigeria lagged behind uptake in Ghana, Kenya and Tanzania (Amu et al., 2018). As fieldwork was drawing to a close, some stakeholders concerned with low uptake, including the Chairman of the Senate Committee on Health, Senator Lanre Tejuoso, were pushing for policy change to make the scheme mandatory for all Nigerians (Ojo, 2018).

Nonetheless, under the present system healthcare public provision is limited. Over 70 per cent of Nigerians lack insurance coverage and rely mainly on out-of-pocket (OOP) payments to access healthcare. Critics argue that this is a major factor in explaining low life expectancy, and the myriad poor health outcomes that affect Nigeria (WHO, 2017). Statistics show that the level of OOP expenditure as a share of total health expenditure in Nigeria remains at 72 per cent, the highest on the continent and one of the highest globally. This poor performance is particularly notable when compared with OOP expenditure in poorer countries in sub-Saharan Africa, such as Kenya (26 per cent) and Gabon (22 per cent). Even countries afflicted with conflict and post-conflict like South Sudan (54 per cent) and Sierra Leone (61 per cent) are still outperforming Nigeria in this respect. 


\subsection{Policy actors involved in UHC Nigeria discourse}

According to Walt (1995), actors involved a health policy process are a group of individuals or organizations with influence who shape health policy in the direction of achieving policy solutions. Policy actors involved in health policy are either state actors or non-state actors could on one side or the other side of the policy process use their influence to determine the policy outcome. Many policy analysts consider state actors such as the federal government, Ministry of Health and other government parastatals the most influential in the policy process due to the influence they possess over the rule of law and state budget. Hall (1978) also mentions a list of hypothetical non-state actors such as interest groups, opposition political parties, the electorate, trade unions, non-governmental organizations, external governmental agencies and UN bodies that as usual participant in a policy process.

What defines the result of a health policy is the role each actor plays and the influence of prominent actors, in combination with the relationship among actors in both state and non-state settings. They have been different actors who have paid crucial roles in the UHC Nigeria implementation process. The ways in which these actors interact with each other have expanded, as have the state and non-state networks concerned with the UHC discourse in Nigeria. The idea of a policy network can be used to describe, explore and understand the interconnections between actors (Lewis-Lettington and Munyi 2004).

Marsh and Rhodes (1992: 260) state ' . . f focusing on policy networks will never provide an adequate account of policy change, because such networks are but one component of any such explanation', focusing on the relationship between state and non-state networks poses questions regarding accountability (for example, who sets the policy agenda), as well as interdependence in policy processes (where sovereignty lies). Using networks allows an exploration of such fundamentals which contributes to understanding changing policy processes and policy environments.

Scholars have used the idea of 'policy networks' to demonstrate the complexity of creating policy and the relationships between different state and non-state actors. The policy networks involved in UHC discourse have through investigation provided insight into policy implementation, and with it, the role of actors involved with the policy. 
In some situations, interactions may be highly contentious, with non-state networks urging the state to shift position. This can enable the federal government or the Ministry of Health to fund projects, programs and policies which are geared towards Universal Health Coverage (UHC) in Nigeria (This Day, 2018). For instance, policy actors such as civil society organizations (CSOs), Nigerian Medical Association (NMA), UN bodies, USAID, the Health Reform Foundation of Nigeria (HERFON) and Economic Community of West African States (ECOWAS) persistent push for the one per cent Consolidation Revenue Fund (1\%CRF) and the Basic Health Care Provision Fund (BHCPF) influenced the National Assembly to shift their policy in Nigeria, relating to its inclusion in the 2014 National Health Act in the 2018 budget (Hafez, 2018).

The inclusion of one per cent of the consolidate revenue fund to the allocation of the health sector in the 2018 budget was hailed as monumental (Onyeji, 2018). Many policy makers believe that the one per cent Consolidation Revenue Fund (1\%CRF) can change the trajectory of the country's health system (Onyeji, 2018). Provided by the 2014 Health Act in 2018 budget, the one per cent of the Consolidated Fund to made available to provide what we called the Basic Health Provision Fund, and that Fund will be invested through Social Health Insurance; Primary Healthcare Revitalization, Accident and Emergency Management and Critical Needs; Research and Development; Recruitment and Training of quality healthcare providers at the primary care level. The implementation of the one per cent Consolidated Fund is one of the major opportunities that has provided the Nigeria health system for achieving the Universal Health Coverage policy (Alawode, 2018).

The NMA was a contributor, with other stakeholders, in getting the healthcare bill through the senate committee on health by making UHC their 'rallying cry' over the year after the introduction to the 2014 National Health Act and beyond the act coming into effect in 2016 after 10 years of consideration. The National Health Act was signed into law in 2014, after 10 years of consideration. The Act aims to establish a framework for the Regulation, Development and Management of a National Health System; to achieve the Universal Health Coverage and meet the Millennium Development Goals (MDGs). The NMA had over the years protested the non-implementation of the National Health Act. They threatened the federal government with a lawsuit as well as embarked on a bi-weekly protest march to urge the lawmakers to include the Basic Health Care Fund in the budget (Onyeji, 2018). 
The NMA is at present involved in influencing UHC health policy formulation in an ad hoc manner. This also involved in many of the government's activities, it is consulted formally by the government only on an ad-hoc basis in respect to UHC policy. This is done by making unsolicited recommendations to government on various health issues and also by making inputs, whenever invited, to some of the UHC committee meetings on policy formulations (Iheamnachor, 2018; Obinna, 2018)

In alternative situations, state and non-state actors create collaborative relationships where the former can achieve policy goals with assistance from the latter, even when they are looking after their own interests (Rhodes 1988; Tantivess and Walt, 2008).

Key policy actors enable collaboration and coordination of activities or efforts, the exchanging of information and the construction of common knowledge (Stone 2001) in ways which support UHC policy outcomes. Such achievements, where they result in several key policies which were formulated and put forward, help enhance UHC implementation in Nigeria. During the Ex-president Olusegun Obasanjo administration (1999-2007) with Prof Eyitayo Lambo as Minister of Health, the agenda was to reform Nigeria's health and bureaucratic systems which to the creation of an NHIS programme with the purpose of attaining an expansive health coverage by 2015 (Umeh, 2015).

The FMoH, State Ministry of Health (SMoH), Federal Government, UN Bodies, development partners, private providers, private employers, HMOs and NMA were key networks involved in moving Nigeria towards Universal Health Coverage (UHC) (Onoka et al., 2013). The agenda was set for solving the problem of access to quality health care services and health status of the citizens through the formation of a National Health Insurance Scheme, which in 1999 was a step towards UHC (assumed to be the solution) (Onoka et al., 2013). Becoming operational in 2005 with the help of the public-private-partnership between the NHIS, Health Maintenance Organizations (HMOs) and other health care providers (Onoka et al., 2013). Whilst the NHIS shapes health insurance policy through the licensing of HMOs which operate the health insurance business, it also accredits health care facilities so that they can provide benefit packages to registered enrolees. HMOs have responsibility for the purchasing of health care services on behalf of the scheme for registered enrolees. To ensure population coverage, the scheme has specific programs for different areas of society. These are the Formal Sector Health Insurance Program for the Federal Civil servants, Urban Self-employed Social Health Insurance Program, Rural Community Social Health Insurance Program, Children under Five 
Social Health Insurance Program (also covers pregnant women), Permanently Disabled Social Health Insurance Program, Prison Inmates Social Health Insurance Program, Tertiary Institutions and Voluntary Participants Social Health Insurance Program and the Armed Forces, Police and other Uniformed Services Insurance Program (captured under the Formal Sector Program). Out of all of these programs, only the Formal Sector Program is functional (Ele et al., 2016).

Those in charge of the scheme are held to account by trade union criticism, mostly from the NMA, over the handling of the NHIS as a health insurance regulator. However, the NHIS has responsibility for accrediting HMOs and health care providers HCPs, as well as ensuring rules of engagement compliance (HMOs and HCPs), it does not the power to enforce these as they were multiple complaints about the HCPs or the HMOs or made by the HCPs about the HMOs or vice versa and are rarely appropriately addressed. Pressure from the NMA and existing trade unions forced the government, through the $\mathrm{FMoH}$, to respond to the unacceptable events within the Nigerian scheme, through commitment and willingness, to achieve comprehensive health sector reforms. A 2006 revised health reform stated that provision of adequate and sustainable finance is crucial for effective, efficient and equitable health system performance. Guided by the National Health Policy, the National Health Financing Policy 2006 is committed health financing fairness and has been recognized as one of the goals of achieving UHC in Nigeria (Campbell et al., 2014).

Despite the World Health Assembly's 2005 resolution on UHC financing, Nigerian progress on the UHC policy, in comparison to their African peers, is still lagging behind. UN body progress metrics suggest that the Nigerian health system requires more than a cosmetic change - it needs radical reform to improve its performance. In 2014, WHO and the World Bank offered a framework for monitoring UHC advances towards a focus on indicators and targets for service coverage including promotion, prevention, treatment, rehabilitation and palliationwith financial protection for all. Pressure on the $\mathrm{FmoH}$ and the government to reach a critical inflection point in its efforts to expand healthcare access, and to move towards UHC resulted in Nigeria's UHC Declaration which was adopted at the Presidential Summit on UHC in 2014 (WHO, 2014).

Collaboration took place between key policy actors to support the government's move towards universal health coverage (UHC). The former President Goodluck Jonathan presented to the WHO and was attended by Executive Governors, Ministers of the Federal Republic, 
Legislators, Health Commissioners, Traditional Rulers, development partners among others, concluded with the Presidential Summit Declaration on UHC. In conjunction with the National Health Insurance Scheme (NHIS), Minister of Health, Minister of State for Health World Bank Representative in Nigeria the meeting assembled top policy actors whose focus was campaigning for effective coverage towards universal health coverage in Nigeria (Awosusi, 2014).

There may be difficulty in pinpointing certain events where interactions have occurred between policymakers, but through using social network analysis and policy process theory the study can identify possible alliances, partnerships, interactions within the UHC discourse (Tantivess and Walt, 2008).

\subsection{Summary}

There is a large literature on UHC, which ranges from case studies of the original UHC countries in the global North to case studies of recent UHC success stories in the global South. Much of the literature takes a normative stance that described the benefits that UHC can provide in terms of improved health and well-being, in reducing social inequality, and contributing to economic growth. While the author is sympathetic to the values that underlie the drive for UHC, the arguments about the value of healthcare coverage for all as a public good are less relevant to this thesis than what we know about the problems of developing and implementing UHC policies. The literature review shows that there are many "overviews" studies of the policy stories of different UHC country stories, and especially accounts of the conditions for successful implementation or what happens when reforms go off track. But our knowledge is thinner when it comes to the detail of what happens when a country that was not a UHC pathfinder responds to current messages from the WHO and others that UHC is possible almost everywhere and initiates a policy debate about how to introduce its own program of UHC reform. That is the gap in respect of which this thesis seeks to make a contribution.

Almost by definition the countries that have already achieved UHC generally possessed more of the prerequisites for major health system reform than those that did not. In a sense the countries now starting out on this path are the more difficult cases, partly in terms of levels of wealth and baseline positions in terms of existing health expenditure and infrastructure, but possibly also in terms of commitment to UHC principles and political will to progress down 
the UHC path. We cannot therefore assume that research reports of policy formation in the UHC pathfinder countries will tell us what will happen when similar processes are enacted in countries like Nigeria. On the one hand the environment is different because the lessons of the pathfinder countries are now being disseminated by a range of international bodies, networks and campaigning groups, and on the other, domestic political, institutional and economic conditions may be less favourable to UHC reform. Nigeria is a wealthy country by African standards, but one whose level of investment in its healthcare system is markedly lower than several regional comparator countries. It therefore makes a useful case study for research on the UHC policy discourse in a country that can be seen as a trend follower rather than a trend setter. 


\section{CHAPTER 3}

\section{ANALYTICAL FRAMEWORK}

\subsection{Introduction}

This study makes use of two frameworks for analysing policy making: Walt and Gilson's (1994) policy process model and social network analysis (SNA), especially as developed by Lazega (1997). The policy process approach was developed by a research group at the London School of Hygiene and Tropical Medicine (Walt, 1994; Gilson et al., 2008; Buse, Mays and Walt, 2012; Gilson, Orgill and Schroff, 2018), and has been widely applied in studies in developing countries, often by doctoral students. SNA is a well-established and highly technical approach with an array of tools for measuring and analysing various relationships within a social network (Borgatti et al., 2013). In the author's view, the two approaches complement each other well. Policy process studies set out to investigate (using the concept of power and Kingdom's multiple stream framework) how actors interact to make policies in particular social contexts, while SNA involves quantitative analysis to delineate the boundaries of a policy network and the patterns of relations between actors within it.

Because identification and description of a policy network is a foundational exercise on which a more in-depth account of the dynamics of influence and power in the policy making process can be built, the empirical chapters that follow will examine the quantitative SNA data before the qualitative interview data. However, because policy process writing is likely to be more accessible to most readers than the more highly technical SNA approach, we will reverse that order in this chapter as we provide greater detail on what the two modes of analysis entail. 


\subsection{The policy process approach}

Two components of the policy process approach that are central to the present study are the policy triangle and the conceptualisation of power in policy making.

The policy triangle is a concept intended to represent central dimensions of the policy process: actors, content, process, and context (Figure 16).

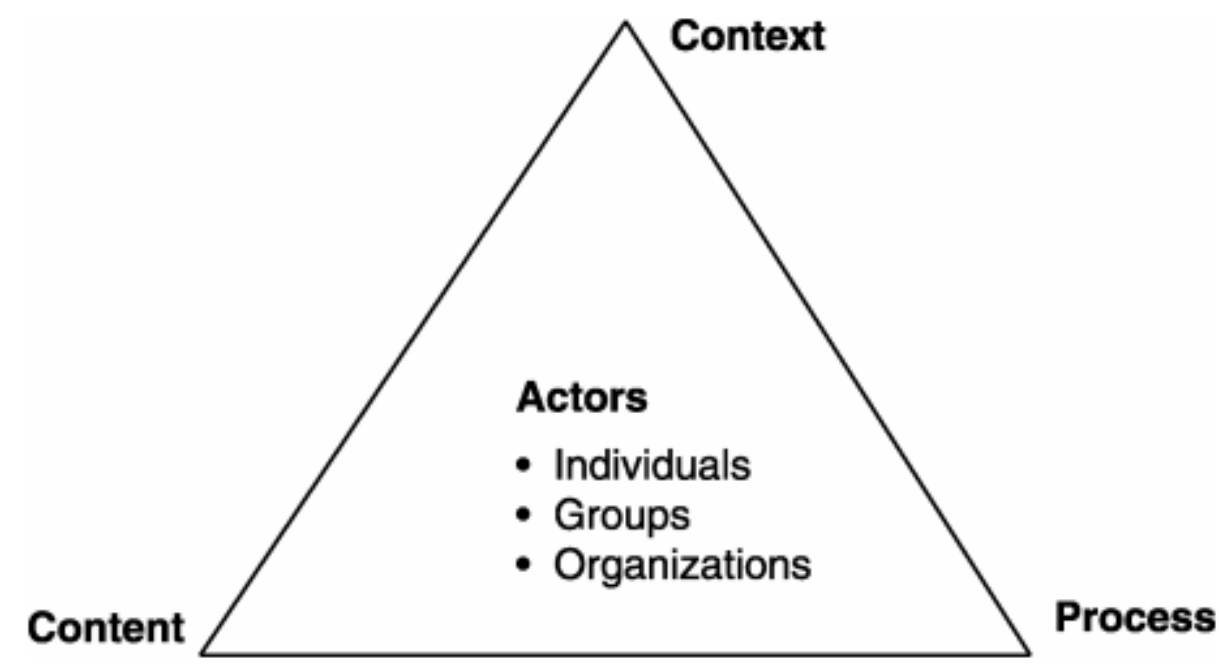

Figure 16: Policy analysis triangle.

Source: Buse, Walt and Mays (2012).

Buse, Mays and Walt (2012: 9) acknowledge that this is a "highly simplified approach to a complex set of inter-relationships" and that the triangle is "like a map that shows the main roads but that has yet to have contours, rivers, forests, paths and dwellings added to it”. They emphasise that the four components must be seen as operating in interaction rather than isolation, and also highlight the power dimension, which they state must be included in any analysis so as to take account of the important place of politics in policy analysis.

Actors are at the centre of the triangle, and include not only individual actors, but organisations, such as civil society groups, international bodies and government departments and parastatals. Actors may form alliances or networks, so that the pattern of relationships is crucially important in policy process studies. Buse, Mays and Walt (2012) maintain that a variety of actors will try to exert influence over the direction of policy, and that their ability to do so will depend largely on the degree of power they can deploy within the contexts in which they operate. 
Policy content may vary on a number of dimensions that make policies more or less hard to deliver, such as complexity, scope, cost, place in the overall government policy programme, and degree of consensus or controversy. Where there is more than one route to a policy objective such as UHC, the selection of the mechanism to bring the desired outcome about, such as public health insurance, may itself be a matter of debate. Even if there is broad agreement that such a mechanism is preferred over alternatives, there may still be considerable controversy about the form of the insurance scheme or schemes selected and the respective roles of public and private sectors.

According to Buse, Mays and Walt (2012: 13) the policy process encompasses the "ways in which policies are initiated, developed or formulated, negotiated, communicated, implemented and evaluated”. They direct attention to the processes, trajectories or chronologies that shape policies as they move from initial conception to rollout in a healthcare system. Many early writers on the policy process, such as Lasswell (1956) and Howlett and Ramesh (2003) identify a number of stages of the "policy cycle” but differ regarding just how many stages are present. Walt et al (2008) opt for the four stages of agenda setting, policy formation, implementation and evaluation. The notion of the policy cycle was intended to describe a characteristic sequence of stages through which a policy passed as it progressed to have real world impact. In recent years the stages approach has been criticized for over-simplifying the complex trajectories, with frequent overlaps or undulations, that real-world policies follow. However, some argue that the "stages heuristic" remains useful for conceptualising the broad sweep of what is inevitably a fluid policy process and helps us to more easily label the literatures concerned with aspects such as agenda setting or implementation (see: Cairney, 2013). The author acknowledges that policy formation, implementation and evaluation may overlap and interact, but takes the view that it remains useful to distinguish implementation from policy formation, not least because policy actors themselves often refer to these aspects of the overall policy process.

One aspect of process that is rarely discussed is the period of time over which a policy is implemented, which as Sabatier (1999) points out can often be very lengthy. Sabatier (1999: 149) writes of the "desirability of longitudinal studies of a decade or more" and suggests that "early implementation studies using time frames of 3-4 years resulted in premature assessments of program performance”. It can be argued that reforms that take too long to implement may suffer from a loss of momentum, as governments or supporting officials change, and the 
favourable combination of circumstances that raised a policy high on a government policy agenda is eroded with the passage of time. This may mean that implementation efforts are driven by different groups at different times, and that there are ebbs and flows in the degree of governmental support. For example, Intaranongpai and colleagues' (2012) study of the Thai UHC reforms over a ten-year period describes how the pendulum swung between top-down and bottom-up influences as the organisational framework changed and new alliances between interest groups were formed. The history of UHC reform efforts in Nigeria is a lengthy one that involves moments of what appeared to be rapid progress and pauses when little advance was possible, and this is part of the background to the ongoing UHC policy debates examined in the present study.

At the top of the policy triangle is context. Context refers to the political, economic and social environment in which policy is formulated. A developing country such as Nigeria provides a very different context from a Western country such as the United Kingdom in terms of geography, GDP, demography, culture, religion, history, and political system. Collins, Green and Hunter (1999) discuss context and health sector reform and remind us of the need to link "policy context with an overall understanding of the policy process, the "messiness" of policymaking, the interrelationship between the contextual factors, [and] the contextual factors interpreted by policy actors” (p.1). The nature of social institutions, both in terms of the formal organisations that make up the governmental and healthcare sectors, and their characteristics ways of operating, is a major contextual factor (Scott, 2013). This extends to the institutional configuration of the healthcare system and the pattern of relationships between the central health ministry and its parastatals or decentralised tiers, which in Nigeria includes the state and local government levels. The present thesis seeks to put into context the collective and individual behaviours of the actors involved at these levels in establishing Nigeria's UHC policy. The act of placing something in context - contextualising it - is both methodological and substantive. From a substantive perspective, it involves the identification of constraints on the behaviour of those involved and the opportunities available both to them and to others. From a methodological perspective, it is an essential part of comparative analysis and is necessary if results are to provide satisfactory answers to the research questions (Silverman and Gubrium, 1994).

Buse, Mays and Walt (2012: 9) emphasize that the policy triangle is a "highly simplified approach to a complex set of inter-relationships" and that it is "like a map that shows the main 
roads but that has yet to have contours, rivers, forests, paths and dwellings added to it”. They caution that the dimensions of actors, content, process and context interact with each other and cannot be analysed in isolation and argue that the framework puts politics at the centre of policy analysis.

Other writers, who are otherwise broadly sympathetic to the policy process approach, have criticised the policy triangle for paying insufficient attention to additional factors that influence policy. For example, Shiffman and Smith (2007) describe a framework of ideas, the power of actors, and environment, and describe "issue characteristics" (specific features of particular issues) that can affect policy. John (1998) and Howlett and associates (2009) produced a framework of ideas, institutions and interests (the "three I's") to show how these forces shape policy making. Both of these frameworks share many of the assumptions of Walt and Gilson's original approach. These friendly critics take the view that the policy process perspective can be enhanced by greater attention to institutional theory, the impact of organisational rules and norms in relation to policy processes, the characteristics of issues, and actors’ purposive action.

Buse, Mays and Walt (2012) argue that politics is “omnipresent” in the policy process, and they see power is a key component of their theoretical framework, especially in relation to the connection between actors and process. Among their concerns are the distribution of power within the network of policy actors, how different groups exercise power, and how power shapes decision making.

There is, of course, a huge political and social science literature on the conceptualisation of power and how this aligns with various overarching theoretical frameworks. The policy process approach defines power as the ability to influence policy decisions, and especially to control use of resources (Buse, Mays and Walt (2012: 20). The three dimensions of power identified by these writers are power to make decisions, power as non-decision making, and power as "thought control”. The first, based on a classic study by Robert Dahl (1961), is concerned with how successful different actors and groups are in exercising direct or indirect influence on policy making. The second aspect of power as non-decision making refers to the ability of elite actors to prevent certain issues from rising on to policy agendas, thus ensuring that they are not widely debated or translated into proposals for reform. The final dimension of "thought control" is based on Steven Lukes' (1974) claim that elites use insidious means to shape public perceptions of reality through socialisation, mass media propaganda and other forms of information control. As the present study is concerned with ongoing implementation of an 
established UHC policy rather than agenda setting, the notion of power as influence over policy decisions is probably most relevant for us, but we will return to the various aspects of power later in the thesis.

Buse and associates (2012: 10) suggest that power is not always manifest in a rational process through which individual actors realise favoured policy objectives, but often in struggles between groups or factions. They suggest that power may not so much reside in individuals, but in "policy networks", sets of connected actors inside and outside government in a particular sector who seek to influence policy (Buse, Mays and Walt, 2012: 91). In a country such as Nigeria, with a well- developed apparatus of central government, and also a relatively strong level of state governments, these networks are quite extensive and have complicated relationships with international bodies and actors in domestic non-governmental organisations. Policy process writers acknowledge the likelihood that network actors will come together in alliances and coalitions, borrowing ideas in part from writers such as Kingdon (1984) (policy entrepreneurs) and Sabatier (1988) (advocacy coalitions). The conclusion must be that making policy is rarely a rational process but is iterative and is influenced by the various actors. Lindblom (1959) described policy making as a process of “muddling through”, while Cohen, March and Olson’s (1972) “garbage can model” portrays organisational decision making as a sometimes-chaotic process, in which solutions may only be loosely connected to problems.

It is worth noting that a number of more recent policy process studies pay greater attention to the network concept. Quissell and colleagues (2015; 2018) examine the emergence of transnational networks to control tuberculosis and pneumonia and argue that network formation can be seen as an outcome of the policy process linked to the alignment of problem, politics, and policy streams, as in Kingdon's (1984) three streams approach. Shearer and associates (2018) look at the issue of whether network structure affects policy innovation, taking the case of how research evidence was utilised in three policy networks in Burkina Faso. This study marks a shift of emphasis from the earlier studies in that it moves more explicitly to utilise SNA tools and concepts, in particular measures relating to network closure and heterogeneity. Interestingly, the wider study (Shearer et al., 2016) combines elements of SNA with the "three I's” framework of institutions, interests and ideas, based on Peter John's (1998) book. The author was unaware of these recent developments when he conceived the idea of combining SNA and the policy process approach, but this parallel development suggests that this may indeed be a feasible and valuable direction for theoretical synthesis. Although the work by the 
LSHTM group is innovative and interesting, their use of SNA tools is so far quite limited. The present research attempts to go a step further by bringing a wider range of SNA techniques into an integrated study.

The present study sets out to examine UHC policy making in Nigeria in context. The policy process approach sets out to unravel the complexity of power and process that underpins the UHC policy discourse. It tries to reconstruct the story of the reform process. SNA approaches the contextualisation of the behaviour of the various actors in a different way by identifying the network in which they operate and measuring various aspects of network relationships. In essence it describes the social environment in which the actors operate, in effect, inductively modelling the structure or relational pattern of the social setting in which the observed action takes place. Participants are clustered in subsets according to criteria that may include political alignment, social ties, obligations, and influence over decisions (Miller and Dingwall, 1997). A brief outline of this approach, and some of SNA tools to be used in this study will now be provided.

\subsection{Power in health policy}

According to Buse, Mays and Walt (2012), power refers to the ability an individual or organization has to influence a desired outcome. In regard to UHC Nigeria implementation, the idea of power is in decisively influencing individuals or groups irrespective of their intentions or will (Buse et al., 2012). Consequently, health systems are influenced by power dynamics underlying societal interactions. This is because power can be assumed by particular groups in a health system who can influence the direction of health policy, and, if powerful group interests are unrepresented in the present institutional structure, they will use persuasion to turn the issue to their advantage (Khan, 2010). Naturally, power is the primary focus of many social and political theories. Additionally, power is occasionally tightly concentrated among particular actors, e.g., policy elites (Wright Mills, 2000).

To recognize how an individual, organization and network derives power is crucial to increase understanding of how and why power flows in a direction or accumulates in a particular group. This understanding also assists awareness of how the power sources are unevenly distributed in addition to how they might be used to improve health policy and system equity. 
The student aimed to provide greater engagement with the power concept to guide other policy analysts focusing on mixed methods analysis in Nigerian UHC discourse. Our focus is the narrow power role in shaping health policy and systems functioning, instead of exploring power as a determinant of health outcomes (e.g., in the context of social position or macroeconomic structures). To analyze power distribution in Nigerian UHC discourse, this section focuses on understanding the nature and forms of power, and how exercising power supports and opposes, facilitates policy change, in addition to how it underpins change resistance (Sriram et al., 2018).

UHC Nigeria analysis draws on power as decision making, a part of the three dimensions of power developed by Steven Lukes (1974) which are as follows: power as decision making; power as non-decision making and power as thought control (Buse, Mays and Walt, 2012). Power as decision making is a dimension of power that emphases the actions of individuals or group’s ability to influence policy decisions (Buse, Mays and Walt, 2012). Hence, this explores the identity of the different groups of policy actors or policy organizations and their relative power which will help understand the politics surrounding the policy process of the UHC process in Nigeria.

This is because the extent of power assumed by a certain group within a health system can directly influence the course of any health policy; meanwhile if powerful groups' interests are not met from the current situation from the institutional structure, they will lobby in other to overturn the issue to their benefit (Khan, 2010).

Robert Dahl's (1961) classic study concludes about who has power by examining the available preferences of interest groups and making a comparison with policy outcomes. He discovered that the resources conferring power on citizens and interest groups varied and that these resources were unequally distributed: while some individuals had plenty of some political resources, they were poor in others. Thus, different individuals and groups were able to exert influence on different policy issues. These findings caused Dahl to conclude that different societal groups, including weak groups, could 'penetrate' the political system and exercise their power over decision makers according to their preferences. While some people directly influence key decisions, defined as the successful initiation or vetoing of policy proposals, most had indirect influence by voting power. The range of resources which can be used in health policy is equally diverse and will be a function of policy content and context (Buse et al., 2012). 
As hypothesized by Lavers and Hickey (2015:10-11), in a competitive political setting where elite policy actors are excluded from the ruling coalition are powerful and where the ruling coalition has little autonomy from its supporters and the population in general, healthcare policies or programmes are more likely to derail. Rulers have an incentive to turn them into clientelist channels of redistribution and sites of rent capture to accommodate the powerful opposition or reinforce the loyalty of their supporters. Implementation of healthcare policies and programmes might also suffer because "the more powerful lower-level factions become, the greater the number of points at which the enforcement of particular rules can be blocked" (Khan, 2010: 65).

However, criticism of Dahl's analysis suggests that his observation on policy issues fail to recognize the possibility that dominant groups exert influence on the policy agenda towards their goal and away from acceptable concerns. On the contrary, the second dimension of power developed by Steven Lukes (1974) articulates differently, power as 'non-decision making' involves "the practice of limiting the scope of actual decision making to safe issues by manipulating the dominant community values, myths and political institutions and procedures” (Bachrach and Baratz, 1963 p. 641).

In this dimension of power, Nye (2002) conveniently differentiates 'non-decision making' into hard and soft power where hard power illustrates the carrot and the stick approach and soft power relies on co-opting actors towards your interests, goals and objectives through resources such as culture, values, ideas, and institutions. Hence, draws attention to what and who persuaded the Nigeran government towards UHC implementation as a policy agenda and this is beneficial in understanding the concept of legitimacy in Nigeria's policy-making process.

Furthermore, the third dimension of power conceptualizes by Lukes (1974) is 'power as though control', and it is a form of power that shapes the preferences of other policy actors and their perceptions of what is in their interest. In this dimension, Lukes consider this form of power as ‘supreme’ and 'most insidious, which manipulates actors away from their interests, perceptions and cognitions or from contesting the policy ideas present to them either because there are no alternative approaches or because the ideas are beneficial and valuable (Buse, Mays and Walt, 2012). 
This highlights the fact that wider bodies of power theory (from Steven Lukes' (2005), a prominent policy theorist, characterized three faces or dimension of power) offering insights into the emergence of power in UHC discourse, how it is channeled, and how power is overtly or covertly expressed. Finally, power taxonomy developed by Steven Lukes (1974) characterized an important domain of power analysis, focusing on how actors might extract power from specific sources such as resources, skills, knowledge, access or individual attributes. Recognizing how individuals, organizations and networks derive power is vital to increase understanding into how and why power flows in a particular direction or accumulates in groups. This understanding also aids awareness of how those power sources are unevenly distributed in addition to how they can be used for improving UHC discourse (Sriram et al., 2018).

\subsection{Multiple stream framework}

The previous section uses the policy process model to explain Nigeria's UHC reform from agenda-setting to policy formulation, policy implementation and policy evaluation. According to Roberts et al. (2004), each policy stage carries the flow of political or non-political events that need a continuous theoretical analysis to capture any policy change.

According to Buse, Mays and Walt, in exploring the flow of events with Nigeria's UHC policy process, a good starting point of an analysis is the agenda-setting stage which identifies how policy problems/solutions get onto the government agenda and how the response to these issues is crucial to the study.

Numerous studies on agenda-setting, commonly use the Hall model and Kingdon's multiple streams framework model, two prominent theories best known for evaluating how problems enter the policy agenda. These two theories are simple and easy in assessing the agenda-setting stage of the policy process. The Hall model responds to the legitimacy, feasibility and support of how the policy issue and the solution enter the government agenda. However, Kingdon's multiple streams framework centres on the policy process and the actors involved, which is a research objective of this thesis (see page 30 ).

Kingdon's Multiple Streams Framework (MSF) suggests that agenda setting is affected by three parallel streams in the policy making process (the problem stream, policy stream and politics stream), which generally take separate paths, but, from time to time, converge so that 
an idea 'whose time has come' moves on to the government's policy agenda. The coming together of streams creates a time-limited 'window of opportunity' when a policy may be translated into legislation and then real-world change. This is illustrated in Figure 17, which is based on the MSF as developed by Ashford et al. (2006).

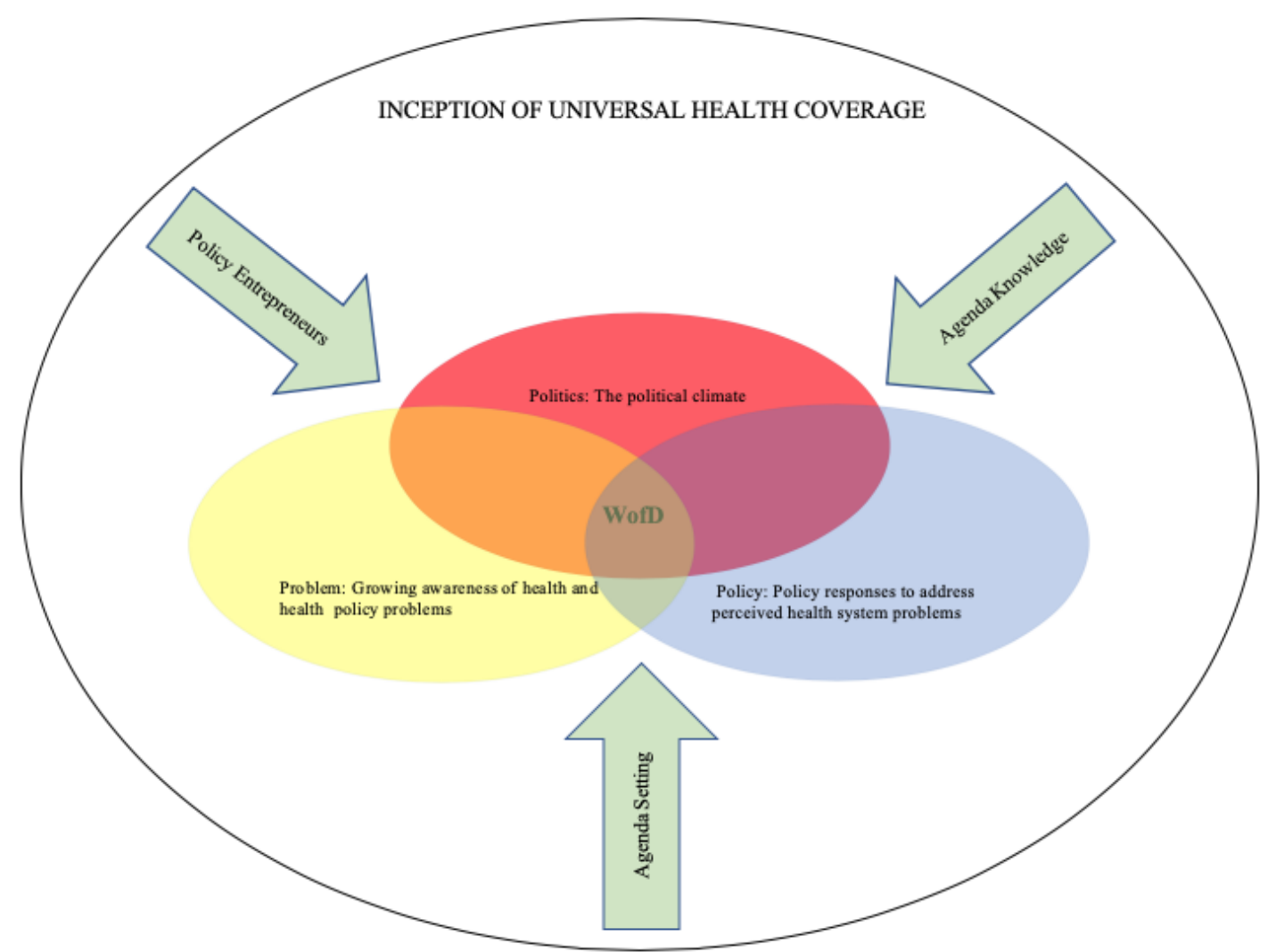

Figure 17: A theoretical framework for understanding how the UHC policy rose to the top of the government's policy agenda. Source Ashford et al. (2006).

Key: WofO* = Window of Opportunity

Kingdon's framework would predict that, during Nigeria's pre-UHC period, the streams flowed along different channels until conditions changed to bring them into at least partial alignment and open a window of opportunity (see in-depth analysis in Chapter 6). These three parallel streams - the problem, policy, and political streams - have the following features (Beland and Howlett, 2016):

1. The problem stream (growing awareness of health and health policy problems) exposes health issues in need of government respond. Policy makers discover these issues 
through health indicators, public opinion or sudden health crisis (like a pandemic, epidemic and endemic) and then work to get government to respond.

2. The policy stream (policy responses to address perceived health system problems) occurs when policy experts scrutinize and suggest policy solutions to the issues. In this stream, ideas are floated around and narrowed down to a particular solution.

3. The political stream (the political climate) involves the political factors that converge the other two streams, such as national elections and pressure group activism programmes.

Kingdon (1984: 21) understands agenda-setting as how "the separate streams of problems, policies, and politics come together at certain critical times. Solutions become joined to problems, and both of them are joined to favourable political forces.” According to Kingdon (1984), these three independent streams flow through a policy window opening, which gets the problem recognised by government action or inaction. The policy window opens under certain circumstances, such as election events, socioeconomic crisis or budget deadlines and is taken advantage of by 'policy entrepreneurs' in a policy subsystem to advance their self-interest (Howlett, 1998).

Policy entrepreneurs are actors existing within the policy subsystem who take the opportunity to influence and shape the outcomes of the three streams and their convergence to their advantage. Furthermore, policy entrepreneurs who have the ears of influential officials are more likely to advance their self-interests because they can propose a set of ideas when the opportunity arises (Zahariadis and Exadaktylos, 2015).

To successfully analyse Nigeria's UHC with Kingdon's MSF, much of the agenda-setting strategy will depend on how various policy entrepreneurs marshal their arguments on the substances of the policy solution to the issue revolving around the health system. "The content of the ideas themselves, far from being mere smokescreens or rationalizations, are integral parts of decision making in and around government” (Kingdon, 1984: 133).

Furthermore, the Kingdon model appeals to Cohen et al.'s (1972) 'garbage can’ theory of organisation which rejects the fact that decision-making occurs in a linear progression where policy makers identify problems, bureaucrats with comprehensive analysis provide several responses and the government officials pick out the best response. The 'garbage can' phrase symbolises where a mixture of problems, solutions and choices are meshed together and 
dumped in a place. For Cohen et al. (1972), deciding on a policy solution to issues is messy, ambiguous and time-consuming to generate the feasible response. Sometimes the phrase 'an idea whose time has come' emphasises how policy makers wait for the right opportunity to present policy solutions to government officials or the wider policy communities.

When compared to other policy making theory, such as the Advocacy Coalition Framework (ACF) and punctuated equilibrium, Kingdon's MSF model is applied mostly around US policy making and less in other countries. Yet, Cairney and Zahariadis (2016) consider the model applicable without difficulty in any country's policy making processes because it suggests a systematic insight to identify multiple stream events and windows of opportunity that brings issues to the any country’s policy agenda (Cairney and Zahariadis, 2016).

Furthermore, Cariney and Jones (2016) suggest that the MSF model can identify several universal elements, such as ambiguity, competition for attention, an imperfect selection process, limited time and a departure from 'comprehensive rationality' and a linear decision-making process within the agenda-setting process.

Howlett et al. (2016) examine the capabilities of the multiple streams framework and ascertain that it is possible to combine the MSF model with other approaches, such as the ACF and policy process theory, to create a representative analysis that captures diverse features (such as the intricacy of policy drivers, involving shift in political ideology and government interventions) during policy making. Their interpretation suggests that combining the MSF with such approaches provides a superior analytical framework for policy making instead of having competing approaches or zero-sum alternatives.

Furthermore, Kenis and Schneider (1991) complement network analysis and policy process analysis by using network analytical methods to identify and reconstruct complex policy games, i.e., relationships or patterns of strategic actions between a set of actors in policy formulation and implementation. In this approach, network analysis can be used as a measurement tool for game-theoretical models. Network sampling methods could be used to specify boundaries for the identification of aggregate or collective actors, and to identify relationships between players, which are fundamental for a given game (e.g., information structures on mutual payoffs).

Similarly, Nagel et al. (2017) integrate the MSF model with the policy network analysis (using social network analysis tools) to examine a recent climate change policy in Germany and Japan and investigate the MSF framework on integrated networks. The findings show a more reliable 
information exchange network connection in Germany than in Japan because an integrated network leads to a more progressive institutionalised policy in Germany compared to Japan. They argue that the integration of policy network analysis with MSF offers a precise and formalised analysis of identifying more or less policy network integration.

MSF is a matured policy framework with recent dynamic development calling for better conceptualisation, operationalisation, and empirical application. To contribute to this momentum, we aim to develop the concept of network integration by enriching it with quantitative policy network analysis. We argue that it is possible to apply policy network analysis to MSF to indicate more or less integrated policy frameworks and that this application improves our abilities to test related assumptions and hypotheses (Nagel, Novotny and Satoh, 2017).

\subsection{Social network analysis}

Social Network Analysis (SNA) describes the relationships connecting people in a given social circle (Chanut et al., 2005; Wasserman and Faust, 2009; Jang, 2012; Borgatti et al., 2013). Its origins lie both in mathematical graph theory and social science. Graph theory makes available a set of abstract concepts and methods for the analysis of graphs that can be utilised to map social networks. Other analytical tools come from social science, including methods used for the visualization and analysis of social networks.

According to Shearer et al. (2016), SNA provides a set of tools for investigation of social behaviours and their effects. The behaviour of the actors can be placed in context by network analysis on the basis of a description and inductive modelling of the structure, or pattern of relationships, of the social setting in which an action takes place. Specific data are collected on exchanges and relationships between network members and analysed with the aid of various pre-specified procedures.

SNA scholars maintain that a great deal of social behaviour is shaped by the structure of social networks (Wasserman and Faust, 2009; Borgatti et al., 2013). Lazega (1997) sees the interactions between actors as having theoretical priority over the individual character of the actors. Social action is seen as being embedded in a network of relations, so that it must be explained in terms of the structure of the network rather than by how the actors direct their 
actions in isolation. Examples include government systems which require politicians and officials to interrelate effectively, and national economies in which actors depend on money that changes hands many times (Borgatti et al, 2013).

SNA may be approached either quantitatively or qualitatively. As well as mapping networks using quantitative techniques, SNA can shed light on the nature and meaning of social interactions within the network (Crossley, 2010; Fuhse and Mützel, 2011; Bellotti, 2015; 2016; Hollstein, 2016). In particular, writers have point to the ability of SNA to shed light on the context in which actors interact by investigating the way the group environment is structured (Lazega, 1997; Bellotti, 2015). Usually, qualitative analysis is introduced as an additional component in a mixed methods study, rather than an alternative to quantitative SNA (Crossley, 2010; Edwards, 2010). According to Wonodi (2012), SNA is well suited to mixed methods research because it helps to place actors' interactions and meanings in the context of the patterns of network ties (see also: Ryan, Mulholland and Agoston, 2014); this works particularly well in the case of interactive networks such as communication or influence networks.

Mixed methods and SNA has become increasingly popular in sociology and political science. It has been used to answer a wide variety of research questions including how social movements are formed and how formal and informal networks come to exist both inside and between institutions (Ryan and D’Angelo, 2017; Ahrens, 2018). Crossley (2010: 2) has argued that, while the formal structural mapping of networks provided by quantitative SNA studies is valuable, there is a risk this neglects social process and agency. Crossley points out that the interactions that produce network "ties" also generate shared meanings, norms and identities that tend to be overlooked in quantitative analysis. He points to the value of studying networks as "social worlds" and suggests that SNA can benefit from incorporating ideas from sociological symbolic interactionist theory and the associated methods of participant observation and qualitative interviewing, which can be combined with more conventional SNA analysis (Crossley, 2010: 2-3). Reason why the thesis uses a qualitative survey.

There is now a growing number of mixed-methods or qualitative network analysis studies of policy networks (Schindler, 2006; Sandström, 2008; Sandström and Carisson, 2008; Schiffer and Hauck, 2010), and this seems a promising line for development. Quantitative data on formal structural properties of networks, such as degree of closure, flows of information, advice, and resources, and presence of brokers who act as bridges between sub-groups, can be 
combined with qualitative data on actors' subjective perspectives, aims, ideologies and vested interests. Such studies have covered both domestic policy networks and supra-national networks, such as those formed by international civil society organisations or regional blocs like the European Union (Schmidt, 2013; Verloo, 2006). Such studies allow structural network characteristics to be visualised and combined with qualitative insights on the dynamics of the policy process and its wider societal context.

SNA typically uses visualisations to illuminate network structures. Wonodi (2012) describes SNA as a research method that uses visual representations to illustrate social phenomena and the interactions that take place between individuals in a social circle. The research method engages with social systems on the basis of the relationship between the actors (or "nodes") that constitute the systems. Emmanuel Lazega (1997), for example, used SNA to describe and inductively map dialogue patterns and relational structures between judges working in the field of patent law in EU countries. He used it primarily for the investigation of, and extraction of information about relationship patterns and ways of exchanging advice and information between judges lobbying for the construction of an EU patent court.
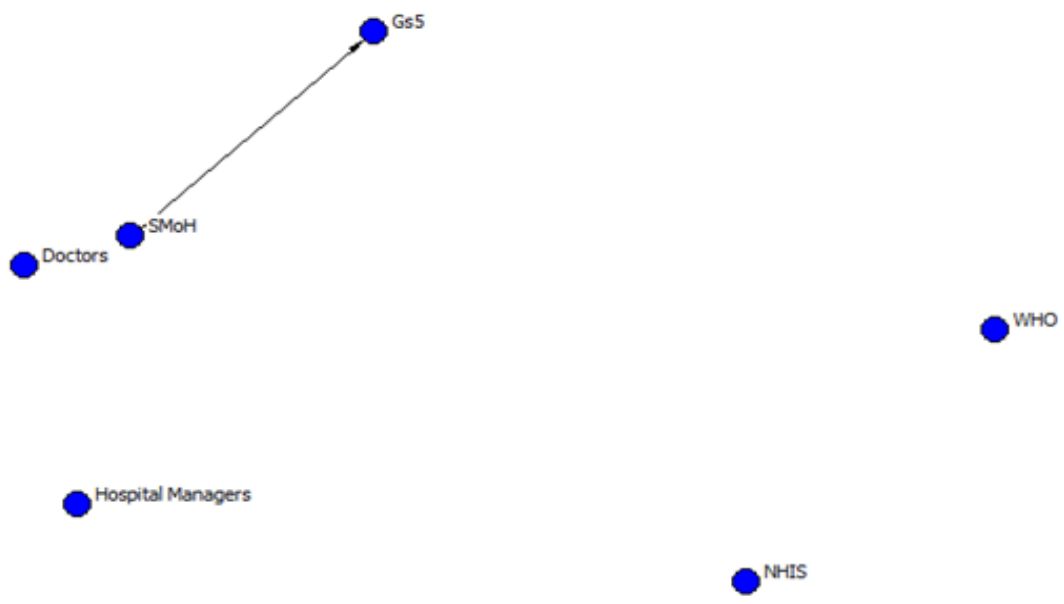

Figure 18: A simple network graph.

An SNA can comprise a number of "nodes" which represent individuals or organisational actors, which are all conceived as active agents. Nodes will most frequently be either individuals - doctors, perhaps, or hospital managers - or collectives (groups of doctors or hospital managers). The connections that join nodes together are relationships that may be based on interactions, beliefs, political interests or friendships, and are described as “ties” (Jang 
et al, 2011). Figure 18 shows a diagram (in blue) containing six nodes, each of which represents policymakers in the Nigerian health system. In this diagram, the only relationship shown is between SMoH and Gs5 and the other four nodes exist in isolation, but when every node has been tied into the social setting it becomes a network circle. The SNA researcher must understand that there may or may not be ties between some nodes while the analysis is in process (Park et al., 2012). The four completely detached nodes shown in Figure 18 are known as 'isolates' which will be discussed further below.

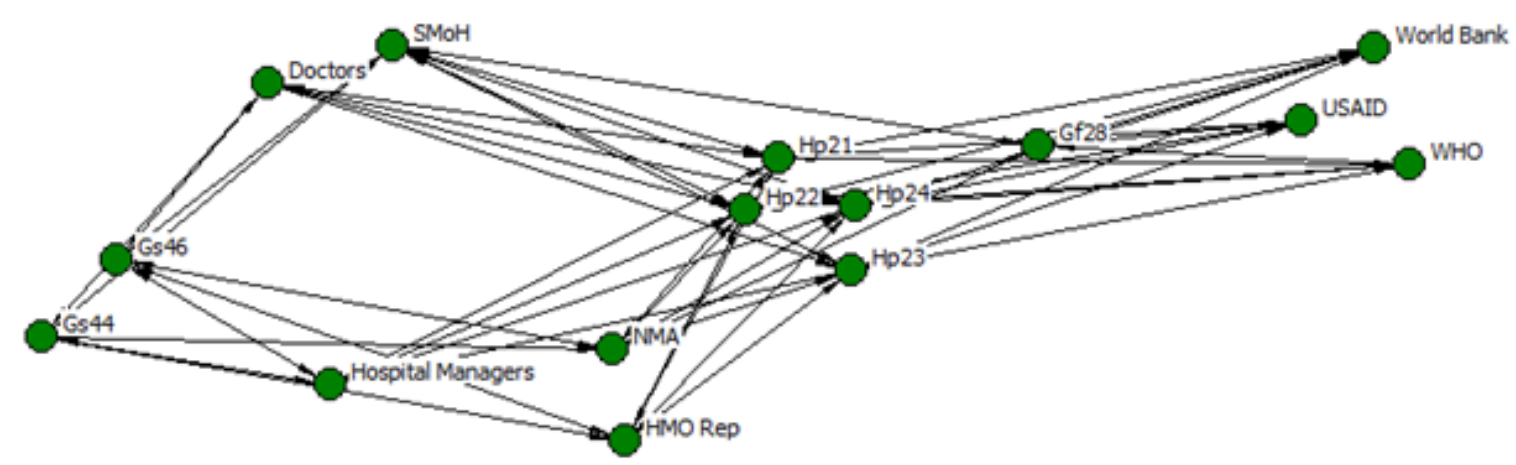

Figure 19: A complete directed network graph.

Networks (such the graph in Figure 19) provide a way to think about relationships between the nodes that constitute the system.

Network analysis has to do with structure and relative position, and a large collection of concepts have been developed to measure structure and position. Various theoretical concepts, such as structural equivalence and centrality (see below) are used to measure and quantify the character of nodes and their ties (Borgatti and Halgin, 2011).

\subsection{Concepts utilised in SNA}

A graph can represent relationships in a social network in a quantitative way and translate data into a visual diagram (Borgatti et al., 2013). SNA has its own specialist language, partly borrowed from mathematical graph theory. For example, a vertex is an endpoint for two or more lines (essentially another term for a node in an SNA graph), while an "edge" is a line segment that connects two vertices (the plural of vertex) together. In SNA edges therefore 
represent ties. Vertices can also describe points or nodes; edges (ties) are connected to two vertices.

The mathematical representation of a graph might be:

$$
(\mathrm{U}, \mathrm{V})=\mathrm{e}(\mathrm{G}) \text {. }
$$

When two vertices (U, V) are linked by an edge e, they are said to be adjacent. Hence, the size of the graph $\mathrm{G}$ is the total number of e (edges, ties or vertices) which makes the size of the or represented in mathematically equation above (Diestel, 1990). Where two vertices (for example, SMoH and Gs5 in Figure 18) connect to the same edge they are said to be incident. Rather confusingly, if the vertex A is connected to an edge e, then A may also be said to be incident on- e (vertex-edge incidence). In graph theory, the "degree” (of connectedness) of a vertex is the number of edges that are incident to the vertex. In plain English, a node that connects with three other nodes has a degree of 3. Graphs may represent ties between nodes as being directed or undirected. A directed graph is a network with arrows attached to the ends of the edges. A directed edge between two nodes (otherwise known as an arc) may be able to reverse direction, depending on the nature of the network activity under consideration. Figure 18, for example, shows a set of vertices (Hospital manager, WHO, SMoH, NHIS, Doctors and Gs5), and one edge between SMoH (the State Ministry of Health) and Gs5 (a State government official of health). Directionality in this arc might indicate, for example, that the $\mathrm{SMoH}$ allocates funds to a state government official Gs5, and not the other way around. However, if the visualisation was constructed to represent patterns of advice-giving rather than financial flows, the arrow on the arc might be reversed.

Every so often, vertices may express attributes such as qualities, motivations, properties or relationships other than actors that tie with actors in the network graph. (Robins, Lewis and Wang, 2012). Figure 18 illustrates a simple unipartite network with one kind of node (policymakers) represented by six actors. These six policymakers are the same type of node with a relationship, an interaction or an edge between the SMoH and Gs5 node can assume different forms of relational data such as advice, coalition or members of a discussion group. In any case, Robins, Lewis and Wang (2012: 377) insinuate that in unipartite networks, "there may be ties between nodes of different types but not between nodes of the same type.” Bipartite networks consist of a two nodal type network where ties/edges connect actor y to organization $\mathrm{z}$ (see Figure 19), and this indicates the variable $\mathrm{X}_{\mathrm{yz}}$ (where, $\mathrm{X}=$ graph, $\mathrm{y}=$ actor and $\mathrm{x}=$ organization). 


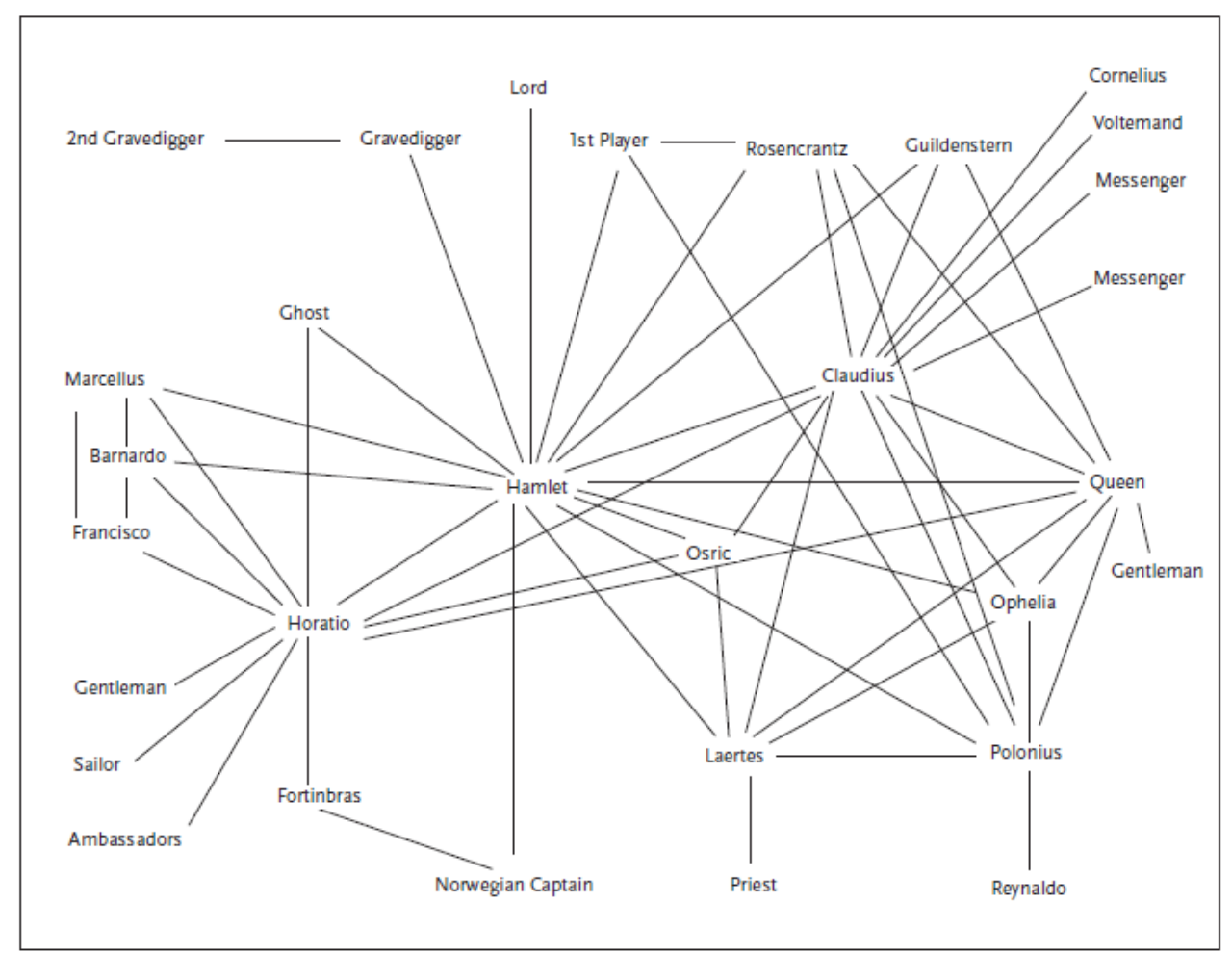

Figure 20: The Hamlet network (Moretti, 2011).

To provide a further illustration of how SNA is conducted, Figure 20 reconstructs the network of actors within Shakespeare’s play, Hamlet. It depicts a typical undirected graph containing edges without directional arrows. An undirected graph is used to describe links and ties where it is not necessary to know the direction or where there is reciprocation. An example might be a graph of the audience members at the premiere of a movie in which, within a network where there is no verbal interaction, individuals are asked whether or not they saw named others.

The network should not be seen as a unitary entity because actors form relationships of different kinds for different purposes, and the same actors relate on multiple dimensions, which can each be said to constitute a different network. As mentioned in an earlier example, the ties in a network concerned with financial flows are likely to be different from ties in a network concerned with the flows of advice, even though many of the individual actors involves are present in both networks.

According to Lazega (1997), social networks typically consist of subsets of actors, who are in regular interaction with each other. Such subsets can be identified, and the nature of their interactions can be measured by attending to two main factors. 
- The distance or density of ties between actors, so that a clique is formed from a subset of actors who have direct, strong ties with each other.

- Structural similarity among actors. In this case, the subset into which similar actors are grouped may be called a cluster, a position, or a clique.

In this way a visual map is created of different subsets or clusters where the subset is made up of actors who interact with each other according to specific criteria (Lazega, 1997). Figure 20, for example, is a representation of Franco Moretti's (2011) analysis of the web of relationships between characters in the play Hamlet. The principal character, Hamlet, is the central node, and the diagram reconstructs the ties/edges between this actor and other actors (nodes) in the network circle (Moretti, 2011). It can be seen that apart from the cluster around the central node (degree 16), there are clusters around the important nodes of Claudius (degree 13) and Horatio (degree 12), as well as some smaller clusters centring on Ophelia and the Queen.

The ties between each node tell us something about the roles of the main characters and the groups that form around them. That is, in the language of SNA, a character can be categorised, to list some possibilities, as a connector, a leader, a bridge, or an isolate. Various concepts are used to characterise relationships between proximate nodes. For instance, two nodes with no direct tie may appear not to be connected, but information may still be passed between them through ties to intermediary nodes. In the Hamlet network a path of this kind may exist between the Priest and Reynaldo via Laertes and Polonious.

According to SNA terminology a path is any sequence of non-repeating nodes that connects the two nodes of interest. Therefore, the sequence involving the Ambassadors, Horacio, Fortinbras and the Norwegian captain is a path, while the sequence encompassing the Queen, Ophelia, Polonius, Queen, Gentleman is not a path because the Queen features twice. The part of the sequence between the Queen and the Gentlemen is referred to a trail, because in a trail a node is revisited, but no edge is traversed more than once. A sequence that moves through an edge more than once is neither a path nor a trail and is referred to as a walk. For example, the sequence Claudius- Queen- Guildenstern- Claudius- Queen- Horatio revisits both a node (Queen) and a tie (between Claudius and the Queen) and is therefore a walk. A walk traverses a sequence of nodes and ties, doubling back to re-visit at least one tie (Borgatti et al., 2013 and Prell, 2012). What defines the length of a path, a trail or a walk is the number of ties/edges 
traversed. In the view of Borgatti et al. (2013) and Prell (2012) all paths eventually lead to a trail, which leads on to a walk.

Components in graph theory are portions of a network that are not connected to each other. They are subgraphs within which any two nodes are connected to each other by paths, but there are no ties to any additional nodes in the wider identified network (the "supergraph"). In essence, a component is a group of nodes that are able to interact, via established ties and which will remain connected if an additional node is inserted. Many real-world networks have multiple components.

Bridges are formed when the tie between two nodes forms the only link ("cut-point") between two positions of a network. If this tie were removed the network would divide into two separate components or networks. In Figure 20, the link between (Claudius, Osric, and Queen) and (Horatio), if broken, will result in two separate subgraphs with Horatio at the centre of one and Claudius at the centre of the other. Isolates, as the term suggests, are nodes that are disconnected from other nodes. In Figure 18, the Hospital manager, WHO, Doctor, and NHIS nodes are not connected to other nodes and so are isolates. SMoH and Gs5 would be termed pendant vertices, meaning that they connect to only one other edge (degree 1). The edge between them is known as a pendant edge, because it links two pendant nodes.

A number of critics have suggested that a gap exists in SNA theory because the emphasis is on description rather than theory (Granovetter, 1979; Burt, 1980; Salancik, 1995). It may be argued that the play network which Moretti (2011) constructs to visualise relations between characters in Hamlet is a descriptive and not a theoretical analysis.

SNA makes available a number of techniques for measuring such network characteristics as autonomy, centrality, cohesion and structural equivalence (Prell, 2012). For example, Boyer et al. (2010), illustrated the usefulness of SNA in investigating relationships between doctors in a French hospital by describing centrality, prestige and clique formation, with the aim of raising healthcare quality and improving staff welfare by achieving better relationships between medical professionals.

SNA analyses commonly seek to attach values to the ties (edges) between actors (nodes) so as to represent the frequency of interaction and the strength of the tie. 
SNA has a number of ways of conceptualising, measuring and analysing actors' positions in the network. Seeing a visual representation of the network can provide a qualitative understanding that would be difficult to gain through numerical data alone. As we saw in the figures above, a network diagram comprises points that represent nodes and lines that represent ties. The points and lines have various characteristics that can be represented through shape, size and colour in order to communicate information about the nodes and their interrelationships. In the next section, the visualisation of social networks will be discussed, and the discussion will distinguish between the elements themselves and the way they are graphically represented. Further examples will be provided to show ways in which certain issues of visualisation can be addressed and (especially in large networks) network complexity can be reduced (McGrath, Blythe and Krackhardt, 1997).

\subsection{Social network analysis and policy process in health context}

Volumes of academic work combining policy network analysis and policymaking theories spanning over three decades and have extended vastly through topics varying from local, to state and to international levels of analysis (Laumann and Pappi, 1976; Schneider, 1992; Thomson et al., 2006; Witte et al., 2000).

"Policy network analysis or policy network" was first termed by Katzenstein (1976) in a study comparing French and United States economic policies (Knoke, 2014).

Other policy theorists add that applying SNA into policymaking can define the policy concerns or consequences using a structural network of actors competing and cooperating within a particular policy environment (Knoke, 2014), and this is because SNA analytic assessments have an array of models, ideologies, and propositions to explain different features of policy networks (Robins, Lewis and Wang, 2012).

This section defining policy network and other corresponding policy concepts adopted by many analysts, examines crucial developments of studies applying policy networks in policy processes and summarizes the origins of policy network. 


\subsubsection{Understanding policy network}

A policy network like any social setting consists of a group of actors interacting in several ways in order to achieve a given objective, where actors within the network serve either as individual persons (such as Bill Gates, Greta Thunberg or the Clintons) or individuals representing organizations (NGOs, External Government Agencies, UN Bodies, political parties, labour unions or legislatures) at the state and global level of analysis (Knoke, 2014). For instance, Kenis and Schneider (1991) perceive the policy network as a set of public and private corporate actors developing relationships through the exchange of information, expertise, trust, and other political resources.

Corresponding policy concepts such as the policy game; the policy regime; the policy sector (see Benson, 1982); the policy domain (see Laumann/Knoke, 1987); the policy system for actors (see Sabatier, 1987); the policy community (see Jordan/Richardson, 1983; Meny, 1989); and the policy arena develop theories in parallel with policy network when describing certain phenomena (Kenis and Schneider, 1991). For instance, a well-known concept, the 'policy domain' shares familiar boundaries with the policy network where actors' predispositions are considered social construct of their environment and their behaviour surrounding decision making occurs as a result of interacting with other domain respondents (Laumann and Knoke, 1987).

Policy domains exist within several sectors such as defence, education, agriculture, social welfare, health, and transportation (Laumann and Knoke, 1987; Burstein, 1991). According to Konke and Laumann (1982: 256), policy domain in any policy process “identified by specifying a substantively defined criterion of mutual relevance or common orientation among a set of consequential actors concerned with formulating, advocating, and selecting courses of action (i.e., policy options) that intend to resolve the delimited substantive problems in question.” Both concepts (the policy network and the policy domain) recognize that a social environment system is bounded by actors' interconnection with each other (Knoke, 2014), while other policy concepts accentuate special features (Kenis and Schneider, 1991). For instance, the policy arena concept examines policy making practices within institutional structures whether focusing on institutional conflict and institutional integration.

The policy network approach combines well with many social science disciplines such as organizational studies, political sciences and policy sciences, which end up expressing a 
diverse understanding of how well network analysis works and what a policy network approach categorically infers in policy analysis (Börzel, 1998; Carlsson, 2000; Kickert et al., 1997). Combining social network analysis with policymaking contributes to the creation of a rich mixture of policy models and theories that can evaluate, detect and tackle policy issues, policy actors and policy solutions with feasible options. For instance, Sabatier and Jenkins-Smith's (1993) advocacy coalitions, Hjern and Porter’s (1993) implementation structures, Jordan \& Schubert's (1992) iron triangles, Heclo's (1978) issue networks, Jordan’s (1990) policy communities, and Rhodes' (1990) sub governments are all policy models or theories included in this category.

Understanding social network analysis in policymaking is required to examine the thesis's research questions and achieve the research objectives. Hence, empirical investigation of UHC Nigeria policy reflects a series of "actions taken by members of a group to further their common interests” (Bogdanor, 1987: 113) and the social interactions among policy actors involved in UHC Nigeria is perceived as "problem-specific entities, organizing a policy area by different forms of collective action" (Sandstrom and Carlsson, 2008: 508). Furthermore, the thesis adopts similar perspective to policy networks as Sandstrom and Carlsson (2008), where networks are structured social environment which involves diverse relationships of policy actors engaged in a process for the collective good of their interests and policy solution.

Despite the significant development of policy networks in policymaking research, several critics suggest that the concept of policy network analysis is incapable of producing substantial metaphorical use and a coherent theory (Carlsson, 2000; Dowding, 1995). They express concern that the concept of policy networks is more or else a descriptive metaphor demonstrating the materialization of certain key political events occurring from a group of interactive actors mostly at a macro level and should not be treated as theoretical models capable of explaining policy change or outcome (Dowding, 1995).

Proponents suggest that the challenging question of adopting network analysis is by asking are beneficial to the topic? A formalised SNA through quantifying social interactions, mapping network structures, and statistically examining social networks increases the potential for the research (see Adam \& Kriesi, 2007). 


\subsubsection{Origins of Policy networks}

This section presents three key geographical origins of the policy network approach in policymaking, which come under a fair amount of dispute by many policy analysts (Peterson, 2003). Richardson (2000: 1006) states "British origins of what is now termed the network approach.” Rhodes (1990: 32) agrees that “American political science was not the major formative influence' on early work which sought to make sense of the British 'post parliamentary' state using network analysis in the late 1970s.”

Firstly, Laumann and Pappi (1976) ushered German literature of community power into new directions. They revealed in a study that the public policies of a small German town were influenced by the collective effort of multiple networks of elites. Their approach began to spread within German public policy analysis seen in studies such as Laumann and Knoke's ‘The Organizational State' (1987), which extended network analysis of power structures to national policy domains. The organizational state research puts forward the idea that national policy domains are complex social networks between formal organizations and not elite individuals. Laumann and Knoke's (1987) reveal that the power structures within a formal organization are organize in complex patterns of networks such as communication, resources, reputations, and political support among organizations with partially overlapping and opposing policy interests. Henceforth, many in Europe have taken into the network approach on policy analysis.

Secondly, in Britain, many political scientists in the 1980s moved closer to policy network analysis as policymaking began to shift away from entrenched sub governments (such as ministerial-level policy domains) responsible for consensual policy agendas. British political scientists' pursuit of network analysis was to identify the dimensions of a policy network structure according to distinguishing features (such as pluralism and corporatism), as issues within national policy domains become complex (Rhodes, 1990; Atkinson and Coleman, 1992; Jordan and Schubert, 1992). According to Richardson (2000: 1009-11), in national domain structures, "more fluid and unpredictable networks emerged in such policy domains as agriculture, civil nuclear power, youth employment, smoking, heart disease, sea defences, and information technology.” Furthermore, the 1990s saw British political scientists shift towards a new direction in intergovernmental and public management practices (Rhodes, 1996). 
Thirdly, the American perspective on policy network analysis on policymaking is entrenched in the German policy network approach. For instance, a study utilizes the network approach on two middle-sized Illinois cities to illustrate how formal organizations positioned centre in the network structure influence and shape policy affairs within the community, which is an exact replica of Laumann and Pappi's (1976) community power structure reiterated in the German origins. In contrast to the French and British policy network analysis, the German and the American share a similar approach because their federal system resembles each other, where states have the decision-making power on policies concerning the national government. Prereunification, Lehmbruch (1984) in the Federal Republic of Germany (FRG) draws ideas from Scharpf's et al. (1976) interlocking politics to investigate corporatist politics, which portrayed the web of inter-organizational networks as crucial in stabilizing collective policy actions.

In earlier studies, analysts were unsure whether to place policy networks either as dependent or independent variables because these research studies unsuccessfully created thorough theories and concept on policy advancement and results (Knoke, 1990). These policy network studies relied more on metaphor and description while lacking heavily on terminological imprecision and proliferation of typologies, especially when the goal was examining policy networks development (Dowdling, 1995; Mikkelson, 2006). Furthermore, as time went by, the policy network generated an abundance of models, ideologies, theory, procedures, and techniques that facilitated the field and prepared the foundation for other forthcoming studies, especially within the health context. For instance, modern policy network studies in health policymaking context include Goldman (2007) assessing in what way did the World Bank link several environmental NGOs alongside the business community in creating a transnational water-policy network; Kohlmorgen et al. (2007) analysis the networks of influence and relationship between southern actors global campaign against HIV/AIDS, tuberculosis and malaria and Oliver's (2012) exploration of power, influence and evidence-base in social networks on policymaking within public health. 


\subsection{Characterizing whole networks}

One immediate issue confronting the analyst is who is in a social network. To identify in advance every actor who participates in a social network is difficult (Wellman and Berkowitz, 1988), as is delineating the boundary of a network. Network research designs can be divided between whole network and personal network forms.

A personal network (often called an ego network) refers to the network immediately surrounding a single actor. A personal network contains a set of focal nodes (ego nodes) and illustrates their ties to others (alters). Each respondent is the centre of his or her network, and the study of personal networks is mainly concerned with the way egos relate to alters or are influenced by them. Belloti (2015), for example, studied the friendship networks of a nonrepresentative sample of 12 male and 11 female single heterosexuals between the ages of 25 and 35 years who lived in Milan. Interviews were focused on the meanings of friendship ties, the resources they offer, the conflicts and constraints involved, and the way they evolve and develop over time. Such person-focused designs are valuable for investigating the social situation of given individuals, but not well suited to the analysis of policy networks.

Whole network designs are more appropriate for policy-oriented research. Such studies examine relationship patterns of a particular kind - control, support, advice - between a defined set of actors. One of the most frequently studied settings for while network research has been the formal organisation, and especially organisational management structures. Common topics include the degree of integration of the organisation, the relationships between autonomy, power and centrality, how patterns of ties influence decisions, and what structures bring competitive advantages. Such studies have found that relationships of friendship, influence and advice can cut across functional, hierarchical and office boundaries so that informal practice may be different from the formal organisational plan, and that such network relationships affect how resources are exchanged and distributed between members (Miller and Dingwall, 1997).

Mainstream SNA studies of this kind tend to involve primarily qualitatively analysis, but there is growing interest in mixed quantitative/qualitative designs. According to Lazega (1997), use of quantitative SNA techniques can add an important missing dimension to qualitative research, but the qualitative component remains equally valuable in preventing the analysis from becoming overly formalistic and divorced from the subjective perspectives of the participants. Lazega (2012: 116) used SNA to provide a visual representation of patterns of dialogue within 
a network of European intellectual property judges, who discuss legal principles, read papers written by other network members and refer to each other's decisions. His study illustrates how a research phase involving analysis of nodes and ties in the network, can be followed by a second phase when behaviour and actor perspectives are examined via qualitative interviews. Lazega's various research studies show how a formal SNA component within this type of mixed-methods approach can contextualise behaviour via description of the network environment, and so provide an analysis that bridges the individual, relational and structural levels (Miller and Dingwall, 1997).

We now turn to discuss three important facets of network analysis and then introduce to the metrics that the method section is planning to use in the analysis: morphology (network and subgroup structure), actor positioning or location within a network, and performance.

\subsubsection{Morphology}

Determining network structure or morphology is one of the primary steps in SNA. As mentioned earlier, determining a network member's position in a relational structure is a way of setting behaviour in its immediate social context. We have mentioned already that networks do not always consist of a single component. Groups of actors interacting with each other to such a degree that they could be regarded as a separate entity are often found embedded in a network (Wasserman and Faust, 2009). In a friendship network, for example, there may exist a group of close friends who socialise together, as well as other actors at the periphery who interact less frequently. At the group level, a network of interacting organisations may have embedded within it a group of organisations subject to common governance arrangements, such as a government department and its parastatals. Relational structures of this type of form building blocks for larger network structures and are known (among other terms) as cohesive groups. A cohesive group is a subgroup within a network where a high percentage of the subgroup's actors share ties that are strong, direct, mutual, frequent and/or positive (Moody and White, 2003). Subgroups within a network may be various described as "components", “cliques,” “blocks,” “core-periphery structures,” and “k-cores”. These subgroups influence the behaviours and perspectives of individuals as well as of the collective and are part of the metrics selected in the methods to evaluate the research questions. 
Cohesion is an important SNA concept, and several techniques are used to measure the degree of cohesiveness of subgroups. Cohesion is by its nature multilevel. It involves mutual ties in dyads (pairs of actors), as well as the embedding of dyads in larger structures, and the roles played by individuals in linking together the larger network. Relational cohesion between dyads is measured in several ways, including the adjacency of nodes, reachability in terms of a path between nodes (fragmentation), and distance between nodes in terms of the number of edges traversed (transitivity). Similarly, cohesion in subgroups can be analysed using multiple techniques, such as summing the results calculated for dyadic cohesion, measuring subgroup graph “density” (in terms of the proportion of dyads that are tied), "transitivity” (in terms of the extent that dyads are connected by an edge), "fragmentation” (in terms of the proportion dyads not able to reach each other) and calculating the average subgroup degree (based on number of ties between a node and other nodes). Prell (2011) cautions that this is a complicated task, so that it is important in subgroup analysis to be clear about the theoretical implications of selecting the chosen measures.

\subsubsection{Positioning}

Several procedures exist to describe the position of actors within a social network. Actors' locations can be described in various ways, for example, by their participation in a clique or cluster or degree of centrality, autonomy, or prestige. These procedures can be used flexibly so that an analysis can move between individual, structural, local and global levels (Miller and Dingwall, 1997). - cohesiveness, while others indicate differences as the shape of the network, such as a strong core-periphery structure or a network split into distinct components.

Measures of centrality aim to determine the structural importance of a node within a network; for example, to identify the most influential actors in a policy network, or the super-spreaders in a disease epidemic. These procedures can be applied to total networks or to network components. A researcher who has collected network data for a whole organisation could elect to calculate centrality scores for nodes at that level, or to do this for particular subgroups, such as organisational departments. This enables the analyst to find well- connected actors, actors who are likely to greatest access to information, or actors best placed to connect with the wider network. A node can be important to a structure in a variety of ways. A bridging node may be important because removing it will disconnect the network into two components, while a node 
with a large number of ties may be a key source of influence within a cluster of network actors. Centrality may also be considered according to the advantages a node receives from where it sits in the network (access to information would be an obvious example). Nodes can have high centrality because they are positioned to catch what is passing between the nodes, or because of their ability to control information flows, either by filtering or modifying messages in a way that is beneficial to the node (Borgatti, 2005).

Centrality is interpreted sociologically in many ways, with various degrees of abstraction. Network centralisation is likely to be associated with hierarchical organisation, in that centrality measures the extent to which activities are dependent upon one or more dominant individuals. A central node may be described as having great power, influence, prestige, prominence and/or autonomy. It may also be described as being a gatekeeper or a leader. Strictly speaking these properties are not inherent properties of centrality, but rather predictions of the consequences that centrality will have, whether for the node itself or for a group in which the node is embedded. Centrality is often used in empirical studies as an independent variable used to predict node outcomes such as health, status, wealth acquisition and satisfaction with life (Prell, 2012). A review of the various measures of centrality is beyond the scope of the present thesis, but five popular measures will now be described.

Degree centrality measures how many adjacent nodes have ties to an actor in a particular network (see Figure 41). Bogatti et al. (2013) regards degree centrality as the most natural form of centrality. As we saw above, the degree of a node is determined by the number of ties it has with other nodes, while taking no account of the value or direction of the ties. Degree centrality measures the involvement of an actor in a network regardless of the influence the actor may have.

Eigenvector centrality expands the idea of degree centrality by calculating values for the ties an actor has with other network actors but taking the degree centrality of those other actors into account. It assigns scores to all the nodes in a network and makes the assumption that a connection to a high scoring node carries more weight than a connection to a low-scoring node (Bonacich and Lloyd, 2001). The basic idea is that an actor connected to a given number of high-scoring actors has a higher centrality score than the same actor if connected to the same number of low-scoring nodes. If, for example, a pupil starts at a new school and wishes to become popular among his or her new peers, one way to achieve this would be to become a friend of the class's most popular student and, in that way, become connected through that most 
popular student to others (Bonacich and Lloyd, 2001). Eigenvector centrality is therefore focused on the value of the scores within a node's local network (Bonacich and Lloyd, 2001). It is possible to take different views of Eigenvector centrality, but many consider it to be a refined version of degree centrality (Borgatti, 1995).

Betweenness centrality quantifies the number of times a node functions as a bridge along the shortest path between two other nodes. It calculates values for all of the ties in a network when calculating a node's score but is concerned with a different aspect of centrality from eigenvector centrality. For example, in a learning network betweenness centrality may be used to determine scores for students who have potential to gain knowledge. A student whose attendance rate is high and is placed between a number of other students with lower attendance rates can choose to withhold or share information obtained from tutorials and has a high centrality score on that basis.

A node's betweenness is zero when it is never along the shortest path between any two other nodes. This can occur when the node is an isolate, or when all other nodes are directly connected to each other. Betweenness reaches its maximum value when the node lies along every shortest path between every pair of other nodes.

Closeness centrality indicates the independence of a node. It is calculated by measuring the average length of the shortest path between the node and all other nodes in the network. The nodes with the shortest sequence of ties to other nodes are those with the most closeness centrality. Briefly, the rationale underpinning this measure is that nodes that are not central will usually have to rely on others to have a message relayed through the network (Borgatti et al., 2013). The degree of closeness centrality a node has indicates how much freedom it possesses to access information easily through any form of network, be it a power network, an influence network, or a communication network (Friedkin, 1981).

Investigating the positional patterns (using centrality) enables the understanding of UHC Nigeria's policy network. The opportunity to visually map the UHC Nigeria policy structure is an issue in research not encountered in other fields of social science. Network analysis provides answers to questions in search of, for instance, are nodes in central positions influential in making a decision, does a more cohesive network encourage affiliations to achieve results, and does a fragmented network spell conflict and contention among subgroups (Robins, Lewis and Wang, 2012). 
Another challenging question of network performance and effectiveness is identifying the relational correlation among two or more network structures. Using the SNA technique called “Quadratic assignment procedure” (QAP) recognises relational elements among pairs of matrices (Bochsler, 2008).

QAP: The "quadratic assignment procedure” (QAP) was coined first by Hubert (1987) for general investigative approach on data but is applied in social network analysis as a statistical analysis to measure the correlation of two matrices (Robins, Lewis and Wang, 2012). The QAP techniques are not explicit to social network analysis and were originally used to explore if the spread of disease was environmentally clustered by the statistician Mantel (1967). The application of QAP in social network analysis evaluates the association between the same set of nodes in two networks. For example, a research question may pursue the association between an organization's informal communication network with formal organizational hierarchy.

According to Krackhardt (1987), QAP can address the above research question on network analysis. For instance, "take two adjacency matrices, y and w, we can produce a statistic of association (e.g., a Pearson correlation coefficient) just by treating the corresponding cells of the two matrices (excluding diagonals) as a data point involving two variables (i.e., $\mathrm{y}_{\mathrm{ij}}$ and $\mathrm{w}_{\mathrm{ij}}$ ) (Robins, Lewis and Wang, 2012: 384).” QAP offers a non-distributional bootstrapping way of analysis to assess the relationship between y and w. The QAP's application in social network analysis extends to both unipartite and bipartite networks structures. Correlation between the two matrices is then calculated the normal way with the researcher given the opportunity to choose an association statistic suitable to the measure of the relationship. within the network. According to Robins, Lewis and Wang (2012), the Pearson correlation suit for valued data than binary data. Furthermore, when the relationship of two matrices is independent of each other, the statistic is consistent with a regular correlation subjected to a standard null hypothesis test, which determines whether there is a connection between two networks or conclude if the value is significantly different from zero. However, self-organizing nature of policy networks mean that there is a form of dependency between network variables (Robins, Lewis and Wang, 2012). The challenge that these questions of network performance and effectiveness present is exciting research subjects for empirical network analysis (Robins, Lewis and Wang, 2012). 


\subsubsection{Performance}

Some SNA studies have investigated the association between network characteristics and organisational performance outcomes (Sandström, 2008; Chambers et al., 2012). For example, Cummings and Cross's (2003) study of a large communications company found that work groups that had strong hierarchies and a clear core-periphery network structure performed less well than those with flat hierarches and a more cohesive shape. Several such studies have aimed to improve performance in the healthcare domain (Aydin et al, 1998; West et al., 1999; Scott et al., 2005).

The idea of interconnectedness is similar to that concept of cohesion discussed above. As we have seen, it is typically investigated using the measures of centrality and density touched upon earlier. We will examine these concepts in more detail, and then go on to consider structural holes, defined as the absence of ties or as the presence of weaker ties within or between networks.

Network centrality and density can be analysed in various ways. Position and patterns of relationships between network actors may be seen as independent variables that shape behaviour and can be measured (Miller and Dingwall, 1997). One approach here is to look at micro-level structures in networks and relate this to organisational outcomes. Some analysts have undertaken a so-called triad census, or census of different configurations of dyads into sets of three actors (triplets), to investigate whether these low-level structures have an impact on how the overall network operates. For example, measuring the proportion of triplets in a network neighbourhood that are closed (i.e., fully connected by three edges), as opposed to only being connected by two edges, is a way of identifying clusters. This section will focus on structural measures that take account of the whole network and consider the degree to which a network remains coherent compared with the degree to which a network breaks apart. A network's cohesion is the degree to which it does not break up into substructures.

Density is assessed by measuring the number of ties in a network as a percentage of the maximum possible number. It amounts to the probable likelihood that a tie will exist between any two nodes chosen at random. In general terms, density counts the number of actual ties a network contains and expresses this as a proportion of the ties that could potentially exist. Higher density scores indicate more dense networks, and therefore the level of interconnectedness (or cohesion) in the network. 
Cohesion measures (such as fragmentation and transitivity) include factors like average distance between pairs of nodes and how many nodes would have to be removed from the network to disconnect a pair chosen at random and the significance of this would be to help measuring the inter-connectedness of small groups within social network settings.

Burt (2000) defines structural holes as the absence of connections or only weak ties in an area of a network. He explains that: "Holes are buffers, like an insulator in an electric circuit. People on either side of a structural hole circulate in different flows of information” (Burt 2000, 353). Those in a position to bridge what would otherwise be separate network components can act as "entrepreneurs" who manage the flow of information and are well-placed to control projects that involve communication between actors on different sides of the "hole" (thus creating entrepreneurial networks). For Burt these bridging actors are rich in social capital and are often able to use their position to influence flows of information and resources. Writers influenced by this social capital perspective, such as Sandström (2008; 69) see structural holes not just as a feature of whole networks, but as something affecting a network's linkage to other networks and its cross-boundary character. The concept of global structural holes is intended to capture the way that policy networks, in particular, are connected to other network constellations and involved in various forms of cross-boundary exchange. Exchange will often be facilitated by actors who are able to act as a bridge between, for example, a domestic policy network and international donor organisations. Sandström (2008: 70) suggests that cross-boundary connections are likely to be more successful when a network contains a diversity of actors from different professions, organisations and sections of society, because this heterogeneity is likely to bring more bridging opportunities.

Sandström (2006) set out to test Burt's predictions about the significance of closure and structural holes by undertaking mixed-method case studies of three Swedish policy networks, centring on a university reform program, the establishment of a research station in a local government region, and a fresh-water fisheries management project managed by an association of fishing rights owners. She hypothesised that a "high performing policy network is a heterogeneous network with a high level of network closure”, and that such a network would be "dense and centralized", but at the same time contain a "diversified set of actors involved in cross-boundary interactions” (Sandström, 2006: 70). The study found that these characteristics could have positive effects, but in different ways. A greater degree of network closure increased the capability to plan and prioritize, thereby enhancing efficiency. On the other hand, 
more heterogeneous networks were better able to mobilize resources from external sources, which improved effectiveness. In Sandström's research SNA was used to measure network characteristics, while an assessment of performance was based on reconstructing the policy stories of the three projects and making a judgement about their degree of success. Indeed, a scoping exercise of studies on network structure and performance in healthcare organisations by Chambers and associates (2012) found only one study that systematically measured a change in practice (involving a quasi-experimental design). Most of the 52 studies reviewed described how SNA was undertaken as a means of assessing performance but reported no follow-up action that might have conclusively shown that shifting towards a more optimal network structure had positive results.

The hypothesis of a strong association between performance and network closure and/or structural holes deserves further investigation but does not at face value appear likely to hold for all network types is all contexts. The two dimensions pull in different directions with the first suggesting the potential value of cohesion, and the second pointing to benefits of a more diffuse network structure with important outward-facing connections. It seems likely that dense, highly centralised networks function well for some purposes, while the strong hierarchies and top-down control that this may involve will do less well when innovation and flexible responses by actors at the periphery of a network are important. Granovetter's (1973) classic paper on "the strength of weak ties" suggests that the latter have valuable bridging functions between a closely-knit group of actors and more distant parts of a social network; they are necessary to ensure that information flows and action can be coordinated for occasional joint projects. A diversity of actors appears to help a network form outward connection with other networks in its environment, but probably involves a network form that is less dense and more dispersed towards its periphery, which will lack the benefits gained by relatively closed networks.

The present study employs SNA mainly for the purposes of describing a policy network and its dynamics, rather than offering any rigorous assessment of the impact of network structure on performance. The intention is to build a picture of network relations that provides a foundation for qualitative investigation of how actors understand and work within the network environment. Given that implementing UHC in Nigeria is an unfinished story and was likely to remain so within the timespan of this research project, it would not have been feasible to utilise a research design that measured performance outcomes in the way that might be possible 
in studies of network structure and success of clinical interventions. Nevertheless, some structural features of the Nigerian UHC policy network appear to have negative or positive impacts on policy development, and this will be discussed in later chapters.

\subsection{Relational perspective of SNA and policy analysis}

Dye (1976: 1) says policy analysis “is finding out what governments do, why they do it, and what differences it makes" while policy network or social network analysis is a wide range of analytic techniques that investigates social interactions within network structures. Both the policy analysis and policy network analysis are two disciplinary fields within the social sciences with the potential for cross-fertilization. The systematic integration of both research methodologies, which are highly relevant to each other, creates the flexibility in addressing the research questions because each research domains covers their weaknesses with their strengths (Kenis and Schneider, 1991). For instance, policy network analysis by itself cannot produce an explicit theory (Collins, 1988) even with its strong affinity to certain policy theories but has the toolset to assess the structural and relational features of a network. In contrast, policy analysis evaluates policymaking process from defining the problems, identifying policy options, implementing policy solutions to evaluating the policy outcomes (Buse, Mays and Walt, 2012).

Using the perspective of Kenis and Schneider (1991), this section looks at three network analysis applications in the study of policymaking:

1. This strategy, i.e., describing and measuring network analysis capacities, can be used for cross-network comparisons to develop (or test) hypotheses which explain the effects of the policy network's aggregate characteristics on specific interactions. This can be achieved by comparisons of cross-national policy networks, or comparisons between different national policy domains or policy processes. The strategy focuses on constructing empirical indicators for measuring network characteristics (for example, density, connectedness, centralisation, asymmetry, fragmentation, etc.) and on building models which relate to the performance of these structural characteristics, or the outcomes which result from specific policy networks. It may be that, in such a hypothesis, the more asymmetrical the exchanged or influenced networks, then the greater the capacity for collective action. 
2. Network analysis can also be used to construct and test formal models on policy making processes. In this research strategy, network analysis is used in the operation of formal models. For example, the model developed by James S. Coleman on exchanged processes within systems of action (Coleman, 1990). Applying a model such as this necessitates a greater amount of information in regard to structural dependencies and resource flows within a set of policy actors. The information can be collected, and the required model indicators constructed, using network analytic tools.

3. Network analysis can also be used to test the hypotheses of theories on policy making which includes structural propositions. For instance, corporatist theory operates on the assumption that specific relational configurations function between large and monopolistic associations, their members, and the state. This contracts with pluralist theory, which assumes different structural arrangements in the policy process. Moreover, governance theories which distinguish between hierarchies and market-like relations feature specific propositions on structural configurations, which can be more precisely identified and described using network analysis. For example, it could be used to determine if an empirical structure of cooperation and information exchange is representative of hierarchical control rather than market coordination. Or, crucially, it could reveal empirical forms of cooperation, including hybrid mixtures of different forms of governance. 


\subsection{Conclusion}

This chapter has outlined a number of theoretical concepts and practical considerations associated with the policy process approach and SNA. Although the two frameworks have different origins and put different degrees of emphasis on qualitative versus quantitative methods, they both point to the importance of actors and their interactions, and the influence of context on courses of action. While the author had been aware of convergence between the two approaches as reflected in the work of Lazega (1997) in utilising SNA to add rigour to qualitative studies, it became apparent from reviewing relevant literature that others too saw value in a synthesis of approaches. Policy process researchers connected with the London group have been incorporating elements of SNA into their recent studies (e.g., Quissell et al., 2018; Shearer et al., 2018), and SNA researchers also see advantages in adding a larger qualitative component (see: Hollstrein, 2016). Therefore, combining the two approaches in a policy study such as the present one seems eminently feasible.

The move towards UHC in Nigeria has extended over many years, and involved different governing administrations, NGOs, and professional and civil society groups. There is a complex interplay between federal, state and local actors, as well as those operating at national and global levels. These actors form an extended and geographically distributed network, which has changed somewhat as UHC was pushed onto the government's policy agenda, and actors have lined up to support or oppose the formulation and implementation of various public health insurance, service delivery and financing reforms introduced with the aim of expanding coverage. Both the structural characteristics of that network, and the subjective perceptions and actions of the actors who populate it, are therefore highly relevant to the UHC policy story and will be explored in the chapters that follow. 


\section{CHAPTER 4}

\section{METHODS}

\subsection{Introduction}

This chapter discusses practical and philosophical issues considered in the design of the study, and goes on to outline the chosen study methods, and selection of location and samples. It outlines the rationale for combining the policy process approach (developed by Walt and Gilson) with elements of Social Network Analysis (SNA) in order to investigate the interactions of elite policy actors involved in developing and debating UHC policy in Nigeria. Ethical issues relevant to the study process are also discussed. In later sections, the sampling strategies and participant recruitment for the two phases of the study - the SNA and qualitative interviews exploring the policy process - are described. This is followed by sections on the data collection process for each phase, how data were analysed, and an explanation of the steps taken to maximise research quality.

\subsection{Outline of the study design}

A well-designed study needs to start with a clear research question and make logical connections between the methods chosen to address that question and the theoretical framework used to make sense of the data (Strauss \& Corbin, 1998). It is also important to ensure that the methods selected, and the type of analysis attempted are compatible with the philosophical assumptions - the ontological and epistemological positions - that guided the study. In seeking to examine how elite actors sought to advance or oppose policies that would bring the Nigeria healthcare system closer to UHC, the author reasoned that subjective perceptions and ongoing constructions of the meaning and significance of particular policy initiatives would be crucially important, and that a predominantly qualitative approach would be needed to get "inside the black box" of the policy process and understand its dynamics. Moreover, the author felt that utilisation of such a qualitative approach would fit with the traditional theoretical framework of interpretive or interactionist sociology from which the approach had emerged (see: Miller and Dingwall, 1997), so that the evolution of policy could be seen as a constructed reality that emerges from social interactions between the key actors involved. However, the author was also mindful that a qualitative interview study that sought 
to explore the dynamics and meaning of policy from the perspectives of multiple actors might not tell us as much as we would like to know about the overall shape of the policy network, the nature and frequency of contacts between the various actors and organisations that make it up, or the way they divide on various issues. For that reason, SNA appeared to the author to provide a valuable additional dimension that could combine with qualitative interviews to provide a more comprehensive understanding of the UHC policy process. In the author's view this combination of methods retains epistemological coherence because both approaches sit within a broadly "realist" position, and more specifically the position of "subtle realism" adopted by a number of qualitative researchers (Hammersley, 1989; Murphy et al., 1998; Pope and Mays, 2000). The constructivism of this study may be seen as a "soft" constructivism that admits the existence of an external world of interests and political manoeuvring and is fully compatible with a SNA that maps patterns of relationships between the actors who are of interest. Thus, in line with the literature discussed in Chapter 2, the chosen study design involved a mixed method approach that sought to understand interactions between key policy actors through the twin lenses of SNA and in-depth qualitative interviews.

\subsection{Research objectives}

The main aim of this study is to examine and elucidate the policy drive to push the Nigerian healthcare system towards achieving UHC. As explained in the previous chapter, the study relies on a combination of SNA and a policy process approach. Using the former it aims to describe the network of actors and organisations that shapes UHC policy, while the latter is utilised to follow the life of the policy through the stages of policy formulation and implementation, paying close attention to who the key actors are at different stages of the process and how they interact. This combined approach aims to elucidate the characteristic features of the UHC policy process, and to identify factors that advance or impede progress towards UHC policy objectives. 


\subsection{Mixed-methods research design}

Sekaran (2009: 4-5) defines research as, “a multi-step process and an organised, systemic, databased, critical, objective, scientific inquiry or investigation into a particular problem undertaken with the purpose of finding answers or solutions to it”. Here, the main objective is to solve past and present problems and to share the knowledge of their solutions (Bassey, 1999; Sekaran, 2009; Saunders, 2012). As was stated earlier, this study sets out to understand the challenges and obstacles that stand in the way of moving towards UHC in Nigeria. The main aim of this study is to explore how policy was developed, advanced, and sometimes slowed in debates between elite actors.

To achieve this, a mixed-methods approach was chosen as the most appropriate approach by which to identify key policy actors, the social networks in which they operate, and their perspectives on, and interpretations of emerging policies of these experiences (Saks and Allsop, 2013). This is because it provides researchers with the means to explore research participants' subjective points of view, while locating those perspectives within wider social structures or patterns. Although mixed method studies typically involve a combination of quantitative and qualitative approaches, it is worth noting that studies that combine different qualitative methods, such as observation and interviews are also common. The present study combines qualitative interviews with SNA (that takes a largely but not wholly quantitative form), but the inspiration for combining these two approaches comes from the work of Lazaga (1997), who sees this approach as a way of adapting a broadly qualitative approach to import greater formality and rigour into some aspects of the analysis.

Mixed-methods research has its critics, who question the epistemological consistency of many such studies, often making the related point that quantitative and qualitative approaches represent two separate paradigms (Morse, 2003; Bryman, 2012). The underlying philosophical assumptions of each method must be carefully addressed to avoid epistemological incoherence (Morse 2003). Additionally, researchers face the risk of making the mistake of using one approach which might overshadow the other throughout the study (Dunning, 2007; Rohlfing, 2008). Finally, a researcher can be criticised for poor understanding and application of a particular method or using one method more effectively than others (Bennett, 2007; Lohmann, 2007). Bryman (2006b) cautions that the use of a mixed-methods approach should not be considered necessarily superior to single method studies. Despite such criticism, mixedmethods studies are becoming increasingly commonplace, largely because of the claimed 
benefits of triangulation or the broadening of the focus of single studies (Denzin, 1978; Tashakkori and Teddlie, 1998; Morse, 2003).

\section{Classifying mixed methods research in terms of priority and sequence}

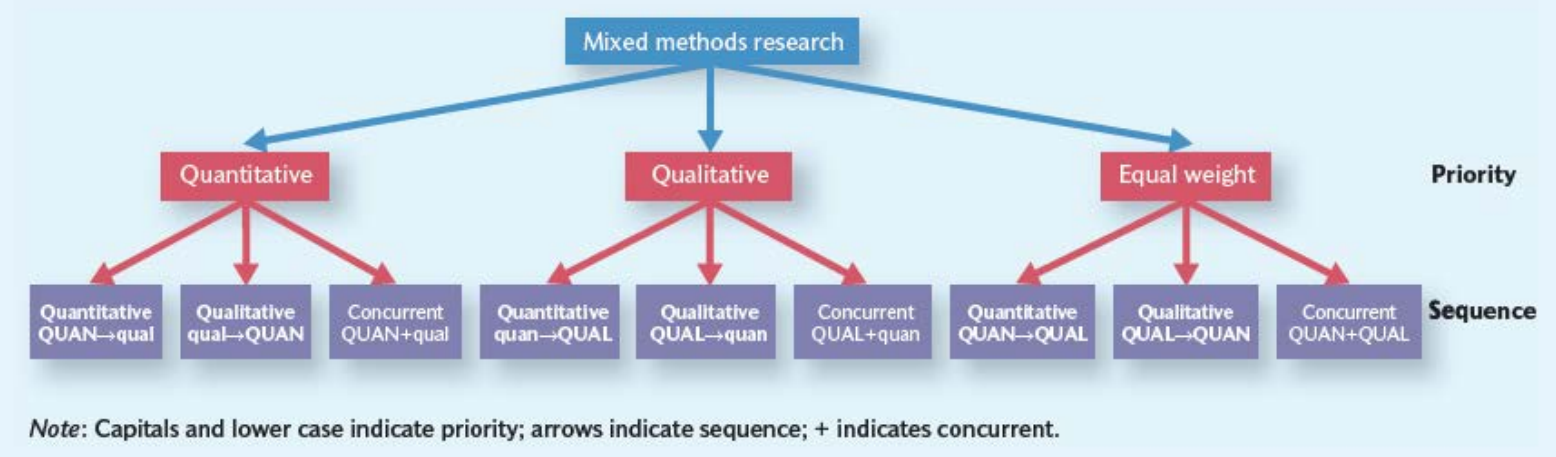

Figure 21: Classification of mixed-methods research.

\section{Source: Bryman (2012: 632).}

Figure 21 shows nine possible classifications which originate from Morgan's (1998) framework. In the diagram, the upper-case word indicates priority, and the lower case indicates the secondary method. For example, QUAL $\rightarrow$ quan indicates that the qualitative component was the main data-collection approach, and the quantitative approach played a more subsidiary role.

Morgan's classification provides a useful indication of the range of mixed-methods study, though as Bryman (2012) observes it may not be easy in practice to establish what the priority and sequence were in a particular study.

\section{Mixed Medtods} Research
Equal weight
Concurrent

Figure 22: Study with equally weighted, combination of quantitative and qualitative methods.

Figure 22 shows where the present study fits into Morgan’s (1998) taxonomy, falling into the seventh QUAN $\rightarrow$ QUAL category. The research uses quantitative SNA analysis to map the terrain for a second stage of in-depth qualitative interviews, both by identifying respondents 
and generating information about the relevant policy network that would help to guide the content of the qualitative interviews.

The QUAN $\rightarrow$ QUAL category is similar to the approach Lazega (1992) (to which the thesis adopts) where he uses SNA to map out the relationships and flows of advice between colleagues in a New England corporate law firm. Lazega (1992) identified an influence network encompassing 71 lawyers in three offices - 36 partners and 35 associates. For the quantitative SNA phase of the study, he distributed questionnaires to partners and associates to map the network and generate quantitative data on relationships, which was later complemented with qualitative interviews that explored network members' perspectives in greater depth. This is essentially the approach that is employed in the present study of the actor network involved in the development of UHC policy in Nigeria.

Overall, the present study seeks to achieve a balance between approaches by combining quantitative data collected using SNA techniques and thematic analysis of qualitative face-face interview data with the same actors. This type of combined approach remains fairly unusual and involves a sequential research design where the application of the quantitative method comes first, before the use of the qualitative method. The two phases are separate, but the second step depends on the outcome of the first step and presents a logical extension of the findings of the first phase (Tashakkori and Teddlie 1998, Morse 2003: 199, Tashakkori and Teddlie 2003).

Furthermore, the point of interface between quantitative and qualitative methods, and how each method serves to the operationalization of the research questions is understood in two directions. Firstly, the SNA analysis focuses on the section of the research questions, which involves revealing policy actors participating in UHC Nigeria discourse; identifying key policy actors within the UHC policy; understanding the relational and interactive pattern between policy actors inside the discourse; differentiating the role of contending actors from actors in support of existing issues and expanding on existing disagreement within the UHC discourse. Secondly, the answers from the first direction generates will enough meat for the policy process analysis to pursue the remaining research question, in which this analysis evaluates, the factors leading to UHC Nigeria initiation, the faces of power exercised in UHC policy making, and the challenges and obstacles facing UHC Nigeria's implementation. Finally, these actions are set to provide significant empirical evidence capable of offering the obstacles and challenges affecting the move towards UHC healthcare coverage in Nigeria. 


\subsection{Quantitative approach}

Wassermann and Faust (1994) state that the use of social network analysis (SNA) as a quantitative approach is an effective way to put the interactions between actors into context through a descriptive and inductive modelling of the relational and structural study of the social environment. Carrington (2014) also suggest that "SNA is fundamentally neither qualitative nor quantitative, nor a combination of the two. Rather, it is structural” (Carrington 2014: 35). The mathematics behind social network analysis - graph theory - does not represent "quantities” but "structures" (Carrington 2014: 35), as in patterns that emerge from interweaving ties (Bellotti, 2015). However, some authors applied the SNA as a quantitative approach to evaluate mathematical, statistical and graphical demonstration of their research outcomes (Noorale, Sale, Marin and Ross, 2018; Freeman, 2004). For example, Neergaard, Shaw and Carter (2005) research utilised quantitative network analysis to present key concerning gender bias in small firm network. Most notably, Gorard (2010: 238) criticized the current norm of defining quantitative research as involving numbers, and qualitative as involving everything else (e.g., text and discourse, images, observations, recordings, etc.).”

Contextualization for the purpose of this section is divided into substantive and methodological dimensions. Here the substantive aspect means recognising some of the limitations put on some individual's behaviour and the specific roles proposed to them, while the methodological dimension compares and generalises findings. Therefore, SNA needs accurate data, such as an interaction between people in a social setting, and this data is analysed using established SNA techniques and usually visually represented in graphs.

The thesis seeks to understand the development of UHC in the context of Nigerian policy making and the patterns of relations between the various stakeholder groups involved. In their volume on "context” in qualitative research, Miller and Dingwall (1997) argue that SNA can provide a strong foundation for subsequent qualitative interviews. Social network analysis is defined as the investigation of the interrelationships between a set of individuals within a particular social boundary (Miller and Dingwall, 1997). Hence, analytically the relationships formed between actors has precedence over actor characteristics. Researchers start with relationships and structures, before they consider the characteristics of actors or actors' behaviour, in order to make sense of the structures emerging for the analysis, to explain the emergence of a particular structure, or to explain the observed behaviour as a function of actors' 
position in the network. The main point is that researchers start with data on relations, adding information on attributes and additional behaviour only at a second stage. The main contribution of this method to theory-building is its capacity to contextualize behaviour by describing relational structures in a way that bridges the individual, relational and structural levels of analysis. The use of network analysis requires two additional but important preliminary tasks: identifying the boundaries of the field of action under examination and mapping out the relationships used to constitute the informal social structure of the setting (Miller and Dingwall, 1997).

With regard to boundaries, rigid delimitations of a social setting are always arbitrary, but network analysis offers systematic ways of defining and redefining the field of action. Usually, social systems do not have clear boundaries, and there is flexibility in SNA to allow researchers to define temporary boundaries after exploration of the process of boundary definition by the members themselves. Two comments can be made regarding the definition of the network relationships involved. First, the description of the relational structure by identification of subsets (cliques or positions) in networks is a way of identifying inductively actors present at a specific moment in a social setting, as well as the relationships or exchanges between them. Second, the description of these relations, or absences of relations, between blocks identifies a system of interdependences between these network actors. The analysis thus sheds light on underlying regulatory mechanisms (Prell, 2013).

\subsection{Qualitative approach}

The second qualitative phase of the study is intended to explore the nature and meaning of interactions between actors in the UHC policy network in more depth. Popay (1992: 100) states that the qualitative approach "explores the meaning people attach to their experiences and identifies and describes the social structures and processes that shape these meanings”. Indepth interviews can produce “thick description” (Geertz, 1973) of events and relationships. Geertz (1973) explains that thick description allows the unravelling of social actions embedded within social norms and ideologies that bring meaning to social behaviour. Bryman (1988:61) suggests that the thick description is "seeing through the eye of the people you are studying". The focus of this research is to provide a contextual understanding of UHC policy processes 
from a policy actor's perspective. This will elucidate the dynamics of the UHC policy process in Nigeria and may reveal patterns that also appear in similar policy networks in other developing countries. Because qualitative studies seek to answer "what", "how” and "why" research questions, the approach is well suited to exploring the health policy making process which is the focus of this study (Silverman, 2001; 2004).

The approach used in the qualitative interviews was tailored to answer what, how and why questions of Nigeria's UHC policy process. As is conventional in qualitative studies, the author planned to use thematic analysis to identify patterns in the interview data and make connections, because the study would need to link to a large existing health policy literature on the policy process and other countries' experiences of implementing UHC.

\begin{tabular}{|c|c|c|c|c|}
\hline \multicolumn{5}{|c|}{ Qualitaive data analysis } \\
\hline $\begin{array}{l}\text { Dimensions of Theme-Identification } \\
\text { Techniques }\end{array}$ & Nature of semi - structured qualitative interviews & Type of Techniques & Theme-Identification Techniques & $\begin{array}{l}\text { Rich } \\
\text { narratives }\end{array}$ \\
\hline Type of data sets & Textmal data & Scrutiny and processing trechniques & $\begin{array}{l}\text { Repetitions; transitions; similarities and dfferences; and } \\
\text { outting and sorting }\end{array}$ & Easy \\
\hline Needed proficiency & Fluent in English Langange & Scrutiny and processing techniques & $\begin{array}{l}\text { Reptitions; similaities and dfferences; and culting and } \\
\text { sorting techniques }\end{array}$ & Easy \\
\hline Fxpected labor & Manual (eyeballing) and time consuming & Scrutiny technique & Repetitions and transitions & Easy \\
\hline $\begin{array}{l}\text { Quantity and quality of themes } \\
\text { required for generation }\end{array}$ & $\begin{array}{l}\text { Identifying number of new themes, subthemes, and } \\
\text { metrathemes. }\end{array}$ & Processing trechniques & Cutting and sarting & Easy \\
\hline Concerns of retiability and validity & \multicolumn{4}{|c|}{$\begin{array}{l}\text { The rediability and validity of the identified themes were a demonstration of maximum darity, judgment } \\
\text { and agreement on the part of the researcher and supervisor }\end{array}$} \\
\hline
\end{tabular}

Table 5: Methods for identifying themes from semi-structured qualitive interviews.

Bryman and Burgess (1994) point out that the criteria employed in the identification of new themes or assessing the relevance of themes identified in past research, are often unclear. The current study is influenced by the framework developed by Ryan and Bernard (2003). This aim is to identify, examine and report the themes and patterns which recur in the data. This technique is used for organising and describing the information source as well as for interpreting the various elements of the research findings. There is no single correct method by which to find themes, but under some conditions certain techniques have greater effectiveness. Ryan and Bernard (2003) evaluate eleven methods on the five dimensions of: (1) type of datasets, (2) proficiency required, (3) expected labour, (4) quantity of themes required for generation, and (5) validity and reliability concerns. These methods are based on use of two sets of techniques (scrutiny and processing techniques), which can be utilised in different combinations according to the characteristics of particular studies to generate themes. Figure 23 suggests how to choose from the various theme identification techniques. 


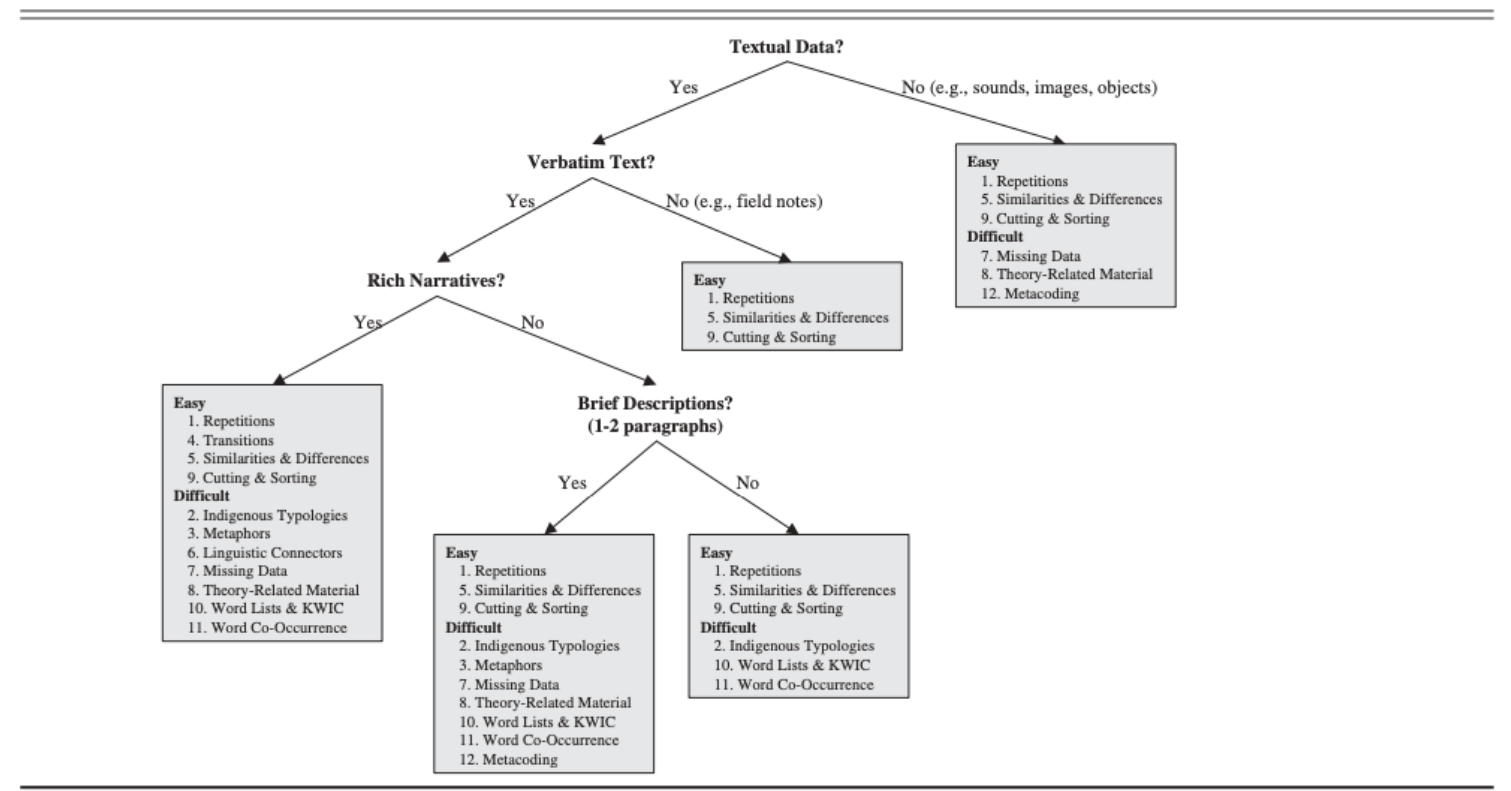

NOTE: KWIC $=$ key words in context

\section{Figure 23: Ways of classifying themes.}

Source: Ryan and Bernard (2003: 102).

Table 5 incorporates part of this framework to illustrate the application of the study dimensions and techniques in a study like the present one that relies on semi-structured qualitative interviews. Looking at Table 5, similarities, differences, and repetitions, and as well as cutting and sorting techniques, are the techniques best suited to discovering themes in a study of this kind. Section 4.13 described how these analytical phases were applied in greater detail. This approach can be employed in regard to several different techniques for analysing qualitative data, such as grounded theory, narrative analysis, critical discourse analysis, and qualitative content analysis. It has also been employed in relation to the systematic review of qualitative research (Thomas and Harden, 2008). 


\subsection{Justification for combining SNA and qualitative interviews}

As mentioned earlier, a common criticism of mixed methods designs is that they combine methods rooted in different epistemological traditions. Development of the ethnographic origin of SNA occurred in the 1920s primarily through collaboration between anthropologists and mathematicians; significant contributors included Jacob L. Moreno, Helen Jennings, and associates (in the 1920s and 30s), Kurt Lewin (in the 1940s), Max Gluckman (in the 1950s), Clyde Mitchell/Thomas Kuhn (in the 1960s), Harrison White (in the 1970s), with recent contributions from Barry Wellman and Patrick Doreian (Otte and Rousseau, 2002).

Categorising SNA as a theory or a metaphor has become a divisive discourse over the years. Earlier theorists used SNA analysis to capture the relevant connections and structural features of social networks (Wellman, 1988). Modern scholars have disputed this stance and offered a well-developed SNA which consists of theoretical concepts such as structural holes (Burt, 1992), centrality (Freeman, 1978), the strength of ties (Granovetter, 1973), and structural equivalence (Lorrain \& White, 1971).

Some have made the discourse surrounding the concept of SNA research ambiguous. Many scholars consider SNA a quantitative method (Crossley \& Edwards, 2016; Hollstein, 2014) and other researchers have suggested that it is qualitative and to view it as quantitative is a complete divergence from the historic origins of SNA (Freeman, 2004).

Although SNA has developed from an ethnographic approach and followed a path different from a qualitative approach influenced by Chicago sociology and anthropology, the quantification that it involves does not imply any assumptions about determinism or causality that contradict the idea of human agency and the emergence of meanings in social interaction.

Hammersley (1999: 73) argues that decisions about what methods to utilise "should depend on the nature of what we are trying to describe, on the likely accuracy of our descriptions, on our purposes...not on our ideological commitment to one methodological paradigm or another”. Sarantakos (2005: 48) suggests that the differences between methods should be seen in context as differences rather than deficiencies. He concludes that arguing that the mixed method approach is deficient based on the differences in its epistemological and ontological deficiencies is like "arguing that ships are not an effective means of transport because they cannot fly, and airplanes are not efficient because they cannot cruise through the sea..." As 
was shown in Chapter 3 a growing number of writers from SNA, the policy process approach, and interactionist sociology see the combination of network analysis and qualitative interviews as a feasible and legitimate way forward, and the author does not believe that this poses major epistemological difficulties.

The study involved two phases of data collection. Phase one involved structured SNA interviews with policy actors. Because this exercise set out to identify the network participants before analysing the nature of the network, it consisted of two rounds of structured interviews. Phase two involved semi-structured qualitative interviews with the same group of individuals. The following section discuss ethical considerations, location of interviews, sampling, data collection, data analysis, and "relevance" in relation to the study.

\subsection{Ethical considerations}

Research governance provides a regulatory framework of principles, requirements and standards of excellence of research activity undertaken in the United Kingdom (DOH, 2005, 2008). These standards offer public reassurance that all research activity is guided by rigorous ethical principles. It is incumbent on all those involved in research nationally and internationally to ensure that all studies receive ethical scrutiny (WMA, 1964). This includes assessing the risks and benefits for anyone participating in research studies, assurances of participant consent, and ongoing governance arrangements for monitoring of studies ( $\mathrm{DOH}$, 2011). The author applied both for ethical approval from the Ethics Committee of the College of Human and Health Sciences (ECCHHS) in Swansea University, and relevant organisations in Nigeria for access and confirmation of ethical approval to conduct the study. Subsequently, the ECCHHS granted ethical approval and written and oral permission for research access was obtained from the relevant Nigerian health organizations (Appendix 2). The latter accepted the ethical approval granted by the ECCHHS as sufficient so that no additional ethical approval in Nigeria was needed.

With regard to access consent the general procedure used was to gain consent at the organisational level, and then one by one from individuals who were identified as target participants. Those approached all agreed to receive details of the study. All participants had the opportunity to ask questions and be given more information about the study throughout their contact with the author after reading a participant information sheet (Appendix 4), and 
before signing a consent form (Appendix 5). The author emphasized to participants that they could opt in or out of the study at any time without giving a reason. Written consent was only sought from study participants once they had been given full information about what their involvement in the study would involve. Participants in this study held top professional and managerial positions within their respective organisations and directorates. Consequently, once they had the participant information sheet and the opportunity to discuss any aspect of the study, the author's view was that they were fully capable of making an informed decision to participate. Before commencing interviews, the student again made a point of asking if consent was still forthcoming and emphasizing that involvement in the study was entirely voluntary. The author had previously emphasized to participants when making presentations about the study and when clarifying participant involvement that he would rather they did not take part in the study if they felt pressured to do so.

Ethical issues must be considered in any research study (Hammersley \& Atkinson, 2007). The social research process has the potential to create conflict between the aim of research and the rights of participants to maintain privacy and confidentiality (Orb et al., 2000). Ethics pertains to doing well and avoiding harm (Hammersley \& Atkinson, 2007). Harm can be prevented or reduced through the application of appropriate ethical principles. Therefore, the protection of participants in any social research study is imperative. The author paid careful attention to specific ethical issues posed by this study, in particular informed consent, the right to maintain privacy, and the right to retain confidentiality (Hammersley \& Atkinson, 2007), which are discussed in the following sections.

It was not envisaged that participants would be at risk of significant harm. The student did however consider that if there was a need for debriefing following any difficult interactions that occurred during interviews, then it would be right to halt and if necessary, end the interview. Another consideration was that participation in interviews was often outside individuals' working hours, on some occasions before or following office hours. The student gave some thought to the possibility of rescheduling interviews should this clash with participants' work duties.

In Nigeria this field of study is mostly located at the head offices of the participants, which are mostly in urban areas in Abuja the state capital and other states in Nigeria, particularly Lagos state. As work offices were the respondents' usual working environment it was the obvious 
place for the interviews to take place, and these were generally convenient and safe places as far as the researcher was concerned. None of the participants were known to the researcher prior to the study.

Assurances of participant anonymity and confidentiality were given for both phases of the study. The risk of breach of confidentiality was carefully considered. All participants were provided with clear information in English (written in English on the Participant Information Sheet) about the confidential nature of this study. In interviews this issue of trust centred on the relationship between the participant and the researcher. Many participants knew of others in the study because snowball sampling had been used to generate an initial list of names. The researcher therefore started each interview in the first phase by discussing ground-rules which included issues of anonymity and confidentiality, and this was repeated again with the second phase interviews. The researcher first clarified the purpose of the study, which was to gather information from elite policy actors with various experiences of the UHC debate in Nigeria and reviewed the overall aims of the study.

The researcher told all participants that they would be given a pseudonym attached to their specific interview or transcript. It was explained that all study documents and transcripts would remain securely stored in a locked cabinet. For the duration of the study, all electronic data were kept on a personal computer located in a university post-graduate research room allocated to the student and password protected. Apart from storage on a PC hard drive, one backup was made and stored on a USB drive kept in a locked cupboard. Regular back up updates were performed.

\subsection{Sampling}

Elite policy actors are pivotal in shaping policy in health care sectors or policy in general. At the onset of the study the researchers were aware that policy actors from government institutions, interest groups, non-governmental organizations (NGOs) were all involved in policy debates about UHC. However, the immediate question to answer was how to go about identifying the network of key policy actors shaping the UHC debate in Nigeria.

According to Prell (2012), it is essential to identify your population in other to get an idea of your network boundary. However, in SNA the notions of population and sampling are often 
complicated as they are connected to the concept of a network boundary. Network boundary is regarded as the line that encircles the social environment made up of the individuals involved in the network of interest. Ascertaining the network boundary is equal to identifying the individuals from a study population. Unfortunately, no ideal solution exists for determining a network boundary, and this is an issue that network analysts have yet to fully resolve (Granovetter, 1976; Knox et al., 2006). Statisticians still debate how best to model social networks when the population or sample is not fully known (David and Snijders, 2002).

The best current practice is to use various known techniques for approximating what the boundary might be. Borgatti et al. (2013) suggests three approaches that a researcher can employ to define the boundary of a network: the realist option, the nominalist option and the snowballing option. In the realist approach the analyst turns to the actors themselves to define their network, but this raises the problem of knowing which actors to approach in advance of knowing who is in the network. In the nominalist approach it is the analyst who defines the network, usually by using a list of employees, documents or media reports to identify key names. The third snowballing approach combines elements of the other two; the researcher identifies key actors who are known to be involved in the area of interest and then asks them to identify others in their network, whom the researcher then approaches. Since there is no perfect solution to network sampling, a pragmatic approach seemed to be to select organizations, departments and individuals on the basis of their apparent involvement in UHC policy making, and then seek to add to this by "snowballing". This resembled the purposive sampling used in much mainstream qualitative research.

Social network data is often incomplete, with some actors or links missing from the dataset. In a whole network social setting, most of this incompleteness emerges from the following sources: the so-called boundary specification problem (Laumann et al., 1983); respondent inaccuracy (Bernard et al., 1984; Brewer and Webster, 1999; Marsden, 1990; Butts, 2003); non-responses in network data collection (Stork and Richards, 1992; Rumsey, 1993; Robins et al., 2004); or via study design (Burt, 1987). Although missing data often occurs in empirical studies, there has been little research which has examined the possible effects of missing links or nodes on the measurable properties of networks. This section aims to highlight the problem of missing data in social network analysis.

One way to approach this is to develop analytic techniques that capture global statistical tendencies, and which do not rely on individual interactions (Rapoport and Horvath, 1961). A 
complementary strategy is to develop remedial techniques that minimise the effect of missing data (Holland and Leinhard, 1973; Robins et al., 2004). Although a definitive statistical treatment is not offered in this paper, we conduct exploratory analyses which are clearly acknowledged and discussed in the methodological section (Costenbader and Valente, 2003). Non-responsive participants are an important limitation of the research.

In a standard sampling situation, for example when drawing a representative sample from a population, special techniques are available to correct parameter estimates for imperfect response rates (Little and Rubin, 2002). The refusal of some participants to reply adds to the limits of the network representation. From the description it seems that the network represents only a partial section of the policy network - that which was reachable by the candidate. This section utilizes Kossinets (2006) 'actor non-response’ principal of missing data mechanisms to discuss the effect of miss data in social network analysis

Actor non-response principal: an examination of these mechanisms was performed in regard to their impact on random bipartite graphs. The results of the simulation reveal that actor nonresponse is an important issue within the study, as it appears from the data collection that network representation is missing a partial section of the policy network.

A crucial issue within network survey research is that of non-responses to the survey. In a standard sampling situation, such as drawing a representative sample from a population, special techniques are available to correct parameter estimates for imperfect response rates (Little and Rubin, 2002). However, there is no such definitive treatment for social network analysis, although the effects of non-responsive participants on some network properties have been previously described (Stork and Richards, 1992; Rumsey, 1993).

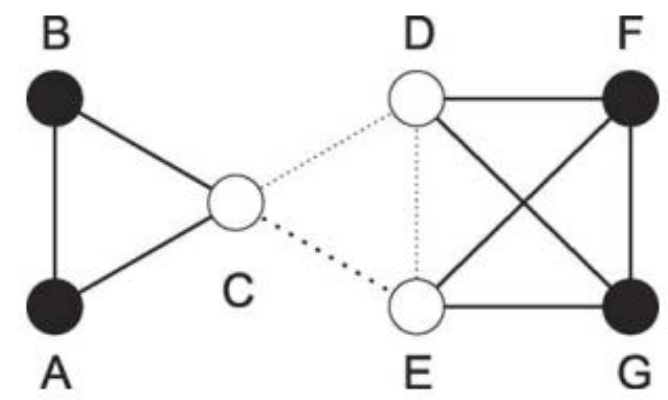

Figure 24: A non-response survey in network. Source: Kossinets (2005) 
While single-mode networks with non-respondents are amenable to statistical treatment (Robins et al., 2004), non-response in networks with multiple interaction contexts (modelled as bipartite graphs) have other particular implications. When collecting data of an affiliation network, the survey asks actors to name peers with whom they interact (that is, ignoring the multiplexity of ties); this allows the non-response effect to be balanced out by reciprocal nominations (Stork and Richards, 1992), for instance, if actor C failed to fill in the network questionnaire (Figure 24). Yet, C's interactants who participated in the survey (A and B) must have reported their interactions with $C$. It is therefore expected that if the number of nonrespondents is small relative to the size of the network, and the researcher does not require all nominations to be reciprocated (as a crude validity check), then the quantity of missing data as a result of non-response should be negligible, or at least small (Kossinets, 2006).

\subsection{Participant selection}

Due to the practical and financial limitations that apply to a single-researcher doctoral project, it was not feasible to interview all the policy actors involved in policy discussions about UHC across the country. However, it was envisaged that a full range of organisations could be included, with a sample of actors from these organisations then being interviewed. As explained above, the elite policy actors involved in this study were first identified using the nominalist approach, and the sample later expanded using the snowballing approach. It was felt that using the nominalist approach alone might potentially omit other important actors involved in UHC policy discussions in Nigeria (Wassermann and Faust, 1994). Thus, to get a better picture of the network as the actors themselves saw it, an element of snowballing was added. Additionally, a contact known to the researcher from Management Science of Health (MSH), who works on UHC implementation in Nigeria, also helped by pointing to possible omissions.

As a first step the researcher prepared a candidate list of actors and organisations, which on a basis of a reading of media reports and documents about UHC in Nigeria appeared likely to be centrally involved in the policy process. It was not anticipated that all these candidates would be accessible for interviews or would necessarily have great influence, but this was seen as the pool of potential network participants to be investigated. 
1. Rockefeller Foundation representative

2. World Bank Group country representative

3. World Health Organization representative

4. International Labour Organization representative

5. Management Sciences for Health Nigeria representative

6. USAID representative

7. Health for All campaign

8. Chief Executive NHIS

9. Health in Africa initiative

10. Minister of Health

11. Minister of Finance

12. Executive Governors

13. Health state commissioners

14. Traditional rulers

15. Hospital managers

16. NHIS board and management

17. Head of Health Workers Association

18. PharmAccess Foundation

19. State Ministry of Health officials

20. Federal Ministry of Health officials

21. 12-member technical group for UHC

22. Permanent Secretary $\mathrm{MoH}$

23. Nigerian Medical Association

24. Senate Committee on Health

25. Health Maintenance Organizations representative

These actors and organisations listed above come from various sectors and strata of Nigerian society, and for the purposes of the study the researcher categorised them in terms of four groupings of:

- Government, including both the federal and state levels and the parastatals

- Labour, including professional organizations and trade unions

- Health Sector, including the NHIS, the public and private hospitals and the HMOs

- International Bodies, including UN organizations and international NGOs. 
The researcher reasoned that the final mix of network participants selected for SNA should include a reasonable balance of all these categories. However, for various practical reasons, including lack of personal contacts and problems of circumventing gatekeepers, the researcher felt unable to proceed to approach all the above actors/organisations immediately, and instead opted to start the study by interviewing a relatively accessible subset of actors who could be asked for their views of who the key policy actors were. To address the research questions, it was necessary for the researcher to gather the views both of these who supported the UHC policy and those who opposed it. This would be a snowballing exercise which would generate a more precise list of names and a better idea of feasible channels of communication to request participation.

In consultation with the key informant from Management Science of Health the researcher identified 25 persons with known involvement in UHC policy discussions, and who included both proponents and opponents of the policy, who would be approached to help generate a list of influential policy network members. Because of the researcher's lack of easy access to international bodies from within Nigeria, and limited contacts at the governmental level, the group for the snowballing exercise was skewed towards the Health Sector and Labour groups. However, it was intended that the final selection of participants included in SNA would be spread more evenly across the categories.

The 25 approached for the round one snowballing interview comprised:

- Government: one senior official from the federal $\mathrm{MoH}$ and one from a state $\mathrm{MoH}$ (n = 2)

- Labour: senior representatives from the medical (1), dental (1) and nursing (3) professions and two from community health organizations $(n=7)$

- Health sector: four senior officers from NHIS, six senior managers from HMOs, five managers from private sector providers or provider associations $(n=16)$.

One candidate (from an association of private sector organisations) declined to participate, but the remaining 24 were interviewed. They were given the list of candidate actors/organisations mentioned previously as possibilities and asked for names of particular influential actors involved in UHC from these or other backgrounds, which the researcher then wrote onto a prepared sheet. The list was intended as an aide memoire of possibilities rather than an exhaustive set of categories so that it was possible for novel names or positions to be written in. 
Interestingly this insider group indicated that several individuals or groups on the nominal list of candidates were not as centrally involved in UHC policy as the researcher had supposed. They suggested that other senior officials had greater hands-on involvement than the Minister or Permanent Secretary in the $\mathrm{MoH}$, or the Minister of Finance. No member of the Senate committee on Health was named as a key actor, and no traditional rulers were named. Representatives from the Rockefeller Foundation, the International Labour Organisation, Management Sciences for Health, the Health for All Campaign, the Health in African initiative, or the PharmAccess Foundation featured on the expanded list of 67 names produced.

Where snowballing is used, the process ideally continues until names start to repeat, and the researcher feels he or she has saturated the possible list of nominations within the network boundary (Bryman, 2008). Bearing in mind the limited resources of a doctoral study, the researcher's initial view had been that it was impractical to include many more than about 40 actors, with around 10 in each of the four chosen categories (Government, Health Sector, Labour and International Bodies). However, the expanded list appeared to include highly relevant persons and so the researcher decided to go ahead with a larger sample than had been tentatively planned and approach all 67 candidate network members.

According to critics of the snowballing approach, its main disadvantage is that the sample is biased by the referring actors (Scott, 2000). Hence, who the researcher approaches first for additional names may determine who is deemed to fall within the network. Individual actors may have their own inner circles, which may be different from the acquaintance circles of other key actors. Knoke and Kuklinski (1982) propose viewing the network as dividing into a number of zones where each sample collected through snowballing is formed out of the first set of nominees put forward until something approximating a full circle of network members emerges, but this was not possible with the resources available in the present study. What was done instead was to include key actors even where this involved more interviews than initially planned.

The list from snowballing comprised 67 candidates from eighteen policy organisations who might take part in stage two SNA interviews. When these were approached 6 stated they were not in fact involved in UHC policy and so were not included, and a further 14 declined to take part. Of these 14, three were from UN bodies, five from Health Maintenance Organisations, and six were from the Labour sector, including one who had held a senior position in the NMA. The 24 policy actors who had participated in snowballing all agreed to complete second round 
SNA interviews and were joined by an additional 23 consenting participants identified from snowballing. Therefore 47 respondents took part in second stage structured interviews concerned with SNA data.

The list of the 47 participants and their roles is shown in Table 7 in the next chapter (see page 153). The breakdown of actors in terms of the four categories mentioned earlier was: Government 12, Health Sector 16, International Bodies 9, and Labour 10. Thus, a reasonable balance was achieved between what appeared at the onset to be key stakeholder groups.

\subsection{The Interview Method}

The present study is primarily an interview study, and so it is worth discussing this method in some detail. Interviews are interactions between an interviewer and a respondent, completed with the purpose of gathering information on a specific set of topics (Harrell \& Bradley, 2009). Interviews are a common method for collecting primary data in scientific studies. First, interviews are an ideal way to collect information from individuals about their own practices, beliefs, or opinions, and they also can be used to gather information on past or present behaviours or experiences (Warren \& Karner, 2005). Second, the method of the interview is suitable for gathering deep, nuanced, and complex data because it allows the interviewees to describe their perspectives in their own words with minimal distortion arising from the researcher's assumptions or the structure of the questions (Britten, 2000).

According to how much control the interviewer will have over the interaction, interviews can be categorised as unstructured, semi-structured, and structured interviews. There are benefits to each of these approaches.

The structured interview exerts considerable control over the content of answers. In structured interviews, the questions are fixed, and they are asked in a specific order (Harrell \& Bradley, 2009). These interviews are often used in quantitative research, and when the researcher has a very large sample and is looking for data that can be generalised to a large population (Harrell \& Bradley, 2009). A face-to-face structured interview is a type of interview in which the interviewer asks a particular set of predetermined questions that have been planned and created in advance. This means that all respondents are asked the same questions in the same order. Structured interviews are also known as standardized interviews, patterned interviews, planned interviews and formal interviews. Since in structured interviews all the respondents 
are asked the same questions, it is easy to quantify and compare their answers. Finally, this type of one-size-fits-all interview can seem a bit cold and impersonal, making it harder to generate rapport between researcher and respondent.

In qualitative unstructured interviews, the researcher has a clear plan and set of topics, but minimal control over how the respondent answers (Harrell \& Bradley, 2009). Collecting information in this manner might lead to very rich and nuanced data, but the process of interviewing, transcribing and analysis can be more time consuming than analysis of closedend structured questions (Harrell \& Bradley, 2009). Usually an interview guide is used, which lists general questions and topics that must be covered. However, this only provides a loose structure, so that most questions are open ended, and additional questions can be added to those on the outline guide. This enables the researcher or the participant to diverge from a standard question in order to pursue an idea or response in more detail (Britten, 2000). Semi-structured interviews allow interviewees freedom to respond in their own way and describe events or concepts in their own words (Morse \& Field, 1996). Using an outline interviewing guide in a flexible way can help not only to cover the necessary questions but also to encourage participants to tell their stories in their interviews (Bryman, 2001).

Hence, the method of semi-structured interviews can help the researcher to delve deeply into a topic and get respondents to develop their answers at greater length. The researcher can use follow-up questions to get respondents to clarify ambiguous statements (Kvale, 1996; Mason, 2002), and a series of linked questions can draw out accounts of specific situations and action sequences (Kvale, 1996). Therefore, the method of face-to-face semi-structured interviews was considered suitable for data collection in this study because it seemed a well-tried way of exploring and understanding the interactions between elite policy makers.

\subsection{Data collection}

As explained earlier, the first phase of the research utilised a social network analysis to provide a "visualisation" of interactions with the UHC policy network, and the nature of the policy process was explored further in second phase qualitative interviews. Additional analysis of relevant documents concerned with UHC policy was undertaken to examine the origins and evolution of Nigerian policy. 
The first phase of data collection took place in Nigeria between March and June 2016. The second phase of data collection commenced immediately after the first and ended in February 2017.

\subsubsection{Phase One}

As explained above, two rounds of SNA interviews were necessary because the researcher had first to identify who was in the network, before then asking that full set of participants about the nature of the network and the ties between them. The first round was designed to generate a list of key UHC policy actors, but also collected some basic background information on the round one informant. The second-round SNA interviews were administered to network members who had been identified in the first-round interviews and agreed to participate. The questions were grouped into six sections concerned with different relationships of the interactions between network actors and were intended to allow the mapping out of the flow of information between them and the nature of the ties between them. These six sets of questions related to six relationships of influence within the network that were examined in the subsequent analysis.

\subsubsection{Phase Two}

Phase two of the data collection involved the use of the semi-structured qualitative interviews to flesh out the meaning that participants attached to their dealings with other network actors and their perspectives on UHC policy. All 47 first-stage SNA participants completed a secondstage semi-structured interview lasting approximately 45 minutes to an hour. This was mostly at a location acceptable to the participants. However, due to visa problems and the need to return to the UK, the researcher ended up conducting the last few interviews by telephone, again though moving through a similar set of loosely framed questions.

The interview guide used was designed according to the research questions of this study and themes derived from the literature review. It was further developed and refined in the light of experience of the early interviews. Over time some questions were changed or added based on emerging themes that arose during preliminary and ongoing analysis and reflection. For example, it became evident that some topics initially included in the topic guide were not deemed important by the respondents and were subsequently dropped. Other topics were 
identified as important and therefore subsequently included. In addition, using a semistructured topic guide allowed for flexibility in the style and order of the questions, allowing topics to be further explored and expanded upon based upon interviewees’ responses (Britten, 2006; Denscombe, 2007). Topic guides were adapted to be slightly different for the interviews which depended on the position of the interviewee (e.g., Government, UN Bodies/External Government Agencies/NGO, Health sector and Labour) (see Appendix 3- Phase two).

With the permission of the participants, each interview was audio recorded using a digital recorder. All interviews were recorded in the English language and the data were transcribed manually on paper and subsequently typed up into text files on your PC. This approach allows the researcher to use direct quotations from the interviewee when presenting the findings and increases confidence that the analysis is based on what respondents actually said.

\subsection{Document review}

Alongside the interviews, documentary analysis was used to provide background, especially on the evolution of UHC policy in Nigeria. This was useful in filling gaps in interview accounts and understanding some of the events or developments mentioned.

Documentary sources can provide information about previous social events and reflect the social behaviours that emerge in a given political, economic or socio-cultural context (Prior 2003). Data from documents can be applied either directly or indirectly, to answer research questions or for the purpose of authentication of what informants say in interviews (Mason 1996; Patton 2002). Such documents usually take the form of written texts collated by an organization or individual for specific purposes (Mason 1996). Many documents are relevant to health policy research, for example papers, articles, official papers, statistical databases and the Internet (Buse, Mays, and Walt 2005). Researchers should not overlook how documents select and represent the information they present (Patton 2002). Hence, a document can be defined as a social artefact that can take many different forms.

In this study documentary analysis was used as a complementary source of data collection to examine relevant documents relating to UHC policy. Official documents relating to UHC policy in Nigeria and the policy process practice in particular were investigated, including government/state documents and reports, regulations and legislations, and another archival 
document. Several documents were gathered from various government/UN agencies mainly the National Health Insurance Scheme (NHIS), Ministry of Health (both Federal/State), UN Bodies and Health and the Planning Secretariats. Both qualitative and quantitative data have been gathered through these sources. The qualitative data have been used to describe Nigeria's path towards achieving UHC through the interaction between actors during the policy process stages. However, studies on the subject of the health policy process in general and in Nigeria are very limited, and published data associated with the topic are also rarely available or easily accessible. Thus, it is worth mentioning that most of the secondary data used in this study were obtained through direct contact with different sources during the two fieldwork periods in Nigeria. Furthermore, every health research must follow ethical procedures and must also appear ethical otherwise views such as political correctness or moral qualities may prevent its approval from peers or society at large (Johnsson et al., 2014). Researchers soliciting documents need to be conscious of their obligation to protect the respondent involved in their research. When the researcher uses documents contained in public archives, then much of the bureaucracy of governance and ethics is redundant. As the documents are in the public domain, they are freely available and the problem of protecting sources does not arise.

\subsection{Analysing the SNA data}

Overall, the data collection included three rounds of interviews gathered in two phases, where the first phase involved two rounds of face-to-face structured SNA interviews, and the second phase involved a third round of semi-structured qualitative interviews.

Only limited analysis was needed for the data gathered from the 24 participants involved in the first round of face-to-face structured SNA interviews, because these were completed primarily to identify further network members (via snowball sampling). There was an identity check and a confirmation of their consent to participate in the study, and some questions on sociodemographic information and personal views about UHC. Data analysis for this first round of SNA interviews took the form of simple descriptive statistics on characteristics, but as this group did not comprise the final network this was used only as a check on the likely demography and balance of the final sample. The main purpose of this round was to expand the pool of candidate network members for the next stage. 47 selected network members participated in the second round of the face-to-face structured interviews. The data collected 
were intended to allow an analysis of the network structure. Again, the researcher interviewed respondents face-to-face and guided them through the structured interview.

\section{Box 1}

Read Relationship - involves those that have read any published work to get ideas from the following network member.

Discussion Relationship - involves those that have taken part and have face-to-face meetings in a UHC discussion forum with any of the following network member.

Coalition Relationship - involves those that have formed a coalition with any of the following persons within the policy network.

Affiliation Relationship - involves those that have followed network members, outside what we might call your coalition of close associates share the same views within the network boundary.

Conflict Relationship - involves those that have followed network members, outside what we might call your coalition of close associates have a conflict of interests within the network boundary.

Advice Relationship - involves those that have taken advice from any of the following network member.

\section{Box 1: Six Relationships of the policy network}

The analysis examined the policy network and the nature of ties between network members. Ties were investigated according to six relationships, comprising the read relationship (read a named actor's work), the discussion relationship (discussed issues with named actor), the coalition relationship (aligned with named actor), the affiliation relationship (attachment to named actor), the conflict relationship (past conflict with named actor) and the advice relationship (took advice from named actor).

A social setting may have key actors positioned in different sections of the network boundary; some actors are located at the centre, others are at the periphery and a few are isolated (Borgatti, 2004). The analysis sought to identify the shape of networks for each of the six relationships using appropriate social network measures that reveal the network structure. Identifying key sub-networks is a step needed to assess the impact of degree of network cohesion and relational patterns on the UHC policy process. Several previous SNA studies have proposed ways of 
identifying the behavioural patterns of cohesive subgroups within their social environment, but there is no single comprehensive model that provides understanding of social and organisational processes (Borgatti, 2013). The chosen SNA analysis examined the six relationships of the policy network taking account of the network structural characteristics.

Structural characteristics: This aspect of SNA evaluates the structural component of the policy network in four stages using SNA techniques to create a taxonomic tree (see Figure 50). The four stages are as follows.

Connectedness stage: This stage evaluates the network size of each policy network and the ratio of connected to unconnected ties within each network. It uses structural measures such as network sampling and it measures the total number of nodes involved in the network boundary (active and inactive nodes), in addition to network density (Moody and White, 2003).

According to Faust (2006), network size, density, transitivity and fragmentation are fundamental network measures used for identifying the distribution of dyadic properties. Network density is the number of ties in each policy network divided by the potential number of ties. For instance, when each respondent's average number of ties is precisely quantifiable, then the total number of ties increases with the network size. If the network size is raised by the power of two, then the potential number of ties increases. The assumption is that the density of each policy network may show the possibility of heavily constrained dyadic distributions. Some dyadic properties have low probabilities, simply because of the density of the policy network (Faust, 2006). Transitivity states that the extent to which two interacting policy actors in the policy network are connected by an edge. Transitivity of an interaction means when there is a link between actor $\mathrm{A}$ to actor $\mathrm{B}$, and actor $\mathrm{B}$ to actor $\mathrm{C}$, there is a link between actor $\mathrm{A}$ to actor C- a friend of my friend is a friend. Fragmentation is the proportion of pairs of nodes that cannot reach each other. For instance, Fragmentation is how shattered the policy network looks when a couple of policy actors are removed from the network.

The problem with using network size, density, transitivity and fragmentation to examine the policy networks is their limited value for identifying cohesive subgroups. The next section covers the issue of cohesion. 


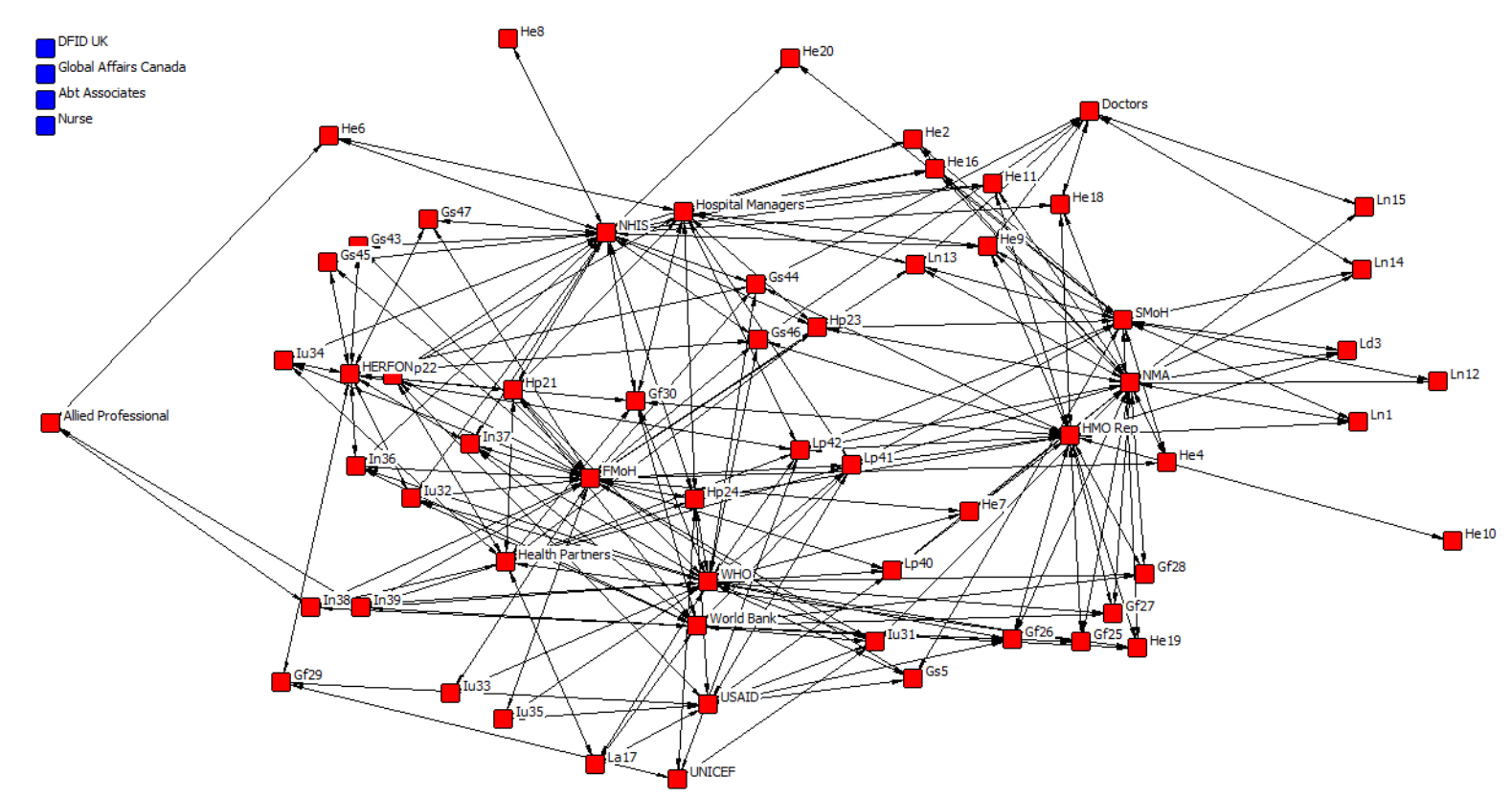

Figure 25: Component analysis of the "advice” relationship of the policy network.

Component of policy network stage: The previous stage evaluates the properties of potential connections and the structural boundary in each policy network. This stage focuses on deconstructing each policy network by progressively removing parts to find the relevant subgroups and examining not just the respondents and their interactions, but the entirety of the network subgraphs. Measures of the cohesiveness of network components are applied at this stage to tackle the issue of cohesion mentioned at the end of the connectedness stage. For instance, Figure 25 gives an example where portions of the policy network are disconnected from each other, where $\mathrm{x}$ represents nodes red and, y represents nodes in blue. Cohesion is a multilevel idea that evaluates all six relationships of the policy network in search of components that are disconnected from one another (Moody and White, 2003).

Subgroups within the network stage: Unlike the previous stage which explores the number of existing components within the subgraphs of all six policy networks, this stage focuses on areas within the suggested components where the actors are structurally related to each other more than they are to the others in the rest of the network (Alvarez-Hamelin et al., 2005). Identifying the policy actors of the cohesive subgroups within a network is a significant issue in social network analysis. Cohesive subgroups are a small group of actors connected with similar tie characteristics (Moody and Coleman, 2015). This section uses cohesive measures such as K- 
core, cliques, and core-periphery in order to find the cohesive subgroups of actors within all six relationships of the policy network.

Key institutions and organisations in policy process stage: Not all individuals are equally important in social networks. Key nodes exist to link a focal unit to other areas within the organisation (power actors), as well as to areas outside of the organisation (gatekeepers and policy actors connected to the power actors). Individuals can also be uncoupled from the rest of the network (isolates). Centrality describes an actor's relative position within the context of his/her social network. Centrality measures have been applied in a diverse range of research studies (Ortiz-Arroyo, 2010). For instance, centrality measures have been applied to investigate influence within inter-organisational networks, the power or competence in organisations, to analyse the structure of terrorist and criminal networks, to analyse employment opportunities and many other areas (Berlusconi et al., 2016). The ability that centrality measures have to determine the relative position of a node within a network has been used in previous research to discover the key players in social networks.

Degree centrality (including indegree and outdegree centrality), betweenness, closeness and eigenvector centrality are among the most popular social network measures used in this stage. These measures focus on discovering a set of key players in the policy network involved in the UHC dialogue in Nigeria. A simple example in Figure 41 illustrates the possible taxonomy outcome of the research analysis on the data collected from the second round of interviews.

This section has presented a brief summary of methods that can be used to identify key actors in the UHC policy network. The development of the taxonomy tree involved an iterative process, where the structural patterns presented in the policy network informed the questions about their content and meaning. The results of this more qualitative aspect of SNA were used to interpret the data, and also to enhance and amend the visualisations. The act of visualising and narrating the networks usually prompts a justification of their changing nature over time. Thus, we are not simply counting the number of ties in each relationship of the policy network but rather, we are trying to understand the dynamic meaning and intensity of the relationships among the policy actors in Nigeria’s UHC discourse (Ryan, 2016). This was then taken a step further in the qualitative interviews. 


\subsection{Analysing the qualitative interviews}

All of the 47 first-stage SNA participants participated in a second-stage semi-structured interview which lasted for between 45 minutes and one hour. Thematic analysis was used to organise data by identifying patterns, and for analysing and reporting themes. This is an inductive approach allowing accessibility and, in theory, flexibility, and can be especially relevant to researchers early in the qualitative research process (Braun \& Clarke, 2006).

Because of this method's flexibility, researchers are able to use their own subjective assumptions (Bristowe et al., 2015) as long as what they are doing, and how and why, is transparent (Attride-Stirling, 2001). Finally, Vaismoradi et al. (2013) have stated that this approach is reliable enough to be used to conduct an introductory study on a novel phenomenon, where the data depends on the quantities of energy and time the researcher must expend on the data collection and analytic process. The raw data consisted of the interview transcripts. As DeSantis and Ugarriza (2000) indicate, the thematic analysis involves searching for, and identifying, commonalities across a set of interviews.

Analysing text consists of several aspects: (1) discovering themes and subthemes, (2) whittling these themes down to a manageable number (i.e., deciding on which themes are important), (3) constructing hierarchies of themes or code books, and (4) linking the themes to theoretical models (Ryan and Bernard, 2003).

This process was iterative, rather than linear with constant moves back and forth between the entire dataset, the coded transcript extracts, the emerging themes/categories and the relevant notes, in line with the usual qualitative analysis processes (Vaismoradi et al., 2013). Although this is not a grounded theory study as such this aspect of the analysis is similar to Glaser and Strauss's (1967) constant comparative method in that the themes identified in transcribed interviews are compared and elaborated as analysis proceeds. Themes emerging from earlier interviews sometimes led to more emphasis being given to those topics in subsequent interviews (Boeije, 2002).

Three scrutiny techniques (things to look for in texts) are described, as is one processing technique, concerned with how to process texts into rich narratives for the qualitative analysis section. These four techniques are applied in different combinations (see Figure 6). 


\begin{tabular}{|c|c|c|c|c|}
\hline \multicolumn{5}{|c|}{ Theme-Idenifization Texthiques } \\
\hline \multicolumn{3}{|c|}{ Scrutiny tethique } & Processing tectninues & \multirow{2}{*}{ Final theme } \\
\hline Repectitions & Transitions & Simbrities and Differences & Cuting and sorting (subthemes) & \\
\hline 'FMaH' & \multirow{3}{*}{ 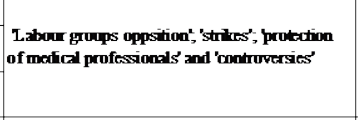 } & \multirow{3}{*}{ 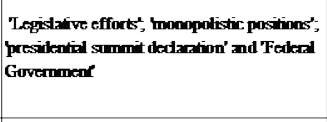 } & \multirow{3}{*}{ 'Potitizal power' } & \multirow{15}{*}{$\begin{array}{l}\text { Pawer and potites in the } \\
\text { UHC policy netwott' }\end{array}$} \\
\hline 'SMaH' & & & & \\
\hline 'csos' & & & & \\
\hline 'Feikenal Governmene & & 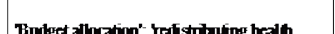 & & \\
\hline UN Bodies' & 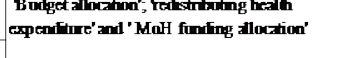 & $\begin{array}{l}\text { expenifines,', MaH funding allocation', } \\
\text { Fealeral Govermment' and 'FMaH' }\end{array}$ & 'Financial power' & \\
\hline USAIIT & & & & \\
\hline 'พно' & \multirow{3}{*}{$\begin{array}{l}\text { 'Legistative efforts, 'monopolistic positions' and } \\
\text { 'presidential summit dedartion' }\end{array}$} & \multirow{3}{*}{ 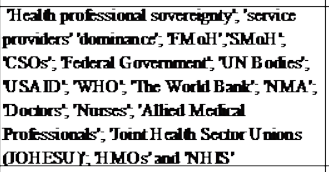 } & \multirow{3}{*}{ 'Professional power' } & \\
\hline 'The World Bank' & & & & \\
\hline 'NMA' & & & & \\
\hline 'Doctors' & \multirow{3}{*}{$\begin{array}{l}\text { Forcign healith experts', program transitions' } \\
\text { and 'tecthica assistanci' }\end{array}$} & \multirow{3}{*}{ 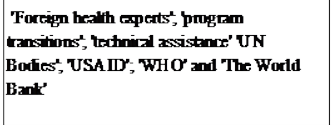 } & \multirow{3}{*}{ 'Power throngh trechniral expertise' } & \\
\hline 'Nurses' & & & & \\
\hline 'Alled Medical Professionals' & & & & \\
\hline 'Shint Health Sextor Unions (IOHESU)' & \multirow{3}{*}{$\begin{array}{l}\text { Health professional sovereignty', 'service } \\
\text { providers' and 'dominance' }\end{array}$} & \multirow{3}{*}{ 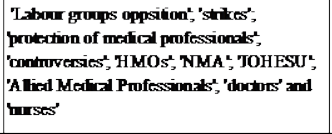 } & \multirow{3}{*}{ 'Power in numbers (vin formation of coalitions)' } & \\
\hline FMOs' & & & & \\
\hline 'NH $\mathbf{s}$ & & & & \\
\hline
\end{tabular}

Table 6: Example of coding techniques used in the analysis.

\subsubsection{Identifying themes in interview transcripts}

This usually involves scrolling through a number of texts and marking relevant passages. This may be done manually by, for example use of different colour fonts or appended code numbers, or electronically by attaching codes to text segments. Bogdan and Biklen (1982) suggest reading the text over at least twice, while Sandelowski (1995) suggests analysing the texts by proofreading the material and underlining key phrases "because they make some as yet undeveloped sense”. The three scrutiny techniques alert the analyst to features to look for.

Repetition: is an easy way to identify themes. For example, in semi-structured interviews with respondents from the labour sector, it was found that the participants repeatedly referred to ideas linked to the mismanagement of health funds, insufficient healthcare spending, selfindulgence and misappropriation. The conclusion was that these ideas were important themes and were displayed alongside other ideas linked to the obstacles and challenges which affect the process of the implementation of UHC in Nigeria. The more the same concept occurs within a text, the more likely it is to be a theme. However, the number of repetitions required to constitute an important theme is an open question which only the investigator can decide upon (Ryan and Bernard, 2003). 
Transitions: refer to naturally occurring content shifts in interview talk may be theme markers. In these semi structured interviews, the conversation moves from one topic to another, which creates transitions. Analysing the features of conversation and discourse, such as the structure of turns at talk in the interview (including, for instance, speaker interruptions, repairs or formulations of gist) can identify these transitions (Silverman, 1993).

Similarities and differences: comparison involves looking at similarities and differences in the presentation of a topic by different respondents or different topics by the same respondent. Careful attention to how a given respondent presents particular issues can help the researcher build themes empirically from the data rather than basing them on prior theoretical assumptions (Charmaz, 2000).

Processing technique: alongside the three scrutiny techniques analysis involves use of a processing technique. This technique is informal and involves organising the textual data from interviews into manageable categories. Traditionally it meant spreading out transcripts on the floor and making a start on marking themes and relevant sections, while in the computer age it may involve entering text files into a qualitative data package or sorting passages from transcripts into different MS Word file folders.

Cutting and sorting: After the initial sifting and marking of transcripts, cutting and sorting involves the identification of relevant quotations and then arranging them into piles of items which go together. However, this technique has many variations. For example, traditionalists who prefer manual analysis might cut out relevant quotes (ensuring that some of the context in which it occurred remains) and paste the passage onto a small index card. On the reverse of each of the cards a note might be made of who said it and where it appeared in the text. Next, the cards are laid out on a table and sorted into piles of similar quotes. Each pile is then named. These are the themes. As the first exploratory data analysis stage, investigators are more concerned with identifying the largest range of themes possible. In later steps, they identify which themes are the most important and require further analysis (Ryan and Bernard, 2003). In recent times many will prefer to automate this process by using a computer application, such as NVivo, to undertake a series of similar steps electronically.

Additionally, this processing phase may also assist in provisionally identifying findings (see Figure 26). The objective here is to present the themes and findings in a concise and logical 
manner. Suitable data extracts can be selected and presented to illustrate the points which the research is demonstrating, without unnecessary complexity.

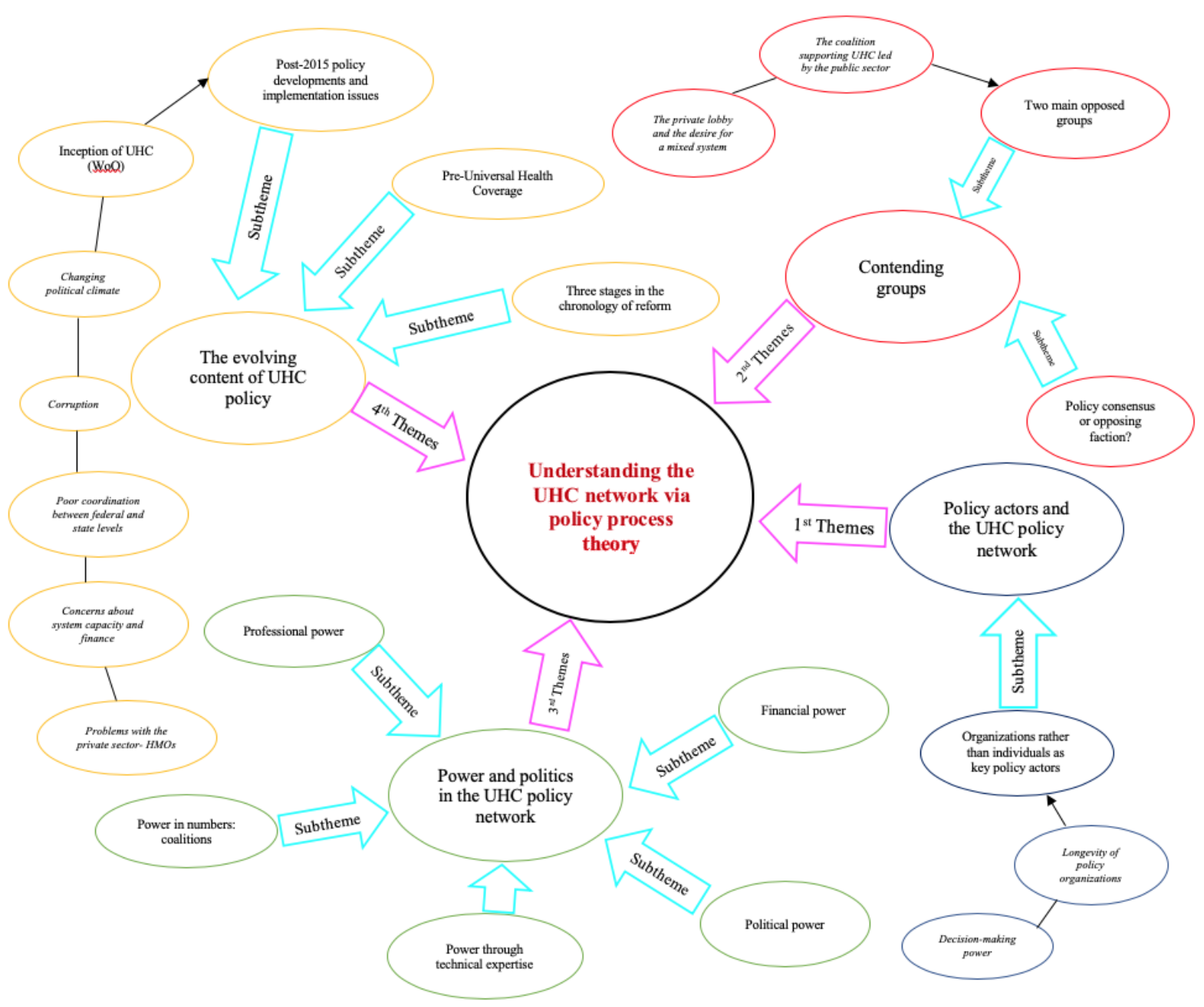

Figure 26: Using thematic analysis to map out findings.

\subsection{Data management}

All the interviews were audio taped and transcribed. Being personally involved in the interviewing process aided in transcription because of the benefit of knowing who is speaking at any particular time. Field notes were taken during the interviews and after each interview a post-interview synopsis was written. This helped during the transcriptions to recreate the research environment and the picture of the events. A journal of all the documents that were found was kept and also a diary of significant events contacts and encounters 


\subsection{Reflexivity}

Reflexivity is concerned with the researcher's awareness of his/her own influence on each stage of the study as it develops (Guillemin \& Gillam, 2004). Reflexivity is more than mere reflection. It involves consideration of every aspect of researcher's interpretive activity beginning with the study rationale and research questions, and continuing with choices about methodology, sampling, data collection methods, approach towards data analysis, and the way the research is documented and disseminated (Denzin, 2008). Reflexivity is concerned with "epistemological accountability" in terms of explaining how researchers have come to their research decisions (Hand, 2003). This involves consideration of the mindfulness by which researchers are able look back more immediately on their personal thoughts and feelings and how these may have affected the research (Corbin \& Strauss, 2008). Walker Read and Priest (2013) advocate that researchers take account of the concept of reflexivity because it is impossible to remove the researcher from the research process or the findings of the study. The concept of reflexivity acknowledges that the orientations of researchers are shaped by their socio-historical locations, including the values and interests that these locations confer on them (Kvale, 2002). Mruck and Mey (2007) explain reflexivity as active acknowledgement by the researcher that her/his own actions and decisions will inevitably impact upon the meaning and context of the action under investigation. Reflexivity requires researchers to improve their awareness of personal prejudices, to reflect on actions taken, their roles and emerging understandings, whilst engaged in the research process (Hammersley and Atkinson 2007). Reflexivity is therefore not an activity that occurs at one point in time, but it is a process that is carried out throughout the entire research process (Ortlipp, 2008).

To improve his reflexive awareness throughout this study, the student maintained a reflexive diary, which recorded his reflexive insights, and the ways in which the study findings will have been influenced by his personal sociocultural background, world view, subjective experiences, and pre-understandings (Nadin and Cassell, 2006). The diary also recorded some analytical memos, and unexpected incidents which happened during the study. The reflexive diary helped the student because it provided an outline of his thinking and reminded him of what had achieved so far and what was still required. The student drew on notes from the diary to look back on some key decision made, and these provided a kind of audit trail for readers. One example of reflexive diary written after participants' interviews are provided in Appendix 12. Moreover, the use of reflexivity also aided the researcher in clarifying his views regarding insider or "domestic" and outsider or "foreign” perspectives on the Nigerian health care system. 
In terms of a study of UHC the status of the researcher as a person living in Nigeria and familiar with the domestic healthcare system and way of life or a person coming from overseas and seeing Nigeria as yet another "foreign" healthcare system worthy of study through a comparative lens, makes a considerable difference to the kind of research produced. Dwyer and Buckle (2009: 60) observe that some researchers occupy "the space between", in that they do not fit easily into either the insider versus outsider, or domestic versus foreign researcher categories. Moreover, these authors suggest that the researcher role itself tends to accentuate ambiguity about which status applies: "We may be closer to the insider position or closer to the outsider position, but because our perspective is shaped by our position as a researcher (which includes having read much literature on the research topic), we cannot fully occupy one or the other of those positions” (Dwyer \& Buckle, 2009: 61).

That was the case for the author, who has a Nigerian background, but had lived away from the country for more than eight years in the UK. He could be seen as having a "outsider" perspective because he had become unfamiliar with the way the Nigerian health system works, and yet still had many connections to Nigerian culture and social norms. Thus, in his case the boundaries between insider and outsider status were blurred and are not as clear cut as the wider literature suggests. This sense of being, at least in part, a stranger to the system had a considerable impact of the experience of fieldwork, and the interpretation of the findings.

\subsection{Ensuring rigor}

The study sought to address the issue of rigour mainly through the use of triangulation. Triangulation is the utilisation of "two or more sources to achieve a comprehensive picture of a fixed point of reference” (Padgett, 2008: 186). Drisko (1997) also points out that qualitative researchers are capable of achieving "completeness" or an exhaustive response to the research question through collecting data from multiple sources (data triangulation) or using multiple analysts to review the data (observer triangulation). To ensure that the outcome of this research was an appropriate and adequate representation of the evidence available, the selected data collection methods were applied in a rigorous manner, as has been illustrated. Critics of mixedmethods research argue that data from exploratory studies are often messy, non-methodical and that the researcher can most often end up seeing what he wants to see through some form of data massaging (Robson, 2002). In the present study three triangulation strategies were applied 
to the data both in collection and analysis to maximise robustness. The triangulation strategies used were:

- Method triangulation. Findings of the study were checked for consistency with the different data collection methods of SNA, qualitative interviews, and documentary evidence.

- Source triangulation. Data sources were collected from policy actors within four different institutions comprising Government, international actors, the health sector and Labour. This helped to clarify meaning and to corroborate observations and interpretations.

- Perspective triangulation. Different theoretical perspectives are used to analyze and interpret the data. This included in particular Walt's policy process approach and SNA techniques.

One of the main criticisms of mixed method research is that sample size is usually small. This study attempted to identify a policy network made up of key actors involved in the UHC policy, by first using a purposive selection of policy actors and then using a snowballing technique to mark out the network boundary so as to include the most important actors. These key policy actors represented the study respondents who were later interviewed face-to-face. As all 47 who completed phase 1 also participated in phase 2, this seemed to the researcher to meet the requirements of ensuring representativeness. The strategy used in arriving at the sample for this study has been rigorous and logical. Persons from each institution were given the opportunity to be included in the study. The result has a good spread of demographic characteristics, including different levels of experience, exposure and clout in the UHC debate. It is also worth mentioning that the data obtained from qualitative interviews are used to increase our insight into social phenomena rather than demonstrate statistical representativeness in any strict sense. The logic is similar to that of the case study where the objective is to produce theoretical generalisations that can be tested in further case studies, rather than to demonstrate statistical generalisation from the study sample to a wider population, or in this case to UHC policy making outside Nigeria (Mitchell, 1983; Yin, 2017).

In the view of Marshall (1990), if a researcher desires to give value and trust to mixed method research, then the following should be done: the research design and methods must be explicitly detailed; the research questions and relevance of data must be rigorously argued and explicit; and the study should be situated in a scholarly context. The methodology as presented has 
demonstrated a robust qualitative strategy that fulfilled the Marshall's criteria and enabled the collection of adequate and appropriate data to achieve the research objective. This notwithstanding the researcher concedes that there are limitations of the study and these are acknowledged in a later chapter of the thesis.

\subsection{Relevance}

Hammersley (1992: 73) identifies two core aspects of relevance, namely “importance of topic” and "contribution to the literature". An evaluation can be made in terms of the importance of the studied topic to a substantive field and its relevance to wider societal values and contexts. Moreover, the relevance of a research is judged by its contribution to the literature. Hammersley (1992: 73) stresses that "research that confirms what is already known well is of little value.” A study must constitute a significant addition to what is established knowledge, either through empirical findings or building theory (Lofland, 1974, Athens, 1984). If the findings and analytical insights are found to be meaningful and have potential to fit situations outside of the study, then a contribution to theoretical development can be made.

Therefore, the research can be judged to be relevant or irrelevant "not only in terms of its relation to some topic of interest but also on the basis of its exemplification of some methodological or theoretical paradigm” (Hammersley, 1992: 73). The student agrees that the relevance of this study will be established if the findings fit with other situations outside of the study, or if readers judge the findings to be meaningful and credible (Sandelowski, 1986). Therefore, the author has attempted to provide sufficient information in regard to the locations and settings of the study whilst maintaining participant confidentiality so that readers can judge the transferability of the findings.

\subsection{Summary}

This chapter has discussed issues involved in combining the methods of SNA and qualitative interviews, and the approaches taken to study design, data collection and data analysis. The next chapter presents findings from the first - SNA - phase of the study. 


\section{CHAPTER 5}

\section{ANALYZING THE UHC POLICY NETWORK}

\subsection{Introduction}

This chapter presents the main findings of the SNA component of the study. It describes key features of the network in which UHC policy is debated, formed and contested, and presents visualisations of aspects relevant to the analysis. It presents the taxonomy for classifying stakeholder groups implicated in the policy discourse on UHC and maps out relationships and cross-cutting patterns of influence.

The characteristics of the UHC policy network were examined by applying various standard SNA measures such as size, density, transitivity, fragmentation, component analysis, k-core, core-periphery measure, cut-points/blocks and centrality, together with a qualitative narrative for each relationship. A four-stage social network analysis (comprising a connectedness stage, component of policy network stage, subgroups within network stage, and key institutions and organisations in policy process stage) was used to examine the degree of interaction between actors and their position as core or peripheral participants in networks or isolates with little network involvement. Actors were classified according to their institutional affiliations as governmental, health sector, international body or labour organisation participants, so that the network relations between these sectors could be examined. The analysis investigated the nature of network relationships by measuring respondents' structured interview responses regarding six relationships of interpersonal contact and influence: reading another network actor's written products, discussing UHC policy with that actor, entering a coalition with them, being formally affiliated with them, taking advice from them, or being in conflict with them. These will be referred to in the chapter as the network relationships or the modalities of engagement. The relationships may overlap and should not be interpreted in an overly rigid way in determining whether an individual is a core or peripheral member of a network; it is the overall combination of relational relationships that determines position in the network (see Bellotti, 2015). This part of the SNA ends with a taxonomy of the four stages of the analysis, summarising how it has examined network connectedness, network components, network subgroups and key network institutions and organisations network connectedness.

The chapter concludes with a qualitative narrative that builds on the quantitative SNA to describe the roles and functions of key sub-networks within the broader UHC policy network. 
It looks in particular at the relative influence of the different institutional sectors and organisations. There is a discussion of key power actors and gatekeepers, and the pattern of relationships between different stakeholder groups in the policy network.

\subsection{The network actors and stakeholder groups}

Table 7 shows the respondents classified according to their institutional affiliations and indicates their network status (in terms of core actors, peripheral actors and isolates) across the six relationships of contact and influence. Table 8 shows groups of actors by sector and institutional affiliation and shows their network status in respect of the six relationships of contact and influence at this institutional level. The analysis involves 47 individual policy actors who each provided data on their relations with other network actors relating to the six relationships mentioned above (Table 7). Additionally, for some aspects of the analysis, the individual actors were aggregated into 18 participating organisations (Table 8), so that in total there are 65 actor nodes. The actors were classified according to their institutional affiliations and internal (Nigerian state sector actors) versus external (Nigerian non-state or foreign actors) involvement as shown in Tables 7 and 8. 


\begin{tabular}{|c|c|c|c|c|c|c|c|c|c|c|c|c|c|}
\hline \multicolumn{14}{|c|}{ Taxonomy of Respondents } \\
\hline \multirow{2}{*}{ Institutions } & \multirow{2}{*}{ Sub-sector } & \multirow{2}{*}{ Policy organizations } & \multirow{2}{*}{ Respondents code } & \multirow{2}{*}{ Internal/sxternal Invoviement } & \multirow{2}{*}{ Sex } & \multirow{2}{*}{ occupation } & \multirow{2}{*}{ Category } & \multicolumn{6}{|c|}{ Policy Networks } \\
\hline & & & & & & & & Read & Discussion & Coalition & Afflilation & Conflict & Advice \\
\hline \multirow{12}{*}{ Government } & \multirow{6}{*}{ Federal } & \multirow[t]{6}{*}{ FMOH } & 25 & Internal & M & (ACHPO) Strategic Planning & Government & Core & Periphery & Periphery & Periphery & core & Periphery \\
\hline & & & 26 & Internal & M & (ACHPO) Strategic Planning & Government & Periphery & Periphery & Periphery & Core & Core & Core \\
\hline & & & 27 & Internal & M & (ACHPO) Strategic Planning-CAO & Government & Core & Periphery & Periphery & Core & Core & Periphery \\
\hline & & & 28 & Internal & M & (ACHPO) Strategic Planning-Assistant Chief & Government & Core & Periphery & Core & Periphery & core & Periphery \\
\hline & & & 29 & External & M & FMoH-scient fic Officer & Government & Core & Periphery & Periphery & Core & Core & Periphery \\
\hline & & & 30 & External & M & FMOH-DOHPRS-HSSD- HFEl-Senior Health Economist & Government & Core & Core & Periphery & Isolate & Core & \\
\hline & \multirow{6}{*}{ State } & \multirow[t]{6}{*}{ sMoH Commissioners } & 43 & Internal & M & Yobe State-Permanent Secretary & State-Government & Periphery & Periphery & Periphery & Periphery & Per phery & Periphery \\
\hline & & & 44 & Internal & M & Gombe State- Permanent Secretary & State-Government & Periphery & Periphery & core & Core & Per phery & core \\
\hline & & & 45 & Internal & M & Adamawa State- Permanent Secretary & State-Government & Periphery & Periphery & Periphery & Periphery & Per phery & Periphery \\
\hline & & & 46 & Internal & M & Borno State- Permanent Secretary & State-Government & Core & Periphery & Core & Core & Per phery & Core \\
\hline & & & 5 & Internal & M & Deputy director of health Lagos State & State-Government & Periphery & Periphery & Periphery & Periphery & Core & Periphery \\
\hline & & & 47 & Internal & M & State-Civil Servant & State-Government & Periphery & Periphery & Periphery & Periphery & Per phery & Periphery \\
\hline \multirow{16}{*}{ Health Sector } & \multirow{4}{*}{ Public- Heath Sector } & NHIS & 23 & Internal & M & General Manager NHIS & Public Health Sector & Core & Core & Core & Periphery & Core & core \\
\hline & & & 21 & Internal & $\mathrm{F}$ & Deputy General Manager NHIS & Public Health Sector & core & core & core & Periphery & Core & core \\
\hline & & & 22 & Internal & M & Assistant General Manager NHIS & Public Health Sector & Periphery & Periphery & Core & Periphery & core & core \\
\hline & & & 24 & Internal & M & Principal Manager NHIS & Public Health Sector & Periphery & Periphery & core & Periphery & Core & core \\
\hline & & HMOs rep & 2 & Internal & M & HMO Manager (Medical Doctor) & Private Health Sector & Periphery & Periphery & Periphery & Core & Per phery & Periphery \\
\hline & & & 78 & Internal & M & 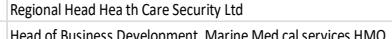 & Private Heath Sector & Core & Periphery & Periphery & Core & Core & 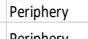 \\
\hline & & & 8 & Internal & M & Head of Business Development, Marine Med cal services HMO & Private Health Sector & Periphery & Periphery & Periphery & Periphery & Isolate & Periphery \\
\hline & & & 9 & External & M & General Manager Clear Line HMO & Private Health Sector & Periphery & Periphery & Periphery & core & Isolate & Periphery \\
\hline & & & 10 & External & $\mathrm{F}$ & Manager Mu ti Shield LTD (Nurse) & Private Health Sector & Periphery & Periphery & Periphery & Periphery & Per phery & Periphery \\
\hline & Private- Heath Sector & & 11 & Internal & M & Chief Operating Officer (HMO) & Private Health Sector & Core & Periphery & Periphery & Periphery & Per phery & Periphery \\
\hline & & & 16 & Internal & M & Quality Assurance Officer (Clearline HMO) & Private Health Sector & Periphery & Periphery & Periphery & Core & Per phery & Periphery \\
\hline & & & 18 & External & M & HMO Manager (Medical Doctor) & Private Health Sector & Periphery & Periphery & Periphery & core & Isolate & Periphery \\
\hline & & & 20 & External & M & СЕО (HMO) & Private Health Sector & Periphery & Periphery & Periphery & Core & Isolate & Periphery \\
\hline & & Hospitals managers & 4 & Internal & M & Chief Operating Officer (Medical Doctor) & Private Health Sector & Periphery & Periphery & Periphery & Core & Isolate & Periphery \\
\hline & & & 6 & Internal & M & Chairman AGPMPN & Private Health Sector & Periphery & Periphery & Periphery & Periphery & Per phery & Periphery \\
\hline & & & 19 & Internal & M & Head Adminstrator KAL & Private Health Sector & Periphery & Periphery & Periphery & Periphery & Per phery & Periphery \\
\hline & & WHO & 34 & Internal & M & WHO Rep & International-UN & core & Periphery & Periphery & Isolate & Isolate & Periphery \\
\hline & UnBodies & World Bank & 35 & Internal & M & World Bank Rep & International-UN & Core & Core & Periphery & Isolate & Isolate & Periphery \\
\hline & & UNICEF & 32 & Internal & M & UNICEF-MNCH-Health Specialist & International-UN & Core & Core & Periphery & Isolate & Isolate & core \\
\hline & & USAID & 33 & External & M & USAID Rep & External GovtAgencies & core & Periphery & Periphery & Isolate & Isolate & Periphery \\
\hline International & External Govt Agencies & DFID UK & 38 & External & M & DFID UKRep & International-NGO & Periphery & core & Periphery & Periphery & Isolate & Periphery \\
\hline & & Global Affairs Canada & 39 & Internal & M & Global Affaris Canada Rep & International-NGO & Periphery & Core & Periphery & Core & Isolate & Periphery \\
\hline & & \begin{tabular}{|l|l|} 
ABT Associates \\
HEREN
\end{tabular} & 37 & 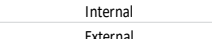 & M & $\begin{array}{l}\text { Abt Associates Rep } \\
\text { HFFFON- }\end{array}$ & International-NGO & Periphery & Periphery & Isolate & Isolate & Isolate & Core \\
\hline & NGos & HERFON & 31 & External & M & HERFON-Executive Secretary & International-NGO & Core & Periphery & Periphery & Isolate & Periphery & core \\
\hline & & Heath Parthers-(PHCUOR) & 36 & Internal & M & HPI- Public Heath Specialist tand PHCUOR Expert & International-NGO & Periphery & core & Isolate & Isolate & Isolate & core \\
\hline & & NMA & 40 & External & M & NMA Pres dent & Labour & Periphery & Periphery & Periphery & core & Isolate & core \\
\hline & Professional Association & & 41 & External & M & NMA Secretary General & Labour & Core & Core & Periphery & Periphery & Isolate & Core \\
\hline & & & 42 & External & M & NMA Select Committee (Consultant Ophthalmologist) & Labour & Periphery & Periphery & Periphery & core & Per phery & core \\
\hline & & Doctors & 3 & External & M & Public Heath (Physician) & Labour & Periphery & Periphery & Periphery & Periphery & Isolate & Periphery \\
\hline Labour & & Nurres & 1 & Internal & M & Community hea thextension worker (CHEW) Nurse & Labour & Periphery & Periphery & Periphery & Periphery & Isolate & Periphery \\
\hline (1) & & & 12 & External & $\mathrm{F}$ & Community hea th officer & Labour & Periphery & Periphery & Isolate & Periphery & Isolate & Periphery \\
\hline & Non-Professional Associat on & & 13 & Internal & $\mathrm{F}$ & Ch ef nursing officer (midwife) & Labour & Periphery & Periphery & Periphery & core & Isolate & Periphery \\
\hline & & & 14 & Internal & $\mathrm{F}$ & Chef nursing officer (nurse) & Labour & Periphery & Periphery & Isolate & Periphery & Isolate & Periphery \\
\hline & & & 15 & Internal & $\mathrm{F}$ & Community hea the extension worker (CHEW) Nurse & Labour & Periphery & Periphery & Isolate & Periphery & Isolate & Periphery \\
\hline & & Allied Professional & 17 & Internal & $\mathrm{M}$ & Dental Officer & Llabour & Periphery & Core & Periphery & Core & Core & Periphery \\
\hline
\end{tabular}

Table 7: Taxonomy of respondents involved in the second round of structured SNA interviews. 


\begin{tabular}{|c|c|c|c|c|c|c|c|c|}
\hline \multicolumn{9}{|c|}{ Taxonomy of Policy Organizations } \\
\hline \multirow{2}{*}{ Institutions } & \multirow{2}{*}{ Sub-sector } & \multirow{2}{*}{ Policy actors } & \multicolumn{6}{|c|}{ Relationship of the Policy Network } \\
\hline & & & Read & Discussion & Coalition & Affiliation & Conflict & Advice \\
\hline \multirow{2}{*}{ Government } & Federal & $\mathrm{FMoH}$ & Core & Core & Periphery & Core & Periphery & Core \\
\hline & State & SMoH Commissioners & Core & Core & Core & Core & Periphery & Periphery \\
\hline \multirow{3}{*}{ Health Providers } & Public- Health Provider & NHIS & Core & Core & Periphery & Core & Core & Core \\
\hline & \multirow{2}{*}{ Private- Health Provider } & HMOs rep & Core & Core & Core & Core & Core & Core \\
\hline & & Hospitals managers & Core & Core & Core & Core & Core & Core \\
\hline \multirow{9}{*}{ International } & \multirow{3}{*}{ UN Bodies } & WHO & Core & Core & Core & Core & Core & Core \\
\hline & & World Bank & Core & Core & Core & Core & Core & Core \\
\hline & & UNICEF & Periphery & Periphery & Periphery & Periphery & Isolate & Periphery \\
\hline & \multirow{3}{*}{ External Govt Agencies } & DFID UK & Periphery & Periphery & Isolate & Periphery & Isolate & Isolate \\
\hline & & Global Affairs Canada & Isolate & Isolate & Isolate & Periphery & Isolate & Isolate \\
\hline & & USAID & Core & Core & Core & Core & Core & Core \\
\hline & \multirow{3}{*}{ NGOs } & HERFON & Core & Core & Periphery & Core & Isolate & Core \\
\hline & & ABT Associates & Isolate & Isolate & Isolate & Periphery & Isolate & Isolate \\
\hline & & Health Partners-(PHCUOR) & Core & Core & Periphery & Core & Core & Core \\
\hline \multirow{4}{*}{ Labour } & Professional Association & NMA & Core & Core & Core & Core & Isolate & Core \\
\hline & \multirow{3}{*}{ Non-Professional Association } & Doctors & Core & Core & Core & Periphery & Isolate & Periphery \\
\hline & & Nurses & Periphery & Periphery & Isolate & Isolate & Isolate & Isolate \\
\hline & & Allied Professional & Periphery & Periphery & Periphery & Periphery & Isolate & Periphery \\
\hline
\end{tabular}

Table 8: Taxonomy of policy organisation involved in the second round of interviews.

The organisational level data, formed by aggregating data on individual actors, was used to examine the degree of influence the various organisations have in the network. There is a risk here that designating an organisation (comprising multiple respondents) as an actor alongside individual actors in a network diagram automatically means the organisation have more ties and more influence than the individual actor nodes. However, it must be remembered that the selection of network participants is based on respondents' own perceptions of who the key network actors are, so that grouping key actors according to organisations is a legitimate step in analysis. 


\subsection{Network size, density, fragmentation and transitivity}

Figure 27 presents a visualisation of the directed and non-valued network in which the red nodes (circle-shaped) represent sixty-three active actors and the blue nodes (square-shaped) represent two inactive actors (isolates). This presents an image of the network size (65 nodes) and the links between actors.

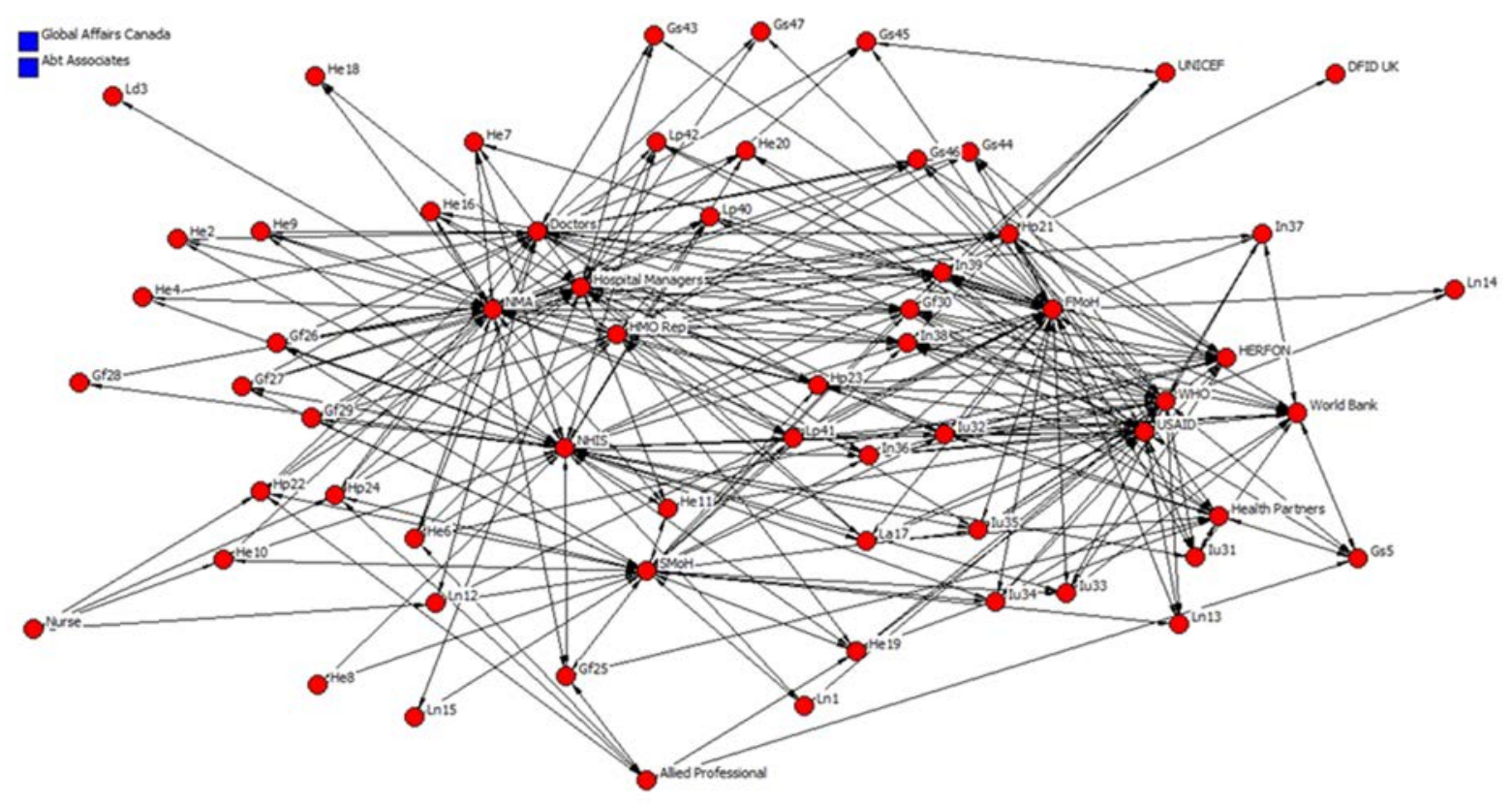

Figure 27: Size of the directed and non-valued policy network.

Figure 27 shows a large number of social interactions within the discussion relationships between policy-actors involved in the UHC policy process. There is also a sizeable amount of social activity between respondents that is concerned with the coalition and affiliation relationships, while the conflict relationship has the lowest social activity out of the six relationships constructed to represent the different modalities of engagement. 


\begin{tabular}{l|c|c|c|c|c|c|c}
\hline Relationship & Size & Density & Number of ties & Average Degree & Average Distance & Fragmentation & Transitivity \\
\hline Read & 65 & 0.332 & 281 & 5.979 & 2.156 & 0.061 \\
\hline Discussion & 65 & 0.314 & 266 & 5.66 & 2.22 & 0.615 \\
\hline Coalition & 65 & 0.177 & 150 & 3.191 & 2.725 & 0.061 \\
\hline Affiliation & 65 & 0.18 & 152 & 3.234 & 2.549 & 0.26 \\
\hline Conflict & 65 & 0.053 & 45 & 0.957 & 3.795 & 0.26 & 0.592 \\
\hline Advice & 65 & 0.239 & 202 & 4.298 & 2.424 & 0.714 \\
\hline
\end{tabular}

Table 9: Measures of cohesion for six relationships of the policy network.

In SNA the intensity of interactions between actors is termed network density, and network density on these six relationships is shown in Table 9. Density is based on the number of active connections reported by respondents. It portrays the level of activity that occurs within the policy network by quantifying the frequency with which activities such as reading the work of others, holding discussions and taking advice (plus the other three of the six relationships) are mentioned. The network analysis in itself does not tell us the precise functions and meanings of these various forms of interaction. However, the "read" and "discussion" relationships have the same density score, while these are denser than the "advice” relationship, which is denser than the "coalition" and "affiliation" relationships (both of these last relationships have the same density score). The "conflict" relationship is the least dense relationship. In comparison to the network density, fragmentation and transitivity are more sensitive in measuring large number of networks along the same cohesion measure lines.

Fragmentation is one minus connectedness, where connectedness is the proportion of pairs of actors interacting with each other through a path in the same component. Fragmentation is interpreted as the proportion of paired actors (within the policy network) that cannot relate with one another in any way possible (Borgatti et al., 2018). The Fragmentation measure is used to evaluate any transformations occurring inside a network either in reality or as part of computing model. Table 9 shows how fragmentation captures cohesion for the network of all six relationships. The "read" and "discussion" relationships have the same fragmentation score, while these are more connected than the "advice" relationship, which is less fragmented than the "coalition" and "affiliation" relationships (both have the same fragmentation score). The “conflict” relationship has the most fragmented score in all six relationships.

This may suggest a kind of hierarchy in the reported (density and fragmentation measures) frequency of the various relationship forms so that more informants talk about reading others' work or holding discussions with them than report taking advice, that these activities are seen 
as more common than joint activities concerned with affiliation or coalition formation, and that conflictual relationships are even less common.

Furthermore, when networks have lots of transitivity, they tend to have a clumpy structure. That is, they contain knots of nodes that are all interrelated. Transitivity refers to the ratio where the number of triads $(\mathrm{A}-\mathrm{C})$ is the numerator, and the denominator is the number of triads (AB) and (B-C). Applying the transitivity to all six relationships of the policy network yields a high score of 0.765 in the "conflict" relationship, which is the highest of all six relationships and the "affiliation" relationship having the lowest transitivity score (0.509). The policy network is characterized as transitive through the presence of quite high transitive scores among all six relationships automatically implying other structural features exist within the policy network.

The network density, fragmentation and transitivity of the policy network shows its cohesiveness, but Friedkin (1981) objects to the use of density, fragmentation and transitivity as a measure of cohesion because it fails to highlight significant cohesive sub-groups within each relationship of the policy network. for instance, most experts suggest that density score in larger networks is only a partial measure of cohesiveness. A possible solution is to investigate the existence of 'components’ within each relationship (Tsvetovat and Kouznetsov, 2011).

\subsection{Network components}

Borgatti et al. (2013) defines a component as the maximum group of actors in which all actors are connected to each other by some path. The term 'maximum' means that the introduction of a new actor into the group would not disrupt the structural integrity of the path. However, this approach is only meaningful if the given network is broken down into several different components or into a component and isolates (see Figure 28). An isolate is regarded as a disconnected component (Tsvetovat and Kouznetsov, 2011). In this context, an isolated node refers to the respondent not named relevant in a relationship but who participated in the interview. Figure 28 shows the component analysis of the read relationship of the policy network where the two nodes in blue squares are isolates (two components) and the nodes represented by red squares are actors that form a single network component. Concerning the concept of cohesion, a network consisting of just one component is a strongly connected graph where every actor can interact with each other via the direction of arcs (Borgatti et al., 2018). 
Findings suggest that every relationship of the policy network have strongly connected graphs and are likely to give more robust subgroups (see Appendix for the other relationships of the policy network).

In this diagram individuals with clear organisational affiliations have been aggregated under their organisational labels to form nodes. This was seen as an aid to identifying possible subgroups within the network that might solidify into components.

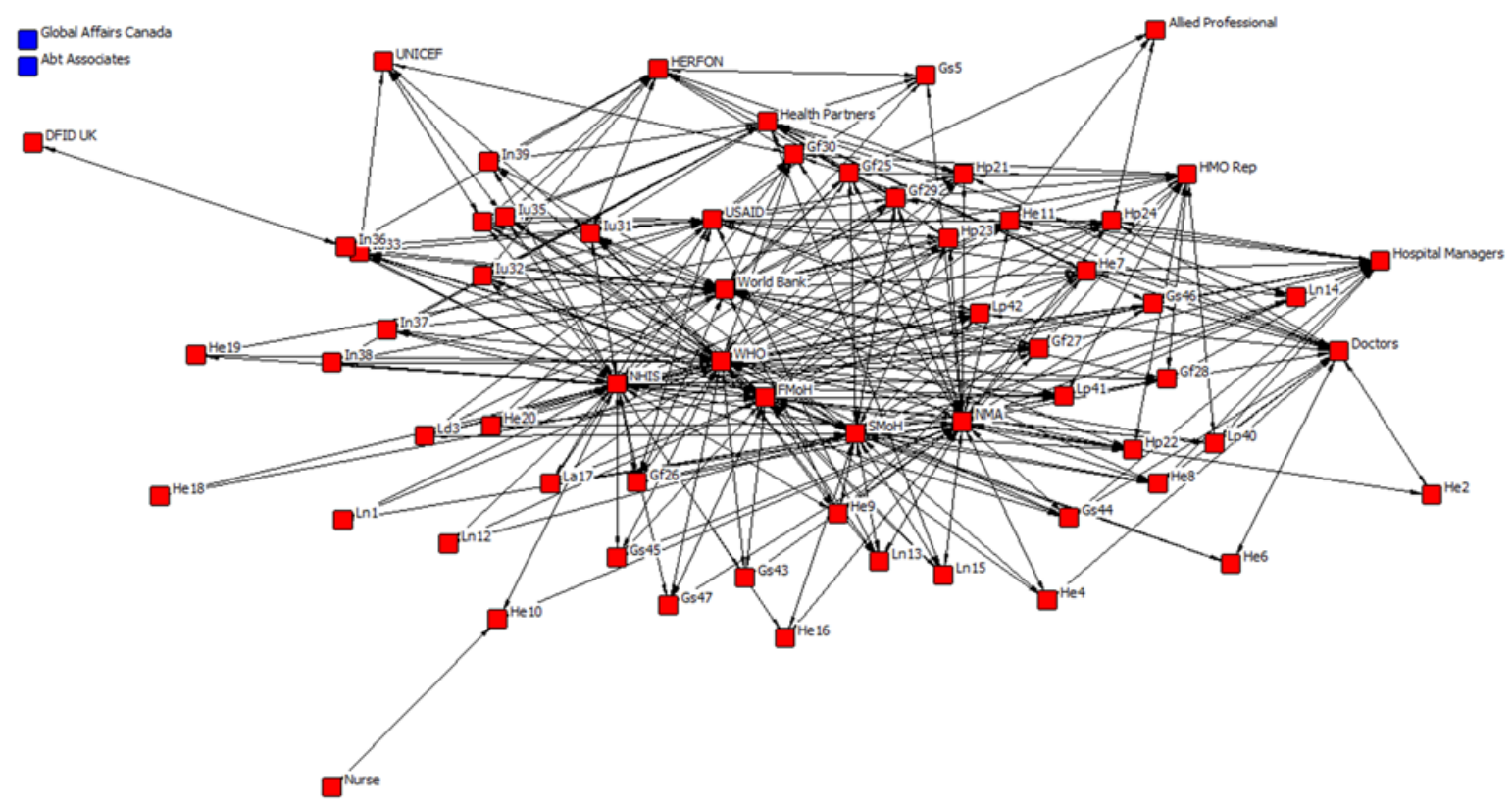

Figure 28: Component analysis of the "read" relationship of the policy network.

Figure 28 presents a visualisation of the component analysis of the 2-mode (bipartite graph) "read” relationship network, using this first relationship as an example. The graph shows that in terms of the "read" relationship the policy network has two components: the two blue squared nodes are isolates disconnected from the strong and well-connected component of cohesive subgroups in the red nodes.

Findings from the component measure showed that for each of the six relationships only one major component of connected actors (nodes) was evident (see Appendix for the other relationships of the policy network).

Borgatti et al. (2013) suggests that the component approach comes with limitations because component analysis is only significant when the networks are broken down into several components (Boland and El-Neweihi, 1995). This approach does not measure the social activities between any cohesive subgroups within the component (Borgatti et al., 2013). Further 
analysis is needed to evaluate the quantitative and qualitative properties of the components of the networks as well as their dyadic relationships (Boland and El-Neweihi, 1995).

This provisional finding that the policy network did not appear to divide into obvious subnetworks but rather took the form of a single network component together with isolates, will be explored in more depth in the next section.

\subsection{Network Components and the 'closeness' of links between actors}

SNA provides various techniques for investigating network components (sub-networks) in more depth. In the absence of obvious cliques, k-core and core-periphery analysis is an alternative approach to further conceptualise and measure the cohesiveness of a network component (see: Prell, 2012). A core-periphery analysis breaks down a network structure into a core group of actors and a periphery group of actors. Therefore, a core-periphery structure indicates a subgroup of actors where those interacting within the core have more control/influence than actors on the periphery (Borgatti et al., 2013). Models of core-periphery structures are described in more detail in Figure 35 - 40. Many experts argue that the k-core measure offers advantages over core-periphery structure analysis for measuring cohesion. Unlike the latter, k-core analysis investigates the extent to which peripheral nodes may nevertheless be linked to the core nodes within the network structure (Zhang et al., 2014).

$\mathrm{K}$-core is a measure of cohesiveness that re-arranges the heterogeneous nodes within a network into clusters of homogeneous sub-groups determined by their k-core score. A k-core is a group of actors, who are all connected to a specified score $(\mathrm{k})$ of other members of the group. The $\mathrm{k}$ value can be varied to require an actor to have a link to more or fewer group members before being deemed a member of the k-core. In the analysis otherwise heterogeneous actors (nodes) with the same k-core score are taken to constitute a subgroup for analytical purposes (Borgatti et al., 2013). Subgroups in this sense are theoretical constructs emerging from the analysis that have no relationship to the classification of actors presented in Figure 29- thus they are not formed on the basis of institutional affiliation, though there may be some overlap. Prell (2012: 159) explains that in deciding what constitutes a k-core subgroup 'as the value of $\mathrm{k}$ becomes lower subgroup sizes will increase, that is, it becomes easier to draw a boundary around the subgroup.' In the present analysis the k score value for inclusion in the core group in each 
relationship was set at seven (for read), seven (discussion), five (for coalition), five (for affiliation), two (conflict) and five (advice).

Figure 29 shows a k-core graph based on the "read” relationship where the core actors (nodes) who read each other's work fall within the red boundary, the peripheral actors (nodes) are inside the black oval and the isolated actors (nodes) are encircled yellow.

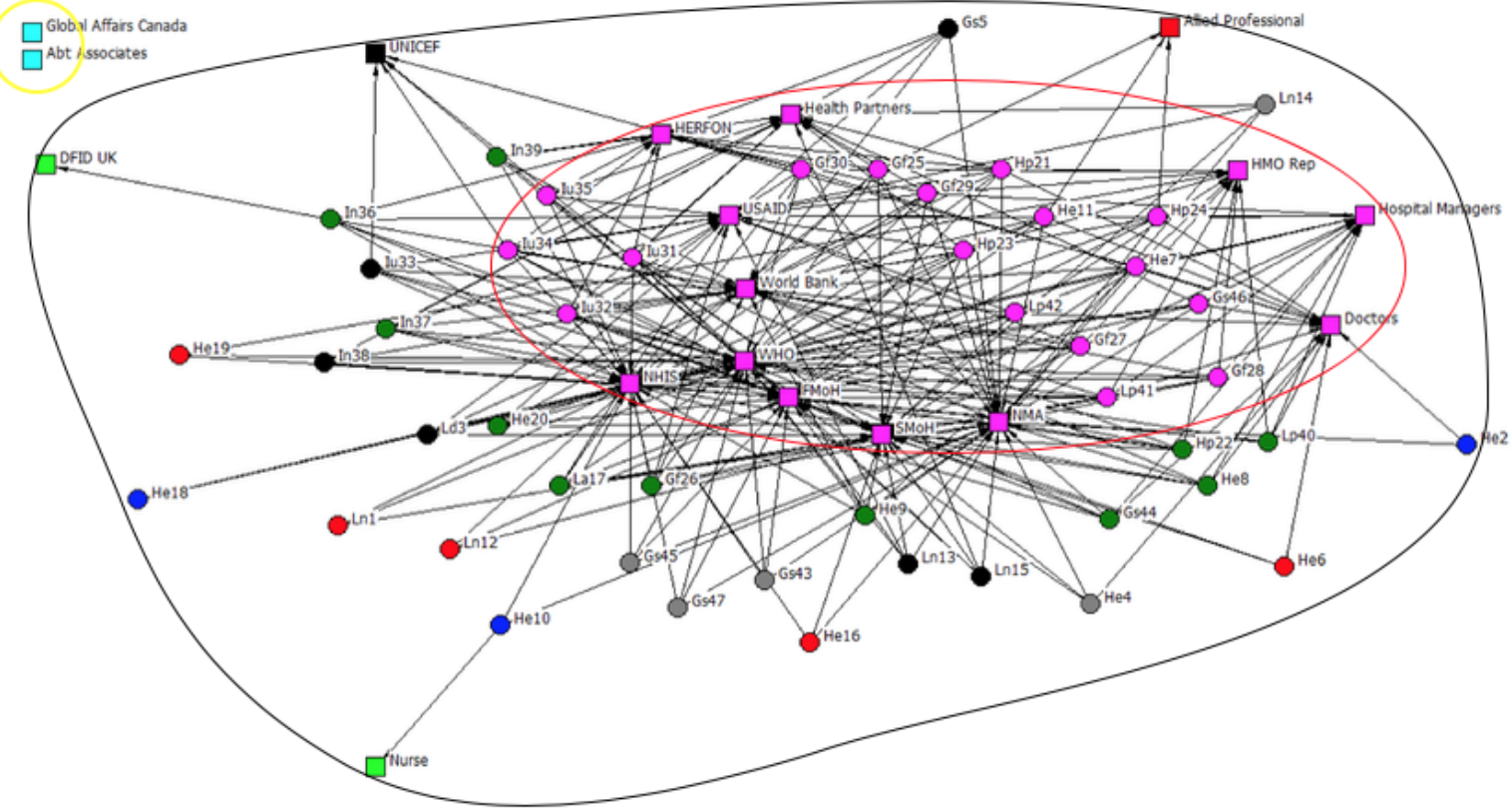

Figure 29: Visualisation of the k-core measure of the (bipartite graph) "read" relationship of the policy network showing the presence of eight subgroups.

Nodes within the red circle are at the core of the relationship, nodes inside the black circle are termed peripheral nodes and the isolates (Subgroup 0 ) are circled yellow.

Key: Core nodes- Subgroup7, Periphery nodes- Subgroup6- Subgroup5- Subgroup4Subgroup3- Subgroup2- Subgroup1, Isolated nodes- Subgroup0.

Although the k-core finding in this case suggests that the network divides into three groups (core, periphery and isolates) with respect to the "read" relationship, for purposes of analysis it was necessary to categorise the nodes into eight subgroups based on k-core scores. Thus, nodes in subgroup7 link to 6 other nodes, nodes in subgroups6 to five other nodes and so on, with nodes in subgroup 0 having no connections to other nodes and constituting the isolates. The k-core analysis confirms the finding from earlier measures that the primary network component divides into two major groups without the isolates (because isolates are nodes that have minimal involvement in a network), core and periphery. Figure 29 illustrates three encircled subgroups of nodes based on k-core scores. Nodes inside the red boundary had a 
score of seven and belong to the core group, while the black boundary line contains peripheral nodes with k-core scores ranging from six to one (each node is represented with their colour codes). Figure 35 illustrates how the core subgroup of the 'read relationship's core-periphery structure contains the same nodes inside the red boundary of Figure 29.

In comparison, the core-periphery analysis and the k-core subgroup7 of the 'read relationship' share similar core subgroup actors- USAID, WHO, SMoh, FMoH, NMA, NHIS, HERFON, World Bank, Health Partners, Doctors, Hospital Managers, HMO rep, Iu31, Iu32, Iu34, Iu35, Gf30, Gf25, Gf29, Gf28, Gf27, Gs46, Hp21, He11, Hp24, He7, Hp23, Lp42 and Lp41 (see Figure 35). Although both measures (core-periphery analysis and k-core analysis) share similar nodes within their periphery subgroup, the k-core measure does better by explicitly defining the connection between periphery nodes to core nodes from those periphery nodes furthest and closest to the core subgroups in the 'read relationship.' The findings relating to the coreperiphery analysis and k-core measure of the other five relationships of the policy network showed a similar resemblance to the 'read relationship' outcome.

Core-periphery analysis and the k-core measure of the 'discussion relationship' show that each has a similar set of core actors in the core subgroup of the core-periphery structure and the kcore score of subgroup7- Doctors, Hospital Managers, NMA, HMO Rep, NHIS, SMoH, Health Partners, USAID, World Bank, WHO, HERFON, FMoH, La17, Iu36, In36, Iu32, Lp41, Hp23, In38, Gf30, In39 and Hp21 (see Figures 30 and 36).

The core subgroup measures for the 'coalition relationship' suggest that key interactions revolve around the Hospital Managers, HMO Rep, SMoH, WHO, Doctors, World Bank, USAID, Hp23, Hp24, Hp22, Hp21, Gf28, Gs46, Gs44 and NMA nodes, seen within the core subgroup of the core-periphery structure and core subgroup 5 of the k-core measure (see Figure 31 and 37).

However, for the 'affiliation' relationship, most core activity surrounds the NHIS, FMoH, SMoH, Hospital Managers, Health Partners, HERFON, USAID, WHO, World Bank, HMO Rep, He16, He7, La17 In39 He18, Gs46, Gs44, He20, Gf26, Gf27, Gf29, Ln13, Lp40, He4, He2, Lp42, He9 and NMA exhibited in the core subgroup of its core-periphery structure and k-core score subgroup5 (see Figures 32 and 38). 
The findings in the 'conflict' relationship suggest that the USAID, World Bank, HMO Rep, Health Partners, NHIS, WHO, Gs5, Gf27, Gf30, He7, Hp22, Gf28, Gf29, Gf25, Gf26, Hp23, Hp24, Hp21, La17 and Hospital Managers' nodes have the most core-to-core interaction surrounding them in the core subgroup of its core-periphery structure and subgroup2 of the kcore score (see Figure 33 and 39). The 'advice relationship' also shows a core-to-core interaction of nodes such as the FMoH, NMA, HMO Rep, USAID, World Bank, WHO, NHIS, Hospital Managers, Health Partners, HERFON, Gf26, Lp40, Iu31, Lp41, Lp42, Hp23, Gs44, Gs46, Hp24, Gf30, Hp21, In37, Iu32, In36 and Hp22 which are represented in both the core subgroup of the 'advice relationship' core-periphery structure and the subgroup of k-core score five (see Figure 34 and 40).

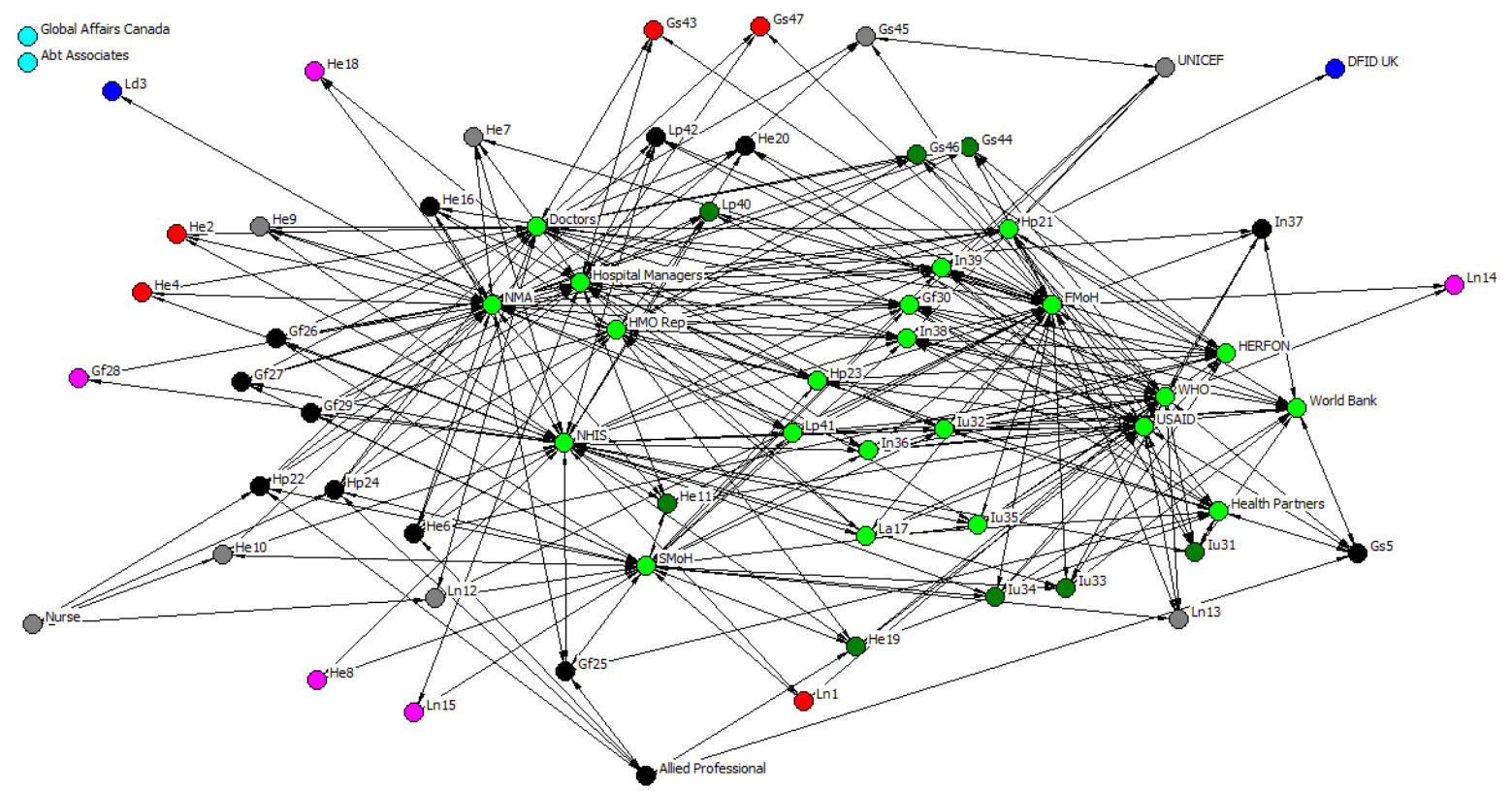

Figure 30: Visualisation of the k-core measure of the (bipartite graph) “discussion” relationship of the policy network showing the presence of eight subgroups.

Nodes within the red circle are at the core of the relationship, nodes inside the black circle are termed peripheral nodes and the isolates (Subgroup 0 ) are circled yellow.

Key: Core nodes- Subgroup7, Periphery nodes- Subgroup6- Subgroup5- Subgroup4Subgroup3- Subgroup2 - Subgroup1, Isolated nodes- Subgroup0. 


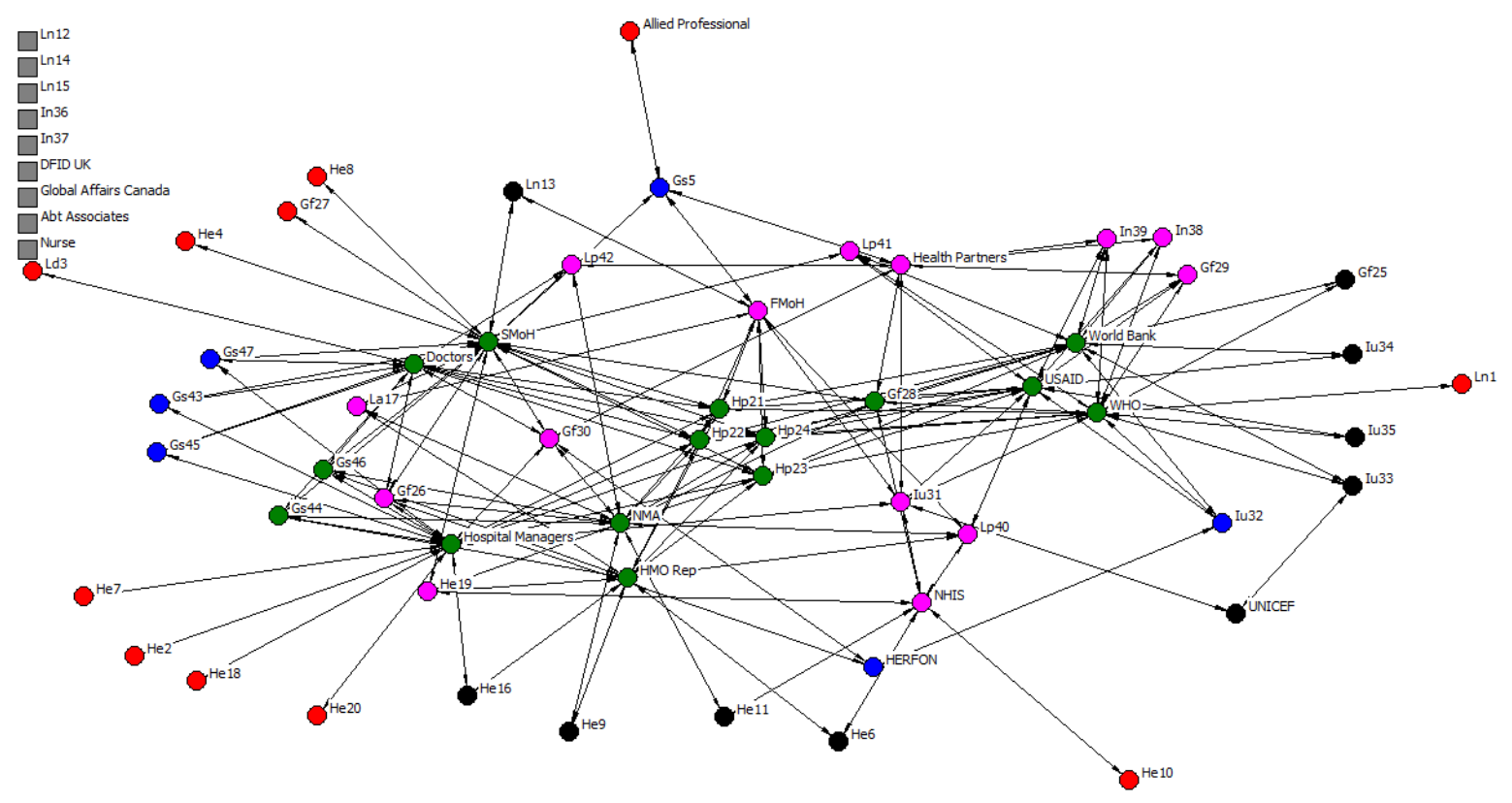

Figure 31: Visualisation of the k-core measure of the (bipartite graph) “coalition" relationship of the policy network showing the presence of eight subgroups.

Nodes within the red circle are at the core of the relationship, nodes inside the black circle are termed peripheral nodes and the isolates (Subgroup 0 ) are circled yellow.

Key: Core nodes- Subgroup5, Periphery nodes- Subgroup4- Subgroup3- Subgroup2Subgroup1, Isolated nodes- Subgroup0. 


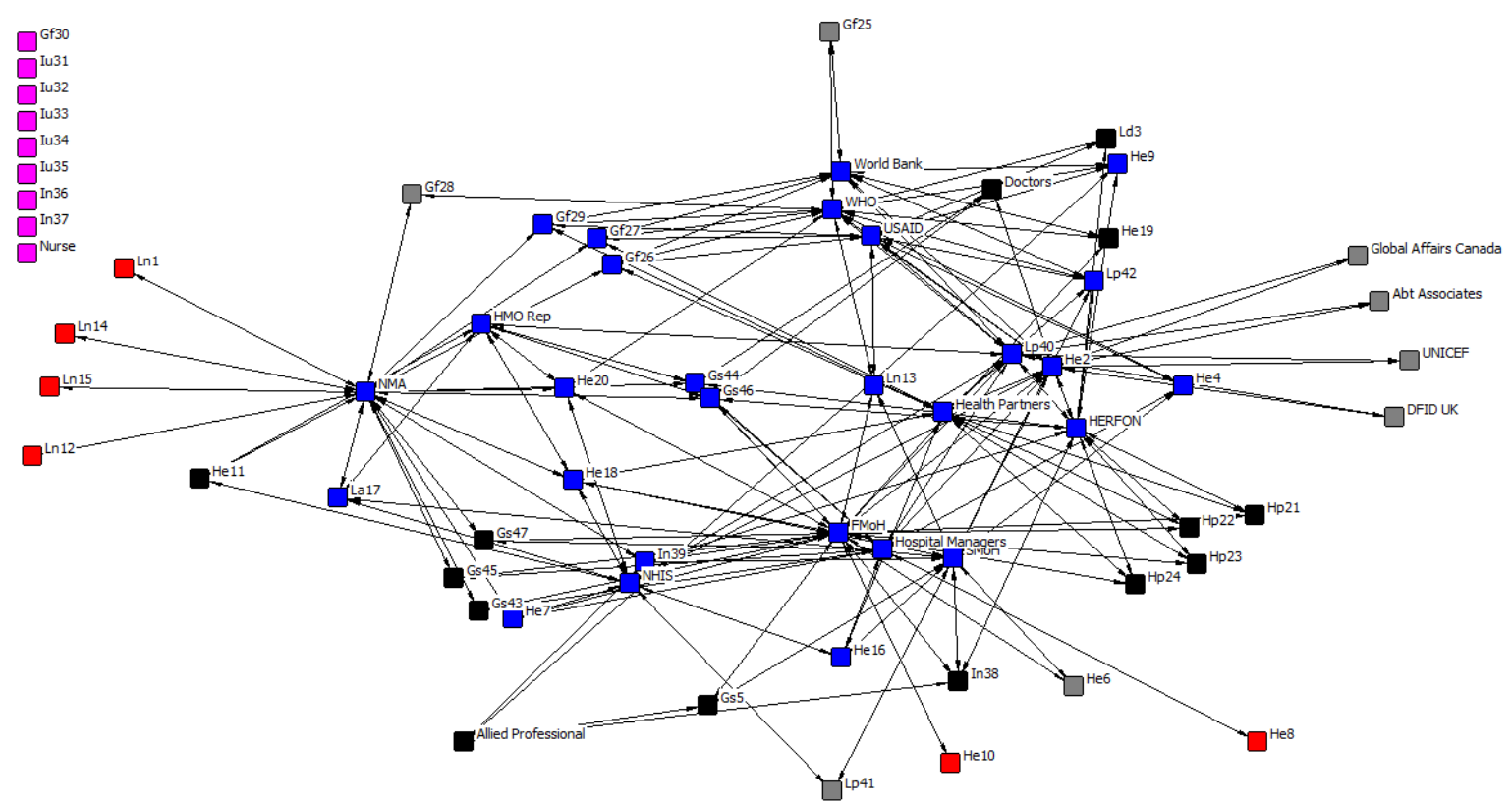

Figure 32: Visualisation of the k-core measure of the (bipartite graph) "affiliation" relationship of the policy network showing the presence of eight subgroups.

Nodes within the red circle are at the core of the relationship, nodes inside the black circle are termed peripheral nodes and the isolates (Subgroup 0 ) are circled yellow.

Key: Core nodes- Subgroup5, Periphery nodes- Subgroup3- Subgroup2- Subgroup1, Isolated nodes- Subgroup 0. 


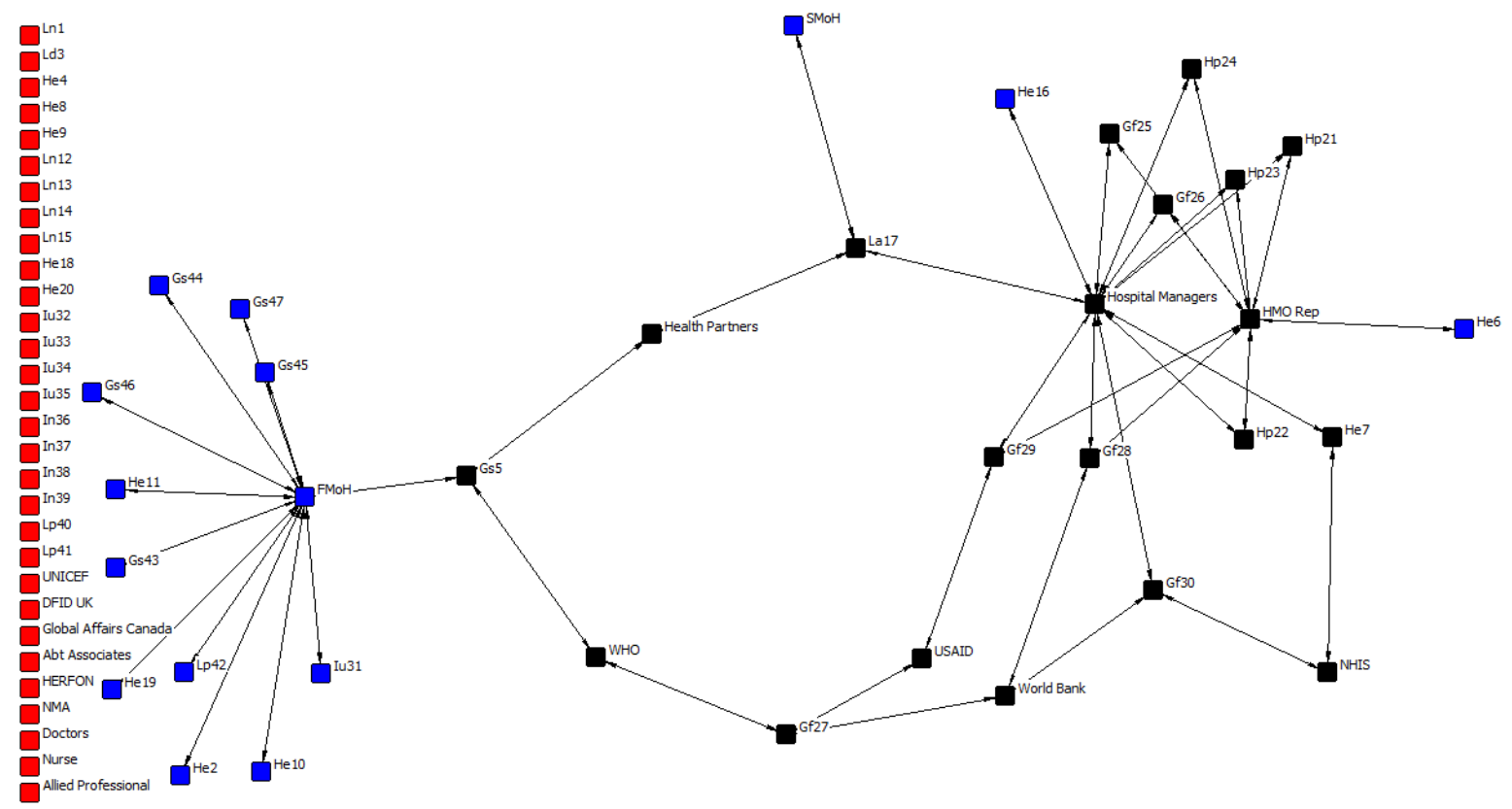

Figure 33: Visualisation of the k-core measure of the (bipartite graph) "conflict" relationship of the policy network showing the presence of eight subgroups.

Nodes within the red circle are at the core of the relationship, nodes inside the black circle are termed peripheral nodes and the isolates (Subgroup 0 ) are circled yellow.

Key: Core nodes- Subgroup2, Periphery nodes- Subgroup1, Isolated nodes- Subgroup0. 


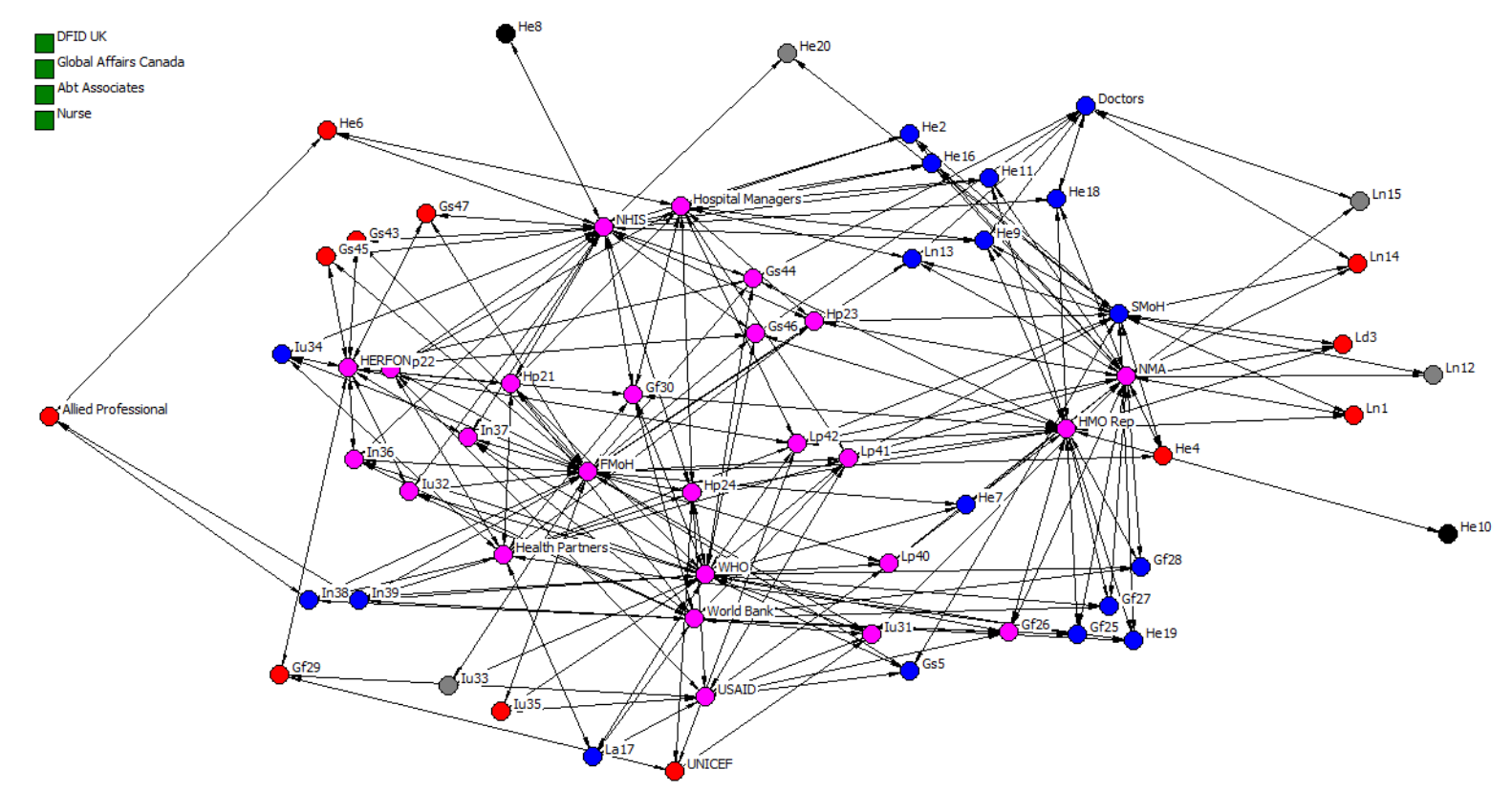

Figure 34: Visualisation of the k-core measure of the bipartite graph "advice" relationship of the policy network showing the presence of eight subgroups.

Nodes within the red circle are at the core of the relationship, nodes inside the black circle are termed peripheral nodes and the isolates (Subgroup 0 ) are circled yellow.

Key: Core nodes- Subgroup5, Periphery nodes- Subgroup4- Subgroup3- Subgroup2Subgroup1-, Isolated nodes- Subgroup0. 


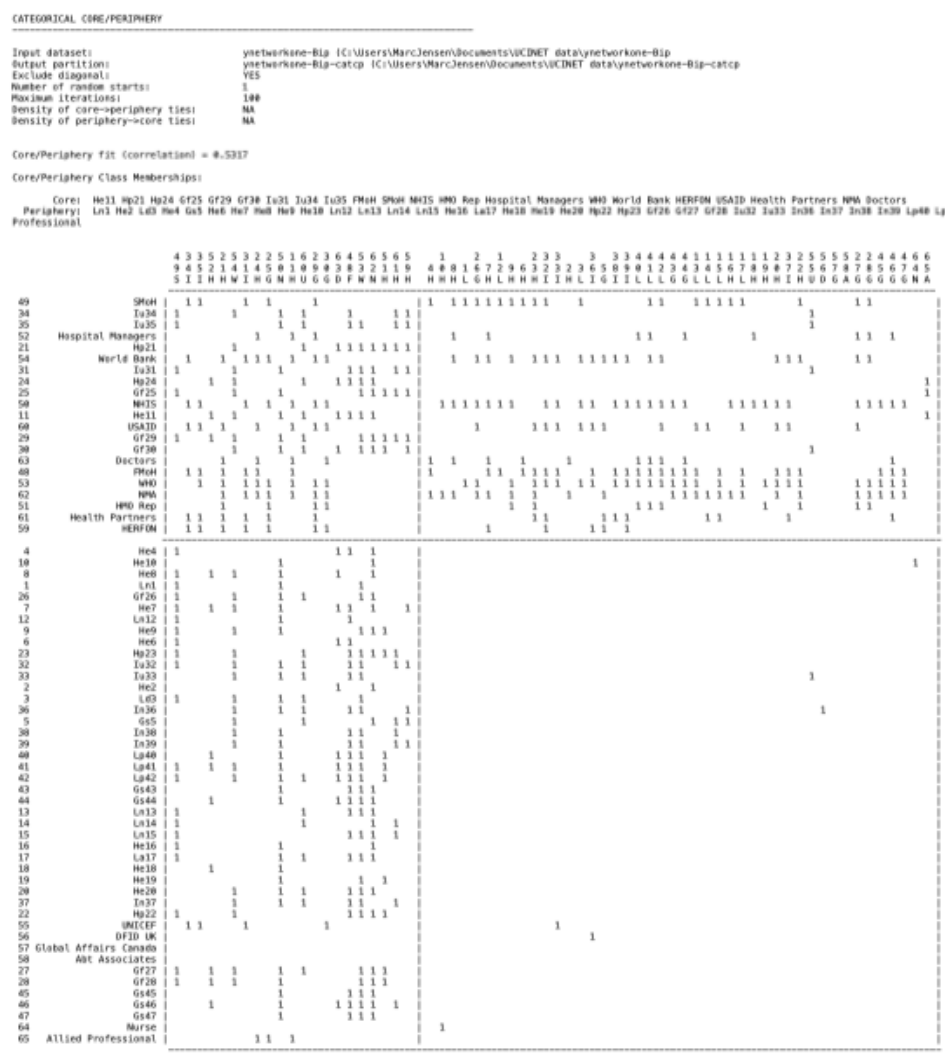

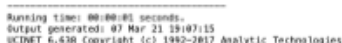

Figure 35: A core-periphery network of the bipartite graph "Read" relationship of the policy network showing the presence of core and periphery subgroups. 


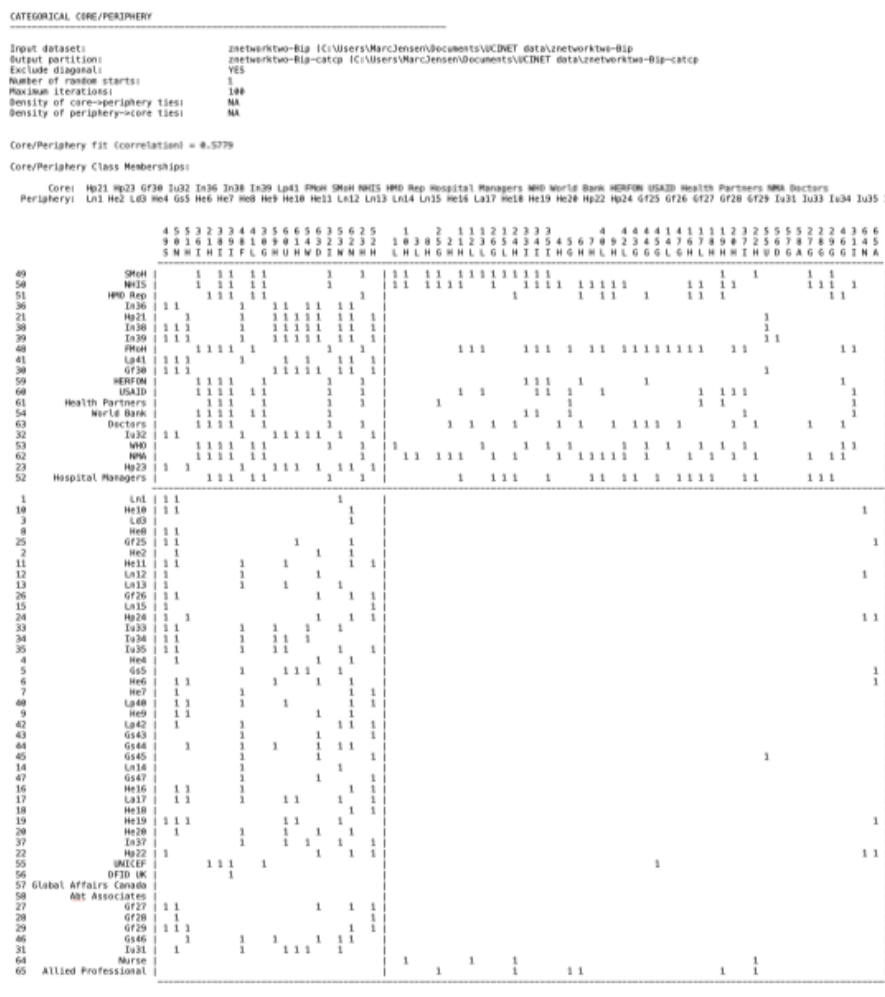

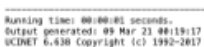

Figure 36: A core-periphery network of the bipartite graph “discussion” relationship of the policy network showing the presence of core and periphery subgroups. 


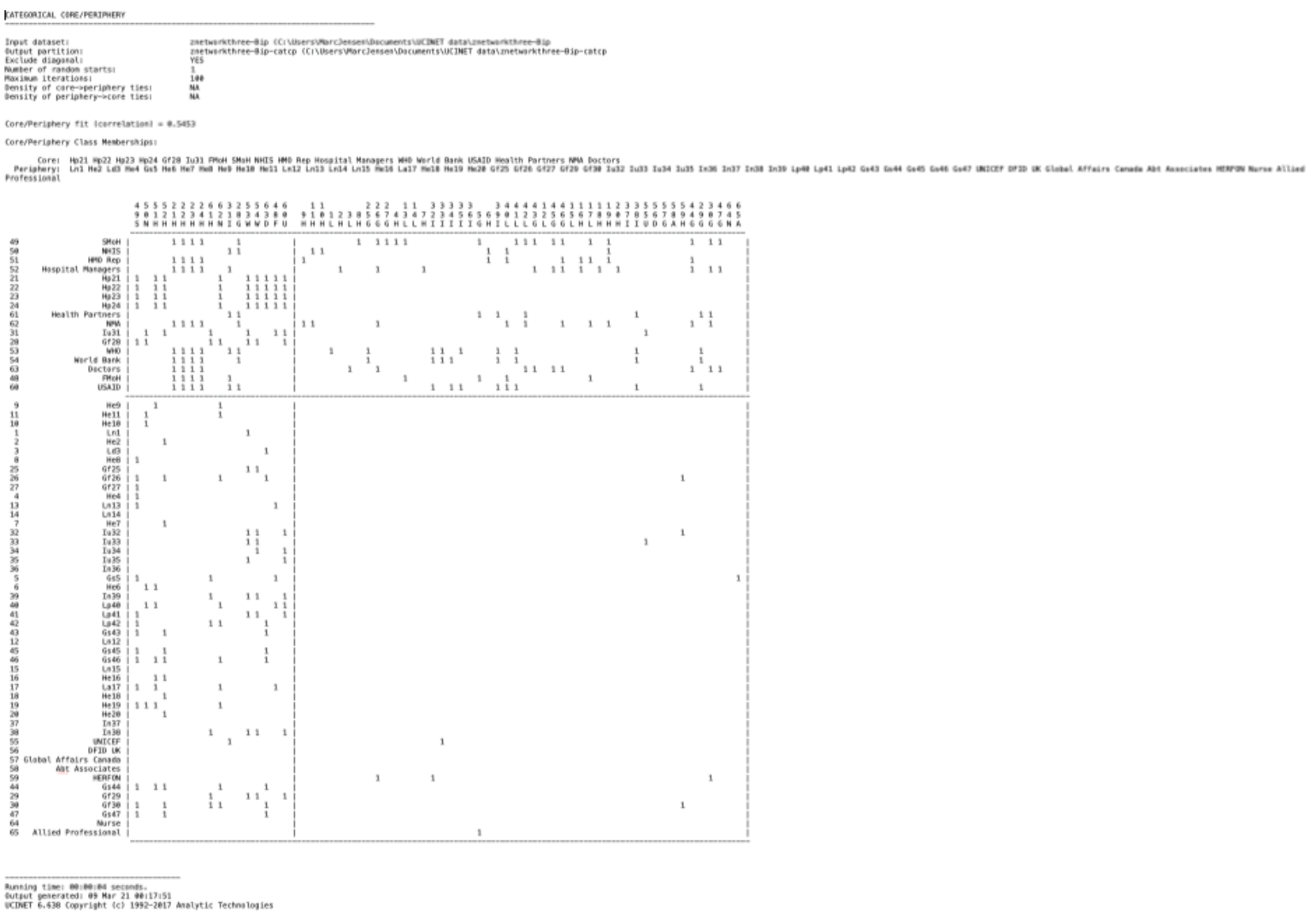

Figure 37: A core-periphery network of the bipartite graph “coalition” relationship of the policy network showing the presence of core and periphery subgroups. 


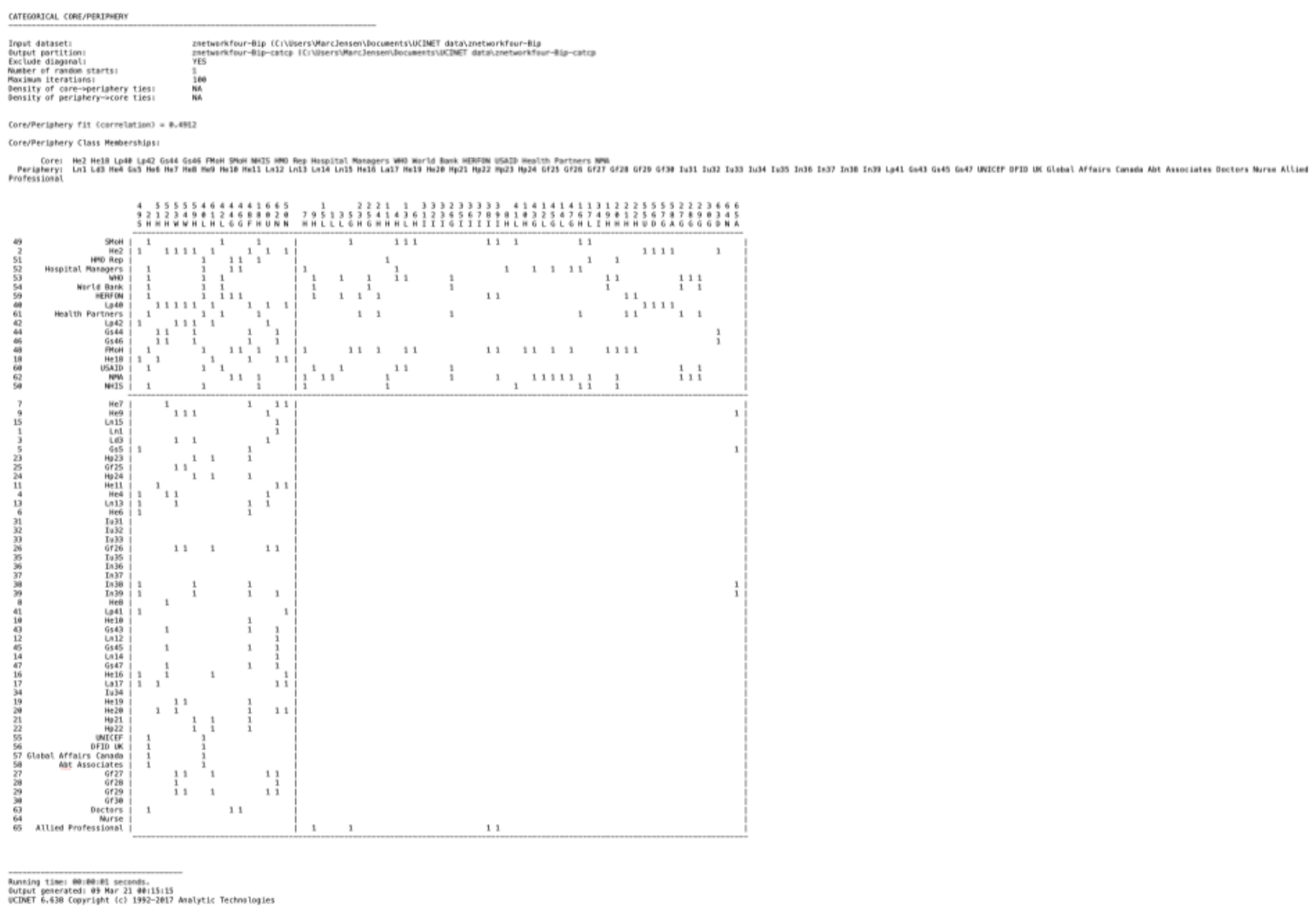

Figure 38: A core-periphery network of the bipartite graph "affiliation" relationship of the policy network showing the presence of core and periphery subgroups. 


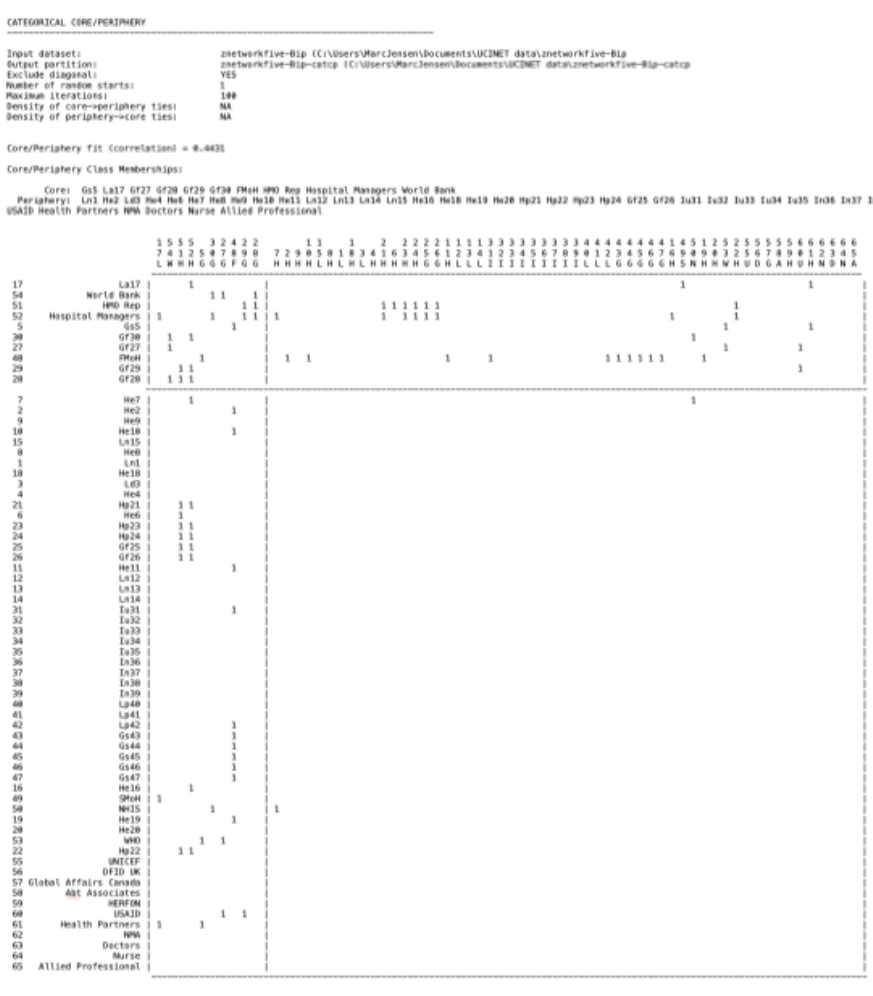

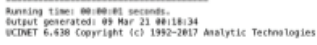

Figure 39: A core-periphery network of the bipartite graph "conflict" relationship of the policy network showing the presence of core and periphery subgroups. 


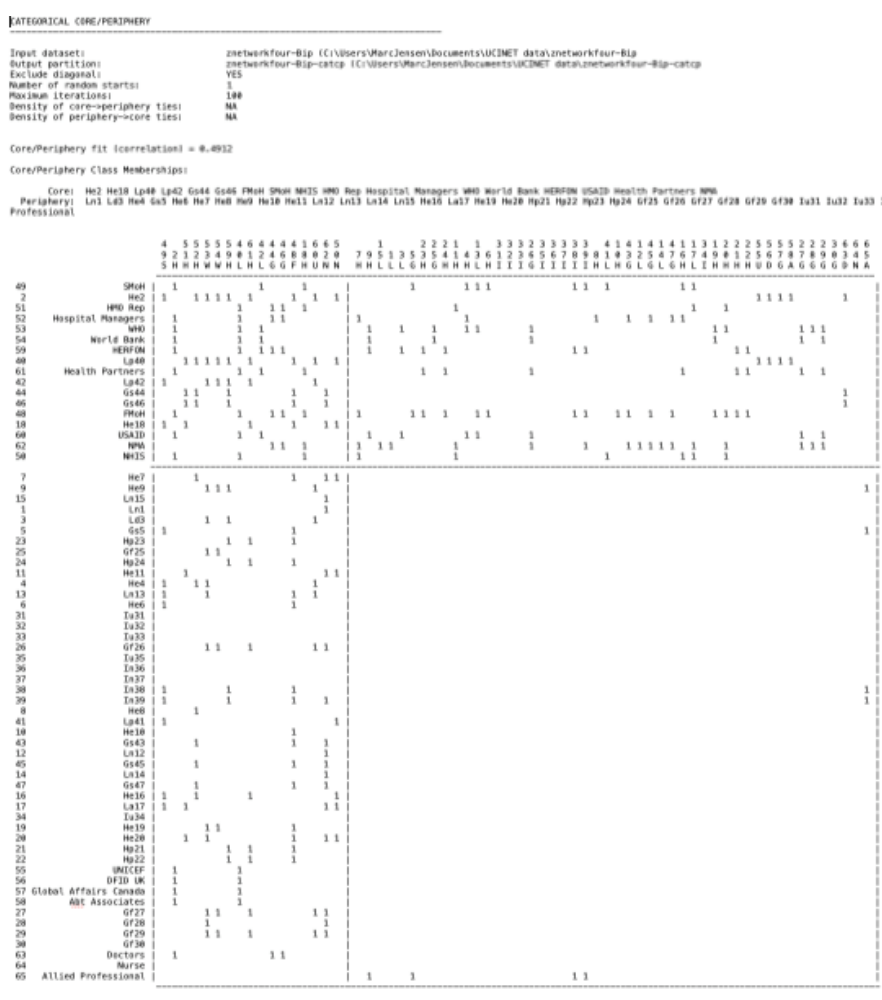

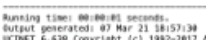

Figure 40: A core-periphery network of the bipartite graph "advice" relationship of the policy network showing the presence of core and periphery subgroups.

The visualisations for the 'read relationship' and the other modalities of engagement show a core group of highly active and central policymakers (albeit a mixture of types of policy actors) surrounded by a group of peripheral nodes - policy actors who engage in a less intensive way. Findings from the k-core measure suggest the core group itself (based on nodes with similar scores) is quite large, heterogeneous and fragmented. These participants were capable of shifting the direction of the policy debate towards their interests by maximizing their influence in comparison to the peripheral actors.

The k-core analysis helps identify a core of highly active participants who differ in the intensity of their links from a group of more peripheral actors, but it does not adequately describe the relative positions of the core actors vis-à-vis their ability to exert influence, and the interactions between them Given that the study research questions include: 'What are the most important actors and networks within the UHC policy process?' these issues need to be addressed. Centrality measures provide a tool to address this issue. Centralisation is characterised by an actor's position within a particular network, thereby addressing the importance of an actor's 
influence within the network (Prell, 2012). The next stage of the SNA focuses on the precise identification of key actor networks for the six relationships of the policy network.

\subsection{Investigating core group interactions via measures of centrality}

\subsubsection{What is centrality analysis?}

In the present study centrality analysis focuses on interaction between actors with the highest k-core scores, what we might term core-core interaction within the core group in the UHC policy network. It measures relations between network actors using various centrality methods such as degree centrality, betweenness centrality, closeness centrality, eigenvector centrality and other variances (Halgin and Borgatti, 2012). Unlike cohesion measures (such as density) and sub-group cohesion measures (such as k-cores), centrality provides a more precise description of an actor's positioning in a network graph. Labels such as prominent, leader, prestigious, and influential are used to characterise the position of an actor within the social circle (Valente et al., 2008).

According to Borgatti et al. (2013), degree centrality is the most intuitive out of the centrality techniques. It measures each relationship by adding the number of ties that an actor receives from other actors. Degree centrality identifies the most influential actor by its degree size. For instance, policy actors or policy organisations with a high degree of centrality in each relationship might theoretically exercise substantial influence within the policy process.

However, degree centrality is the least powerful measure of centrality. The other three centrality measures (betweenness centrality, closeness centrality, and eigenvector centrality), unlike degree centrality, take into consideration the rest of the network when calculating the score for a given participant. A small network of highly central policymakers in the UHC policy process can be identified and separated from their colleagues using these centrality measures.

We will now review findings generated by the different centrality measures listed above. The following sub-sections deploy the tools of degree centrality, betweenness centrality, closeness centrality and eigenvector centrality to identifying key actors for each of the six modalities of engagement (the relationships) used in this network analysis. The findings show that each centrality measure identified two key classifications within the core-periphery structure of the policy network; these are the "domestic” and the “foreign” sub-networks. 


\subsubsection{Degree centrality and power actors}

According to Prell (2012), degree centrality is mostly interpreted as a measure of influence and power in a social setting. One might predict that nodes (i.e., actors) with higher degree centrality have better access to UHC information and resources or have the influence to steer the policy process towards their own objectives. The findings show that the degree centrality of all six network relationships breaks the core sub-group into two key sub-networks (foreign and domestic). We have given the designation 'power actors' to the nodes with the highest degree centrality values in both the foreign and domestic sub-networks. Figures 29 and 41 provide visualisations of the k-core and degree centrality measures for the "read" relationship (which is again selected as our example). Readers will recall that in Figure 29, the k-core measure identified three cohesive sub-groups (core, periphery and isolates) in the read network where the core sub-group is encircled in red, the periphery in yellow and the isolates in black. If the red section of Figure 29 is over-laid on Figure 41, there are seven prominent core nodes with a higher degree of centrality and these are actors from the WHO, USAID, the World Bank, FMoH, NMA, NHIS and SMoH. These seven nodes form the key network actors within the core sub-group based on degree centrality. The first three are foreign organisations, while the last four are domestic. These organisations are respectively the most influential foreign and domestic actors within the UHC policy network on the "read" relationship.

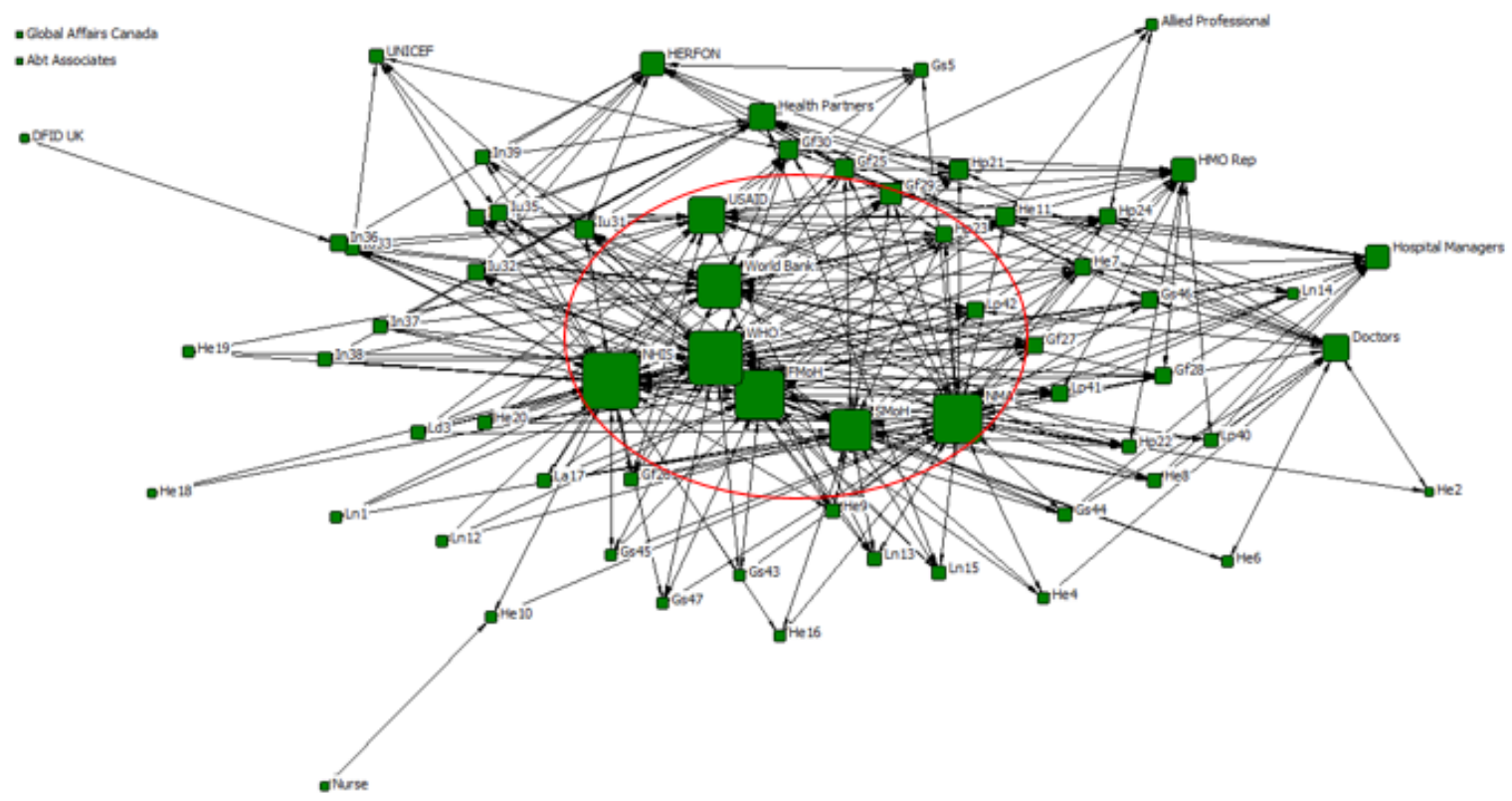

Figure 41: Visualisation of the degree centrality measure of the 2-mode (bipartite graph) “read" relationship of the policy network. 
The policy network holds directional and non-valued data possible for the indegree and outdegree centrality measure on all six relationships. The indegree centrality evaluates the number of interactions a node receives from other nodes, and outdegree centrality measures the opposite. Indegree centrality often interprets prestige or popularity, and outdegree centrality interprets expansiveness (Borgatti et al., 2018). Findings show that the indegree and outdegree centrality score of every node in all six relationships have the same degree centrality score, and this has no significant impact on the degree centrality's interpretation. It is important to note that the drawbacks of using degree, in-degree and outdegree centrality to calculate meaningful links among actors in the same policy network do not account for missing actors. It increases the likelihood of bias which is sensitive to the representation of the network. For instance, the degree, in-degree and outdegree centrality calculate based on the given network data and where the more representative the network is, the decrease in a bias of the network interpretation (Borgatti et al., 2018).

\subsubsection{Betweenness centrality and the gatekeepers}

Betweenness centrality measures the number of times that an actor acts as a bridge along the shortest path between two other actors. This aspect of centrality emphasises the ability to control access to information so as to influence decisions affecting UHC policy. In general, it measures the potential control that key actors have over the flow of information. Therefore, actors with high betweenness scores are capable of connecting two actors at different sub-graph levels of a network. 'Gatekeepers' are in a position to threaten the network with the disruption of operations. For example, inside the red core circle of the "read" relationship, Figure 42 shows seven prominent nodes with a higher betweenness centrality measure. The NHIS and NMA nodes (circled red in Figure 42) have the highest betweenness scores in this graph. 


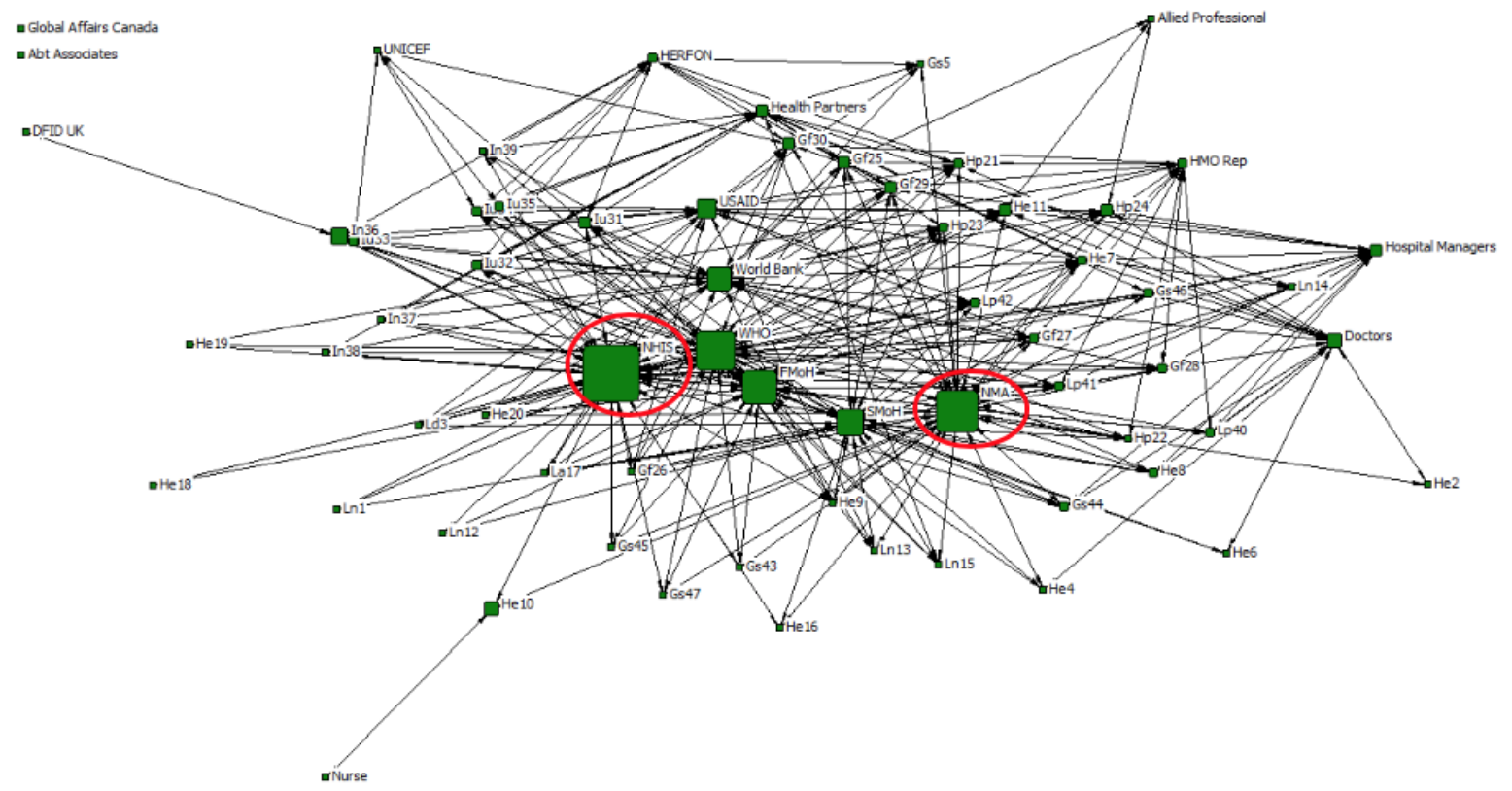

Figure 42: Visualisation of the betweenness centrality measure of the 2-mode (bipartite graph) “read” relationship of the policy network.

SNA explores weak points in networks by examining what would happen if certain nodes were removed. A situation in which removal of an actor would cause a network structure to divide into unconnected parts is termed a "cut point", while the divided parts thus created are terms "blocks". Figure 43 shows the cut-point and block measure of the "read" relationship where there is a core-to-periphery connection between the core subgroups-NHIS-In36-DFIDUK and the core subgroups-NMA-HE10-Nurse. Both the NHIS and the NMA therefore appear to exercise substantial power as a bridge or gatekeeper controlling core-to-periphery connections in the network. 


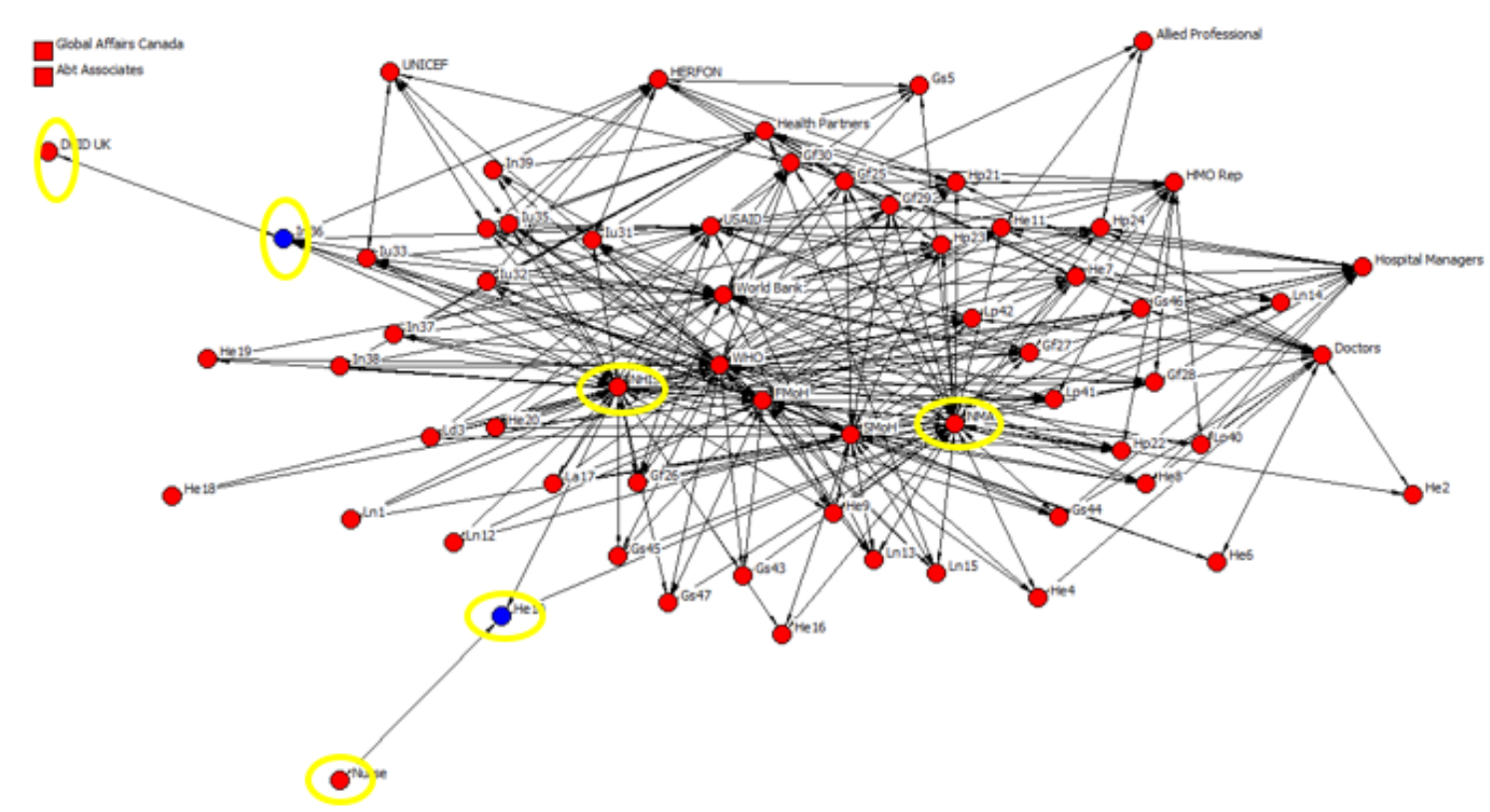

Figure 43: Visualization of the blocks and cut-points of the 2-mode (bipartite graph) “read" relationship of the policy network.

Note: This is computed with three blocks, each containing one member and two cut-points (Iu36 and He10) isolating nodes nurse and DFID UK all circled yellow.

The findings of the betweenness centrality measure are similar to that of degree centrality; there are two key networks with nodes either belonging to a foreign or domestic organisation. WHO, USAID and the World Bank nodes have the highest betweenness centrality within the foreign network and FMoH, NMA and NHIS have the highest betweenness centrality within the domestic network?

It is important to note that the policy network variables are vulnerable to omission errors such as missing edges and nodes, which affect the betweenness centrality identification of key actors and determine node-level scores. Missing data can make policy actors in the policy network seem less significant or more important than they appear (Borgatti et al., 2018). Therefore, the study attempts to resolve this limitation by collecting data above the minimum size required for a network boundary.

\subsubsection{Closeness centrality and influence through proximity}

Closeness centrality calculates the shortest paths between all nodes, then assigns each node a score based on the sum of its shortest paths. Closeness centrality scores are used to rank each actor (node) based on their 'closeness' to all other actors within the network, and thus to 
identify the key actors who are best placed to influence other actors in the network. Closeness centrality is best measured within a well-connected network (Prell, 2012). This is an "inverse" measure of centrality, meaning that a node with a higher score is highly peripheral (vice versa for nodes with a lower score). The underlying assumption is that if a policymaker is not central, he/she generally tends to depend on individuals linked to policymakers at the centre of network to stay informed about the UHC policy discourse (Freeman, 1979). Thus, if we examine the DFID UK, Allied Professional, and Nurse nodes we can see that on average across all six relationships of the policy network these have the highest closeness centrality scores. This suggests that these are highly peripheral actors in the UHC policy dialogue and tend to rely on the information coming from peripheral policy actors received from the central actors (see Figure 44).

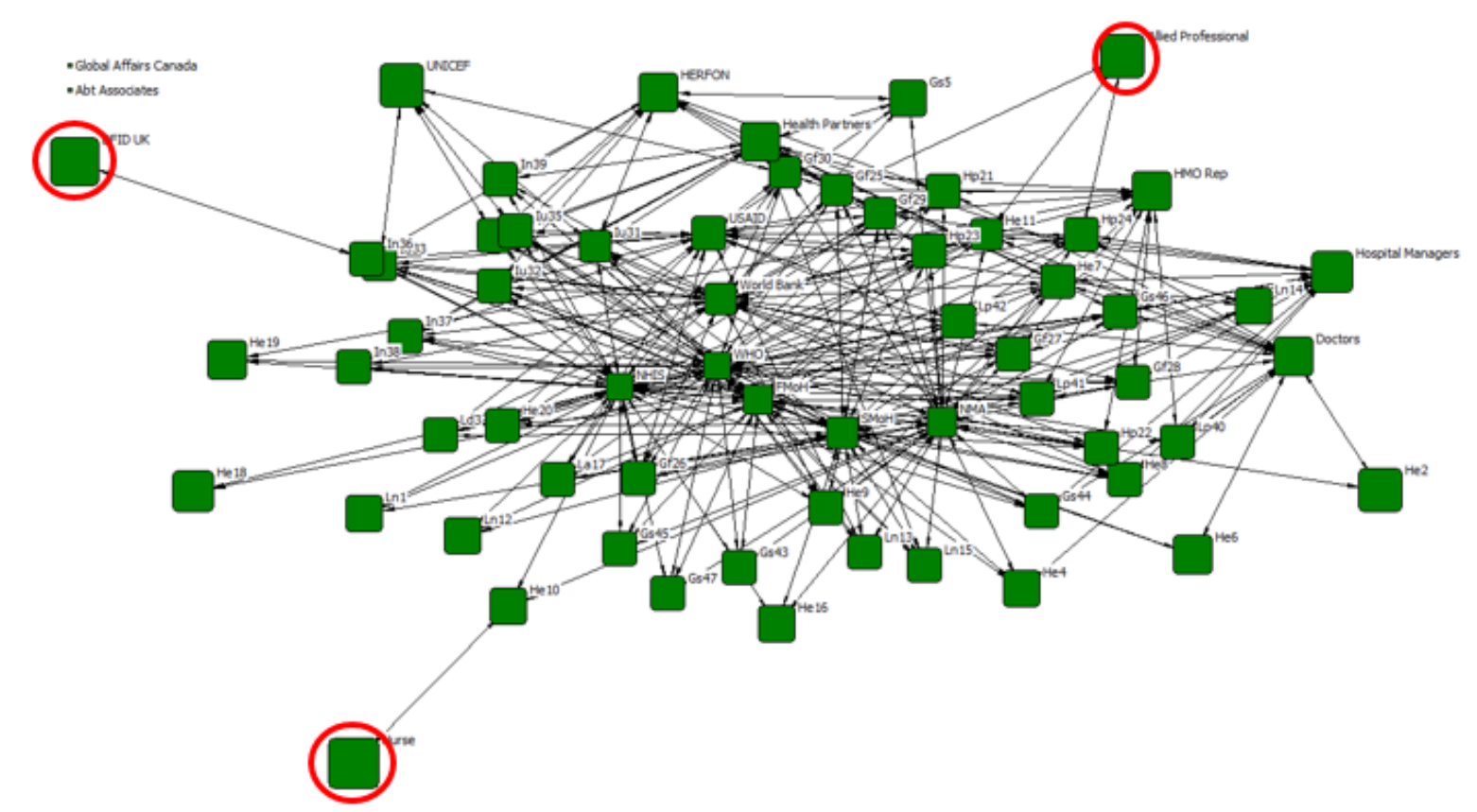

Figure 44: Visualization of closeness centrality of the 2-mode (bipartite graph) "read" relationship of the policy network.

Measuring disconnected networks with closeness centrality is very problematic. Fortunately, all six relationships of the policy network are well-justified without variance on the closeness centrality scores, an issue the centrality suffers with, which leads to poor correlations with any variable. This is why closeness centrality is of limited use as a centrality measure (Borgatti et al., 2018). 


\subsubsection{Eigenvector centrality and how policy actors link to power actors}

Eigenvector centrality is a variant of degree centrality. It examines nodes that are immediately adjacent to actors with high degree centrality or who are identified as powerful actors on other measures (Bonacich and Lloyd, 2001). This measure can be seen of as a more refined version of degree centrality (Borgatti, 1995). It identifies nodes directly connected to influential nodes or nodes with high degree centrality. The underlying idea is that policy actors who have a close connection with power actors are more influential than policy actors who have no such connection. Policy actors with higher eigenvector centrality are connected to the power actors in all of the six relationships of the policy network. Unlike the earlier classifications of the core sub-group via other centrality measures, the group of actors with maximum eigenvector centrality includes a high proportion of participants who do not work in the larger policy organisations. Thus Hp21, Hp22, Hp23, Hp24 have the maximum value of this centrality (see Figure 45). The nodes that link to the nurse, allied professionals, DFID UK, ABT associates and Global Affairs Canada are not connected to any power source, and so have low eigenvector centrality.

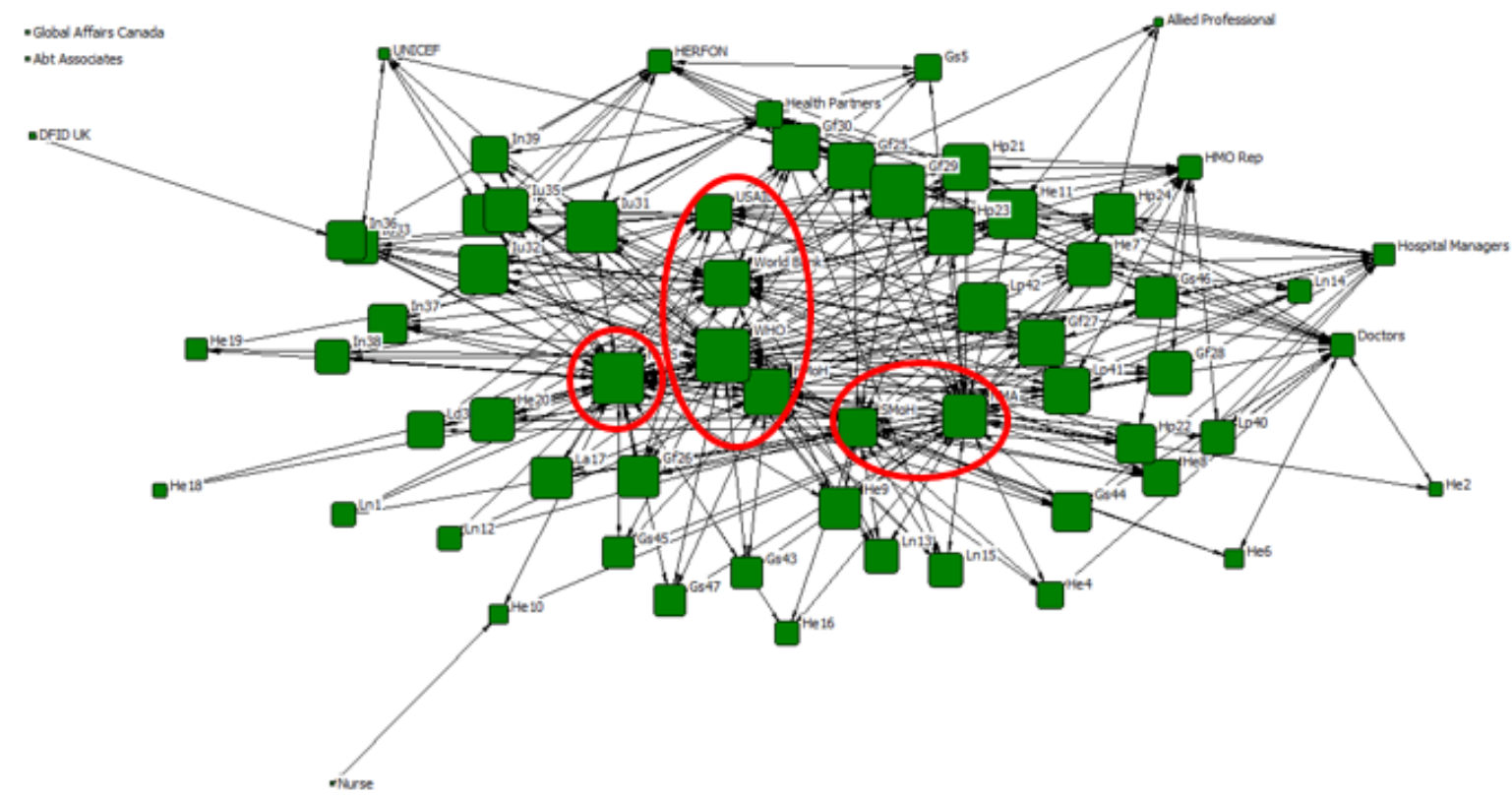

Figure 45: Visualisation of the eigenvector centrality measure of the 2-mode (bipartite graph) “read” relationship of the policy network. 
An important issue with eigenvector centrality is that in disconnected networks it will assign zeros to all members of the smaller components. Furthermore, to have an ideal eigenvector measure, the actor with the largest eigenvalue should be at least two or three times larger than the actor with the second largest eigenvalue. Otherwise, the measure will need more than one eigenvector to appropriately interpret the position of actors within the network, and it is not the case for all six relationships in the policy network.

\subsection{Identifying Multi-relational Policy Actors within the Policy Network}

This section applies a QAP correlation run on a UCINET analysis where all six relationships of the policy network are manually overlapped and compared using their topological and functional properties. It is important to note that not all policy actors have equal impact in a policy network. Some actors might be unique due to their multi-relationship connectedness.

A QAP correlation of the all the networks representing each relationship will give robust results on the validity of these name generators and will present a clearer analysis of the relationship overlap. The idea of the Quadratic Assignment Procedure (QAP) is to identify the value of the measure of association when there really isn't any systematic connection between the two relations (Hanneman and Riddle, 2005). The QAP technique correlates the two matrices by effectively reshaping them into two long columns as described above and calculating an ordinary measure of statistical association such as Pearson’s r. Borgatti and associates (2018) call this the 'observed' correlation. According to Borgatti, Everett and Johnson (2018: 147), "to calculate the significance of the observed correlation, the method compares the observed correlation to a reference set of thousands of correlations between thousands of pairs of matrices that are just like the data matrices but are known to be independent of each other.” To construct a p- value, it simple counts the proportion of these correlations among independent matrices that were as large as the observed correlation. As elsewhere, we typically consider a p-value of less than 5 per cent to be significant (i.e., supporting the hypothesis that the two matrices are related) (Borgatti, Everett and Johnson, 2018). It was decided to hold back the "conflict” relationship for separate analysis, because of its controversial nature, and it will be discussed in a separate section. 

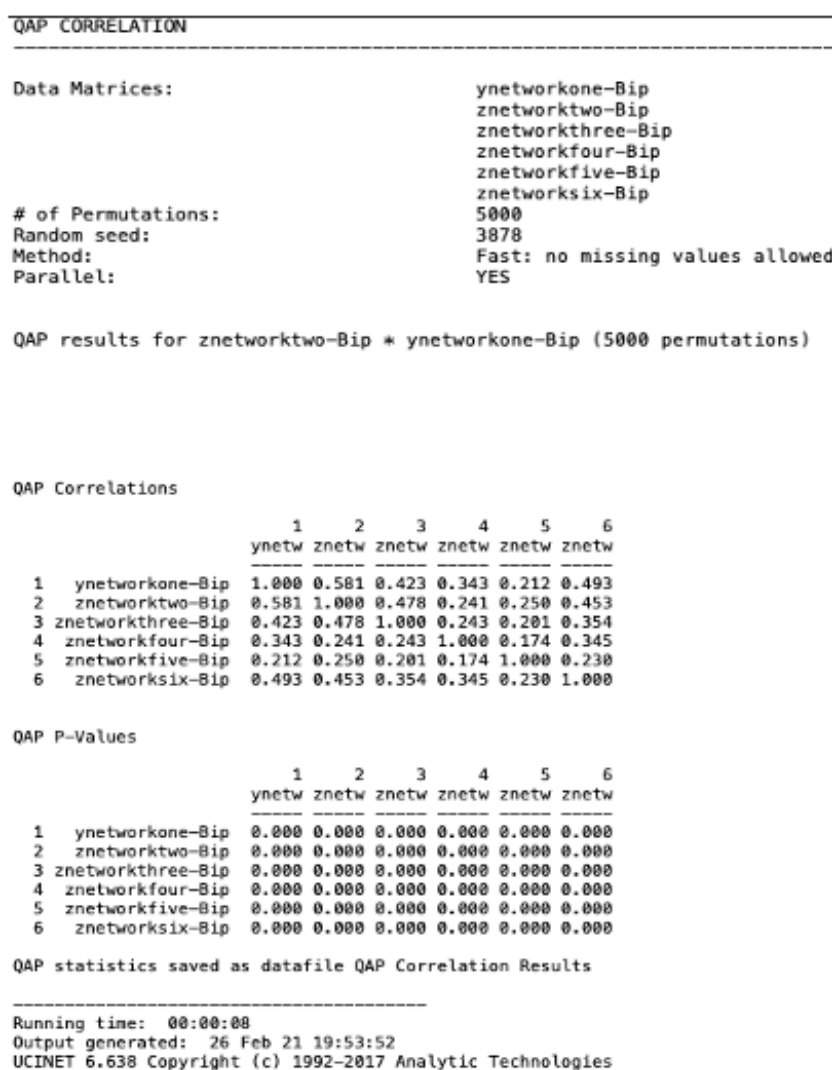

Figure 46: Association between all the networks representing each relationship by a QAP correlation.

Key: Read relationship- ynetw1/ynetworkone; Discussion relationship- znetw2/znetworktwo; Coalition relationship- znetw3/znetworkthree; Affiliation relationship- znetw4/znetworkfour; Conflict relationship- znewt5/znetworkfive; Advice relationship- znetw6/znetworksix.

To illustrate the policy network's QAP correlation, the student ran all relationship in the policy network described in Box 1. SNA measures such as density, centrality, and size activity were employed to characterize and investigate their similarities and differences. The findings relating to centrality showed the importance of a highly active and well-connected set of policy actors in all six relationship core groups. Reconstitution and the overlapping of all five analysed relationships show that the correlation between the network of 'Read' relationship and the network of 'Discussion' ties is 0.581 , and it is highly significant (see Figure 46). We note, for example, that there is an observed simple matching of 0.581 (i.e., if there is a 1 in a cell in network one, there is a $58.1 \%$ chance that there will be a 1 in the corresponding cell of network two). This would seem to indicate association. The results support the hypothesis that the two kinds of ties are related. The moderate and significant QAP correlation (0.493) shows there is a relationship between the number of policy actors that take advice from network members 
they have read and the extent to which they interact with one another. This is not surprising but does reflect the tendency to interact more with actors with whom you have read any published work.

One thing to note in the output is that 5000 permutations were used in this run. It is important to run a large number like this in order to stabilize the p-value. Since the permutations are random, if we only used a handful of them, each time we ran the program we would get a slightly different p-value (but the correction would always be the same). The larger the sample of permutations, the less the variability in p-values (Borgatti, Everett and Johnson, 2018).

\subsection{Conflict in the Policy Network}

The conflict relationship was excluded from the overlapping analysis of the other five relationships of the policy network because of its uniqueness as a measure of tension and controversy within the policy network. Its function is to explore disputes among the policymakers within the ongoing policy discourse concerning UHC. Conflict during the process of making and legislating health policy occurs regularly among stakeholders. Conflict is not new to the health care system, which is a fragmented landscape with many players who from time to time will have divergent interests and objectives (Marcus, Dorn, \& McNulty, 2013).

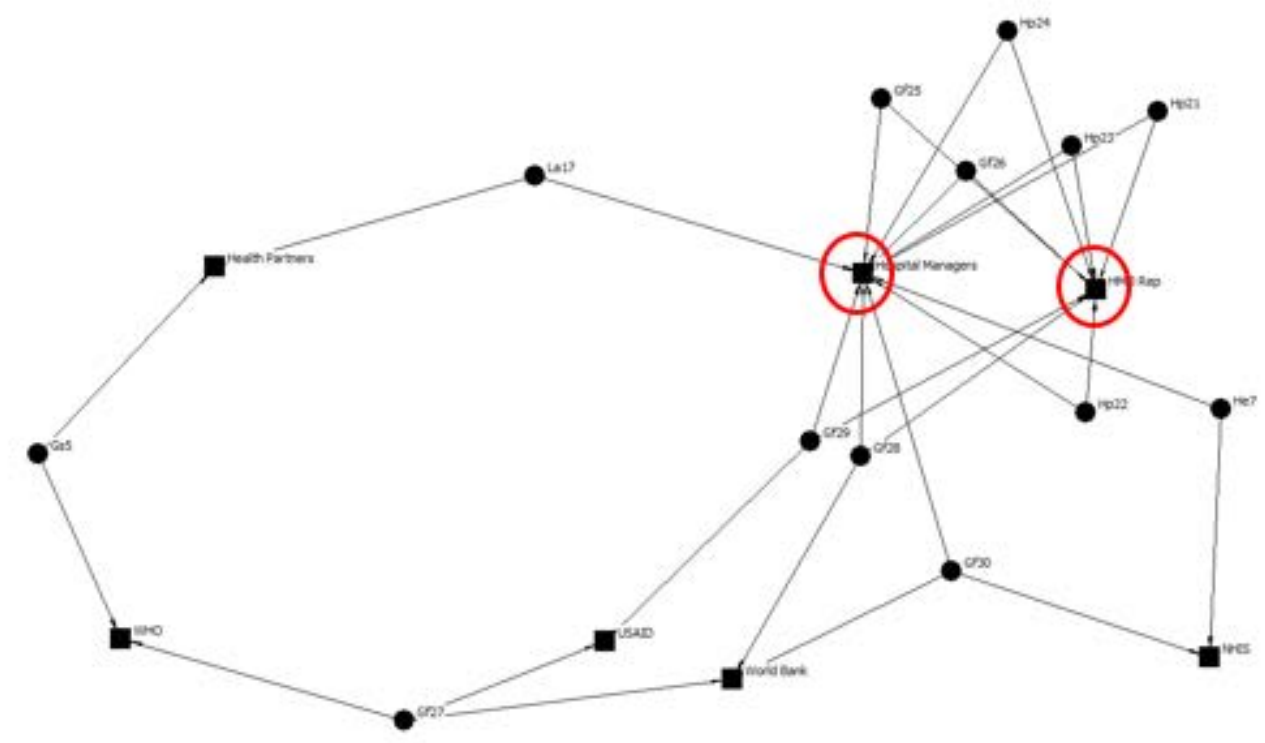

Figure 47: Visualisation of the core group of the 2-mode (dichotomised graph) “conflict” relationship of the policy network. 
The findings in this section focus on policy actors, outside of their coalition of close associates that are involved in conflict concerning UHC policy. K-core, isolates, cut-point/block, and centrality are the primary SNA measures used to gather key findings on the "conflict" relationship (see Figures 47-49). The findings show that the conflict relationship has the highest number of isolated nodes in the policy network. Isolates are considered to be components that are disconnected from a network. Figure 48 shows 30 isolated nodes disconnected from the "conflict” relationship, which would suggest that much of the criticism or opposition to UHC policy comes from individuals not connected to the core group of policy actors.

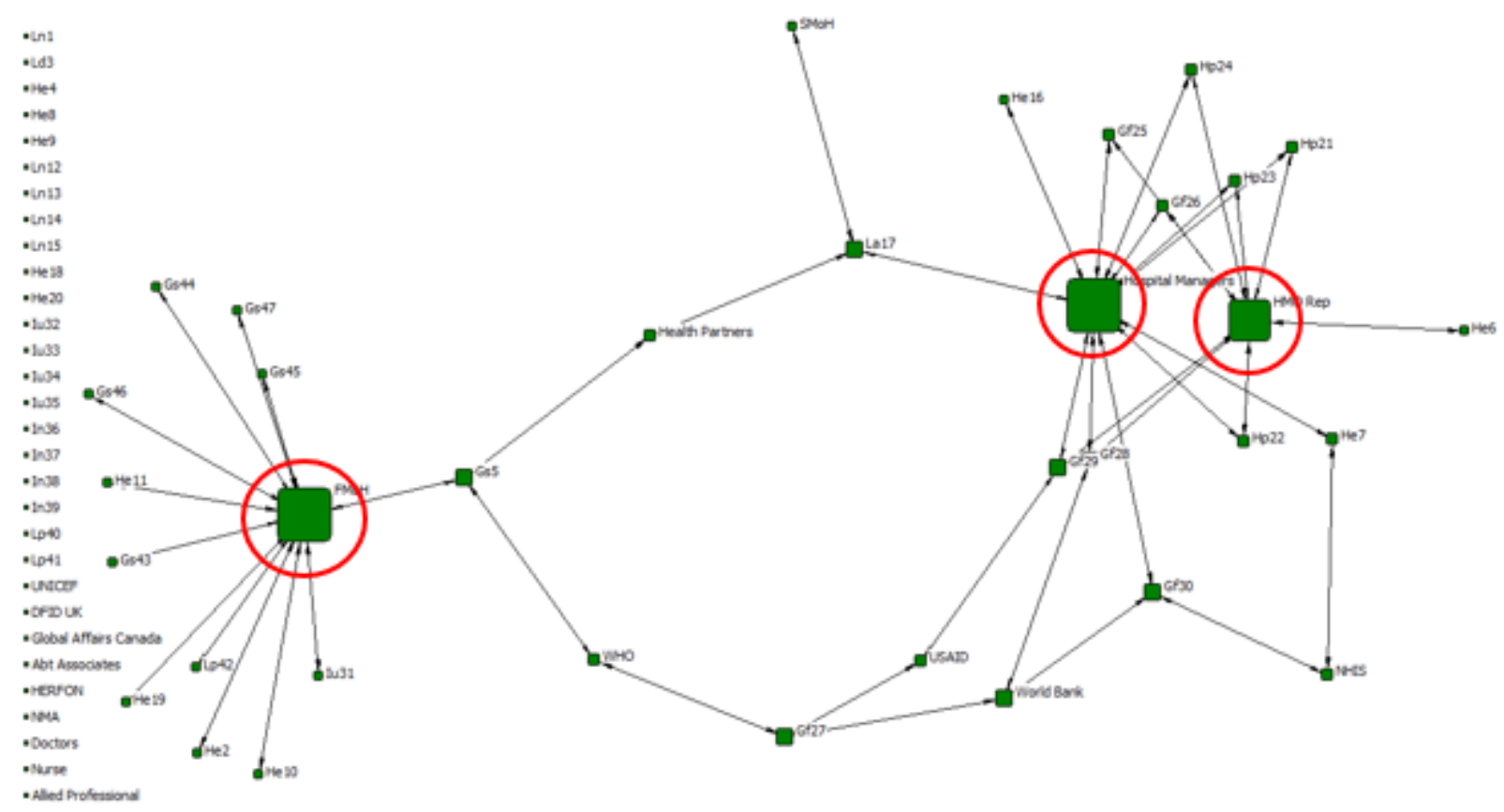

Figure 48: Visualisation of degree centrality measure of the 2-mode (bipartite graph) “conflict” relationship between respondents.

Analysis of the cut-points/block and degree centrality measures identifies three prominent nodes within the conflict relationship: the $\mathrm{FMoH}$, hospital managers, and the HMO representatives (see Figure 48 and 49). This indicates that these actors (nodes) were more likely to be reported by other nodes as being in conflict with them. K-core analysis suggests that several respondents (nodes) reported that they were in conflict with the hospital managers and the HMO representatives. Thus, in the core group of the "conflict" relationship only two (hospital managers and the HMO representatives) of the three prominent nodes (i.e., not including the $\mathrm{FMoH}$ ) are present (see Figure 47). This suggests that hospital managers and 
HMO representatives are the two most prominent nodes in the core group of the conflict relationship (see Figure 47). It is unclear whether this means both were the most likely actors to be involved in disputes about UHC policy or merely the most likely to report or be reported as being in conflict with other nodes.

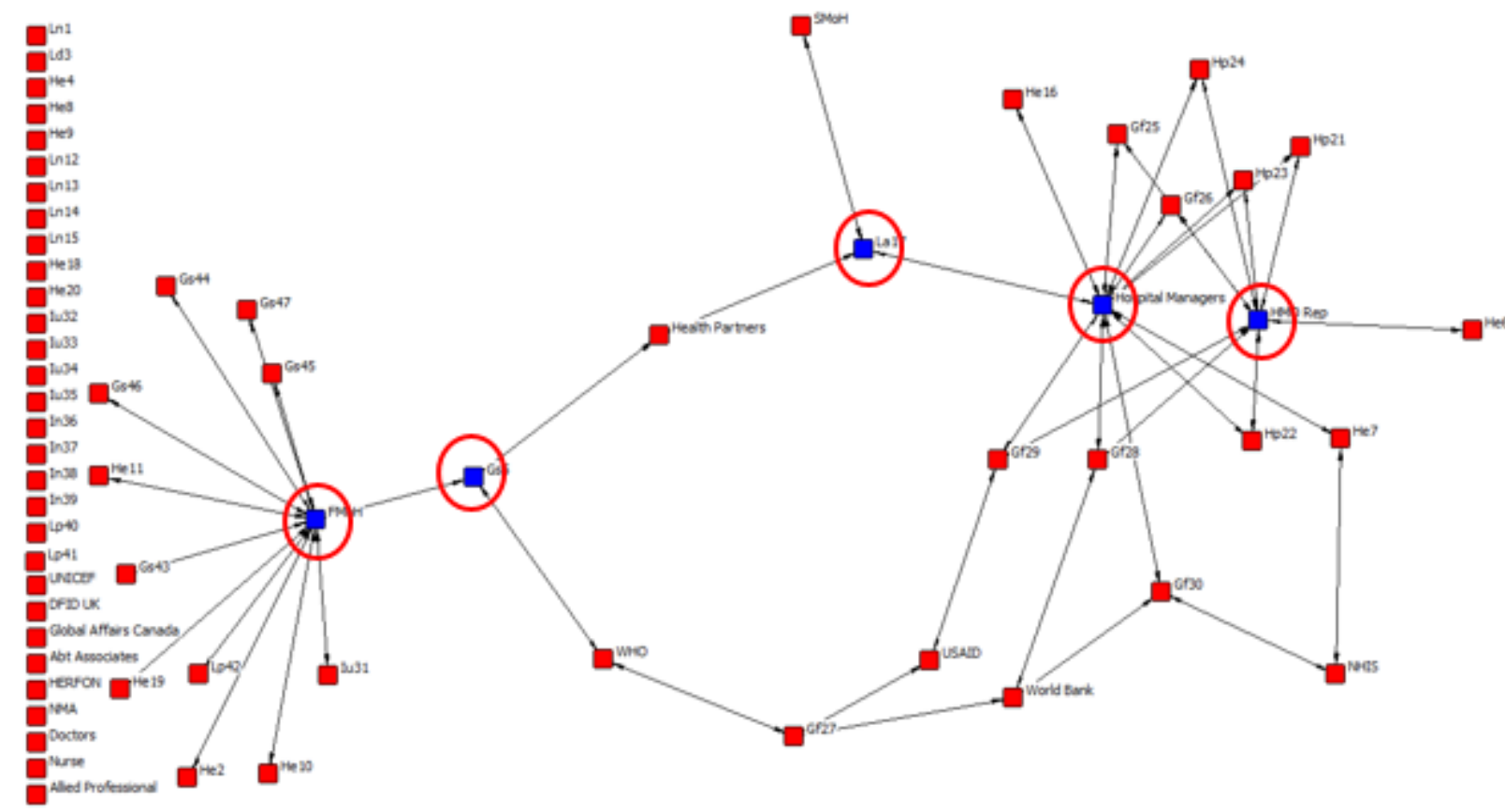

Figure 49: Visualization of cut-points/block measure of the 2-mode (bipartite graph) “conflict” relationship of the policy network.

\subsubsection{Peripheral Policy Actors}

Earlier in the chapter we saw how, employing the k-core measure to examine network “components”, the policy network can be divided into 'the core sub-group' and the 'peripheral' actors. The peripheral actors are confined to an orbit around the augmented core sub-group (see Figure 29). However, these nodes are connected to nodes within the core sub-group, which suggests some form of core-to-periphery relationship. These nodes do not cluster. There are several linear core-to-periphery interactions. However, to identify strategic peripheral nodes within each policy network, the cut-point between blocks and the closeness centrality measures were applied to every pair of peripheral ties that is adjacent to a core node.

A cut-point/block point is a point whose removal would increase the number of components by dividing the sub-graph into two or more separate blocks with no connection to each other 
(Scott, 2017). The findings show that every policy network has a different cut-point/block outcome that is peculiar to their interactions.

\begin{tabular}{|c|c|c|c|c|}
\hline Policy network & \multicolumn{2}{|c|}{ Closeness centrality } & Cut-point/Block & Core subgroups \\
\hline \multirow{2}{*}{ Read relationship } & \multirow{14}{*}{ 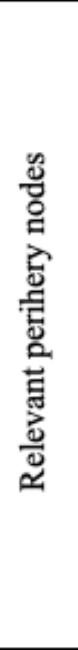 } & DFID UK & In36 & NHIS \\
\hline & & Nurse & He10 & NMA \\
\hline \multirow{2}{*}{ Discussion relationship } & & Ld3 & \multicolumn{2}{|c|}{ NMA } \\
\hline & & DFID UK & \multicolumn{2}{|c|}{ In39 } \\
\hline \multirow{3}{*}{ Coalition relationship } & & $\mathrm{Ld} 3$ & \multicolumn{2}{|c|}{ Doctors } \\
\hline & & $\mathrm{He} 7-\mathrm{He} 2-\mathrm{He} 18-\mathrm{He} 20$ & \multicolumn{2}{|c|}{ Hospital managers } \\
\hline & & Ln1 & \multicolumn{2}{|c|}{ WHO } \\
\hline \multirow{2}{*}{ Affiliation relationship } & & $\mathrm{He} 8$ & \multicolumn{2}{|c|}{ Hospital managers } \\
\hline & & Ln1-Ln-Ln14-Ln15-Ln12 & \multicolumn{2}{|c|}{ NMA } \\
\hline \multirow{3}{*}{ Conflict relationship } & & $\mathrm{He} 6$ & \multicolumn{2}{|c|}{ HMO rep } \\
\hline & & $\mathrm{SMoH}$ & \multicolumn{2}{|c|}{ La17 } \\
\hline & & He16 & \multicolumn{2}{|c|}{ Hospital managers } \\
\hline \multirow{2}{*}{ Advice relationship } & & $\mathrm{He} 8$ & \multicolumn{2}{|c|}{ NHIS } \\
\hline & & $\mathrm{He} 10$ & \multicolumn{2}{|c|}{ HMO rep } \\
\hline
\end{tabular}

Table 10: Relevant periphery nodes of six relationships of the policy network using the closeness centrality and cut-point/block measures.

The significant peripheral nodes for each given relationship were identified by comparing their closeness centrality score together with their cut-point positioning. Closeness centrality is a measure that inter alia indicates the least central peripheral nodes within a graph. For example, the node with the highest closeness score is the most peripheral node, while the node that has the lowest closeness score is the most central. Table 10 shows a summary of the relevant peripheral nodes identified using the closeness centrality and cut-point/block measures in all six relationships of the policy networks (see Appendix 7 for an example of all diagrams regarding the cut-points of the discussion relationship).

\subsubsection{Isolated Policy Actors}

One significant finding from the analysis so far is that the UHC network contains a number of isolated policy actors, actors who differ in their relationship to the core and peripheral network components according to various criteria. Crucially their vertices (the nodes in the visualisations) have no connection in the network in at least some relationships. These are termed "isolates". 
Isolates may be a single node or multiple nodes that are not connected to the network of interest in at least some relationships that are of interest. Identifying isolated policy actors on the periphery of a policy network is essential, as the present network analysis requires knowledge of actors that did not participate in the UHC discourse in particular relationships. The findings suggest that the "conflict" relationship has the highest number of isolates followed by the "coalition" and "affiliation” networks. The "read" and "discussion” networks have the fewest isolates.

\subsubsection{The taxonomy used in the analysis}

Figure 50 shows the framework developed to construct a taxonomy of the policy network. This framework helped to inform the qualitative analysis in the next chapter, based mainly on interview transcripts, needed to answer the research questions. The qualitative analysis can explore and describe the network in conjunction with the (mainly quantitative) social network analysis.

\begin{tabular}{l} 
Connectedness stage \\
\begin{tabular}{|l|l|} 
Component of policy network \\
stage
\end{tabular} Subgroups within network stage \\
\hline $\begin{array}{c}\text { Key institutions and organisations } \\
\text { in policy process }\end{array}$
\end{tabular}

Figure 50: Taxonomy of the policy network analysed in four stages. 


\subsection{Qualitative narrative}

This final chapter section aims to draw out implications of the SNA analysis for the UHC policy process by focusing on the parts played by power actors, peripheral actors, gatekeepers or brokers, isolated actors, and policy actors connected to the power actors.

To investigate and identify relevant sub-networks, the second round of the SNA interviews proper examined the way information was exchanged. Tables 7 and 8 (above) displayed the full structural network taxonomy, showing first the respondents and their organisations, and then grouping these according to institutions - sector, subsector and organisation. Table 11 build on this institutional taxonomy by identifying key policy organisations with the highest average centrality scores (using three measures) in the policy network.

\begin{tabular}{|c|l|l|l|r|r|r|}
\hline \multirow{2}{*}{ Cohesive subgroups } & \multicolumn{1}{|c|}{ Policy actors } & \multicolumn{2}{|c|}{ Institutions } & \multicolumn{2}{c|}{ Sub-sector } & \multicolumn{2}{c|}{ Centrality } \\
\cline { 3 - 7 } & & & & \multicolumn{1}{c|}{ Degree } & Betweenness & \multicolumn{1}{c|}{ Eigenvector } \\
\hline \multirow{3}{*}{ Foreign } & WHO & International & UN Bodies & $18(28 \%)$ & 155.23 & 0.1778 \\
\cline { 2 - 7 } & USAID & International & External Govt Agencies & $13.2(20 \%)$ & 64.42 & 0.1324 \\
\cline { 2 - 7 } & World Bank & International & UN Bodies & $13.2(20 \%)$ & 76.92 & 0.123 \\
\hline \multirow{5}{*}{ Domestic } & FMoH & Government & Federal & $19.2(30 \%)$ & 229.34 & 0.1564 \\
\cline { 2 - 7 } & SMoH & Government & State & $14.3(25 \%)$ & 159.54 & 0.1498 \\
\cline { 2 - 7 } & NMA & Labour & Professional Association & $19(29 \%)$ & 215.36 & 0.1696 \\
\cline { 2 - 7 } & NHIS & Health Provider & Public- Health Provider & $14.7(23 \%)$ & 158.1 & 0.176 \\
\hline
\end{tabular}

Table 11: Centrality of key institutions for all relationships of the policy network.

In Table 11, the nodes exhibiting higher centrality scores were grouped into the two key networks of domestic and foreign networks based on degree, betweenness and eigenvector centrality measure scores (with the exclusion to the closeness centrality measure because it focuses on the most peripheral policy actor). This can be considered alongside six tables illustrating the centrality scores of the key networks in each relationship of the policy network (see Tables 12-17), which include other actors that displayed similar features within their cohesive sub-groups. The key organisation on all six relationships of the policy network are the large nodes inside the red circle red in Figure 41: the FMoH, NMA, NHIS, and SMoH and the WHO, USAID, World Bank. Using the categories of the taxonomy, and based on centrality scores, the FMoH is the dominant power actor, while the NMA, WHO and are influential gatekeepers or brokers controlling the flow of ideas and information, and the World Bank, SMoHs and USAID bring influence to bear through their close connections with the power actor. 


\begin{tabular}{|c|c|c|c|c|c|c|}
\hline \multicolumn{7}{|c|}{ Matrix: Read Relationship } \\
\hline \multirow{2}{*}{ Cohesive subgroups } & \multirow{2}{*}{ Institutions } & \multirow{2}{*}{ Sub-sector } & \multirow{2}{*}{ Policy actors } & \multicolumn{3}{|c|}{ Centrality } \\
\hline & & & & Degree & Betweenness & Eigenvector \\
\hline \multirow{4}{*}{ Domestic } & Health Providers & Public- Health Provider & NHIS & $36(55.4 \%)$ & 368.45 & 0.186 \\
\hline & \multirow{2}{*}{ Government } & Federal & $\mathrm{FMoH}$ & $30(46.1 \%)$ & 202.57 & 0.159 \\
\hline & & State & $\mathrm{SMoH}$ & $26(40 \%)$ & 151.25 & 0.136 \\
\hline & Labour & Professional Association & NMA & $30(46.1 \%)$ & 256.43 & 0.154 \\
\hline \multirow{3}{*}{ Foreign } & \multirow{3}{*}{ International } & \multirow{2}{*}{ UN Bodies } & WHO & $35(53.8 \%)$ & 248.31 & 0.194 \\
\hline & & & World Bank & $27(41.5 \%)$ & 131.34 & 0.16 \\
\hline & & External Govt Agencies & USAID & $21(32.3)$ & 83 & 0.121 \\
\hline
\end{tabular}

Table 12: Centrality of key institutions for the "read" relationship of the policy network.

\begin{tabular}{|c|c|c|c|c|c|c|}
\hline \multicolumn{7}{|c|}{ Matrix: Discussion Relationship } \\
\hline \multirow{2}{*}{ Cohesive subgroups } & \multirow{2}{*}{ Institutions } & \multirow{2}{*}{ Sub-sector } & \multirow{2}{*}{ Policy actors } & \multicolumn{3}{|c|}{ Centrality } \\
\hline & & & & Degree & Betweenness & Eigenvector \\
\hline \multirow{6}{*}{ Domestic } & \multirow[b]{2}{*}{ Health Providers } & Public- Health Provider & NHIS & $30(46.1 \%)$ & 284.45 & 0.18 \\
\hline & & Private- Health Provider & Hospital Managers & $26(40 \%)$ & 221.59 & 0.162 \\
\hline & \multirow{2}{*}{ Government } & Federal & $\mathrm{FMoH}$ & $28(43.1 \%)$ & 234.12 & 0.176 \\
\hline & & State & $\mathrm{SMoH}$ & $24(36.9 \%)$ & 191.94 & 0.151 \\
\hline & \multirow{2}{*}{ Labour } & Professional Association & NMA & $28(43.1 \%)$ & 266.82 & 0.169 \\
\hline & & $\begin{array}{l}\text { Non-Professional } \\
\text { Association }\end{array}$ & Doctors & $22(33.8 \%)$ & 148.2 & 0.138 \\
\hline \multirow{3}{*}{ Foreign } & \multirow{3}{*}{ International } & UN Bodies & WHO & $21(32.3 \%)$ & 111.76 & 0.148 \\
\hline & & External Govt Agencies & USAID & $19(29.2 \%)$ & 68.03 & 0.144 \\
\hline & & UN Bodies & World Bank & $12(18.4 \%)$ & 23.76 & 0.098 \\
\hline
\end{tabular}

Table 13: Centrality of key institutions for the "discussion" relationship of the policy network.

\begin{tabular}{|c|c|c|c|c|c|c|}
\hline \multicolumn{7}{|c|}{ Matrix: Coalition Relationship } \\
\hline \multirow{2}{*}{ Cohesive subgroups } & \multirow{2}{*}{ Institutions } & \multirow{2}{*}{ Sub-sector } & \multirow{2}{*}{ Policy actors } & \multicolumn{3}{|c|}{ Centrality } \\
\hline & & & & Degree & Betweenness & Eigenvector \\
\hline \multirow{3}{*}{ Domestic } & Government & State & $\mathrm{SMoH}$ & $21(32.3 \%)$ & 373.55 & 0.227 \\
\hline & Health Providers & Private- Health Provider & Hospital Managers & $17(26.1 \%)$ & 308.77 & 0.181 \\
\hline & Labour & Professional Association & NMA & $15(23.1 \%)$ & 148 & 0.19 \\
\hline \multirow{3}{*}{ Foreign } & \multirow{3}{*}{ International } & UN Bodies & WHO & $15(23.1 \%)$ & 197.32 & 0.164 \\
\hline & & External Govt Agencies & USAID & $14(21.5 \%)$ & 135.961 & 0.167 \\
\hline & & UN Bodies & World Bank & $13(20 \%)$ & 122.14 & 0.151 \\
\hline
\end{tabular}

Table 14: Centrality of key institutions for the "coalition" relationship of the policy network.

\begin{tabular}{|c|c|c|c|c|c|c|}
\hline \multicolumn{7}{|c|}{ Matrix: Affiliation Relationship } \\
\hline \multirow{2}{*}{ Cohesive subgroups } & \multirow{2}{*}{ Institutions } & \multirow{2}{*}{ Sub-sector } & \multirow{2}{*}{ Policy actors } & \multicolumn{3}{|c|}{ Centrality } \\
\hline & & & & Degree & Betweenness & Eigenvector \\
\hline \multirow{3}{*}{ Domestic } & \multirow{2}{*}{ Government } & Federal & $\mathrm{FMoH}$ & $21(32.3 \%)$ & 344.51 & 0.25 \\
\hline & & \begin{tabular}{|l|} 
State \\
\end{tabular} & $\mathrm{SMoH}$ & $12(18.4 \%)$ & 116.98 & 0.138 \\
\hline & Labour & Professional Association & NMA & $19(29.2 \%)$ & 347.61 & 0.182 \\
\hline \multirow{5}{*}{ Foreign } & \multirow{5}{*}{ International } & UN Bodies & WHO & $14(21.5 \%)$ & 143.93 & 0.184 \\
\hline & & External Govt Agencies & USAID & $10(15.4 \%)$ & 53.81 & 0.15 \\
\hline & & UN Bodies & World Bank & $9(13.8 \%)$ & 53.22 & 0.135 \\
\hline & & \multirow{2}{*}{ NGOs } & HERFON & $13(20 \%)$ & 115.98 & 0.179 \\
\hline & & & Health Partners & $12(18.4 \%)$ & 92 & 0.173 \\
\hline
\end{tabular}

Table 15: Centrality of key institutions for the "affiliation" relationship of the policy network. 


\begin{tabular}{|c|c|c|c|c|c|c|}
\hline \multicolumn{7}{|c|}{ Matrix: Conflict Relationship } \\
\hline \multirow{2}{*}{ Cohesive subgroups } & \multirow{2}{*}{ Institutions } & \multirow{2}{*}{ Sub-sector } & \multirow{2}{*}{ Policy actors } & \multicolumn{3}{|c|}{ Centrality } \\
\hline & & & & Degree & Betweenness & Eigenvector \\
\hline \multirow{3}{*}{ Domestic } & Government & Federal & $\mathrm{FMoH}$ & $12(18.4 \%)$ & 308 & 0.004 \\
\hline & \multirow{2}{*}{ Health Providers } & \multirow{2}{*}{ Private- Health Provider } & Hospital Managers & $12(18.4 \%)$ & 281.78 & 0.345 \\
\hline & & & HMO rep & $9(13.8 \%)$ & 56.98 & 0.278 \\
\hline Foreign & International & UN Bodies & World Bank & $3(4.6 \%)$ & 41.56 & 0.065 \\
\hline
\end{tabular}

Table 16: Centrality of key institutions for the "conflict" relationship of the policy network.

\begin{tabular}{|c|c|c|c|c|c|c|}
\hline \multicolumn{7}{|c|}{ Matrix: Advice Relationship } \\
\hline \multirow{2}{*}{ Cohesive subgroups } & \multirow{2}{*}{ Institutions } & \multirow{2}{*}{ Sub-sector } & \multirow{2}{*}{ Policy actors } & \multicolumn{3}{|c|}{ Centrality } \\
\hline & & & & Degree & Betweenness & Eigenvector \\
\hline \multirow{5}{*}{ Domestic } & \multirow{2}{*}{ Government } & Federal & $\mathrm{FMoH}$ & $24(36.9 \%)$ & 286.85 & 0.193 \\
\hline & & State & $\mathrm{SMoH}$ & $15(23.1 \%)$ & 123.51 & 0.097 \\
\hline & \multirow[b]{2}{*}{ Health Providers } & Public- Health Provider & NHIS & $22(33.8 \%)$ & 295.41 & 0.162 \\
\hline & & Private- Health Provider & HMO rep & $19(29.2 \%)$ & 198.47 & 0.152 \\
\hline & Labour & Professional Association & NMA & $22(33.8 \%)$ & 273.29 & 0.153 \\
\hline \multirow{3}{*}{ Foreign } & \multirow{3}{*}{ International } & \multirow{2}{*}{ UN Bodies } & WHO & $23(35.4 \%)$ & 230.04 & 0.199 \\
\hline & & & World Bank & $15(23.1 \%)$ & 89.51 & 0.129 \\
\hline & & External Govt Agencies & USAID & $15(23.1 \%)$ & 50.72 & 0.08 \\
\hline
\end{tabular}

Table 17: Centrality of key institutions for the "advice" relationship of the policy network.

Table 11 shows the FMoH occupying the most prominent position in all of the policy networks, with an average of 30 per cent degree centrality score, leading to its classification by the author as the main power actor. Approximately 30 per cent of UHC policy discourse goes through the FMoH node in all six relationships of the policy network, and its average degree centrality measure is roughly one per cent more than the next largest node, which is the NMA, two per cent more than the WHO node, and seven per cent more than the NHIS node.

On average, these four policy organisations (FMoH, NMA, WHO and NHIS) have the highest degree centrality measure in every cohesive sub-group. These may be seen as the key policy organisations with the ability to influence a wide range of policymakers engaged in the UHC policy discourse.

The FMoH facilitates UHC policy development through national, state or local advisory bodies, through affiliation with other policymakers and stakeholders, and by indirectly providing reading materials as well as discussion forums that contribute to the policy discourse. As explained by Onoka et al. (2015), the NHIS is a decentralised public health authority set up by the FMoH to improve the health of all Nigerians at an affordable cost by overseeing a social 
health insurance scheme and various other prepayment systems. The NHIS supports achieving universal coverage to improve health outcomes for Nigerian people. It is mandated to provide social health insurance for enrolees and oversees a system where health care services from contracted providers are funded mainly from contributions made by scheme participants, but with some additional subsidy from federal tax revenues. However, the NHIS is a public body influenced by the FMoH in many ways, such as the appointment of its secretary general, the funding subsidy provided to the NHIS and the provision of a progressive legislative environment for the NHIS to deliver on its mission. This makes the FMoH the most powerful actor in the policy network.

According to the National Strategic Health Development Plan Framework (NCH, 2009), the FMoH has a mandate as the national coordinating authority on health issues in Nigeria. It is the highest decision maker in Nigeria's health sector through the National Council on Health, which brings together various groups of stakeholders and policy actors for participation in policy formation and implementation.

Among the international agencies/UN Bodies seeking to promote universal coverage in Nigeria, the World Health Organisation (WHO) is the most influential foreign actor providing technical support towards achieving UHC in Nigeria. The WHO has formed partnerships with various stakeholders in a number of projects that aim to improve UHC in Nigeria (WHO, 2017). It is the third most powerful actor in all six relationships of the UHC policy network and the most powerful actor among the foreign organisations. However, its absence from the nexus of conflictual relationships, shows that, whether through design or because of a lack of lack of influence on local actors, it has little involvement in domestic debates about the role of HMOs and the private sector.

The application of SNA to the six relationships of the UHC highlights the role that the FMoH, NMA, WHO and NHIS play as key actors exerting influence and creating alliances within the UHC policy debate. It provides ways of quantifying the power these key policy organisations exercise, and how relationships to these central actors' help determine the degree of influence that other policy organisations and actors can bring to bear.

Table 11 shows the actors with the highest average betweenness centrality scores for all six relationships of the policy network. FMoH, again, has the highest betweenness score in the network. Most of its gatekeeping or brokerage occurs when it acts to liaise between other actors, 
meaning that the $\mathrm{FMoH}$, more than any other organisation, facilitates the exchange of information between state and non-state groups, international and national partners and local and state groups.

The newly elected President of Nigeria recently highlighted the importance of the FMoH in rejuvenating primary health care (PHC) as a pathway towards a stronger health care system and a prerequisite for achieving UHC in Nigeria. To improve the societal benefits for Nigerians, the $\mathrm{FMoH}$ facilitates its global relations by collaborating with different groups of healthcare partners to support its policy strategies. The FMoH organises various forums or platforms in conjunction with health bodies such as the Public Health Foundation of Nigeria and the UK's Faculty of Public Health (a public health association with charity status), which provides the opportunity for various stakeholders to network and discuss UHC-related issues.

A second key organisation, because of its connecting role, is the NMA. It is responsible for forming ties between the FMoH and the labour workforce. NMA, as an association, is the most powerful professional representative body for healthcare professionals in Nigeria and it mediates the exchange of information between medicine and other health professions.

NMA, as a gatekeeper or broker, has the potential to control the flow of information on UHCrelated policy guidelines and frameworks coming from the $\mathrm{FMoH}, \mathrm{SMoH}$ NGOs and UN bodies to doctors and related professional networks. For example, the NMA node reaches its maximum score when it lies along the shortest paths connecting actors from the labour sector and international bodies, government and health providers. The findings show that the FMoH and the NMA control much of the flow of information between policy organisations and actors, and thus influence any learning which might occur in the process of UHC policymaking.

Turning to foreign network actors, the World Health Organisation acts as a key gatekeeper in all six policy relationships of the network. It is a UN body extensively involved in the implementation of UHC in many LMICs. One source of influence is that it provides technical liaison support to central departments such as the FMoH and actors in the state Ministries of Health. It also functions as an international consultant to other non-governmental organisations, as a gatekeeper of global resources available to support healthcare system development, and as a channel for disseminating knowledge generated by global health policy discourses. Together with the NMA and the NHIS, WHO is one of three gatekeeper organisations, that alongside 
the $\mathrm{FMoH}$, act as brokers to control the exchange of ideas and information within the policy network.

The implementation of UHC in Nigeria is a dynamic process that brings together multiple policymakers and key stakeholders with different vested interests, and differing visions of how Nigerian citizens can achieve access to affordable healthcare (Onoka Hanson and Hanefeld, 2015). Conflict arises in any decision-making process, especially where there are continuous social interactions. Conflict is a dynamic process that can be positive or negative, healthy or dysfunctional, within the domain of policy making (Birkland, 2016). Figure 47 shows the visualisation of the lines of conflict in the policy process. Figures 47 to 49 demonstrate how conflict is centred on three policy actors (in red circles) - the FMoH, hospital managers, and the HMO representatives - these are the three policy organisations that have the highest degree centrality in the graph. This appears to reflect a domestic tension between the FMoH and private health care providers regarding how health care coverage can best be extended.

The underlying tensions between government plans and the ambition of private sector organisations to increase the volume of paid-for services they provide as Nigeria moves towards UHC have become clearer since fieldwork ended. In July 2017, the FMoH indicated that it wished to see a curtailment of the role of Health Maintenance Organisations (HMOs) in Nigeria. The Minister of Health, Professor Isaac Adewole, cited the results of a two-day investigation hearing organised by the House of Representatives Committee on the healthcare service compliance rate of HMOs. The investigation found that many HMOs had mismanaged funding from the NHIS, mistreated enrolled patients, and neglected their responsibilities to offer affordable care to vulnerable patient groups (Ovuakporie and Nwabughiogu, 2017). The lines of disagreement were illustrated when the Chairman of the Health and Managed Care Association of Nigeria (HMCAN), Dr Babatunde Ladapo, responded by saying that HMOs could play an important role in achieving the global benchmark of UHC for Nigeria. He argued that the problems found had arisen because of the federal government's failure to make HMOs follow the rules and stated that it was not the HMOs' responsibility to manage the social health insurance scheme (Ifijech, 2017).

The SNA yielded several important findings concerning the key policy actors, the patterns of influence, and knowledge-sharing relationships found in the network. Senior government officials were at the centre of UHC policy and decision-making and were also influential in controlling the flow of ideas and information within the network. For this reason, the FMoH 
has been classified as the main power actor. Six other organisations or organisational groups were close to the centre of the network. The NMA, WHO and the NHIS acted as brokers of ideas and information, while the SMoHs, World Bank and USAID exerting influence through their close connections of the Ministry. The FMoH, NMA and the WHO in particular, are in positions to influence the UHC policy process, but may need support from other policy actors with whom they are connected if particular initiatives are to succeed. These alignments will differ according to the sectors and issues involved. Thus, the HMOs, unions, doctors and other health professions may all exercise power from time to time in their spheres of influence. Much of this is to be expected, but certain organisations were less prominent than might have been predicted. The World Bank, which has been a major player in UHC reform because it controls substantial development funding is not currently providing any substantial funding to support Nigerian UHC and was less central to UHC policy development in the period studied than was the WHO. Considering the decentralised organisation of the Nigerian healthcare system with 34 SMoHs having considerable devolved authority over the organisation of state health services, these state bodies occupied a less important position within the central cluster of policy organisations than might have been expected. The fact that the SMoHs were not a major source of advice, suggests that engagement with the FMoH was not as close as might have been anticipated, and this may connect with qualitative findings presented in the next chapter. The position of the HMOs and professions towards the periphery of the networks on many of the six influence relationships suggests that, despite fears about the malign power of the private healthcare sector in the Nigerian public insurance system, its influence was on the wane in the period studied. Interesting the low scores of the international bodies on the "conflict" relationship suggest that they have avoided entanglement in the controversy about the role of the private sector; whether because of a desire to maintain a neutral status or other reasons they have not taken sides.

From the quantitative analysis, we can see that the results of the SNA can usefully complement the findings from the qualitative analysis. The SNA method can illuminate the general patterns of interaction between the policy actors and provide a series of 'snapshots' of the various relationships of the UHC policy network. Identifying the policy actors and the policy network framework provides a map of the network and its subgroups that is a foundation for more detailed examination of the perspectives of those concerned. The study now seeks to shed further light on the associated relationships, views and interactions by discussing findings from the qualitative interviews. 


\section{CHAPTER 6}

UNDERSTANDING THE UHC NETWORK VIA POLICY PROCESS THOERY

\subsection{Introduction}

This chapter examines the findings from the semi-structured interviews which were carried out face-to-face, and which comprise the second primary data collection method used in this research. It describes the perspectives of the respondents regarding the main aspects of how UHC policy is financed, formed and contested. The theoretical framework used to examine the formation and implementation of UHC policy combines elements of Walt (1994) and Gilson's (2012) policy process approach with Kingdon’s (2010) Multiple Streams Framework (MSF). The chapter identifies certain stages in the change and implementation processes of Nigerian UHC policy.

The chapter considers aspects of the policy triangle approach (Walt, 1994), including the key actors and - most importantly - the power relationship. The chapter considers various relationships of power and how ability to marshal certain forms of power affected the way actors engaged in the policy discourse. It considers whether implementation depends mainly on rational-technical planning or is more realistically viewed as the outcome of struggles between contending groups of actors exercising their power as policy develops (Gilson et al., 2018). Such an analysis requires an examination of the role of key policy actors, and how they interact with each other in the UHC network in Nigeria

The Multiple Streams Framework (MSF) is useful because it allows us to identify policy entrepreneurs, their engagement in networks, and their ability to use opportunities for agenda setting to move items onto the formal Government policy agenda for UHC-related reforms. The framework describes how the characteristics of policy issues together with current political circumstances, can lead to the opening and closing of 'windows of opportunity' for moving issues onto the agenda to begin the policy process. The MSF describes the interaction of three 'streams' implicated in this process: the problem stream, the policy stream and the politics stream. Policies only rise on to the policy agenda of the government when the three streams run in the same direction.

Kingdon (2010) states that these three streams flow along different channels and are independent of each other until a policy window opens. Solutions are then connected to 
problems, and both are joined to favourable political forces, leading the streams to converge. An issue rises towards the top of an official (or institutional) agenda actor in the relevant departments and agencies begin to address it. This framework offers a useful way of understanding the shape and nature of the policy process within UHC Nigeria.

The sections that follow will examine the actors, the groups into which they align, their relative power, and the evolving content of policy - the chronology of the policy story. Each will be examined taking account of specific features of the Nigerian context. The chapter will conclude with consideration of actors' views on the future of UHC in Nigeria and developments since fieldwork ended.

\subsection{Policy actors and the UHC policy network}

The development of UHC policy in Nigeria occurred within a policy network characterised by the heterogeneity of prominent actors, including those of government (Federal, State, and local), NGOs, UN Bodies, labour groups, multilateral agencies, think tanks, the private health sector, and medical professionals. Participants involved in the semi-structured interviews come from many different policy institutions represented within the UHC discourse in Nigeria.

There is the assumption among many participants that the involvement of a diverse range of policy actors is beneficial for the development of UHC policy and will magnify the efforts towards achieving UHC in Nigeria. This may occur through various public and private-sector organizations, resources, and influences motivated by various inter-related interests, ranging, for example from the provision of affordable health services, and increases in health system budgets, to the creation of new opportunities for private healthcare providers.

Some participants shared views such as:

"In order for the beneficiaries of our health system to access quality health services they need to appreciate the Federal Government's and different health parastatals' effort which encourages foreign health organizations involvement into achieving UHC policy in the country." (RC.28)

"If I am to mention key organizations, I would mention HERFON, Nigerian Health Watch, WHO, World Bank, UNICEF, FMoH, Federal Government even different bilateral agencies most notable USAID, DFID. These actors were key supporting of the different aspect of the advocacy.” (R26) 
"Different policy makers were involved in the process... but to my knowledge common health experts like NMA, HMOs, WHO, FMoH, SMoH, NHIS and the National Assembly were 70 per cent involved in setting the policy agenda towards UHC implementation in the country." (RC.13)

This section focuses on the reflections of participants about the range of policy actors involved and how they helped shape the discourse on UHC policy. Of course, within Walt and Gilson's (1994) policy process framework, policy actors are a key component in the policy triangle and the power dynamics of policy formation and implementation.

\subsubsection{Organisations rather than individuals as key policy actors}

Given that policy actors are so critical, one significant early finding was that named individuals were seen by most respondents as being less important than organisational representatives. In line with Weber's (1978) classic theory of the bureaucratisation of charisma, respondents suggested that it was institutions rather than individuals that develop consistent policies over time, so that it was the collective organisational position rather than a particular individual office holder that was important. This would suggest that charismatic individuals and ‘leadership’ are less important in UHC policy making than convergent organisational positions that have a fair degree of consistency over time. Usually these are positions that align with a policy agenda that is being heavily pushed by international bodies like WHO and the World

Bank. This general feeling is illustrated in comments from the following participants:

"So, I can list for you from the government side the names of prominent individuals involved the UHC policy process. Yet, I feel that naming prominent individuals is irrelevant because they work for the government and they represent that part of the institutions and when they retire or move elsewhere their title and representation changes. But the government's (as an organization invested in UHC) agenda stays the same, and I don't know how useful it will be for you if I name each government representative." (RC.30)

"I know a number of them if you want their names in specific.... but having names of notable health professionals in my opinion does not make sense but rather you will achieve more if you focus on the agenda and interests of vital government parastatals, NGOs, labour groups and research institutes involved in the UHC discussions and policy process in Nigeria." (R41)

Many participants believe that it is the organizations that are best seen as the key actors because it is collective organisational perspectives rather than individual perspectives that has greatest influence on the UHC discourse, and that even when forceful personalities are involved this 
remains the case. However, it is important to acknowledge that this does simplify the issue to some extent. Individuals are unable to be disentangled from the organisations where they work; an organisational group is formed of various people, not all of whom speak with a singular voice, so that individual beliefs and values may vary (Buse et al., 2012: 9).

When respondents mentioned which policy-actors were influential they named a range of organisations, and their relative importance was not immediately clear.

"The Government, Ministry of Health and NHIS are very important in Nigeria's UHC development." (RC.32)

"With poor domestic funding from the government, we continue to discuss financing health services to move towards UHC in Nigeria while mobilising financial support from the World Bank as a catalyst towards UHC development." (RC.35)

"UHC is a health policy heavily backed by all tiers of government with the promise that Nigerians must have access to universal health care. The government promises to implement this policy in order to improve access to basic health services and basic health outcomes like malaria, HIV/AIDS and Life expectancy." (RC.40)

"Then in terms of the private sector we have a thousand and one HMOs that are doing one thing or the other in different areas in order to push the health system towards universal coverage." (RC.3)

"The FMoH ensure the government sectors get involved in UHC at the grassroots level through the primary health care sector.” (RC.23)

When the interviews are considered as a whole, and also bearing in mind the SNA findings, it was perhaps predictable that respondents identified The Federal Government, the FMoH and other Ministry of Health parastatals, HMOs and NHIS, the WHO, ILO and World Bank as highly influential actors shaping the development of the UHC policy. The range of organisations mentioned suggests that international as well as domestic bodies have an influence in policy networks responsible for achieving UHC in Nigeria. Thus, the challenge is in part to examine how the various organizations interact and whether their influence is confined to various stages or aspects of policy. This is a complex task because of the large number of actors, or individuals, involved in implementing the UHC process in Nigeria. This section now moves on to consider further interview extracts to suggest how the various players fit into the policy process. It explores the roles of the various bodies in the UHC policy discourse, from where they derive their power and how that power is exercised. 
To help our understanding of why certain organizations are seen as the main players, it is helpful to consider the two relationships of longevity of participation and organisational decision status. Both can be seen as a basis for claims to authority and legitimacy. The first relationship assumes that influence can be gauged by the duration, continuity and intensity of participation in the policy discourse about UHC. The second relationship of decision-making status is concerned with whether an organisational has a specific constitutional, legal or political mandate to be involved in policy formation in some designated area or if its structural position in the field of institutions means that it is inevitably involved. The two may overlap to some extent in that organisations able to exert strong influence over decisions are likely to maintain continuing involvement in the UHC policy discourse over time. However, there are also organisations that lack power over decisions but still maintain a place in the policy network over time, and organisations that have decision power at a particular juncture but then move into the background as policy discussions continue.

\title{
6.1.1.1 Longevity of policy organizations
}

Longevity refers to the fact that some policy organisations remain as significant actors in the policy network, while others are prominent at certain times but then fade into the background. This is important because longevity may be an issue missed in a snapshot image of the policy network produced by an SNA.

One participant described the way some organisations drifted in and out of the 'policy discussion' at different stages of policy development, while others maintained steady involvement:

\begin{abstract}
"So, there are a lot of policy organizations that I have never heard about and some others that I have heard about whose contributions to the UHC agenda enabled several policies such as the signing of the Act. Many of these organizations have left the policy discussion while the rest that are still around are very much contributing to UHC. These organizations have been around before and after the 2014 UHC inception, they start from the government, legislators, Ministry of Health, international donors and UN organizations like WHO. These actors have been around from the beginning as others have fizzled out mainly from the private health sector." (RC.31)
\end{abstract}

International organisations might be more or less involved at different times but did remain as long-term players in the wider policy environment. 
"International organizations that have been there for us and I hope we will continue to have them for a long time are the WHO and the World Bank. They have done a lot in sponsoring the country financially and technically supporting the health system move towards UHC agenda." (RC.29)

This relationship recognizes key policy organizations on the basis of the duration of their participation in policy development. The longevity of policy organizations involved in the Nigerian UHC discourse is relevant because it demonstrates a commitment to supporting, or in some cases stalling, the process over a long period of time. Informants suggested that the UHC policy process underwent changes in the degree of involvement of several stakeholders, as institutions (involved in healthcare) and health organizations either left or joined discussion about the policy initiative. These respondents stated that policy organizations that remain at the centre of things as policy developed included the Federal Government, MoH, WHO, World Bank, and Legislative Committee on Health. However, these differed in the degree of power they had to influence key decisions, with some having continuing involvement but only limited influence.

\subsubsection{Decision-making power}

This brings us to the second relationship of status and power in the decision-making process. There are a range of organisations whose statutory powers and duties, professional mandates or role under international treaties or agreements would seem to put them at the heart of UHC policy making. In many cases they are indeed at the centre of the policy dialogue, but in others their involvement is less central than might have been expected. This suggests that an organisational actor's location in the wider network, its alignment with other actors, and its contingent ability to exercise influence at a particular time need to be considered alongside its formal organisational mandate.

This discussion revisits the insider/outsider typology which is frequently used to differentiate the status of interest groups in the development of policy. Marsh et al., (2009) suggest that the term 'insider group' is associated with a specific style of policymaking and that it is important to avoid conflating group strategy and group status. Grant's (1978) typology of policy organizations distinguished between insider and outsider groups according to the type of contact made and the tactics used to approach government or other influential bodies. In the 
Nigerian context, participants commented on the close relationships between the 'parastatals' (the linked state departments) involved in UHC policy discussions:

"The NHIS is not different from the government... you should also understand that NHIS, NTHC, CDA and NAFDAC have been contributing policies related to the UHC and they are all Ministry of Health parastatals under the federal government. That said, I think one of the unsung heroes also is - not as a person but an organization - is NAFDAC that has also been dealing with issues around quality drugs because if you are pushing for access to care and Nigeria does not have quality drugs and quality care. NAFDAC has also contributed a lot... These organizations are involved immensely both at the federal and state level of the MoH." (RC.22)

"The policymakers in Nigeria that are key in making sure that the UHC policy is implemented start from the government itself; the federal government and then the legislators since they have the power to decide what the enacted policies are, after which the FHoM and SMoH will deliver the policies to local communities through the primary health care, since it's an avenue closer to the people." (RC.9)

Such responses suggest that the core network for policy development does indeed involve a small number of central departments and agencies, and that it is these actors, who retain close links with government, that have greatest influence. Insider groups in the UHC discourse are policy actors who are an official part of the government machinery and are recognised by Ministers as legitimate players in the policy-making circle. These organisations are regularly consulted and are expected to form part of the decision-making process for reform in the health system. Such insider groups either singly or working together become involved in testing policy ideas, overseeing pilot schemes, and otherwise developing UHC policy. Thus, certain key organisations - the Federal Government, the $\mathrm{FMoH}$ and its parastatals, the Legislative Committee on Health, SMoH, NHIS, and NAFDAC had high legitimacy to enact policies connected to UHC.

In contrast to this inner circle, participants described how certain peripheral organisations had more of the character of outsider groups. Some of these are peripheral because they 'chip in' input at certain stages of policy development, and so lack longevity of participation.

"First of all, the advocacy for UHC in Nigeria started with Good-luck Jonathan's signed declaration on UHC in 2014 which also saw the implementation of the 2014 National Health Act. Yet, the pursuit of UHC existed more than ten years prior to this event. This particular advocacy was led by Health Reform Foundation of Nigeria (HERFON) in collaboration with the Department of Planning, Research and Statistics of the Federal Ministry 
of Health (FMoH) and of course there were different partners that chip in efforts - both local and international organizations." (RC.31)

"Part of our role as the NMA within the UHC campaign is to challenge the government to organise favourable conditions for medical practitioners across the country. We advocate for policies that increase the benefits for both medical personnel and various local communities. We do this by forming difficult alliances with other labour groups with the other health labour unions banding themselves under the Joint Health Sector Unions (JOHESU) banner and other organizations invested in the health system.” (RC.40)

Other organisations remain prominent actors in the policy network over time, but only have real influence at certain stages, as when international bodies provide direct technical support on particular policy developments or operate as brokers that bring in external consultants or specialist partners for short periods when the need arises.

"I see the World Health Organization as a foreign stakeholder that brings together bilateral agencies and international organizations with their technical strategies, exchanges and several initiatives to support the government in a successful UHC implementation.” (RC.26)

The outsider groups include both domestic and international policy organizations with specialist expertise that progressively contributed to the UHC discourse over time, but which are external to the government policy-making process. Perhaps the highest profile policy organizations regarded as outsider groups within the UHC discourse are the WHO, USAID, World Bank, NMA and HERFON. For instance, the development of a national communications strategy for UHC required WHO, USAID, World Bank, Results for Development (R4D) and HERFON to work with the FMoH to produce the necessary plan and guidance. The FMoH commissioned three states (Abia, Osun, and Niger States), financed by the basic health care provision fund, to undertake a pilot scheme designed to help Nigerians better understand the value of UHC in their community (Onyedinefu, 2019).

Participants recognise that different actors have different roles or mandates that affect their mode of participation in the UHC discourse. This is not just a straightforward question of relative power, but of the legitimate limits of influence for particular bodies, and an agreed division of labour in the policy-making process. Thus, the international bodies - UN bodies such as WHO, World Bank and ILO, and national bodies such as USAID - must tread carefully in balancing the promotion of global policies against incursion on national sovereignty, and 
generally frame their activities as technical support that draws on the expertise of the international health policy community. This is a different role from that of domestic governmental bodies, which have a democratic mandate to make policy, and non-governmental organisations such as the NMA and HERFON, which have a place in policy discussions because of their influence over the profession and wider public. While all have some influence, the international bodies and domestic NGOs are, relatively speaking, regarded as outsider groups whose level of influence over UHC policy relies on getting the ear of policy insiders, such as the central government departments and their health parastatals.

\begin{tabular}{|c|c|c|c|c|c|c|c|}
\hline \multirow{2}{*}{ Qualitative Findings } & \multirow{2}{*}{ Institutions } & \multirow{2}{*}{ Sub-sector } & \multirow{2}{*}{ Policy Organizations } & \multicolumn{4}{|c|}{ SNA Findings } \\
\hline & & & & Domestic & International & Core & Peripheral \\
\hline \multirow{4}{*}{ Insider Group } & \multirow{3}{*}{ Government } & \multirow{2}{*}{ Federal } & $\mathrm{FMoH}$ & $\mathrm{X}$ & & $\mathrm{X}$ & \\
\hline & & & NAFDAC & & & & \\
\hline & & State & $\mathrm{SMoH}$ & $\mathrm{X}$ & & $\mathrm{X}$ & \\
\hline & Health Sector & Public-Health Sector & NHIS & $\mathrm{X}$ & & $\mathrm{X}$ & \\
\hline \multirow{5}{*}{ Outsider Group } & \multirow{4}{*}{ International } & \multirow{2}{*}{ UN Bodies } & WHO & & $\mathrm{X}$ & $\mathrm{X}$ & \\
\hline & & & World Bank & & $\mathrm{X}$ & $\mathrm{X}$ & \\
\hline & & External Govt Agencies & USAID & & $\mathrm{X}$ & $\mathrm{X}$ & \\
\hline & & NGOs & HERFON & $\mathrm{X}$ & & & $\mathrm{X}$ \\
\hline & Labour & Professional Association & NMA & $\mathrm{X}$ & & $\mathrm{X}$ & \\
\hline
\end{tabular}

Table 18: The nature of the UHC network.

Overall then the interviews paint a picture in which policy is largely steered by a cluster of insider organisations comprising the Federal Government, the Legislative Committee on Health, FMoH, SMoH, and NHIS, and outsider organisations - such as NAFDAC, USAID, World Bank, NMA and HERFON - that remain highly visible in the policy network but exert real influence only from time to time. Classified into insider and outsider groups, the key policy organizations mentioned in the interviews fall into a similar pattern to the core/periphery and domestic/international network patterns emerging from the SNA (see Table 18). The policy organisations seen by respondents as the insider groups (Federal Government and its parastatals such as the Legislative Committee on Health, FMoH, SMoH, NHIS, and NAFDAC) are present in the core and domestic category of the SNA framework. The policy organizations, such as the WHO, USAID, World Bank, NMA, and HERFON, listed as outsider groups by respondents were split between domestic and international and between core and periphery in the SNA.

However, alongside this broad similarity there were some differences between the findings from the qualitative interviews and the SNA. Interview respondents tended to categorize policy organizations according to their perceived ability to influence decision making within the UHC 
discourse, while the SNA identified the most influential policy organizations according to their position, location, interactions, and metrics in a social network. This meant, there was less consensus among respondents in the interviews than in the SNA, and that a more complex picture of the extent of tensions and conflict within the policy network emerged.

\subsection{Contending groups}

Nigeria's push towards UHC is attracting interest from international health policy experts and increasing optimism about the prospects of successful reform. Yet the interesting finding here is that different stakeholders' favour different visions of what a UHC system means. Within the UHC discourse, conflict between policy organizations occurs due to contrasting interests and opinions about the approach that best fits the Nigeria context. Respondents' answers on this theme reveal several areas of tension between policy organizations arising from opposing ideas about how UHC should be developed, handled and especially the balance between public and private sector involvement in the policy.

\subsubsection{Policy consensus or opposing factions?}

In the interviews, some of the participants described how policy actors shared common goals and values when it came to UHC. Several participants maintained that there is an atmosphere of togetherness in the Nigerian UHC discourse that is encouraged by the federal government in the hope of preventing fragmentation of the policy network and promoting a rational process by considering options and selecting the best policies to hasten UHC policy development. This resonates with the SNA findings which suggest a relative lack of conflict within the policy network, except in respect of the role of the private sector. In this view there is a division of labour whereby the FMoH exercises substantial control over key stakeholders such as the USAID, Health, Finance and Governance (HFG), Results for Development (R4D) and Nigeria Health Watch (NHW) so as to develop the UHC strategy as a cooperative endeavour. 
The comments below illustrate this view:

"Our goal within the government is to limit fragmentation among key stakeholders during the policy process and make sure that we all align our efforts together so that we can move forward.” (RC.28)

"At the point of implementing the National Health Act to the one percent of the Consolidated Revenue Fund (CRF) if we the legislative arm of the health sector had not worked with other health groups both domestic and international... I don't think we would have been able to get to this point where we see the Basic Health Care Provision Fund (BHCPF) considered for implementation.” (RC.32)

"So, we have those that have contributed immensely from the two arms of the senate - the upper and lower house. They have contributed a lot to making sure our UHC agenda is properly structured and there is an easy pathway to implementing UHC in Nigeria." (RC.25)

However, other participants refute this claim of a unified cooperative approach, especially where peripheral organisations are involved:

"Many of these policy organizations have provided massive efforts to accelerate the country's UHC agenda. We have a thousand and one NGOs in different areas of the health sector that prefer the freedom to individually do their thing to push the Nigerian health system towards universal coverage. These organizations like to work separately from other organizations because it gives them the space to achieve more progress rather than been dragged along by others." (RC36)

Respondents like the one just quoted argued that many organizations prefer the freedom to individually work on their ideas or concepts to support UHC. There was a suggestion that they prefer to avoid accountability for other policy organizations' incompetent and unproductive efforts to advance the UHC agenda, while focusing on their own favoured approach or area of work. Another respondent explained that the complexity of UHC policy necessarily involves many different organisations working on different facets that are difficult to bring together until policy development work has reached a certain stage and agreement on a way forward is possible:

"Some participants are giving the wrong impression that UHC in Nigeria is just one policy with several actors coming together to achieve a policy outcome... That is not true. From my understanding the movement towards UHC in Nigeria has various sections with several developing policies in formulation and interests involved end up in engaging one another to achieve results that suit everyone. For instance, take the NHIS programme for 12 or 
13 years since its inception. Even though everybody agrees and believes that the scheme provides an avenue for access to health care to all Nigeria, this was not achievable during the 13 years because all 36 states in Nigeria refused to join NHIS because the government was advocating for a central riskpooling fund. Since the health system is decentralised with the SMoHs having the ability to independently make decisions and plan healthcare on behalf of their state, they did not think it was legally right for the government to establish the NHIS as a central risk pool. After several agitations from the SMoHs over the years, it was not until 2015 that both the state and federal government came up with a policy to decentralise the NHIS. That now gave rise to the State Health Insurance Scheme and within several years we have got up to 75 per cent of states have established a Health Insurance Scheme at the moment. It took over thirteen years of back and forth between the government and SMoH to reach this conclusion." (RC.5)

Another respondent echoed this view of a broad policy process with many strands and a multiplicity of actors with different interests and different roles in the policy process:

"UHC policy in Nigeria is very broad and it is difficult to tell you every contending group which exists within the policy process because there are many components of the UHC policy that has different aspects with plenty of policy actors having different interests and functions. Hence, I would disagree with other participants suggesting a form of unity among policy actors within the policy process. So, does it mean that all the actors including the government and non-government are always in unison about the various policy interventions and their implementation towards UHC in Nigeria? The answer is no! For instance, the organized labour headed by the NMA refused to pay part of workers' salaries into the risk pool of the NHIS because they felt that their money would go into the hands of the HMOs. According to them, the scheme is designed to charge higher premiums for a higher standard in the delivery of care because of immense private sector participation in the scheme. All the HMOs and providers are privately owned. And that is why they forced the implementation of the National Health Act which increases public financing into healthcare." (RC.42)

These respondents argue that policy development is shaped by contending groups, which contest ideas and seek to influence the UHC discourse to suit their own interests. In particular there is scepticism about the motives of HMOs and private providers. The large number of interest groups involved in the policy discourse creates problems for the central government departments because it is difficult to create an environment where developments in different parts of the policy network are synchronous. The case mentioned in the last extract was recalled by several respondents as an example where conflict between contending groups surfaced. 
This case concerns how Nigerian labour groups, such as the NMA, opposed the government's expansion of the NHIS program from federal workers to workers in the informal sector. Such an expansion needed non-federal workers to enrol in the NHIS scheme by paying contributions from their salaries, which according to the NMA might end up in the pocket of HMOs (the private health sector). This led to several disputes between the NMA and HMOs, which saw the NMA call on doctors to boycott HMOs, and direct a series of strikes against the scheme, and threaten to create an alternative health service provider focused on universal coverage. There is a widespread perception in Nigerian society that HMOs focus more on profits than affordable treatments. This encouraged the NMA to campaign for a National Health Act, which would provide a framework for the regulation, development and management of a national health system and set standards for providing health services.

The Act established the Basic Health Care Provision Fund, the target of which is to substantially increase revenue and improve Primary Health Care (PHC) services. The Nigerian government and the Nigerian Medical Association had argued over the content of a future National Health Act for over ten years before it was enacted in 2014. When the law came into effect, its improved access to health care by combining a Community Based Health Insurance Scheme (CBHIS) with the broader State Health Insurance Framework in order to provide coverage for the informal sector population, including poor and underserved communities. Under an agreement between the government and $\mathrm{SMoH}$, the new scheme (CBHIS) was added to the existing state health insurance framework in every local government area to stimulate demand for health insurance across the State. The CBHIS predominantly targets informal sector workers and offers a package of prepaid healthcare services for enrolled community members. The intended outcome is to convert the current prevalent out-of-pocket payments for healthcare into prepayments which are more progressive and equitable. Over 75 per cent of states in Nigeria have adopted this scheme in order to extend coverage (Fadlallah et al., 2018). Based on the interviews, participants can be categorized into three different groups. Group A consists of participants whose perception of the UHC discourse in Nigeria presents policy organizations as working together cooperative to develop a largely consensual approach to UHC policy. According to these participants, the Federal Government of Nigeria provides an environment within the health system that limits fragmentation between policy organizations and encourages close inter-organizational relationships within the policy network. Participants in Group B said the opposite; they denied that a unified approach existed. They argued that the size and diversity of the policy network results in argument and conflict between (at least some) 
policy organizations due to their differing interests, ideologies, and approaches to achieving UHC in Nigeria. In Group C, participants are somewhat on the fence between Group A and Group B arguments. They acknowledge that there have been government efforts to create a cooperative environment that brings together stakeholder efforts towards achieving universal coverage but say that in reality contention between policy organizations is reflected in differences in approach that are hard to reconcile.

\subsubsection{Two main opposed groups}

Just how UHC should be provided has long been a matter of controversy for many Nigerians, and perhaps the most important line of division concerns the role of the public and private sectors. Consider two interview extracts, which at face value may seem to only contain a subtle difference of emphasis, but illustrate the way the argument is often framed:

"The NHIS is very important to making UHC a reality in Nigeria. We provide the working tools, prepaid from government." (RC.23)

"NHIS gives a quality health care service by pushing tight regulations on Nigerian health providers to make the basic health insurance available for many Nigerians.” (RC.20)

The first extract casts the state as a system manager and direct provider, while the second presents the state as a regulator of public and private providers. While a large number of participants maintained that the National Health Insurance Scheme is the key mechanism by which to achieve UHC in Nigeria, the two camps disagree when it came to the balance between public and private provision. The insurance mechanism itself is relatively uncontroversial, since as well as being supported by reports from the World Bank and WHO (PWC, 2019) it is familiar to Nigerians because the NHIS has already run for more than fifteen years and covers $3 \%$ of the population. The major point of difference that divides participants concerns the preferred type of healthcare provider to delivery UHC to the population through the NHIS.

The two viewpoints are reflected in the following data extracts, the first from a respondent from the private sector and the second from a government official:

"We are leading the advocacy for UHC post-2015 SDGs and developing several frameworks to increase the health coverage to about 30 percent with better basic health packages for individual Nigerians. We are in talks with the 
government to improve regulations that encourage the expansion of health insurance coverage through an increase of health providers from the private sector... Because, to achieve this 30 percent, the government needs all the help it can get especially from health providers to resolve health financing and the universal coverage gap.” (RC.9)

"The health insurance scheme is slow and losing its effectiveness. For me... that's why I consider that the Federal Government and people in power need to take charge of providing health services outside the civil servant bubble, using the extra funds enabled from the Act... To also improve on the laws and operational guidelines to prevent inadequate ownership by stakeholders and a poor enrolment strategy by many HMOs destroying the progress of health coverage in Nigeria.” (RC.41)

The interviews show that the framework to deliver a health insurance scheme and extend coverage to underserved populations is a divisive issue amongst policy organizations, mainly revolving around the respective roles of the state and the private sector. Those who see the road to UHC involving an expanded public sector are opposed by a private lobby calling for a mixed system. We now turn to consider how a state-level insurance scheme, the State Health Insurance Scheme (SHIS), was implemented in various Nigerian states, paying attention to how conflict between the two opposing groups played out. The SHIS aims to fund basic care and inject new energy into the primary health care system through the construction of a network of functional health centres providing basic services across every Nigerian state.

\subsubsection{The private lobby and the desire for a mixed system}

One issue as policy on the SHIS was developed was how far the SHIS and the HMOs should cooperate, and whether services were best delivered in publicly provided health centres or a mixed system. Advocates of a mixed system argued that private healthcare providers should be free to register and manage new enrolees and participate in the management of the insurance scheme (Global Health Sciences, 2016). Proponents tended to argue that private sector involvement was necessary to ensure adequate finance for the scheme:

"It will be difficult for states to adopt universal coverage for their people because of the cost attached to it, the financial budget for health in some states can't provide the basic health package of the health scheme. But with the inclusion of more private health providers, their invested participation would ease the burden for some states and encourage the development of health coverage for the community." (RC.11) 
Some also suggested that closer links between the NHIS and private health providers of a kind that might develop after cooperation regarding state level insurance, would encourage the private sector to take on greater responsibility for the construction, financing and maintenance of operational risks, and therefore improve and increase the provision of healthcare services for others besides insured civil servants.

"From 2015, 2016 to 2017... Throughout these years, the government focused on achieving universal health coverage for ordinary Nigerians. There were funding and financial arrangement difficulties due to the economic instability of the country. However, in the last two years, we are advocating for an increase in the Ministry of Health's engagement with the private sector to encourage private investment into various states that have adopted the state insurance scheme. In my opinion, private investment in health is needed to stabilize the funding and financial volatility within each state. This is precisely what the Dangote group is doing through their Bank of Industry, which injects billions of Naira into the private health sector, making capital, equity, and loans available for healthcare start-ups. I firmly believe that encouraging more private participation like Dangote into the system will ease the burden on government and speedily increase our chances of achieving universal coverage for many." (RC.7)

The private sector argues that a mixed model under a well-regulated SHIS would advance progress towards UHC in Nigeria, partly by increasing the number of beneficiaries and the size of the risk pool, vital if national health insurance scheme is to be the mechanism for widening coverage. Advocates argue that, to achieve this, states must adopt strategies encouraging resident participation in the schemes. This may involve measures like tying service eligibility by the state to scheme participation. (Global Health Sciences, 2016).

\subsubsection{The coalition supporting UHC led by the public sector}

On the other side of the argument, the NMA has expressed its concerns about the involvement of HMOs in any SHIS scheme. The Association suggests that HMO participation would be likely to result in rising premiums, defeating the purpose of the SHIS which aims to reduce healthcare costs. There were some concerns among respondents about the drawbacks of a mixed system:

"Funding misappropriation, no accountability, inappropriate capacities and regulatory incompetency at the top while lack of adequate professional expertise and lack of training and empowerment at the bottom are the 
characteristics of HMOs... This is what is adding to the burden of out-ofpocket payments and premiums on enrolees for healthcare services." (RC.41)

"Poor sub-nationals and private sector buy-ins; inadequate regulations and frameworks prevent many of the enrolees from getting access to medical treatments." (RC.3)

The above participants and others pointed out that NHIS had barely increasing coverage above $5 \%$ of the population. They claimed that problems have arisen from factors such as low individual enrolment, a number of corruption scandals, some HMO failures, mismanagement of NHIS funds, and poor enrolee contributions collected were too low to keep the scheme viable.

The NMA instead supports an SHIS healthcare initiative led by the public sector and using the Basic Health Care Provision Fund (BHCPF) via the National Health Act to provide sustainable healthcare to individuals in different states. Part of the requirement is that states should enrol and provide healthcare to registered individuals paid for by federal government funds under provision of the Health Act. The NMA pressured the government for meaningful engagement to prevent HMO involvement by advising doctors not to participate in the SHIS (Onyeji, 2018).

\subsection{Power and politics in the UHC policy network}

Exercising power is a critical element of Walt and Gilson's policy analysis triangle framework. The understanding of power is vital in the analysis of UHC discourse in Nigeria (Sriram et al., 2018). Power is implicitly and explicitly manifested in many ways via the interactions of health system actors at local, national and global levels, particularly when negotiating priorities and the allocation of resources (Sheikh et al., 2014). This section considers these relationships using social or political theories to consider power and politics within the UHC discourse.

Power is a complex and controversial concept. While some scholars argue that it is tightly concentrated among particular actors, as for example, within a power elite (Mills, 1956), others like Dahl (1957) maintain that it is dispersed more broadly among individuals and groups, who are competing for influence (Buse et al., 2012).

One definition of power is "the ability to influence people, and in particular to control resources" (Buse et al., 2012: 21). Concerning UHC implementation, power becomes manifest in the 
ability to control individuals or groups, regardless of their intentions (Buse et al., 2012). Particular groups in a health system can determine the direction of health policy, as they seek to use argument and persuasion to get their preferred outcome (Khan, 2010), or conversely to block policies that are not seen to be advantageous to the relevant group.

For example, Nigeria's Oral Health Policy (OHP) went through three decades between 1984 and 2009 when attempts to get improved oral health onto the government policy agenda failed to command support. According to Etiaba et al. (2015), the external influence of international bodies such as the WHO played a substantial role in getting domestic policy actors to acknowledge the importance of the OHP and successfully implementing the policy. This illustrates the amount of power the World Bank and WHO wielded in certain sectors and certain time periods, including the adoption of OHP in Nigeria. The policy led to a reduction in oral health diseases, an expansion of the medical workforce, and improvements in coordination of oral health service delivery (Etiaba et al., 2015).

This section aims to explore various aspects of power based on the qualitative interviews. The interview extracts suggest that the power sources in the UHC policy process divide into multiple relationships, namely political power, financial power, professional power, power through technical expertise and power in numbers (via formation of coalitions). This can be linked to Steven Lukes' (2005) concept of the three faces of power. It can be argued that in the UHC policy discourse power can be analysed according to (a) how it emerges (b) how it is channelled, and (c) how it is overtly or covertly expressed.

\subsubsection{Financial power}

Power in the Nigerian health sector comes primarily from political office or state power deriving from access to financial resources such as taxation, oil revenues, and using that power to influence decision-making about UHC policy.

The importance of control of budgets can be seen in interview extracts such as the following:

"You will agree with me that nobody has power more than that person who is holding the money, so if you are a budget holder you have the power. So, it's only when you have the power and money you will start talking about the political will. In fact, only in a meeting yesterday with the $\mathrm{SMoH}$, they told us 
that they don't think that anything like political will exists. If they have plenty of funds, then they can forget political will.” (RC.30)

Power as decision making focuses on individual and group acts which influence policy decisions. Robert Dahl's (1961) classic study investigates the distribution of power by examining the preferences of interest groups and making a comparison with policy outcomes. He discovered that the resources conferring power on citizens and interest groups varied and that these resources were unequally distributed: while some actors had plenty of certain political resources, they were poor in others. Thus, different individuals and groups were able to exert influence on different policy issues. These findings led Dahl to conclude that different societal groups, including weak groups, could 'penetrate' the political system and exercise a degree of power over decision makers depending on the resources they could bring to bear in that policy area. The range of resources which can be used in health policy give different groups more or less power in influencing policy and will be a function of policy content and context (Buse et al., 2012). Several respondents argued that money is a driving factor in the progress of UHC in Nigeria, and access to financial resources constitutes the decisive power shaping policy within the UHC discourse. The interviews suggested that the core institutions wield more influence than outsider groups in the UHC policy process because they can access the financial resources needed to move UHC forward.

In recent times financial support for the implementation of UHC in different national contexts has come from both international and domestic sources. As another respondent points out recent changes that have placed a greater emphasis on funding from national budgets have changed the balance of power between domestic and international organisations in the policy network.

"Now for me, you know the difference between MDGs and SDGs, is that MDGs was funded from external funding. The other one will require huge domestic resources and less international funding. Now characterizing these policies. Some of the policies that we directed to the MDGs were not like one years or two years or three-year policy. They were like a 10-year plan that did not end even post 2015-MDGs. Some of them spilled over into the era of SDGs and then of course their implementation continues until that date. Apart from the fact that these policies were overlaps, the failures of the MDGs made us face reality and make the government own up to its responsibility, which is to commit more resources to the health system. Which is why the 1\% CRF came on board. It didn't just come from government actually started putting down strategies to achieve the $1 \%$ CRF. It was the policy overlap started making the government responsible for its duties." (RC.32) 
Unlike the MDGs initiative, which depended on funding from international health donors, UN agencies and other external sources, current UHC developments in Nigeria depend on domestic government expenditure and achieving UHC has been estimated to require 15 per cent of total government expenditure to be committed to health system. This is so despite exhortations for international donor stakeholders to fulfil agreed targets for Official Development Assistance (ODA) to Nigeria (The World Bank, 2017). For example, the Federal Ministry of Health’s implementation of the MDGs in Nigeria began when they pledged to apply the savings accrued from the Paris Club Debt Relief Deal in 2005 to pro- poor programmes and projects. Several policies, programmes and projects aimed at MDG achievement have been implemented by diverting savings from external debt relief to implement MDGs programmes and projects (OSSAP-MDGs, 2015).

The above respondent, like several others interviewed, takes the view that, given the failure of the MDG initiative, an increase in domestic expenditure provides a stronger foundation for UHC than does dependence to foreign funding. Several respondents mentioned the importance of core government funding:

"Health financing affects all the health systems building blocks towards UHC and this is my view I believe that health financing... appropriate health financing is a gateway towards UHC. And just checking there is no country that has achieved universal coverage without putting appropriate health financing in place." (RC.29)

"My view here is that for Nigeria to be able to achieve UHC, which I think is very possible, Nigeria must get health financing right. So that all the effort around decentralizing health insurance ensures that each State establishes a functional health insurance scheme, and the direction all our efforts are headed in right now is towards the Basic Health Care Fund which will serve as a subsidy to the State Health Insurance Scheme (SHIS). It will also provide additional funding for the primary health care and input in terms of the operational cost of primary healthcare facilities. Hence, without appropriate domestic funding it is impossible to have these systems in place to work the way you want." (RC.5)

The above quotations make the point that such funding must be channelled through an effective health financing system, something that has been continually emphasized by international advocates for UHC, such as the Joint Learning Network. Thus, several participants mentioned the importance of matters such as the design of health insurance schemes (about which there are differing views), the rational allocation of resources to support services that bring the most health gain, pooling of funds, increases in transparency and accountability, introducing 
strategic purchasing, and strengthening managerial capacities at federal and state level- all affecting implementation, and ultimately UHC progress.

The diminishing flow of international funding and greater reliance on domestic budgets meant, for many respondents, that it was the Ministry that had become the main driver of UHC, even if the international bodies continued to push in that direction.

“To answer your question, I think those that are providing persuasive support are partners in the UN agencies, the CSOs and development partners. These are powers trying to drive and force the government to implement the policy into the health system. But when it comes to the main driver of this initiative in this country you talk about the federal government of course. They, the Ministry of Health and other federal agencies have the power to drive this initiative to fruition.” (RC.31)

This suggests that the widespread perception (mentioned earlier in the chapter) that power centers upon the Federal Government and its parastatals, such as the $\mathrm{FMoH}, \mathrm{SMoH}$, the National Primary Health Care Development Agency (NPHCDA) and NHIS is linked to the view that only they have political and financial authority to allocate resources to support new policy developments. As one respondent said it is the Ministry that occupies the central position in policy development:

"The FMoH plays a vital role by making the health services accessible to the whole country." (RC.9)

This is not to say that there is always a common view within the inner network of government ministers, departments and agencies. Some respondents suggested that where there were differences with Federal Government Cabinet policy, the FMoH is sometimes sufficiently powerful to bring about a change in direction. The FMoH has primary responsibility for the downstream allocation of health sector departmental budgets which is received from the annual budget. While it must operate within the envelope of the national health budget, it can influence how it is spent. The FMoH also has responsibility for liaising on UHC policy with domestic bodies, such as SMoH and NHIS, and international bodies, such as the World Bank Group. Just as the FMoH can sometimes influence Ministers, an informant suggested that the NHIS can sometimes push back to maintain a consistent approach when government policy statements diverge from the direction of existing policy. 
"The main stakeholders are not always consulted when decisions are to be made i.e., the main people concerned are being boycotted (overlooked) during decision making. To me, I think the NHIS plays a major role in making UHC a reality by being consistent at every step of the debate." (RC.6)

This was further explained by another participant:

"Leave the federal government for now. I think the National Primary Health Care Development Agency (NPHCDA) and NHIS have found themselves in key positions within the UHC policy process, mandated to them by the Ministry of Health to do things that will make UHC achievable in the country." (RC.41)

Other participants also maintained that the NHIS and NPHCDA wield influence alongside the FMoH. They suggest that this comes mainly via their formal responsibilities in a number of specific areas relevant to UHC. For example, the NHIS's powers span regulating private health insurance companies to create health coverage for vulnerable groups and the formal sector, mainly via social health insurance schemes. It gains influence partly through its close association with the private health sector, which has considerable muscle due to its size, as reflected in the number of treatment facilities, health maintenance organisations, pharmaceutical manufacturers, and other entities (Adewole et al., 2017).

\subsubsection{Political power}

Political forces play a dominant role in shaping the delivery of health services for many Nigerians. The transition into democracy illustrated how a change in the political environment opened the way for major improvements in the healthcare system. Health services have often been something of a 'political football' by which opposing political parties could seek to attract votes and form governments, and sometimes this has meant that politicians have promoted certain interest groups within the health system to gain their support (Sriram et al., 2018). This political relationship featured in the accounts of respondents, such as the following:

"So, I am going to take that it from a different perspective. Firstly, UHC is the right thing to do, as it is the responsibility of the Nigeria government to take care of the health sector...the introduction of UHC is mainly a political issue used as a political tool by our political leaders that want to show results to their constituents, and some of those results can be achieved through health care outcomes. You see many time political leaders can be worried about the 
results of health surveys because they do not want health surveys to come out as bad as they use to be. So, the question is 'what have you achieved during your political tenure?' So, they use significant health policies, such as the UHC policy to increase their political currency - if there is a promise of improvement in health care. Lastly... the international health bodies such as the World Bank, WHO and UNICEF persuaded the federal government to start on a current health development programme and this enabled the Goodluck Jonathan administration to use its commitment to UHC as a political tool to influence voters for their re-election campaign.” (RC.23)

Many participants identified political authority as the dominant source of power that exists within the UHC discourse. According to participants, the federal government is the sole political actor that has the ability to establish a legislative framework that can shape the direction of UHC implementation and also set aside financial resources for the policy. Thus, some participants maintained that recent progress towards UHC can be attributed mainly to the Goodluck Jonathan administration's commitment to the policy at the presidential summit in Abuja Nigeria on 10th March 2014. The push towards UHC in Nigeria was given fresh impetus at this summit because the federal government decided to commit to the policy in a high-profile international forum (WHO, 2016).

\subsubsection{Power through technical expertise}

One way that policy organizations outside government can play a bigger role is through providing specialist technical expertise. Their participation may be attractive to governments because of the legitimacy their perceived expertise brings when they give a policy their support. UN and international aid bodies feature prominently in this category and respondents mentioned them frequently in the context of advice, knowledge sharing, technical strategies to achieve UHC, and policy 'push':

"The major drivers trying to advise the government towards achieving UHC are the World Bank, WHO, Bill and Melinda Gates Foundation, UNICEF, DFID, USAID. These are the major the drivers that push the UHC agenda because they advocate strongly to the government for the increase in health financing, reducing the chances of corruption and strengthening of primary health care, which all address the issues of equity in the health system." (RC.35)

"I see the World Health Organization as a foreign stakeholder that brings together the right foreign stakeholders and the technical strategies to deliver 
their desire to exchange and share several initiatives from other countries' successful UHC implementation.” (RC.26)

“... the WHO and other international organizations want to produce the same health services as in other developing countries by introducing UHC in the health system." (RC.38)

"So maybe what I can do is share the list of the attendance of the people that participate in the national advisory committee for the Basic Health Care Provision Fund (BHCPF). I attended that meeting this afternoon and I can tell you from the participants in attendance they have a lot of authority and power in health so that you could see it and feel it. The Minister of Health was present, so were UN agencies such as the World Bank, WHO and UNICEF, several international donor agencies such as DFID, USAID. But I didn't see the Canadians (but they are part of the committee). Bill and Melinda Gates Foundation were also there, and several private sector groups such as the Dangote group. There were a lot of powerful people involved whose bottom line is to focus on achieving UHC in Nigeria.” (RC.32)

"The WHO continues to work closely with the FMoH on various technical aspects which improve UHC progress." (RC.32)

According to the respondents an array of technical experts is involved in supporting the UHC policy process, with most being affiliated with the various UN bodies, NGOs, international health organizations, private health sector groups, civil society organizations, and academic institutions. It is important to note though that such actors exercise influence as specialists in the overall division of labor, rather than direct decision makers, and that this influence generally takes the form of technical support and consultation for the Ministry of Health. Their strength comes from the range of specialist expertise they can offer, so that their involvement will often take the form of episodic contact in relation to particular problem issues. Generally, their recommendations are consistent with current international initiatives and shared knowledge disseminated through various global or regional UHC networks and reflects the way that UHC has now risen near the top of the policy agendas of leading organizations such as the World Bank and WHO.

Relating this back to our earlier mention of Lukes' (2005) three faces of power, it might be argued that the de facto consensus among the leading international organizations represents the most insidious relationship - what he terms 'thought control' - even if the motivations of the organizations are altruistic. The new consensus in favour of UHC can be powerful in shaping 
perceptions, cognitions and preferences so that actors accept the value of a movement that has currency far beyond Nigeria. Joseph Nye (2004) spoke of soft power as achieving influence in world politics, where an actor's values, culture and institutional prestige are the political currencies promoted to get what they want. It can be argued that these policy organizations, both domestic and foreign, while separate from the national institutions of government, nevertheless use soft power to exert indirect influence on the UHC policy process. Of course, they do not convince everybody, and as already mentioned there is a powerful domestic lobby associated with the private medical sector that remains resistant to the international push towards UHC. We have seen already that participants see the WHO, the World Bank, the Bill and Melinda Gates Foundation, DFID and USAID, as being among the key external agencies influencing Nigerian UHC policy.

Achieving universal health coverage in Nigeria depends on willingness to learn from the welldocumented experiences of other countries in achieving UHC. Much of this information is transmitted via collaborative action from multiple foreign stakeholders, organizations and institutions working together to find more sustainable solutions. Although policy organizations such as the UN bodies, external government agencies, and NGOs are outside the government's sphere of influence in Nigeria's health care, they still deliver the power through technical expertise to determine the shape of Nigeria’s UHC implementation.

\subsubsection{Power in numbers: coalitions}

The notion of power in numbers concerns the ability policy actors have to assemble coalitions of multiple actors or groups to advance or block a policy. This can be positive or negative. According to one participant:

\footnotetext{
"Needless to say, universal coverage for Nigerians and financing of the health system have been victims of the federal government's lack of commitment to improvements in health policies related to UHC development... That said it is our responsibility as the Nigerian Medical Association to hold the government in check by reaching out to several allies such as other union health groups with the aim to fight for the realisation of UHC and proper working conditions for medical professionals in Nigeria.” (RC.40)
}

Some respondents viewed labour groups, such as the NMA, as occupying a strong position in the UHC discourse because they could pressurize the Federal Government by gathering support 
from other labour groups to oppose policy developments they disliked. The NMA favors implementing UHC, and makes constant efforts to increase policy awareness, in the general population. Its views are represented in the public political discourse, political party manifestos, campaigns and media debates. For example, the NMA has influenced government by pointing out these shortcomings (such as the concerns about poor coverage, alleged corruption, alleged mismanagement of funds contributed by subscribers and inhumane treatment of enrolees), and so contributed to the reigning back of the Health Management Organisations' (HMOs') role in UHC.

A participant from the labour sector vented his frustrations about the NHIS's operations in Nigeria:

"The NHIS has yet to reach its full potential, with just less than five than per cent of the population with health coverage, those people in that organization need to get their act together and do their jobs properly if we are to achieve UHC in this country." (RC.41)

Complaints have been made by the NMA about attempts by the previous and present NHIS leadership to undermine NHA authority. They have suggested that these leaders are too close to HMO representatives who are on the same Health \& Managed Care Association of Nigeria (HMCAN) agency board. The NMA insisted that this situation leads to conflicts of interest that hinder NHIS progress and urged the government to order cessation of fund disbursement to the HMOs. The NMA states that the NHIS was established to provide care to Nigerians regardless of their ability to pay, employment status or other life situations. However, the number of Nigerians covered by the NHIS after 12 years is 1.5 per cent of the population, which compares unfavourably with countries like Kenya and Rwanda which have achieved better outcomes. NMA pressure on the federal government led to the suspension of top officials in NHIS and the committee for health investigated several HMOs for possible infractions (Nigeria Health Watch, 2017).

The NMA's influence comes from its central position in medical and other professional networks and its ability to coordinate action of other labour groups. As the following extract suggests, forming alliances with other groups increases its influence in policy debates: 
"Part of our role as the NMA is to organize favourable conditions for medical practitioners and access to basic health services Nigerians across the country. We advocate for policies to the government that increase the chances of improving health insurance coverage around lower-income communities in many parts of the country. Although difficult, we use any means necessary by creating awareness compelling the government comes on board improving health as we do all this in alliance with other groups whose interest is aligned." (RC.42)

Constituent professional specialties (including physicians like this respondent) affiliated with the NMA may also feel their power is enhanced by the Association's collective 'clout' and wider influence.

"As part of the NMA, we drew government's attention to selected health issues that will impact positively on the health indices of the nation and the state." (RC.40)

The NMA uses 'power in numbers' by marshalling support from other professional associations in the health sector as well as donor agencies, community leaders and traditional leaders to persuade the government to act, or not act, so as to ensure that UHC develops in its preferred direction.

The NMA frequently criticizes Nigeria's slow progress towards UHC and blames the government for poor political leadership, lack of commitment, and inadequate budgetary allocations, which results in a poor access to healthcare and heavy reliance on out-of-pocket payments. The kinds of pressures described above have long-term impact, but only work slowly to challenge the inaction of the government in advancing UHC policy. The NMA was particularly influential in securing the enactment of the 2014 Health Act, which advanced the Association's objective of creating earmarked funding that would support an extension of coverage. Thus, it lobbied successfully for the inclusion of an additional one per cent of the Consolidated Revenue Fund (CRF) into the 2014 National Health Act budget:

“... the Act made specific provisions for the establishment of Basic Health Care Fund which is a first-line charge on the consolidated revenue of the Federal Government. This fund is to be primarily deployed into our primary healthcare which has been so neglected in the past." (RC.30)

The extra one per cent will support a range of new developments, including expanded social health insurance, primary healthcare revitalization, accident and emergency services, and recruitment and training of staff at the primary care level. Commentators suggest that it was 
only through the NMA's influence on the public sector unions, and the threat of damaging strikes, that the Nigerian government was persuaded to put forward legislation (Onyeji, 2018). This raises the question of whether the power dynamic between the NMA and the federal government creates an unhealthy situation whereby the federal government complies with the NMA's demands because it fears protests, strikes and walkouts. The Association has been criticised for its willingness to use a strike weapon that may short-term damage on the healthcare system by stopping patients getting care, but it does appear to have given the HNA power beyond it formally mandated role.

\subsubsection{Professional power}

According to Buse et al. (2012), one structural characteristic of many health systems around the globe is the dominant position of the medical profession (see also: Freidson, 1970; Alubo and Hunduh, 2017). Almost all participants said that medical doctors were powerful actors in the Nigerian health system. They occupy leading positions in many areas of the health sector, such as the senior management echelons of the FMoH, SMoHs, NMA, UN Bodies, Federal Government and the HMOs, and they are at the centre of the UHC discourse.

Participants say that the level of access the medical profession possesses in UHC policy discussions gives them the professional power to shape, control, and regulate the pace at which UHC is implemented. While many doctor respondents expressed strong support for a version of UHC driven by state investment, many in senior positions wanted a policy that opened the door for significant private-sector involvement and was not leading to any rapid extension of coverage. This stance was important because allied health professionals and nurses habitually rely on the lead set by doctors when framing their own policy positions on UHC. Respondents see doctors as the dominant professional group, mainly because of the authority conferred by their perceived expertise in a spectrum of matters relating to health, ranging from the drugs required in a basic benefits package to the design of effective health promotion and disease prevention measures. This cultural authority is illustrated in the following interview extracts:

"Well, doctors, of course, are the major custodians of health for the Nigerian population and the Nigerian Medical Association - among its reason for existence - is to safeguard the integrity of other health professionals and the wealth of the nation through events, talks, health awareness programmes, conferences and advocacy for better health coverage, which is conveyed to the Federal Government and FMoH.” (RC.40) 
"The reality is that the Nigeria Medical Association in conjunction with the Joint Health Sector Unions (JOHESU) are just bunches of medical doctors who believe that as a healthcare workers group, their one obligation is to serve the common will of those who are in need of health services in Nigeria." (RC.40)

Many respondents agreed that professional power within the UHC dialogue is primarily understood by participants to involve doctors' power to influence other health professionals by shaping their policy stance on health care. Doctors themselves believe that their collective power can influence policy makers:

"As a medical doctor who has been well active in the UHC policy process over the years, the 2014 National Health Act policy, with a lot of contributions from the NMA I must say, has a lot of promise if implemented properly in making UHC possible in our health system." (RC.41)

"We, the doctors, are today calling for the immediate government response by saying that the time to implement the Health Act is now; we can no longer wait any further." (RC.3)

According to Friedson's (1970) four factor definition of medical dominance, the privileged position of the medical profession within the UHC discourse is manifested through the professional autonomy of doctors, through their pivotal role in the economics of health services, through dominance over allied health occupational groups, through administrative influence, and through the collective influence of medical associations (Kenny and Adamson, 1992). The interviews suggest that though many practicing doctors express strong ideological support for UHC, medically qualified officials and senior managers, as well as those in the private-forprofit sector, are slowing progress towards UHC by taking policy down the complicated path of an insurance system that gives a large role to private organisations. 


\subsection{The evolving content of UHC policy}

We now turn to consider the trajectory and evolution of UHC policy as presented in the interviews, and how this fit with the theories of the policy process mentioned previously.

The first stage of the policy process cycle (Walt, 1994; Buse et al., 2012) is agenda-setting. Getting UHC onto the Nigerian Government's health policy agenda was a critical milestone because there had been a long period when providing healthcare to the wider population had been regarded as economically infeasible. Although Nigeria was experiencing high morbidity and mortality from both communicable diseases (malaria, typhoid, and HIV/AIDS) and emerging diseases of affluence, the initiatives that emerged in the late 20th century focused on particular diseases or sectors rather than attempting comprehensive health system reform. Lack of progress in improving health outcomes mobilized numerous policy actors to address these problems. One issue to consider is what happened in the debates and dialogue that then occurred to open a "window of opportunity" for future health system transformation. Here Kingdon's Multiple Streams Framework (MSF) suggests a framework for analysing what occurred as UHC came on to the Nigerian health policy agenda. The evolving content of UHC policy is discussed in many interviews, which touch upon the goals of the UHC policy, policy development, and possible problems surrounding the policy process. We will consider these interview accounts in conjunction with the timeline of health system reform shown in Figure 51. 


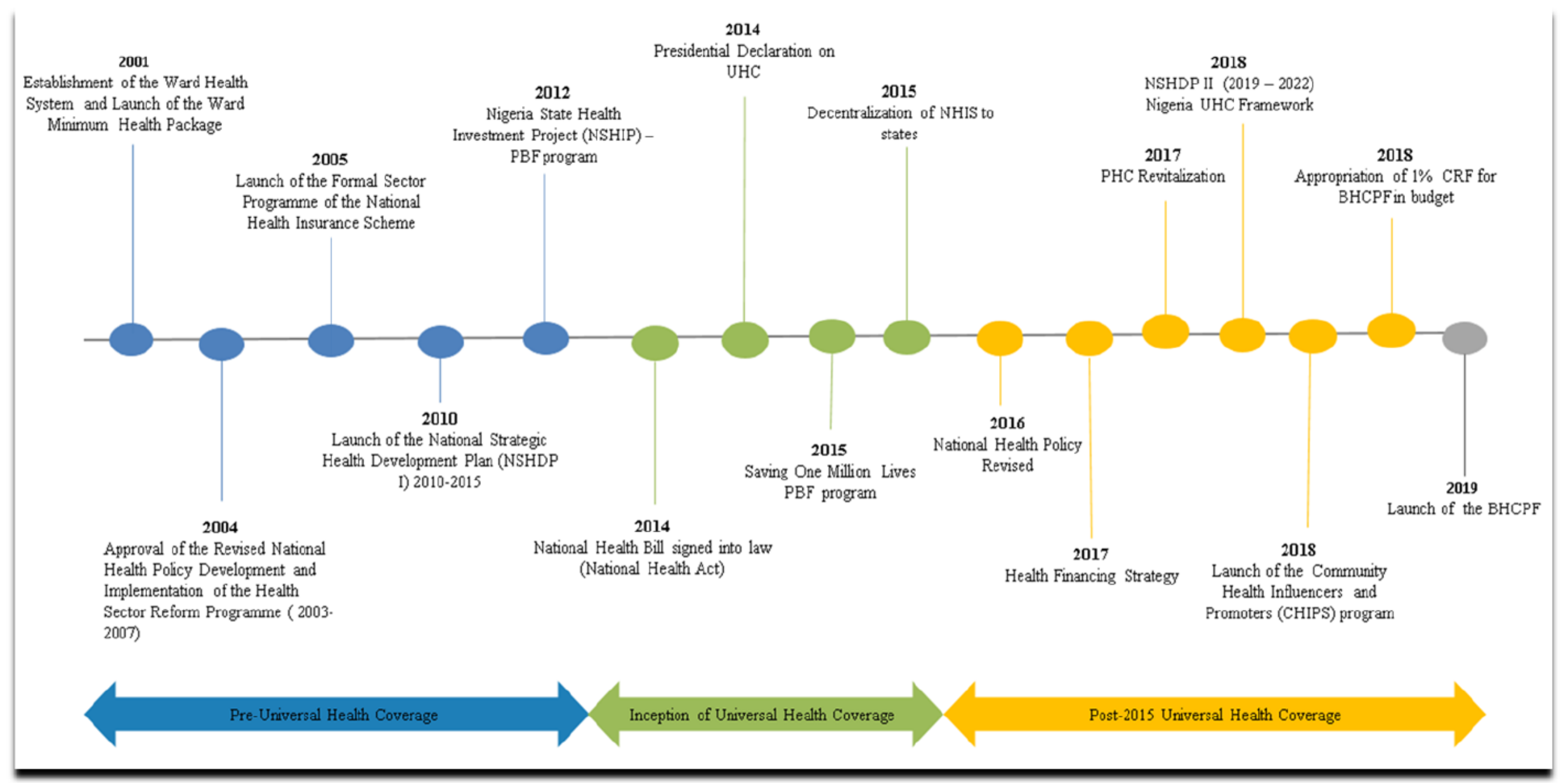

Figure 51: A timeline of progress towards UHC in the Nigeria health system. 
The interviews describe a timeline of how the pathway towards achieving targets and developing UHC policy occurred. Figure 51 has been constructed to reflect the view emerging from the interviews that there were three stages of UHC policy development, namely: pre UHC, when the NHIS, NHP and NHSDP were formed; the inception of the UHC policy and the window of opportunity; and post-2015 policy development.

\subsubsection{Pre-Universal Health Coverage}

The pre-UHC period can be viewed as the time when widening healthcare coverage emerged as a something that might climb towards the top of the policy agenda. According to respondents, health experts in the pre-UHC period were preoccupied with a range of public health issues, such as the health impacts of poverty, the maternal mortality ratio (MMR), infant mortality rate (IMR), high rates of child mortality, and health-related disease indictors. According to official estimates the poverty rate stood at $69.2 \%$ in 1997 , while the MMR was one of the poorest in the world at 630 maternal deaths per 100,000 births and child mortality under five years was 191 deaths per 1000 lives in 2000 (Nwanolue, et al., 2014; USAID, 2012 and Igbuzor, 2013).

Quotations like the following give a good idea of respondents' views of this period.

"If the previous government was fully committed to our health system there is an 80 per cent possibility that it would have significantly addressed the primary healthcare needs of Nigerians. This would also have eased the burden of focus on other NCDs. Remember more than 80 per cent of health needs are related to PHC issues and so if people can access quality health services at an affordable rate in any state in Nigeria the effort of the government will be lauded by the people of Nigeria." (RC.27)

"As it is the responsibility of the government to take care of our failing health system, there was the urgent need for capable health policies and programmes needed to tackle the burden of disease happening in Nigeria... and of course the performance of the health sector in Nigeria using key service delivery indictors such as the skilled birth attendance, immunisation rates and the other indictors shows how the burden of disease directly affects the health outcomes of the infant mortality rate, maternal mortality rate and the life expectancy in the country, which have been very low. Even after the efforts of several health policies implemented by the health sector the health outcomes of the country remain the same." (RC.34) 
"The fact is that the previous health situation in Nigeria was awful and many health experts felt it wasn't acceptable for a country of this status. The government also was not satisfied with health outcomes in the country. Therefore, they were looking into a change agenda in the health sector that would ensure that every Nigerian will be provided with proper health services despite their background.” (RC.1)

Many participants suggest that international health bodies drew the government and policymakers' attention to these deteriorating health outcomes and called for policy actions to improve equitable access to health services. This gave rise to several health policy measures (seen in Figure 51) that were designed to enhance equity, efficacy and efficiency.

Figure 51 shows a decade of subsequent policy initiatives in this period which began with the establishment of the ward health system (WHS) in 2001, followed by the national health policy (NHP) in 2004, the national health insurance scheme (NHIS) in 2005, the national strategic health development plan framework 2009- 2015 (NSHDP 1) in 2010 and lastly the Nigeria State Health Investment Project (NSHIP) in 2012.

Some participants reflected that these developments provided a useful baseline for later policies linked to UHC but needed to be integrated within a more comprehensive reform plan.

"At that time, the FMoH set up commendable health programs and policies which focused on integrating the ward system into localized primary care components to strengthen health promotion, while other policies invested in several health projects which boost state health delivery. In my opinion, such policies, programs, and projects undertaken by the previous governments would be relevant to our current policy direction.” (RC.29)

"Without a doubt UHC is one of the most significant health policies introduced into our health system. Before its introduction into the health system, healthcare in Nigeria was tackled in bits and pieces, you had the National Strategic Health Development Plan, you had the National Health Policy, these campaigns were embarked to address the existing public health issues." (RC.47)

Other participants all echoed the view that these early developments were important milestones on the path to comprehensive reform:

"NHIS is one of the significant health policies implemented in increasing healthcare coverage, by increasing health coverage which provides a basic health package; some Nigerians can get access to these health services." (RC.21)

"I am certain off the top of my head I can give you individual policies that have been put forward that are at their formative stage, but I know that the 
implementation of the national health policy has made a lot of positive contributions to the health indices because we have seen many other health initiatives come and go and none with the prowess of the NHP." (RC.5)

“The previous National Strategic Health Development Plan was designed to aid the MDGs through the improvement of several primary health care facilities across many communities but came up short in its objectives which later affected the country's targets towards the MDGs." (RC.27)

Public opinion was also a significant factor in this period when support for comprehensive healthcare system reform grew. As the following respondent suggests, politicians were not blind to the potential electoral popularity of widening healthcare coverage, and this may well have played a part in the success of Goodluck Jonathan’s Democratic People’s Party.

"I am glad that after so much domestic and international calls for better affordable health for many Nigerians, the Jonathan administration held to its own commitment which is to adopt the Universal Health Coverage policy into the health system, and I hope this commitment extends into the new administration health agenda for healthcare improvement." (RC.32)

This participant hints that the Jonathan administration adopted a policy that was seen to have widespread international and domestic support because it was likely to gain votes in the upcoming election. Health reforms have often been described as part of 'populist' political programmes, although this can also be seen as language that conservative critics use to suggest that UHC reforms are financially infeasible (Wangkiat, 2019).

Kingdon's framework would predict that during the pre-UHC period, the streams flowed along different channels until conditions changed to bring them into at least partial alignment and open a window of opportunity (see Figure 17). The following sections explores how specific issues brought the UHC policy initiative to near the top of the health reform agenda.

In terms of the problem stream, health issues in Nigeria caught the attention of policymakers in various ways, such as monitoring the health status, evaluating existing policies, and public opinion, which initiated a reaction from the government. There was also global pressure from various international health bodies such as the WHO, the World Bank and UNICEF. For instance, to alleviate Nigeria's overstrained health system, the World Bank suggested a path towards UHC via an improved strategic planning framework and development of health insurance (The World Bank Group, 2016). However, many participants say that the three key policy strategies of the pre-UHC period - the National Strategic Health Development Plan 
Framework (NSHDP 1), the National Health Policy (NHP) and the National Health Insurance Scheme (NHIS)) - did little to improve the country's health outcomes. Participants say that these policies never achieved their targets and brought no significant improvement in the health status of the population.

For instance, if we consider some of the key health outcomes data at the end of the MDG period in 2015, Nigeria's infant mortality rate was 72.7 deaths per 1,000 live births, the contraceptive prevalence rate was 15.1\% (2013), health expenditure was 3.9\% of GDP (2013); the prevalence of HIV was 3.17\% (2014), and life expectancy at birth was 52.55 years (2014), all of which are significantly lower compared to other countries in Sub-Saharan Africa (WHO, 2015).

Respondents suggested that the implementation of the three aforementioned policies feel considerable short of their projected targets, and this in itself was seen as generating a series of policy problems:

"The previous National Strategic Health Development Plan was designed to aid the MDGs through the improvement of several primary health care facilities across many communities but came up short in its objectives which later affected the country's targets towards the MDGs." (RC.27)

"While UHC remains a promising policy for our health system, the execution of several previous health policy frameworks has been lacking over the years. There was a lack of sincerity on the part of the government to own up to the failures of the NHIS scheme and all the other bottleneck health policies which resulted in a low-level of enrolees, health maintenance organization problems and many unsolved health concerns in the country." (RC.40)

Respondents suggested that the inadequacy of these programmes in addressing the multiple health problems of Nigeria has resulted in a failure to achieve policy objectives. For example, after NHIS launched in 2005, the scheme appears to have failed in realising its core objective of making healthcare affordable to Nigerians. Although good strategies were introduced to improve scheme coverage, the scheme was not implemented well which led to less than 5 per cent of the Nigerian population, primarily federal government workers and their dependents, being covered (Onwujekwe et al., 2019).

The policy stream involves discussion and debate about policy options for addressing the issues emerging within the problem stream. At this time, driven by a global momentum, many health systems in LMICs were embarking on major healthcare system reforms intended to achieve 
UHC. Several international organisations portray UHC as a global movement in which countries that have successfully implemented UHC reforms provide a model for other LMICs to emulate, especially when it comes to baseline pre-requisites and financing mechanisms (WHO, 2016; Meessen et al., 2019). In the push to respond to Nigerian health issues within the problem stream, no policy reform united as wide a range of stakeholders as the call to implement UHC. Against the background of a strong push in this direction from World Bank, WHO, ILO and others, many domestic policymakers saw a UHC policy initiative as a means to address the shortcomings Nigeria’s healthcare system.

Thus, according to this participant:

"Well with substantial evidence, it was necessary to implement universal health coverage because the health indices of Nigeria were less than impressive. So now, we are experiencing a higher rate of infant mortality, maternal mortality and the prevalence of diseases has obvious impact or burden on the economic indices of the country. There is no questioning the fact that a poor health sector exhibiting poor health indices is anathema to meaningful development for the country - even to the viability of the government of the day. It was an inescapable necessity to develop policies which reacted to the failing health indices in order to improve development." (RC.33)

In this policy stream, multiple possibilities were explored and were narrowed down to one policy initiative, UHC delivered via an expansion of health insurance. This stood out as an attractive policy option at this time, not just because of UHC's rising global prominence, but because the policy offered the prospect of addressing Nigeria poor health outcome indices, putting right the failures to achieve MDG targets and making a start in charting a path towards achieving the SDG health targets.

This convergence of external international pressure with the need to find a policy that would assist domestic policy actors in finding solutions to their own problems comes across in several interview extracts. Some put more emphasis on how the policy offered solutions to problems faced by domestic policy actors.

"Well as I said the need for universal health coverage was self-evident because of Nigeria's abysmal indices and... I supposed by UHC you are talking about it in its current form because there has always been this idea that UHC in this country came in the form of free healthcare that was not sustainable. I suppose you're asking about UHC in its current form with the health insurance model - that Third Republic event which occurred under Former President Olusegun Obasanjo's 1999 to 2000 tenure. Like I said earlier the indices were abysmal, there were the MDGs which created a new partnership for African development, since it was clear that a new push was 
required to the present course of our health system. There had to be a policy change and the old ways were not just working, there was a great deal of advocacy with demands from the international health community such as the UN Bodies and other multi-lateral agencies encouraging local support to renew the push for UHC through NHIS." (RC.28)

Others put more weight on the influence of the international bodies in suggesting a policy direction.

"Let me just tell you from my point of view, the government didn't really understand the real intricacies of universal health coverage. What persuaded the government to go down the UHC route was global pressure whose objective is to increase basic healthcare coverage for many within LMICs. So, the policy is just being driven down into the Nigerian health system, it's not that the national, sub-national or local level is driving us towards UHC. Not at all. It's the pressure that was put down by the international community on the government to adopted UHC policy in order to tackle its health issues." (RC.41)

The majority view is that the external organisations - the World Bank, and WHO - were highly influential at this time in suggesting the outline of a policy that offered the prospect of solving certain problems facing domestic policy actors. Some domestic organisations such as HEFRON and various CSOs played an important role in cooperating with the international bodies to tailor a UHC policy to Nigerian situation, by combining finance through health insurance with a plan to develop primary healthcare infrastructure. According to this account the international bodies therefore had maximum influence at this early stage, mainly through suggesting the feasibility of an outline policy that had been successfully implemented in several other LMICs. These bodies remained as active plyers in the wider policy network, but as time passed and the $\mathrm{MoH}$ and the parastatals took the lead in driving UHC policy, the international bodies throttled back their policy push to take on a more passive technical support role.

The politics stream involves specific political events that developed independently of the first two streams. These events make convergence of all three streams possible so as to create the right climate for a policy to rise to the top of the government's policy agenda (Buse et al., 2012). The interviews suggest that political calculations ahead of the 2015 presidential election in Nigeria were the final important factor in advancing the UHC policy.

In the run-up to the 2015 election politicians in all the main parties would have been increasingly aware of growing interest in UHC in policy circles. By 2014 the administration of 
President Goodluck Jonathan had been in place for around four years, and it was only in that year that it made an explicit policy commitment at a presidential summit to fund the move to UHC. At the 2014 Summit on UHC in Abuja, Jonathan stated that for UHC to be introduced to Nigeria, health insurance needed to be compulsory. He signalled the government's political commitment to providing equitable, good quality and affordable healthcare for all Nigerians, though acknowledging that there are challenges which meant that this would take time (WHO, 2014).

The policy featured prominently in the election campaign as the contending political parties scrambled for political points to sway voters to their side. The main parties all saw benefits in supporting a policy that addressed voters' concerns about the poor quality and accessibility of Nigerian healthcare, and the pressure on policy makers to move on from failure to make expected progress on the MDGs to do better in the SDG era (Vos et al., 2014).

\subsubsection{Inception of UHC (and Window of Opportunity)}

Two elements from Figure 17 not so far considered are policy entrepreneurs and agenda knowledge. The theoretical concepts of policy windows and policy entrepreneurs were recognised in interviews with stakeholders. Participants identified four key factors that contributed to the rise of UHC to the top of the policy agenda: the influence of policy entrepreneurs; increasing knowledge about and visibility of feasible policy strategies; the recognition of the importance of increased public finance as a prerequisite for UHC; and the salience of health as a policy issue in the political environment.

Factor one- the influence of policy entrepreneurs promoting UHC:

Several respondents said that the rise of the UHC policy depended on the evidence of a coalition of policy advocates, spanning both the international organisations and domestic bodies (such as the HEFRON and CSOs). These informants maintain that their efforts to persuade were instrumental in placing UHC on the policy agenda:

"To answer your question, I think that those that are providing support include development partners, HEFRON and the CSOs. They are trying to drive and force the government to do what it's supposed to do in terms of implementing UHC in Nigeria. To me these are the various [influences]." (RC.44)

These organisations are seemed like policy entrepreneurs because, acting in accord with their self-defined missions, they push UHC onto the government policy agenda. For instance, the 
role of HERFON, a non-governmental organization, was to collaborate with various international health agencies to help develop and advocate for health reform strategies and a UHC policy design that fits into the health system model of Nigeria. The CSOs sought to influence the policy design by campaigning for more focus on equity and public involvement, consonant with their citizen-led philosophy. They sought to get policy makers to pay greater attention to the health needs of the most marginalized and vulnerable people in Nigeria. These domestic policy entrepreneurs were critical in helping localise policy templates coming from the international bodies for the Nigerian context.

Factor two - growing knowledge about and visibility of feasible policy strategies:

While general support for UHC policies by the international bodies was backed up by evidence from a number of national case studies of successful UHC reforms, there was a need to develop detailed plans for an insurance-based approach for Nigeria. A considerable amount of preparatory work was necessary to build a "road map” for Nigeria:

"Yes, the agenda was to send the UHC policy proposal to the federal government through the Ministry of Health... And, before its approval into the health sector, the government had to understand the intricacies of the UHC policy..., Hence, government set up a technical working group involved in building a road map for UHC Nigeria...” (RC.31)

To formulate feasible policy strategies to achieving UHC, the government launched a health committee in collaboration with development partners and other stakeholders such as the NMA, HEFRON and the CSOs to focus on the technicalities of assimilating UHC into the Nigerian health sector.

Factor three - recognition of the importance of increased public finance to resolve public health problems:

Over time there was a growing recognition that earmarked public budgets to support the extension of coverage was essential if progress towards UHC was to be made. According to participants:

"Through strong advocacy, we teamed up with non-government organisations such as the HEFRON, who are based in Abuja. We pressed the government on the need to improve the accessibility of quality health services to average Nigerians. The National Health Act policy advocated making primary health care functional for at least 24 hours a day. It advocated that our funds should be released from the national budget - an incremental increase from the 3 to 
4 per cent while making available the one percent Consolidated Revenue Fund (CRF) for health. So, we have been advocating this Act to least raise the health budget beyond the 4 per cent that we are currently having within the health system." (RC.42)

Of course, we (the HEFRON organization) led the coalition that resulted in the enactment of the 2014 National Health Act. We led. In fact, the reason for this was because of Nigeria's bad performance in the World Health Report in 2000 that made us set up specific agencies that will metamorphize to help improve the Nigerian health system. So, we believed that there must be a legislative overarching mechanism to support UHC. That's why we set up the Health Act and we continued to advocate for funding, ownership, transparency and then good governance in health." (RC.31)

"Among other policies, I would say that the 2014 National Health Act, if implemented, would provide, or let's say create, further financial allocation towards the health sector, which can be beneficial to the progress of UHC in the country. The Act contains the Basic Health Care Provision Fund (BHCPF) which is at a pilot stage conducted in selected states in the country whereby the result of its performance is bound to radically shift health financing towards UHC. The Basic Health Care Provision Fund (BHCPF). Remember, I previously said that proper health financing would be good for the progress of UHC in our health system.” (RC.45)

Several participants stated that the Nigerian Medical Association (NMA), various civil society organisations, and the Health Reform Foundation of Nigeria (HERFON) were instrumental in the enactment of the 2014 National Health Act. The Act authorizes an additional injection of public expenditure into the health system via the Basic Health Care Provision Fund (BHCPF), and the one per cent consolidated revenue fund (CRF), which will help finance UHC in Nigeria. Among other things, these additional expenditures will go into expanding the basic minimum package of health services available to Nigerians under the National Health Insurance Scheme (NHIS), combined with a drive to improve and maintain the network of primary healthcare facilities across the country (FMoH et al., 2017).

Factor four - the salience of health as a policy issue in the political environment:

The 2015 presidential election campaign brought healthcare coverage to the centre of the political debate in a way that had not happened before. It led politicians to make connections between extending coverage and the poor healthcare outcomes of the past that raised public expectations. According to a senior administrator:

"It will be fascinating if at the end of the day if we can implement UHC in this country. Since the political elites have been unable to provide Nigerians with 
proper health care... I think the commitment to UHC around this period is a massive gift that makes Nigerians optimistic about health improvements in the future." (RC.43)

The political debate raised awareness among both political stakeholders and the general public of policy options for UHC. Kingdon's concept of “agenda knowledge” (or knowledge acquisition) is related to awareness of the emergence of feasible solutions at a specific time. In the run-up to the election the growing focus on health led the Goodluck Jonathan government to sign into law health legislation that had been debated since 2014. This brought health to the centre of the election campaign, and in the view of several informants put in place important building blocks such as finance necessary to make progress towards UHC. A survey of 130 health professionals carried out in 2015 found that 86.2 per cent claimed good awareness of the 2014 National Health Act, with almost as many have a positive opinion of it, although their detailed knowledge of its provisions was much less good (Enabulele and Enabulele, 2016). Translating technical proposals into proposals in a political campaign - "political facts" - is a crucial step in building support for a reform policy.

Several respondents explained that the financial provisions of the Act, together with measures to extend primary care provision to vulnerable population groups such as women, children and older people, were its most important sections.

"To me... the signed 2014 Health Act created an avenue for health experts and the Ministry of Health to further nudge the federal government into making a stance on UHC commitment... Because, with additional financing from the Act and global pieces of evidence that the UHC policy can improve our failing health outcomes, committing to the UHC policy on the healthcare agenda is the right timing for the Good-luck Jonathan administration." (RC.24)

"But it was not until 2014 when President Jonathan signs the 2014 National Health Act which allowed the opportunity to define the health system of the country... and in that Health Act, it defines the Basic Health Care Provision Fund which is supposed to inject extra financial funds into the health sector in order to increase health coverage to some of the poorest and most vulnerable Nigerians." (RC.40)

"To be honest UHC in Nigeria is still very new... apart from declaring UHC in 2014, it's around 2015 that is when the discussion of UHC started and was taken seriously by decision-makers in the health sector. One of the pointers or indictors that led to this serious discussion is when Nigeria developed a new strategy - the National Health Act which is mainly designed to support Nigeria 
achieve UHC. So, through that Act, all of the discourse is about revitalizing the primary health care linking it up to the secondary and tertiary level, so that the quality of health delivery is strong. surrounding UHC in Nigeria." (RC.30)

The passage of the Act marked a breakthrough for the groups campaigning for UHC, but the window of opportunity opened was seen to be time-limited because of the possibility that the outcome of the election would halt progress. There was therefore a push to secure as many government commitments as possible on the use of the new budgets and the planned expansion of coverage before the election. There was a perception that the Act itself locked in some progress, but that more needed to be done:

"Because of the change of government administration in Nigeria, health policies such as UHC held by the previous administration might be affected by the objectives of the newly elected administration. When there is a new administration in power, they have the ability to change or scrap previous policies of the last government. For example, Nigeria's constitution runs a four-year presidential term... a new government can discard a four-year policy by readdressing these issues, which not only wastes a lot of time but hinders the progress of the health system. This is why the Jonathan government signed the Health Act in order to safeguard our commitment to UHC from any change in administration because Nigeria does not have a stable government or system. Stability is a big issue in Nigeria and again with the lowest healthcare allocation in Africa, our funding issues might be resolved with this Act thereby having a positive effect on UHC implementation.” (RC.3)

"The third factor is the inconsistency on government policies because of changes from one government to another which are the main challenges." (RC.19)

This accounted for the sense of urgency felt by domestic policy actors, such as the HEFRON and the CSOs, to enact and then make the most of the Health Act before the window of opportunity closed.

\subsubsection{Post-2015 policy developments and implementation issues}

In the March 2015 general election Goodluck Jonathan’s People’s Democratic Party (PDP) was defeated by the All-Progressives Congress (APC), an alliance of four opposition parties headed by the retired Major General, Muhammadu Buhari. Apart from the health issue the election debates had been dominated by concerns about Boko Haram activity in the north, the negative impact of falling world oil prices on national revenues, and slow government progress 
in fighting corruption and improving infrastructure, all of which had counted against the PDP. The new president pledged to continue to prioritize the health sector for funding, carry on with coverage extensions through the NHIS, such as free surgery for 10,000 vulnerable NIHS beneficiaries, and maintain the focus on improved basic care for all through improvements in primary care infrastructure (WHO, 2016). However, increasing economic problems and a series of corruption scandals involving APC politicians, meant that health dropped down the policy agenda. The government was heavily preoccupied with the continued squeeze on global oil prices, which affected Nigeria's main export commodity and depressed the revenues available for public expenditure, including the health budget.

Respondents' views on progress towards UHC after 2015 were mixed but often gloomy, with many feelings that momentum had slowed:

"It depends on which category of Nigerians you are talking about on how the process of UHC is moving in the country. I think you can classify them into those that are policymakers, healthcare industries and those that are receiving healthcare. I think the healthcare industries are pushing and technically assisting the policymakers on how to go about UHC and some Nigerians are open to the idea of UHC in their community. But the policy process needs to gather more momentum in providing more coverage to individuals because many are still without health coverage." (RC.25)

"We have not progressed as much as we should and sadly this is because of the lack of capacity in terms of understanding the design... also people are very sceptical about doing things differently and trying to experiment on what they are not very sure of." (RC.5)

Some participants say that the post-2015 period is mainly about moving from policy design to implementation and grappling with the changes needed to widen coverage. Many were worried about the pace of change and the commitment of the government. Improvements in primary care remained quite limited, while the NHIS is still lagging behind the projected increase in health coverage for the uninsured. According to a Guardian (2019) article, written around the time of Buhari's election for a second term, the government's promise to move towards UHC had been slow to materialise. It will be difficult to achieve coverage for all in Nigeria before 2030 if the PHC system fails to improve and enrolment in the NHIS remains voluntary. Despite the provisions of the 2014 Act, many respondents mentioned finance as a continuing barrier to progress.

"I think for me... implementing UHC with the funding framework of our health system limits its process. We don't have an appropriate funding allocation that would allow implementing UHC the way it should be when compared to other 
countries. Just two months ago the finance minister came to tell us that the government does not have the resources to allocate to the health sector to help implement UHC on a large scale. So, one of the ways the government chose to implement UHC is to start in the first two years focusing on rebuilding functional primary health care facilities throughout the country as a way of making coverage easier for the NHIS to provide. According to the minister, this would cost the government 5-6 billion dollars per annum for a large-scale implementation which is a lot of money the government is not willing to spend...” (RC.26)

Other participants insisted that the slowdown in the momentum of UHC Nigeria is not just about finance, but about phasing implementation to give priority to primary care and expanding health insurance. These respondents argued that the planning process was itself defective and that politicians and civil servants had an over mechanistic view of developing policy in stages, which meant that the reform vision was being lost as participants squabbled about incremental change at the margin.

Like policymakers in many countries those in Nigeria operate with model for policy development that assumes a logical linear flow between discrete phases, so that they first define objectives, before identifying policy options and assessing the costs and benefits of the various paths. There are valid reasons for thinking in this way since it represents a basic Nigerian democratic model: politicians have goals, which are translated (via consultation and lobbying) into governmental action plans, which are executed by healthcare officials. This is a basic input/output model, and it possesses strong intuitive appeal.

Unfortunately, policy development in Nigeria has been affected by lack of capacity to manage a complex policy that extends over different levels of the national and state healthcare systems and a multiplicity of actors, as well as political pressures arising from reduced export earnings, Islamist insurrection in the north, and squabbles in the governing circles. Working out how polices to extend coverage at different levels of the system has proved more challenging that reform advocates anticipated.

"I think I would say that we are in the rudimental stages... put it in terms of conceptualisation, understanding the policy concept and adopting policies at different levels and putting in resources to begin to implement and then monitoring the level implementation of policies that are supposed to enable UHC in the health system." (RC.30) 
A gap has emerged between the idealised planning process and the reality of a fragmented healthcare system negatively affected by lack of resources and opposing visions of the meaning of UHC (Hongoro et al., 2018). Some participants said that achieving UHC will hang in the balance if policymakers do not resolve these challenges. Five significant challenges came up recurrently in the interviews: the changing political climate, concerns about finance and system capacity, poor coordination between federal and state levels, problems with the private sector (especially HMOs), and corruption. Of course, from the government's perspective such challenges in the health policy domain do not exist in a vacuum, and issues from other policy domains will from time-to-time command greater attention.

\subsubsection{Changing political climate}

Following the 2015 election the new government launched a series of policies affecting the healthcare system, including the National, Strategic Health Development Plan II (NSHDP) 2018-2022, a Primary Health Revitalization initiative, and the operationalisation of the Basic Healthcare Provision Fund (BHCPF), which included the one per cent Consolidation Revenue Fund (1\% CRF). Via such policies, the Buhari administration seemed to signal its commitment to re-energising the drive towards UHC (NBS, 2017). However, the interview extracts below show that key stakeholders in senior positions in the health sector were highly sceptical about the government's "political will” to advance the UHC policy at a time of economic constraint:

"Lack of political will within the present (Buhari) administration is a major obstacle coupled with the financial crisis the country is facing, since we are yet to recover from the previous global recession." (RC.28)

"The lack of political will keeps repeating itself as an inescapable obstacle to the progress of UHC in Nigeria. The reason being that the new administration is filled with many officials, politicians, legislators, and policymakers who do not take healthcare as the top priority when it comes to dealing with issues affecting the country. We, as the custodians of health in Nigeria, have to make them see the value of providing better healthcare to Nigerians." (RC.42)

"The lack of political will on the government part is a major obstacle to us policy makers in our efforts to carry out the implementation process for this policy." (RC.35)

Comments like those above suggest that many participants were doubtful that the Buhari administration had the same enthusiasm for UHC as the previous government. They suspected 
that their pledges to continue progress towards UHC (which continued in the run-up to the 2019 general election) were mere lip service.

One participant raised the issue of whether finance came first and political will was only meaningful if resources were available:

"Finally, when we are talking about political will concerning the health system governance and stewardship, if the budgetary allocations for health were adequate, we would not consider the political will as a major obstacle to UHC progress in Nigeria.” (RC.30)

Whether or not one factor is dependent on the other, it seems evident that without both political will and an adequate budget there will be little immediate progress towards UHC.

\subsubsection{Concerns about system capacity and finance}

The concern about the influence of an over-mechanistic conception of policy development mentioned earlier was partly based on the observation that Nigeria's attempts to move towards UHC did not progress in a linear process following the conventional stages or cycle models, but that instead policymakers oscillated back and forth between agenda-setting, policy formulation and implementation. For instance, in the words of a senior manager within the NHIS:

"There have been mechanisms in place that formulate policies that are passed to legislators... but the implementation is yet to be processed. So, the formulation mechanism that guides the conduct of achieving UHC in the country is mostly on hold." (RC.24)

According to this participant, policy makers have not harnessed Nigeria's full health system capacity in the post-2015 period. His concern is that continual failures to implement changes that improve health services delivery leads to a perception of crisis and a back-and-forth regression in several rounds of policy discourse (oscillating between tweaking plan and trying to implement the revised policy design) that lead to a form of policy stasis. Two economist respondents were concerned that delays and stop-start policies meant that headline changes were often not supported by necessary changes in downstream parts of the healthcare system they would affect:

"The federal government did not release funding from the last budget. But in this year's budget, the federal government released some money and approved 
the [Finance] Bill to get the one per cent consolidation revenue fund going after so many years of stagnation after the signing of the [2014] Act. As we speak, we are just trying to put the processes in place so that that structure is there from the federal government down to all the states - so that people understand it." (RC.31)

"There is a lack of capacity in the health system to handle the demands of the UHC policy. Many of the states in Nigeria do not have the required capacity or structure to be able to implement or roll out the Basic Healthcare Provision Fund. For instance, states are required to have the State Health Insurance Scheme (SHIS) which does not exist in many of the states... yet these states do not have the capacity to run or implement the SHIS, and the public health sector of many states does not have the financial ability to achieve the scheme. The majority of the people within these states are under the informal sector which financially finds it hard to provide health coverage." (RC.30)

These two respondents appear to see these deficiencies as stemming from systemic weaknesses that will be hard to overcome in the immediate future. Other participants agree that there are problems but think these can be overcome step by step given time.

"UHC needs a step-by-step approach since our technical capacity is not up to par, but with assistance from the UN bodies most of our policy makers can little by little learn how to formulate the right policies which can assist UHC implementation in our health system rather than rush into its implementation... and I also heard it is not an easy policy to go into. ” (RC.32)

The forgoing extracts represent two schools of thought among respondents: those who say that UHC in Nigeria is still in its early stages so that patience is needed, and implementation should not be rushed, and more sceptical participants who argue that Nigeria's health system is incapable of achieving universal coverage until several pressing issues are addressed. These latter issues include agreeing on a funding mechanism that would support the policy, increasing health system capacity to allow the rollout of new programs, and achieving better coordination of Federal and State Ministry of Health programs.

\subsubsection{Poor coordination between federal and state levels}

Interviewees state that the level of public health expenditure for health is insufficient to support the necessary infrastructure, service improvements and operational expenses required for achieving UHC. This means that central government allocations to the states have often been late, leading to delays in change at state level: 
"Several states are yet to adopt mandatory health insurance schemes; other states have either passed this into their state law or begun to enrol individuals in their state. However, the one concern for many states, especially for those yet to adopt the programme, is the issue surrounding the level of available funding allocated to health from the federal and state government. I think the government is not releasing enough funding for us to put in place an effective structure in order for us to enrol more individuals." (RC.5)

Once the FMoH has distributed the central health budget to key parastatals the rest is allocated to the SMoHs, which is not enough to fund the SHIS adequately. A participant explained that:

"And the constraint is that... well that depends on the national budget, we see a lot of diversions of money towards other sectors rather than health where the distribution of the national budget filters through to other sectors leaving no money left for health." (RC.3)

Some respondents complained that the Federal Government consistently gives lower priority to health in its budget allocations than to other departments of state, for instance the Ministries of Works, Power, Housing and Transportation. These may or may not be taking money from healthcare in a competitive way, since some policy initiatives in these areas may themselves impact on health. Another respondent argued that improved coordination between federal and state governments in planning service development was crucial if extra funding was to bring the maximum benefit:

"From all the list of challenges I have given you, I can gladly say that the government is aware of most of them, and they are trying to solve them little by little. For instance, the Federal Government is coming up with several funding programmes for PHC and infrastructure development in the health sector. But, without a strong relationship between the $\mathrm{FMoH}$ and $\mathrm{SMOH}$, improving funding into the sector will discourage better engagement with health experts." (RC.31)

Several participants argued for better coordination of the budget planning process to facilitate the uplift over time of annual health budgets that could support UHC.

\subsubsection{Corruption}

There are various impediments to achieving UHC in Nigeria, but several participants maintained that the root cause is mismanagement of limited financial resources by the $\mathrm{MoH}$, which often involves corruption. Five participants shared their views on corruption as follows: 
"The first on my top list of challenges is corruption, which is massively affecting the implementation of UHC policy in Nigeria, and I'm referring to that at the government level where the leaders at the top care less about making Nigeria move forward, not to talk about the effect of which trickles down into the health sector." (RC.6)

“Corruption is an inevitable impediment to this policy.” (RC.7)

"And again, corruption too, our leaders prefer to use money for something else rather than to use it for health and the 'I don't care' attitude is a common thing in Nigeria's system, which is a major constraint we have... corruption. If we can deal with this thing will be better." (RC.14)

"Corruption is a disease that has genetically been inherited for generations, to the extent that is has become the reflection of the society in which it functions." (RC.17)

"The goal of achieving UHC in Nigeria is very clear and it is understood by many policy-makers. But there are people who are very sceptical, they are not sure of how it will form, and they are afraid that corruption will take all the money for health. They feel that there is corruption from down the road at the primary health level up to the world development committee, and that may affect the prospects of UHC in Nigeria." (RC.32)

These participants consider corruption to be the main reason why the financial expenditure for health remains insufficient, and mismanagement of government funds for health will continue to affect the budget allocations necessary for progress towards UHC. Participants suggested that corruption undermined the planning and allocation of resources in the $\mathrm{MoH}$ system, and thus harmed preparations for UHC. They claimed that the effect of corruption further downstream in the system affects many health service programs and pushes patients towards private health care. Respondents suggested that this partly accounts for Nigeria's high levels of out-of-pocket (OOP) payments, which drive the lower-class and sick towards impoverishment. Eradicating corruption in the healthcare sector will increase the chances of achieving UHC, reducing out-of-pocket spending and catastrophic health expenditure linked to serious illnesses (Kamorudeen and Bidemi, 2012; Aregbesola, 2016; Orisadare, 2018). 
The recent accreditation of private health insurance companies saw the governing council of NHIS delist at least 23 out of 57 HMOs because they failed to achieve the standard requirements necessary for operational services, training activities, and maintenance services. The decision was made after 57 HMOs underwent an accreditation process required by the NHIS. The health system possesses about 60 HMOs with 600 functioning branches. The budget allocated for public health insurance is estimated to be about N28 billion in the 2019 budget, and yet this sector only covers that section of the population, estimated at just over 5\%, that has health insurance. This led some respondents to question the significance of HMOs' role in expanding coverage, and some maintained that they were actually an impediment to progress in implementing UHC. A recent NGO report (Onyeji, 2019; Akinbode et al., 2019) suggests that HMOs have failed to live up to their potential. Several of the same shortcomings listed in this report were mentioned by respondents:

"We are hopeful that we would soon get it right as a nation. But the NHIS as it is presently constituted needs to be unbundled and replaced with a more efficient framework that removes the middlemen - HMOs - who are presently one of the banes of the scheme's workability. Sustained funding by government usually does not get to the heart for those that need health care due to way funds are mishandled." (RC.40)

There were suggestions that the corruption mentioned earlier extended to the HMOs, so that government monies were being creamed off without translating into extended coverage for NHIS beneficiaries. Some participants blamed the government for creating an environment that facilitates corruption, where HMOs allegedly have the opportunity to embezzle government funds as cover for providing private health insurance coverage. They think this environment leads to complacency within the private health sector, where many HMOs lose sight of their responsibility as health providers, leading to poor service delivery, financial irregularities and unaffordable care.

At the time of research fieldwork there were tensions within the Federal Ministry of Health after senior officers had been accused of disregarding public service rules and possible malfeasance. The Guardian (2017) reported that high ranking officers from the National Health Insurance Scheme (NHIS), certain Health Maintenance Organizations, and the National Primary Health Care Development Agency (NPHCDA) were investigated over corruption 
allegations. Many higher-level policymakers called for the suspension of these officials after their alleged corrupt practices were reported in the media.

\subsection{What will it take to achieve UHC?}

Many interviewees remained optimistic about the prospects of progress on UHC, even while acknowledging that current health outcomes require improvement. However, views differed about what it will take to achieve UHC, how it should be implemented, and the impediments to progress. Such differences were generally related to professional background and interests.

The vision of the future for many participants looks forward to a time when all Nigerians have access to affordable healthcare. Participants state that every Nigerian deserves the right to basic health services with adequate social protection backed by good governance.

The following comments represent the views of several interviewees who are optimistic about the future:

"To me I think the future is bright and I say this because of the SHIS program. At the rate we are going before the end of this year, every state will have mandatory health insurance and once that happens there will be a domino effect of many states beginning to implement the SHIS. However, what this means is that... if for example, a state like Kano refuses to adopt the implementation of the SHIS, the government should make it illegal to do so and make them aware that they are breaking the law. So, I think the SHIS is a game changer once many states begin to adopt mandatory health insurance. I also think that social media could play a big part for the future of UHC in Nigeria because I know a lot of doctors in Nigeria who use this medium to get information across to people in order for them to prioritize their health. Once Nigerians start making health a priority then you can rest assured that when you tell them to pay a small fee of 15,000 naira per annum for health insurance this can provide some basic minimum of care for them and their families... Because if you walk into a health facility for a caesarean section, it will cost an individual a fee of 200,000 naira an operation. This is the trade-off. So, I am certain of a better future where the Nigerian health sector will achieve UHC soon." (RC.45)

"My view is that if properly implemented with well-structured monetary and evaluation systems in place - and with a judicial process for managing funds, risks and to prevent corruption in the chain across the agencies - the Basic Health Care Provision Fund for the first time can provide the opportunity for Nigerians to provide primary health care on their doorsteps free of charge, and that will significantly improve healthcare outcomes in Nigeria." (RC.29) 
"I am assured about the future of UHC in Nigeria but it's not going to be easy or straightforward to achieve this task. It will need putting systems in place to revitalize the health system. For instance, imagine going to your village to witness a 24-hour functioning primary health centre with constant electricity, running water and the basic commodities needed for a woman to deliver a child, immunize the child, take care of the child's injures if needed and all of that. I think that would be a significant leap if a system was put in place. Also, this system must reduce out-of-pocket expenditure, catastrophic expenditure of many families in the villages. We have estimated that it will save a family in Nigeria over 10,000 naira every month if a proper system is implemented, which will be a huge boost to the economy. I know that this will work, but it will take time to implement it because it's not a one-year, two-year program... I am hoping that before we get midway into the SDGs the system will start working. I am not 100 per cent sure even with the Basic Health Care Provision Fund that Nigeria will achieve the SDGs. We need more significant investments and getting the primary health care and tertiary care to work. Making sure our people are educated, employed, fight poverty/illiteracy and all of this are complementary issues that affects our health outcomes. Then when these systems are functioning, the health sector can easily achieve UHC." (RC.35)

This group of participants offers a progressive opinion focused on building a sustainable system which will secure the prospects of UHC Nigeria. These participants propose the implementation of mandatory laws requiring or forcing states in the country to adopt an SHIS program. They reason that making the SHIS scheme mandatory for states increases the probability of expanding health coverage because, when fully implemented, the scheme can reach many individuals in various local communities, opening up access to essential health services.

Some participants in this group think the best prospect for UHC Nigeria is through the implementation of a well-structured performance management and evaluation framework with safeguards for managing risks and achieving key health policy objectives. For example, the Basic Health Care Provision Fund, initiated by the National Health Act to substantially increase financial revenue into the health system, needs a well-structured financial management strategy to ensure accountability at national, state, local levels.

Other participants highlight the need to address issues outside of the health sector which might impede UHC’s development. Factors such as poor education, climate change, transportation, housing, and national security may all influence health. 


\subsection{Conclusion}

Health policy emerges from the places where politics and policy development intersect. The study of policy formation on its own focuses on the content, context and process of the discourse (Hill, 2014). Policies can be described and evaluated, and alternatives explored. To analyse policy success or failure, consideration must also be given to politics; the factors that affect interactions between decision-makers as policy is adopted, elaborated and implemented. This chapter reflects on the policy process concerned with UHC in Nigeria and the nature of power and politics that surround the UHC discourse.

Informants acknowledge that Nigeria has not yet achieved UHC but suggest that the government is developing policies that open the way for a major extension of coverage. Many respondents believe that Nigeria can achieve UHC in the coming years, providing that the health system can overcome certain challenges and obstacles. Although participants have different opinions of what UHC entails and how it can best be implemented, they all share a similar objective, which is to provide social and financial risk protection schemes for poor and vulnerable populations as a basic human right. Universal health coverage is the ultimate expression of fairness and one of the most powerful social equalisers among all policy options (Sakolsatayadorn and Chan, 2017). The next chapter discusses what the findings reveal about the UHC policy process in Nigeria. 


\section{CHAPTER 7}

\section{DISCUSSION}

\subsection{Introduction}

The first section of this discussion chapter provides a summary of the main findings from the study. Next the chapter turns to provide answers to the seven research questions listed in Chapter 1. Later sections consider what the research adds to the existing literature on UHC policy development, and the methodological limitations of the study.

\subsection{Overview of the study}

The research objectives are:

1. To investigate the roles and interactions of policy actors in the UHC policy process and how they impede or advance progress in extending coverage.

2. To investigate the extent of consensus or disagreement between influential actors and interest groups on the direction of UHC policy, and how that has affected progress.

3. To identify the barriers and obstacles which affect Nigeria's process of health policy towards achieving UHC.

These objectives were pursued by examining the actions and interactions of key actors who make up the network concerned with the development of UHC policy in Nigeria, and especially the obstacles and challenges that may help explain the slow pace of progress towards UHC in that country. This was undertaken through a study divided into two phases, which respectively examined formal aspects of the network using SNA techniques, and the nature of the policy process as revealed through qualitative interviews.

As mentioned in Chapter 5 (analysing the UHC policy network), the respective positions of, and relations between, actors who had been identified as part of the policy network were examined using a range of SNA measures. This enabled the researcher to map out the key organisations implicated in the development of UHC policy, the nature of their relationships and their relative influence in determining outcomes. 
These actors and organisations enjoyed different degrees of access to information, to professional support and material resources, in part at least according to their location and connections to other network actors around them. Relationships within the network were investigated using six relationships that were assumed to indicate different forms of influence or power, named reading another's written products, taking advice, discussion, coalition, affiliation, and conflict). These patterns of relationships created opportunities for influence but might also restrict a policy actor's scope for action because of obligations to, or conflict with others in, the network (Emirbayer \& Goodwin, 1994).

The SNA analysis suggests that, while the six UHC policy network relationships point to a complex mix of relationship forms, a fairly stable subset of seven organisations have high centrality and influence across most relationships. Other organisations and actors have high centrality only on some relationships. In essence the UHC discourse is substantially shaped by three important categories of actors: which the author has termed power actors, gatekeepers/brokers, and actors who have close connections to the power actors. There may be variations in the roles of less central actors because of their relationship to the core and peripheral network components according to several criteria. It is important to identify isolated policy actors on a policy network’s periphery, and to map whether there are some relationships of the UHC discourse in which they become more involved. For instance, conflict with the UHC discourse had the fewest number of involvements amongst policy actors, leaving the FMoH, hospital managers and HMO representatives prominent as those most likely to be involved in policy disputes. By contrast, those policy actors whose influence comes from their close connection to the Ministry (the power actor) have a more limited role in policy network information exchange.

In general terms, senior government officials have most influence over UHC policy development and decision-making, and also a degree of control over the transfer of information within the policy network. Their influence highlights that government officials both drive and obstruct the policy process involved in achieving UHC in Nigeria. However, coalitions and connections to other actors are also important. The FMoH, NMA and to a lesser extent the WHO and NHIS have influence over UHC policy processes but might require support from other connected policy actors if particular initiatives are to succeed. One rather surprising finding is that the SMoHs, while in the inner circle of influential organisations, are neither a power actor or a key broker, and we will return later to say more about their position. 
The second findings chapter - Understanding the UHC network via policy process theory (Chapter 6) - investigated the policy process relating to UHC policy in more depth, using qualitative interview data. It drew on both mainstream policy process theory and Kingdon's (1984) multiple streams framework to examine how policy evolved and the degree to which a window of opportunity opened - and remained open - for UHC reform. From the perspectives of various actors, the aim of the framework is to increase the awareness of what occurred when UHC emerged onto the Nigerian health policy agenda. More recent expositions of the MSF (Ridde, 2009; Boswell and Rodrigues, 2016; Zahariadis and Exadaktylos, 2015) suggest that full convergence of the politics, problem and policy streams predicted in Kingdon's seminal book may not always be present when a policy moves forward, and that conditions may change between the time of initial policy formation and further development of implementation, and the Nigerian story fits this picture of generally favourable conditions at certain times giving way to times when barriers to further progress emerged. The period leading up to the 2014 Health Act was a time when a number of positive factors supporting UHC came together and represented a breakthrough for groups campaigning for reform. But the window of opportunity was widely perceived to be time-limited because of the possibility that the election outcome would halt progress. Despite a push to secure firmer government commitments on securing new budgets and the planned expansion of coverage, progress since the change of government once again appears to have slowed.

Chapter 6 utilised qualitative interview accounts to examine how UHC policy was formed and contested, and also to understand the engagement of various relationships of power that could be harnessed by policy actors within the UHC policy discourse. Policy development occurred within a policy network characterised by the heterogeneity of prominent actors (see Table 13), with collective organisational perspectives proving more important and enduring than the perspectives of individual actors, even when forceful personalities were involved. While international as well as domestic organisations influenced the policy process, the international bodies generally had decisive influence only at certain key stages and in relation to particular issues, rather than have the continuous and wide-ranging control that the Federal Government, the FMoH and its parastatals and to a lesser extent the NHIS, exerted. Two relationships that differentiate the organisations at the centre of policy from those with more peripheral, issue specific, or episodic involvement are longevity of participation and organisational decision status. Both provide a foundation for claims to authority and legitimacy, and key organisations 
typically have both longstanding involvement and a formal mandate to take part in policy making.

Power manifests in a number of ways through the interactions of actors in the health system at local, national and global levels, particularly during the negotiation of priorities and allocating resources (Sriram et al., 2018). The power sources in the UHC policy process can be divided into political power, financial power, professional power, power through technical expertise and power in numbers (via formation of coalitions), which were all discussed in Chapter 6 .

At the time of the study UHC was a key issue in the election campaign and ongoing political debates. A large group of pro-reform informants acknowledged that Nigeria has not yet achieved UHC but argued that policies being developed by the then government would significantly extend coverage. They believed that Nigeria would soon be able to achieve UHC because changes underway since the 2014 Act would help overcome the challenges and obstacles that had blocked progress. However, others were more sceptical and saw the obstacles to reform as more intractable, expressing divergent views about the role of the private sector in achieving UHC, and worried about the degree of political commitment to push reform through. While almost all participants express support in principle for UHC it was clear that different groups had different understandings of what UHC entails and how it should be implemented.

The chapter now turns to a review of the research questions, and how far the study has provided answers to them. Readers will recall that the research questions were as follows:

1. Which influential actors and organizations are shaping Nigerian UHC policy?

2. What are the patterns of power relationships and influence in the policy network?

3. What are the roles of international and domestic actors in shaping policy?

4. Is there any disagreement in the policy network over how to pursue UHC, or the feasibility or desirability of UHC policies?

5. What gains and setbacks have so far been encountered, as seen by policy actors?

6. How far along the UHC path do policy actors think Nigeria has travelled, and how do they assess the prospects of achieving adequate coverage?

7. What general lessons can be provided by Nigeria regarding the policy process associated with UHC reform? 


\subsection{RQ 1: Who are the influential actors and organizations shaping UHC policy in Nigeria?}

Policymaking is a complex process that involves actors and organisations negotiating in pursuit of their interests and marshalling different types of evidence to support their policy positions (Russ et al., 2014). We have seen that the policy network in Nigeria is characterised by the heterogeneity of prominent actors, including those from government (federal, state, and local), NGOs, UN bodies, labour groups, multilateral agencies, think tanks, the private health sector, and the medical and nursing professions. This mix of organisations appears typical of the type of policy network that shapes health system reform in low- and middle-income countries (Khan et al., 2017). Interestingly however, the study respondents reported that the main areas of policy under debate depended more on the positions developed by the various organisations than on particular named individuals. The SNA data suggest that collective organisational perspectives and patterns of relationships take a broadly consistent form and that individual actors tend to operate within the envelope of an organisational position.

Patterns of relationships within the network revolve around three key entities: a major power actor, gatekeepers (or brokers), and policy actors connected to the power actor. On the periphery of the network other actors in more isolated positions may become involved more intermittently under certain conditions via their contacts with well-connected actors. These isolated actors on the periphery of the network tended to participate in some modalities of engagement more than others. For instance, when the "conflict" relationship was examined, it appeared that many preferred to take a more neutral position in respect of the major policy disputes about the role of the private sector, disputes in which the main actors were the $\mathrm{FMoH}$, hospital managers and HMO representatives.

The three categories of power actor, brokers, and policy actors connected to the major power actor were identified on the basis of SNA centrality scores. The principal power actor, defined as the organisation that had the highest degree centrality in every cohesive sub-group, was the $\mathrm{FMoH}$. In reality, as the qualitative analysis indicates, the FMoH may be seen as a kind of shorthand for the Ministry itself and the other departments of the Federal Government, which may sometimes exert influence via this channel. The policy brokers - the main agencies in the network that control the flow of ideas and information - are the NMA, WHO and NHIS. These three categories were not entirely separate, as the $\mathrm{FMoH}$ (the power actor) also operates as a 
broker to connect actors such as professional bodies with information providers such as the WHO. Other actors closely connected to the central actors and exercising some influence because of this are USAID and the World Bank. All these organisations have the potential to influence or shift decisions on given issues affecting UHC policy, but not with equal force over time. The Federal Ministry has decisive control over the overall policy process, while the brokers are key actors with continuing influence on the content of policy, but not the same decisive authority. The World Bank and USAID had more episodic issue-specific influence based on their close connections to the other listed organisations.

The seven key organisations listed above were the key actors exercising the greatest influence within the policy network, but this did not apply uniformly across all six relationships of influence (the modalities of engagement). As mentioned above, the "conflict” relationship was the most obvious exception, and this was excluded from an exercise undertaken to assess the degree of overlap/correspondence of centrality on each of the other five relationships (read, advice, discussion, coalitions, affiliation). Mantel’s (1967) Quadratic Assignment Procedure (QAP) method was used to assess the extent of overlap of relationships across the five relationships. This showed that on both the "advice" and "read" relationships six of the seven key organisations had high centrality scores, with the only other organisations emerging as prominent in these relationships being HMOs for “advice”. Interestingly, the SMoHs scored low on the "advice" relationship, and less well on other relationship that most other key organisations, suggesting that there are not as closely involved in key decisions as might have been expected (see Table 11). The seven central bodies read each other's documents and share advice, but interestingly influential actors might also read the work of non-influential actors without taking their advice. For example, Table 11 shows that two government officials (Gs46 and Gf30) read the works of non-influential actors such as hospital managers and doctors, presumably without giving so much weight to their advice that they are allowed a central role on other influence relationships. The seven key organisations feature as prominent nodes in the centrality diagrams for all five relationships and are prominent in both the "advice" and "read" relationships. The pattern with the other three relationships shows a more diffuse set of influence relationships. Perhaps most interestingly, on the "discussion” relationship SMoHs, hospital managers, doctors, health partners and the NMA were prominent nodes alongside the seven key organisations, suggesting more intense interaction on this relationship than say on the "advice” relationship. The "coalition” and "affiliation” relationships are more indicative of the support given to the central organisations, or the formation of opposing groups of 
stakeholders. Regarding the “coalition” relationship (Figure 31), hospital managers are shown to be important supporting actors, while in terms of affiliation (Figure 32) HERFON and health partners are important allies.

SNA can also help to identify influence channels. In particular, it shows the roles played by the FMoH, NMA, WHO and NHIS in creating alliances in the UHC policy debate. These are the main policy brokers or gatekeepers in the policy network, with the highest betweenness centrality scores in all six policy relationships. Most of their brokering occurs through facilitating contact or information flows between actors, meaning that the FMoH assists with exchanging information between state and non-state groups, international and national partners and local and state groups. The NMA, as a gatekeeper, has control over the information flow on UHC-related policy guidelines and frameworks received from the $\mathrm{FMoH}, \mathrm{SMoH}$, NGOs and UN bodies to doctors and other relevant professional networks. The World Health Organisation is a key gatekeeper of global resources supporting development of the healthcare system and acts as a channel for disseminating knowledge generated by global health policy discourse to domestic organisations. Perhaps surprisingly the World Bank and USAID - the policy actors connected to the power actor - have lesser roles in facilitating information exchange.

Unsurprisingly the study confirms that senior government officials are able to substantially shape the direction of policy, and along with WHO facilitate the transfer of information within the policy network. This means they are in a position both to drive policy forward and at times to obstruct progress towards achieving UHC. The NMA, WHO and NHIS are brokers whose influence permeates the UHC policy debate and supply a flow of information to network actors. In general, the analysis shows that the FMoH, NMA, WHO and the NHIS all act as brokers to feed ideas and information influencing UHC policy development but may need support from connected policy actors for particular initiatives to be successful.

\subsection{RQ 2: What are the patterns of influence and power relationships within the policy network?}

Power is relative and relational and has an impact at levels that range from macro policy making to locally implemented interventions. Several studies have drawn attention to power influences affecting international health reforms, which extend from conditionality in donor financing 
(Kentikelenis et al, 2016), to control over consultancy expertise (Lee et al., 2002), and rare instances such as in the Thailand case where domestic policy makers were able to push ahead with UHC reform despite World Bank advice that this was infeasible (See: Evans et al., 2012). Power is often a neglected factor in recent work that emphasises evidence-based strategies to guide decision-making, which focus more on issues of knowledge transfer rather than politics (Sriram et al., 2018).

This study shows that while some organisations have enduring and continuous influence, the exercise of power is quite widely dispensed. The international bodies, which are usually seen as powerful actors in global terms, only seem to exert real influence at certain critical times and usually in relation to specific issues. For example, the World Bank Group, which the research anticipated would be a powerful actor in the Nigerian context, was less central to the policy network than the WHO. The qualitative interviews suggested that the circle of policy "insiders" centred on governmental bodies at the national and state levels as well as the NHIS and NMA. Healthcare unions and frontline health workers had more episodic influence, often in local contexts and in relation to workforce and service delivery policies, something that has been reported elsewhere (Gore and Parker, 2019).

To summarise the content of section 6.3, power in the UHC policy process has multiple relationships, and depends on actors' ability to make authority claims based on their political connections, control over finances, professional authority, technical expertise or critical mass (“power in numbers”, usually via the formation of coalitions).

The study categorised policy actors according to their location in four sectors or sets of institutions: government, the health sector, international bodies and labour organisations (see: Table 7). The qualitative interviews added depth to an examination of how these interact and their relative ability to shape policy. Those interviews support the general picture emerging from the SNA that government - in the shape of the FMoH and the Federal Government more generally - is the dominant actor determining the direction of policy, and that key organisations from the other sectors (WHO, HMA, NHIS, SMoHs, the World Bank and USAID) also have significant roles, with the HMOs, labour unions, doctors and other health professions getting involved from time to time. Much of this is fairly predictable, but certain actors mobilised less power to steer events than might have been anticipated. The limited role of the World Bank has already been mentioned, and the fact that the SMoHs had relatively low centrality scores within the inner circle is also significant. The peripheral position of the HMOs and professions 
on many issues suggests that the private healthcare sector has not managed to steer the UHC reform process as much as some respondents feared.

Probably few readers will be surprised that government largely controls the reform process. The literature tells us that health system reform requires competent governance based upon technical knowledge, ethical probity, political skills, and strategic vision (Frenk, 2011). However, the picture of reform in developing countries in past decades did not always take this form, and there are several case studies where structural adjustment policies and conditionality imposed by the IMF and World Bank overshadowed the domestic policy programmes of national governments. That has not been the case in Nigeria, where both successes and problems in the reform process are largely the responsibility of the governing politicians and administrators. The major step forward - the 2014 National Health Act - is among the Goodluck Jonathan government's major achievements, although its enactment may have been more to do with an attempt to court electoral popularity than with any longstanding commitment to the ideal of UHC. Although the Act has not broken the logjam that stops UHC becoming an immediate reality, the guarantee of earmarked funding through the BHCPF and $1 \%$ CRF are likely to make a big difference in future years.

Part of the FMoH's power comes from the coordinating role it performs in mediating communication between other actors and information flows. The Ministry is responsible for technical support to the health system, and inter-organisational relations regarding health policy and provision of services. It is normally the channel via which the Federal Government communicates with external policy organisations, and it mediates the flow of technical expertise from the UN bodies and international health organisations to the SMoHs, domestic NGOs, private health sector groups, civil society organisations, and academic institutions. Against these sources of power, its main area of weakness is that it is financially dependent on the Federal Government's allocation of healthcare funds, so that it is in competition with other government departments when it comes to gaining the resources needed to take forward initiatives linked to UHC.

Another important finding about the role of government is that the FMoH has considerably more prominence in UHC deliberations than do the 34 State Ministries of Health, even though Nigeria has a decentralised healthcare system, in which the States have considerable discretion when it comes to local patterns of access and service delivery. As we saw earlier, while the 
SMoHs are one of the seven key organisations, they had lower centrality scores than most others, and scored particularly low when it came to the "advice" relationship. This SNA finding about network structure chimes with the qualitative interview data finding that the SMoHs were not as closely involved as they might have been in deliberations regarding health insurance system development. In many cases they appeared to have been slow to implement feasible State health insurance schemes under the umbrella of the NHIS, and there was little commonality of approach at the State level. This is one factor that may help explain the slow progress in increasing the number of citizens covered by public health insurance and progress towards achieving UHC.

The three most influential institutions from the health sector as measured by their centrality scores in the SNA were the NHIS, HMO representatives and hospital managers. The NHIS has a brokerage role in mediating communications between government and health sector organisations, which reflects its position as a decentralised public health authority responsible for the oversight of the various public health insurance systems. Its tasks of fund management and organising HMO and provider registration, accreditation and monitoring have become more complicated as new and decentralised schemes have been established and private sector involvement has increased. Acting on behalf of the FMoH the NHIS can provide conditional funding to support the development of State insurance schemes or reduce financial deficits, but as we have seen this is an area of weakness which may have impeded UHC development and may be reflected in the patterns of relations shown in the SNA. Onoka, Hanson and Hanefeld (2015) argue that decentralised State public health insurance made most progress when the FMoH liaised directly with the States to get new programmes off the ground but ran into problems when the NHIS took over the leadership role. The qualitative interview from the present study confirms Onoka and colleagues' suggestion that the growing role of the HMOs caused discomfort at State level and reduced State support for public health insurance. This fits with the finding from the SNA analysis that it was HMOs and private sector hospital managers who scored highest on the "conflict" relationship. Admittedly SNA analysis in itself does not reveal the nature of that conflict, but the qualitative analysis shows frequent tension between the HMOs, the NMA and other actors.

The effective implementation of UHC in Nigeria undoubtedly involves close cooperation between domestic policy actors and international institutions. External non-state policy organisations can influence policy processes because of the control they have over technical 
expertise, and (to a lesser extent in the Nigeria case) donor finance. Governments are willing to accept inward knowledge transfer because of the added legitimacy this gives to policy. Promoting UHC in LMICs has moved near to the top of the agendas of all the major international health sector bodies in recent years, and there a fair degree of consensus about the broad shape of national policies that should be encouraged, though also a recognition that one size does not fit all. There is a well-established corpus of evidence from pathfinder countries like Thailand, Mexico and Ghana that has been collated by organisations such as the Joint Learning Network for UHC and fed to WHO, World Bank, USAID and ILO, and can be passed on by collaborating with countries such as Nigeria (Campos and Reich, 2019).

Yet there are challenges as well as opportunities. These challenges include the overshadowing of the existing programmes in these countries, ignoring the capacity of their national health systems, providing poor advice based on ideology or the non-transferability of lessons from other countries (Bowser et al., 2014). For example, there have been mixed results from the research into whether externally funded vertical programmes (for example HIV treatment programmes) strengthen or weaken current health systems (Yu et al., 2008). Probably the mistakes of the past have led the major international bodies to be more cautious, and in the period covered by the study the WHO and World Bank in particular appear to have taken a restrained stance centring mainly on technical expertise necessary to develop the building blocks required to develop sustainable financing mechanisms, a viable health insurance system and the necessary health delivery infrastructure and professional workforce. From respondents accounts the WHO did push very hard in an earlier period to encourage the Nigerian government to bring UHC onto the policy agenda, but in recent years is involved more episodically in relation to particular technical challenges. ILO, which is a major player in supporting UHC policy development in Southeast Asia, featured less in the Nigerian health policy network than had been expected.

The institutions making up the labour sector generally lacked sufficient power to change the direction of policy in isolation but could sometimes bring more influence to bear by forming alliances. Medical professionals have attempted to influence UHC policy at different stages of the policy cycle to minimise their losses and maximise the gain from the proposed changes. The NMA has often taken the lead in assembling coalitions with other professional associations, unions or private sector bodies to push policy in its desired direction, harnessing what in Chapter 6 the author termed "power in numbers". Among other moves, the NMA has tried to bring together medical and non-medical professional agencies to influence policy on healthcare 
system regulation and maintain professional autonomy. It can resist policy implementation by using the cultural authority of the medical profession (Campos and Reich, 2019) and also the threat of wider industrial action. Some critics have raised the question of whether the power dynamic between the federal government and the NMA has led to an unhealthy situation where the federal government is complicit with the NMA's demands because it fears walkouts, strikes, and protests. The Association has come in for criticism for being so enthusiastic in its use of industrial action as a weapon to cause short-term damage to the healthcare system by halting patient care, but this appears to have been an effective tactic in preserving its position near the centre of the policy network.

\subsection{RQ3. What are the respective roles of domestic and international actors in shaping policy?}

Part of the answer to this question has already been provided by the brief review of the relative influence of the international bodies and three categories of domestic actors above and will not be repeated here. As has been explained, the Federal Government has been the key actor in taking decisive steps forward, such as the statement at the Presidential Summit on Universal Health Coverage (UHC) in 2014, and the subsequent enactment of the Health Act. These created a window of opportunity for concrete steps to widen coverage, but, as has also been argued, such "windows" are time-limited and at the present time it looks as though momentum has again been lost. However, if we focus in on this limited step in the path towards UHC it is clear that international and domestic organisations also played a part in building pressure through multi-sectoral advocacy, which pushed the government to make commitments to the benefits and feasibility. Much lobbying was done to gain attention for the issue. Moreover, the government's support for the UHC appears to have had a pragmatic, more than an ideological basis. Commitments were made in the run-up to the 2014 presidential election, and the electoral appeal of the policy was a definite factor.

Because of the complexities and intricacies of health coverage expansion on a larger scale, it is difficult for government going forward to implement concrete reforms without the domestic and international partnerships of which the policy networks are comprised. Apart from the recent change of government, differences in view among stakeholders about how to develop 
public health insurance and controversy about the role of the private sector, along with difficult economic conditions help explain why progress towards UHC has again slowed.

The typology in Table 20 shows where the various organisations sit in terms of their status and power to influence UHC policy development. It distinguishes those who can be considered members of an insider group from an outsider group, and which domestic and international bodies fall into each category.

The table again shows how UHC policy is primarily steered by a number of insider organisations comprised of the Federal Government, the Legislative Committee on Health, FMoH, SMoH, and NHIS. Outsider organisations - for example the WHO, USAID, World Bank, NMA and HERFON - remain highly visible in the policy network, but only occasionally exert real influence as non-state actors, and then typically on particular issues or aspects of reform.

The authority and legitimacy of the government to make final decisions on public policies is its most influential resource. As outsiders, non-state actors (domestic and international) have different organisational priorities and aims as well as technical resources and have the greatest voice when the issues of concern lie within their domain. This means that, in terms of continuous involvement and steering of reform, the state actors were generally able to dominate the policy process. The influence of health officials over civil society members can be seen at all policy stages. For example, the determination to embark on a path towards UHC through a particular form of public health insurance came from state actors rather than bodies like WHO and World Bank, which were setting out a wider range of policy options.

\subsection{RQ4. Are there disagreements within the policy network over the approach to UHC to be pursued, or the feasibility or desirability of UHC policies?}

Some degree of disagreement is unavoidable in any health policy process and the implementation process of UHC in Nigeria is no exception. Disagreement and tension form part of the decision-making process of healthcare policies, and inevitably result in some degree of discomfort for those in the policy network. Often disagreement between actors will translate into disagreement between rival coalitions of policy organizations. At worst, disagreement may 
lead to a slowing of the policy process because of relationship breakdown amongst actors in the network (Klofstad, Sokhey and McClurg, 2019).

We saw from Chapter 6 that almost all actors express in-principal support for UHC, even if they do not share the same vision of what it will entail. Nigeria's poor performance on a number of indicators of morbidity and preventable early deaths lead many to think that UHC reform offered the best route to health improvement (Onwujekwe el al., 2019). The inclusion of UHC among the targets spelled out in Sustainable Development Goals was a further factor encouraging many stakeholders to get behind the policy. The SDG target of achieving UHC in Nigeria by 2030 is very challenging (WHO, 2019), and politicians of all the main parties recognise that failure to meet the target will have a political cost.

Cost has long been seen as one of the biggest obstacles to implementing UHC, and as discussed above, the passing of the 2014 National Health Act that enshrined increase health expenditure in law through the Basic Health Care Provision Fund (BHCPF) and the 1\% Consolidated Revenue Fund received broad support from a range of stakeholders. Yet the translation of this new legal structure into improved health infrastructure, medicines availability and workforce improvement, alongside better public health insurance that covers more people is complicated and generates many disputes about means to the desired end of UHC. The financing arrangement was one of the big issues that gave rise to disagreements among participants. Against those respondents who believed that the injection of additional finance would enable politicians to translate policy rhetoric into policy reality (Aregbashola, 2018), others contended that affordability and financial sustainability remained huge issues. The present level of public expenditure on health, which is much less than the $15 \%$ of government expenditures allocated to health deemed necessary in the Abuja Declaration. Exercises conducted by WHO (2011) and USAID (2013) before the present study began suggested that Nigeria was one of a number of Sub-Saharan countries that would meet the target only with great difficulty.

Doubts about affordability led to differences of view about just what UHC would entail in terms of who would be covered and for what range of services. As we saw in Chapter 2, UHC is often represented using a cube with three relationships of coverage (population covered, services provided and extent of financial coverage), with of which might be varied in different reform designs (Cotlear and Rosemberg, 2018) (see Figure 2). For some respondents this suggested the necessity of trade-offs, on the basis that a LMIC could not provide everything for everyone, free of charge (compare: Ochalek et al., 2020). Many concluded that even if the aim was to make progress on all three coverage relationships, a phased approach to 
implementation was more feasible than a single "big-bang” reform. A step-by-step approach is dependent upon efforts to gain political support from (and sometimes confront) specific interest groups, and also relies on developing institutional and technical capacities in the move towards implementing UHC. This meant that much of the conflict within the policy network involved disagreement about specific incremental reforms that in themselves were just small steps towards the end goal.

As shown by the SNA and confirmed by the qualitative interviews the main conflicts in the study period involved the respective roles of the public and private sectors in healthcare delivery and the development of public health insurance schemes. Those who saw the path to UHC coming through an expanded public sector generally opposed lobbying for a mixed system. On the other side of the argument were those who argued that private sector involvement in well-regulated health insurance would hasten the progress of UHC by increasing the number of beneficiaries and the size of the risk pool, which is crucial for the financial viability of a national health insurance scheme. Often the positions taken reflected organisational positions and interests, with HMOs, other private providers and a section of the medical profession lining up in favour of a mixed system, and many officials, NGOs and public sector doctors favouring a predominantly publicly funded and managed system.

Although actors who aligned directly with the private sector were a minority in the UHC policy network, and in many cases appeared disconnected from the core power actors, support for a large role for the private sector clearly extended into the upper reaches of government. President Buhari has expressed the view that public-private partnerships are critical in generating the necessary finance to extend coverage (Infrastructure Concession Regulatory Commission, 2020). Moreover, the large size of the Nigerian private sector in relation to the public sector meant that it would not be ignored. It can be argued that the ongoing conflict and tension between supporters of a mainly public route to UHC and those who favoured private sector involvement did not reach any early resolution because these were very powerful and well-matched groups. There was effectively a situation of "countervailing power" (Galbraith, 1952) where neither group was able to establish dominance and direct the UHC reforms decisively in their preferred direction. But in contrast to the positive policy dynamic that Galbraith had believed emerged in the USA from the twin centres of power of business versus trade unions and consumer organisations, the conflict in Nigeria appears to have led to policy stasis and lack of progress. The situation more closely resembles Light's (2010) description of 
American health care, where the medical profession's domination of the medical market was significantly reduced through federal legislation, and cost controls imposed by insurance companies and the federal government programmes. In the US case, this ongoing conflict without a clear winner has led to continuing system tensions and the frustration of reform initiatives (of which the curtailment of Obama's Affordable Care Act reforms is the latest example). It seems that in Nigeria as well an intractable conflict between powerful interest blocs poses a major obstacle to early movement towards UHC.

\subsection{RQ5. What are the main gains and setbacks to date, as seen by policy actors?}

Before turning to discuss what the respondents themselves considered to be the main positives and negatives to date, it is worth summarising the findings from a systematic assessment of progress towards UHC in eleven countries undertaken by the Government of Japan and the World Bank (Maeda et al., 2014; Reich et al., 2016). This exercise assembled data on countries that had committed to UHC as a key national aspiration and categorised them into four groups based on stage of progress. Countries in group 1 have set achieving UHC as a national policy agenda but so far made little progress; countries in group 2 have made significant progress towards UHC yet still face substantial coverage gaps; countries in group 3 have recently achieved many UHC policy goals but now need to deepen and sustain coverage; and countries in group 4 have mature UHC health systems but must still adjust their national policies to meet changing circumstances (Reich et al., 2016). It seems clear from the criteria used in this study that Nigeria falls into group 1, as a country in the early stages of UHC with very low population coverage. The country's status with regard to UHC policies and programmes is that it still focuses on setting agendas for developing new systems and piloting new programmes. Implementing UHC in Nigeria has been a lengthy, and sometimes low-priority and conflictridden process that has been affected by unexpected contingencies.

In the eyes of most respondents that major steps so far have been: (1) the establishment of NHIS and decision to make health insurance the primary mechanism for achieving UHC; (2) the 2014 National Health Act; and (3) the earmarked budgets created by the Act.

As mentioned earlier in the thesis, the scope the NHIS has widened with the Formal Sector Social Health Insurance Programme, now operating alongside other programmes such as one for the informal sector and a voluntary programme for certain disadvantaged groups (Sunday 
et al., 2018). Respondents who support the NHIS in its present form see it as offering a staged progression towards increased coverage over time. However, opponents criticize it for not achieving expected milestones. Coverage in Nigeria remains low, with less than $5 \%$ of the population having health insurance) and over 65\% of healthcare funded from households' outof-pocket payments (Aregbeshola, 2018). In spite of this criticism, many in the policy network still think that the NHIS is the most promising route to UHC, providing that it is appropriately managed. As noted earlier the role of the private sector and the relations between the NHIS and state insurance schemes remain areas of contention.

UHC policy optimists remain positive about the National Health Act, seeing it as a viable framework for fast-tracking progress towards UHC, and praising the financial provisions in particular. The Act strengthens the NHIS's position as 50\% of the Basic Health Care Provision Fund (BHCPF) is to be managed by the National Health Insurance Scheme (NHIS) to provide basic health insurance for vulnerable populations.

The above developments show that there has been substantial government support for UHC, even if this has ebbed and flowed over time, but the more critical respondents believe that further implementation delays are likely. Among the factors highlighted by participants that block or set back further progress are the uncertain political climate, system capacity, problems in coordinating action at federal and state levels, poor financial management, and problems associated with HMO participation in health insurance.

Among the more critical respondents there was a large strand of the scepticism regarding the "political will” of the government to push forward UHC policy at a time of economic constraint. While it was noted that the Buhari administration appeared as keen on UHC in its election campaign as the previous government, some respondents maintained that their pledges were just lip service. Given the centrality of government in the policy network, the absence of political will, would mean that real progress towards UHC in the short term is unlikely.

The critics also expressed concerns about system capacity, claiming that, even since the financing changes introduced in the wake of the 2014 Health Act, policymakers have failed to use the full capacity of the healthcare system. They are concerned with what they see as the constant failure to introduce new measures to improve services, leading to policy stasis. They claim that policy development is bedevilled with circular arguments and constant attempts to amend plans. Generally, respondents divide into the two groups of those who suggest that UHC in Nigeria is still in its early stages, requiring patience and gradual implementation, and sceptics 
who argue that unresolved problems are blocking further progress. The main problems, as seen by these informants, are disagreement about the specifics of funding mechanisms, failure to increase healthcare system capacity sufficiently to permit new program rollouts, and poor coordination of Federal and State Ministry of Health insurance programmes.

This latter issue of poor coordination between federal and state levels appears to be one of the main factors impeding progress on UHC. Even with the new budgets created by the 2014 Act, the Nigerian healthcare system remain under-funded, and central government allocations to the states are frequently late, causing delays in changes at the state level. In most years the FMoH distributes the bulk of the central health budget to key parastatals, leaving insufficient funding for the SHISs in particular. Respondents suggested that more funds are needed and that even the improved coordination of service development planning between state and federal government levels is needed if extra funding is going to have the maximum effect. As mentioned previously, the SNA findings on relationship patterns within the policy network suggest that contacts of this kind are relatively infrequent and weak.

According to respondents, mismanagement of limited financial resources at federal and state levels frequently involves corruption. This undermines resource planning and allocation by the FMoH and is harmful to UHC preparations. Informants stated that systemic corruption affects several health services programmes and drives patients away from public facilities towards using private healthcare. A study within the same research group as the present study at Swansea University found that "dual practice” doctors in Nigerian public hospitals frequently push patients towards their private facilities (Eze, 2019). Our findings indicate a widespread perception that corruption acts as a barrier to healthcare access, which negatively affects progress towards UHC. Measuring and tracking corruption in the healthcare sector is important to guarantee that there is progress towards achieving UHC (Hsiao et al., 2019).

For some respondents the important role that HMOs have in the health insurance schemes is another major impediment to progress. This is partly about probity, but perhaps more centrally about the perverse incentives that prevent these private organisations from expanding coverage to the whole population. Respondents claimed that there is a corruption aspect whereby government funds channelled to HMOs fail to be translated into services for the intended. But more crucially many respondents point to the HMOs failure to boost the number of Nigerian people covered by health insurance. They claim that HMOs profit more than enough from the 
status quo, leading to complacency and a lack of incentive to extend services or coverage further. This means that the overall management and operation of Nigerian public health insurance is flawed, and that major problems are building (Falade, 2020).

\subsection{RQ6. How far along the path towards UHC do policy actors believe Nigeria has travelled, and what is their assessment of the prospects of achieving adequate coverage?}

Respondents divided into optimists and pessimists. There is optimism amongst many network members about the timescale for achieving UHC in Nigeria, yet others are doubtful that the 2030 SDG target can be achieved. The former state that the progression of UHC implementation may have slowed because of problems like limited finance, capacity, and lack of political commitment, whilst the latter believe that little real progress has being made in addressing these issues. In the 2010-15 health financing plan for Asia Pacific region, WHO (2009: VI) identified four indicators of progress towards UHC: total health expenditure of $4 \%$ to $5 \%$ of GDP; reducing out-of-pocket spending levels below 30\%-40\% of total health expenditure; ensuring that over $90 \%$ of the population is covered through risk-pooling prepayment schemes; and achieving close to $100 \%$ coverage of poor and vulnerable persons with safety net schemes. As the time of the study, Nigeria was close to the GDP requirement (spending 3.9\% of GDP on health), but well short on the other indicators of progress. Out-ofpocket spending was $72 \%$ of total health expenditure; prepayment and risk pooling schemes covered less than $10 \%$ of the population, and less than $39 \%$ of poor and vulnerable persons had coverage (WHO, 2019). The known disparities between benchmarks for UHC progress and Nigeria's actual performance gave the more pessimistic stakeholders plenty of ammunition to support their assessment that Nigeria was unlikely to achieve UHC in the foreseeable future.

Although many interviewees remained optimistic about continuing progress, these respondents differed in their views about what was required to achieve UHC, and how barriers could be overcome. Many see access to healthcare as a human right which must be pursued notwithstanding current obstacles. A sizeable group believe the best route to expanding the coverage of public health insurance is for government to make it mandatory for all and put more pressure on the states to roll this out in a reasonable time scale. Others talked of the need to achieve the SDG targets on UHC and argued that stronger management was needed to take this forward. High levels of out-of-pocket payments are seen as a particular problem, with 
several respondents say that financial management in particular must be strengthened. None of the respondents interviewed believed that an immediate "big bang" reform that would introduce UHC in a single step was feasible: those who believe that UHC will come envisage that this will be through incremental change in a series of progressive steps.

\subsection{RQ7. What general lessons can Nigeria provide regarding the policy process associated with UHC reform?}

Nigeria is a country that in terms of national wealth and income appears to have high potential to achieve UHC, but for various reasons has made only limited incremental gains. It has adopted the general strategies recommended by major international bodies such as WHO, while also trying to fashion a path suited to Nigerian conditions. The lessons that it may provide come in several areas.

The first area of interest concerns social health insurance as a route to UHC. Although the healthcare systems of some UHC countries include a large component of public tax-financed and directly provided services, and others give a bigger space to the private sector both regarding providers and voluntary private medical insurance, most also make some use of social health insurance. However, the evidence base remains thin concerning how public health insurance can best be developed, the choice between tax and contributions funding, whether a single national scheme or multiple and possibly decentralised schemes are preferably, and whether the private sector can have a major role in a publicly managed scheme.

It is difficult to reach any conclusion other than that Nigerian public health insurance has performed poorly. Coverage remains disappointingly low, and the way forward for the insurance system remains one of the main areas of controversy among policy actors. Where a country such as Turkey consolidated multiple pre-existing social health insurance schemes into a single public insurance scheme (Atun, 2015) and Thailand left in place existing schemes for civil servants and workers in formal employment alongside its universal coverage scheme (Evans et al., 2012), Nigeria has built on its original scheme for public-sector employees (the original NHIS) in a more piecemeal way. The introduction of supplementary schemes for particular population groups under the NHIS umbrella introduced additional complexity, and complexity has been accentuated by decentralisation and the attempt to develop SHIS. When a large element of private sector involvement via the HMOs is added to this mix, the picture 
becomes very complicated, and system fragmentation, problematic relations between federal centre and state periphery, and fierce disagreements about the role of HMOs, all appear to have slowed progress towards UHC. Therefore, one lesson from the Nigerian experience is that a simpler, more integrated public health insurance system design is likely to bring better results. A second area where lessons can be learned concerns the importance of adequate and sustainable finance for UHC reform, and how different approaches to finance meet with success or failure. Generally speaking, public funding is needed in LMICs to subsidise health costs for poor and chronically sick populations and improve equity of access. Critically, the expansion of health coverage from the formal sector into the informal sector, where much of the population does not have regular, salaried employment, makes it difficult to collect revenues from direct taxes or health insurance contributions. Funding must instead come from government revenues mainly sourced from indirect taxes (e.g., value added taxes), which then need to be levied selectively to reduce their regressive impact on the poor. In a period of falling government income from oil and gas exports, the inability of government to get poorer service users to share costs puts a huge burden on public finances, which again is a major factor in Nigeria’s slow progress towards UHC.

Progress so far has centred mainly on giving greater priority to health financing in national budget allocations, mainly by ring-fencing funding through the Basic Health Care Provision Fund (BHCPF) and the earmarking of 1\% of the Consolidated Revenue of the Federation for health. These funds represent only a small incremental increase in the overall health budget. Arguably the main intention is to try to get Nigeria over a crucial early threshold for widening coverage by developing primary care infrastructure in geographically remote areas and extending coverage to rural populations who presently get little or no access to modern healthcare. The limited progress made so far again suggests that steps taken to date may have been insufficient.

Perhaps because Nigerian policy makers are still trying to put basic building blocks of UHC in place, less attention has been paid to the issue of gaining maximum health improvement from the budget that is available, and how financing mechanisms can be designed to properly incentivize providers and patients to use the system wisely. Much of the debate in countries whose UHC schemes cover the bulk of their populations, is concerned with matters such as primary care gatekeeping, and closed-end funding mechanisms such as weighted diagnosisrelated groups, capitation and pre-specified pay-for-performance. These mechanisms are 
intended to ensure that the system can deliver UHC as reasonable cost. Probably these topics will feature more in debates about financing as a greater proportion of the Nigerian population is brought under the umbrella of public health insurance. The relatively intensive involvement of the private sector in Nigeria compared with most UHC pathfinder countries may mean that financing mechanisms designed to control costs are resisted, and that is likely to be part of the future UHC story. The present study suggest that attention should be paid to efficient use of budgets as well as re-allocation of budgets between sectors. Ensuring probity in a state sector notorious for its levels of corruption is part of this, but the overhaul of healthcare financing arrangements that several high-level respondents advocated would also make a difference.

The trajectory of UHC reform in Nigeria suggests additional lessons concerning the appropriate roles of the public and private sectors in UHC reform. The government has determined that the best way to expand coverage quickly is through public-private partnership between the NHIS and the HMOs. The rationale for this is that while the NHIS has an official mandate to oversee the health insurance system, it makes sense to harness the expertise of the HMOs in enrolling members and arranging healthcare provision. As has been documented earlier in the thesis, critics of this arrangement point to a number of issues such as corruption, tension between HMOs and unions or professional associations, the non-remittance of private payments to health providers, poor customer service, and inaccessibility to enrolees at critical moments of need, all of which indicate that PPPs are not working as well as intended.

The author's view, as expressed above, is that PPPs add complexity to an already fragmented health insurance system, and that a comprehensive review and reappraisal of the framework for delivering public health insurance in Nigeria is needed. If it is determined that HMOs should continue to have a prominent place in a reformed system, then the role of government as regulator needs to be strengthened. There is a strong case for a public agency that sets standards concerning probity, fair competition, affordability, service quality, and is responsible for monitoring performance against these standards. That is to say that PPPs do not imply "less government”, but rather a different and more active governmental role (Jamali, 2004; Scharle, 2002). It is not clear that the NHIS, as the existing system manager, is in a position to also act as regulator. Instead, government should consider creating a semi-autonomous, arm's-length agency to undertake this role. This could be based on the model found in the English NHS with the arm's-length bodies Monitor and Care Quality Commission, though in the Nigerian context a single body is likely to be sufficient. 


\subsection{What the research adds to the wider literature}

Although there are an increasing number of published studies of LMICs that aspire to achieve UHC, the majority of these focus on pathfinder countries that have made substantial progress (the Stage 2 or 3 countries in Reich and colleagues' (2016) paper), rather than countries that aim for UHC but have progressed less quickly (Stage 1 countries). Yet arguably, it is this latter group that can provide the most valuable lessons on barriers, obstacles and possible solutions as more LMICs and LICs seek to take the UHC path. Nigeria falls clearly in the Stage 1 country category. The author is unaware of any past research examining the Nigerian UHC policy process in the detail presented here, or using the combination of methods utilised, and so this study makes a unique contribution in that regard. The study uses primary data to dig deeper into the policy process than is common in studies that use secondary sources, discussing the key policy organisations, the nature of the Nigerian UHC policy network, patterns of interaction between players, and the exercise of power among policy actors.

While questions about the role of the private sector in Nigeria's national health financing system have been touched upon in the existing literature, no previous research has examined this topic using a combination of policy process theory and both qualitative interview and SNA data. Onoka, Hanson, and Hanefeld's (2015) research covers some of the same ground but relies on a more restricted data set of 35 in-depth interviews, admittedly with a similar range of institutions. The present study interviewed a larger set of policy stakeholders, who describe controversies over the involvement of HMOs and private providers in which many of them have been involved first-hand. Both the present study and the one by Onoka and colleagues are closer to the key actors than are papers based on media accounts and secondary data.

Although the present study is not unique in seeking to combine the policy process approach with more formal SNA, applying this approach in the health policy field remains unusual, and means that the author often needed to work out how to combine these methods with little in the way of guidance from previous studies. In outline the SNA provided a snapshot of patterns of relations with the policy network at a particular time, and allowed formal measurement of relative influence, alignments and conflict in the network using standard SNA concept such as centrality and clustering. The qualitative interviews provided a window on the subjective perspectives, policy aims and strategies of these same actors, and allowed the researcher to reconstruct a picture of the development of policy over time so as to tell the story of how UHC policy came to figure high on government policy agendas. The findings chapters mention other 
connections between this research and other research findings that cumulatively begin to flesh out the story of the development of Nigerian UHC policy and its link to the 2030 SDGs agenda.

Chapter 5 on the policy network and its structure provides original quantitative data on network characteristics that would have been very difficult to infer from a conventional observational or interview study. The chapter presents a four-stage policy network taxonomy that identifies the roles played by three categories of key actors - power actors, brokers, and policy actors connected to the power actors - as well as peripheral or isolated actors. The visualisations of network structure constitute unique data that are a valuable part of a mixed methods study. SNA has been deployed in other health policy and healthcare delivery studies (Blanchet and James, 2012; Chambers et al., 2012; Hindhede and Aagaard-Hansen, 2017), but rarely in combination with a more conventional policy approach. The present study provides another case to add to the small group of policy process studies also incorporating an SNA component that have emerged from the LSHTM group in recent years (e.g., Shearer, et al., 2014; 2016; Quissell et al., 2018).

Chapter 6 follows a fairly conventional policy process approach, and the main intention is to add an additional and useful country case study to the stock of policy process studies, rather than offering any great theoretical advance. The present study borrows elements of Kingdon's MSF, as well as ideas from Sabatier and Weible’s “advocacy coalition” framework but prefers not to incorporate the "3-Is" framework of interests, ideas and institutions (based on John, 1998) that has influenced some recent LSHTM policy process studies. This is because the Nigerian case involves an incomplete implementation process, thus raising the question of why a window of opportunity has not been taken and suggesting that MSF can aid understanding. The present study draws on the main elements of the MSF, such as the three streams, windows of opportunity and policy entrepreneurs, to try to make sense of the policy process in a developing country but does not seek to contribute to the sophisticated theoretical debates about the development of MSF that can be found in recent literature (e.g., Vos et al., 2014; Jones et al. 2016). The story here is one of a window of opportunity that was opened due to a perceived problem of poor health indicators and the efforts of determined policy entrepreneurs, but led only to incremental progress and now appears to be closing. In so far as the Nigerian case study provides theoretical insights, these are that windows of opportunity are important at the stage of policy implementation as well as agenda setting, that there are interesting questions 
about how long windows stay open and why they close, and that pushing through a major UHC reform may require a series of windows over time before it can be achieved.

Windows of opportunity close for various reasons ranging from subversion by interest groups that oppose reform, poor cooperation or coordination between those charged with implementing change, and general loss of momentum due to factors such as economic constraint. Studies of just why a planned policy reform is not carried through to full implementation are still thin on the ground, especially in cases where there is no clear policy U-turn but developments that progress the reform have diminished to a trickle. The present study gives an outline of the many factors that have close down the window of opportunity provided by that 2014 Health Act in Nigeria. The main components of the policy story seem much less than ideal: UHC comes to the top of the Government's policy agenda because of a global drive towards UHC, the SDG targets and a perception that poor health indicators revealed fundamental problems in the Nigerian healthcare system; an existing public sector insurance scheme is chosen as the primary vehicle for achieving UHC; instead of developing a comprehensive strategy to revamp public health insurance, the government opts for piecemeal extensions and later for decentralisation to state level; the major role handed to the HMOs only accentuates fragmentation and conflict in the system, and all this takes place in a period of domestic political instability (with an armed Islamist movement creating disturbance in the north) and worsening economic conditions. Additional uncertainty was introduced because of a change of government at just the time when the 2014 Act were expected to begin to bring about changes that would widen coverage. Policy optimists continue to believe that UHC will come in Nigeria, but momentum has gone.

And yet it would be too simple to portray the Nigerian experience as a straightforward case of policy failure, because it is not yet clear that the UHC policy is dead. In the second decade of the 21st century the inability of a national government to deliver on UHC policy is not enough in itself to consign the policy to failure, because the external push for UHC is so strong. The SDGs remain in place, and there are a powerful coalition of international organisations led by the WHO and World Bank that continue to support the policy. Significantly the drive for UHC cannot be presented as an attempt by the rich countries of the Global North to push the poorer countries of the Global south into adopting an unaffordable policy that they are unlikely ever to achieve, because some of the strongest UHC advocates are themselves from the South. Much of the accumulated stock of UHC guidance and resources circulating within networks 
such as the Joint Learning Network for UHC now takes the form of South-South knowledge transfer. Nigerian stakeholders who remain optimistic about achieving UHC may thus have good grounds for thinking that new windows of opportunity will appear before too long, and that achieving UHC through a series of incremental steps remains a realistic goal. Therefore, it can be argued that, while the present case study finished before the Nigerian UHC policy story has come to an end, it provides an account of an ongoing policy trajectory that, in combination with future research, will aid understanding of the long-term unfolding of a major health reform process.

The argument has been made that the main contribution of the thesis to the literature is to add another useful case UHC study, in this case not one that reports a reform success story but rather a struggle to maintain momentum for a policy that risks being derailed. The other atypical and perhaps valuable feature of the study is that, even when compared with other policy process studies, it has a strong empirical basis in a large corpus of qualitative interviews conducted in the field. While several recent volumes or initiatives on UHC include collections of country case studies, these generally take the form of compilations of national statistics that demonstrate the gains in coverage, reduction in out-of-pocket payments and catastrophic health expenditure, and health indices (e.g., World Bank, 2018; Balabanova, McKee and Mills, 2011). In the view of the author the literature will be strengthened if, in addition to these "overview" country profiles, researchers undertake more field-based case studies that engage directly with front-line actors and shine a light inside the policy process by collecting first-hand accounts. The present study sets out to make a modest contribution in that area, with the additional novelty of combining qualitative inquiry with a more formal SNA study component.

\subsection{Limitations of the study}

Some of the study's limitations were touched on previously, particularly in the methods chapter when the data collected from the face-to-face semi-structured interviews and structured SNA interviews were discussed. The combination of SNA and qualitative interviews represents a relatively new approach and one where there is no consensus about a robust method for combining the two kinds of data. At present each researcher undertaking a mixed-method study of this kind must seek their own way forward, and as a neophyte researcher the author concedes that his chosen strategy may not solve all the problems that bringing the methods together raises. 
Possibly, with the benefit of hindsight more sustained consideration could be given to exactly which SNA measurements can be best aligned with the qualitative data, and whether qualitative questions could be more precisely framed to mesh with emerging SNA findings. In the present study analysis of SNA data was done towards the end of the study period at almost the same time as analysis of qualitative data, so SNA findings were not available when the quantitative interviews were conducted. This was mainly because of the logistics of a Ph.D. study in which only a short period could be spent on fieldwork in Nigeria before return to the UK for analysis and writing up.

Other possible limitations of the study relate to the study sample and issues of representativeness, the robustness of the policy network boundary, and the political and societal context in which the study was conducted. Sampling and the selection of participants who are deemed to be part of the network (identifying the network boundary) are related issues. As all the participants included in the phase 1 SNA study also completed phase 2 qualitative interviews, the network became the sample for those interviews.

One limitation of the study may be that the sample size is small. Given that Nigeria is a country of about 196 million people, with an extensive bureaucracy under the Federal Ministry of Health at the state and facility levels, as well as a large private sector, it would certainly have been possible to map out a larger network including inter alia more state and HMO actors. Against this, the number of participants interviewed was at the upper end of what was feasible for a lone researcher in a Ph.D. study.

The use of snowball sampling brought the sample size higher, and also generated a good spread of nominations, leading to a diverse set of respondents. Gaps may still exist. For example, only one "allied professional” was identified as having involvement in the network. This could be because allied professionals are not (or at least are not recognised as being) engaged in UHC policy development, or because the study sampling strategy missed them. Another possible area of imbalance in sample selection is gender. There were roughly four times as many male as female participants ( $81 \%$ versus 19\%). The researcher had anticipated that there might be a preponderance of males in elite policy roles in Nigeria and tried to guard against ignoring influential female actors by inviting the inclusion in the study of nurses and other professions in which women are well represented. However, nominations put forward at the snowballing stage did not include many females, and the author suspects that the sample gender mix merely reflected the male-centric nature of health policy making in Nigeria. 
Lack of agreement on a clear methodology for identifying the boundary of the network (and which actors to include) is seen by many critics as a general problem in SNA studies. It must be conceded that the present study opted for a pragmatic rather than definitive solution to this problem by using the snowballing technique to get known policy actors to name other participants in their policy network (see Lewis 2009). The possibility remains that snowball sampling might miss some targets and introduce selection bias. To reduce the chances of this happening interaction between the putative network members was examined carefully at the analysis stage, utilising, using six separate relationships to map influence relationships between actors and to confirm where the network boundary lay (Wasserman and Faust, 1994). As readers will recall, the six relationships of interpersonal contact and influence measured in the structured interviews were: reading another network actor's written products, discussing UHC policy with that actor, entering a coalition with them, being formally affiliated with them, taking their advice, or being in conflict with them. These are referred to in Chapter 5 as the network relationships or the modalities of engagement. There may be some overlap in the relationships, and it is necessary to look across the overall pattern of linkages to determine whether an individual is a core or peripheral member of a network. In other words, it is the overall combination of relational relationships which determines the network position (Bellotti, 2015).

Some readers may object that the patterns of influence that emerge from the SNA component of the study are based on actors' perceptions rather than some more concrete measurement of impact on policy outcomes, and the author accepts that reliance on subjective assessments of participants' roles within the network is a limitation. Such assessments in the structured interviews may indeed have over- or under-estimated the influence of certain actors and affected the measurements of such things as network density, centrality and the strength of connections between nodes. However, there is strong corroboration from multiple informants of the central role of certain key actors, including the Federal Ministry for Health, NMA and WHO, which consistently scored highly for influence on multiple relationships. Interestingly, several of the nominees from snowballing who declined to participate came from these key organisations, so that adding responses these missing respondents would probably have increased rather than decreased the high centrality scores recorded for the organisations. The respondents selected undoubtedly included actors from relevant national and international organisations, who were clearly centrally involved in UHC policy development. Put another 
way many participants were clearly key policy makers, actors whose participation is likely to increase the real-world impact of studies of this kind (see: Onoka, Hanson, and Hanefeld, 2015).

The issue of representativeness can be considered at both the in-country and between-country levels. While the author believes he has engaged with a reasonably wide and representative set of actors from national organisations, it must be conceded that most respondents come from Nigeria’s Capital Territory and largest cities, rather than being distributed across the 36 states, which all have their own health ministries and differ in their local policies on matters such as the specifics of public health insurance schemes. Yet as UHC policy is driven largely from the centre it can be argued that this national - rather than regional focus - provides an accurate picture of the policy process at the level that counts. The research may be regarded as a case study that sheds light on the operation of a particular policy network at a particular time, so that findings may or may not be generalisable to other potential social networks that emerge as the UHC policy story continues, but these would need to be investigated via additional case studies. Analysis of sub-networks at state level or within the medical profession or private sector might expand and extend the insights that the present research provides, and again could be subjects for others to research in future.

Turning to international representativeness, we may observe that Nigeria is an interesting case example, whose experience is relevant to some but not all of the many other countries now embarking on UHC reform. Nigeria was not among the pathfinder nations in the wave of UHC reform in LMICs after the millennium - it lags behind say Thailand, Mexico, Turkey, or Brazil, which are all near to consolidating UHC. However, pitfalls and obstacles encountered along the slow and uneven path towards UHC in Nigeria may provide lessons for several other countries that are only inching slowly towards that goal. In terms of its representativeness of this group of countries, only some characteristics are shared but there will be certain common obstacles and barriers, and insofar as Nigeria has made progress in overcoming these, the solutions offered will be helpful to others.

One factor that may determine whether there are lessons for other countries is the extent to which the political, economic and organisational context of reform in Nigeria was so atypical that it followed its own distinctive path. Several writers have argued that context - the differing combinations of situational, structural, cultural and environmental factors that apply in a particular case - may limit generalisability (Leichter, 1979; Collins, et al., 1999; Buse et al., 2002). Nigeria does have its own distinctive policy story, shaped inter alia by its trajectory 
since independence, the oscillation between military and civilian governments, the programmes adopted by its political leaders and parties, the economic impact of falling world oil prices, and the chosen route of achieving UHC by public insurance with significant private sector involvement. Reforms are affected by societal values and governance arrangements and at times the Nigerian policy story may have affected by issues of probity and maladministration. With the above issues in mind, the lessons that emerge need to be considered critically, and may not apply to all contexts, but they should provide a valuable point of comparison for case studies of the implementation of UHC reforms elsewhere. 


\section{CHAPTER 8}

\subsection{Introduction}

This chapter summarises the study's primary concerns and main conclusions. This research has examined the UHC policy process in Nigeria and collected empirical data to add to the subject knowledge, which is currently gaining awareness, but which is not yet well-documented in Nigeria. To achieve the objectives of the research two frameworks were developed to analyse the move towards UHC: Walt and Gilson's (1994) policy process model and social network analysis (SNA), especially as developed by Lazega (1997). This final chapter summarises the conclusions emerging from the study, makes recommendations for further policy development, points to areas for future research, and provides a postscript to update readers on events since fieldwork was completed.

\subsection{What the study found}

This study examined the challenges and obstacles which have affected progress towards UHC in Nigeria. It used a combination of qualitative interviews and SNA to investigate how Nigeria's UHC policy was initiated, developed, and implemented in the health system, and to identify the key policy actors, their roles and their social interactions within the policy network. The policy story encompasses the reasons for the appearance of UHC on the government's health agenda, the decision to use national health insurance as the primary mechanism for achieving UHC, the intertwining of the UHC and SDG policies, and progress under the Goodluck Jonathan and Muhammadu Buhari administrations. The main conclusions of the study fall under a number of headings.

Firstly, the study used SNA and qualitative interviews to investigate the roles and interactions of policy actors, and how they impeded or advanced progress in extending healthcare coverage. The policy actors were found to be a diverse group where varying interests that translated into certain recurrent lines of division, particularly with regard to the respective roles of the public and private sectors in the operation of Nigeria's public health insurance schemes. 
The power that the contending groups are able to mobilise, together with the mediating role of the Federal Government, have a crucial bearing on outcomes. Arguably the failure of either the supporters of public provision or their private sector counterparts to exert dominant influence is one of the factors that account for the slow progress towards UHC to date. In a situation of countervailing power (Galbraith, 1952), where two sets of political actors must accept the influence of the other side, an uneasy compromise has emerged where UHC is pursued through the mechanism of a social insurance system in which the HMOs have a central role.

Five different forms of power were discussed in order to understand how key policy actors mobilise influence: professional, political, financial, power through technical expertise, and power in numbers. Each provides a basis for bring power to bear within the policy process, and this sheds light on how different actor attributes and ways of applying influence within the policy network can impede or facilitate progress towards UHC.

The SNA analysis suggested that the interactions in which power was deployed were crucially affected by position in the network, and especially by actors' status as power actors (defined in terms of multiple connections to others), gatekeepers/brokers, and actors who have close connections to the power actors. These relationship patterns created opportunities for influence, yet they may also have restricted the actionable scope of policy actors because of obligations to, or conflict with others in, the network (Emirbayer \& Goodwin, 1994).

Another conclusion reached from the interviews is that there are markedly different assessments about the prospects for the ultimate success of the UHC policy, so that respondents broadly divided into the two categories of optimists and pessimists.

Optimists were encouraged by the commitment of the government, the $\mathrm{MoH}$ and other stakeholders to the expansion of health coverage and suggested that implementing UHC can be better achieved through continuing incremental progress or a 'phased approach'. They believed that this approach fitted into the Nigerian health system model of gradual improvement through targeting. This is because the policy aims to improve health and prevent financial impoverishment for the most impoverished and vulnerable using the limited resources allocated to the health sector. Their preferred strategy is to make steady progress towards UHC through a series of incremental steps designed to expand health coverage for the majority of low-income Nigerians. 
Pessimists in the policy network have loftier ambitions and think progress is too slow to have a transformative effect on the healthcare system. Irrespective of how committed the government is to achieve UHC in Nigeria, they think momentum is slowing to the point where on-the-ground changes do little to improve services for poorer people. They point out that many Nigerians have difficulty in accessing essential health services, and they little faith that healthrelated SDGs will be achieved by 2030. Pessimists argue that progress towards UHC depends on the ability of policymakers to overcome five significant challenges which emerged from the interviews: the changing political climate, concerns about finance and system capacity, poor coordination between federal and state levels, problems with the private sector (especially HMOs), and corruption. In their view these issues taken together act as a bottleneck to the UHC implementation process, leading to a degree of policy stagnation.

The study indicates that, although pressure from international bodies was a significant factor in the early deliberations about putting UHC on the government policy agenda, and although the SDGs remain a major preoccupation for policy makers, it is domestic actors and particularly the Nigerian government that is determining the direction of current policy. International data and information about the experiences of universal coverage in other countries do continue to feed into policy debates in Nigeria but are used selectively in support of positions that reflect domestic concerns.

Finally, the study has set out to inform the policy discourse concerning the challenges involved in implementing UHC in Nigeria. It provides a snapshot of relationship patterns within the policy network at a specific time, and sets out to measure relative influence, alignments and conflict in the network using standard SNA concepts such as centrality and clustering. The qualitative interviews give insights into the subjective perceptions of individuals, and the policy aims and strategies that lie behind their actions. The interview accounts have allowed the researcher to construct an overview of policy development over time to tell the story of how the UHC policy came on to government policy agendas and progressed to the present time. 


\subsection{Policy recommendations}

The Federal Government (FG) and the MoH need to make a sustained effort to overcome the existing challenges and obstacles to out from the qualitative data which have impeded the implementation of UHC in Nigeria. This section presents recommendations based on the findings from this study, which might help to take the UHC process forward.

1. 'Power as decision making' in health policy is conceptualized by Steven Lukes (1974) to mean the ability of a policy actor or a group of policy actors to influence policy decisions according to a desired result. Respondents' reactions from the qualitative data would suggest that the Federal Government (FG) assumes two dimensions of power sources in UHC Nigeria's policy process namely political power and financial power. The SNA analysis also puts the FMoH as a power actor central to the UHC Nigeria policy network. These findings insinuate FG's political power and the FMoH's (as a power actor) can be linked with Lukes' (2005) concept of 'power as decision making' because both the Nigerian FG and MoH plays a central role in shaping all aspects of the health system such as pursuing consistent policies and practices across all levels of government health functions and increasing the standard of quality health programmes beneficial to the health sector (Corrigan, Eden and Smith, 2003). The first recommendation at the national level is to undertake a government review of existing public health insurance schemes with a view to rationalizing and consolidating the overall framework of social health insurance. Social insurance is the primary mechanism chosen in Nigeria to implement UHC, and so it is important to ensure that the arrangements in place rest on firm foundations. The existing system has evolved from the original core NIHS scheme for public employees in an incremental and piecemeal way, and the multiplicity of smaller schemes and variable engagement of the States in developing state-level programs means that it has become complex and unwieldy. A more integrated and comprehensive scheme will need to attend to some specific issues mentioned in the remaining recommendations.

2. Like any decision-making process, conflict is common to the health system, and it often occurs among actors involved in the policy process (Marcus, Dorn, \& McNulty, 2013). The SNA finding reveals a conflicted relationship within the UHC policy network regarding the discourse surrounding the function of the health insurance framework. Although the analysis did not specify the root cause of the conflict, it identified three 
prominent policy actors (the FMoH, hospital managers, and the HMO representatives) involved in the conflicted relationship, and these actors are responsible for steering the health insurance scheme towards achieving UHC. Hence, the thesis recommends that the insurance framework needs to balance the existing benefits offered to middle and upper-income beneficiaries with greater gains for the poor. As the existing programs mainly cover civil servants and those in formal employment they mostly cater for the better-off segments of Nigerian society. However, if UHC is to achieve maximum health gain for the wider population it can have most impact by improving the situation of lower-income groups. Particularly in country situations such as that of Nigeria, where finance and other obstacles mean that UHC has to proceed step by step rather than in a single "big bang" reform, it is important to ensure that the poor are not left behind. Commentators increasingly write of achieving "progressive universalism”, meaning that every step along the path to UHC should bring as much benefit to the poor as to the rich (Gwatkin and Ergo, 2011; Jamison et al., 2013).

The qualitative analysis uses Grant's (1978) typology of policy organizations to define policy actors within UHC Nigeria's network into insider and outsider groups. Findings demonstrate that cooperation between insiders and outsiders can influence and shift UHC discourse towards achieving progressive universalism. Both policy actors in both groups can actively participate in Nigeria's political policymaking in the health system, e.g., by calling for legislative shift progressive universalism.

Ensuring improved coverage for the poor is a crucial step in a country like Nigeria, but it is also important that targeted pro-poor programs do not halt progress towards full UHC. There is a strong argument that universal programmes have greater sustainability than targeted programmes, since a broader population segment will support provision that covers everybody. There is a danger that countries see a settlement that delivers something short of full coverage but containing a strong pro-poor policy strand as an acceptable endpoint, and it would be a mistake for Nigerian policy makers to take this position.

3. In reiteration, the financial power links to Steven Lukes' (1978) concept of 'power as decision making' shows that the Federal Government has the ability to mobilize the necessary fiscal resources to solve UHC Nigeria policy problems. Since health 
financing is one of the building blocks of health system, its level of functionality has direct effect on the overall functioning of the health system. In terms of finance, it will be important to safeguard and indeed increase the budget of the $\mathrm{MoH}$, as strengthening its core functions are critical for further reform progress and it is the main channel for allocating resources to the public healthcare system. The federal government's current health budget allocation is insufficient to meet the 2014 Abuja target of $15 \%$ of general government spending. The health sector capital allocations at the federal level have been in decline for several years (relative to budget size), falling from a high of $5.97 \%$ in 2012 to 3.33\% in 2019 (Budgit, 2019). Fitch Solutions reports that by 2021 healthcare expenditure in Nigeria is predicted to reach an estimated 2.94\% of the country's GDP (Medic West Africa, 2019), and the position has been eroded since then by sharp cuts in the 2020 budget allocation (see Postscript). International observers have pointed out that the chronic insufficiency of domestic health budgets results in a large deficit in the critical inputs needed to implement UHC (World Bank, 2016).

Many of the parastatals operating under the $\mathrm{MoH}$ umbrella need significant budget uplifts, and the federal government therefore needs to substantially increase the funds allocated to the Basic Healthcare Provision Fund (BHSPF), which is perhaps the single most important source of monies for UHC implementation. In the present financial climate, budget increases are, of course, politically very difficult, and the $\mathrm{MoH}$ should as far as possibly strengthen its existing efficiency savings and cost improvement programs so as to demonstrate that additional funding will translate into real coverage improvements rather than merely adding fat to the system.

4. Reference from the qualitative data has already been made to the problems of fragmentation and coordination that exist with the present piecemeal organization of public insurance schemes. Mathauer, Saksena and Kutzin (2019, p. 1) argue that insurance pooling, "the accumulation and management of prepaid financial resources on behalf of some or all of the population", is a relatively neglected aspect of UHC finance in the literature but actually a key policy instrument. In terms of the classification of approaches put forward by these authors, Nigeria is at best a fragmented system with population segmentation through different pools for different socio-economic groups, complicated by different schemes for different geographical localities and a mix of mandatory and voluntary enrolment. At present these problems 
are particularly acute in the insurance schemes targeted specifically at the poorest population groups, the community-based health insurance (CBHI) schemes aimed at the informal sector in local areas. If greater insurance system integration is to be achieved then the sustainability and sufficiency of these schemes needs to be improved through a more coordinated and standardized approach, and one key element here is better risk pooling, organized at state level.

The CBHI schemes operating under the NIHS umbrella aim to extend coverage to lowincome groups but are very diverse and uptake to date has been disappointing (Odeyemi, 2014). One problem is that due to the variety of groups covered, benefits offered and funding sources the insurance pool to be covered is fragmented. Risk pool fragmentation occurring through devolution of scheme oversight to state and local level poses particular dangers, with some schemes tottering on the brink of collapse because of small member numbers and inadequate finance. While it appears infeasible to bring informal sector workers and dependents into the main FSSHIP section of the NHIS, these are the groups for whom UHC would make the greatest difference. It is desirable to undertake a systematic assessment of the characteristics of this group, its health status and risk profile, and the budget needed to deliver a basic package of services (Etiaba et al., 2018). Given that recruitment to CBHI schemes is voluntary, there is a danger of adverse selection in that the chronically sick are more likely to sign up than the healthy, and it is necessary to have a sufficiently large pool of members to spread risk and plan an appropriate level of public subsidy to supplement pre-paid finance from contributions. Scale is needed if sufficient health infrastructure and resources are to be put in place, and many of the existing schemes are too small to support this. State governments should consider integrating the various CBHI programs into an overarching framework in which members of all schemes have access to common services in the local healthcare system, and member contributions from multiple schemes are pooled in a common fund, which is then topped up to the necessary level by donor and charitable finance as well as a state government subsidy.

This is broadly in line with WHO (2000) recommendations that UHC countries work towards larger rather than smaller public insurance pools, a more diverse mix of risk within pools, and compulsory rather than voluntary pools. Ideally the CBHI schemes should shift to mandatory membership for all with contributions adjusted to community situation and individual means (Smith and Witter, 2004), but this is probably not 
immediately achievable in Nigeria. Equalization of benefits between CBHI schemes and consolidation of existing insurance pools and funds would be important first steps. Given the huge care deficit in the informal sector, some targeting, and prioritization will be necessary. This can be done mainly through use of the state subsidy to fund scheme membership for poorer beneficiaries, but also via some redistribution of prepaid funds (Mathauer, Saksena and Kutzin, 2019).

\subsection{Future research recommendations}

The obvious and first priority for future research in this area is to follow the policy story through to the point where UHC is implemented, at least in nominal terms. Such a study would carry on with the main research questions addressed here concerning facilitators and obstacles in keeping UHC at the top of policy agendas until guaranteeing legislation is enacted and would further investigate the (changing?) nature of the policy network and the interest blocs, the interaction of problem, politics and policy streams, and the contextual and other factors that determine the outcome. Of course, this might well turn out to be a story of policy failure rather than policy success.

One interesting theoretical aspect that could be pursued in a further study concerns the question of how long a "window of opportunity” for a major health system reform, such as the move to UHC, remains open. As touched upon in the literature review there has been recent interest in extending Kingdon's (1984) three streams model (originally developed in relation to agenda setting) to also apply to the policy implementation stage (for example: Ridde 2009; Boswell and Rodrigues, 2016; Sager and Thomann, 2017), and additional empirical case studies would be invaluable as these are thin on the ground in the health sector. Sabatier (1999. P. 149) has written of the "desirability of longitudinal studies of a decade or more", and others have suggested that major health reform efforts may last for ten to twenty years (Intaranongpai et al., 2012; Al-Abri, 2019). Where there is an extended reform push as with UHC in Nigeria, we do not know whether it is more appropriate to theorize that a succession of policy windows open and close until an opportunity to complete implementation is taken, or if the problem for policy makers is to stretch a single window over a long time period. Evidence is also scarce about how windows close and how terminal the consequences are for a headline policy like 
UHC, or its various constituent strands. The Nigerian case provides a fascinating setting for future MSA studies of this kind.

Apart from the task of telling the story of UHC reform at macro level there are, of course, many aspects of downstream healthcare system development relevant to UHC that warrant ongoing investigation. For example, these could include perceptions of corruption in health insurance schemes, and the experiences and attitudes of government officials and private health providers to the various forms of misappropriation of public healthcare funds. More research is needed to determine how far corrupt activity is damaging the ability of the healthcare system to deliver high quality care to those who will benefit most. This is an area where a mixed methods study combining SNA analysis and qualitative analysis could shed light on the social networks that allow malfeasance to persist, whether that is in governmental, private or quasi-private healthcare systems.

As the building blocks for a UHC system fall into place there will be scope for studies that assess the sustainability and benefits of the various system components, both on the financing and service delivery sides. While there is an extensive literature on financing models for UHC, and theorising about their relative strengths and weaknesses, much less has been written about how resource allocation operates in practice, with detailed country case studies using data from frontline actors. A study of resource allocation in Nigeria, starting with an examination of how the federal health allocation is distributed within the Ministry of Health and then examining the channelling of funds to the parastatals, the states and specific UHC-related projects, would fill an important gap.

In the longer term, research will be needed to examine how Nigeria fits into the spectrum of developing countries that are attempting healthcare coverage reforms. Studies of Nigeria's experience of implementing UHC, including challenges overcome and any areas of particular success, will be invaluable to other aspiring UHC countries when knowledge is exchanged through forums such as the Joint Learning Network for UHC. The idea of "good health at low cost" is an attractive one that has encouraged important comparative studies of countries that have achieved better health and social outcomes than others at similar income levels (Halstead, Walsh, \& Warren, 1985). Twenty-five years on, the Good Health at Low-Cost project revisited these places, but also examined progress in a new wave of reforming countries that included Bangladesh, Ethiopia, Kyrgyzstan, and Thailand (Balabanova et al., 2011). It is not too much 
to expect that a case study of progress in Nigeria might be added to the UHC country studies in future comparative publications of this kind.

\subsection{Postscript}

Following his 2015 election victory, President Muhammadu Buhari confirmed his commitment to the policy push towards UHC and achieving the targets associated with the SDGs. However, despite regular statements reiterating support for UHC, progress was slow in Buhari’s first term in government. The centrepiece of the National Health Act 2014, the Basic Health Care Provision Fund (BHCPF), which had been seen as the fundamental funding measure to support UHC, did not come on stream immediately. Funds for the BHCPF were appropriated by the National Assembly for the first time in the 2018 budget. At that time the federal government earmarked N55.1 billion from the Consolidated Revenue Fund to the BHCPF, while additional funding of around \$30 million over several years was pledged by the Bill and Melinda Gates Foundation, the Global Financing Facility (a body launched by the World Bank and UN to support the SDGs), and the UK Department for International Development (DFID). In 2018 a Second National Strategic Health Development Plan (2018-2022) was published and a scheme to give extra funding to high-performing states - the Save One Million Lives Programme for Results - was unveiled. Even after the announcement of the budget allocation, the rollout and implementation of the BHCPF was somewhat delayed (Premium Times, 2019). This came during a period where state health budgets were highly constrained and many primary healthcare centres were struggling to stay open. It was not until May 17, 2019 that the first tranche of BHCPF money was credited to the NHIS, the Nigeria Centre for Disease Control (NCDC), and the National Primary Health Care Development Agency (NPHCDA).

Health policy had been somewhat overshadowed by wider economic problems and the Boko Haram insurgency in the North, and Buhari's All Progressives Congress Party, which claimed to have done well in these areas, won a comfortable victory in the general election of 26 February 2019. In an open letter welcoming Buhari's second term, Nigeria Health Watch (2019) gave credit for the progress made with the BHCPF but noted that on-the-ground improvements to support UHC remained sparse, that the leadership of the NIHS would be critical for progress, but that "four years have been wasted on quarrels".

A furore erupted in October 2019 when the Buhari government submitted 2020 budget proposals to the National Assembly that included swingeing cuts in health expenditure 
(Vanguard, 2019, October 15). The proposed capital expenditure of N46 billion for the Ministry of Health was N4 billion less than the 2019 allocation and N10 billion down from 2018. More damagingly for UHC, the proposed allocation for the BHCPF fell to N44.5 billion, compared to N55.1 billion in 2019. Overall, the budget left Health with the eleventh largest budget in the Ministerial pecking order. Within an aggregate 2020 government budget of N10.33 trillion, the proposed allocation to health was 0.4 percent, far short of the Abuja Declaration target of 15 per cent.

Tensions escalated further in June 2020, when in the light of falling oil prices, curtailed government revenues and the economic downturn associated with the COVID-19 pandemic, the government announced a downward revision of the 2020 budget. The allocation for the BHCPF was further cut from N44.4 billion to N25.5 billion, said by critics to be likely to highly consequential as the fund is the main source of primary care funding. The opposition People's Democratic Party and the Nigeria Labour Congress both condemned this move, pointing out that health and education were suffering more than most other areas of public expenditure, and suggesting that this reflected the low priority the Buhari administration gave to social policy (Abu et al., 2020, June 4; Vanguard, 2020, June 3). The budget cuts appear to directly affect the achievement of UHC and the SDGs and are likely to have a disproportional effect on poor persons who might otherwise have been brought under the coverage umbrella (Abu et al., 2020, June 4).

The COVID-19 pandemic is having a catastrophic impact on the most vulnerable communities around the world, and threatens the progress of other health initiatives, such as HIV, TB and malaria programmes. By the end of May 2020 Nigeria became the third African country to record over 10,000 cases of COVID-19. The national response is being led by the Presidential Task Force (PTF) and the Nigeria Centre for Disease Control (NCDC), and they have announced a stimulus package to support businesses hit by the coronavirus pandemic. Some of the budget cuts has been returned to the health sector in the form of a share of this stimulus funding for the private healthcare sector, together with an initial allocation of \$6bn to support the response to COVID-19 (NCDC, 2020), but is clear that the overall reduction in finance will have a severe impact. The larger picture is that the attention of the Nigerian government, the FMoH and the states has massively shifted from expanding health coverage to responding to the COVID-19 pandemic, and that any immediate progress towards implementing UHC has been put on the back burner (The Global Fund, 2020). 
In the period since the study some technical changes have occurred in the measurement of progress towards SDG targets, which affect how Nigeria's status is assessed. Two updated indicators have been adopted by the UN Statistical Commission. The first concerns SDG indicator 3.8.1, coverage of essential health services, and the second the proportion of households with high health expenditure as a share of total household income (SDG indicator 3.8.2) (Hogan et al., 2018).

SDG indicator 3.8.1 is the preferred choice for tracking the progress of UHC coverage in Nigeria. Coverage of essential health services is measured using a series of tracer interventions. These include reproductive, maternal, new-born and child health, infectious diseases, noncommunicable diseases, and service capacity and access among the general and the most disadvantaged populations (WHO, 2019).

Following the definition of SDG 3.8.1, four indicator categories were established: reproductive, maternal, new-born, and child health (RMNCH), infectious diseases, non-communicable diseases, and service capacity and access. 16 tracer indicators were selected for the index, this includes four from each of the categories of reproductive, maternal, new-born, and child health (RMNCH); infectious diseases; non-communicable diseases; and service capacity and access (see Figure 52).

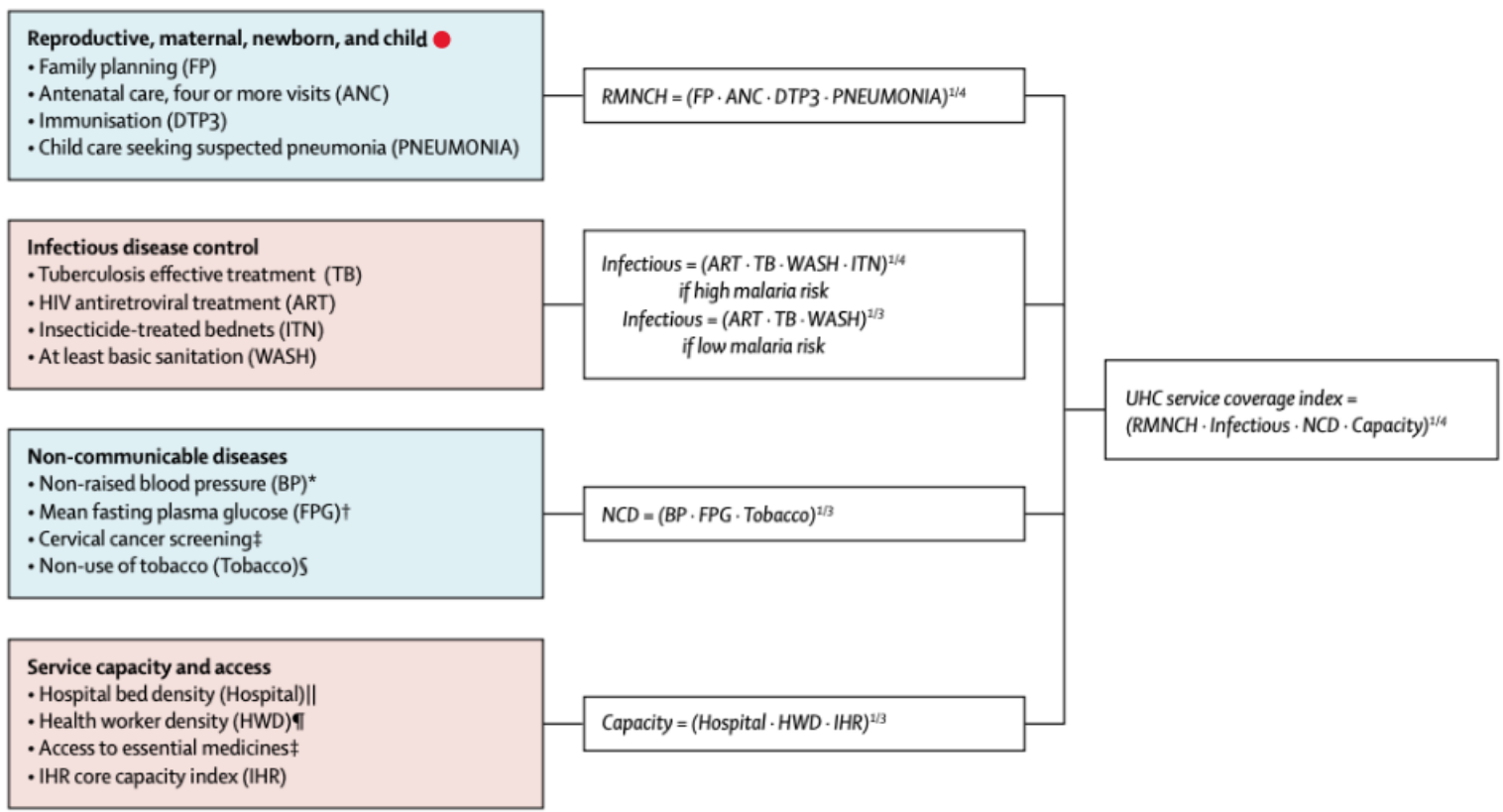


Figure 52: Indicators for universal health coverage service index. Source: (Hogan et al., 2018). Key- the red dot indicates reproductive, maternal, new-born, and child health (RMNCH).

In a study by Hogan et al. (2018), indicator data for 183 countries in the period 2010-2015 were analysed. In this exercise Nigeria ranks 166th out of the 183 countries measured with a UHC service coverage index of 39, while the highest country's UHC service coverage index is above 80. The current World Health Statistics report (WHO, 2020) shows that there has been little improvement. Nigeria’s UHC service coverage index increased slightly from 39 in 2015 to 42 in 2020, but it remained towards the bottom of the league table of listed countries.

From this it can be seen that Nigeria still has a long way to go to achieve UHC. Looking forward to 2030, much work is required to achieve UHC and the associated SDG targets in Nigeria, and more effort is needed from policymakers in tackling the obstacles and challenges identified by this study. 


\section{REFERENCES}

Abramovitz, M. (1986). Catching Up, Forging Ahead, and Falling Behind. The Journal of Economic History, 46(2), 385-406. http://www.jstor.org/stable/2122171

Abu, A., Jimoh, A.M., Olaniyi, S., Omolaoye, S., Nwannekanma, B., Olatunji, K., \& Ogugbuaja, C. (2020, June 4). Outrage over cut in health, education budgets. The Guardian. Retrieved from https://guardian.ng/news/outrage-over-cut-in-healtheducation-budgets/

Adebulu, T. (2020). Private Partnerships Can Help Nigerian Hospitals Improve Maternal Health; Here's How. Nigeria Health Watch. Retrieved from: https://nigeriahealthwatch.com/public-private-partnerships-can-help-nigerianhospitals-improve-maternal-health-heres-how/

Adefolaju, T. (2014). Traditional and orthodox medical systems in Nigeria: The imperative of a synthesis. American Journal of Heath Research, 2(4), 118-124.

Adeloye, D., David, R. A., Olaogun, A. A., Auta, A., Adesokan, A., Gadanya, M., Opele, J. K., Owagbemi, O., \& Iseolorunkanmi, A. (2017). Health workforce and governance: The crisis in Nigeria. Human resources for health, 15(1), 32. https://doi.org/10.1186/s12960-017-0205-4

Adewole, D.A., Dairo, M.D., \& Bolarinwa, O.A. (2016). Awareness and Coverage of the National Health Insurance Scheme among Formal Sector Workers in Ilorin, Nigeria. African Journal of Biomedical Research, 19(1), 1-10.

Adewole, D.A. \& Osungbade, K.O. (2016). Nigeria National Health Insurance Scheme: A highly subsidized health care program for a privileged few. International Journal of Tropical Disease \& Health, 19(3), 1-11.

Adewole, D.A., Akanbi, S.A., Osungbade, K.O., \& Bello, S. (2017). Expanding health insurance scheme in the informal sector in Nigeria: awareness as a potential demandside tool. Pan African Medical Journal, 27, 52.

Agency for Healthcare Research and Quality. (2016). 'The six domains of health care quality.' AHRQ website. $\quad$ www.ahrq.gov/professionals/quality-patientsafety/talkingquality/create/sixdomains.html [accessed 20 January 2021].

Ahrens, P. (2018). Qualitative network analysis: A useful tool for investigating policy networks in transnational settings? Methodological Innovations, 11, 1-9. doi: $10.1177 / 2059799118769816$.

Akinbode, J.O., Sokefun, E.A., \& Aremu, M.O. (2019). Appraisal of Health Maintenance Organisations' Performance in the Nigerian Healthcare Service Sector. Journal of Healthcare Engineering, Article ID- 6820609. doi.org:10.1155/2019/6820609

Al-Abri, M. (2019). Comparative Study of the Implementation of Regional Health Plans in Oman. Unpublished Ph.D. thesis. Swansea: Swansea University, College of Human \& Health Science.

Alcalde-Rabanal, J.E., Nigenda, G., Bärnighausen, T., Velasco-Mondragón, H.E., \& Darney, B.G. (2017). The gap in human resources to deliver the guaranteed package of 
prevention and health promotion services at urban and rural primary care facilities in Mexico. Human Resource Health, 15(1):49.

Alesina, A., \& Glaeser, E.L. (2004). Fighting Poverty in the US and Europe: A World of Difference. Oxford: Oxford University Press.

Alubo, O., \& Hunduh, V. (2017). Medical dominance and resistance in Nigeria's health care system. International Journal of Health Service, 47(4), 778-794.

Alvesson, M., \& Karreman, D. 2007. Constructing mystery: Empirical matters in theory development. Academy of Management Review, 32(4): 1265-1281.

Amadeo, K. (2020). Universal Health Care in Different Countries, Pros and Cons of Each: Why America Is the Only Rich Country Without Universal Health Care. The balance. Retrieved from https://www.thebalance.com/universal-health-care-4156211

Amoah, P. A. \& Phillips, D. R. (2018). Health literacy and health: Rethinking the strategies for universal health coverage in Ghana. Public Health, 159, 40-49.

Amu, H., Dickson, K.S., Kumi-Kyereme, A. \& Darteh, E.K.M. (2018). Understanding variations in health insurance coverage in Ghana, Kenya, Nigeria, and Tanzania: Evidence from demographic and health surveys. PLOS ONE, 13(8), e0201833. doi: 10.1371/journal.pone.0201833

Aregbesola, B.S. (2016). Institutional corruption, health-sector reforms, and health status in Nigeria. Lancet, 388, 757.

Aregbeshola, B.S. (2017). Enhancing political will for universal health coverage in Nigeria. MEDICC Review, 19, 42-6.

Aregbeshola, B.S. (2018). NHIS as a source of health financing towards UHC in Nigeria. International Health Policy. Retrieved July, 20, 2018, from https://www.internationalhealthpolicies.org/blogs/nhis-as-a-source-of-healthfinancing-towards-uhc-in-nigeria/

Aregbeshola, B. (2018). A Tax-based, Non-contributory, Health-Financing System Can Accelerate Progress toward Universal Health Coverage in Nigeria. MEDICC Review, 20, 40-45.

Asakitikpi, A.E. (2019). Healthcare coverage and affordability in Nigeria: An alternative model to equitable healthcare delivery. London: Intech Open. Retrieve from https://www.intechopen.com/books/universal-health-coverage/healthcare-coverageand-affordability-in-nigeria-an-alternative-model-to-equitable-healthcare-delive

Ashford, L., Smith, R., De Souza, R., Fikree, F., \& Yinger, N. (2016). Creating windows of opportunity for policy change: incorporating evidence into decentralized planning in Kenya. Bulletin of the World Health Organization, 84, 669-672.

Atkinson, M.M., \& Coleman, W.D. (1989). 'Strong states and weak states: Sectoral policy networks in advanced capitalist economies.’ British Journal of Political Science, 19: 47-67.

Atun, R., de Andrade, L.O.M., \& Almeida, G. (2014). Health-system reform and universal health coverage in Latin America. Lancet, 385, 1230-47.

Atun, R. (2015) Transforming Turkey’s Health System - Lessons for Universal Coverage. New England Journal of Medicine, 373(14): 1285-89.

Avila, C., Connor, C. \& Amico, P. (2013). Universal Coverage of Essential Health Services in Sub-Saharan Africa: Projections of Domestic Resources, USAID Office of Health Systems Bureau for Global Health. Bethesda, Maryland: ART Associates.

Awosusi, A., Folaranmi, T., \& Yates, R. (2015). Nigeria's new government and public financing for universal health coverage. Lancet Global Health, 3(9), e514-e515.

Aydin, C.E., Anderson, J.G., Rosen, P.N., Felitti, V.J, \& Weng H.C. (1998). Computers in the consulting room: A case study of clinician and patient perspectives. Health Care and Management Science, 1, 61-74. 
Baciu, A., National Academies of Sciences, Engineering, and Medicine, Health and Medicine Division, Board on Population Health and Public Health Practice, Committee on Community-Based Solutions to Promote Health Equity in the United States, Negussie, Y., Geller, A., \& Weinstein, J. N. (2017). Communities in Action: Pathways to Health Equity (Eds.). National Academies Press (US).

Badr, E., Mohamed, N. A., Afzal, M. M., \& Bile, K. M. (2013). Strengthening human resources for health through information, coordination and accountability mechanisms: the case of the Sudan. Bulletin of the World Health Organization, 91(11), 868-873. https://doi.org/10.2471/BLT.13.118950

Balabanova, D., McKee, M., \& Mills, A. (2011). Good health at low cost 25 years on: What makes a successful health system? London: LSHTM.

Barua, B., \& Moir, M. (2020). Comparing Performance of Universal Health Care Countries, 2020. Fraser Institute. http://www.fraserinstitute.org.

Beattie, A., Yates, R., \& Noble, D.J. (2016). Accelerating progress towards universal health coverage in Asia and Pacific: Improving the future for women and children. BMJ Global Health, 1(Supp 2), e000190. doi:10.1136/bmjgh-2016-000190

Belló, M., \& Becerril-Montekio, V.M. (2011). The health system of Argentina. Salud Pública de México, 53(2), s96-s108.

Bello, R.J., Damas, J.J., Marco, F.J., \& Castro, J.S. (2017). Venezuela's health-care crisis. Lancet, 390, 551.

Bellotti, E. (2015). Qualitative Networks: Mixed methods in sociological research ( ${ }^{\text {st }}$ Ed.). London: Routledge.

Bellotti, E. (2016). Ties and narratives: Qualitative methods and visualizations in the study of friendship networks. Sociological Research Online, 21(2), 2. Retrieved from http://www.socresonline.org.uk/21/2/2.html

Benjamin, M., Haendel, M. (1991). Cuba: A healthy revolution? Links, 8(3): 3-6.

Benson, J.K. (1975). A framework for policy analysis. In: Rogers, D.L., \& Whetten, D.A., Interorganizational Coordination: Theory, Research, and Implementation (pp. 137201). Ames: Iowa Sae University Press.

Bernard, H.R., Killworth, P., Kronenfeld, D., Sailer, L. (1984). The problem of informant accuracy: The validity of retrospective data. Annual Review of Anthropology, 13, 495517.

Birkland, T.A. (2016). An Introduction to the Policy Process: Theories, Concepts, and Models of Public Policy Making ( $4^{\text {th }}$ Ed.). London: Routledge.

Blanchet, K., \& James, P. (2011). How to do (or not to do) ... a social network analysis in health systems research. Health Policy Planning, 27(5), 438-46. doi:10.1093/heapol/czr055. Epub 2011.08.12.

Bloom, D.E., Canning, D., \& Sevilla, J.P. (2004). The effect of health on economic growth: A production function approach. World Development, 32, 1-13. doi: 10.1016/j.worlddev.2003.07.002

Bloom, D.E. (2011). 7 billion and counting. Science, 333, 562-569. doi:10.1126/science. 1209290

Bloom, D. E., Khoury, A., \& Subbaraman, R. (2018). The Promise and Peril of Universal Health Care. Science, 361(6404): eaat9644.

Bochsler, D. (2008) A QAP Network Analysis of Intergovernmental Cooperation between Swiss Cantons. In: Friemel T.N., (Eds.), Why Context Matters. VS Verlag für Sozialwissenschaften. https://doi.org/10.1007/978-3-531-91184-7_8

Boerma, T., Eozenou, P., Evans, D., Evans, T., Kieny, M-P., \& Wagstaff, A. (2014). Monitoring progress towards universal health coverage at country and global levels. PLoS Med, 11, e1001731 
Bogdanor, V. (1987). The Blackwell Encyclopaedia of Political Institutions (Eds). New York: Blackwell Reference.

Boland, P.J., \& El-Neweihi, E. (1995). Measures of component importance in reliability theory. Computers and Operations Research, 24(4), 445-463. doi:10.1016/03050548(94)00053-B

Bonacich, P., \& Lloyd, P. (2001). Eigenvector-like measures of centrality for asymmetric relations. Social Networks, 23, 191-201.

Bonell, C., Jamal, F., Melendez-Torres, G.J., \& Cummins, S. (2015). "Dark logic”: Theorising the harmful consequences of public health interventions. Journal of Epidemiology Community Health, 69, 95-8. doi:10.1136/jech-2014-204671

Borgatti, S.P. (1995). Centrality and AIDS. Connections, 18(1), 112-114.

Borgatti, S.P. (2005). Centrality and network flow. Social networks, 27(1), 55-71.

Borgatti, S.P., \& Halgin, D. (2011). Mapping culture: Freelists, pilesorting, triads and consensus analysis. The Ethnographer's Toolkit, 3, 1-60.

Borgatti, S. P., Everett, M. G., \& Johnson, J. C. (2013). Analyzing social networks. London: Sage.

Borzel, T.A. (1998). 'Organizing Babylon: On the different conceptions of policy networks.' Public Administration, 76: 253-73.

Boswell, C., \& Rodrigues, E. (2016). Policies, Politics and Organisational Problems: Multiple Streams and the Implementation of Targets in UK Government. Policy and Politics, 44(4), 507-24.

Boyer, L., Belzeaux, R., Maurel, O., Baumstrarck-Barrau, K., Samuelian, J.C. (2010). A social network analysis of healthcare professional relationships in a French hospital. International Journal of Health Care Quality Assurance, 5, 460-469.

Boudreau, R. (2017). The Plausibility of Universal Health Care in the United States. Journal of Clinical Research \& Bioethics, 8, 298.

Bowser, D., Sparkes, S.P., Mitchell, A., Bossert, T.J., Bärnighausen, T., Gedik, G., \& Atun, R. (2014). Global fund investments in human resources for health: Innovation and missed opportunities for health systems strengthening. Health Policy Planning, 29(8), 986-97.

Brandes, U. (2016). Network Positions. Methodological Innovations, 9, 1-19. doi: $10.1177 / 2059799116630650$

Brearley, L., Marten, R., \& O’Connell, T. (2016). Universal Health Coverage: A commitment to close the gap. Save the Children international: Save the Children. Retrieve from https://assets.rockefellerfoundation.org/app/uploads/20150530121203/uhc-close-thegap-report.pdf

Brewer, D.D., Webster, C.M. (1999). Forgetting of friends and its effects on measuring friendship networks. Social Networks, 21, 361-373.

Budgit. (2019). Proposed 2019 budget analysis. Retrieved June 2, 2020, from https://yourbudgit.com/wp-content/uploads/2019/03/2019-Budget-Analysis.pdf

Burstein, P. (1991). 'Policy domains: Organization, culture, and policy outcomes.' Annual Review of Sociology, 17: 327-50.

Burt, R.S. (1987). A note on missing social network data in the General Social Survey. Social Networks, 9, 63-73.

Burt, R.S. (1992). Structural Holes: The Social Structure of Competition. Cambridge: Harvard University Press.

Burt, R.S. (2000). The network structure of social capital. Research in Organization Behavioural, 22, 345-423.

Burt, R. S. (2001). Structural holes versus network closure as social capital. In: Lin, N., Cook, K. and Burt, R.S. (Ed.) Social capital: Theory and research (pp. 30-56). New York: Walter de Gruyter, Inc., pp. 31-56. 
Buse, K., Walt, G., \& Mays, N. (2012). Making health policy (2 ${ }^{\text {nd }}$ Ed.). Maidenhead: Open University Press.

Buse, K., Walt, G., \& Mays, N. (2012, p. 9). Making health policy ( $2^{\text {nd }}$ Ed.). Maidenhead: Open University Press.

Buse, K., \& Walt, G. (2000). Global public-private health partnerships: Part I - a new development in health? Bulletin of World Health Organization, 78(4), 509-61.

Butts, C.T. (2003). Network inference, error, and informant (in)accuracy: A Bayesian approach. Social Networks, 25, 103-140.

Butts, C.T. (2008). Social Network Analysis: A Methodological Introduction. Asian Journal of Social Psychology, 11, 13-41.

Cairney, P. (2013). Standing on the shoulders of giants: How do we combine the insights of multiple theories in public policy studies. Policy Studies Journal, 41(1), 1-21.

Campbell, J., Buchan, J., Cometto, G., David, B., Dussault, G., Fogstad, H., Fronteira, I., Lozano, R., Nyonator, F., Pablos-Méndez, A., Quain, E. E., Starrs, A., \& Tangcharoensathien, V. (2013). Human resources for health and universal health coverage: Fostering equity and effective coverage. Bulletin of the World Health Organization, 91(11), 853-863. https://doi.org/10.2471/BLT.13.118729

Carlsson, L. (2000). 'Policy networks as collective action.' Policy Studies Journal, 28: 502-20.

Carrington, P.J. (2014). Social network research. In: Domínguez, S., \& Hollstein, B., (Eds), Mixed methods social networks research (pp. 35-64). Cambridge: Cambridge University Press. doi:10.1017/CBO9781139227193

Campos, P.A., \& Reich, M.R. (2019). Political Analysis for Health Policy Implementation. Health Systems and Reform, 5(3), 224-235.

Cavagnero, E. (2008). Health sector reforms in Argentina and the performance of the health financing system. Health Policy, 88, 88-99.

Chambers, D., Wilson, P., Thompson, C., \& Harden, M. (2012). Social network analysis in healthcare settings: A systematic scoping review. PloS One, 7(8), e41911. doi: 10.1371/journal.pone.0041911

Chapman, A.R. (2016). Assessing the universal health coverage target in the sustainable development goals from a human rights perspective. BMC International Health Human Rights, 16(1), 33.

Chambers, D., Wilson, P., Thompson, C., \& Harden, M. (2012). Social Network Analysis in Healthcare Settings: A Systematic Scoping Review. PLoS One, 7(8), e41911.

Chanut, C., Boyer, L., Robitail, S., Horte, C., Jacqueme, B., Giusiano, B., Mabriez, J.C. \& Auquier, P. (2005). Applying social network analysis to the health system (orig. title L'analyse des réseaux sociaux appliqué au systeme e). Sant publique, 17(3), 403-415.

Chen, L., de Haan, A., Zhang, X., \& Warmerdan, W. (2011). Addressing vulnerability in an emerging economy: China's new cooperative medical scheme (NCMS). Rev Can Etudes Dev, 32(4), 399-413.

Cheng, T., \& Wilson, W. (2015). Universal health coverage: An overview and lessons from Asia. Harvard Public Health Review, 5, 1-12. Retrieve from http://harvardpublichealthreview.org/wp-content/uploads/2015/04/HPHRv5-ChengUniversal-Health-Coverage-Copy.pdf

Cleopatra, I., \& Eunice, K. (2018). Household catastrophic health expenditure: Evidence from Nigeria. Microeconomics and Macroeconomic, 6(1), 1-8. doi: 10.5923/j.m2economics.20180601.01

Cohen, M.D., March, J.G. \& Olsen, J.P. (1972). A garbage can model of organizational choice. Administrative Science Quarterly, 17(1), 1-25.

Collins, R. (1988). Theoretical Sociology. San Diego: HBJ. 
Collins, C., Green, A. \& Hunter, D. (1999). Health sector reform and the interpretation of policy context. Health Policy, 47(1), 69-83.

Cooper, C., Booth, A., Varley-Campbell, J., Britten, N., \& Garside, R. (2018). Defining the process to literature searching in systematic reviews: A literature review of guidance and supporting studies. BMC Medical Research Methodology, 18(1), 85. doi:10.1186/s12874-018-0545-3.

Costenbader, E., Valente, T.W. (2003). The stability of centrality measures when networks are sampled. Social Networks, 25, 283-307.

Cotlear, D., Nagpal, S., Smith, O., Tandon, A., \& Cortez, R. (2015). Going universal: How 24 developing countries are implementing universal health coverage from the bottom up. Washington, DC: World Bank.

Cotlear, D., \& Rosemberg, N. (2018). Going Universal in Africa: How 46 African Countries Reformed User Fees and Implemented Health Care Priorities. Universal Health Coverage (UNICO) studies series; no. 26. Washington, DC: The World Bank.

Crossley, N. (2010). The social world of the network: Combining quantitative and qualitative elements in Social Network Analysis. Sociologica, 1. doi:10.2383/32049

Crossley, N., \& Edwards, G. (2016). Cases, mechanisms and the real: The theory and methodology of mixed-method social network analysis. Sociological Research Online, 21(2), 217- 285.

Culyer, A.L. (2018). Health economics and health technology assessment. Health Economics and Sustainable Medicine, 46(7), 379-382.

Cumming, G.S., Bodin, O., Ernstson, H., \& Elmqvist, T. (2010). Network analysis in conservation biogeography: Challenges and opportunities. Diversity and Distributions, 16, 414-425. Retrieved from https://doi.org/10.1111/j.1472-4642.2010.00651.x

Cummings, J.N. \& Cross, R. (2003). Structural properties of work groups and their consequences for performance. Social Networks, 25, 197-210.

Dahl, R.A. (1957). The Concept of Power,” Behavioural Science, 2(3), 201-15.

Dahl, R.A. (1961). Who governs? Democracy and power in an American city. New Haven, CT: Yale University Press.

Dahl, R. (1989). Democracy and its critics. New Haven: Yale University Press.

Dayan, M., Gardner, T., Kelly, E., \& Ward, D. (2018). How good is the NHS \{Picture\}? Nuffield Trust. https://www.ifs.org.uk/uploads/HEAJ6319-How-good-is-the-NHS180625-WEB.pdf [accessed 20 January 2021].

Department of Health \& Social Care. (2020). How the NHS charges overseas visitors for NHS hospital care. Retrieved from https://www.gov.uk/government/publications/how-thenhs-charges-overseas-visitors-for-nhs-hospital-care/how-the-nhs-charges-overseasvisitors-for-nhs-hospital-care

Diestel, R. (1990). Graph Decompositions: A study in infinite graph theory. Oxford: Clarendon Press.

Dimitrova, D., \& Akinola, S. (2019). How Africa and Asia are joining forces on universal healthcare. Geneva: World Economic Forum. Retrieved from https://www.weforum.org/agenda/2019/04/universal-health-coverage-uhc-asia-africakenya-thailand-japan-egypt/

Dionne, F., \& Mitton, C. (2018). Health Care Trade-Offs: A Necessary Reality for Every Health System. Retrieved from https://www.healthaffairs.org/do/10.1377/hblog20180316.120106/full/

Dowding, K. (1995). 'Model or metaphor? A critical review of the network approach.' Political Studies, 43: 136-58. 
Dunn, G. (2018). The impact of the Boko Haram insurgency in Northeast Nigeria on childhood wasting: A double-difference study. Conflict and Health, 12(1), 6. doi:10.1186/s13031018-0136-2

Durkheim, E. (1895/1964). The rules of sociological method and selected texts on sociology and its method. London: Macmillan.

Durokifa, A. A., \& Abdul-Wasi, B. M. (2016). Evaluating Nigeria's Achievement of the Millennium Development Goals (MDGs): Determinants, deliverables, and shortfalls. Africa's Public Service Delivery \& Performance Review, 4(4), a147.

Dutch Ministry of Health, Welfare and Sport. (2008). "Obligatory Health Insurance." http://www.minvws.nl/en/folders/z/2008/obligatory-health-insurance1.asp [accessed 20 January 2021].

Dutch Ministry of Health, Welfare and Sport. (2008). "Risk Adjustment under the Health Insurance Act in the Netherlands." http://english.minvws.nl/en/reports/z/2008/riskadjustment-under-the-health-insurance-act-in-the-netherlands.asp [accessed 20 January 2021].

Dye, T.R. (1976). Understanding public policy. Englewood Cliffs: Prentice Hall.

Edwards, G. (2010). Mixed-Method Approaches to Social Network Analysis. Review paper, ESRC National Centre for Research Methods. Retrieved January 25, 2020, from http://eprints.ncrm.ac.uk/842/

Emirbayer, M., \& Goodwin, J. (1994). Network analysis, culture, and the problem of agency. American Journal of Sociology, 99(6), 1411- 1454.

Enabulele, O., \& Enabulele, J.E. (2016). Nigeria's National Health Act: An assessment of health professionals' knowledge and perception. Nigeria Medical Journal, 57(5), 2605.

Esping-Andersen, G. (1990). The Three Worlds of Welfare Capitalism. Princeton, New Jersey: Princeton University Press.

Etiaba, E., Uguru, N., Ebenso, B., Russo, G., Ezumah, N., Uzochukwu, B., \& Onwujekwe, O. (2015). Development of oral health policy in Nigeria: An analysis of the role of context, actors and policy process. BMC Oral Health, 15, 56.

Etiaba, E., Onwujekwe, O., Honda, A., Ibe, O., Uzochukwu, B., \& Hanson, K. (2018). Strategic purchasing for universal health coverage: Examining the purchaser-provider relationship within a social health insurance scheme in Nigeria. BMJ Global Health, 3, e000917.

Evans, T.G., Chowdhury, A.M.R., Evans, D.B., Fidler, A.H., Lindelow, M., Mills, A., \& Scheil-Adlung, X. (2012). Thailand's Universal Coverage Scheme: Achievements and Challenges. An independent assessment of the first 10 years (2001-2010). Thailand: Health Insurance System Research Office.

Everett, M.G., \& Valente, T.W. (2016). Bridging, brokerage and betweenness. Social Networks, 44, 202-208.

Ewelike, U. (2020). UHC: Nigeria’s Political Will, a Catalyst! Nigeria Health Watch. https://nigeriahealthwatch.com/destination-uhc-nigerias-political-will-a-catalyst/ [assessed 28 December 2020]

Eze, B.S. (2019). Dual Practice of Medical Professionals in Public Hospitals in Southeast Nigeria: An Economic and Policy Analysis. Unpublished Ph.D. thesis. Swansea: College of Human \& Health Sciences, Swansea University.

Fabricant, S.J., Kamara, C.W., \& Mills, A. (1999). Why the poor pay more: Household curative expenditures in rural Sierra Leone. International Journal of Health Planning and Management, 14(3), 179-99.

Fadlallah, R., El-Jardali, F., Hemadi, N., Morsi, R.Z., Samra, C.A.A., Ahmad, A., Arif, K., Hishi, L., Honein-AbouHaidar, G., \& Akl, E.A. (2018). Barriers and facilitators to 
implementation, uptake and sustainability of community-based health insurance schemes in low- and middle-income countries: A systematic review. International Journal for Equity in Health, 17(1), 13.

Falade, T. (2020). Health Insurance: Doctors, Patients, Others Expose Scam, Corruption in HMOs. Independent. Retrieved from February 15, 2020, from https://www.independent.ng/health-insurance-doctors-patients-others-expose-scamcorruption-in-hmos/

Federal Ministry of Health. (2016). National health Policy 2016: Promoting the health of Nigerians to accelerate socio-economic development. Abuja: Federal Ministry of Health

Federal Ministry of Health., National Health Insurance Scheme., \& National Primary Health Care Development Agency. (2017). Basic Healthcare Provision Fund: Harmonized guidelines for the administration, disbursement, monitoring and fund management of the Basic Healthcare Provision Fund. Abuja: FMoH. Retrieve from http://www.health.gov.ng/index.php?option=com content\&view=article\&id=143\&Ite mid $=515$

Fenby, J. (2010). The General: Charles De Gaulle and the France He Saved. London: Simon \& Schuster.

Fenwick, T., \& Edwards, R. (2010). Actor network Theory and Education. London: Routledge.

Freeman, L.C. (1978/1979). Centrality in social networks: Conceptual clarification. Social Networks, 1(3), 215-239.

Freeman, R.E. (2004). The Stakeholder Approach Revisited. Zeitschrift für Wirtschafts-und Unternehmensethik, 5: 228-241.

Freidson, E. (1970). Profession of medicine: A study of the sociology of applied knowledge. New York: Harper \& Row.

Frenk, J. (2010). The global health system: Strengthening national health systems as the next step for global progress. PLoS Med, 7(1), e1000089.

Frenk, J. (2015). Leading the way towards universal health coverage: A call to action. Lancet, $385,1352-8$.

Friedkin, N.E. (1981). The development of structure in random networks: An analysis of the effects of increasing network density on five measures of structure. Social Networks, 3(1), 41-52.

Fuhse, J. \& Mützel, S. (2011). Tackling connections, structure, and meaning in networks: Quantitative and qualitative methods in sociological network research. Quality and Quantity, 45, 1067-89. doi:10.1007/s11135-011-9492-3.

Galbraith, J.K. (1952). American Capitalism: The Concept of Countervailing Power. Boston: Houghton Mifflin.

Gilson, L., Buse, K., Murray, S.F. \& Dickinson, C. (2008). Future directions for health policy analysis: A tribute to the work of Professor Gill Walt. Health Policy and Planning, 23, 291-293.

Gilson, L. (2012). Health policy and systems research: A methodology reader. Geneva: World Health Organization.

Gilson, L., \& Agyepong, I.A. (2018). Strengthening health system leadership for better governance: What does it take? Health Policy Planning, 33(suppl_2), ii1- ii4.

Gilson, L., Orgill, M., \& Shroff, Z.C. (2018). A health policy analysis reader: The politics of policy change in low- and middle-income countries. Geneva: World Health Organization.

Glaser, B., \& Strauss, A. (1967). The Discovery of Grounded Theory: Strategies for Qualitative Research. Mill Valley, CA: Sociology Press. 
Global Burden of Disease Collaborative Network. (2016). Global Burden of Disease Study 2015 (GBD 2015) Life Expectancy, All-Cause and Cause-Specific Mortality 1980-2015. Seattle, United States: Institute for Health Metrics and Evaluation (IHME).

Goldman, M. (2007). 'How "Water for All!” policy became hegemonic: The power of the World Bank and its transnational policy networks.' Geoforum, 38: 786-800.

Gorard, S. (2010). Serious doubts about school effectiveness. British Educational Research Journal, 36(5): 745-766. doi:10.1080/01411920903144251

Gore, R., \& Parker, R. (2019). Analysing power and politics in health policies and systems. Global Public Health, 14(4), 481-488. doi:10.1080/17441692.2019.1575446

Gottret, P., \& Schieber, G. (2006). Health financing revisited [Digital image]. Washington, DC: World Bank.

Gottret, P., \& Schieber, G. (2006). Health financing revisited. Washington, DC: World Bank. Granovetter, M.S. (1973). The strength of weak ties. American Journal of Sociology, 78(6), 1360-1380.

Grant, W. (1978). Insider Groups, Outsider Groups and Interest Group Strategies in Britain. Warwick: University of Warwick Department of Politics Working Party no. 19

Greer, S.L., \& Méndez, C.A. (2015). Universal health coverage: A political struggle and governance challenge. American Journal of Public Health, 105(Suppl 5), S637 - 9.

Grindle, M.S., Thomas, J.W. (1991). Public choices and policy change: The political economy of reform in developing countries. Baltimore and London: Johns Hopkins Press.

Gupta, I., \& Chowdhury, S. (2014). Public Financing for Health Coverage in India: Who Spends, Who Benefits and At What Cost? Economic and Political Weekly, 49(35), 5963.

Gupta, R.D., \& Shahabuddin, A.S.M. (2018). Measuring Progress Toward Universal Health Coverage: Does the Monitoring Framework of Bangladesh Need Further Improvement? Cureus, 10(1), e2041.

Gwatkin, D. R. \& Ergo, A. (2011). Universal health coverage: Friend or foe of health equity? Lancet, 377(9784), 2160-2161.

Halgin, D. S., \& Borgatti, S. P. (2012). An introduction to personal network analysis and tie churn statistics using E-NET. Connections, 32(1), 36 - 48.

Halstead, S.B., Walsh, J.A., \& Warren, K.S. (1985). Good Health at Low Cost. New York: Rockefeller Foundation.

Hanneman, R.A., \& Riddle, M. (2005). Introduction to social network methods. Riverside: University of California, Riverside

Heclo, H. (1978). Issue Networks and the Executive Establishment. In: King, A., The new American political system (Ed.) (pp. 87-124). Washington D.C.: American Enterprise Institute.

Higgs, R. (1987). Crisis and Leviathan: Critical Episodes in the Growth of American Government. New York: Oxford University Press.

Hindhede, A.L., \& Aagaard-Hansen, J. (2017). Using social network analysis as a method to assess and strengthen participation in health promotion programs invulnerable areas. Health Promotional Practice, 18,175-83.

Hjern B., Porter D.O. (1983) Implementation Structures: A New Unit of Administrative Analysis. In: Holzner B., Knorr K.D., \& Strasser H., (Eds.), Realizing Social Science Knowledge. Institut für Höhere Studien — Institute for Advanced Studies IHS-Studies: Physica, Heidelberg. https://doi.org/10.1007/978-3-662-41492-7_19

Hogan, D.R., Stevens, G.A., Hosseinpoor, A.R., \& Boerma, T. (2018). Monitoring universal health coverage within the Sustainable Development Goals: Development and baseline data for an index of essential health services. The Lancet Global Health, 6, e152-e168. doi:10.1016/S2214-109X (17)30472-2 
Holland, P.W., \& Leinhard, S. (1973). Structural implications of measurement error in sociometry. Journal of Mathematical Sociology, 3, 85-111.

Hollstein, B. (2014). Mixed methods social networks research: An introduction. In: Dominguez, S., \& Hollstein, B., (Eds.), Mixed methods social networks research: Design and applications (pp. 3-34). Cambridge, UK: Cambridge University Press.

Hollstrein, B. (2016). Qualitative approaches. In John Scott \& Peter J. Carrington (Eds.) The Sage Handbook of Social Network Analysis (pp. 404-416). London: Sage.

Hongoro, C., Rutebemberwa, E., Twalo, T., Mwendera, C., Douglas, M., Mukuru, M., Kasasa, S., \& Ssengooba, F. (2018). Analysis of selected policies towards universal health coverage in Uganda: The policy implementation barometer protocol. Archive Public Health, 76, 12. doi:10.1186/s13690-018-0258-4

Howlett, M., Ramesh, M., \& Anthony Perl, A. (2009). Studying Public Policy: Policy Cycles and Policy Subsystems ( $3^{\mathrm{RD}}$ Eds). Toronto: Oxford University Press.

Hsiao, W., Li, M., \& Zhang, S. (2014). Universal health coverage: The case of China. Boston: Harvard School of Public Health.

Hsiao, A., Vogt, V., \& Quentin, W. (2019). Effect of corruption on perceived difficulties in healthcare access in sub-Saharan Africa. PLOS ONE, 14(11), e0224915.

Huss, R., Das, M., Ebenso, B., Rawat, B., Onwujekwe, O., Russo, G., Blok, L., Weerasak, P., \& Mirzoev, T. (2014). Participation of policy actors in the development of health policies in India and Nigeria and the implications for the role of evidence in policymaking. BMC Health Service Research, 14,27. doi:10.1186/1472-6963-14-S2P27

Iatridis, D.S. (1990). Cuba's Health Care Policy: Prevention and Active Community Participation. Social Work, 35(1): 29-35. https://doi.org/10.1093/sw/35.1.29

Ifijeh, M. (2017, March 2). HMOs are critical to success of health insurance. ThisDay. Retrieved from https://www.thisdaylive.com/index.php/2017/03/02/hmos-are-criticalto-success-of-health-insurance/

Ikegami, N., Takaku, R., Bessho, S., and Nishimura, S. (2014). Universal health coverage for inclusive and sustainable development: Lessons from Japan (English) (Chapter 3., pp. 41-55). Washington, DC: World Bank Group.

Institute for Healthcare Improvement. (2018). 'The IHI Triple Aim'. IHI website. www.ihi.org/Engage/Initiatives/TripleAim/Pages/default.aspx [accessed 20 January 2021].

Infrastructure Concession Regulatory Commission. (2017). Draft PPP Manual for Nigeria. Retrieved from: http://www.icrc.gov.ng/assets/uploads/2017/12/PPP-Manual.pdf

Infrastructure Concession Regulatory Commission. (2020). Latest News. Retrieved from: https://www.icrc.gov.ng/

Intaranongpai, S., Hughes, D. \& Leethongdee, S. (2012). The Provincial Health Office as performance manager: The shifting balance of power in the local health system after the Thai UC reforms. International Journal of Health Planning and Management, 27(4), 308-326.

Jamison, D.T., Summers, L.H., Alleyne, G., Arrow, K.J., Berkley, S., Binagwaho, A., Bustreo, F., Evans, D., Feachem, R.G., Frenk, J., Ghosh, G., Goldie, S.J., Guo, Y., Gupta, S., Horton, R., Kruk, M.E., Mahmoud, A., Mohohlo, L.K., Ncube, M., Pablos-Mendez, A., Reddy, K.S., Saxenian, H., Soucat, A., Ulltveit-Moe, K.H., Yamey, G. (2013). Global Health 2035: A world converging within a generation. Lancet, 382, 1898-955.

John, P. (1998). Analysing Public Policy. London: Pinter.

Johnsson, L., Eriksson, S., Helgesson, G., \& Hansson, M.G. 2014. Making researchers moral: Why trustworthiness requires more than ethics guidelines and review. Research Ethics, 10(1), 29-46. 
Jordan, G. (1990). 'Sub-government, policy communities and networks: Refilling the old bottles?' Journal of Theoretical Politics, 2(3): 319-38.

Jordan, G., \& Schubert, K. (1992). 'A preliminary ordering of policy network labels.' European Journal of Political Research, 21: 7-27.

Kamorudeen, A., \& Bidemi, S. A. (2012). Corruption in the Nigerian public health care delivery system. Sokoto Journal of the Social Sciences, 2(2), 98-114.

Katzenstein, P. (1976). 'International relations and domestic structure: Foreign economic policies of advanced industrial states.' International Organization, 30: 1-45.

Kaur, M. (2016). Application of mixed method approach in public health research. Indian Journal of Community Medicine, 41, 93-97. doi:10.4103/0970-0218.173495

Kenis, P., \& Schneider, V. (1991). Policy networks and policy analysis: Scrutinizing a new analytical toolbox. In Marin, B., \& Mayntz, R. (Eds.), Policy Networks: Empirical Evidence and Theoretical Consideration (pp. 25-59). Boulder, CO: Westview Press.

Kenny, D., \& Adamson, B. (1992). Medicine and the health professions: Issues of dominance, autonomy and authority. Australian Health Review, 15(3), 319- 34.

Kentikelenis, A.E., Stubbs, T.H. \& King, L.P. (2016). IMF conditionality and development policy space, 1985-2014, Review of International Political Economy, 23(4), 543-582, DOI: $10.1080 / 09692290.2016 .1174953$

Kickert, W.J.M., Klijn, E.H., \& Koppenjan, J.F.H. (1997). Managing Complex Networks: Strategies for the Public Sector (Eds.). London: Sage.

Kieny, M.P., Bekedam, H., Dovlo, D., Fitzgerald, J., Habicht, J., \& Harrison, G. (2017). Strengthening health systems for universal health coverage and sustainable development. Bulletin World Health Organization, 95(7), 537-9. doi:10.2471/BLT.16.187476 PMID: 28670019

Kingdon, J.W. (2010/ [2003]/ [1984]). Agendas, Alternatives, and Public Policies (2 ${ }^{\text {nd }}$ Ed.). New York: Harper Collins.

Khan, M.H. (2010). Political Settlements and the Governance of Growth Enhancing Institutions. Research Paper Series on Governance for Growth. School of Oriental and African Studies, University of London: London.

Khan, A.A., Khan, N.U., Bile, K.M., \& Awan, H. (2010). Creating synergies for health systems strengthening through partnerships in Pakistan: A case study of the national eye health programme. East Mediterranean Health Journal, 16(Suppl), 61-68.

Knoke, D. (1990). Political Networks: The Structural Perspective. Cambridge: Cambridge University Press.

Knoke, D. (2014). Policy networks. In the SAGE handbook of social network analysis (pp. 210222). Thousand Oaks: Sage Publications Ltd. https://www.doi.org/10.4135/9781446294413

Knoke, D., \& Laumann, E.O. (1982). “The social structure of national policy domains: An exploration of some structural hypotheses.” In: Peter V. Marsden and Nan Lin (Eds.), Social Structure and Network Analysis (pp. 255-70). Beverly Hills: Sage.

Kohlmorgen, L., Wolfgang, H., \& Sonja, B. (2007). 'Networks and governance: Transnational networks as a basis for emancipatory politics in global society?’ Peripherie, 27(105-6): 8-34.

Konish, L. (2019, February 11). This is the real reason most Americans file for bankruptcy. Retrieve from https://www.cnbc.com/2019/02/11/this-is-the-real-reason-mostamericans-file-for-bankruptcy.html

Kossinets, G. (2006). Effects of missing data in social networks. Social Networks, 28(3), 247268. https://doi.org/10.1016/j.socnet.2005.07.002

Krackhardt, D. (1987). “QAP Partialling as a Test of Spuriousness.” Social Networks, 9, 17186. 
Krishna, A. (2013). The mixed news on poverty. Current History, 112(1), 20-25.

Krugman, P. (2021). Learn About Universal Health Care: Definition, Advantages and Disadvantages. Masterclass. https://www.masterclass.com/articles/what-is-universalhealth-care\#what-is-universal-health-care [accessed 7 May 2021].

Kruk, M.E. (2013). Universal health coverage: A policy whose time has come. BMJ, 347, f6360. Kumar, R. (2019). Public- private partnership for universal health coverage? The future of free health in Sri Lanka. Globalization and Health, 15(Suppl1), 75. doi:10.1186/s12992019-0522-6

Kutzin, J. (2013). Health financing for universal coverage and health system performance: Concepts and implications for policy. Bulletin of the World Health Organization, 91, 602-611.

Kutzin, J., \& Sparkes, S.P. (2016). Health systems strengthening, universal health coverage, health security and resilience. Bulletin of the World Health Organization, 94(1), 2. doi:10.2471/BLT.15.165050 PMID: 26769987

Langlois, E.V., Tunçalp, Ö., \& Norris, S.L. (2018). Qualitative evidence to improve guidelines and health decision-making. Bulletin of the World Health Organization, 96, 79-79A. doi:10.2471/BLT.17.206540

Lasswell, H. (1956). The decision process: Seven categories of functional analysis. Bureau of Governmental Research, College of Business and Public Administration, University of Maryland: College Park

Laumann, E.O., Marsden, P.V., Prensky, D. (1983). The boundary specification problem in network analysis. In: Burt, R.S. \& Minor, M.J. (Eds.), Applied Network Analysis (pp. 18-34). London: Sage.

Laumann, E.O., \& Knoke, D. (1987). The Organizational State: Social Choice in National Policy Domains. Madison: University of Wisconsin Press.

Laumann, E.O., \& Pappi, F.U. (1976). Network of collective action: A perspective on community influence systems. New York: Academic Press.

Laumann, E.O., \& Knoke, D. (1987). The organizational state: A perspective on the social organization of national energy and health policy domains. Madison: University of Wisconsin Press.

Lavers, T., \& Hickey, S. (2015). Investigating the political economy of social protection expansion in Africa: At the intersection of transnational ideas and domestic politics." ESID Working Paper $n^{\circ} 47$.

Lazega, E. (1997). Network analysis and qualitative research: A method of contextualization. In Gale Miller and Robert Dingwall, Context and Method in Qualitative Research (Eds., pp. 119-138). London: Sage.

Lazega, E. (2012). Mapping Judicial Dialogue Across National Borders: An Exploratory Network Study of Learning from Lobbying Among European Intellectual Property Judges. Utrecht Law Review, 8(2), 115-128.

Lee, K. and Goodman, H. (2002). Global policy networks: the propagation of health care financing reform since the 1980s. In: Lee, K. Buse, K., and Fustukian, S. (Eds.) Health Policy in a Globalising World (97-119). Cambridge: Cambridge University Press.

Lee, W., Yi, G., Jung, D., Kim, M., \& Moon, I. (2014). Network analysis approach to study hospitals' prescription patterns focused on the impact of new healthcare policy. IEEE International Conference on Systems, Man, and Cybernetics (SMC), 2643-2650. doi:10.1109/SMC.2014.6974326

Lehmbruch, G. (1984). 'Concertation and the structure of corporatist networks: Order and conflict in contemporary capitalism.' In: John H. Goldthorpe (Eds), Order and Conflict in Contemporary Capitalism (pp. 60-80). Oxford: Oxford University Press. 
Leichter, H.M. (1979). A comparative approach to policy analysis: health care policy in four nations. Cambridge: Cambridge University Press.

Lia, C., Yub, X., Butler, J.R.G., Yiengprugsawan, V., \& Yu, M. (2011). Moving towards universal health insurance in China: Performance, issues and lessons from Thailand. Social Science \& Medicine, 73(3), 359-366.

Light, D.W. (2010). Healthcare professions, markets, and countervailing powers. In: Bird, C., Conrad, P., Fremont, A. and Timmermans, S. (Eds). Handbook of Medical Sociology (pp. 270-289). Tennessee, TN: Vanderbilt University Press.

Lim, J. (2019). Is Universal Health Coverage Always the Best Solution to Health Challenges? E-International Relations. https://www.e-ir.info/2019/04/19/is-universal-healthcoverage-always-the-best-solution-to-health-challenges/ [accessed 20 January 2021].

Limwattananon, S., Vongmongkol, V., Prakongsai, P., Patcharanarumol, W., Hanson, K., Tangcharoensathien, V. \& Mills, A. (2011). The equity impact of Universal Coverage: Health care finance, catastrophic health expenditure, utilization and government subsidies in Thailand. Nonthaburi: International Health Policy Program, CREHS. Retrieved from http://www.crehs.lshtm.ac.uk/thai biafia 19jul.pdf

Lindblom, C.E. (1968). The Policy Making Process. Englewood Cliffs, NJ: Prentice Hall.

Lindblom, C.E. (1959). The science of muddling through. Public Administrative Review, 19, 79-88.

Little, R.J.A., Rubin, D.B. (2002). Statistical Analysis with Missing Data (2 ${ }^{\text {nd }}$ ed). New Jersey: Wiley-Interscience.

Lloyd-Sherlock, P. (2005). Health sector reform in Argentina: A cautionary tale. Social Science and Medicine, 60,1893-903.

Locke, E. A. 2007. The case for inductive theory building. Journal of Management, 33(6): 867890.

Lorentzen, P., McMillan, J., \& Wacziarg, R. (2008). Death and development. Journal of Economic Growth 13, 81-124. doi:10.1007/s10887-008-9029-3

Lorie Konish, L. (2019). This is the real reason most Americans file for bankruptcy. Retrieved from https://www.cnbc.com/2019/02/11/this-is-the-real-reason-most-americans-filefor-bankruptcy.html

Lorrain, F., \& White, H. C. (1971). Structural equivalence of individuals in social networks. Journal of Mathematical Sociology, 1, 49-80.

Lukes, S. (1974). Power: A Radical Approach. London: Macmillan.

Lukes, S. (2005). Power: A radical view ( ${ }^{\text {nd }}$ Ed). Basingstoke: Palgrave Macmillan.

Lynch, R., \& Altenburg-van den Broek, E. (2010). The drawbacks of Dutch-style health care rules: lessons for Americans [Picture]. The Heritage Foundation. https://www.heritage.org/health-care-reform/report/the-drawbacks-dutch-style-healthcare-rules-lessons-americans/\#_ftnref20 [accessed 20 January 2021].

Maciel, C.O. (2018). Social network analysis and dyadic identification in the classroom. Revista de Administracão Mackenzie, 19(1), 1678-6971. doi:10.1590/eRAMG180051

Maeda, A., Araujo, E., Cashin, C., Harris, J., Ikegami, N., \& Reich, M.R. (2014). Universal health coverage for inclusive and sustainable development: A synthesis of 11 country case studies. Washington, D.C.: World Bank.

Maguire, D. (2019). The NHS needs to be more productive - or is it more efficient? The King's Fund. $\quad$ https://www.kingsfund.org.uk/blog/2019/03/nhs-productive-or-efficient [accessed 20 January 2021].

Management Sciences for Health. (2020). Health for all: Campaign for Universal Health Coverage in Africa. Retrieve from https://www.msh.org/our-work/projects/health-forall-campaign-for-universal-health-coverage-in-africa 
Mantel, N. (1967). “The Detection of Disease Clustering and a Generalized Regression Approach.” Cancer Research, 27: 209-20.

Maps of World. (2020). Nigeria political map. Retrieved $26^{\text {th }}$ February, 2020, from https://www.mapsofworld.com/nigeria/nigeria-political-map.html

Marcus, L.J., Dorn, B.C., \& McNulty, E.J. (2013). Renegotiating Health Care: Resolving Conflict to Build Collaboration ( $2^{\mathrm{ND}}$ Ed.). California: Wiley.

Marsh, D., Toke, D., Befrage, C., Tepe, D., \& McGough, S. (2009). Policy networks and the distinction between insider and outsider groups: The case of the countryside alliance. Public Administration, 87(3), 621-638.

Marsden, P.V. (1990). Network data and measurement. Annual Review of Sociology, 16, 435463.

Marten, R., McIntyre, D., \& Travassos, C. (2014). An assessment of progress towards universal health coverage in Brazil, Russia, India, China, and South Africa (BRICS). Lancet, 384, 2164-2171.

Maruthappu, M., Watkins, J., \& Noor, A.M., (2016). Economic downturns, universal health coverage, and cancer mortality in high-income and middle-income countries, 19902010: A longitudinal analysis. Lancet, 388, 684- 695.

Mathauer, I., Saksena, P. \& Kutzin, J. (2019). Pooling arrangements in health financing systems: A proposed classification. International Journal Equity Health, 18, 198. https://doi.org/10.1186/s12939-019-1088-X

Meny, Y. (1989). Formation et transformation des policy communities: L' exemple francais. In: Meny, Y., Ideologies Parties and Politiques \& Groupes Sociaux (pp. 355-366). Paris: Presses de la Fondation Nationale des Sciences Politiques.

McGrath, C., Krackhardt, D. \& Blythe, J. (2003). Visualizing Complexity in Networks: Seeing both the forest and the trees. Connections, 25(1), 37-47.

McKee, M., \& Stuckler, D. (2011). The assault on universalism: How to destroy the welfare state. British Medical Journal, 34: d7973.

McKee, M., Balabanova, D., Basu, S., Ricciardi, W., \& Stuckler, D. (2013). Universal health coverage: a quest for all countries but under threat in some. Value in Health, 16, 39-45.

Mc Kenna, H., Dunn, P., Northern, E., \& Buckley, T. (2017). How health care is funded. London: The King's Fund. Retrieve from https://www.kingsfund.org.uk/publications/how-health-care-is-funded

Meessen, B., Akhnif, E.H., Kiendrébéogo, J.A., Alaoui, A.B., Bello, K., Bhattacharyya, S., Dini, H.S.F., Dkhimi, F., Jean-Paul Dossou, J-P., Kelley, A.G., Keugoung, B., Millimouno, T.M., Zambruni, J.P., Rouve, M., Sieleunou, I., Godelieve van Heteren. (2019). Learning for universal health coverage. BMJ Global Health, 4, 1-6. doi:10.1136/bmjgh-2019-002059

Medic West Africa. (2019). 2019 Health market insights: Nigeria. Lagos: Informa markets. Retrieved from https://www.medicwestafrica.com/content/dam/Informa/medic-westafrica/english/2019/HealthcareInsights.pdf.

Mesa-Lago, C. (2017). The Emerging Non-State Sector in Cuba's Economy. Latin American Research Review, 52(1): 150-156. doi.org/10.25222/larr.92

Mikkelsen, M. (2006). 'Policy network analysis as a strategic tool for the voluntary sector.' Policy Studies, 27: 17-26.

Miller, G., \& Dingwall, R. (1997). Context and Method in Qualitative Research. London: Sage. Mills, W.C. (1956). The Power Elite. Oxford: Oxford University Press.

Mills, C.W. (1956/2000). The Power Elite. Oxford: Oxford University Press.

Missoni, E. (2010). Attaining universal health coverage: A research initiative to support evidence-based advocacy and policymaking. Milano: Egea. Retrieved from 
https://www.medicusmundi.ch/de/bulletin/mms-bulletin/zugang-zu-medikamentenfur-alle/debatte/attaining-universal-health-coverage-through-primary-health-care

Missoni, E., \& Solimano, G. (2010). Towards Universal Health Coverage: The Chilean Experience. Geneva: World Health Organization.

Mitchell, J. C. (1983). Case and Situation Analysis. The Sociological Review, 31(2), 187-211. Molina-Azorín, J. F. (2016). Mixed methods research: An opportunity to improve our studies and our research skills. European Journal of Management \& Business Economics, 25(2), 37-38.

Molloy, A., Gardner, T., \& Thorlby, R., (2017). Quality of care in the English NHS: In the balance. The Health Foundation. https://www.health.org.uk/publication/electionbriefing-quality-care-english-nhs [accessed 20 January 2021].

Monaghan, M. (2011). Evidence Versus Politics: Exploiting Research in UK Drug Policy Making? Bristol: The Policy Press.

Montgomery, K. (2020). Differences Between Universal Coverage and Single-Payer. VerywellHealth. $\quad \underline{\text { https://www.verywellhealth.com/difference-between-universal- }}$ coverage-and-single-payer-system-1738546 [accessed 20 January 2021].

Moody, J., \& White, D.R. (2003). Structural Cohesion and Embeddedness: A Hierarchical Concept of Social Groups. American Sociological Review, 68, 103-127.

Moreno-Serra, R., \& Smith, P.C. (2012). Does progress towards universal health coverage improve population health? Lancet, 380, 917-923.

Moreno-Serra, R., \& Smith, P.C. (2015). Broader health coverage is good for the nation's health: Evidence from country level panel data. Journal of the Royal Statistical Society Series A (Statistics in Society), 178 (1), 101-124. doi:10.1111/rssa.12048

Moretti, F. (2011). Network Theory, Plot Analysis. New Left Review, 68, 1-64.

Moretti, F. (2011). Network Theory, Plot Analysis [Photograph]. New Left Review, 68, 1-64.

Mueller, M., \& Morgan, D. (2017). New insights into health financing: First results of the international data collection under the system of health accounts 2011 framework. Health Policy, 121(7), 764-769.

Muhammad, F., Abdulkareem, J.H., \& Chowdhury, A.A. (2017). Major public health problems in Nigeria: A review. South East Asia Journal of Public Health, 7(1), 6-11.

Nachuk, S. (2013). Evolution of the Joint Learning Network. Retrieved from https://www.rockefellerfoundation.org/blog/evolution-joint-learning-network/

National Bureau of Statistics. (2015). Millennium Development Goals Performance Tracking Survey Report. Abuja: OSSAP-MDGs, The Presidency.

National Health Insurance Scheme. (2019). NHIS operational guidelines. Abuja: NHIS.GOV.NG. Retrieved December 3, 2019, from https://www.nhis.gov.ng/file/repository/NHIS_OPERATIONAL_GUIDELINES.pdf

Navarro, V. (1989). Why some countries have national health insurance, others have national health services, and the US has neither. Social Science Medical, 28: 887-98.

Neergaard, H., Shaw, E., \& Carter, S. (2005). The impact of gender, social capital and networks on business ownership: A research agenda. International Journal of Entrepreneurial Behaviour \& Research, 11: 338-357. doi:10.1108/13552550510614999

Ndie, E.C. (2013). Evaluation of National Health Insurance Scheme (NHIS) awareness by civil servants in Enugu and Abakaliki. International Journal of Medicine and Medical Sciences, 5(7), 356-358. doi:10.5897/IJMMS2013.0920

NHS Improvement. (2018). 'Performance of the NHS provider sector for the month ended 31 December 2017.' NHS Improvement website. https://improvement.nhs.uk/documents/2471/Performance_of_the_NHS_provider_sec tor for the month ended 31 December.pdf [accessed 20 January 2021]. 
Nicholson, D., Robert Yates, R., Warburton, W., \& Fontana, G. (2015). Delivering Universal Health Coverage: A guide for policymakers. Imperial College of Science: WISH Foundation. Retrieved from https://www.imperial.ac.uk/media/imperialcollege/institute-of-global-health-innovation/public/Universal-health-coverage.pdf

Nigeria Health Watch. (2017, June 29). Are HMOs Needed in Nigeria? Public Hearing Scrutinizes Their Existence. Nigeria Health Watch. Retrieve from https://nigeriahealthwatch.com/are-hmos-needed-in-nigeria-public-hearingscrutinizes-their-existence/

Nigeria Health Watch (2019, May 29) Mr. President, as you begin this journey, please remember that without health, we have nothing. Nigeria Health Watch. Retrieved from: https://nigeriahealthwatch.com/dear-president-buhari-without-health-we-havenothing/

Nigerian Centre for Disease Control. (2020). COVID-19 situation report: Situation report 119. Retrieved June 26, 2020, from https://ncdc.gov.ng/diseases/sitreps/?cat=14\&name=An\%20update\%20of\%20COVID -19\%20outbreak\%20in\%20Nigeria

Nooraie, R.Y., Sale, J.E.M., Marin, A., \& Ross, L.E. (2018). Social Network Analysis: An Example of Fusion Between Quantitative and Qualitative Methods. Journal of Mixed Methods Research, 14(1): 110-124. doi:10.1177/1558689818804060

Nwani, V. (2015). Assessing the penetration of health insurance scheme in Nigeria. International Research on Medical Sciences, 3(1), 1-5.

Nye, J. (2002) The Paradox of American Power: Why the World's Only Superpower Cannot Go It Alone. Oxford: Oxford University Press.

Nye, J. (2004). Soft Power and American Foreign Policy. Political Science Quarterly, 119(2), 255-270.

Ochalek, J., Manthalu, G., \& Smith, P.C. (2020). Squaring the cube: Towards an operational model of optimal universal health coverage. Journal of Health Economics, 70. doi: 10.1016/j.jhealeco.2019.102282

O'Connell, T., Rasanathan, K., \& Chopra, M. (2014). What does universal health coverage mean? Lancet, 383, 277-279. doi:10.1016/S0140-6736(13)60955-1

Odeyemi, I.A. (2014). Community-based health insurance programmes and the National Health Insurance Scheme of Nigeria: Challenges to uptake and integration. International Journal for Equity in Health, 13, 20. https://doi.org/10.1186/1475-927613-20

OECD. (2017). Bridging the Gap: Inclusive Growth 2017 Update Report. Paris; OECD Publishing.

OECD. (2017). 'Understanding the determinants of health' in OECD, Health at a glance 2017: OECD indicators. Paris: OECD Publishing. https://read.oecd-ilibrary.org/socialissues-migration-health/health-at-aglance [accessed 20 January 2021].

OECD. (2018). 'Health expenditure and financing'. OECD. Stat website. http://stats.oecd.org/Index.aspx?DataSetCode=SHA [accessed 20 January 2021].

OECD. (2018). 'Health status.' OECD. Stat website. http://stats.oecd.org/index.aspx?DataSetCode=HEALTH_STAT [accessed 20 January 2021].

OECD. (2018). 'Health expenditure and financing'. OECD. Stat website. http://stats.oecd.org/Index.aspx?DataSetCode=SHA [accessed 20 January 2021].

OECD/European Observatory on Health Systems and Policies, (2019). The Netherlands: Country Health Profile 2019, State of Health in the EU. Brussels: OECD Publishing, Paris/European Observatory on Health Systems and Policies. 
Office of the Senior Special Assistant to the President on Millennium Development Goals (MDGs). (2015). Nigeria Millennium Development Goals End-Point Report 2015. Abuja: OSSAP-MDGs, The Presidency. Retrieve from https://www.undp.org/content/dam/undp/library/MDG/english/MDG\%20Country\%20 Reports/Nigeria/Nigeria MDGs Abridged Sept30.pdf

Office of the UN High Commissioner for Human Rights., \& World Health Organization. (2008). The right to health. Geneva: United Nations. Retrieve from https://www.ohchr.org/Documents/Publications/Factsheet31.pdf

Ojo, J. (2018, April 11). Nigeria and the challenges of Universal Health Coverage. Punch Nigeria. Retrieved from https://punchng.com/nigeria-and-the-challenges-of-universalhealth-coverage/

Okafor, C. (2016). Improving outcomes in the Nigeria healthcare sector through public- private partnership. African Research Review, 10(4), 1. http://dx.doi.org/10.4314/afrrev.v10i4.1

Okpani, A. I. \& Abimbola, S. (2015). Operationalizing universal health coverage in Nigeria through social health insurance. Nigerian Medical Journal, 56(5), 305-310. doi:10.4103/0300-1652.170382

Oleribe, O.O., \& Taylor-Robinson, S.D. (2016). Before Sustainable Development Goals (SDG): Why Nigeria failed to achieve the Millennium Development Goals (MDGs)? The Pan African Medical Journal, 24, 156.

Oliver, K., KA., U., \& Kislov, R. (2012). Policy, evidence and theory: Contextualising a glossary of policymaking. Journal of epidemiology and community health. https://researchonline.lshtm.ac.uk/id/eprint/4646435

One by One. (2020). One by One: Target 2030 | Access Challenge. Retrieve from https://www.onebyone2030.org/

Onoka, C.A., Hanson, K., \& Hanefeld, J. (2014). Towards universal coverage: a policy analysis of the development of the National Health Insurance Scheme in Nigeria. Health Policy and Planning, 30(9), 1105-1117. doi:10.1093/heapol/czu116

Onuobia, F. \& Okoro, O. J. (2020). Nigeria. In Friere, A. L. Sombra, T.L. and dos Santos Neto, R.D. The Public-Private Partnership Law Review ( $6^{\text {th }}$ ed). London: The Law Reviews. Retrieved from: https://thelawreviews.co.uk/edition/the-public-privatepartnership-law-review-edition-6/1225139/nigeria

Onwujekwe, O., Ezumah, N., Mbachu, C., Obi, F., Ichoku, H., Uzochukwu, B., \& Hong Wang, H. (2019). Exploring effectiveness of different health financing mechanisms in Nigeria; what needs to change and how can it happen? BMC Health Services Research, 19, 661. doi:10.1186/s12913-019-4512-4

Onyedibe, K.I., Goyit, M.G., Nnadi, N.E. (2012). An evaluation of the national health insurance scheme (NHIS) in Jos, a north-central Nigerian city. Global Advance Research Journal of Microbiology, 1(1), 5-12.

Onyedinefu, G. (2019, August 8). FCT,15 states access N51bn Basic Health Provision Fund. Business Day. Retrieved from https://businessday.ng/health/article/fct15-states-accessn51bn-basic-health-provision-fund/

Onyeji, E. (2018, December 21). NHIS: NMA directs doctors to shun state health insurance schemes. Premium Times. Retrieve from https://www.premiumtimesng.com/news/topnews/302357-nhis-nma-directs-doctors-to-shun-state-health-insurance-schemes.html

Onyeji, E. (2018). Nigerian govt., medical doctors disagree on implementation of National Health Act. Premium Times. Retrieve from https://www.premiumtimesng.com/news/top-news/259313-nigerian-govt-medicaldoctors-disagree-implementation-national-health-act.html 
Onyeji, E. (2019, September 21). Minister cautions HMOs against contributing to NHIS service failure. Premium Times. Retrieved from https://www.premiumtimesng.com/health/health-news/353579-minister-cautionshmos-against-contributing-to-nhis-service-failure.html

Ooms, G., Latif, L.A., Waris, A., Brolan, C.E., Hammonds, R., \& Friedman, E.A. (2014). Is universal health coverage the practical expression of the right to health care? BMC International Health Human Rights, 14, 3.

Oreh, C.A. (2017). Universal Health Coverage: Is leaving no one behind in a pipe dream? American Journal of Public Health Research, 5(5), 159-162. doi:10.12691/ajphr-5-5-4

Orisadare, A.D. (2018). Nigeria's fight against corruption and the extent to which it has tackled it. IOSR Journal of Humanities and Social Science, 23(11), 39-45.

Osuchukwu, N.C., Osonwa, K.O., Eko, J.E., Uwanede, C.C., Abeshi, S.E., \& Offiong, D.A. (2013). Evaluating the impact of National Health Insurance Scheme on health care consumers in Calabar metropolis, southern Nigeria. International Journal of Learning and Development, 3(4), 30-45.

Otte, E., \& Rousseau, R. (2002). "Social network analysis: a powerful strategy, also for the information sciences.” Journal of information Science, 28(6), 441-453.

Ottersen, T., \& Schmidt, H. (2017). Universal health coverage and public health: ensuring parity and complementarity. American Journal of Public Health, 107(2), 248-250.

Ovuakporie, E., \& Nwabughiogu, L. (2017). Health insurance: FG calls scrapping of HMOs. Vanguard. Retrieved for https://www.vanguardngr.com/2017/06/health-insurance-fgcalls-scrapping-hmos/

Padilha, A., Kasonde, J., Mukti, G., Crisp, N., Takemi, K., \& Buch, E. (2013). Human resources for universal health coverage: Leadership needed. Bulletin World Health Organization, 91: 800-0A.

Paek, S. C., Meemon, N. \& Wan, T. T. (2016). Thailand's universal coverage scheme and its impact on health-seeking behaviour. Springerplus, 5, 1952.

Park, N., Lee, S., \& Kim, J. H. (2012). Individuals' personal network characteristics and patterns of Facebook use: A social network approach. Computers in Human Behaviour, 28(5), 1700-1707. doi: 10.1016/j.chb.2012.04.009

Penchaszadeh, V. B., Leone, F., \& Rovere, M. (2012). The Health System in Argentina: An Unequal Struggle between Equity and the Market. Italian Journal of Public Health, 7(4), $350-8$.

Peterson, J. (2003). Policy Networks. IHS Political Science Series: 2003, No. 90. [Working Paper]

Petrosino, A., Turpin-Petrosino, C., \& Buehler, J. (2003). Scared straight and other juvenile awareness programs for preventing juvenile delinquency: A systematic review of the randomized experimental evidence. The American Academy of Political and Social Science, 589, 41-62. doi:10.1177/0002716203254693

Poole, A. (2011). Political Economy Assessments at Sector and Project Levels: How-To Note. Washington D.C.: World Bank.

Prell, C. (2012). Social Network Analysis: history, theory and methodology. London: Sage.

Premium Times (2019, January 8) Buhari rolls out Basic Health Care Provision Fund, Premium Times. Retrieved from: https://www.premiumtimesng.com/health/healthnews/304833-buhari-rolls-out-basic-health-care-provision-fund.html

PWC. (2019). Sustainability of State Health Insurance Schemes in Nigeria Beyond the Launch. Retrieve from https://www.pwc.com/ng/en/assets/pdf/sustainability-state-healthinsurance-nigeria.pdf 
Quissell, K. \& Walt, G. (2015). The challenge of sustaining effectiveness over time: The case of the global network to stop tuberculosis. Health Policy and Planning, 31(suppl 1), 116. doi:10.1093/heapol/czv035

Quissell, K., Berlan, D., Shiffman, J. \& Walt, G. (2018). Explaining global network emergence and nonemergence: Comparing the processes of network formation for tuberculosis and pneumonia. Public Administration and Development, 38(4), 144-153. doi:10.1002/pad.1836

Ramke, J., Gilbert, C.E., Lee, A.C., Ackland, P., Limburg, H., \& Foster, A. (2017). Effective cataract surgical coverage: An indicator for measuring quality-of-care in the context of Universal Health Coverage. PLoS ONE, 12(3), e0172342.

Ranabhat, C.L., Kim, C.B., Singh, A., Acharya, D., Pathak, K., \& Sharma, B. (2019). Challenges and opportunities towards the road of universal health coverage (UHC) in Nepal: a systematic review. Archive of Public Health, 77, 5. doi:10.1186/s13690-0190331-7

Rapoport, A., \& Horvath, W.J. (1961). A study of a large sociogram. Behavioral Science, 6, 279-291.

Reeves, A., McKee, M., \& Stuckler, D. (2015a). The attack on Universal Health Coverage in Europe: Recession, austerity, and unmet needs. European Journal of Public Health, 25(3), 364-365.

Reeves, A., Gourtsoyannis, Y., Basu, S., McCoy, D., McKee, M., \& Stuckler, D. (2015b). Financing universal health coverage effects of alternative tax structures on public health systems: Cross-national modelling in 89 low-income and middle-income countries. Lancet, 386(9990), 274-280. doi:10.1016/S0140-6736(15)60574-8

Reich, M.R., Harris, J., \& Ikegami, N. (2016). Moving towards universal health coverage: Lessons from 11 country studies. Lancet, 387, 811-816.

Reich, M.R., Harris, J., \& Ikegami, N. (2016, p.812). Moving towards universal health coverage: Lessons from 11 country studies [Digital image]. Lancet, 387, 811-816.

Reis, A.A. (2016). Universal Health Coverage - The Critical Importance of Global Solidarity and Good Governance: Comment on Ethical Perspective: Five Unacceptable Trade-offs on the Path to Universal Health Coverage. International Journal Health Policy Management, 5(9), 557-559. doi:10.15171/ijhpm.2016.61

Rhodes, R.A.W. (1990). Policy Networks: A British perspective. Journal of Theoretical Politics, 2 (3), 291-318.

Richardson, J., \& Jordan, A.G. (1983). Overcrowded policymaking: Some British and European Reflections. Policy Sciences, 15, 247-268.

Richardson, J.J. (2000). “Government, interest groups and policy change.” Political Studies, 48: 1006-25.

Ridde, V. (2009). Policy implementation in an African state: An extension of Kingdon's multiple-streams approach. Public Administration, 87(4), 938-954.

Ritchie, J., \& Lewis, J. (2003). Qualitative Research Practice: A Guide for Social Science Students and Researchers. London: Sage.

Roemer, M.I. (1993). National health systems throughout the world. Annual Review Public Health, 14, 335-53.

Robins, G., Pattison, P., Woolcock, J. (2004). Missing data in networks: Exponential random graph ( $\mathrm{p} *$ ) models for networks with non-respondents. Social Networks, 26, 257-283.

Robins, G., Lewis, J.M., \& Wang, P. (2012). Statistical network analysis for analysing policy networks. Policy Studies Journal, 40 (3), 375-401.

Rodwin, V.G. (2003). The health care system under French national health insurance: lessons for health reform in the United States. American Journal of Public Health, 93(1), 3137. https://doi.org/10.2105/ajph.93.1.31 
Rubinstein, A., Zerbino, M.C., \& Cejas, C. (2018). Making Universal Health Care Effective in Argentina: A Blueprint for Reform. Health Systems \& Reform, 72, 1-11.

Rumsey, D.J. (1993). Nonresponse models for social network stochastic processes (Markov chains). The Ohio State University: Ph.D. Thesis.

Ryan, G. W., \& Bernard, H.R. (2003). Techniques to Identify Themes. Field Methods, 15(1), 85- 109.

Ryan, L. (2016). Looking for Weak Ties: Using a Mixed Methods Approach to Capture Elusive Connections. The Sociological Review, 64(4): 951-969. doi:10.1111/1467-954X.12395

Ryan, L., \& D’Angelo, A. (2017). Changing Times: Migrants Social Network Analysis and the Challenges of Longitudinal Research. Social Networks, 53, 148-158.

Ryan, L., Mulholland, J., \& Agoston, A. (2014). Talking ties: Reflecting on network visualisation and qualitative interviewing. Sociological Research Online, 19(2), 16. doi:10.5153/sro.3404.

Sabatier, P.A. (1987). Knowledge, policy-oriented learning, and policy change: An advocacy coalition framework. Knowledge, Creation, Diffusion, Utilization, 8, 649-692.

Sabatier, P. A. (1988). An advocacy coalition framework of policy change and the role of policy-oriented learning therein. Policy Sciences, 21(2-3), 129-168.

Sabatier, P., \& Jenkins-Smith, H. (1993). Policy Change and Learning. Boulder-Colorado: Westview Press.

Sabatier, P. (1999). Theories of the policy process. New York: Avalon.

Sabatier, P. (2007). Theories of the policy process. Boulder, CO: Westview Press.

Sager, F. \& Thomann, E. (2017). A Multiple Streams Approach to Member State Implementation Research: Politics, Problem Construction and Policy Paths in Swiss Asylum Policy. Journal of Public Policy, 37(3), 287-314.

Sakolsatayadorn, P., \& Chan, M. (2017). Breaking down the barriers to universal health coverage. Bulletin World Health Organization, 95(2), 86.

Salancik, G. (1995). Wanted: A Good Network Theory of Organization. Administrative Science Quarterly, 40, 345-349. doi:10.2307/2393642

Sales, M., Kieny, M. P., Krech, R., \& Etienne, C. (2013). Human resources for universal health coverage: from evidence to policy and action. Bulletin of the World Health Organization, 91(11), 798-798A. https://doi.org/10.2471/BLT.13.131110

Sandelowski, M., \& Barroso, J. (2003). Classifying the Findings in Qualitative Studies. Qualitative Health Research, 13(7), 905-23. doi:10.1177/1049732303253488

Sandström, A. \& Carlsson, L. (2008). The performance of policy networks: The relation between network structure and network performance. Policy Studies Journal, 36(4), 497-524.

Sandström, A. (2008). Policy Networks: The Relation Between Structure and Performance (Unpublished Ph.D. Thesis). Luleå, Sweden: Luleå University of Technology, Department of Business Administration and Social Sciences, Division of Political Science. Retrieved January 25, 2020, http://www.divaportal.org/smash/get/diva2:989924/FULLTEXT01.pdf

Savedoff, W.D. (2012). Transitions in Health Financing and Policies for Universal Health Coverage. Washington D.C.: Results for Development Institute.

Scharpf, F., Reissert, B., \& Schnabel, F. (1976). Politikverflechtung: Theorie unde Empirie des kooperativen Foderalismus in der Bundesrepublik. Kronberg, Germany: Scriptor Verlag. 
Schieber, G., Baeza, C., Kress, D., \& Maier, M. (2006). Financing health systems in the 21st Century. In D.T Jamison, J.G. Breman \& A.R. Measham ( $2^{\text {nd }}$ Ed.), Disease control priorities in developing countries (pp. 225-242). Washington, DC: World Bank.

Schiffer, E., \& Hauck, J. (2010). Net-Map: Collecting social network data and facilitating network learning through participatory influence network mapping. Field Methods, 22(3), 231-249. doi:0.1177/1525822X10374798

Schindler, D.W. (2006). Recent advances in the understanding and management of eutrophication. Limnol Oceanogr, 51, 356-63.

Schmidt, V, A. (2013). Democracy and Legitimacy in the European Union Revisited: Input, Output and Throughput. Political Studies, 61(1), 2- 22.

Schmidt, H., Gostin, L., \& Emanuel, E. (2015). Public health, universal health coverage, and sustainable development goals: Can they coexist? Lancet, 386(9996), 928-930. doi/10.1016/S0140-6736(15)60244-6

Schneider, V. (1992). 'The structure of policy networks: A comparison of the "chemicals control” and “telecommunications” policy domains in Germany.' European Journal of Political Research, 21(1-2): 109-29.

Scott, J. (2017). Groups, factions and social divisions. In J. Scott ( ${ }^{\text {th }}$ Ed.), Social network analysis (pp. 113-135). London: Sage. doi.org/10.4135/9781529716597.n7

Scott, J., Tallia, A., Crosson, J.C., Orzano, A.J., Stroebel, C., DiCicco-Bloom, B., O’Malley, D., Shaw, E., \& Crabtree, B. (2005). Social network analysis as an analytic tool for interaction patterns in primary care practices. Annals of Family Medicine, 3(5), 443448.

Scott, W.R. (2013). Institutions and Organizations: Ideas, Interests, and Identities. London: Sage.

Seervai, S., Shah, A., \& Osborn, R. (2017). How Other Countries Achieve Universal Coverage. To the Point: The Commonwealth Fund. Retrieved from https://www.commonwealthfund.org/blog/2017/how-other-countries-achieveuniversal-coverage

Seervai, S., Shah, A., \& Osborn, R. (2017). How Other Countries Achieve Universal Coverage [Digital image]. To the Point: The Commonwealth Fund. Retrieve from https://www.commonwealthfund.org/blog/2017/how-other-countries-achieveuniversal-coverage

Sen, A. (2015, January 6) Universal healthcare: The affordable dream. The Guardian. Retrieved from https://www.theguardian.com/society/2015/jan/06/-sp-universalhealthcare-the-affordable-dream-amartya-sen

Shearer, J.C., Abelson. J., D Kouyaté, B., Lavis, J.N. \& Walt, G. (2016). Why do policies change? Institutions, interests, ideas and networks in three cases of policy reform. Health Policy and Planning, 31(9), czw052.

Shearer, J.C., Lavis, J.N., Abelson. J., Walt, G. \& Dion, M. (2018). Evidence-informed policymaking and policy innovation in a low-income country: Does policy network structure matter? Evidence \& Policy A Journal of Research Debate and Practice, 14(3), 381-401. doi:10.1332/174426418X15330477583836

Sheikh, K., \& Porter, J.D.H. (2011). Disempowered doctors? A relational view of public health policy implementation in urban India. Health Policy Planning, 26(1), 83-92.

Sheikh, K., George, A., \& Gilson, L. (2014). People-centred science: strengthening the practice of health policy and systems research. Health Research Policy and Systems, 12, 19.

Shepherd, D. A., \& Suddaby, R. (2017). Theory building: A review and integration. Journal of Management, 43(1), 59-86.

Shiffman, J., Skarbalo, M., \& Subotic, J. (2002). Reproductive rights and the state in Serbia and Croatia. Social Science and Medicine, 54, 625-42. 
Shiffman, J., \& Smith, S. (2007). Generation of political priority for global health initiatives: A framework and case study of maternal mortality. Lancet, 370, 1370-9.

Silke, A., \& Kriesi, H. (2007). The Network Approach. In: Sabatier, P.A., Theories of the Policy Process (Ed). Boulder, CO: Westview Press, 129-54.

Silverman, D., \& Gubrium, J.F. (1994). Competing strategies for analysing the contexts of social interaction. Sociological Inquiry, 64(2), 179-198.

Sims, M., Maxwell, R., Bauld, L., \& Gilmore, A. (2010). Short term impact of smoke-free legislation in England: retrospective analysis of hospital admissions for myocardial infarction. BMJ, 340, c2161. doi:10.1136/bmj.c2161

Smith, P.C., Witter, S.N. (2004). Risk Pooling in Health Care Financing: The Implications for Health System Performance. Health, Nutrition and Population (HNP) discussion paper. Washington, DC.: World Bank. Retrieved from https://openknowledge.worldbank.org/handle/10986/13651

Sriram, V., Topp, S.M., Schaaf, M., Mishra, A., Flores, W., Rajasulochana, S.R., \& Scott, K. (2018).10 best resources on power in health policy and systems in low- and middleincome countries. Health Policy Planning, 33, 611-621.

Stigler, F.L., Macinko, J., Pettigrew, L.M., Kumar, R., \& van Weel, C. (2016). No universal health coverage without primary health care. Lancet, 387(10030), 1811. doi.org:10.1016/s0140-6736(16)30315-4

Stork, D., \& Richards, W.D. (1992). Nonrespondents in communication network studies: problems and possibilities. Group and Organization Management, 17 (2), 193-209.

Sunday, E-A., Salau, G., Akutu, G., Akpan, A., Nsoh, I.A., Chikeruba, A., Adewale, M., Julius Osahon, J., \& Egbejule, M. (2018). Amid soaring cost, affordable healthcare still elusive in states. The Guardian. Retrieved from https://guardian.ng/saturdaymagazine/cover/amid-soaring-cost-affordable-healthcare-still-elusive-in-states/

Szabo, S., Nove, A., Matthews, Z., Bajracharya, A., Dhillon, I., Singh, D.R., Saares, A., \& Campbell, J. (2020). Health workforce demography: A framework to improve understanding of the health workforce and support achievement of the Sustainable Development Goals. Human Resource Health, 18: 7. https://doi.org/10.1186/s12960020-0445-6

Tahsina, T., Ali, N.B., Siddique, A.B., Ahmed, S., Rahman, M., \& Islam, S., (2018). Determinants of hardship financing in coping with out of pocket payment for care seeking of under five children in selected rural areas of Bangladesh. PLoS One, 13(5), $1-17$.

Tandon, A., Murray, C.J.L., Lauer, J., \& Evans, D. (2000). Measuring overall health system performance for 191 countries. Geneva: World Health Organization.

Tangcharoensathien, V., Mills, A., \& Palu, T. (2015). Accelerating health equity: The key role of universal health coverage in the Sustainable Development Goals. BMC medicine, 13,101-204.

Tangcharoensathien, V., Pitayarangsarit, S., Patcharanarumol, W., Prakongsai, P., Sumalee, H., Tosanguan, J., \& Mills, A. (2015). Promoting universal financial protection: How the Thai universal coverage scheme was designed to ensure equity. Health Research Policy System, 11: 25. https://doi.org/10.1186/1478-4505-11-25

Tikkanen, R., George, A.W., Djordjevic, A., Mossialos, E., Williams II, R.D. (2020). “The 2020 International Profiles of Health Care Systems: A Useful Resource for Interpreting Country Responses to the COVID-19 Pandemic,” To the Point (blog): Commonwealth Fund. https://doi.org/10.26099/bwxx-4121

The Commonwealth Fund. (2017). Mirror, mirror 2017: International comparison reflects flaws and opportunities for better US health care. New York: The Commonwealth 
Fund. www.commonwealthfund.org/interactives/2017/july/mirror-mirror/ [accessed 20 January 2021].

The Commonwealth. (2020). Nigeria: History. The Commonwealth. Retrieved from https://thecommonwealth.org/our-member-countries/nigeria/history

The Health Foundation. (2017). 'What does the public think about NHS and social care services?'

Health

Foundation. www.health.org.uk/sites/health/files/Polling2017_web.pdf [accessed 20 January 2021].

The Global Fund. (2020). Global fund survey: Majority of HIV, TB and Malaria programs face disruptions as a result of COVID-19. Retrieved from https://www.theglobalfund.org/en/covid-19/news/2020-06-17-global-fund-surveymajority-of-hiv-tb-and-malaria-programs-face-disruptions-as-a-result-of-covid-19/

ThisDay. (2018, April 11). THISDAY and the journal to universal health coverage. ThisDay. Retrieved from https://www.thisdaylive.com/index.php/2018/04/11/thisday-and-thejourney-to-universal-health-coverage/

Thomas, J., \& Harden, A. (2008). Methods for the thematic synthesis of qualitative research in systematic reviews. BMC Medical Research Methodology, 8, 45. https://doi.org/10.1186/1471-2288-8-45

Thomson, R., \& Hosli, M.O. (2006). "Explaining legislative decision-making in the European Union.” In: Robert Thomson, Frans N. Stokman, Christopher H. Achen \& Thomas Konig (Eds.), The European Union Decides. Cambridge: Cambridge University Press.

Thomson, R., Stokman, F.N., Achen, C.H., \& Konig, T. (2006). The European Union Decides (Eds.). Cambridge: Cambridge University Press.

Tsvetovat, M., \& Kouznetsov, A. (2011). Social Network Analysis for Start-ups: Finding connections on the social web. Cambridge: O’Reilly Media.

Valente, T.W., Gallaher, P., \& Mouttapa, M. (2000). Using social networks to understand and prevent substance use: A transdisciplinary perspective. Substance Use and Misuse, 39(10-12), 1685-1712.

UNAIDS., Federal Ministry of Health., \& National Agency for the Control of AIDS. (2019). Press release: New survey results indicate that Nigeria has an HIV prevalence of $1.4 \%$. UNAIDS.

Retrieved

from

https://www.unaids.org/en/resources/presscentre/pressreleaseandstatementarchive/201 9/march/20190314_nigeria

United Nations Development Programme. (2019). Beyond income, beyond averages, beyond today: Inequalities in human development in the 21st century, Human Development Report 2019. New York: United Nations Development Programme.

United Nations. (2019). Resolution Adopted by the General Assembly. United Nations. Retrieved October 10, 2019, from https://undocs.org/en/A/RES/74/2

Vanguard (2019, October 15) Furore over Buhari's 2020 health budget proposal. Vanguard. Retrieved from: https://www.vanguardngr.com/2019/10/furore-over-buharis-2020health-budget-proposal/

Vanguard. (2020, June 3). PDP rejects slash in health, education budgets, berates APC, Buhari presidency. Vanguard. Retrieved https://www.vanguardngr.com/2020/06/pdp-rejectsslash-in-health-education-budgets-berates-apc-buhari-presidency/

Verloo, M. (2006). Multiple Inequalities, Intersectionality and the European Union. European Journal of Women's Studies,13(3), 211-28.

Verrecchia, R., Thompson, R., \& Yates, R. (2019). Universal health coverage and public health: A truly sustainable approach. Lancet Public Health, 4(1), e10-11.

Vigneron, E. (2012). Inégalités de santé, inégalités de soins dans les territoires français [Territorial and social healthcare inequalities in France]. Bulletin de l'Academie nationale de medecine, 196(4-5), 939-952. 
Vos, B., Lagasse, R., \& Levesque, A. (2014). Putting new-born hearing screening on the political agenda in Belgium: Local initiatives toward a community programme- A qualitative study. Health Research Policy System, 12,32. doi: 10.1186/1478-4505-1232.

Walt, G. (1994). Health policy: An introduction to process and power. London: Zeb.

Walt, G. \& Gilson, L. (1994). Reforming the health sector in developing countries: The central role of policy analysis. Health Policy and Planning, 9, 353-70.

Walt, G., Shiffman, J., \& Schneider, H. (2008). 'Doing' health policy analysis: methodological and conceptual reflections and challenges. Health Policy and Planning, 23, 308-17.

Walt, G., Shiffman, J., Schneider, H., Murray, S.F., Brugha, R., \& Gilson, L. (2008). Doing health policy analysis: Methodological and conceptual reflections and challenges. Health Policy Planning, 23(5), 308-17.

Wagner, A. (1958). Three extracts on public finance. In: Musgrave, R.A., Peacock, A.T., (Eds.), Classics in the Theory of Public Finance. London: Macmillan.

Wagstaff, A. (2007). Social health insurance re-examined. Washington, DC: World Bank. Retrieved from http://documents.worldbank.org/curated/en/914391468178446567/Social-healthinsurance-reexamined

Wagstaff, A., Flores, G., Hsu, J. (2018). Progress on catastrophic health spending in 133 countries: A retrospective observational study. Lancet Global Health, 6, e169-79. doi:10.1016/S2214-109X (17)30429-1

Wangkiat, P. (2019, December 9). Populist UHC now a feather in Thailand's cap. Bangkok Post. Retrieve from https://www.bangkokpost.com/opinion/opinion/1811469/populistuhc-now-a-feather-in-thailands-cap

Wasserman, S., \& Faust, K. (2009). Social Network Analysis: Methods and Applications. Cambridge: Cambridge University Press.

Weber, M. (1978). Economy and Society: An Outline of Interpretive Sociology. CA: University of California Press.

Wellman, B. (1988). Structural analysis: From method and metaphor to theory and substance. In: Wellman, B., \& Berkowitz, S.D. (eds.) Social structures: a network approach (pp. 19-61). Cambridge: Cambridge University Press.

Wellman, B., \& Berkowitz, S. D. (Eds.). (1988). Structural analysis in the social sciences, Vol. 2. Social structures: A network approach. Cambridge: Cambridge University Press.

West, E., Barron, D.N., Dowsett, J., \& Newton, J.N. (1999). Hierarchies and cliques in the social networks of health care professionals: Implications for the design of dissemination strategies. Social Science \& Medicine, 48(5), 633-646.

Witte, J.M., Reinicke, W.H., \& Benner, T. (2000). 'Beyond multilateralism: Global public policy networks.’ Internationale Politik und Gesellschaft, 2: 176-188.

Wirtz, V.J., Hogerzeil, H.V., \& AL Gray, A.L. (2017). Essential medicines for universal health coverage. Lancet, 389, 403-476.

Wonodi, C., Privor-Dumm, L., Aina, M., Pate, A., Reis, R., Gadhoke P., Levine, O. (2012). Using social network analysis to examine the decision-making process on new vaccine introduction in Nigeria. Health Policy Planning, 27(suppl_2), ii27-38.

World Bank. (2016). Universal Health Coverage (UHC) in Africa: A framework for action. Washington, D.C.: World Bank Group.

World Bank. (2018). Going Universal: How Countries are Implementing Pro-Poor Universal Healthcare Reforms, Universal Healthcare Studies Series (UNICO). Washington, D.C.: World Bank Group. Retrieved from: https://www.worldbank.org/en/topic/health/publication/universal-health-coveragestudy-series 
World Bank. (2018). World Bank Country and Lending Group [Photograph]. Retrieve from https://datahelpdesk.worldbank.org/knowledgebase/articles/906519-world-bankcountry-and-lending-groups

World Bank. (2020). Current health expenditure (\% of GDP) between 2000 to 2016- South Africa, Nigeria, Uganda, Tanzania, Kenya, Zambia, Sierra Leone, Rwanda, Gambia [Charts].

Retrieved from https://data.worldbank.org/indicator/SH.XPD.CHEX.GD.ZS?locations=ZA-NG-UGTZ-KE-ZM-SL-RW-GM\&name_desc=false

World Health Organization. (2000). The World Health Report 2000 Health Systems: improving performance. Geneva: World Health Organization.

World Health Organization. (2008). Universal coverage- three dimensions [Digital image]. World Health Organization. Retrieved December 15, 2019, from https://www.who.int/health_financing/strategy/dimensions/en/

World Health Organization. (2009). Health Financing Strategy for the Asia Pacific Region? Manilla: World Health Organization.

World Health Organization. (2010) Health Financing: The Path to Universal, 2010 World Health Report. Geneva: World Health Organization.

World Health Organization. (2011). The Abuja Declaration: Ten Years On. Retrieved from: https://www.who.int/healthsystems/publications/abuja report aug 2011.pdf?ua=1

World Health Organization. (2013). Arguing for universal health coverage. Geneva: World Health Organization.

World Health Organization. (2013). Research for universal health coverage. Geneva: World Health Organization. Retrieve from http://apps.who.int/iris/bitstream/10665/85761/2/9789240690837 eng.pdf?ua=1

World Health Organization. (2013). The world health report 2013: Research for universal health coverage. Geneva: World Health Organization.

World Health Organization. (2014, chapter 8). Making fair choices on the path to universal health coverage: Final report of the WHO Consultative Group on Equity and Universal Health Coverage. Geneva: World Health Organization.

World Health Organization. (2014). Presidential summit on Universal Health coverage ends in Nigeria. Retrieved from http://www.msh.org/sites/msh.org/files/presidential_summit_declaration_on_universa $\underline{1 \text { health_coverage_in_nigeria.pdf }}$

World Health Organization. (2016). Global strategy on human resources for health: workforce 2030. Geneva: World Health Organization.

World Health Organization. (2016). Strategizing national health in the 21st century: A handbook. Geneva: World Health Organization.

World Health Organization. (2017). Universal health coverage (UHC). Geneva: World Health Organization.

World Bank. (2017). Tracking universal health coverage: 2017 Global Monitoring Report. Geneva: World Health Organization and International Bank for Reconstruction and Development / The World Bank.

World Health Organization. (2018). Health policy. Geneva: World Health Organization.

World Health Organization. (2018). Monitoring Sustainable Development Goals -Indicator 3.8.2. World Health Organization. Retrieve from https://www.who.int/health_financing/topics/financial-protection/monitoring-sdg/en/

World Health Organization. (2018). Monitoring Sustainable Development Goals -Indicator 3.8.2 [Digital image]. World Health Organization. Retrieve from https://www.who.int/health financing/topics/financial-protection/monitoring-sdg/en/ 
World Health Organization. (2018, goal.3.8). SDG 3: Ensure healthy lives and promote wellbeing for all at all ages. Retrieved from https://www.who.int/sdg/targets/en/

World Health Organization. (2018). World Health Statistics 2018: monitoring health for the sustainable development goals. Geneva: World Health Organization.

World Health Organization. (2019). Primary Health Care on the Road to Universal Health Coverage: 2019 monitoring report. Geneva: World Health Organization.

World Health Organization. (2019). Universal health coverage (UHC). Retrieved from https://www.who.int/news-room/fact-sheets/detail/universal-health-coverage-(uhc)

World Health Organization. (2019). World health statistics 2019: Monitoring health for the SDGs, Sustainable Development Goals. Geneva: World Health Organization.

World Health Organization. (2020). World Health Statistics 2020: monitoring health for the SDGs. Geneva: World Health Organization.

World Health Organization, International Bank for Reconstruction and Development, \& The World Health Organization, International Bank for Reconstruction and Development, \& The World Bank. (2015). Tracking Universal Health Coverage: First Global Monitoring Report. Geneva: World Health Organization and International Bank for Reconstruction and Development / The World Bank.

World Health Organization/World Bank. (2014). Monitoring Progress Towards Universal Health Coverage at Country and Global Levels: Framework, Measures and Targets. Geneva: World Health Organization.

World Health Organization/World Bank. (2015). Tracking universal health coverage: First global monitoring report. Geneva: World Health Organisation.

Xie, J., Sreenivasan, S., Korniss, G., Zhang, W., Lim, C., \& Szymanski, B.K. (2011). Social consensus through the influence of committed minorities. Physical Review E, 84(1), 1130. doi:10.1103/PhysRevE.84.011130

Xie, K. (2012). "Empirical Study of the National Health Service in U.K. and the U.S. Medical Care System." International Journal of Financial Research, 3(1): 33-39.

Yin, R.K. (2017). Case Study Research and Applications Design and Methods (Ed 6 ${ }^{\text {th }}$ ). London: Sage.

Yousefi-Nooraie, R., Dobbins, M., Brouwers, M., \& Wakefield, P. (2012). Information seeking for making evidence-informed decisions: A social network analysis on the staff of a public health department in Canada. BMC Health Service Research, 12, 118.

Yu, D., Souteyrand, Y., Banda, M.A., Kaufman, J., \& Perriëns, J.H. (2008). Investment in HIV/AIDS programs: Does it help strengthen health systems in developing countries? Global Health, 4(1), 8.

Zahariadis, N. and Exadaktylos, T. (2016). Policies that succeed and programs that fail: Ambiguity, conflict, and crisis in Greek higher education. Policy Studies Journal, 44(1), 59-82.

Zhang, W., Zhang, Q., Yu, B., \& Zhao, L. (2014). Knowledge map of creativity research based on keywords network and co-word analysis, 1992-2011. Quality and Quantity, 49, 1023-1038. doi:10.1007/s11135-014-0032-9

Zhang, C., Rahman, M.S., Rahman, M.M., Yawson, A.E., \& Shibuya, K. (2019). Trends and projections of universal health coverage indicators in Ghana, 1995-2030: A national and subnational study. PLoS ONE, 14(5), e0209126. 


\section{APPENDICES}

\section{Appendix 1: Overview of reviewed Literature.}

\begin{tabular}{|c|c|}
\hline Author & Aim \\
\hline Adefolaju. (2014) & $\begin{array}{l}\text { Using literature reviews, this paper examines the structures and features } \\
\text { of both medical systems in Nigeria to fimd a convergence that will be } \\
\text { advantageous to the populace. }\end{array}$ \\
\hline A dew ole et al., (2016) & $\begin{array}{l}\text { The study assesses ooverage levels of the scheme amongst formal } \\
\text { sector workers in Nigeria to gange the extent of coverage of the scheme } \\
\text { and derive suitable solutions. }\end{array}$ \\
\hline A dew olc and Osung back, (2016) & $\begin{array}{l}\text { An assessment of the three dimensions of universal health coverage in } \\
\text { the geo-political zone of sonth west Nigeria is essential for detemining } \\
\text { the gaps in these areas. These will be of assistance for policymakers in } \\
\text { expanding the scheme. }\end{array}$ \\
\hline Amoah and Phillips, (2018) & $\begin{array}{l}\text { Investigating hew health literacy may operate in the context of universal } \\
\text { health coverage (UHC), using evidencr-based rescarch. This artice } \\
\text { addresses this lacuna in Ghana, tortay onc of the Affica's mosit } \\
\text { promising health systcus. }\end{array}$ \\
\hline Amu ct al, (2018) & $\begin{array}{l}\text { This study makes sure that some African countries have implemented } \\
\text { health insurance schemes to ensure universal access to health care. } \\
\text { Iere, we have examined variations in health insurance coverage in } \\
\text { Ghama, Kenya, Nigeria, and Tamzania. }\end{array}$ \\
\hline A tur et al, (2015) & $\begin{array}{l}\text { The article examines reforms which aim to strengthen Latin } A \text { merican } \\
\text { health systems to reduce inequalities in health acoess, and outoomes } \\
\text { which focus on expanding universal health cowerage, particalarly for } \\
\text { pror citizens. }\end{array}$ \\
\hline
\end{tabular}

Study details

However, the aftermath of colonisation in Nigeria has resulted in two healthcare systemistraditional and orthodex - which appear to operate at cross purposes in meeting people's health needs. While orthodox practice is officially recongnized, traditional practioe is derided by the authonies. Y CL a sugnificant proportion of the population (approximatcly $70 \%$ ) stl patronse the traditional health practitiners. The two forms of medical practice have come to remain, thercfore it is logical to explore the posssibility of both being avalable to the peoplc for improved healthcare delivery.

This is a cross-sectional, descriptive survey conducted amongst formal sector workers in torin K wara State, Nigeria. Participants were selected using a stratified sampling technique. Logistic regression analysis indicates that respondents with post-seoondary ectucation and in federa civil service were over nine and three times more likely, respectively, to be aware of the scheme than others. Coverage of the scheme amongst respondents was unimpressive. There remains a kot to be done to fast track the expansion of the scheme among this sector of the population.

Secondary data on health indices such as life expectancy at birth, infant and under 5 mortality rates, maternal mortality ratio and infectious diseases prevalence, were acoessed from the

World Bank website. The scheme funding was limited to federal government contributions, while the beneficiary's contribution was nil, with a resultant shortfall of about one-third of the expected total fund The scticne's population cosverage in the seruthwest yone was peror, the distribution of the crolecs across acredited healh facilites was grossly skewed, and the schiculc was inadequalcly funded These findings have ncgalive miplications on service
delivery efficiency, as well as equitable acoess to quality healthcare services. Stakeholders need to address such gaps to achieve universal health coverage

This is a cross-sectional study, using structured interviews to gather data from 779 rural and This is a coss sectional stidy, using structured interviews to gather dana from 779 rural and

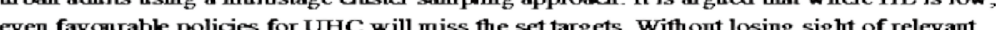
sociocuttral elementes, enhancing IL should be the key stratepy for policies aimed at bridging health inequalities and imlproving UIIC.

Bivariate and multivariale techniques were used to analyse data from Ghanaian demographic and health surveys (2014), Kenya (2014), Nigeria (2013), and Tanzania (2015). The results indicate that coverage was highest in Ghana (Femiakes $=62.19 \%$, Males $=19.1 \%$ ) and lowest valo and sacans

In I atin America, health-system reforms have resulted in a unique approach to universal heath coverage, underpinned by principles of equity, solictarity, and collective action to ovcromme social inequalities. In the majority of countries studied, govennment financing conabled supplyside interventions to be introduced to expand inswance coverage for uninsured citicens w wh defined and enlarged benefits packages and to scale up health service delivery. 


\begin{tabular}{|c|c|c|}
\hline Author & Aim & Study details \\
\hline Bloom et al., (2004) & $\begin{array}{l}\text { An estimation of the production function model of aggregate economic } \\
\text { growth, including two variables identified by inicroeconominsts as } \\
\text { fumdannental components of human capital work experience and health. }\end{array}$ & $\begin{array}{l}\text { The main finding is that good health has a positive, sizable, and statistically significant effect } \\
\text { on aggregate output, even when the experience of the workforce is controlled for. Life } \\
\text { expectancy effect in growth regressions appears to be a real productivity effect of hbour, and } \\
\text { is not the result of life expectancy acting as a proxy for worker experience. }\end{array}$ \\
\hline Bloom, (2011) & $\begin{array}{l}\text { An evaluation of reductions in mortality, followed (but with a ha) by } \\
\text { equally marked reductions in fertility, causing the world population to } \\
\text { double between } 1960 \text { and } 2000 \text {. }\end{array}$ & $\begin{array}{l}\text { Despite alannist predictions, population increases throughout history have not been } \\
\text { econonically catastrophic. Findings indicate that population changes to age structure have } \\
\text { resulted in increased prosperity. Demographic changes continue to have profoumd } \\
\text { repercussions for hunan wellbeing and progress, some of which can be mediated through } \\
\text { policy intervention. }\end{array}$ \\
\hline Bloom et al., (2018) & $\begin{array}{l}\text { Reviewing global health priorities and which is required in terns of } \\
\text { skills, funds, and technology to achieve healthcare access for all. }\end{array}$ & 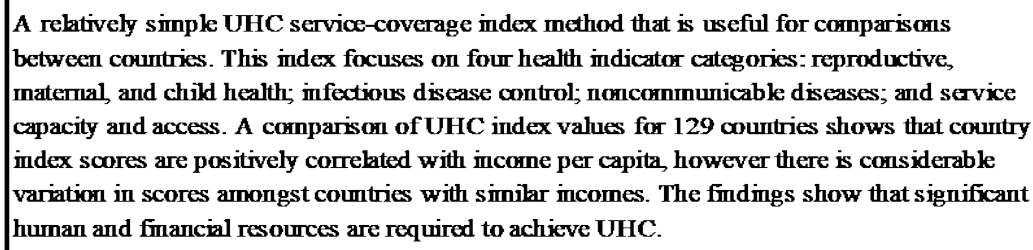 \\
\hline Boudreau, (2017) & $\begin{array}{l}\text { The study considers the issue of healthcare in this country, including the } \\
\text { current expenditure, hack of access, comparable international efforts, and } \\
\text { state proposed changes which have shown some success in } \\
\text { impleanenting wiversal healthcare. }\end{array}$ & $\begin{array}{l}\text { This study focuses on how innovation and govenance have tried to deternime workable } \\
\text { paradigns for refornimg healthcare. The focus on a simgle-pay er or universal health care } \\
\text { systemn has been unsuccessful in facilitating legislative change; therefore, elements of both } \\
\text { arguments will be considered when addressing the best methods to improve access to care and } \\
\text { affordability. A proposed phan will be introduced for considering the best options to improve } \\
\text { the following: } 1 \text {. Affordability; } 2 \text {. Access to care; } 3 \text {. Long-tern viability; } 4 \text {. State and federal } \\
\text { cooperation; } 5 \text {. Distribution of funding or services. The proposed plan addresses these issues } \\
\text { and presents phausible approaches. }\end{array}$ \\
\hline Cavagnero, (2008) & $\begin{array}{l}\text { The study exarnimes the inpact on the three inter-rehated fumctions of } \\
\text { revenue collection, pooling, and purchasmg/provision of health } \\
\text { services. }\end{array}$ & 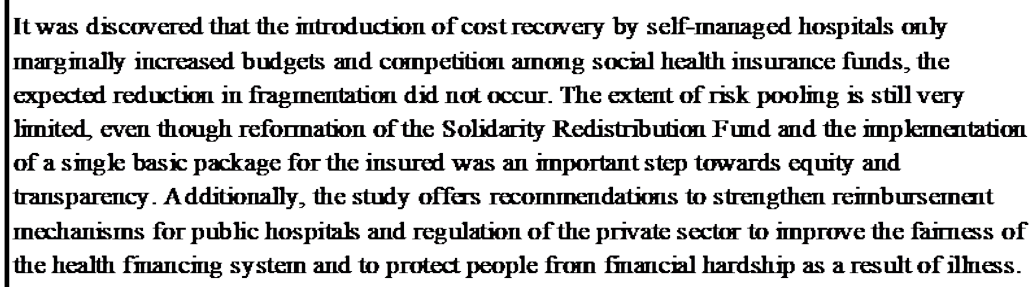 \\
\hline Cleopatra and Eurice, (2018) & $\begin{array}{l}\text { This study perforned an examination of the imcidence and intensity of } \\
\text { catastrophic health expenditure; and, its determinants amongst Nigerian } \\
\text { households. }\end{array}$ & $\begin{array}{l}\text { Descriptive statistics and logistic regression were applied across various thresholds. The } \\
\text { results indicate that the mincidence and intensity of catastrophic health expenditure is high, } \\
\text { particularly amongst households in the poorest quantile, those in nral areas, fenale-headed } \\
\text { households, households with uneducated household heads, households with memployed } \\
\text { heads, and those without health insurance. However, it is lowest among households in South } \\
\text { West Nigerin. Additionally, the risk of incurimg catastrophic health expenditure was lower } \\
\text { among households in the richest quantile, households headed by an employed person, } \\
\text { households located in wban areas, households with no hospitalized member and households } \\
\text { who utilized private hospitals. }\end{array}$ \\
\hline
\end{tabular}




\begin{tabular}{|c|c|c|}
\hline A uthor & Aim & Study details \\
\hline Cotlear et al, (2015) & $\begin{array}{l}\text { A naly sing the experiences of coumtries based on a systematic data } \\
\text { collection effort that captured in great detail how coumtries have } \\
\text { implemented uiversal health coverage reforns. }\end{array}$ & $\begin{array}{l}\text { It was found that programmes in } 24 \text { coumtries are "new, massive and transfomnational'. Most } \\
\text { were hunched in the past decade, cover more than } 2 \text { billion people, and all have fundamentally } \\
\text { changed the way in which health systems operate. Each of the programmes ains to overcome a } \\
\text { legacy of inequality by tackling gaps in both fimancing and provision. }\end{array}$ \\
\hline Cotlear et al., (2018) & $\begin{array}{l}\text { An exarnmaton or key health fmancmig polices used by Afncan } \\
\text { countries and measurements of the existence of regional patterns in the } \\
\text { use of these policies. The ain is to add value to the existing literature by } \\
\text { providing a systematic portrayal of the health financing policies which }\end{array}$ & $\begin{array}{l}\text { The findings of this paper discuss the cost estinations of inplementing a broader benefit } \\
\text { package for the advancernent of miversal health coverage. The paper concludes with a } \\
\text { discussion about the next steps to advance universal health based on its findings. }\end{array}$ \\
\hline Culyer ot al, (2018) & $\begin{array}{l}\text { Health technology assessments (HTA) are techniques to help decision- } \\
\text { makers make better decisions in terms of the impact on a nation's } \\
\text { health. }\end{array}$ & $\begin{array}{l}\text { The article outlimes the primcipal HTA features; it enphasizes the basic economic analysis } \\
\text { which forns its foundation, the integration of economic and other evidence, how it is best } \\
\text { conducted, and its role as an aid to, instead of a substitute for, careful thinking. }\end{array}$ \\
\hline Durokifa \& A bdul-Wasi, (2016) & $\begin{array}{l}\text { This article detaik how Sustaimable Development Goals (SDGs) } \\
\text { succeeds MDGs by reiterating questions including, how well did } \\
\text { MDGs perform in developing coumtries? Were the aims of the MDGs } \\
\text { met? If MDGs cannot achieve } 8 \text { goak, how possible will SDGs } 17 \\
\text { goaks be realized? }\end{array}$ & $\begin{array}{l}\text { The study used secondary data to evaluate the MDG era in Nigeria, and to what extent they } \\
\text { achieved their set target The study indicates that although the MDG era in Nigeria recorded } \\
\text { small progress towards targeted goals, it did not meet the required plausble targets. Therefore, } \\
\text { as a very effective way of achieving sustamable development, the study reconnmends good } \\
\text { govemance and goals prionitization according to the needs of the courtry. }\end{array}$ \\
\hline Gupta and Chowdhury, (2014) & $\begin{array}{l}\text { The article analy ses the govenument's share of financial resources for } \\
\text { health across different agents, particularly focusing on health coverage } \\
\text { resources. }\end{array}$ & $\begin{array}{l}\text { The analysis imdicates that the present health coverage systern is madequate and imiquitous, } \\
\text { with various systerns operating at different costs. It suggests that finances should be } \\
\text { consolidated and move moving towards a more unified system to realise the benefits of } \\
\text { efficiency gams. }\end{array}$ \\
\hline Hogan et al, (2018) & $\begin{array}{l}\text { The study developed an index to measure SDG 3.8.1, and describes } \\
\text { methods for conpiling the index, and reports baselime results for } 2015 \text {. }\end{array}$ & $\begin{array}{l}16 \text { tracer indicators were chosen for the index, including four from each of the categories of } \\
\text { reproductive, maternal, new-bom, and child health; infectious diseases; non-communicable } \\
\text { diseases; and service capacity and access. On average, coumtries had primary data since } 2010 \\
\text { for } 72 \% \text { of the fimal set of indicators. The median national value for the service coverage index } \\
\text { was } 65 \text { out of } 100 \text { (range } 22-86 \text { ). The index Correlate highly with other sumnnary measures of } \\
\text { health, and after gross national imcone and mean years of adult education were controlled for, } \\
\text { was associated with } 21 \text { additional years of life expectancy over the observed range of country } \\
\text { values. }\end{array}$ \\
\hline Hsiao et al, 2014 & $\begin{array}{l}\text { This paper uses a theory of political econorny to analyse Chima's policy } \\
\text { changes and accomplishments, and consists of four main sections }\end{array}$ & $\begin{array}{l}\text { The study summarizes, based on quantitative evidence, the impacts of Chima's UHC in terns } \\
\text { of equitable access to health care, quality and affordability, health outcomes, and financial risk } \\
\text { protection from high and/or catastrophic medical expenses. A though China's UHC was a } \\
\text { great actievernent, there are stark disparities between uban and rural residents in Chima, } \\
\text { together with high health expenditure inflation rates which stem from inefficiency and waste in } \\
\text { the health care system. }\end{array}$ \\
\hline
\end{tabular}




\begin{tabular}{|c|c|}
\hline Author & Aim \\
\hline Ikegami ef al, (2014) & $\begin{array}{l}\text { The study's objectives are to analyse trends of the extent to which fiscal } \\
\text { disparities between the CHI and employment-based programmes have } \\
\text { been mitigated by tax subsidies and transfers from employment-based } \\
\text { programimes to pay for the costs of elders, and the limitations in } \\
\text { mamiaming premimim rates equity (the share of wage income kvied as } \\
\text { preminms) under multiple SHI programmes. }\end{array}$ \\
\hline Limiwattanamon et al, (2011) & $\begin{array}{l}\text { The objective was am assessment of the equity impact of achieving } \\
\text { universal coverage (UC) on the distribution of benefits of public heath } \\
\text { service utilization, healthcare finamcing burdens (measured by finamcing } \\
\text { incidence), and risk protection from expensive medical care costs } \\
\text { (fimancial calastrophe). }\end{array}$ \\
\hline Lloyd-Sherkock, (2006) & $\begin{array}{l}\text { The paper shows that Argentinim health refoms worsened pre-existing } \\
\text { weaknesses in the health sector. }\end{array}$ \\
\hline Lorentzen ef al., (2008) & $\begin{array}{l}\text { An analysis of various cross-national and sub-national data, we argue } \\
\text { that high adult mortahity reduces economic growth by shortening time } \\
\text { horizons. }\end{array}$ \\
\hline Maede et al., (2014) & $\begin{array}{l}\text { A report was prepared summarising UHC for each country, based on a } \\
\text { common analytical framework, focusing on three themes: (a) the } \\
\text { political economy amd policy process for adopting, achieving, and } \\
\text { sustaming UHC; (b) health fmancing policies for enhancing heath } \\
\text { coverage, and (c) humam resouroes for health policies to achieve UHC. }\end{array}$ \\
\hline Maruthappu et al., (2016) & $\begin{array}{l}\text { Estimating the effects of changes in unemployment and PEH on cancer } \\
\text { mortality and identified how universal health coverage (UHC) affected } \\
\text { these relationships. }\end{array}$ \\
\hline Mc Kee et al, (2013) & $\begin{array}{l}\text { The article describes how many developed and developing countries } \\
\text { have pursued universal health care. }\end{array}$ \\
\hline
\end{tabular}

\section{Study detaik}

We reveal that the fiscal disparities between and within the two broad types of SHI programmes are now likely to increase, the long-tem objective being to mege the programmes which should have been considered some time ago, including standardizing the methods of levying premimis.

The findings reveal that fmancing of the Thai healthcane system was equitable before UC The findings reveal that fmancing of the Thai healthcare systemi was equitable before UC
policy was implemented, but became more so after UC was introxtuced. Two key influences on the progressivity of an overall heathcare $f$ incis progressive diect tax payments a d a share reduction of megressive houschold oitof-pocket

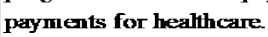

It criticises the reform agenda content and how it was produced, arguing that these were reforms whereby public health considerations were less significant than conforming to the wider model of neo-liberal social and economic development prevailing at the time. It also highlights issues with the implementation of the reform agenda, which reduced the coherency of the reforms. The paper then examines the impact of the crisis, noting links with the precoding reforms. A a number of insights and lessons of potential value are identified for other countries pursuing similar policies.

Considering in detail the age patterns of mortality and endogeneity issues, the findings

advocate that a greater risk of death during the prime productive years is linked to higher levels advocate that a greater risk of death durng the prime productive years is linked to higher levels
of risky behaviour, highe fertility, and bwer investment in physical capital, even when infant mortality is controd for. In our megressions, adult mortality explaims atmost all of Africa's growth tragedy.

All countries can aspire to UHC for the inprovenent of the health and welfare of all their citiens and to help realize inclusive and sustamable development. Low-income countries still have the opportunity to aim for UHC, as they can start building institutional capacily, lean from other countries' experiences, and adapt innovative approaches to speed up coverage
expansion. UHC priorities, strategies, and implementation plans will differ between countric expansion. UHC priorities, strategies, and implementation plans will differ between countries,
dependent upon keal context. However, the key policy messages listed earlier can help every country to develop and refme their own UHC approach, especially to make their policies accountable and tramsparent.

A 1990-2010 kngitudinal analysis of aggregated mortality data for breast cancer in women, prostate cancer in men, and colorectal cancers in men and women, with survival rates excoding $50 \%$, into a treatable cancer class. In addition, asgregated da for hing and cancer class. Interpreting these results shows that unemployment increases are associated with increases in camcer mortality; UHC sems to protect agamst this effect PFH increases linked to reduced cancer mortality. Acoess to heathcare conld be the basis of this effect. We estimate that the 2008-10 economic cisis was associnted with approximately 260,000 excess

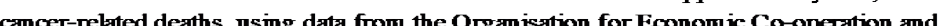
Development akone.

An analysis of progress towards universal health care, combining a review of the existing theories and new empinical analysis, identifies five crucial factors: the strength of organize labour and left-wing parties, adequate economic resouroes, absence of societal divisions, weakness of oppositional institutions (such as organized medicine), and windows of opportunity. After noting the substantial benefits of universal health care, the article conchudes with an analysis of how it has come under threat in some European countries and a waming about the risks posed by current radical austerity policies 


\begin{tabular}{|c|c|c|}
\hline Author & Aim & Study details \\
\hline McKenna et al , (2017) & $\begin{array}{l}\text { An explanation of the main modeds for fimancing healthcare taxation, } \\
\text { private health insurance and social health insurance. }\end{array}$ & $\begin{array}{l}\text { The study outlimes the workings of each modd in its purest fonn, while recognising that most } \\
\text { countries typically pay for health care using a number of methods. The findings indicate no } \\
\text { countries, induding the UK, reties on a single funding source for health care. Typically, } \\
\text { countries use one of the three main funding modes to pay for health care. All countries utilise } \\
\text { user charges to pay for a proportion of overall costs. The precise combination of funding } \\
\text { sources develops over time based on a coumtry's context, history and social values. Whatever } \\
\text { moded is used, there are inevitable debates about its effectiveness and efficiency. However, } \\
\text { there is no evidence that one funding mode, or mixture of funding mechanisms, is imherently } \\
\text { superior to others. }\end{array}$ \\
\hline Moreno-Sera \& Smith, (2015) & $\begin{array}{l}\text { An evaluation at the national leved of exonometric evidence on health } \\
\text { coverage effects on mortality outcomes. }\end{array}$ & $\begin{array}{l}\text { A large paned data set of countries was examined using instrunental variable spexifications } \\
\text { specifically allowing for potential reverse causality and unobserved country-specific } \\
\text { characteristics. The study used a number of proxies for the health systen coverage kvel. } \\
\text { Results show that expanded health coverage, especially via higher levels of publicty funded } \\
\text { health spending results in lower child and adult mortality, with a greater effect on child } \\
\text { mortality in poorer countries. }\end{array}$ \\
\hline Muelles and Morgan, (2018) & $\begin{array}{l}\text { The articde presents the first results of health spending and financial data } \\
\text { collected under the new framework and highlights where SHA } 2011 \text { is } \\
\text { a more useful tool for policy analysis, by compleanenting data on health } \\
\text { expenditure fimancing schenes with infornation about their reverue } \\
\text { streans. }\end{array}$ & $\begin{array}{l}\text { The study describes the major conceptual changes in health financing and highlights why } \\
\text { comprehensive analyses based on SHA } 2011 \text { offers a more complete description and } \\
\text { comparison of health financing across countries, facilitates a more meaningful discussion of } \\
\text { fiscal sustainability of health speriding by analysing compulsory public scheme reverues and } \\
\text { hedps to charify governments' roles in financing health care. }\end{array}$ \\
\hline Muharnmad et al., (2017) & $\begin{array}{l}\text { This study uses a qualitative approach to adequately describe the ains } \\
\text { and objectives. }\end{array}$ & 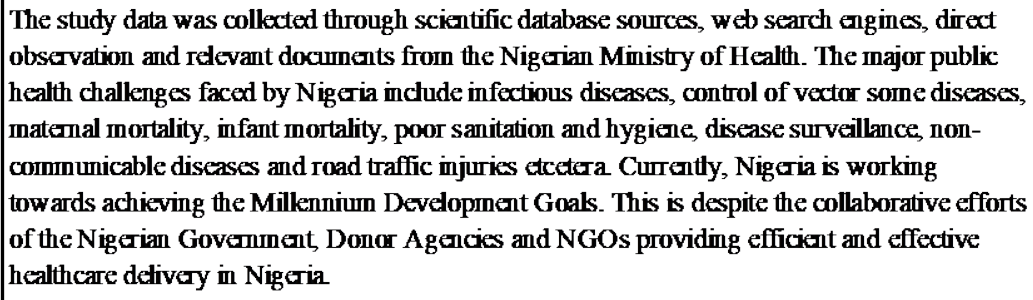 \\
\hline Ndie, (2013) & $\begin{array}{l}\text { This study presents an evaluation of the aw aresess of the National } \\
\text { Health Insurance Scheme (NHIS) by civil servants in Enugu and } \\
\text { Abakaliki }\end{array}$ & $\begin{array}{l}\text { The questionaire was used to evaluate the awareness of the National Health Insurance } \\
\text { Scheme (NHIS) by civil servants living in Enugu and Abakaliki. The results indicate that the } \\
\text { leved of aw areness was very low, with most respondents being unaware of the mode of } \\
\text { payment and benefits of the NHIS, and were of the opmion that NHIS may be unsuccessful } \\
\text { Nigeria. The recommendation is that the operators of the NHIS shouk undertake to educate } \\
\text { the citienry on the mode of operation and benefits of the NHIS. }\end{array}$ \\
\hline Nwani, (2015) & $\begin{array}{l}\text { This study assessed the penetrative kevel of National Health Insurance } \\
\text { Scheme (NHIS) in Nigeria. }\end{array}$ & $\begin{array}{l}\text { Using a sample of } 500 \text { respondents comprising medical service users and providers, the } \\
\text { findings reveal that aw areness, compliance and coverage of the schene is still low } 10 \text { years } \\
\text { after its inplennentation in Nigeria. This research opens a new wave of calls for regulatory } \\
\text { innovations and legislative actions for addressing the shortcomings in penetration and } \\
\text { coverage which camently impede the workings of NHIS in Nigeria. }\end{array}$ \\
\hline
\end{tabular}




\begin{tabular}{|c|c|c|}
\hline Author & Ain & Study detaik \\
\hline Odeyemi, (2014) & $\begin{array}{l}\text { The study's objective is to review the current status of CBHI in SSA so } \\
\text { as to highlight the issues affecting its successful integration into the } \\
\text { NHIS of Nigeria and more widely in developing countries. }\end{array}$ & 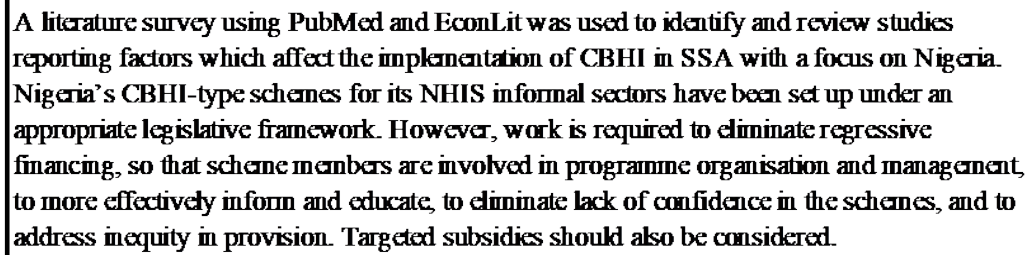 \\
\hline Okpani and Abimbola (2015) & $\begin{array}{l}\text { This article demonstrates a viable path to UHC by expanding social } \\
\text { health imsurance in Nigeria. }\end{array}$ & $\begin{array}{l}\text { It is argued that cncouraging the states which are semi-autonomous federating units to setup } \\
\text { and manage their own insurance schenes presents a unique opportumity to rapidly scale up } \\
\text { prepaid coverage for Nigerians. The article shows that the federal structure of Nigeria } \\
\text { prescribes a sharimg of responsibilities for healthcare in the three tiers of government, which } \\
\text { presents serious challenges in significantly extending social insurance to uncovered groups. } \\
\text { The article recommends that instead of allowing this govenance structure to hinder } \\
\text { progression towards UHC, it should be used to accekrate the process by supporting states in } \\
\text { establishing and managing their own insurance funds while encouraging integration with the } \\
\text { National Health Insurance Schene. }\end{array}$ \\
\hline Onoka et al,. (2014) & $\begin{array}{l}\text { This article examines why and how a national health insurance (NHI) } \\
\text { proposal which targets universal health coverage (UHC) in Nigeria } \\
\text { devedoped over time. }\end{array}$ & $\begin{array}{l}\text { The study included document reviews, in-depth interviews, a revicw of predininary analysis } \\
\text { by revevant actors and a stakeholder analysis approach. Nigeria's experiale shows that where } \\
\text { political leaders have interest in a UHC-retated proposal, their strong political leadership } \\
\text { greatly enhances the pace of the policy process. However, public officials should carefully } \\
\text { guide policymaking processes involving private sector actors, so that strategies compromising } \\
\text { the chances of achieving UHC are not introduced. }\end{array}$ \\
\hline Onyedibe et al., (2016) & $\begin{array}{l}\text { The ain of the study was to deternine the proportion of Nigerian adults } \\
\text { who were enrolled in the schene, how satisfied they were with the } \\
\text { quality and availability of services withim the schene, and the factors } \\
\text { responsible for the dismal health indices in the country despite the } \\
\text { schene. }\end{array}$ & $\begin{array}{l}\text { Questionnaires were randomly assigned to } 200 \text { adult respondents in Jos metropolis. The } \\
\text { findings reveal that only } 24 \% \text { of adults were arrolled in the scheme. } 82 \% \text { of anrolled } \\
\text { respondents ware aw are of NHIS and prefer it to a foe paying system. } 26 \% \text { of earolexs were } \\
\text { dissatisfied with the scheme. }\end{array}$ \\
\hline Osuchukwu et al, (2013) & $\begin{array}{l}\text { This objective of the study was to evaluate the inpact of National } \\
\text { Health Insurance Scheme (NHIS) on healthcare consuners in Calabar } \\
\text { inctropolis, southern Nigeria. }\end{array}$ & $\begin{array}{l}\text { A pre-tested, 43-iten questionnaire was designed and administered to } 200 \text { respondents using } \\
\text { the household survey and patient exit surveys. The results demonstrate that respondents were } \\
\text { predominantly males (58.0\%), Christians (94.5\%), married (56.0\%), civil servants (39.5\%), } \\
\text { had tertiary levd of education (60.5\%) and were aged } 30-34 \text { years (27.5\%). 89.0\% of } \\
\text { respondents were aware of the schene, howeve schene eirolneat was only } 37 \% \text {. }\end{array}$ \\
\hline Penchaszadeh et al, (2012) & $\begin{array}{l}\text { This study perforns an exploration of the modem healthcare system of } \\
\text { Argentina which was developed from } 1945-1955 \text {, a period of economic } \\
\text { bonanza characterized by industrialization, rapid ubanization and } \\
\text { activist labour organizations. }\end{array}$ & $\begin{array}{l}\text { In the 1980s and 1990s, the health system experienced further transfornations, as neoliberal } \\
\text { policies dictated a reduction of state involvenent in social services in favour of privatization } \\
\text { and decentralization of healthcare. This micreased fragmentation, mexuity and inefficacy, as } \\
\text { healthcare is more prone to the economic interests of private corporations (insurance and } \\
\text { phannaceutical industries), trade union bureaucracies and the medical professional and } \\
\text { technology establishments. }\end{array}$ \\
\hline
\end{tabular}




\begin{tabular}{|c|c|c|}
\hline Author & Ain & Study details \\
\hline Ramke et al, (2017) & $\begin{array}{l}\text { The aim is to define and demonstrate effective cataract surgical coverage } \\
\text { (eCSC), one of the UHC indicators combining a coverage measure } \\
\text { (cataract surgical coverage, CSC) with quality (post-operative visual } \\
\text { outcome). }\end{array}$ & 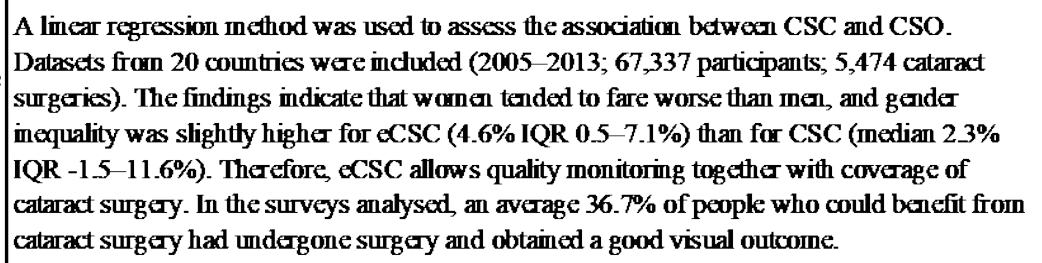 \\
\hline Recves et al., (2015b) & $\begin{array}{l}\text { An investigation of how altemative tax systens affect the breadth, } \\
\text { depth, and height of health system coverage. }\end{array}$ & $\begin{array}{l}\text { A cross-national longitudinal fixed effects moded for assessing the relationships betwern total } \\
\text { and different types of tax revenue, health systen coverage, and associated child and matemal } \\
\text { health outcomes in } 89 \text { low-income and middle-income coutries from } 1995-2011 \text {. The results } \\
\text { mdicate that consunption taxes, a more regressive forn of taxation that might reduce the } \\
\text { affordability of essential goods for the poor, were associated with increased rates of post- } \\
\text { neonatal mortality, infant mortality, and unde-5 mortality rates. No adverse associations were } \\
\text { detected with taxes on capital gains, profits, and income, which tend to be more progressive. }\end{array}$ \\
\hline Reich et al, (2016) & A systematic assessment of global UHC experiences. & $\begin{array}{l}\text { The study identified common challenges, opportumities and useful insights for how to progress } \\
\text { UHC. The study showed the complexities of UHC, that it is fraught with chalkenges, many } \\
\text { possible pathways, and various pitfalls yet it is feasible and achievable. Progression towards } \\
\text { UHC is a long-tern policy engagennent rexuining both technical knowhedge and political know- } \\
\text { how. Texhnical solutions must be utilised together with pragnatic and innovative strategies } \\
\text { that address the context of the national political economy. }\end{array}$ \\
\hline Rocmer, (1993) & $\begin{array}{l}\text { The article examines the specifics of such systeans in both industrialized } \\
\text { and developing countries. }\end{array}$ & $\begin{array}{l}\text { Every country has a national health system, reflecting is history, economic development, and } \\
\text { its dominant political ideology. As a result, several types of health systems exist. The findings } \\
\text { suggest that a country's type of health system depends on the characteristics of each of the } \\
\text { component parts of the system. }\end{array}$ \\
\hline Rubinstein et al, (2018) & $\begin{array}{l}\text { The article includes an overview of the healthcare system in Argentina, } \\
\text { then introduces the driving forces for refom, and fimally analyses four } \\
\text { key issues where our action plan can implement health reforns in } \\
\text { Argentina. }\end{array}$ & $\begin{array}{l}\text { Overall, our ultimate aim is to provide actual, rather than aspirational UHC in Argentima by } \\
\text { strengthenimg provincial health systenns through enforcing public insurance schenes; using an } \\
\text { explicit priority-setting approach to make health coverage decisions; reducing health disparities } \\
\text { in coverage and outcomes, and prioritize health problems; and build a primary care oriented } \\
\text { healthcare system. }\end{array}$ \\
\hline Tahsina et al $\mathbf{l}_{\supset}$ (2018) & $\begin{array}{l}\text { An investigation of the deterninants of financial hardship in coping } \\
\text { with OOP, adopted for the secking of healthcare for under-five } \\
\text { chikhood illnesses in rural Bangladesh. }\end{array}$ & 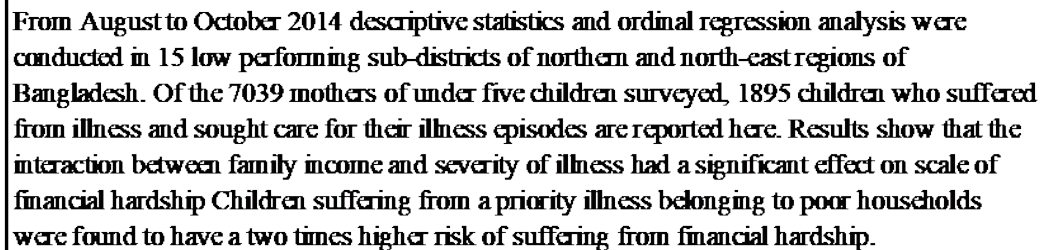 \\
\hline
\end{tabular}




\begin{tabular}{|c|c|c|}
\hline Author & Aim & Study details \\
\hline Wagstaff, (2007) & $\begin{array}{l}\text { The study details how SHI fares in health care dedivery, revenue } \\
\text { collection, covering the fornal sector, and its impacts on the labour } \\
\text { market. }\end{array}$ & $\begin{array}{l}\text { It is argued that SHI does not necessarily detive good quality care at a low cost, in part } \\
\text { because of poor regulation of SHI purchasers. It indicates that there can be substantial costs of } \\
\text { collecting reverues, even in the fornal sector where nonearolment and evasion are frequent, } \\
\text { and that while SHI easily covers the fornal sector and the poor, it fares badly in covering the } \\
\text { non-poor infornal sector workers until the economy is more highly developed. In addition, the } \\
\text { ffindings also argue that SHI can have negative effects on the labour market }\end{array}$ \\
\hline Wagstaff et al., (2018) & $\begin{array}{l}\text { The aim is to extinate the global incidence of catastrophic health } \\
\text { spending trends from } 2000-2010 \text {, and associations between catastrophic } \\
\text { health spending and macroeconomic and health system variables at the } \\
\text { national kevel. }\end{array}$ & $\begin{array}{l}\text { A retrospective, observational study of health spending using household survey data Of } 1566 \\
\text { potentially suitable household surveys, } 553 \text { passed quality checks, covering } 133 \text { countries } \\
\text { from 1984-2015. Findings reveal that the incidence of catastrophic payments positively } \\
\text { correlates with GDP per person and the share of GDP spent on health. Incidence correlated } \\
\text { negatively with the share of total health spending chamelled through social security funds and } \\
\text { other govenment agencies. }\end{array}$ \\
\hline Wagstaff et al, (2018) & $\begin{array}{l}\text { This study measures instances of inpoverishment when the } \\
\text { consunption of a housetiok, including out-of-pocket spending on } \\
\text { health which is greater than the poverty line; but its consumption, } \\
\text { excluding out-of-pocket spending, is less than the povety lime. }\end{array}$ & $\begin{array}{l}\text { The study measures the incidence and depth of impoverishment as the differences betwean } \\
\text { poverty head count and poverty gap, with and without out-of-pocket spending included in } \\
\text { housechold total consunption. The findings reveal inpoverishment as a result of out-of-pocket } \\
\text { spending, even in countries where the entire population is covered by a national health service. } \\
\text { Incidence negativety correlates with the share of total health spending channelled through } \\
\text { social security funds and other government agencies. }\end{array}$ \\
\hline Wirtz et al., (2017) & $\begin{array}{l}\text { The research questions which are explored are: What progress has been } \\
\text { achieved? What challenges still rexuire addressing? Which lessons have } \\
\text { becal karned to inforn future approaches? How can essential medicines } \\
\text { policies be used to promote UHC and contribute to the global } \\
\text { sustainable development agenda? }\end{array}$ & 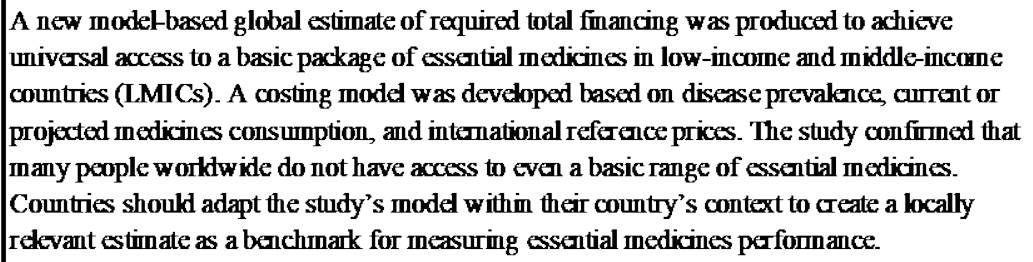 \\
\hline Zhang et al., (2019) & $\begin{array}{l}\text { An investigation of the progress of UHC indicators in Ghana from } \\
1995-2015 \text { and future predictions, up to } 2030 \text {, which assesses the } \\
\text { probability of UHC targets being reached. }\end{array}$ & $\begin{array}{l}\text { The study assessed financial hardship by calculating catastrophic health payments and } \\
\text { impoverishment and used a Bayesian regression moded to estinate trends, future projections, } \\
\text { and the probabilities of achieving UHC targets by } 2030 \text {. There is also an assessment of wealth- } \\
\text { based inequalities and regional disparities. Inequality measures and subnational assessment } \\
\text { revealed that catastrophic expenditure experienced by wealth quimtiles and regions are unequal. } \\
\text { Significant inprovenent are observed in health sevice coverage and financial risk protection } \\
\text { over the years. However, mequalities across wealth quintiles and regions renain a cause of } \\
\text { concem. }\end{array}$ \\
\hline
\end{tabular}


Appendix 2: Ethical approval for the National Health Research Ethics Committee in Nigeria

The Chairman,

National Health Research Ethics Committee of Nigeria

Federal Ministry of Health

Federal Secretariat Complex

Shehu Shaqari Way

Garki

Abuja

P.M.B. 083, Garki- Abuja

Dear Sir/Madam,

Title: Obstacles and challenges affecting the move towards universal healthcare coverage in Nigeria

My name is Jenson Gawain Fofah, a PhD research student at Swansea University, United Kingdom. I wish to submit the above proposed research project for the ethical approval by the Research Ethics Committee. The aim of the research is to examine Nigeria's path towards Universal Health Coverage through studying the interactions of the policy actors involved the policy process.

The research has the potential to generate evidence of the impact of the Universal Health Coverage since its introduction in Nigeria. This is for academic purposes only.

I enclose the following documents:

- The proposal

- Study tools

- Information sheet and consent form.

I look forward to your approval.

Kind regards

Jenson Gawain Fofah 


\section{Appendix 3: Interview guides used in the study}

PHASE ONE: Structured interview for the assessment of the obstacles and challenges affecting the move towards universal health coverage in Nigeria.

Dear respondent,

I am a PhD research student at Swansea University, United Kingdom and am in Nigeria to carry out a research study on the obstacles and challenges affecting the move towards universal health coverage in Nigeria. The overall aim of this research study is to examine Nigeria's path towards UHC through the interactions of the policy actors involved in the policy process. The outcome of this research could assist policymakers involved make the right decision on Universal Health Coverage in Nigeria.

You have been selected to complete this questionnaire because you have been identified as someone with knowledge about the policy.

Your participation is voluntary, and you can decline to answer any question you do not feel comfortable with. A gentle reminder before we begin, I assure you that the entire information gathered in this questionnaire will be treated with absolute confidentiality. Your anonymity will be safeguarded, both by with-holding your name and being careful not to publish other information that might identify you. I will be careful not to pass on such information in interviews with other respondents, or in my project reports. After the study is completed, the content of any recorded interviews will be destroyed. If you have any questions, you may please ask at any time.

\section{SNA interview: Round One}

Section A: Background information and consent Information to be completed by the investigator

Consent: Please before beginning the interview explain the consent to the respondent

\begin{tabular}{|c|c|}
\hline $\begin{array}{l}\text { Questions } \\
\end{array}$ & Code \\
\hline $\begin{array}{l}\text { A1. Has the respondent given consent to complete the } \\
\text { interview? } \\
1=\text { YES } \\
2=\text { NO } \\
\text { If YES, proceed to A2, If NO end the interview }\end{array}$ & \\
\hline $\begin{array}{l}\text { A2. Is the respondent involved in the Universal Health } \\
\text { Coverage (UHC) policy process in Nigeria? } \\
1=\text { YES } \\
2=\text { NO } \\
\text { If YES, proceed to A3, If NO end the interview }\end{array}$ & \\
\hline A3. Respondent contact details & $\begin{array}{l}\text { Email } \\
\text { Address: } \\
\text { Mobile } \\
\text { number:- } \\
\text { Work } \\
\text { number: }\end{array}$ \\
\hline $\begin{array}{l}\text { A4. Respondent ID } \\
\text { e.g. 001, for first respondent, } 002 \text { for second respondent } \\
\text { etc. }\end{array}$ & \\
\hline
\end{tabular}

Section B: Socio-demographic details of respondent (Insert code number only). 
In this section, we would like to ask you some questions about your personal profile. Your name:

ID:

\begin{tabular}{|l|l|}
\hline \multicolumn{1}{|c|}{ Questions } & Code \\
\hline B1. Sex of respondent? & \\
1= Male & \\
2= Female & \\
\hline B2. What is your professional background? & \\
1a= Government Official (federal level) & \\
1b= Government Official (state level) & \\
2= HMO Manager & \\
3= NHIS worker & \\
4= Doctor & \\
5= Nurse/Allied Professional & \\
6= Other & \\
If 6, please specify & \\
\hline B3. What is your current position? & \\
\hline B4. Length of time in current position? & \\
\hline B5. Which category best describe yourself? & \\
1= External involvement in UHC policy & \\
2= Internal involvement in UHC policy & \\
\hline
\end{tabular}

Section C: Personal views of respondent (Insert code number only).

The next few questions are about your personal views on Universal Health Coverage related issues.

\begin{tabular}{|l|}
\hline \multicolumn{1}{|c|}{ Questions General Understanding } \\
\hline \multicolumn{1}{|c|}{ Code } \\
\hline C1. Are you familiar with the Ministry of Health's policy on \\
Universal Health Coverage (UHC)? \\
1= Yes \\
2= No \\
If YES, proceed to C2, if NO answer C6 only \\
\hline C2. Do you understand the term- UHC? (general \\
description, details will follow later in the interview) \\
1= Yes \\
2= No \\
\hline C3. Do you think the topic of UHC is prominent in health \\
policy circles in the country? \\
1= Yes \\
2= No \\
\hline C4. Is Nigerian Government policy on UHC moving in the \\
right direction? \\
1= Yes \\
2= No \\
\hline
\end{tabular}


C7. How long have you been involved in discussion about UHC policy in Nigeria?

$1=$ Less than one year

$2=2-5$ years

$3=6-10$ years

$4=11-15$ years

C8. Which stages of the UHC policy process have you been involved in? (you may select more than one)

$1=$ Agenda setting

$2=$ Policy formulation

$3=$ Implementation

$4=$ All of the above

C9. Which institutions or policy actors have the strongest influence on the UHC policy debates? (You may select more than one)

$1=$ Government

$2=$ Health providers

$3=$ Labour sector

$4 \mathrm{a}=$ NGOs

$4 \mathrm{~b}=\mathrm{UN}$ Bodies

\section{Controversies}

C10. Overall would you say you are broadly in favor or against the development of UHC policy in Nigeria?

$1=$ Favor

2= Against

C11. How much coverage? Which statement most closely matches your view:

$1=$ Nigerian public health insurance should concentrate on those in state and formal sector employment.

$2=$ Nigerian public health insurance should include the above, plus targeted healthcare for the poor.

$3=$ Nigerian public health insurance should be extended to cover the whole population.

$4=$ Other

If 4 , then please specify.

C12. If Nigeria is to expand health coverage, which statement would you favor the most:

1= A 'Phased approach' to implement UHC over a number of years.

2= A ‘Big Bang approach’ going straight to implementing full UHC.

$3=$ Other

If 3 , then please specify.

\section{SNA interview: Round Two}

Section A: Mapping the flow of interaction among policy actors. 
The following set of questions is concerned with different relationships of interactions among policy actors involved within UHC discourse in Nigeria. The results will help me to map out the flow of information among them and better understand how policy actors influence each other.

Please take a look at the following lists of policy actors (below) and tick those who are involved in each policy process

\section{Questions:}

F1. Did you read any of the published work of the following persons in making your own contribution to UHC policy?

F2. Did you take part in a workshop or other discussion forum with any of the following persons in making your own contribution to UHC policy?

F3. Did you form a coalition with any of the following persons in making your contribution to UHC policy?

F4. Do any of the following persons, outside what we might call your coalition of close associates share the same views with regards to UHC policy?

F5. Do any of the following persons: outside what we might call your coalition of close associates have a conflict of interests with regards to UHC policy?

F6. Did you seek advice from any of the following persons in making your own contribution to UHC policy?

PHASE TWO: Semi-structured qualitative interview for the assessment of the obstacles and challenges affecting the move towards universal health coverage in Nigeria.

Interview Guideline Questions

This section features four different categories of interview guideline questions tailored to the four categories of policy actors involved in the policy process of UHC in Nigeria.

Category one- Government

Introduction

Section A. Interviewee Socio-demographic Background

1. What is your occupation?

2. What is your present position?

3. When did you first get involved in the UHC policy? 
4. Have you been continually involved?

Section B. Major Questions

5. What is the meaning of Universal Health Coverage?

6. What persuaded the government to go down the UHC route and who or what were the principal influences?

7. How would you describe the importance of implementing UHC in Nigeria?

8. Is implementing UHC the most significant health policy the government has introduced in the last 15 years in Nigeria?

9. How would you characterize the view of the Nigerian people towards UHC?

10. What organizations have provided support for the government in implementing UHC?

11. What would be your own main criticisms of the UHC policy?

12. In your opinion what are the main obstacles and challenges affecting Nigeria's progress towards achieving universal health care?

Agenda setting regarding UHC policy:

13. How did UHC get onto the policy agenda in Nigeria?

14. What role did the government play at this stage?

15. How much resource was allocated by your governmental organization to pursue the UHC policy?

16. Who were the key actors in the agenda setting? How were they involved?

17. Which stakeholders had the most influence at this stage?

18. How much were external forces involved at this stage?

19. How were policies selected and forwarded to the government for approval?

20. What policies were selected for government approval?

21. What major obstacles and challenges did the government face at this stage of the policy process?

Formulation of UHC policy:

22. What role did the government play at this stage of the policy process? 
23. How were particular policies selected, developed, and put into effect, and how were financial arrangements planned?

24. Who were the key actors in policy formulation? How were they involved?

25. What was the mechanism through which the policy was formulated?

26. What major obstacles and challenges did the government face at this stage of the policy process?

Implementation of the UHC policy:

27. What role did the government play at this stage of the policy?

28. How have those changes in policy affected your governmental organisation?

29. What aspects of the UHC policy have been most difficult to implement?

30. How are the implemented policies going to be evaluated, and what methods and criteria will be used for their evaluation?

31. What major obstacles and challenges did the government face at this stage of the policy process?

32. How is the budget planned for implementing the UHC policy?

33. How far would you say we are towards implementing the UHC policy in Nigeria?

Category two- Health Providers

Introduction

Section A. Interviewee Socio-demographic Background

1. What is your occupation?

2. What is your present position?

3. When did you first get involved in the UHC policy?

4. Have you been continually involved?

Section B. Major Questions

1. What is the meaning of Universal Health Coverage?

2. What persuaded the government to go down the UHC route and who or what were the principal influences? 
3. How would you describe the importance of implementing UHC in Nigeria?

4. Is implementing UHC the most significant health policy the government has introduced in the last 15 years in Nigeria?

5. How would you characterize the view of the Nigerian people towards UHC?

6. What organizations have provided support for the government in implementing UHC?

7. What would be your own main criticisms of the UHC policy?

8. In your opinion what are the main obstacles and challenges affecting Nigeria's progress towards achieving universal health care?

Agenda setting regarding UHC policy:

9. How did UHC get onto the policy agenda in Nigeria?

10. What role did your organization play at this stage?

11. How much resource was allocated by your organization to pursue the UHC policy?

12. Who were the key actors in the agenda setting? How were they involved?

13. Which stakeholders had the most influence at this stage?

14. How much were external forces involved at this stage?

15. What policies did your organization put forward during the agenda setting regarding UHC policy?

16. What policies did your organization disagree with at this stage of the policy process?

17. What major obstacles and challenges did the government face at this stage of the policy process?

Formulation of UHC policy:

18. What role did your organization play at this stage of the policy process?

19. How were particular policies selected, developed, and put into effect, and how were financial arrangements planned?

20. Who were the key actors in policy formulation? How were they involved?

21. What was the mechanism through which the policy was formulated?

22. Did you think that the policies formulated at this stage were sufficient to address the health needs of the people?

23. What major obstacles and challenges did the government face at this stage of the policy process? 
24. How far would you say we are towards implementing the UHC policy in Nigeria?

Implementation of the UHC policy:

25. What role did your organization play at this stage of the policy?

26. How have those changes in policy affected your organisation?

27. What aspects of the UHC policy have been most difficult to implement?

28. To what extent do you think the Government's policy address the needs of the Nigerian people?

29. What major obstacles and challenges did the government face at this stage of the policy?

30. How far would you say we are towards implementing the UHC policy in Nigeria?

Category three- Labour sector

Introduction

Section A. Interviewee Socio-demographic Background

1. What is your occupation?

2. What is your present position?

3. When did you first get involved in the UHC policy?

4. Have you been continually involved?

Section B. Major Questions

5. What is the meaning of Universal Health Coverage?

6. What persuaded the government to go down the UHC route and who or what were the principal influences?

7. How would you describe the importance of implementing UHC in Nigeria?

8. Is implementing UHC the most significant health policy the government has introduced in the last 15 years in Nigeria?

9. How would you characterize the view of the Nigerian people towards UHC?

10. What organizations have provided support for the government in implementing UHC?

11. What would be your own main criticisms of the UHC policy?

12. What other labour group did you form an alliance with during the entire UHC policy debates? 
13. In your opinion what are the main obstacles and challenges affecting Nigeria's progress towards achieving universal health care?

Agenda setting regarding UHC policy:

14. How did UHC get onto the policy agenda in Nigeria?

15. What role did your organization play at this stage?

16. How much resource was allocated by your organization to pursue the UHC policy?

17. Who were the key actors in the agenda setting? How were they involved?

18. Which stakeholders had the most influence at this stage?

19. How much were external forces involved at this stage?

20. What policies did your organization put forward during the agenda setting regarding UHC policy?

21. What policies did your organization disagree with at this stage of the policy process?

22. What major obstacles and challenges did the government face at this stage of the policy process?

Formulation of UHC policy:

23. What role did your organization play at this stage of the policy process?

24. How were particular policies selected, developed, and put into effect, and how were financial arrangements planned?

25. Who were the key actors in policy formulation? How were they involved?

26. What was the mechanism through which the policy was formulated?

27. Did you think that the policies formulated at this stage were sufficient to address the health needs of the people?

28. What major obstacles and challenges did the government face at this stage of the policy process?

Implementation of the UHC policy:

29. What role did your organization play at this stage of the policy?

30. How have those changes in policy affected your organisation?

31. What aspects of the UHC policy have been most difficult to implement? 
32. To what extent do you think the Government's policy address the needs of the Nigerian people?

33. What major obstacles and challenges did the government face at this stage of the policy process?

34. How far would you say we are towards implementing the UHC policy in Nigeria?

Category Four- NGOs and UN Bodies

Introduction

Section A. Interviewee Socio-demographic Background

1. What is your occupation?

2. What is your present position?

3. When did you first get involved in the UHC policy?

4. Have you been continually involved?

Section B. Major Questions

5. What is the meaning of Universal Health Coverage?

6. What persuaded the government to go down the UHC route and who or what were the principal influences?

7. How would you describe the importance of implementing UHC in Nigeria?

8. Is implementing UHC the most significant health policy the government has introduced in the last 15 years in Nigeria?

9. How would you characterize the view of the Nigerian people towards UHC?

10. What organizations have provided support for the government in implementing UHC?

11. What would be your own main criticisms of the UHC policy in Nigeria?

12. In your opinion what are the main obstacles and challenges affecting Nigeria's progress towards achieving universal health care?

Agenda setting regarding UHC policy: 
13. How did UHC get onto the policy agenda in Nigeria?

14. What role did your organization play at this stage?

15. How much resource was allocated by your organization to pursue the UHC policy?

16. Who were the key actors in the agenda setting? How were they involved?

17. Which stakeholders had the most influence at this stage?

18. What technical support did your organization provide at this stage?

19. What major obstacles and challenges did the government face at this stage of the policy process?

Formulation of UHC policy:

20. What role did your organization play at this stage of the policy process?

21. How were particular policies selected, developed, and put into effect, and how were financial arrangements planned?

22. Who were the key actors in policy formulation? How were they involved?

23. What was the mechanism through which the policy was formulated?

24. Did you think that the policies formulated at this stage were sufficient to address the health needs of the people?

25. What major obstacles and challenges did the government face at this stage of the policy process?

Implementation of the UHC policy:

26. What role did your organization play at this stage of the policy?

27. What aspects of the UHC policy have been most difficult to implement?

28. To what extent do you think the Government's policy address the needs of the Nigerian people?

29. What major obstacles and challenges did the government face at this stage of the policy process?

30. How far would you say we are towards implementing the UHC policy in Nigeria? 
College of Human and Health Sciences

Swansea University, United Kingdom.

Project Title: The obstacles and challenges affecting the move towards universal health coverage in Nigeria.

\section{Introduction}

My name is Jenson Gawain Fofah, a $\mathrm{PhD}$ research student at Swansea University, United Kingdom. I am in Nigeria to carry out a research on Nigeria's path towards Universal Health Coverage. I formally invite you to partake in this research. Please, carefully read the participant information below. Please feel free to ask questions if anything is unclear or if you would like further information. Take time to decide whether or not you wish to take part.

Thank you for taking time to read it.

\section{Purpose of the project}

This research examines Nigeria's path towards Universal Health Coverage through the interactions of the policy actors involved the policy process. Furthermore, the research will use a social network analysis to illustrate a visual interaction between policy actors at every stage of the policy process in Nigeria. The research will also gather information of the impact of the Universal Health Coverage since its introduction in Nigeria.

\section{Why have I been selected/do I have to take part?}

You have been selected based on your suitability for this study. It is up to you to decide whether or not to take part; you will not suffer any disadvantage if you decide not to participate. If you do decide to take part, you will be given this information sheet to keep and will be asked to sign a consent form. It is important to note that if you decide to take part, you are still free to withdraw at any time without giving a reason.

\section{What will happen to me if I take part?}

You will be asked to take part in a face-to-face interview and complete two questionnaires. The interview and the completion of two questionnaires will take less than 50 minutes. The first questionnaire involves the use of a snowballing technique with the intent of selecting significant policy actors involved in Universal Health Policy in Nigeria. The second questionnaire involves collecting data from policy actors for mapping out the social interactions among actors at every stage of the political process. The face-to-face interview will be recorded if you permit it using recording devices such as tape-recorded, telephone and 
so on. It is important for the research if the interviews are recorder because it ensures reliability and validity.

\section{Will my participation be kept confidential?}

Confidentially is considered as one of the top priorities of this research. In fact, any information collected, which includes the content of the interview recording, shall be held with extreme confidentiality. We will provide anonymity either to your name or any form data that might identify you during the data collection. After transcription, the content of recording shall be destroyed.

\section{What are the potential risks to you in taking part?}

There are no potential risks concerned in this research. However, you will be obligated to take time off your schedule to participate in this research.

\section{What happens next?}

If you are in agreement in participating in this research, you required signing a consent form to confirm co-operation.

Researchers contact details

Jenson Gawain Fofah,

College of Human and Health Sciences,

Swansea University,

Singleton Park, Swansea,

SA2 8PP,

UK.

Thank You

\section{Appendix 5: Participant Consent Form}




\section{Participant Consent Form}

Title of project: The obstacles and challenges affecting the move towards universal health coverage in

Nigeria.

Notice: Please read each statement, and if you agree with the statement, please tick and initial the box. If you agree to participate in the study, at the bottom of the form please also write your name in block capitals, the date and sign the form. Please also provide your address and telephone number for further contact and indicate which times would be best for me to contact you.

\begin{tabular}{|c|l|c|}
\hline No. & \multicolumn{1}{|c|}{ Statement } & Please tick box \\
\hline $\mathbf{1}$ & $\begin{array}{l}\text { I confirm that the researcher has provided me with the participant information } \\
\text { sheet (Date: }\end{array}$ & $\begin{array}{l}\text { I. I have read and understood the information. } \\
\text { I understand that my participation is voluntary and that I am free to withdraw } \\
\text { from the project at any time, up to the point of completion, without having to give } \\
\text { don't want my data to be used, any data that have been collected from me will be } \\
\text { destroyed. }\end{array}$ \\
\hline $\mathbf{3}$ & $\begin{array}{l}\text { I understand that any information recorded in the investigation will remain } \\
\text { confidential and no information that identifies me will be made publicly available. }\end{array}$ \\
\hline $\mathbf{5}$ & $\begin{array}{l}\text { I consent to being audio recorded as part of the project } \\
\text { I consent to being a participant in the project }\end{array}$ \\
\hline
\end{tabular}

Name of participant:

Name of researcher:
Date: Signature:

Date: Signature: 
Appendix 6: Examples of SNA measures from the read relationship of the policy network.

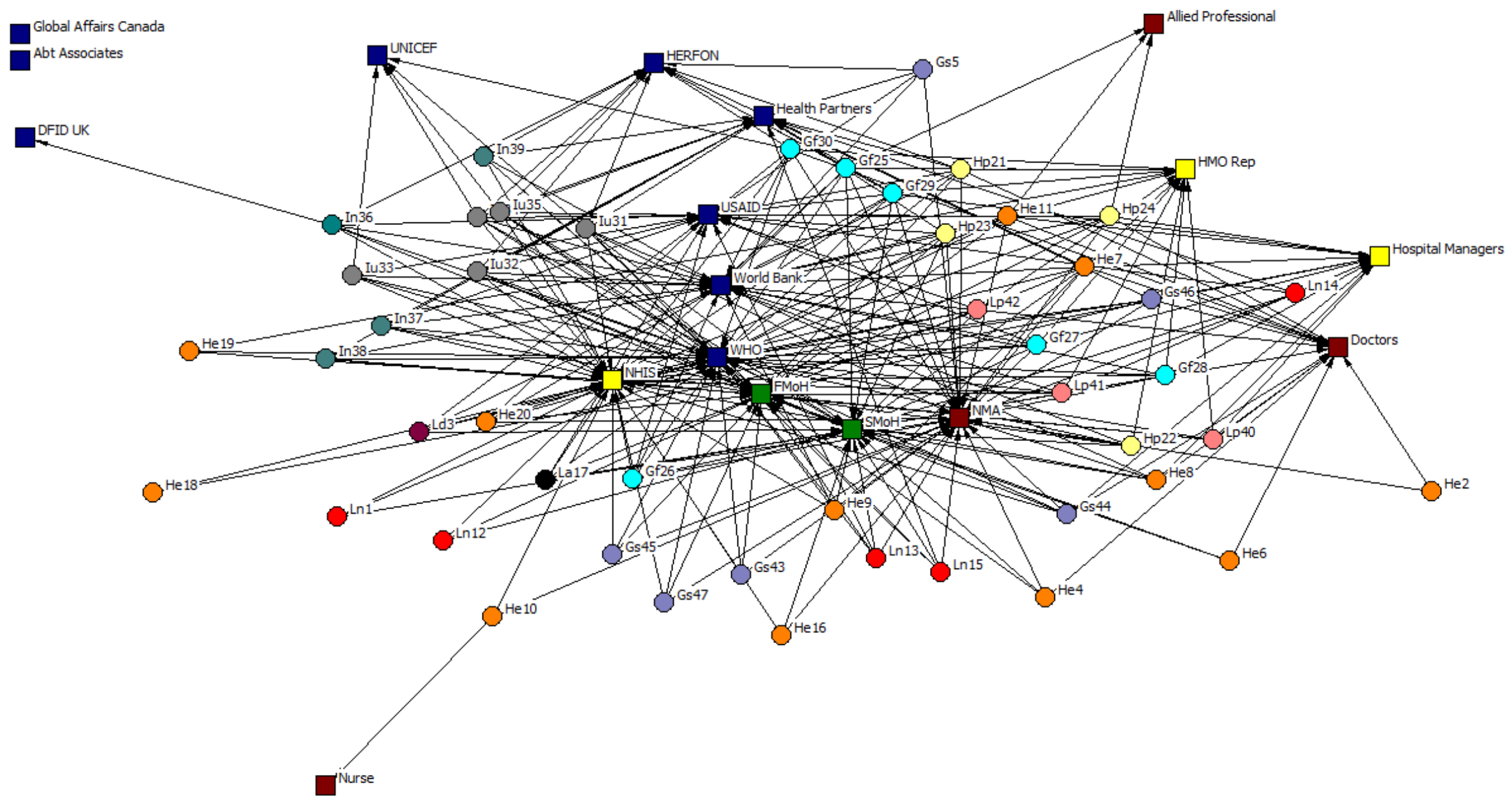

Figure 53: Visualization of 2-mode (dichotomized graph) “read” relationship of the policy network. 


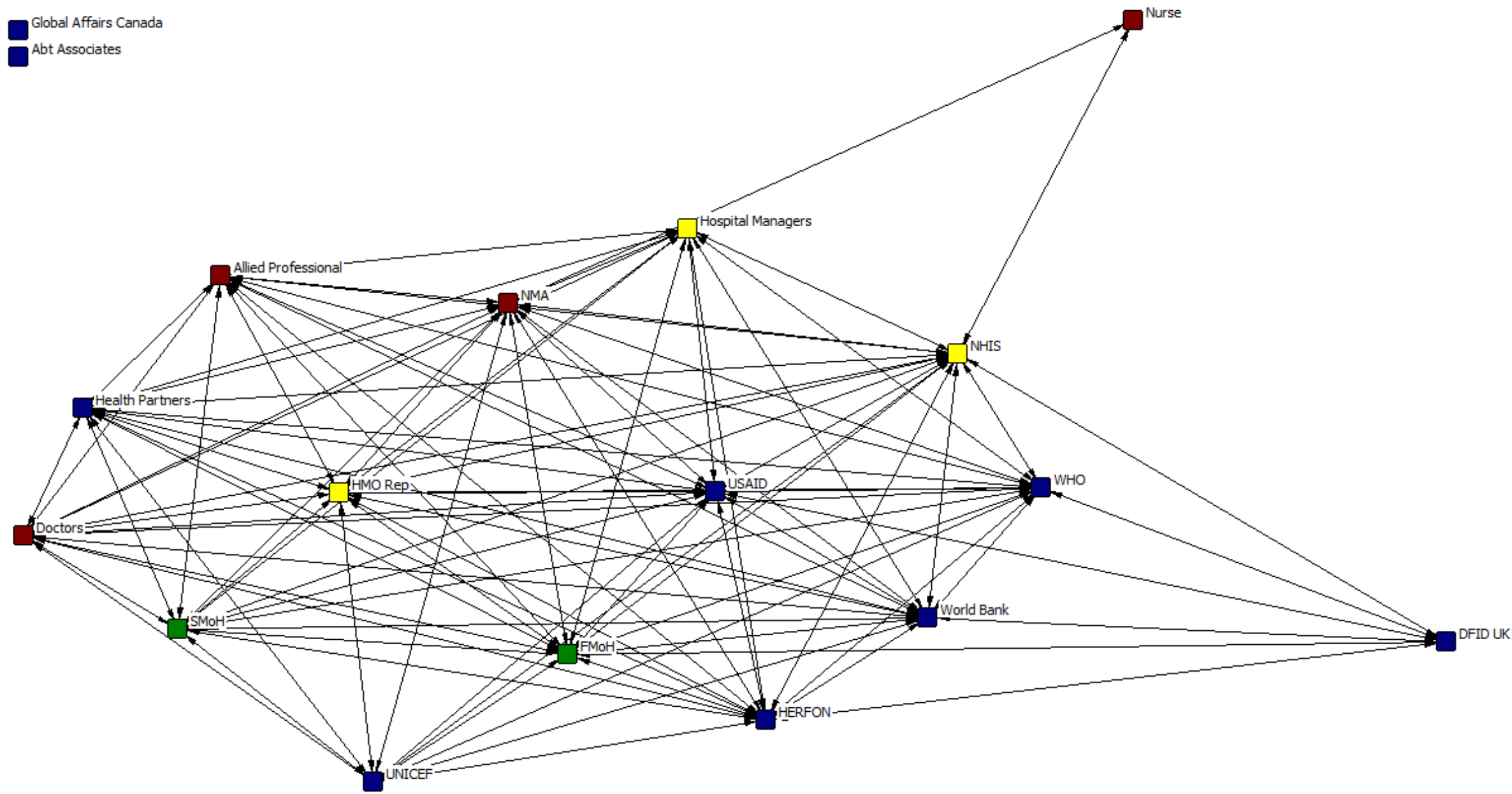

Figure 54: Visualization of (policy organisation-to-policy organisation) “read” relationship of the policy network. 


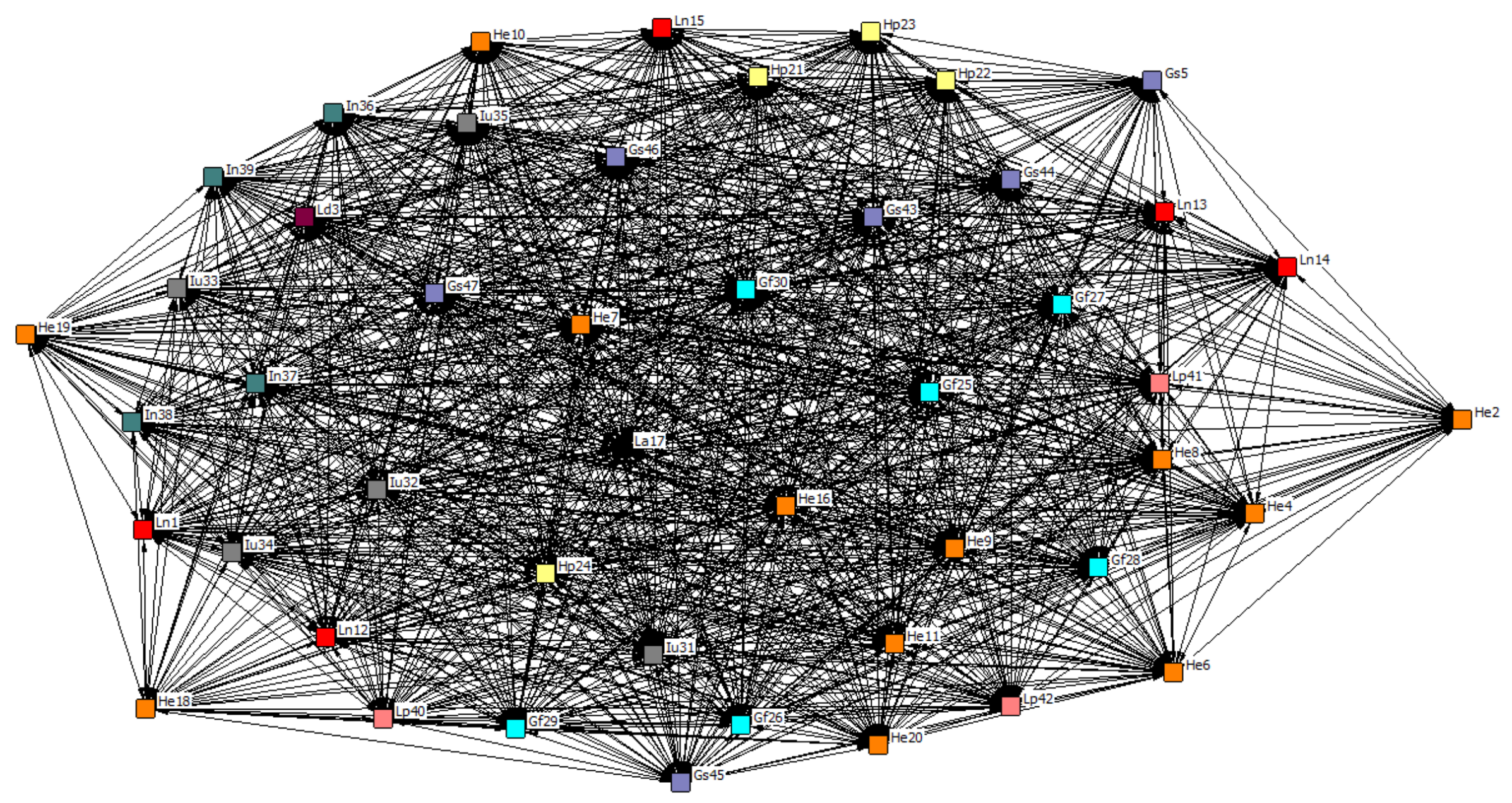

Figure 55: Visualization of (respondent-by-respondent) “read" relationship of the policy network. 


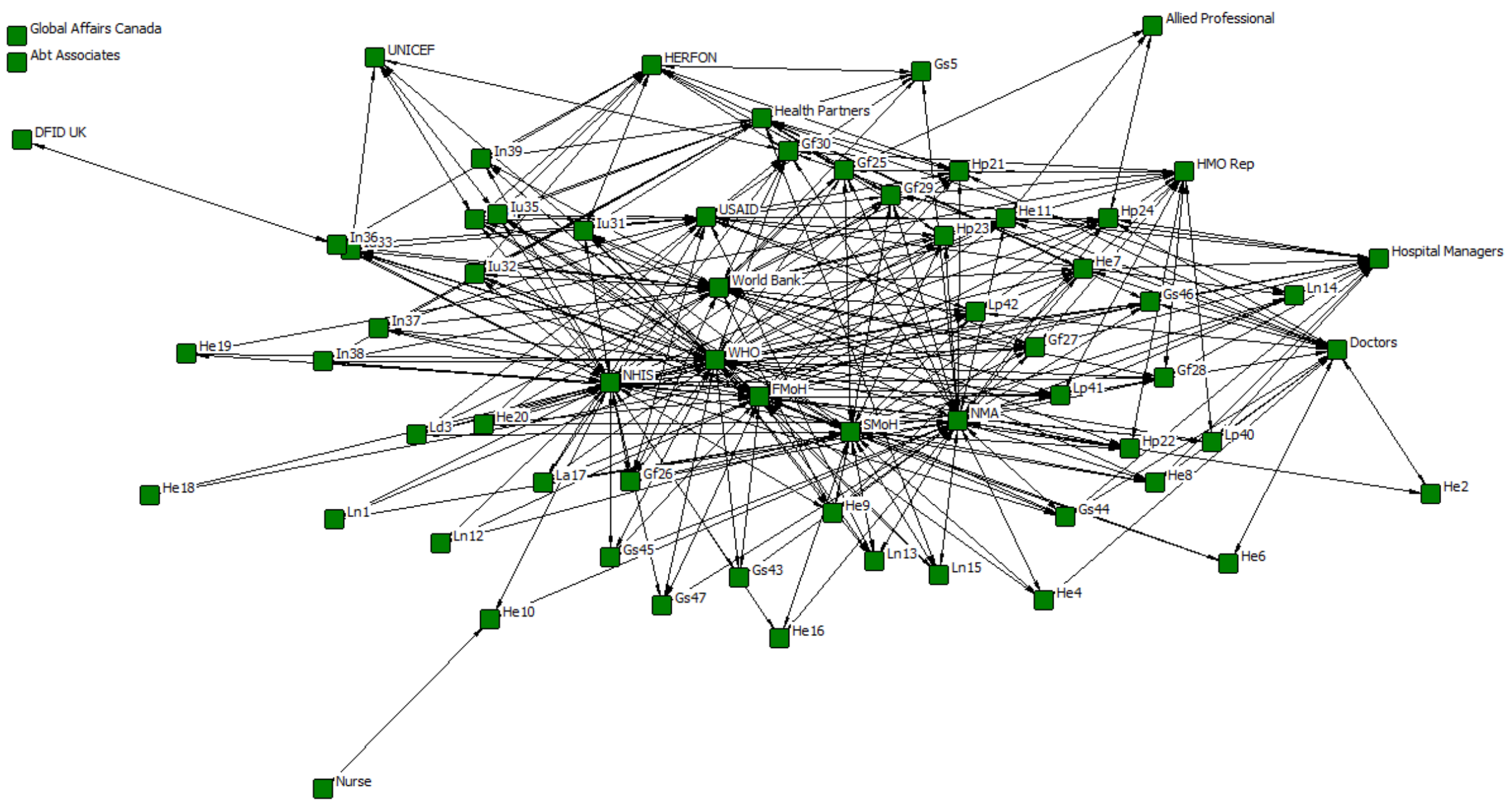

Figure 56: Visualization of (respondent-to-policy organisation) “read” relationship of the policy network. 
Appendix 7: Examples of SNA measures from the discussion relationship of the policy network.

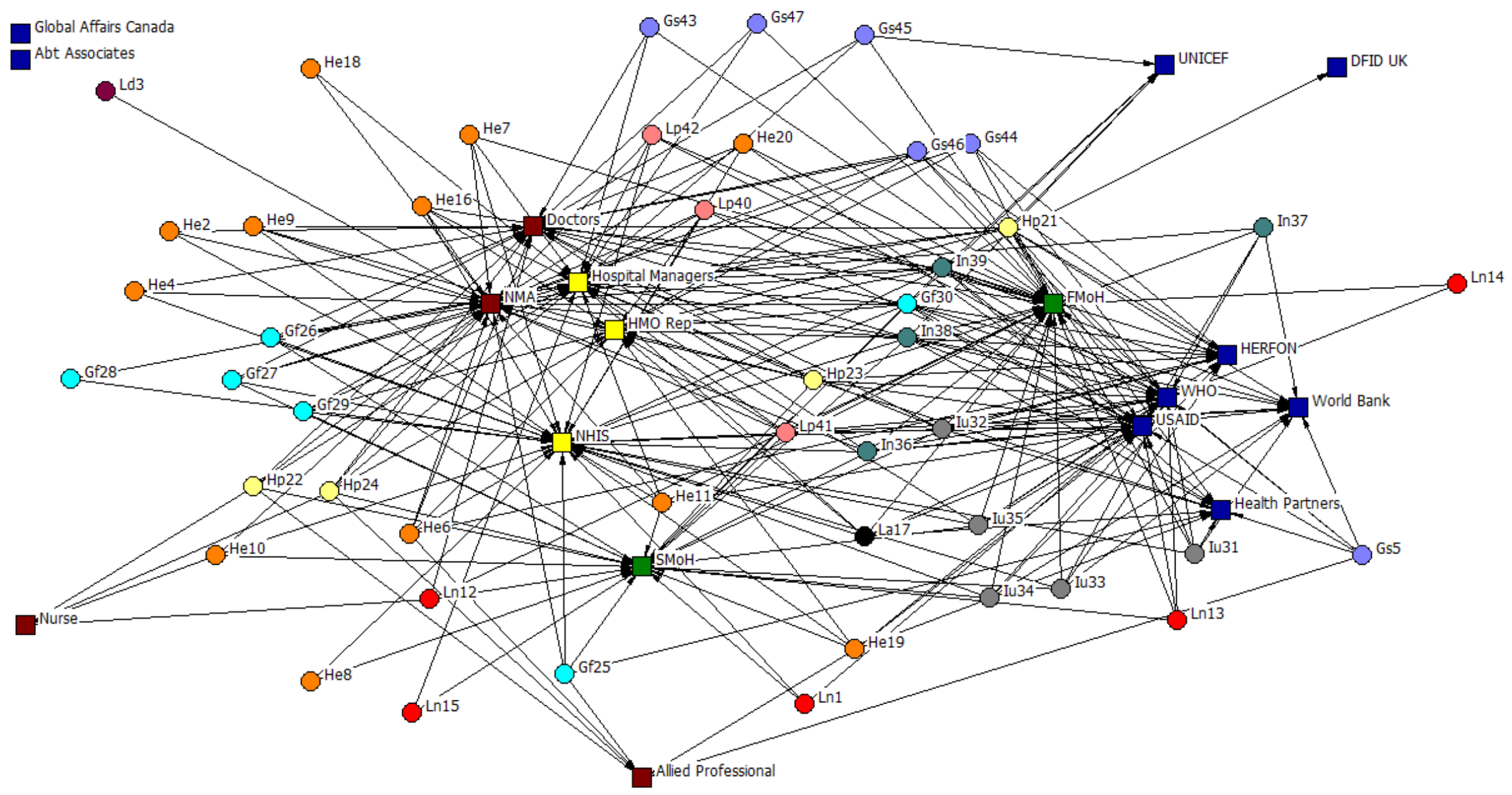

Figure 57: Visualization of (dichotomized graph) “discussion” relationship of the policy network. 


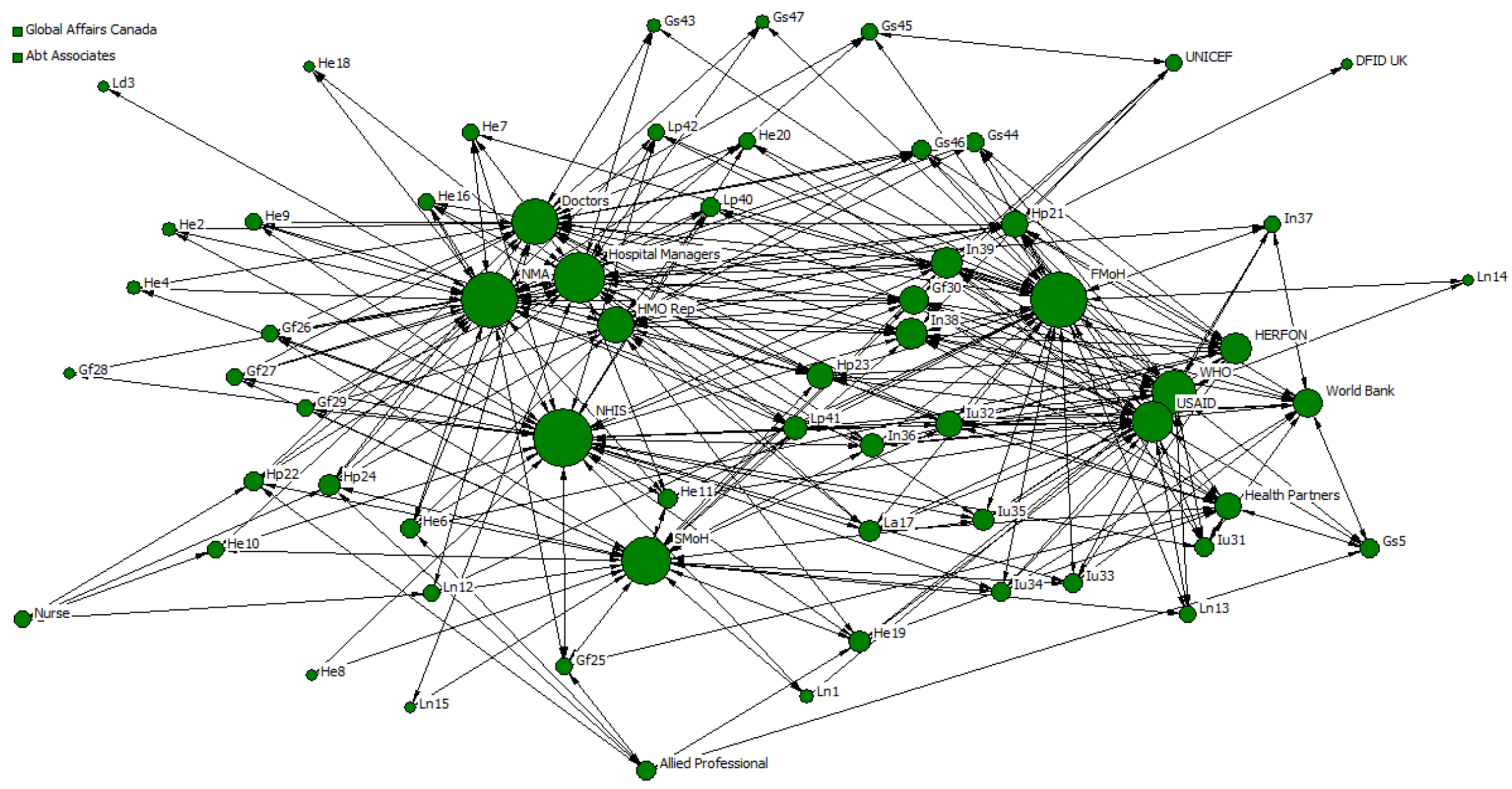

Figure 58: Visualization of the degree centrality measure of (bipartite graph) “discussion” relationship of the policy network. 


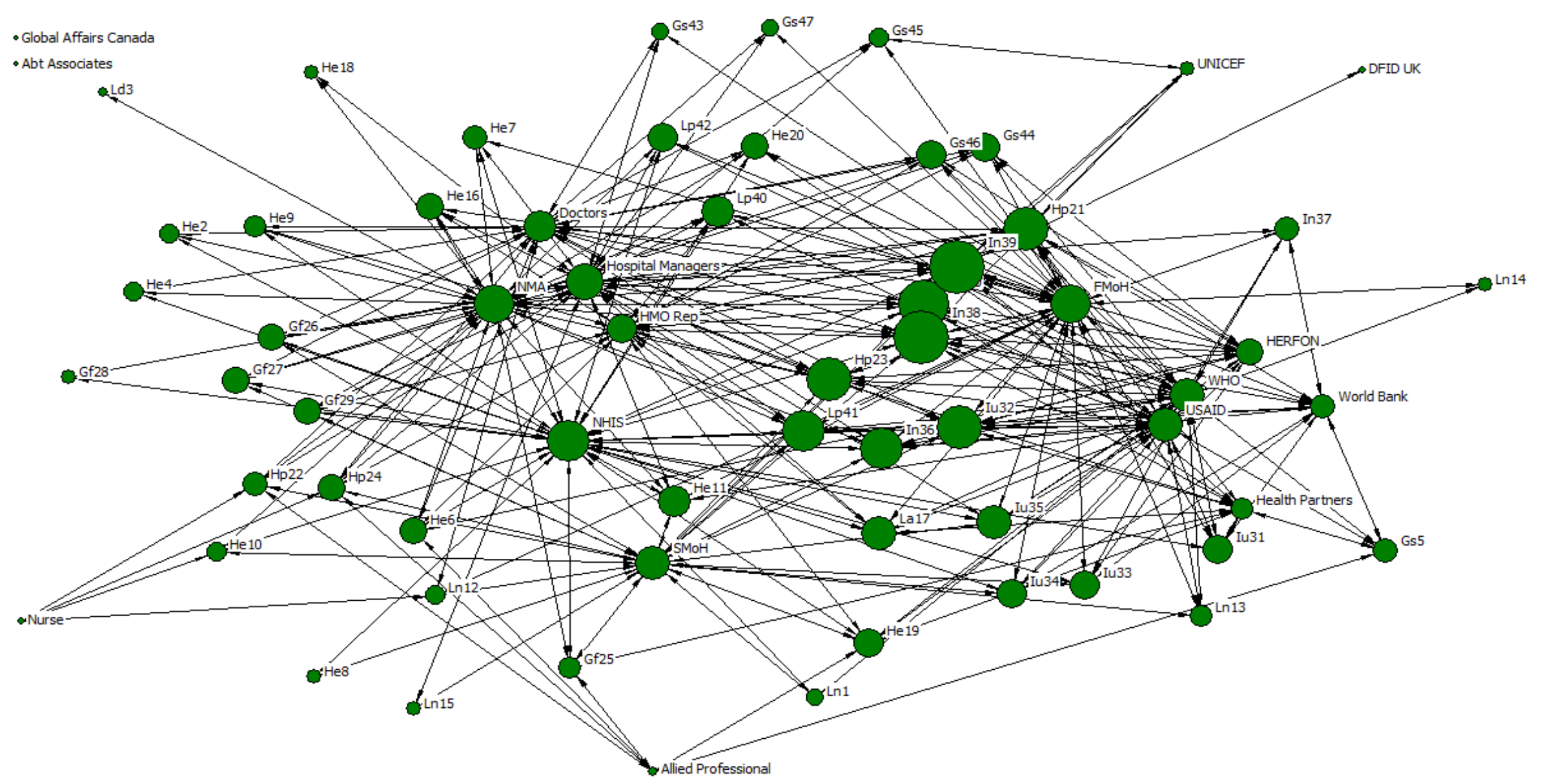

Figure 59: Visualization of the eigenvector centrality measure of the (bipartite graph) “discussion” relationship of the policy network. 


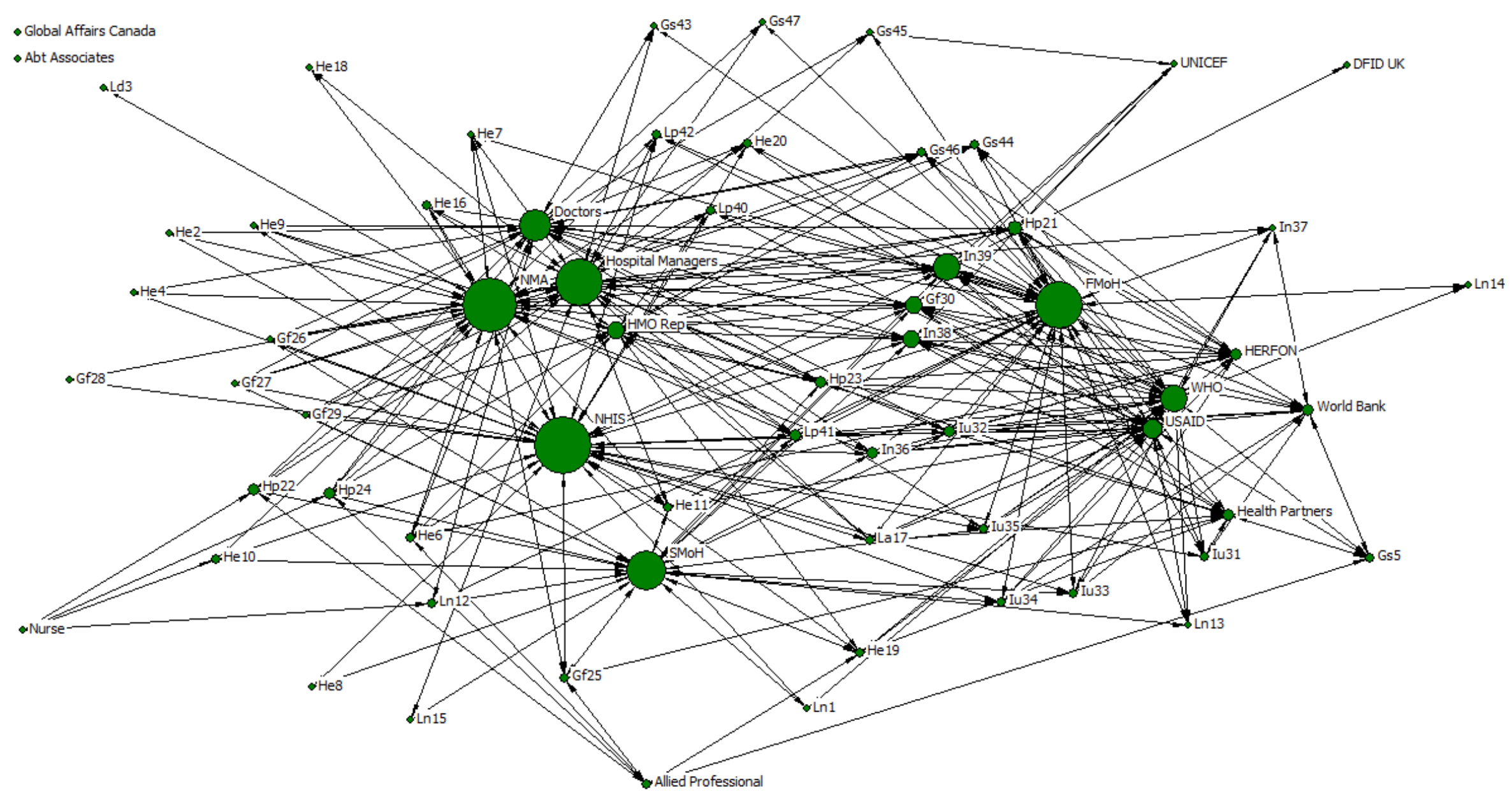

Figure 60: Visualization of the betweenness centrality measure of the (bipartite graph) “discussion” relationship of the policy network. 


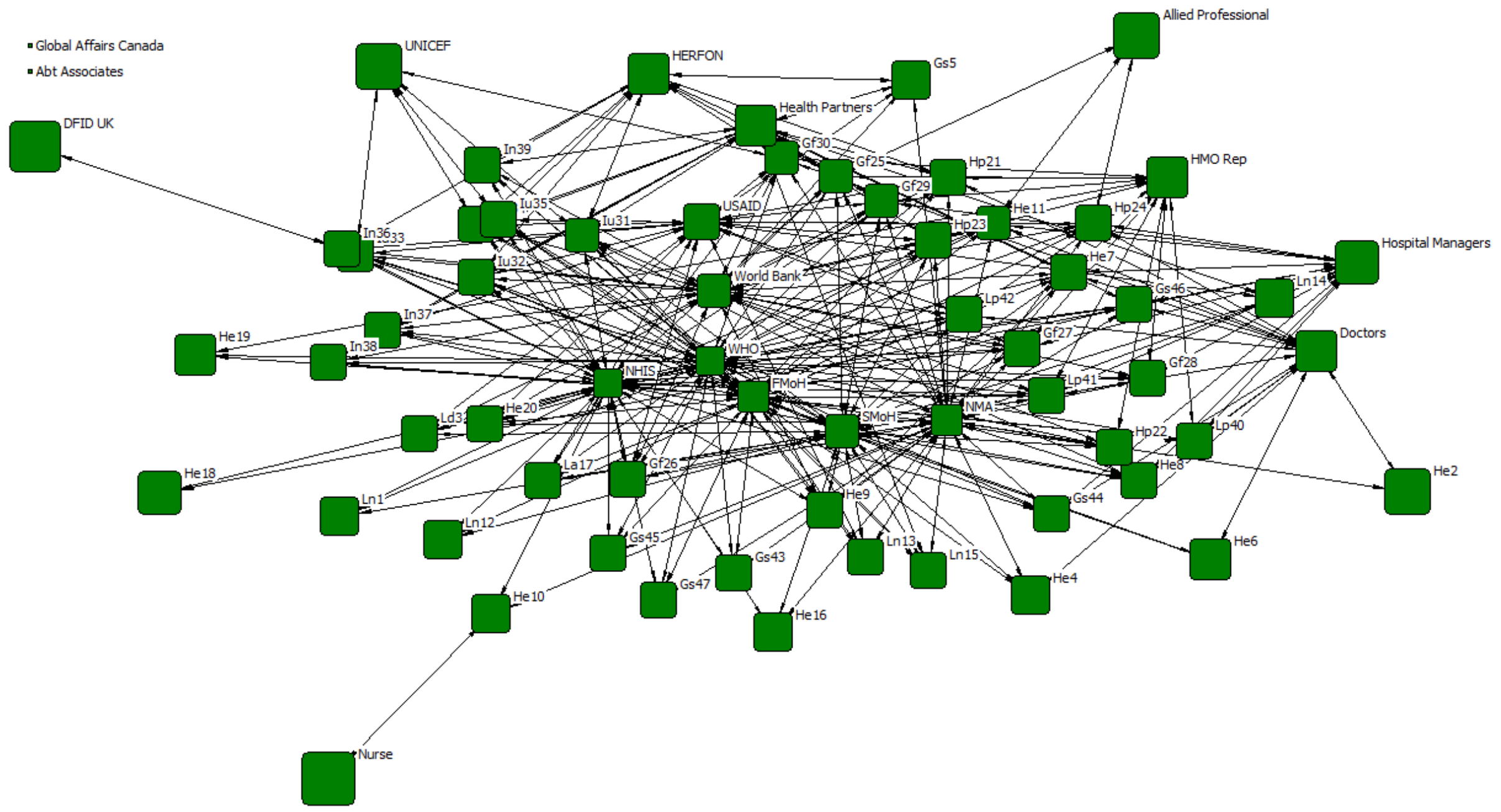

Figure 61: Visualization of the closeness centrality measure of the (bipartite graph) “discussion” relationship of the policy network. 


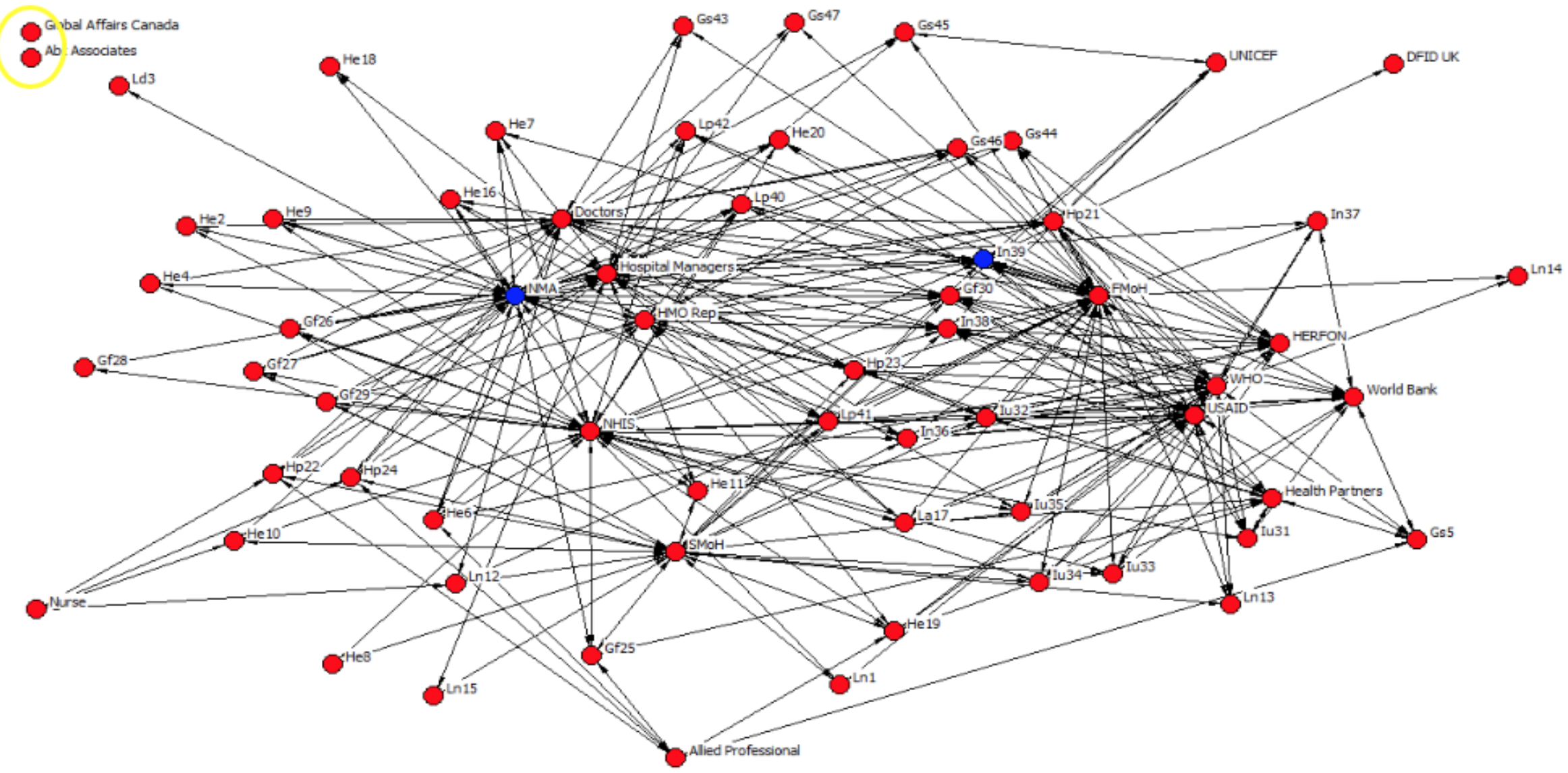

Figure 62: Visualization of the blocks and cut-points of the (bipartite graph) “discussion” relationship of the policy network.

Note: This is computed with four blocks, each containing two isolating members (Ld3 and DFID UK) and two cut-points nodes (NMA and In39) isolating nodes Global Affairs Canada and Abt Associates all circled yellow. 


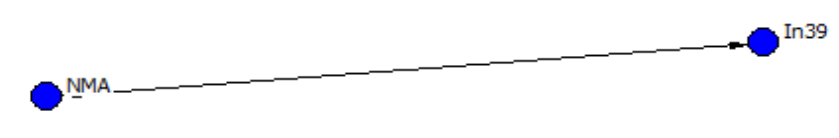

Figure 63: Visualisation of the cut-points of the (bipartite graph) “discussion” relationship of the policy network.

Note: This is computed with one block containing two cut-points node (NMA and In39). 


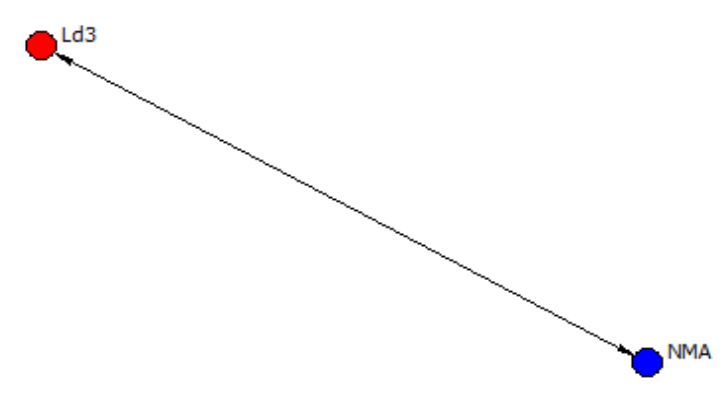

Figure 64: Visualization of the cut-point of the (bipartite graph) “discussion” relationship of the policy network (a).

Note: This is computed with two blocks, each containing one isolating member and one cut-point node (Ld3 and NMA). 


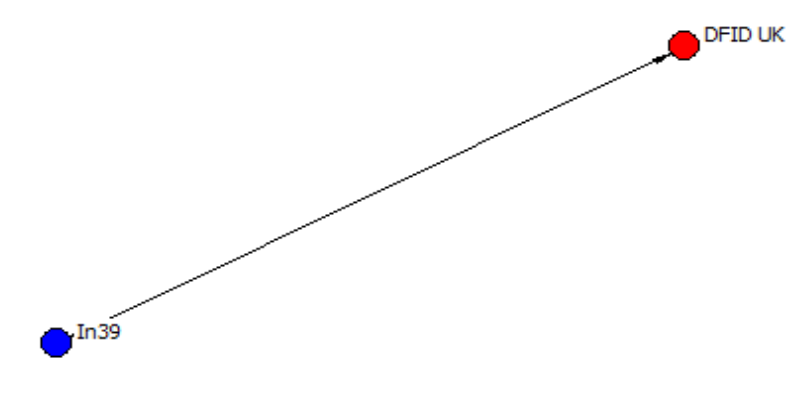

Figure 65: Visualisation of the isolate and cut-point of the (bipartite graph) “discussion” relationship of the policy network (b). Note: This is computed with two blocks, each containing one isolate member and one cut-point node (DFID UK and In39). 


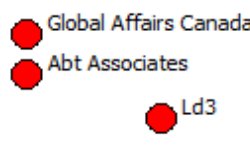

Figure 66: Visualization of the isolates and isolates cut-points of the (bipartite) “discussion” relationship of the policy network.

Note: This is computed with three blocks, each containing two isolating nodes (Global Affairs Canada and Abt Associates) and two isolating members (Ld3 and DFID UK). 
Appendix 8: Examples of SNA measures from the coalition relationship of the policy network.

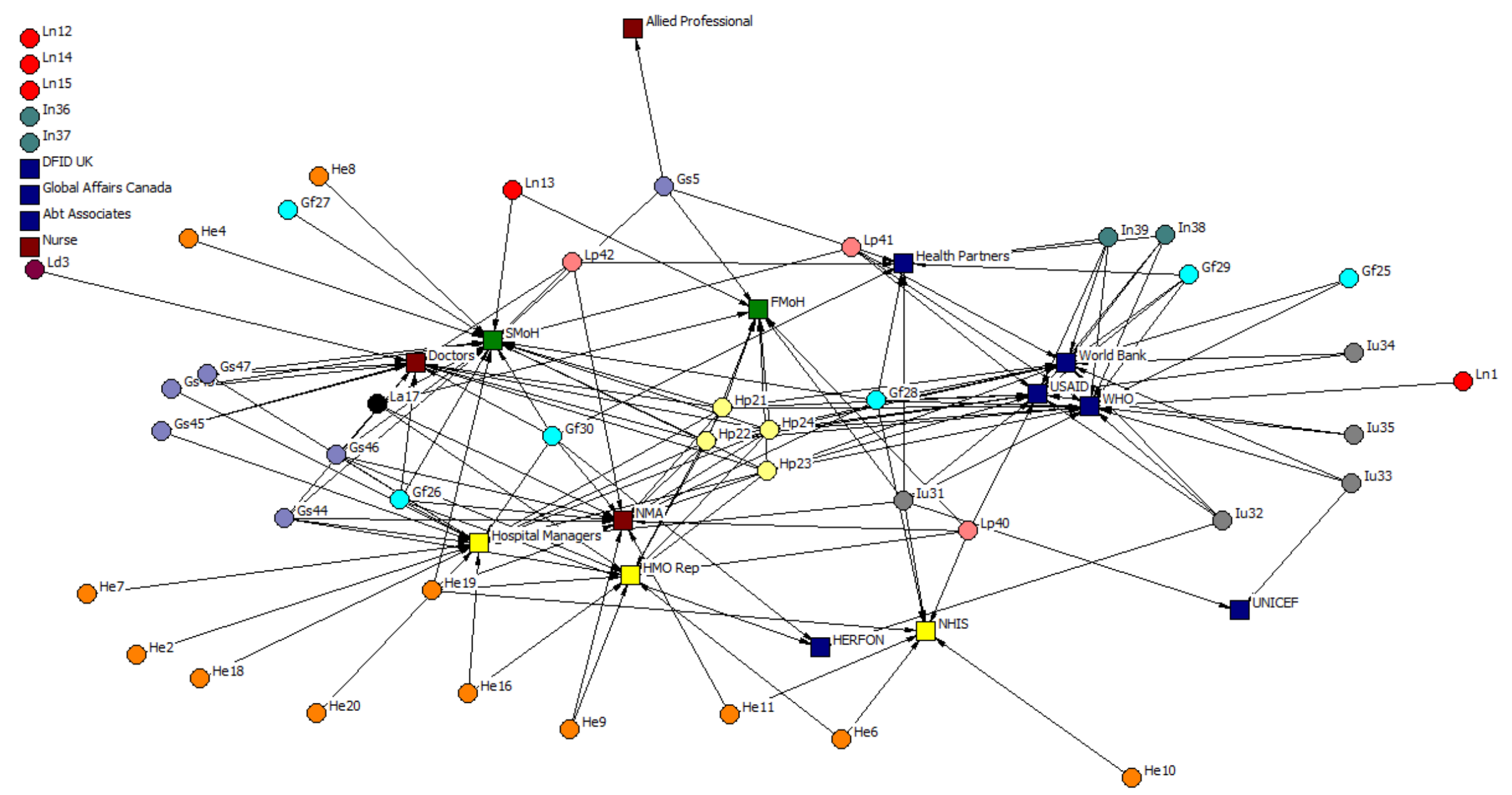

Figure 67: Visualization of (dichotomized graph) “coalition” relationship of the policy network. 


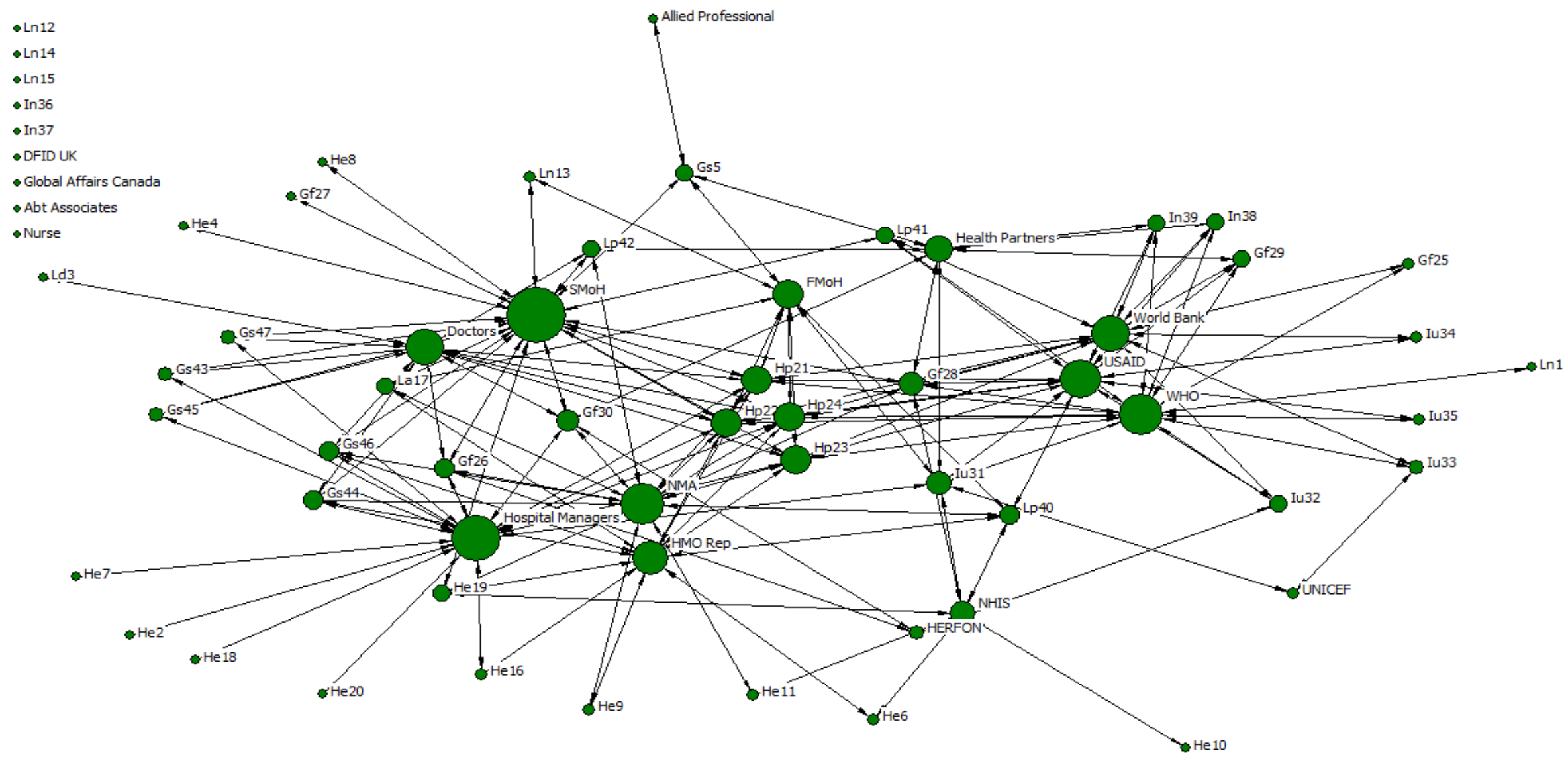

Figure 68: Visualization of the degree centrality measure of the (bipartite graph) “coalition” relationship of the policy network. 


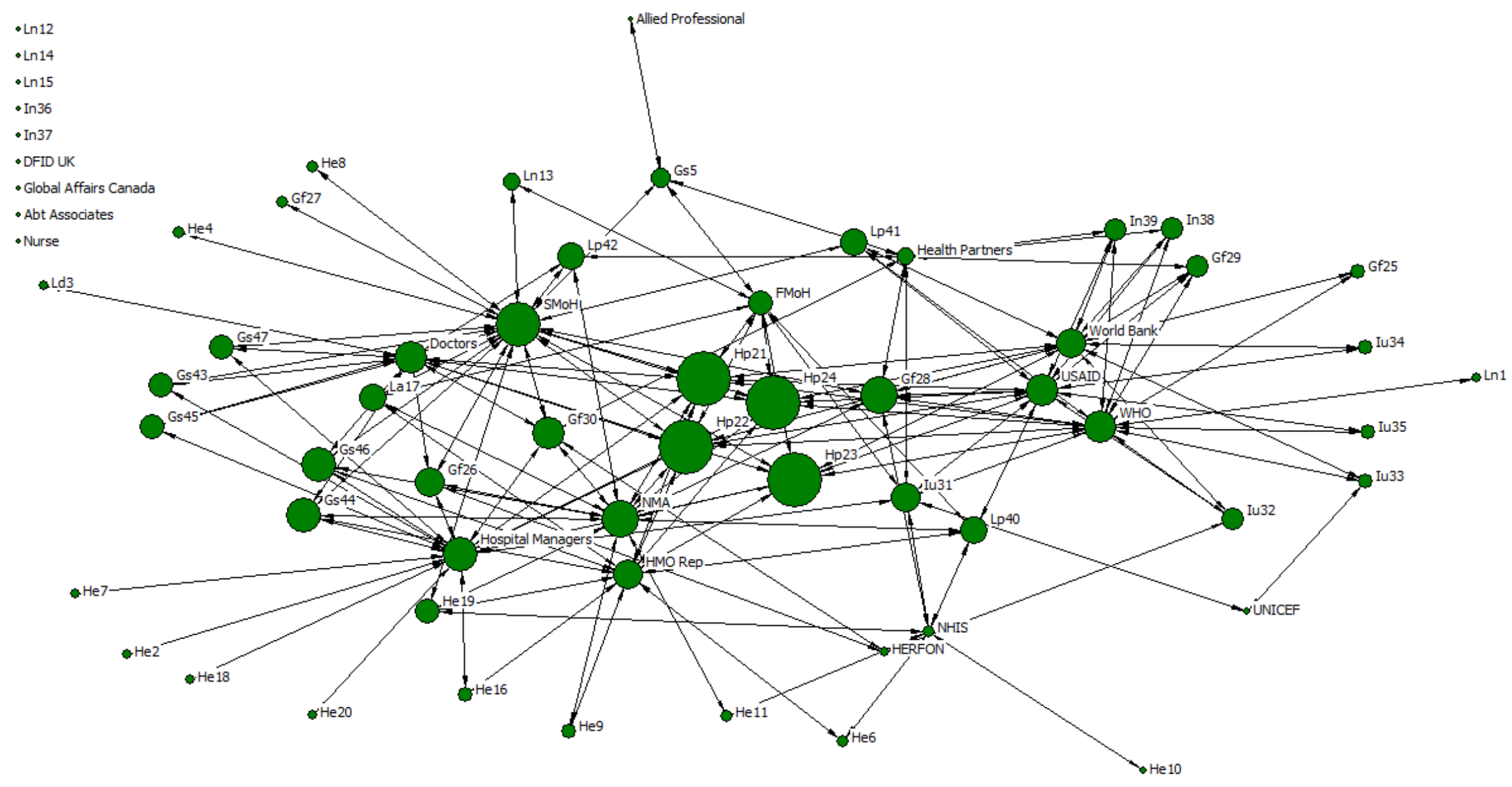

Figure 69: Visualization of the eigenvector centrality measure of the (bipartite graph) “coalition” relationship of the policy network. 


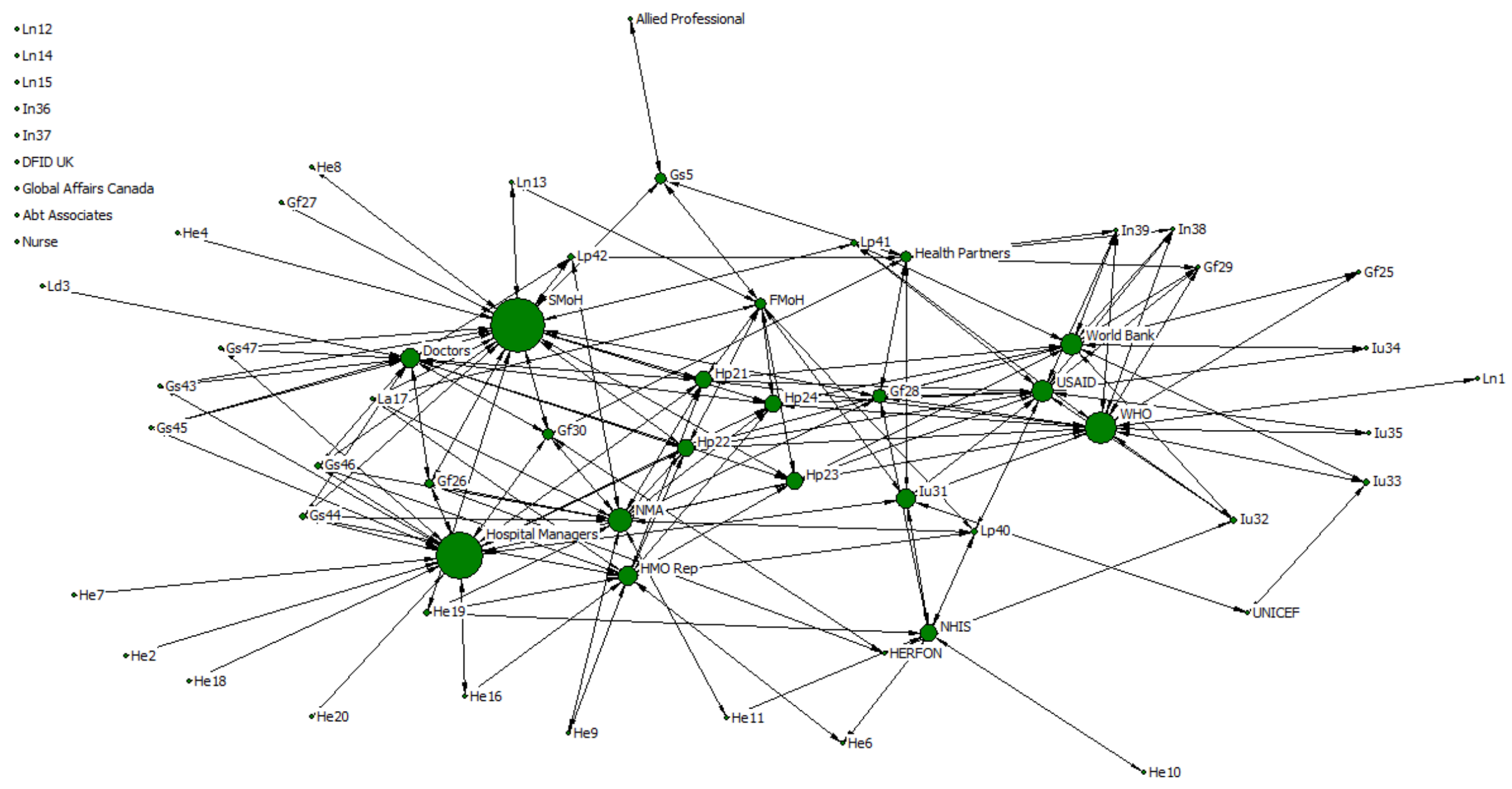

Figure 70: Visualization of the betweenness centrality measure of the (bipartite graph) “coalition” relationship of the policy network. 


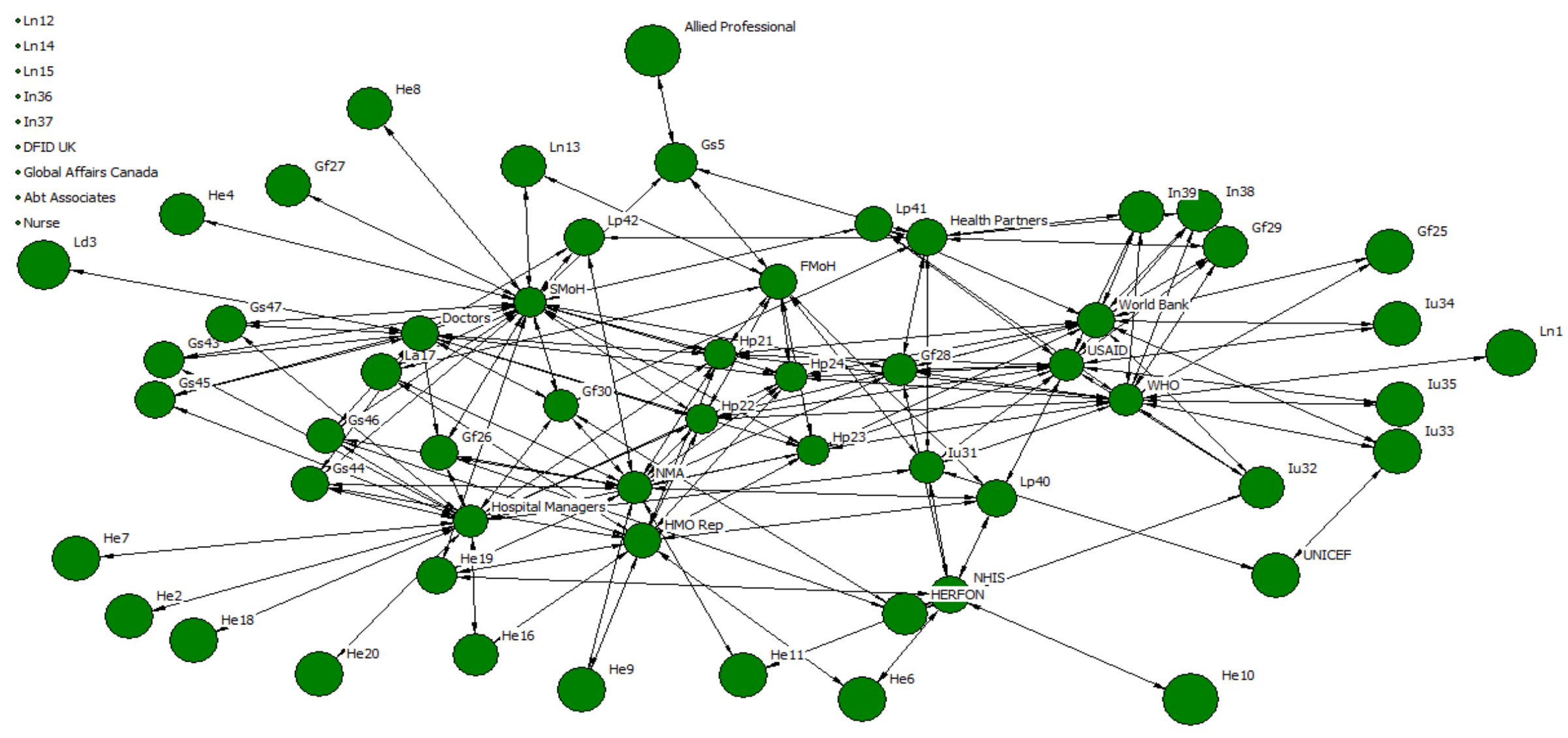

Figure 71: Visualization of the closeness centrality measure of the (bipartite graph) “coalition” relationship of the policy network. 
Appendix 9: Examples of SNA measures from the affiliation relationship of the policy network.

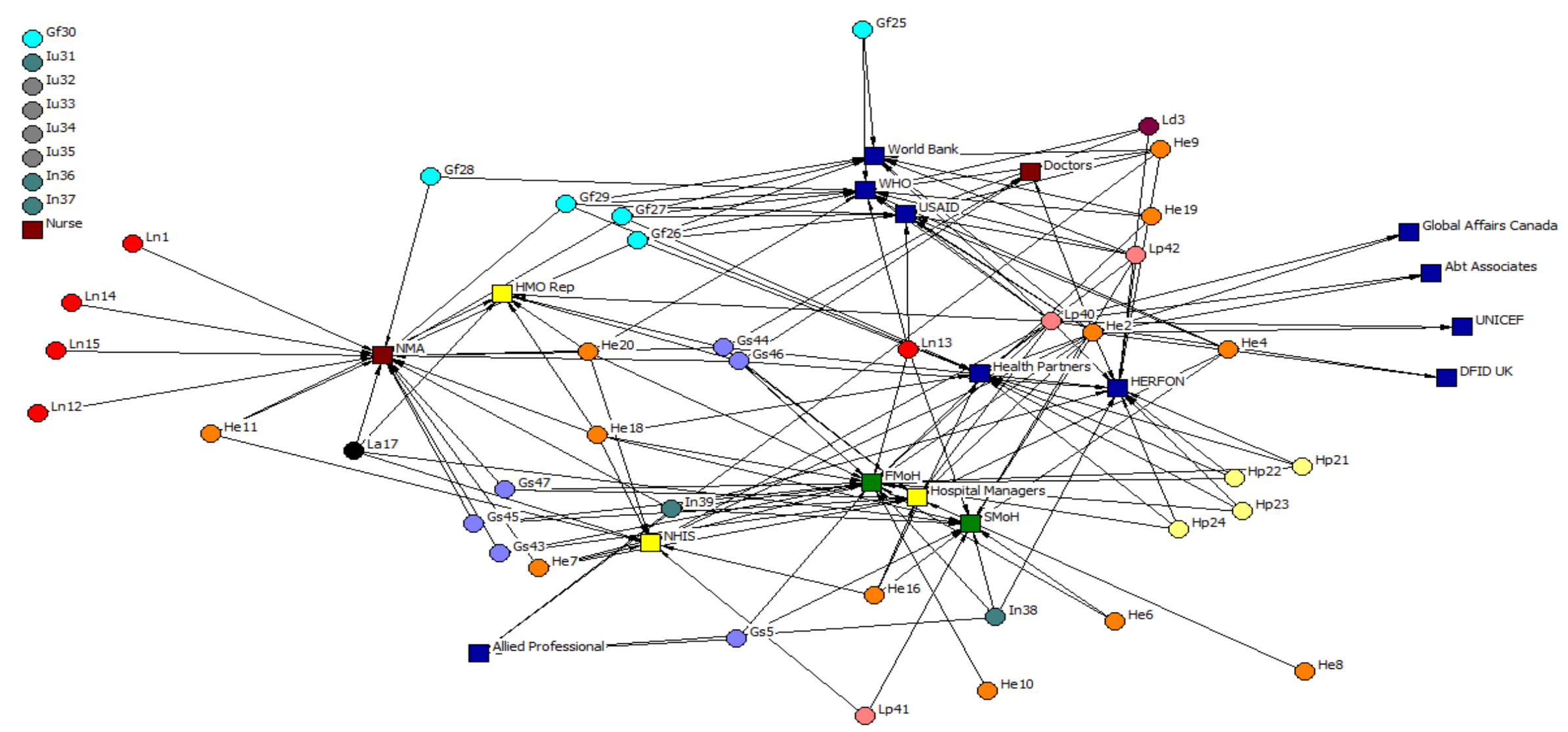

Figure 72: Visualization of (dichotomized graph) “affiliation” relationship of the policy network. 


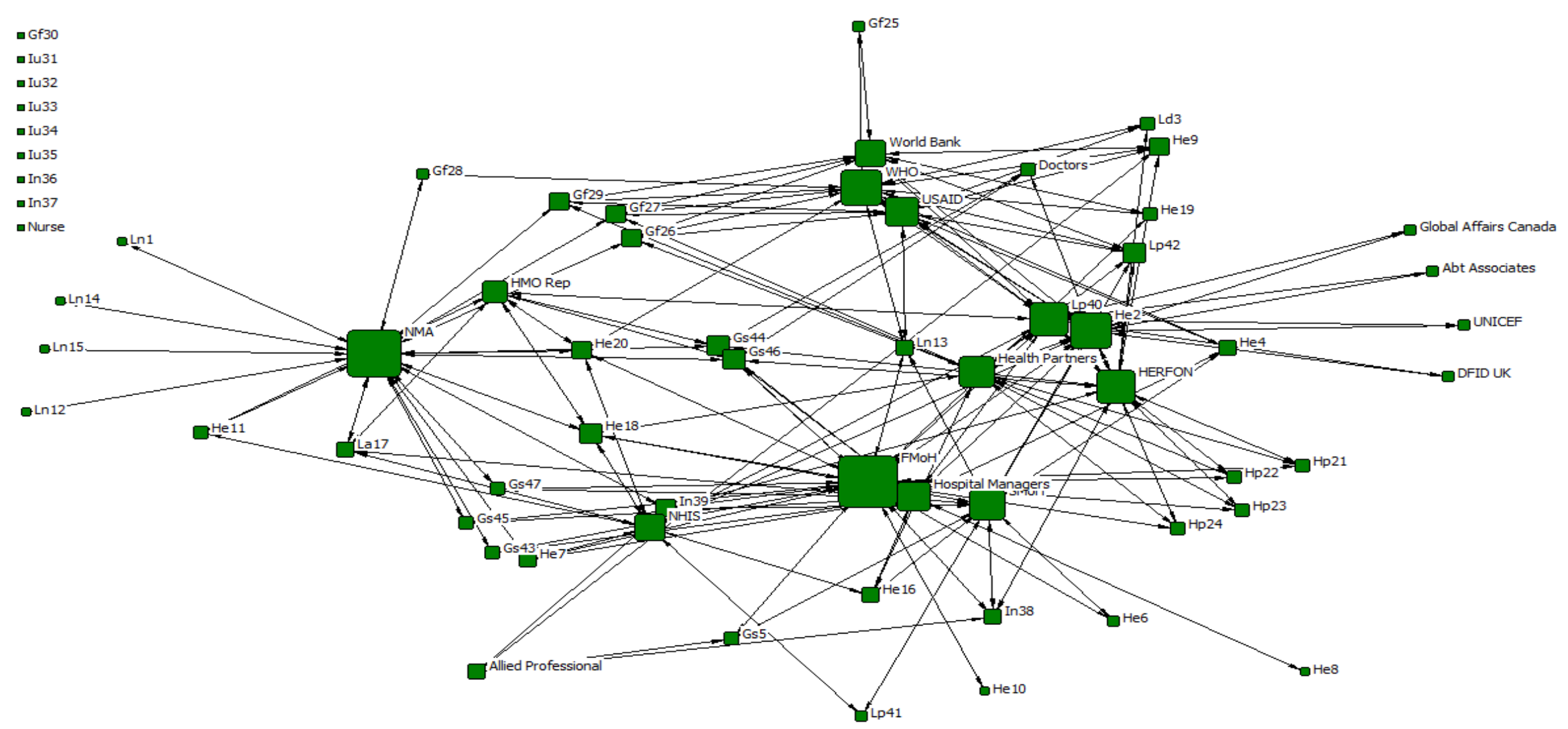

Figure 73: Visualization of the degree centrality measure of the (bipartite graph) “affiliation” relationship of the policy network. 


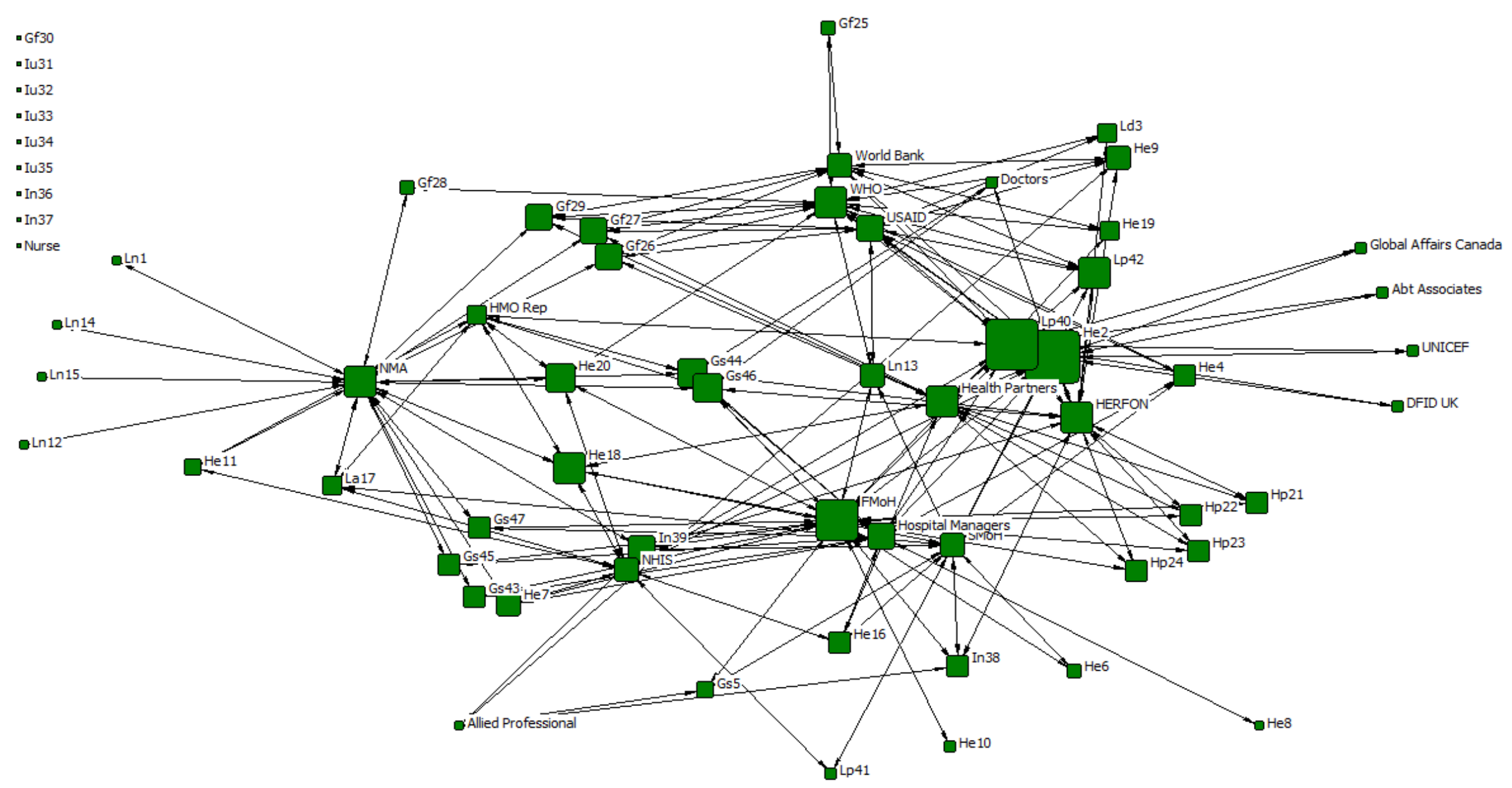

Figure 74: Visualization of the eigenvector centrality measure of the (bipartite graph) “affiliation” relationship of the policy network. 


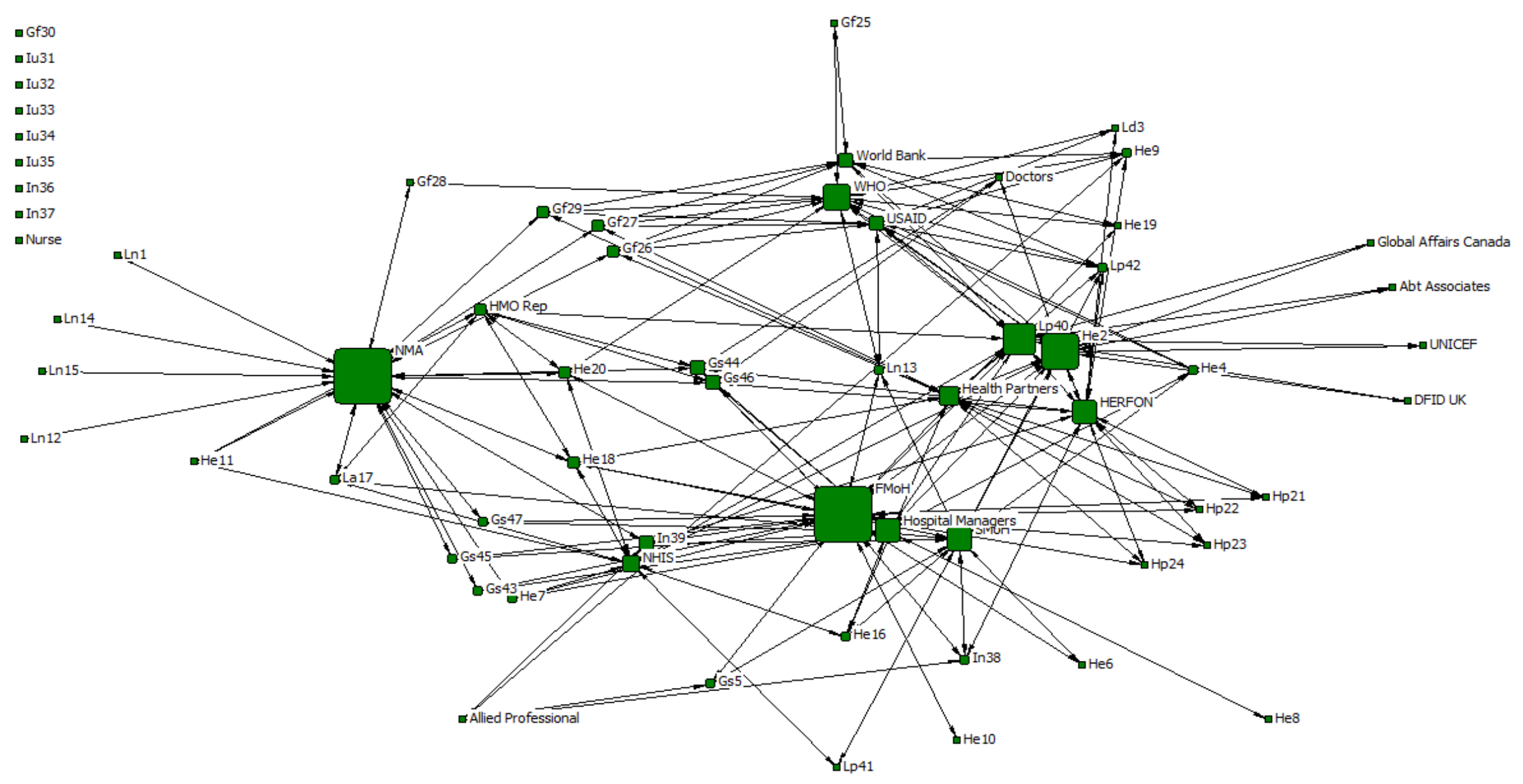

Figure 75: Visualisation of the betweenness centrality measure of the (bipartite graph) "affiliation" relationship of the policy network. 


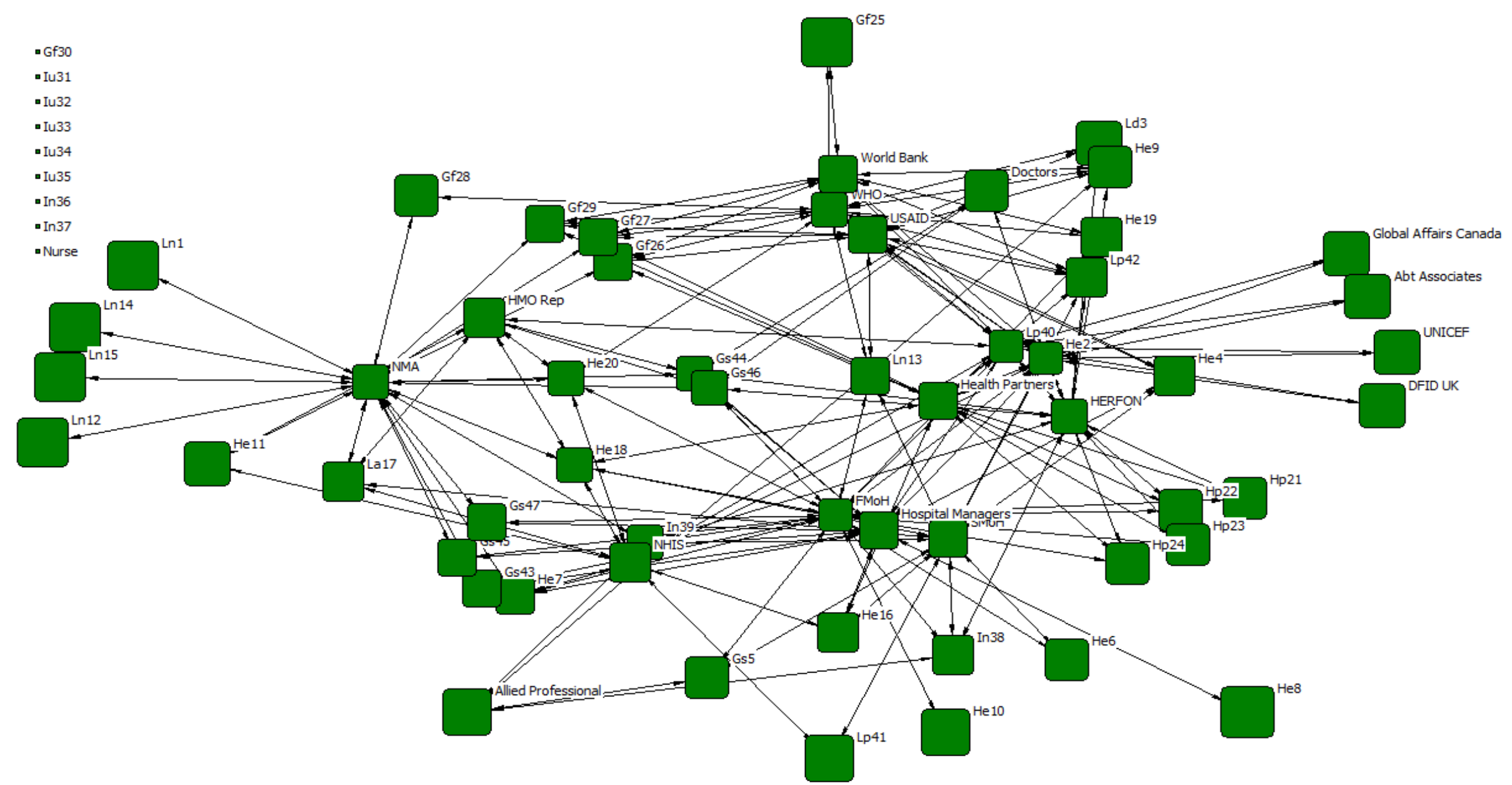

Figure 76: Visualization of the closeness centrality measure of the (bipartite graph) “affiliation” relationship of the policy network. 
Appendix 10: Examples of SNA measures from the conflict relationship of the policy network.

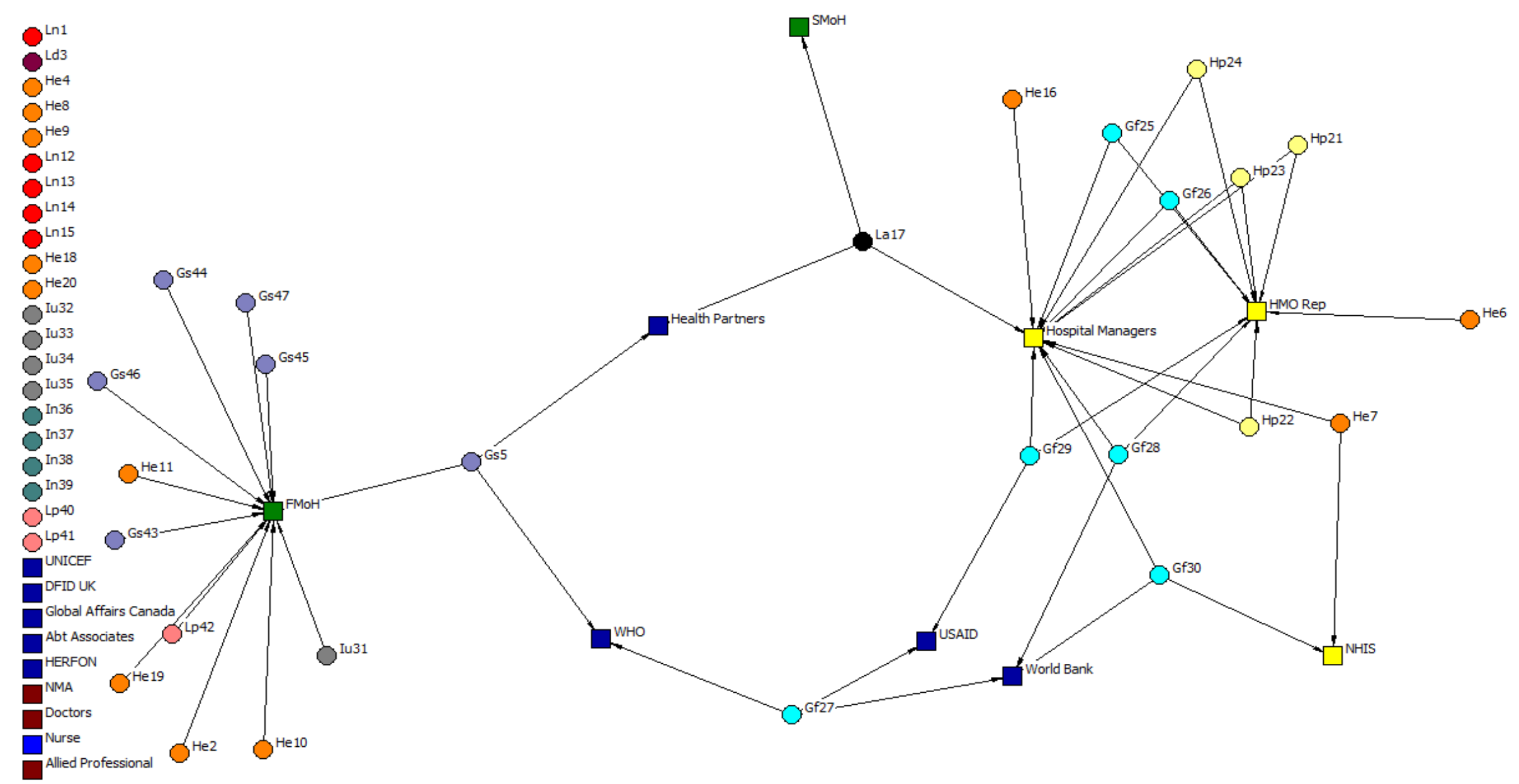

Figure 77: Visualization of (dichotomized graph) “conflict” relationship of the policy network 


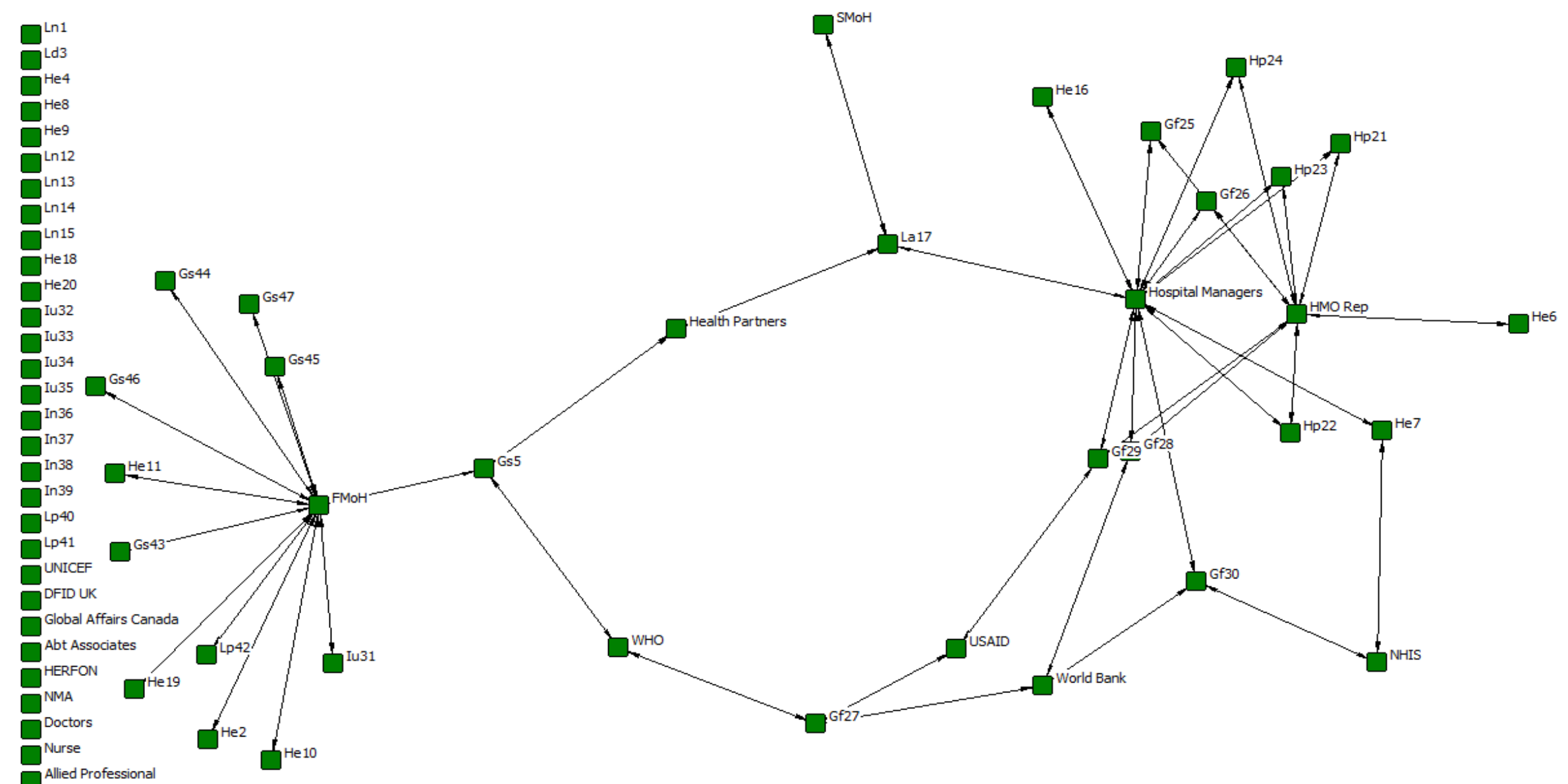

Figure 78: Visualization of (respondent-to-policy organisation) “conflict” relationship of the policy network. 


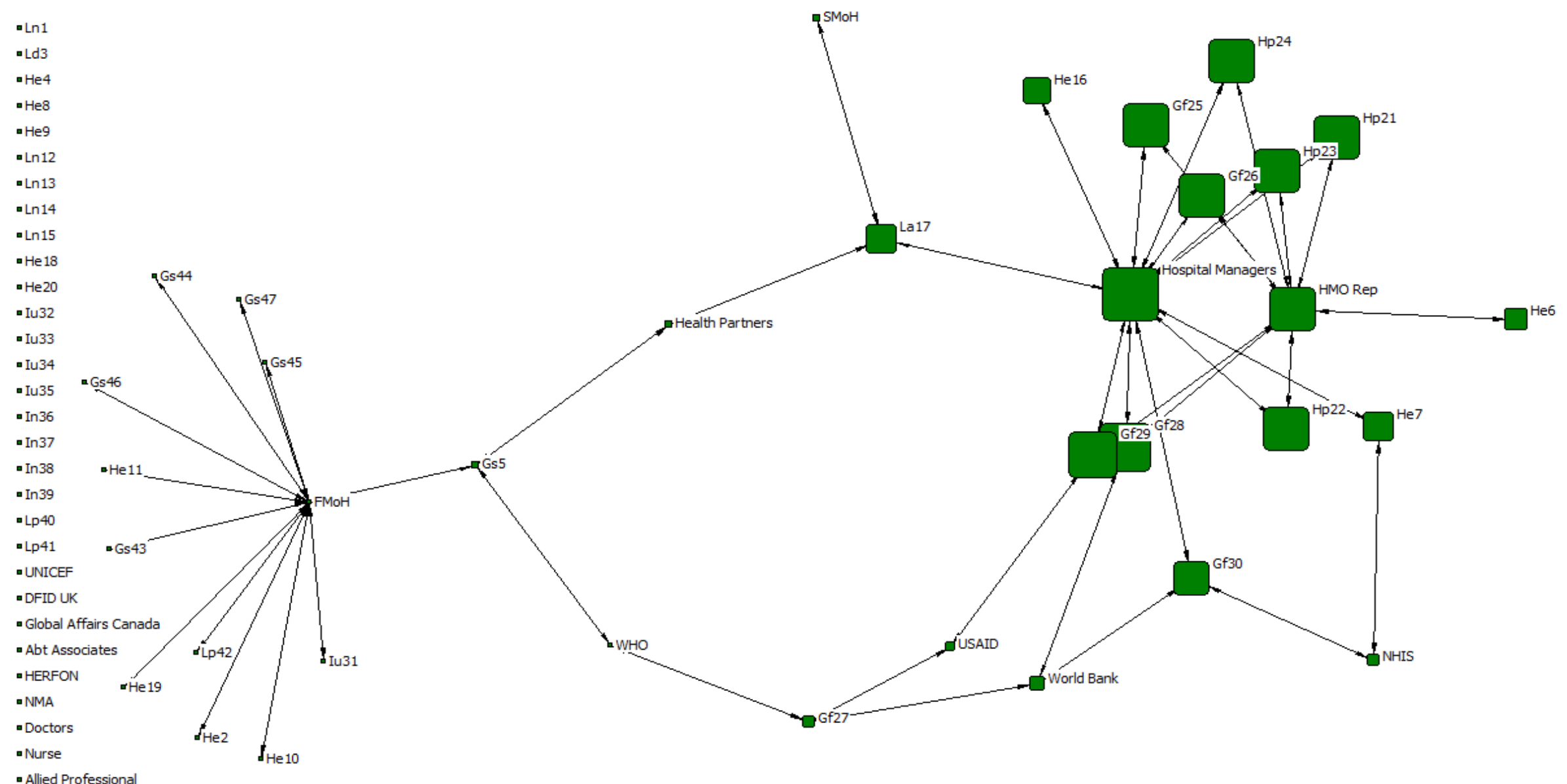

Figure 79: Visualization of the eigenvector centrality measure of the (bipartite graph) “conflict” relationship of the policy network. 


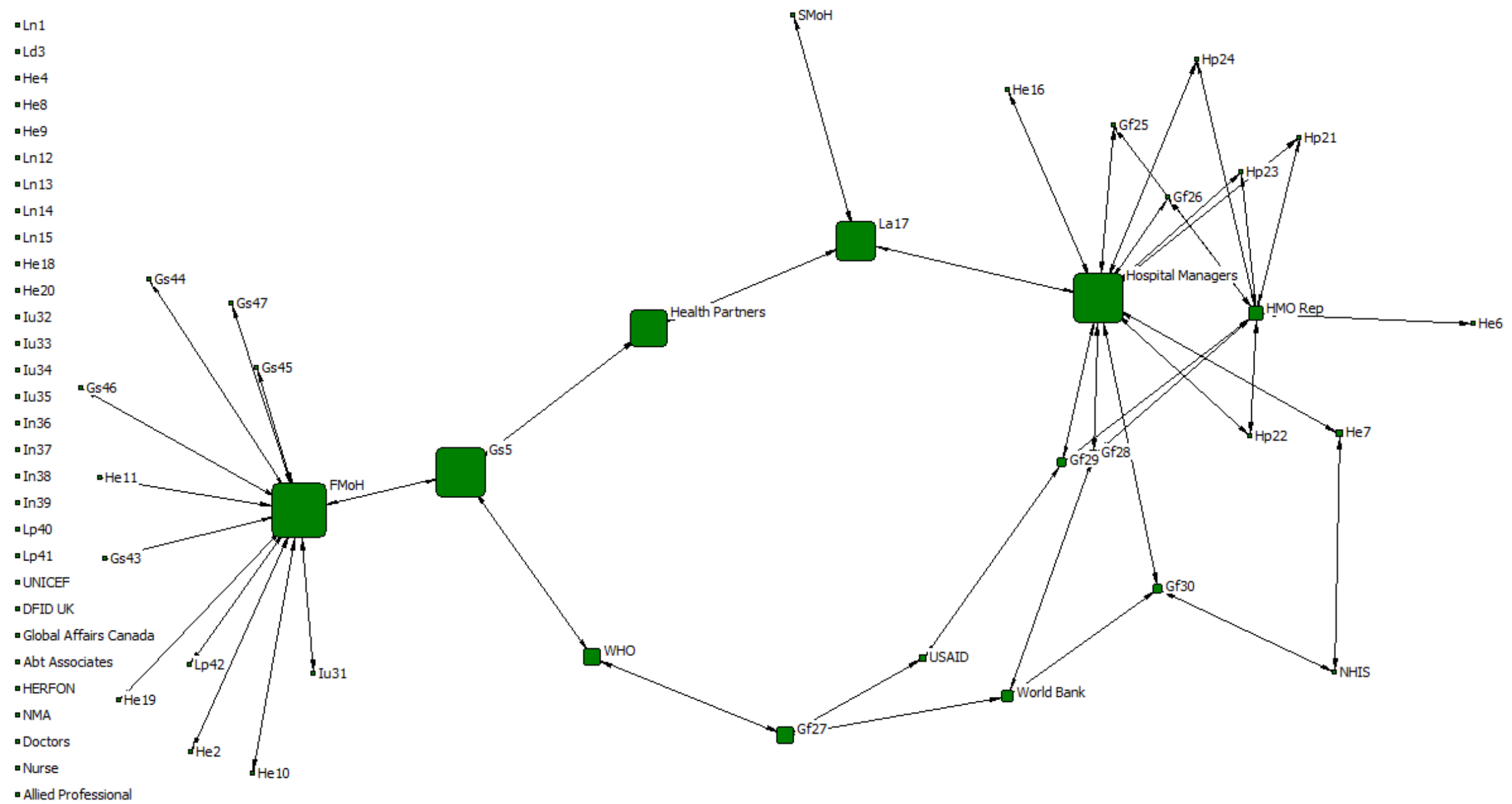

Figure 80: Visualization of the betweenness centrality measure of the (bipartite graph) “conflict” relationship of the policy network. 


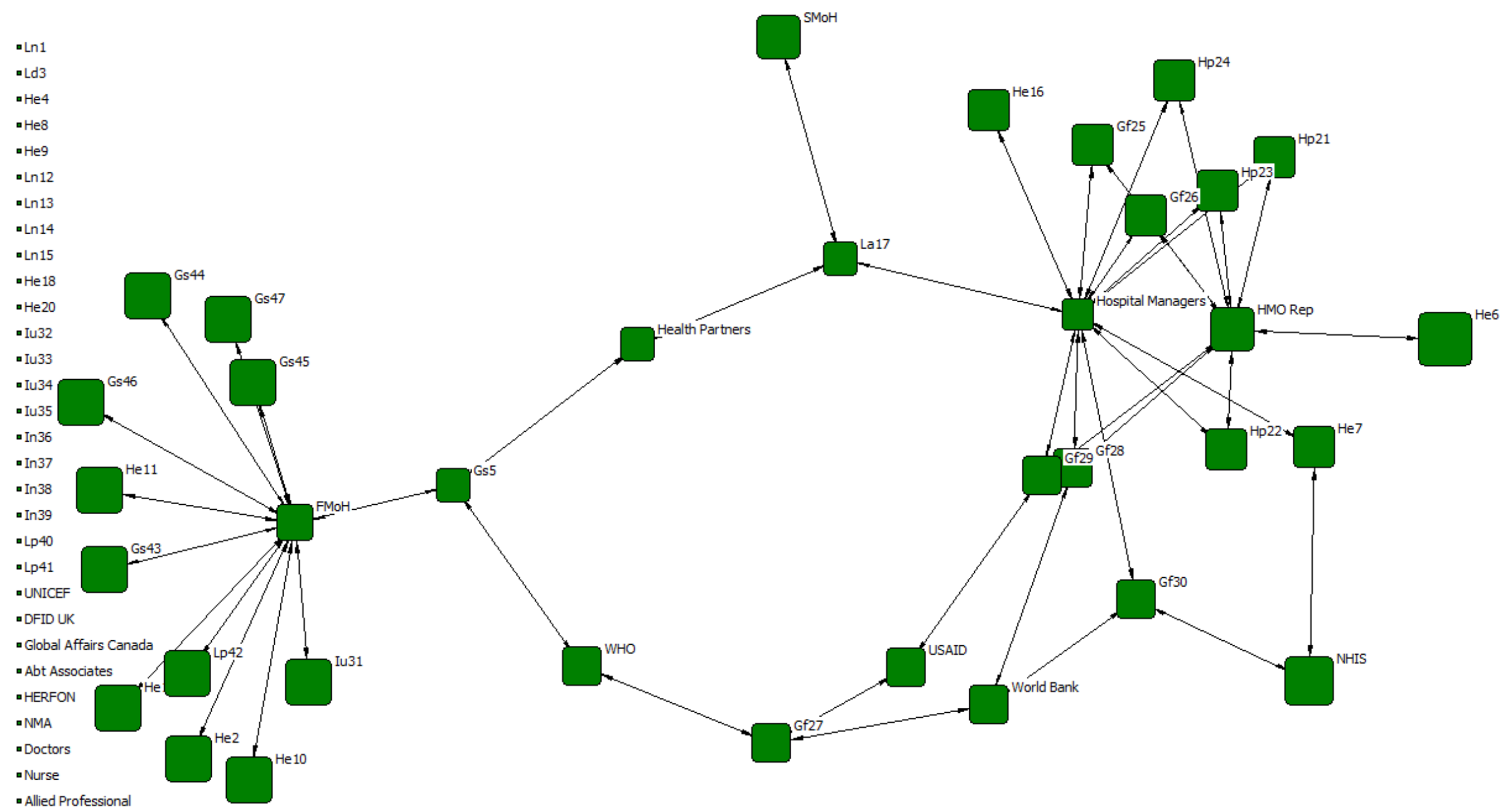

Figure 81: Visualisation of the closeness centrality measure of the (bipartite graph) “conflict” relationship of the policy network. 
Appendix 11: Examples of SNA measures from the advice relationship of the policy network.

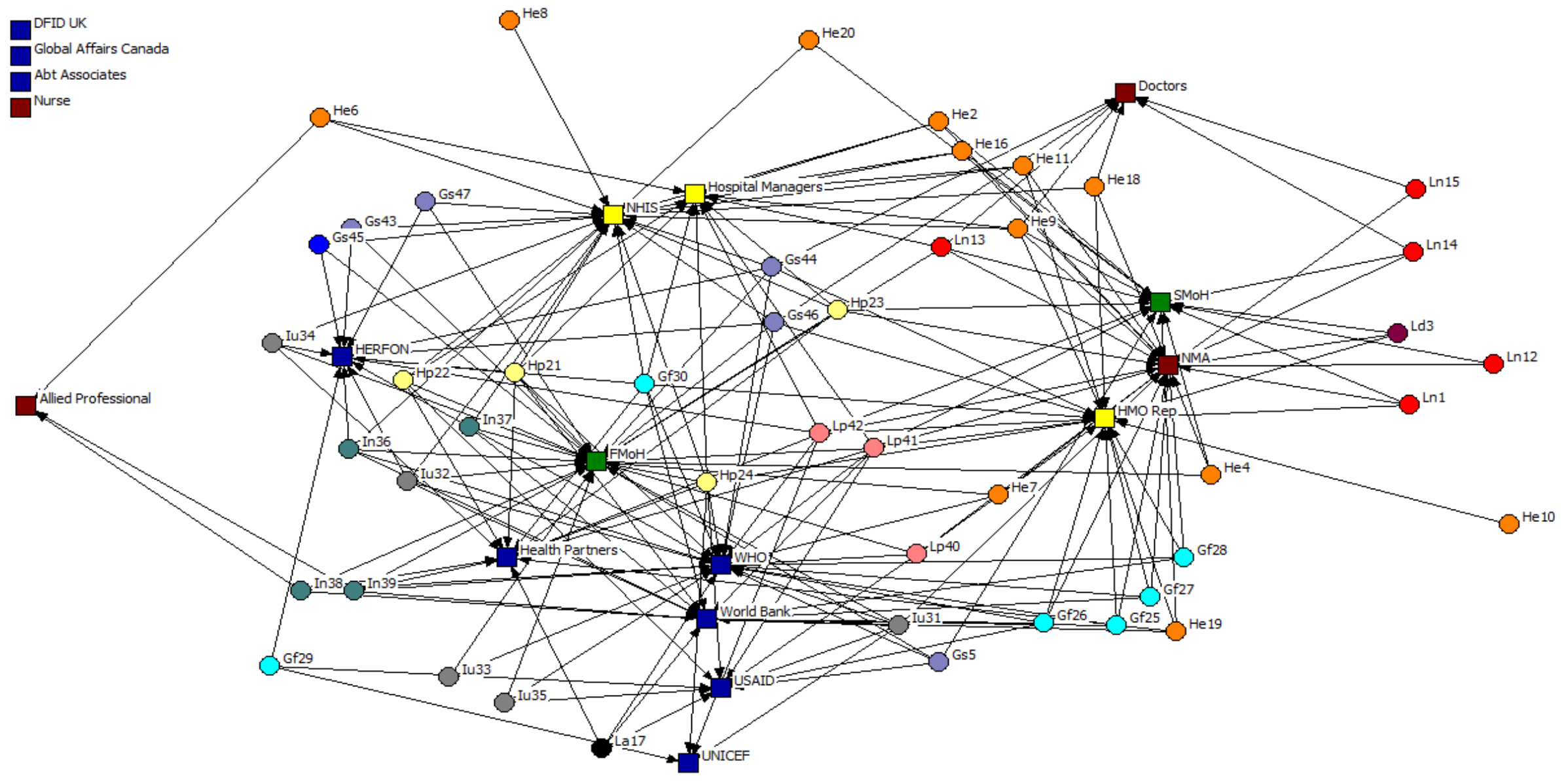

Figure 82: Visualization of (dichotomized graph) “advice” relationship of the policy network. 


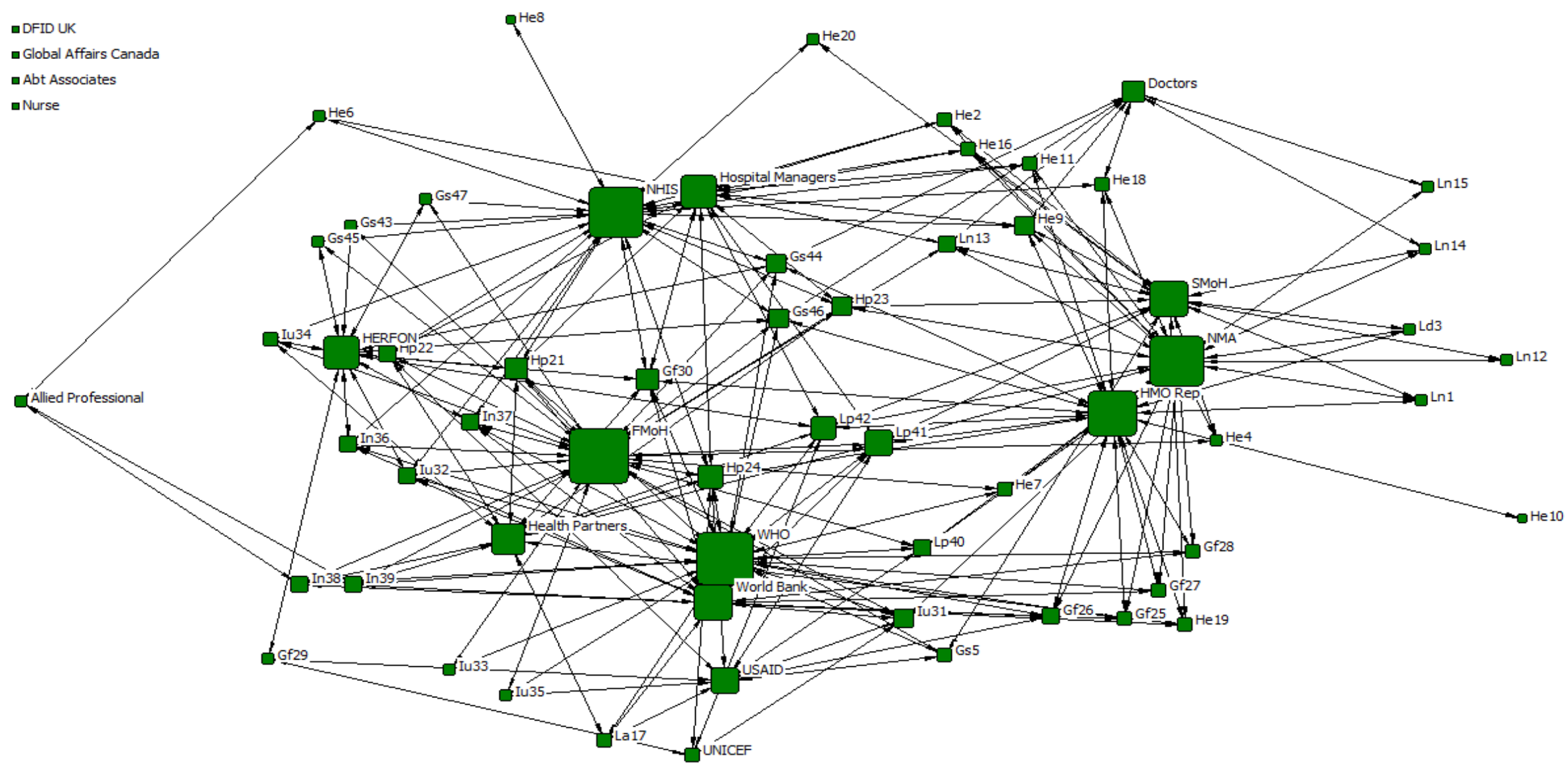

Figure 83: Visualization of the degree centrality measure of the (bipartite graph) “advice” relationship of the policy network. 


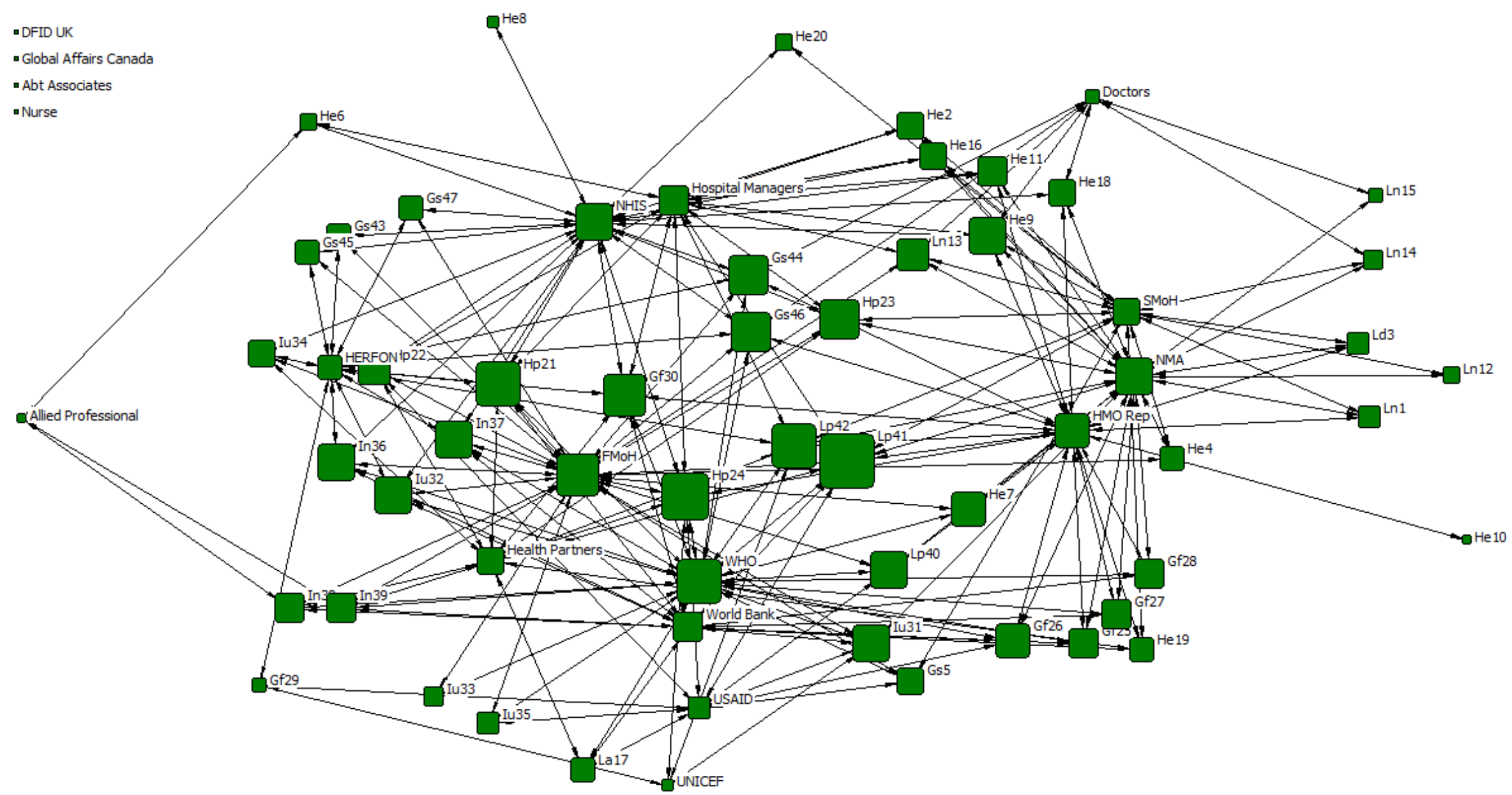

Figure 84: Visualization of the eigenvector centrality measure of the (bipartite graph) “advice” relationship of the policy network. 


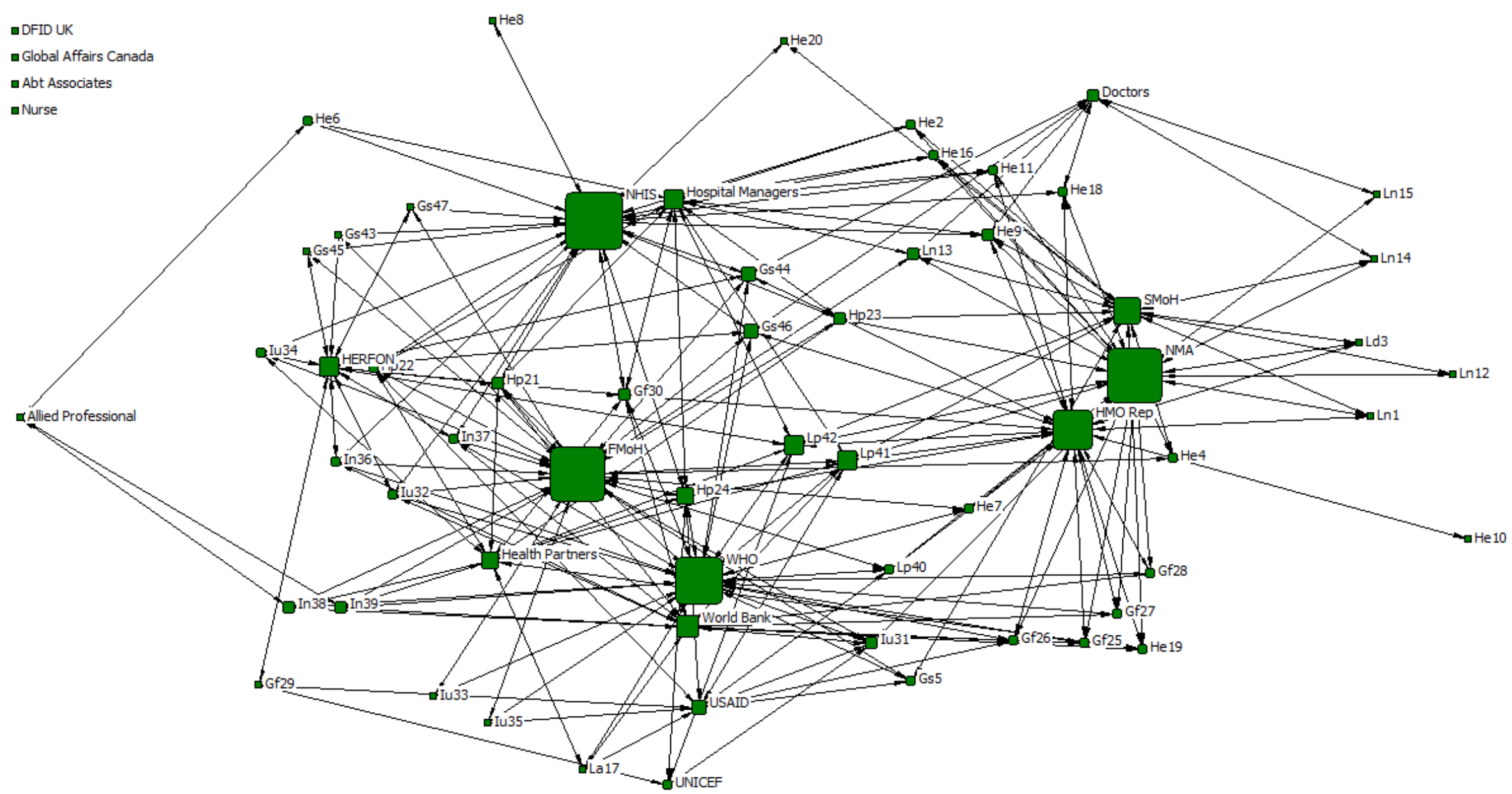

Figure 85: Visualisation of the betweenness centrality measure of the (bipartite graph) "advice” relationship of the policy network. 


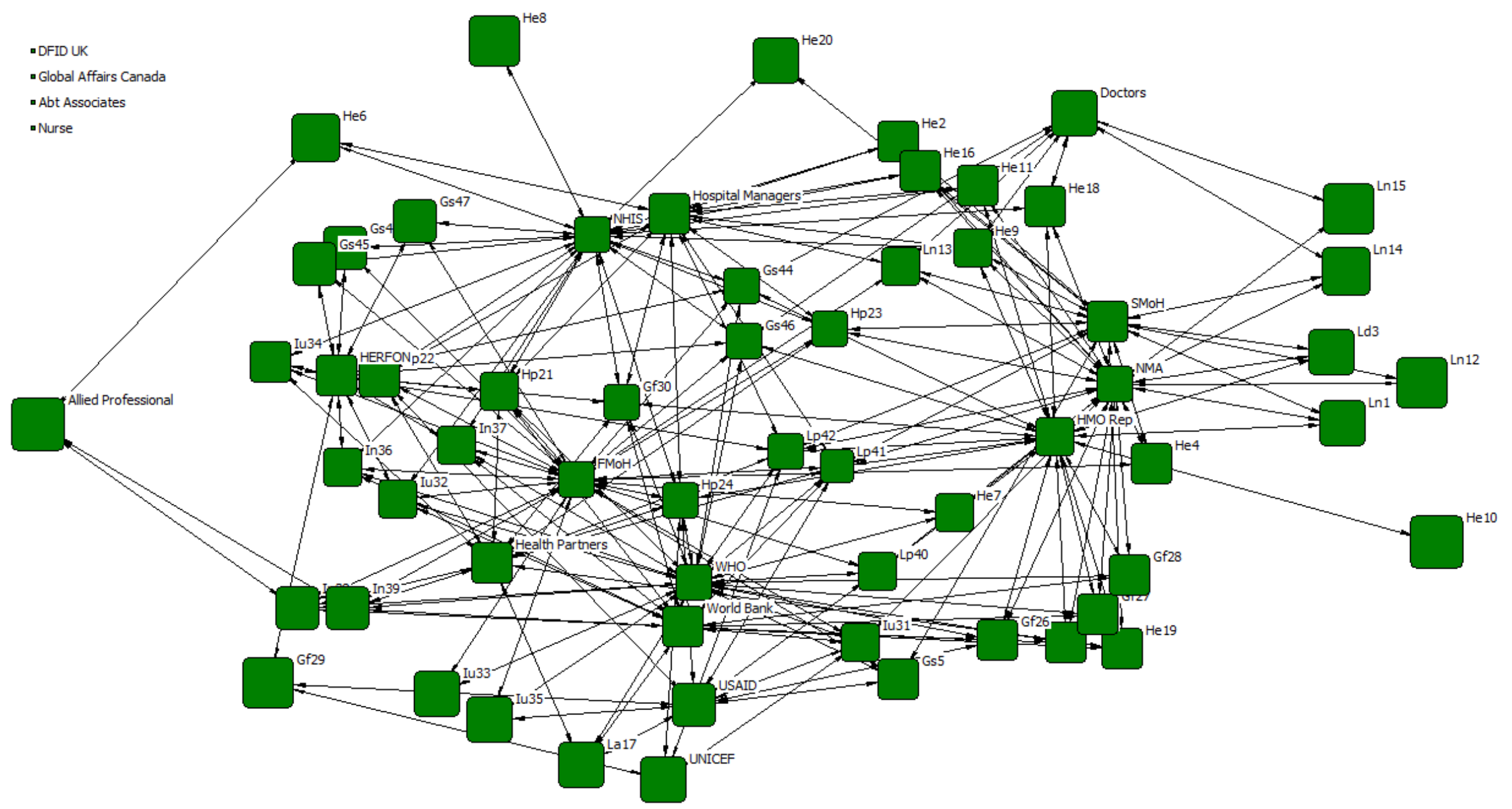

Figure 86: Visualisation of the closeness centrality measure of the (bipartite graph) “advice” relationship of the policy 
Appendix 12: Example of a reflexive diary

\section{dialkshop's \\ WETIN LAWYERS DEY DO...SEF?}

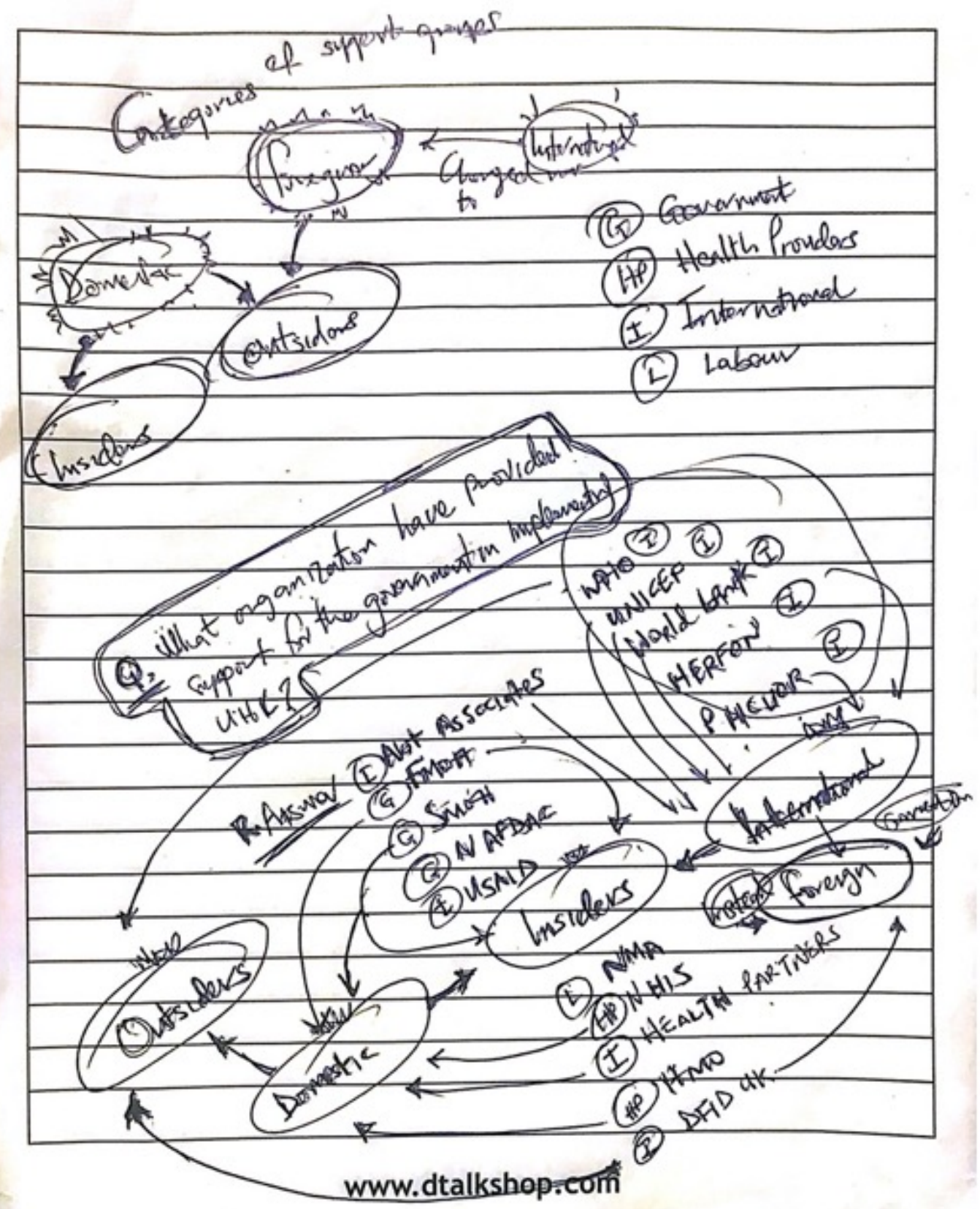

Scanned with CamScanner 